Keywords: Tank Farm

Ammonia

Partial Pressure

Retention Time: Permanent

\title{
Ammonia Henry's Law Constants in SRS High Level Waste Pump Tanks
}

\author{
R. F. Swingle \\ M. R. Poirier \\ C. J. Berry \\ T. B. Edwards \\ J. E. Young
}

Publication Date: January 4, 2001 
This document was prepared in conjunction with work accomplished under Contract No.

DE-AC09-96SR18500 with the U.S. Department of Energy.

\section{DISCLAIMER}

This report was prepared as an account of work sponsored by an agency of the United States Government. Neither the United States Government nor any agency thereof, nor any of their employees, makes any warranty, express or implied, or assumes any legal liability or responsibility for the accuracy, completeness, or usefulness of any information, apparatus, product or process disclosed, or represents that its use would not infringe privately owned rights. Reference herein to any specific commercial product, process or service by trade name, trademark, manufacturer, or otherwise does not necessarily constitute or imply its endorsement, recommendation, or favoring by the United States Government or any agency

thereof. The views and opinions of authors expressed herein do not necessarily state or reflect those of the United States Government or any agency thereof.

This report has been reproduced directly from the best available copy.

Available for sale to the public, in paper, from: U.S. Department of Commerce, National Technical Information Service, 5285 Port Royal Road, Springfield, VA 22161, phone: (800)

553-6847, fax: (703) 605-6900, email: orders@ntis.fedworld.gov online ordering: http://www.ntis.gov/ordering.htm

Available electronically at http://www.doe.gov/bridge

Available for a processing fee to U.S. Department of Energy and its contractors, in paper, from: U.S. Department of Energy, Office of Scientific and Technical Information, P.O. Box 62, Oak Ridge, TN 37831-0062, phone: (865 ) 576-8401, fax: (865) 576-5728, email: reports@ adonis.osti.gov 


\section{SUMMARY}

Testing has been completed to determine the Henry's Law constants for ammonia in SRS High Level Waste (HLW) Pump Tanks. The results obtained using a multiple headspace extraction gas chromatography method showed a high degree of variability, but were in reasonably close agreement with data obtained by Pacific Northwest National Laboratory (PNNL) on one Hanford HLW salt solution. The results did not provide the relief from PNNL's restrictive Henry's Law constants that was initially expected, largely because the concentrations of the salt solutions in the pump tanks were found to be of approximately the same concentration as those tested at PNNL. Without a significant reduction of the salt solution concentration or temperature, the Henry's Law constants will continue to be restrictive. The results did support extrapolation of the PNNL model to $90{ }^{\circ} \mathrm{C}$.

\section{INTRODUCTION}

The High Level Waste Tank Farms store and process high-level liquid wastes from a number of sources including F- and H-Canyons. These wastes are made alkaline prior to transfer to the Tank Farm and are subject to acceptance based on their composition. These wastes may contain significant concentrations of ammonia from flushing of the process vessel vent system. The Authorization Basis for the Tank Farm limits ammonia concentrations in canyon receipts to control flammability in pump tanks and waste tanks. However, during flushing of the canyon process vessel vent systems, the current limits pose significant operational restrictions. It was originally thought that the current limits based on data obtained by Pacific Northwest National Laboratory (PNNL) ${ }^{1,2}$ on a Hanford salt solution might be overly conservative with respect to salt solutions normally found in SRS Pump Tanks. However, on investigation of the possible range of concentrations based on canyon transfer data, it was found that pump tank salt solution concentrations probably did not differ significantly from the salt solution tested by PNNL. Additionally, the PNNL data also only go as high as $70{ }^{\circ} \mathrm{C}$. Concentration, Storage and Transfer Engineering (CSTE) requested the Savannah River Technology Center (SRTC) to examine ammonia partial pressures and Henry's Law constants over less concentrated salt solutions $\left(0.4-2 \mathrm{M} \mathrm{OH}^{-}\right)$, as well as at temperatures up to $90{ }^{\circ} \mathrm{C}{ }^{3}$ This report documents the work performed as originally described in the task technical plan. ${ }^{4}$

\section{DISCUSSION}

\section{Ammonia Vapor-Liquid Equilibrium Experimental Program}

PNNL previously used a vapor pressure measurement technique to measure the Henry's Law constants of ammonia over sodium hydroxide and two different Hanford simulated HLW salt solutions. ${ }^{1}$ That method was initially considered to expand the data to better cover SRS HLW pump tank salt solutions and to extend the temperature range up to $90^{\circ} \mathrm{C}$. There was a concern that the manner in which PNNL added ammonia to the simulated salt solutions might lead to experimental inaccuracies and that the tests could not be performed at temperatures as high as $90{ }^{\circ} \mathrm{C}$. PNNL added aqueous ammonia to the salt solution after measuring the vapor pressure of water above the salt solution. This addition of extra water could have diluted the salt solution which would have increased the vapor pressure of water over the solution that could have manifested itself as high ammonia vapor pressure. Therefore, another method was considered. 
Recently, a multiple headspace extraction (MHE) method of headspace gas chromatography (HSGC) was developed by Zhu and Chai of the Institute of Paper Science and Technology to automate the measurement of vapor-liquid equilibrium of volatile solutes over a solvent phase. ${ }^{5}$ Since the equipment needed to carry out these measurements was already available on-site, it was decided to use this technique. The equipment used to make these measurements included an HP-7963 automatic headspace sampler connected to an HP-5890 gas chromatograph owned by the Environmental Sciences and Technology Department (ESTD) of SRTC.

Initially the gas chromatograph was calibrated for the method using a methanol-water solution and comparing with data provided by Zhu and Chai. ${ }^{5}$ Researchers then ran the tests by placing small amounts of the salt solution containing varying quantities of ammonia into vials specifically designed for the headspace sampler. Each vial was heated to the appropriate temperature and agitated for 30 minutes to equilibrate the ammonia. A sample was pulled and analyzed for ammonia concentration. The vial was allowed to re-equilibrate for 10 minutes and was sampled again. This equilibration-sampling process was repeated ten times as the ammonia concentration decreased. The Henry's Law Constants were calculated using the results of the multiple concentration analyses (see equations $1-12$ below).

Table 1 gives the contents of the salt solutions that were used in this experimental program along with the salt solutions used by PNNL for comparison. We prepared three salt solutions ranging from $0.4 \mathrm{M} \mathrm{NaOH}$ to $2 \mathrm{M} \mathrm{NaOH}$ coinciding with $\mathrm{NaNO}_{2}$ concentration range of $0.8 \mathrm{M}$ to $0 \mathrm{M}$, respectively $\left(\mathrm{Na}^{+}\right.$ concentration from 6.1 to $6.9 \mathrm{M}$ ). This covered the concentration ranges anticipated in the pump tanks as based on the canyon waste compliance $\operatorname{plan}^{6}$ and some undocumented canyon transfer data. The concentration of $\mathrm{NaNO}_{3}$ used in the simulated salt solutions tested was based on the maximum expected from the waste compliance plan. The $\mathrm{Na}_{2} \mathrm{SO}_{4}$ and $\mathrm{NaAl}(\mathrm{OH})_{4}$ concentrations were based on the average of results from undocumented canyon transfer data. Total ammonia concentration was not expected to have a significant effect on the Henry's Law constants for ammonia in the salt solutions. However, we tested three different ammonia concentrations to verify this. The three different ammonia concentrations were approximately $0.5,2.0$ and 3.5 molal in ammonia. A temperature range of $30-90{ }^{\circ} \mathrm{C}$ was initially requested to be tested. The instrument used to conduct the testing has a realistic minimum temperature of $\sim 50{ }^{\circ} \mathrm{C}$. Therefore a temperature range of $50-90{ }^{\circ} \mathrm{C}$ with intervals of $10^{\circ} \mathrm{C}$ was used. The data obtained from these tests were then compared to the model developed by Pitzer ${ }^{7}$ and used by Norton and Pederson at PNNL.

Table 1. Concentration (M) of Simulated Waste Solutions

\begin{tabular}{|c|c|c|c|c|c|}
\hline Component & Solution & Solution & Solution & PNNL Simulant A & PNNL Simulant B \\
\hline & $\underline{1}$ & $\underline{2}$ & $\underline{\mathbf{3}}$ & & \\
\hline $\mathrm{NaOH}$ & 0.4 & 1.0 & 2.0 & 2.3 & 1.7 \\
\hline $\mathrm{NaNO}_{3}$ & 4.1 & 4.1 & 4.1 & 3.7 & 1.7 \\
\hline $\mathrm{NaNO}_{2}$ & 0.8 & 0.4 & 0 & 3.2 & 2.0 \\
\hline $\mathrm{NaAl}(\mathrm{OH})_{4}$ & 0.35 & 0.35 & 0.35 & 2.2 & 0.43 \\
\hline $\mathrm{Na}_{2} \mathrm{CO}_{3}$ & - & - & - & 0.6 & 0.2 \\
\hline
\end{tabular}


WSRC-TR-2000-00226

Revision 0

Page 4 of 18

January 4, 2001

$\mathrm{Na}_{2} \mathrm{SO}_{4}$

$\underline{0.23}$

$\underline{0.23}$

$\underline{0.23}$

Total $\mathrm{Na}^{+}$

6.1

6.3

6.9

12.6

6.2

Experimental Theory

The multiple headspace method assumes a basic mass balance for the solute gas in the vessel. Equation [1] gives the mass balance for the gas.

$$
m_{1}=C_{G 1} V_{G}+C_{L 1} V_{L}
$$

where $m_{1}=$ the total mass of the solute gas in the vessel,

$C_{G 1}$ and $C_{L 1}=$ the concentration of the solute gas in the vapor and liquid phases initially, respectively and $V_{G}$ and $V_{L}=$ the volumes of the vapor and liquid phase, respectively.

Equation [2] gives the mass of the solute gas released each time a sample is pulled from the vessel $\left(m_{E X, \text { solute }}\right)$.

$$
m_{E X, \text { solute }}=\varphi C_{G} V_{G}
$$

where $\varphi=$ the fraction of the gas in the vapor space released when the vessel is sampled.

The total mass of the solute gas remaining in the test vessel after any given sample is pulled $\left(m_{n}\right)$ is represented by equation [3].

$$
m_{n}=\left(C_{G n} V_{G}+C_{L n} V_{L}\right)=m_{1}-V_{G}\left(\varphi_{1} C_{G 1}+\varphi_{2} C_{G 2}+\ldots .+\varphi_{n-1} C_{G(n-1)}\right)=m_{1}-V_{G} \sum_{1}^{n-1} \varphi_{i} C_{G i}
$$

where $m, C_{G}, C_{L}, V_{G}, V_{L}$ and $\varphi$ are as described above. Knowing that test vessel is pressurized to the same pressure prior to pulling each sample, it can be assumed that the value of $\varphi$ remains constant for any given set of GC operating conditions. Given a Henry's Law Constant as described by equation [4],

$$
H_{C}=\frac{C_{V}}{C_{L}}
$$

equation [3] may be rewritten as equation [5].

$$
C_{G n}\left(V_{G}+\frac{V_{L}}{H_{C}}\right)=m_{1}-\varphi V_{G} \sum_{1}^{n-1} C_{G i}
$$

Given that the solute concentration in the headspace, $C_{G}$, is proportional to the measured GC peak height, $A$, (i.e., $A=f C_{G}$ ), equation [5] can be expressed as 
Page 5 of 18

January 4, 2001

$$
\sum_{1}^{n-1} A_{i}=a+b A_{n}
$$

with

$$
a=\frac{f m_{1}}{\varphi V_{G}}
$$

and

$$
b=-\frac{1}{\varphi}\left(1+\frac{1}{H_{c}} \frac{V_{L}}{V_{G}}\right)=-\frac{1}{\varphi}\left(1+\frac{1}{\beta H_{c}}\right)
$$

where

$$
\beta=\frac{V_{L}}{V_{G}}
$$

Zhu and Chai indicate that plotting of data against a fit derived using equation [6] should give an excellent linear fit. ${ }^{5}$ These plots were used to filter out potentially bad data. Rearranging equation [8] gives

$$
\varphi=\frac{-1}{b}\left(1+\frac{1}{\beta H_{c}}\right)
$$

and

$$
H_{C}=\frac{1}{(1+\varphi b) \frac{V_{G}}{V_{L}}} \text {. }
$$

Using a system with known Henry's Law constants, equations [6] and [10] may be used to calculate $\varphi$ for a given set of GC operating conditions (constant temperature and $\beta$ ) and with the value for $\varphi$, equations [6] and [11] may be used to calculate values of $H_{c}$ for any other system run under the same GC operating conditions.

It should be noted that Henry's Law constants are discussed in two forms within this report: a dimensionless value discussed above and a value with units of mole/kg*atm used by PNNL. The dimensionless value can be converted to the value with units using equation [12].

$$
H_{C}=\frac{1}{H_{c, P N N L} R T_{K}}
$$


where $H_{c} \quad=$ Henry's Law Constant (dimensionless),

$H_{c, P N N L}=$ Henry's Law Constant (mole $\left./ \mathrm{kg}^{*} \mathrm{~atm}\right)$,

$R \quad=$ Ideal Gas Constant $(0.08205$ L*atm/g-mole*K) and

$T_{K} \quad=$ Temperature in Kelvin.

\section{Experimental Results}

A methanol-water system was used to calibrate the GC for $\varphi$. The $H_{c}$ data used to calculate the values of $\varphi$ for the GC as operated were taken from Reference 5. Figure 1 provides a plot of the experimental $\varphi$ values along with a fit of the data. Appendix A provides more detail on the derivation of the $\varphi$ values used as well as the experimental data.

\section{Figure 1. Values of $\varphi$ Derived from a Methanol-Water System}

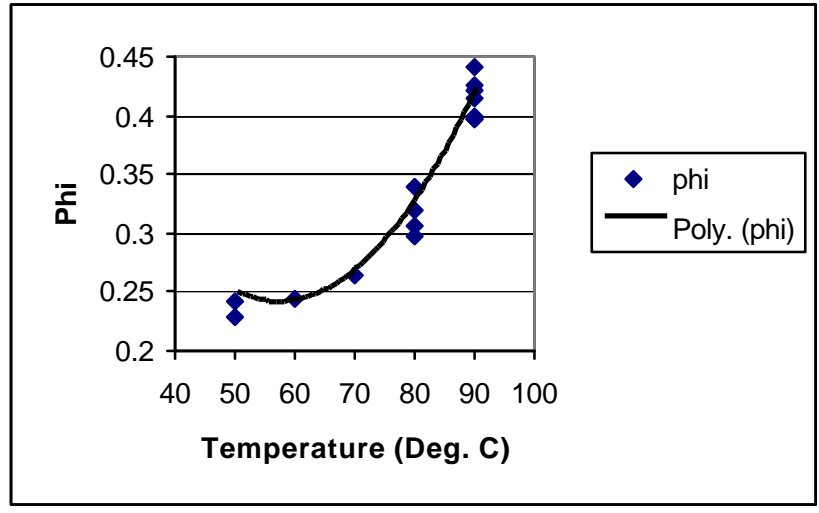

Once the values of $\varphi$ for the system parameters used was determined, the salt solutions described above were run using the procedure described above. The resulting GC peak areas were fit to equation [6]. The resulting slopes were used to calculate values for $H_{C}$ from equation [11]. The resulting values of $H_{c}$ showed considerable variability and were screened as described in Appendix A. The $H_{c}$ values for screened data are shown in Figures 2, 3 and 4 for salt solutions 1, 2 and 3, respectively.

Figure 2. Experimentally Derived Values of $\boldsymbol{H}_{c}$ for Screened Data from Salt Solution 1 
WSRC-TR-2000-00226

Revision 0

Page 7 of 18

January 4, 2001

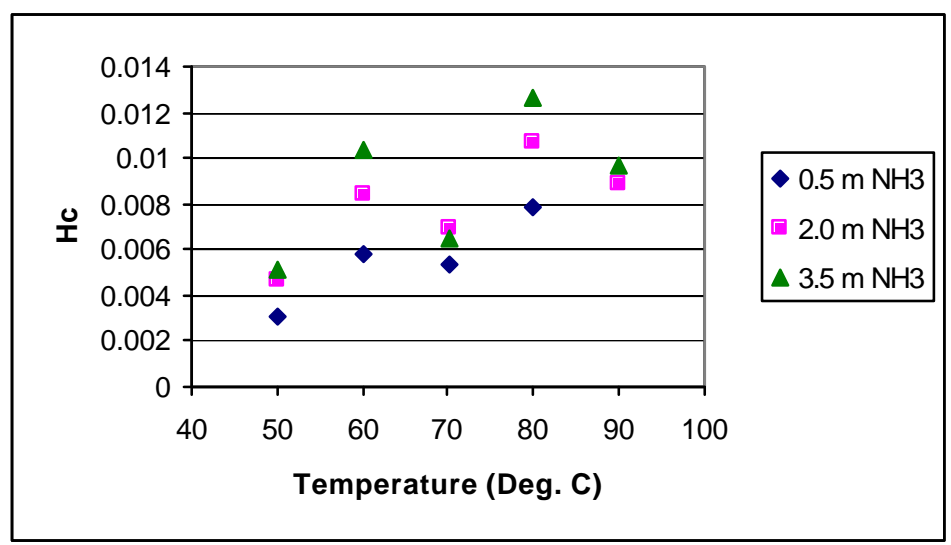


Figure 3. Experimentally Derived Values of $H_{c}$ for Screened Data from Salt Solution 2

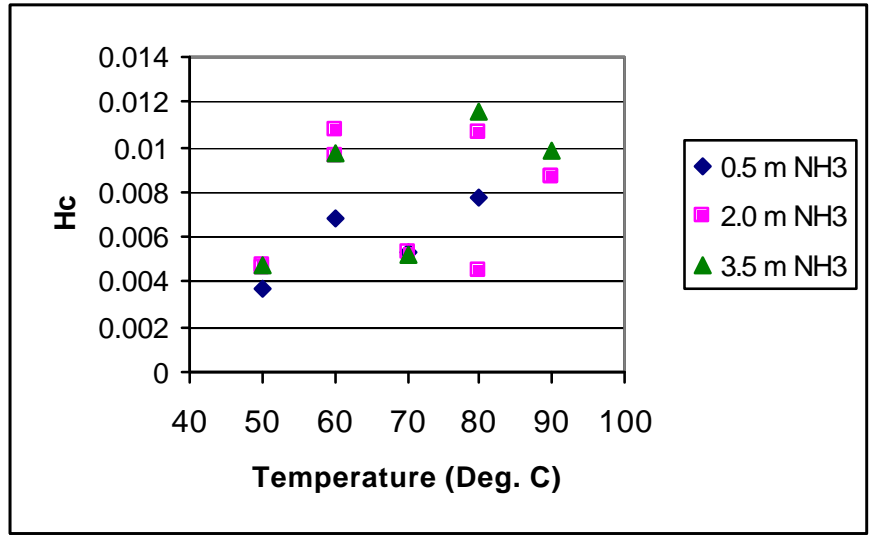

Figure 4. Experimentally Derived Values of $\boldsymbol{H}_{c}$ for Screened Data from Salt Solution 3

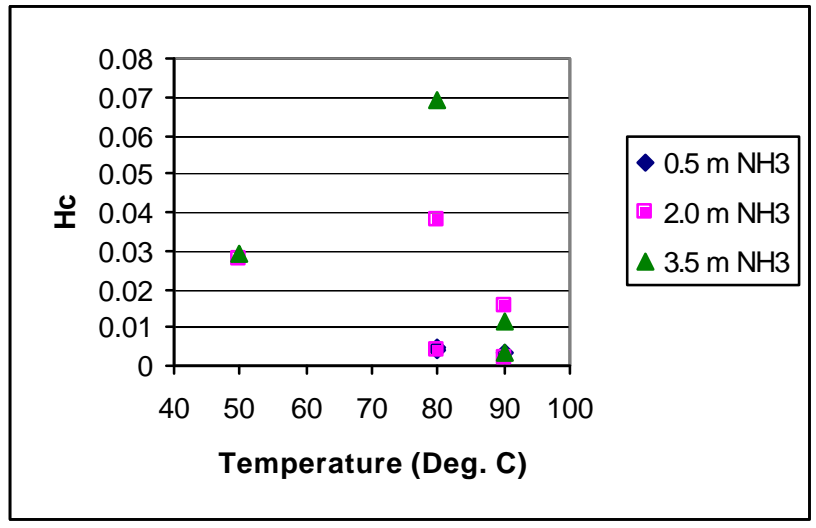

There is some indication of an effect of ammonia concentration on $H_{c}$ for salt solution 1, but no indication of an effect of ammonia concentration on the value of $H_{c}$ for either salt solution 2 or 3 . For salt solution 1 , with one exception, all of the $\mathrm{H}_{\mathrm{c}} \mathrm{s}$ for $0.5 \mathrm{~m} \mathrm{NH}_{3}$ are the lowest at each temperature, the $2 \mathrm{~m} \mathrm{NH}_{3} \mathrm{H}_{\mathrm{c}} \mathrm{s}$ are next and the $3.5 \mathrm{~m} \mathrm{NH}_{3} \mathrm{H}_{\mathrm{c}} \mathrm{s}$ are the highest. In theory, the effect of ammonia concentration on the value of $H_{c}$ for ammonia ought to be very small for an ideal solution.

Because of the considerable variability of data, even after screening, and because the ammonia concentration should have very little effect on the values of $H_{c}$, we decided to average the data at each temperature for each salt solution. These values were then regressed linearly. Figures 5, 6 and 7 show the average Henry's Law Constants at each temperature along with the regressed fit for the data for salt solutions 1, 2 and 3 respectively. These results indicate very poor predictability of these $\mathrm{H}_{\mathrm{c}}$ models developed using temperature alone. The results for Salt Solution 3 show little (if any) temperature effect. The average value at $60{ }^{\circ} \mathrm{C}$ for Salt Solution 2 prevents a statistically significant relationship between these $\mathrm{H}_{\mathrm{c}}$ values and temperature. Even the Salt Solution 1 results show a great deal of scatter, and the fit of the Hc values to temperature is not statistically significant at a $95 \%$ confidence level. 
WSRC-TR-2000-00226

Revision 0

Page 9 of 18

January 4, 2001

Figure 5. Fit of Average Temperature Data for $H_{c}$ for Salt Solution $1\left(R^{2}=0.629\right)$

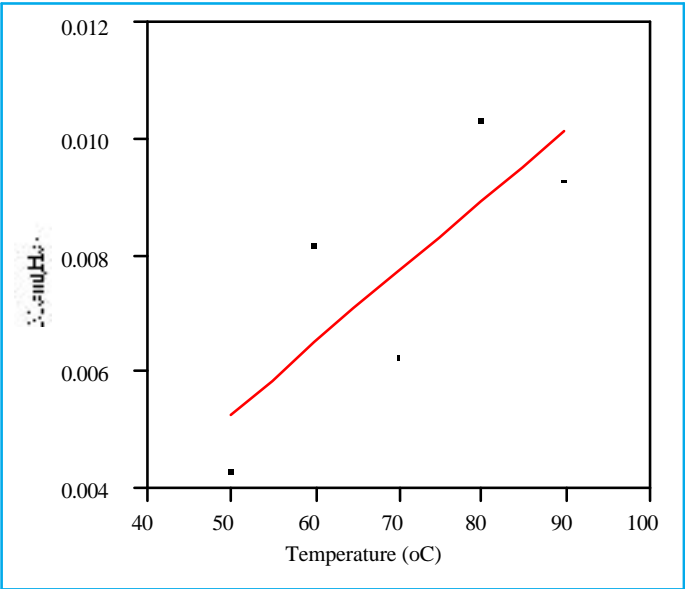

Figure 6. Fit of Average Temperature Data for $H_{c}$ for Salt Solution $2\left(\mathbf{R}^{2}=\mathbf{0 . 2 9 2}\right)$

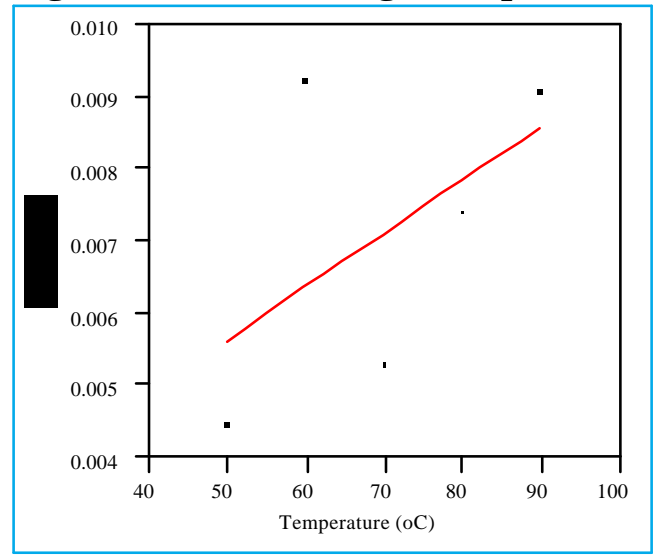

Figure 7. Fit of Average Temperature Data for $H_{c}$ for Salt Solution $3\left(R^{2}=\mathbf{0 . 6 7 2}\right)$

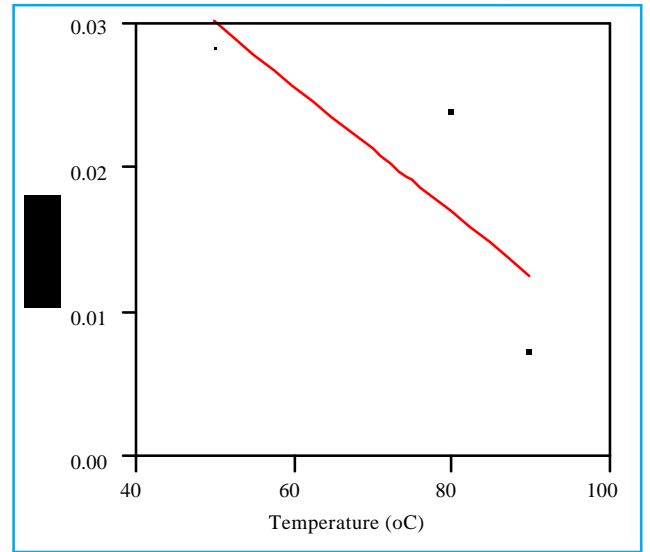


The model of $\mathrm{H}_{\mathrm{c}}$ as a function of both temperature and $\mathrm{NH}_{3}$ molal concentration for Salt Solution 1 is investigated in Figures $8-10$. These figures provide (for each NH3 molal concentration) plots of the experimental $\mathrm{H}_{\mathrm{c}}$ values, the model predictions for the $\mathrm{H}_{\mathrm{c}}$ values, the upper and lower prediction limits for the average $\mathrm{H}_{\mathrm{c}}$ values, and predictions based upon the PNNL model.

Figure 8. Fit of Salt Solution 1 Data at an Ammonia Concentration of 0.5 Molal

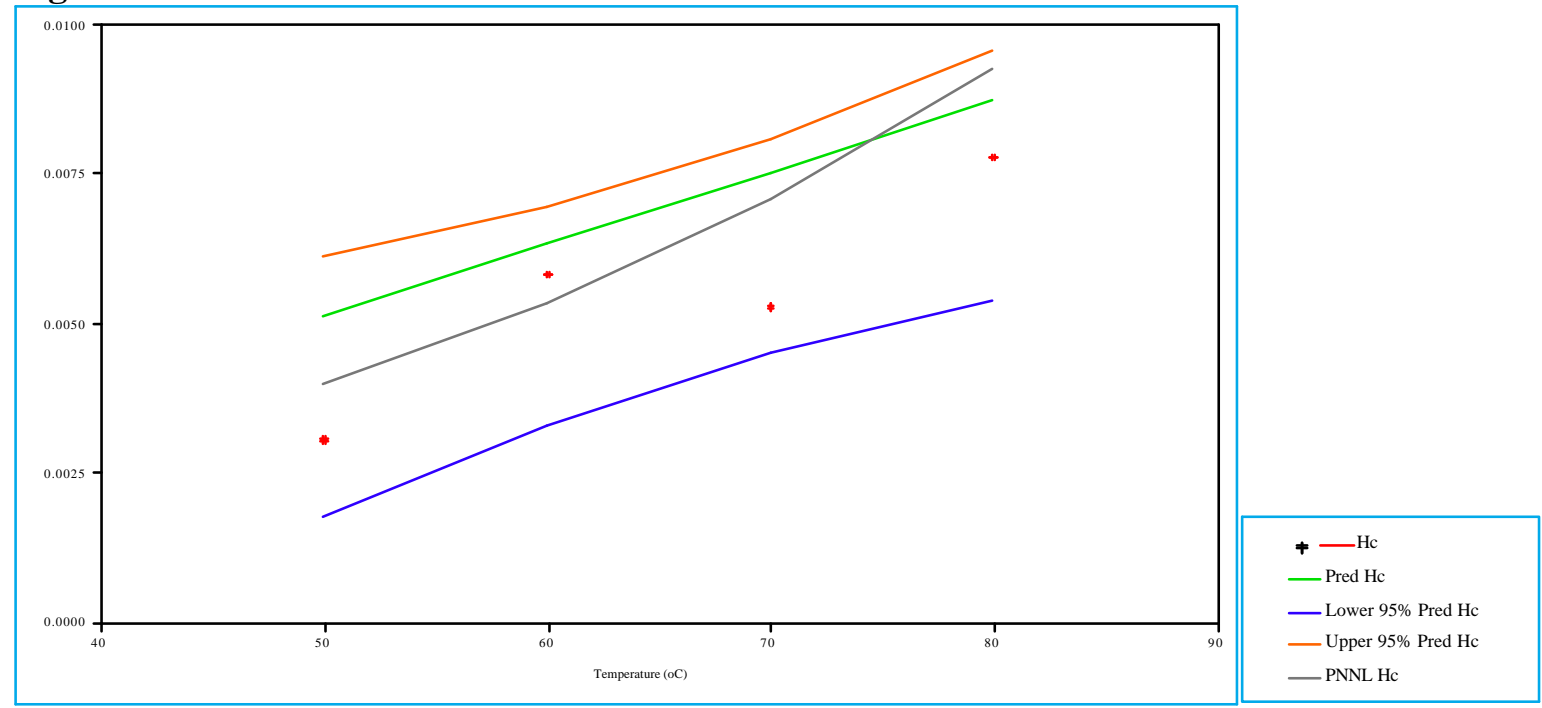

Figure 9. Fit of Salt Solution 1 Data at an Ammonia Concentration of 2.0 Molal

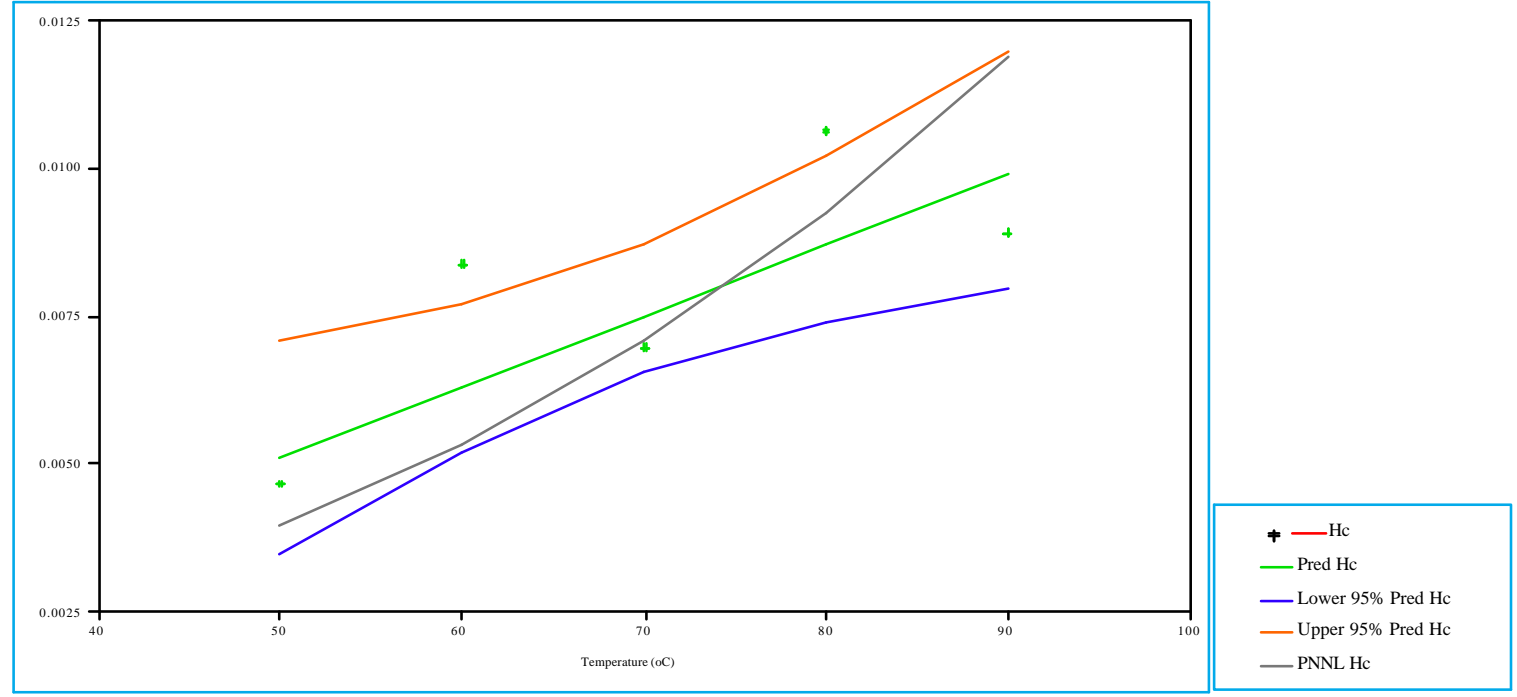


Figure 10. Fit of Salt Solution 1 Data at an Ammonia Concentration of 3.5 Molal

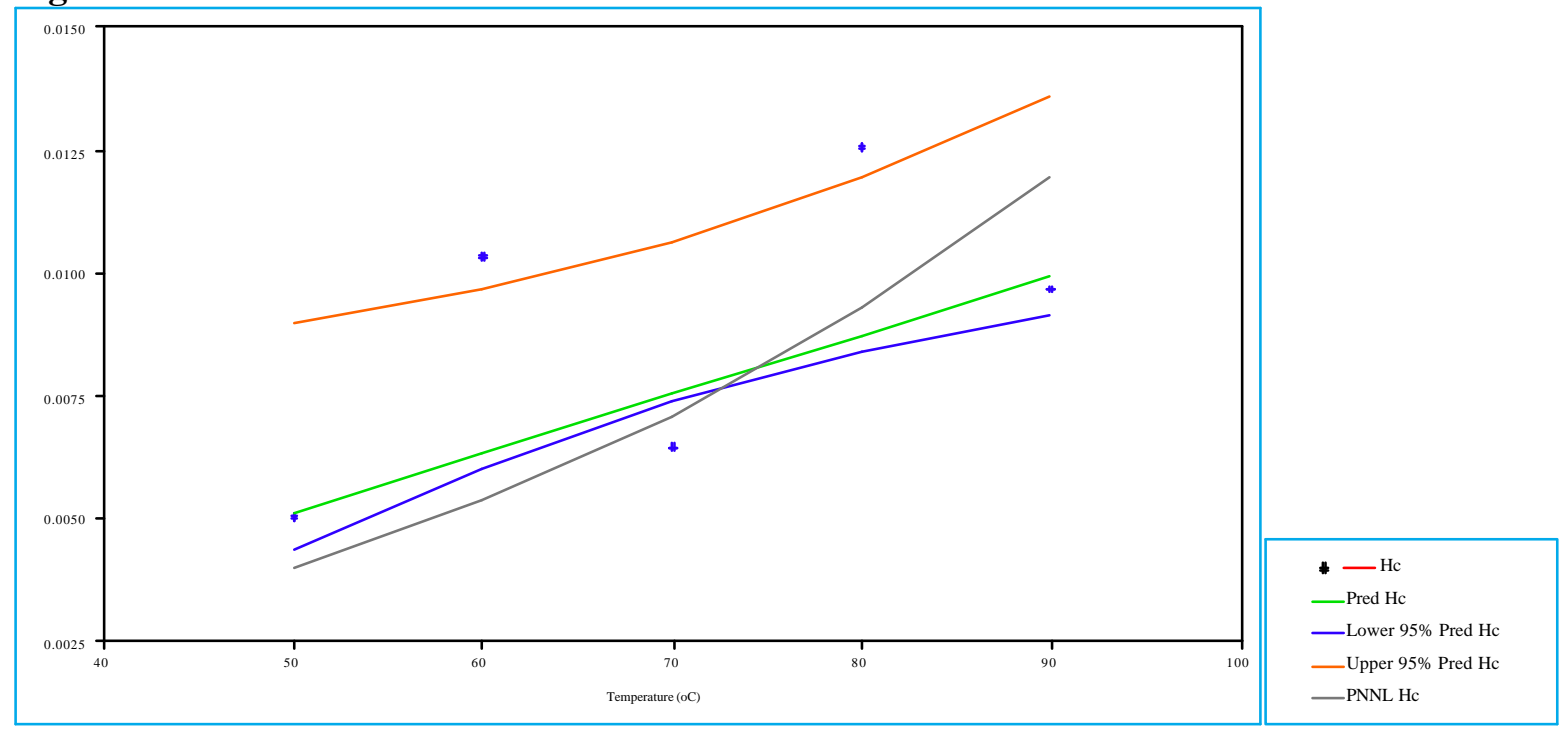

Figure 11 provides plots of the average fits for all three salt solutions along with a plot of the PNNL fit to their Simulant B data. This graph shows that the PNNL data are very similar to the data for SRTC simulated salt solutions 1 and 2.

Figure 11. $H_{c}$ Fits for All Three SRS Simulated Salt Solutions

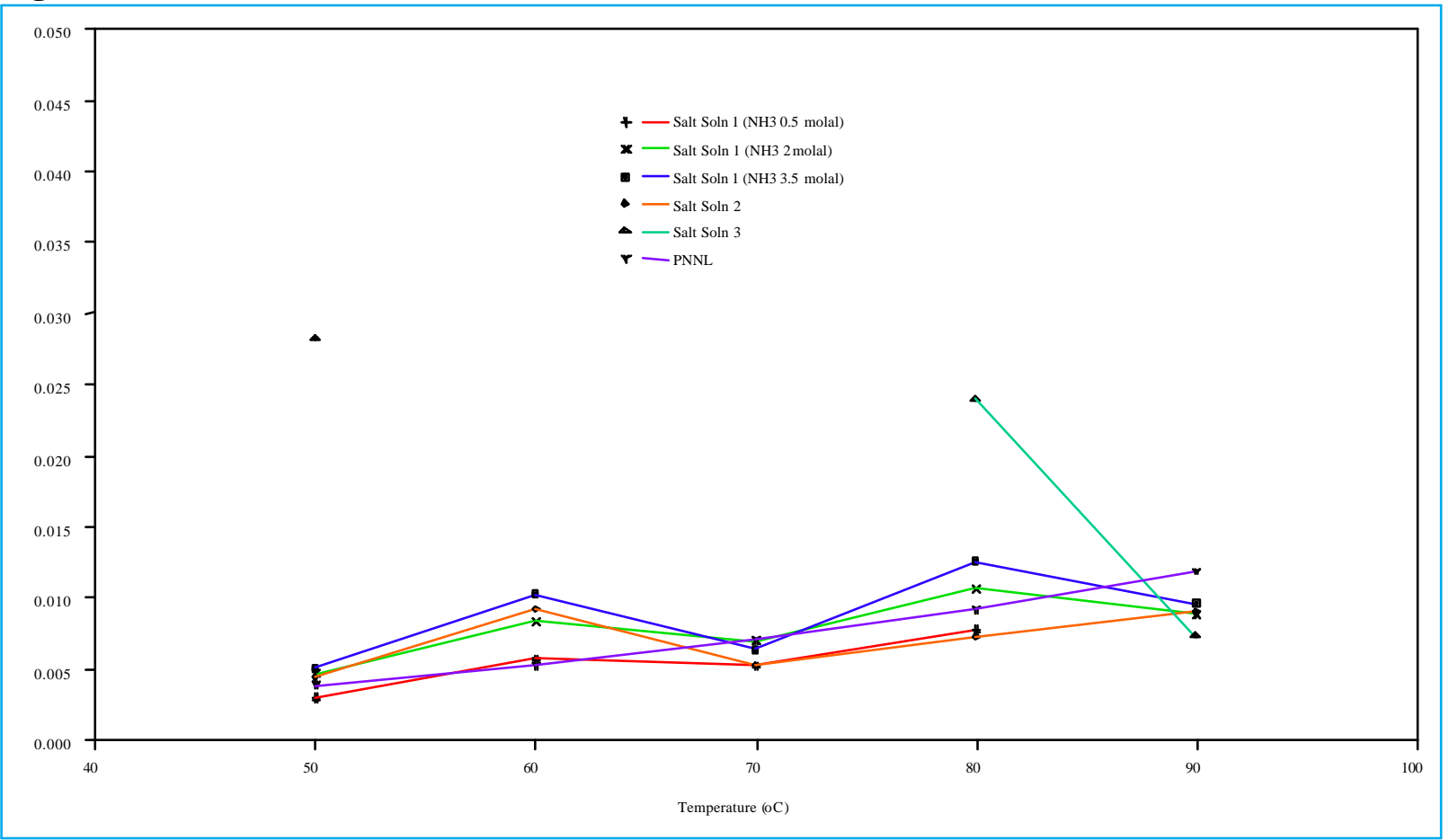


WSRC-TR-2000-00226

Revision 0

Page 12 of 18

January 4, 2001

\section{Modeling Ammonia Vapor-Liquid Equilibrium}

Because of the variety of waste streams present in the SRS high level waste system, developing a model to predict ammonia vapor-liquid equilibrium (VLE) is valuable to the SRS HLWD. The authors evaluated the Pitzer model ${ }^{7}$ for predicting ammonia VLE in SRS waste tanks. The model predicts thermodynamic properties of electrolytes. It can be applied to high ionic strength solutions, and to single and mixed electrolyte systems. It has been used to predict ammonia VLE in electrolytes. ${ }^{1,8}$ Clegg and Brimblecomb determined model constants for a variety of salts over a temperature range of $0-40{ }^{\circ} \mathrm{C} .{ }^{8}$ Norton and Pederson compared model prediction with experimental data for Hanford waste streams and found good agreement with $0-7$ molal $\mathrm{NaOH}$ solutions and a 6.2 molar salt solution over a temperature range of $25-$ $70{ }^{\circ} \mathrm{C}^{1}$

The Henry's Law constant is defined by equation [13].

$$
K_{H}=\frac{\gamma\left(\mathrm{NH}_{3}\right) m\left(\mathrm{NH}_{3}\right)}{p\left(\mathrm{NH}_{3}\right)},
$$

where $\mathrm{K}_{\mathrm{H}}$ is the Henry's Law constant in pure water, $\gamma$ is the activity coefficient for ammonia, $\mathrm{m}$ is the ammonia concentration in the aqueous phase (in molality), and $\mathrm{p}$ is the ammonia partial pressure.

The Henry's Law constant for pure water is described by equation [14]

$$
\ln \left(\mathrm{K}_{\mathrm{H}}\right)=-8.09694+3917.50 / \mathrm{T}\left({ }^{\circ} \mathrm{K}\right)-0.00314 \mathrm{~T}\left({ }^{\circ} \mathrm{K}\right)
$$

The activity coefficient can be calculated with the Pitzer model which is described by equation [15].

$$
\ln \gamma_{\mathrm{NH} 3}=2 \mathrm{~m}_{\mathrm{NH} 3} \lambda_{\mathrm{NN}}+2 \mathrm{~m}_{\mathrm{MX}}\left(\mathrm{v}^{+} \lambda_{\mathrm{NM}}+\mathrm{v}^{-} \lambda_{\mathrm{NX}}\right)+6 \mathrm{~m}_{\mathrm{MX}} \mathrm{m}_{\mathrm{NH} 3}\left(\mathrm{v}^{+} \mu_{\mathrm{NNM}}+\mathrm{v}^{-} \mu_{\mathrm{NNX}}\right)
$$

where $\lambda_{\mathrm{NN}}$ is the ammonia self interaction parameter, $\lambda_{\mathrm{NM}}$ is the ammonia-cation interaction parameter, $\lambda_{\mathrm{NX}}$ is the ammonia-anion interaction parameter, $\mu_{\mathrm{NNM}}$ is the second order ammonia-cation interaction parameter, and $\mu_{\mathrm{NNX}}$ is the second order ammonia-anion interaction parameter.

The ammonia self interaction parameter is defined by equation [16]

$$
\lambda_{\mathrm{NN}}=0.033161-21.12816 / \mathrm{T}\left({ }^{\circ} \mathrm{K}\right)+4665.1461 / \mathrm{T}\left({ }^{\circ} \mathrm{K}\right)^{2}
$$

The first and second order interaction parameters have been compiled for a number of salts and are generally additive for multi-component electrolytes. ${ }^{8}$

The temperature dependency of the ammonia self interaction parameter is described in equation [16]. The other interaction parameters were assumed to be independent of temperature. ${ }^{1}$ Table 2 shows the values of the interaction parameters used in this analysis. 
WSRC-TR-2000-00226

Revision 0

Page 13 of 18

January 4, 2001

Table 2. Ion Interaction Parameters

\begin{tabular}{ll} 
Ion & $\lambda_{\mathrm{N}, \mathrm{i}}$ \\
\hline $\mathrm{Na}$ & 0.0175 \\
$\mathrm{OH}$ & 0.103 \\
$\mathrm{AlO} 2$ & $0.103 *$ \\
$\mathrm{NO} 3$ & -0.01 \\
$\mathrm{NO} 2$ & -0.003 \\
$\mathrm{SO} 4$ & 0.140
\end{tabular}

* This value is an estimate. ${ }^{3}$ No data available for aluminate ion

The model predictions were compared with ammonia VLE measured in this program and by PNNL. ${ }^{1}$

Table 3 shows the conditions under which the model was compared with experimental data.

Table 3. Test Conditions

\begin{tabular}{|c|c|c|c|c|c|}
\hline \multirow[b]{2}{*}{ Species } & \multicolumn{5}{|c|}{ Concentration (molality) } \\
\hline & PNNL & PNNL & PNNL & $\underline{\text { SS\#1 }}$ & $\underline{\mathrm{SS \# 2}}$ \\
\hline $\mathrm{NH}_{3}$ & 0.573 & 0.549 & 0.859 & 0.57 & 0.57 \\
\hline $\mathrm{NaOH}$ & 0.5 & 1.0 & 2.10 & 0.61 & 1.52 \\
\hline $\mathrm{NaNO}_{3}$ & 0 & 0 & 0.54 & 6.34 & 6.34 \\
\hline $\mathrm{NaNO}_{2}$ & 0 & 0 & 2.11 & 1.20 & 0.61 \\
\hline $\mathrm{NaAlO}_{2}$ & 0 & 0 & 2.50 & 0.53 & 0.53 \\
\hline $\mathrm{Na}_{2} \mathrm{CO}_{3}$ & 0 & 0 & 0.25 & 0 & 0 \\
\hline $\mathrm{Na}_{2} \mathrm{SO}_{4}$ & 0 & 0 & 0 & 0.35 & 0.35 \\
\hline
\end{tabular}

Figures 12 - 15 show the comparison of the Henry's Law constants predicted by the model and measured experimentally for the conditions listed in Table 3. The agreement between the model and the experimental PNNL data is good. The difference between the predicted and measured Henry's Law constants is $<10 \%$ at $25{ }^{\circ} \mathrm{C}, 15 \%$ at $50{ }^{\circ} \mathrm{C}$, and $25 \%$ at $70{ }^{\circ} \mathrm{C}$. The agreement between the model and the SRS data is reasonable given the variability of the experimental method.

Figure 12. Comparison of Model and Experimental Data (1 molal $\mathrm{NaOH}$ and 0.573 molal $\mathrm{NH}_{3}$ (PNNL)) 
WSRC-TR-2000-00226

Revision 0

Page 14 of 18

January 4, 2001

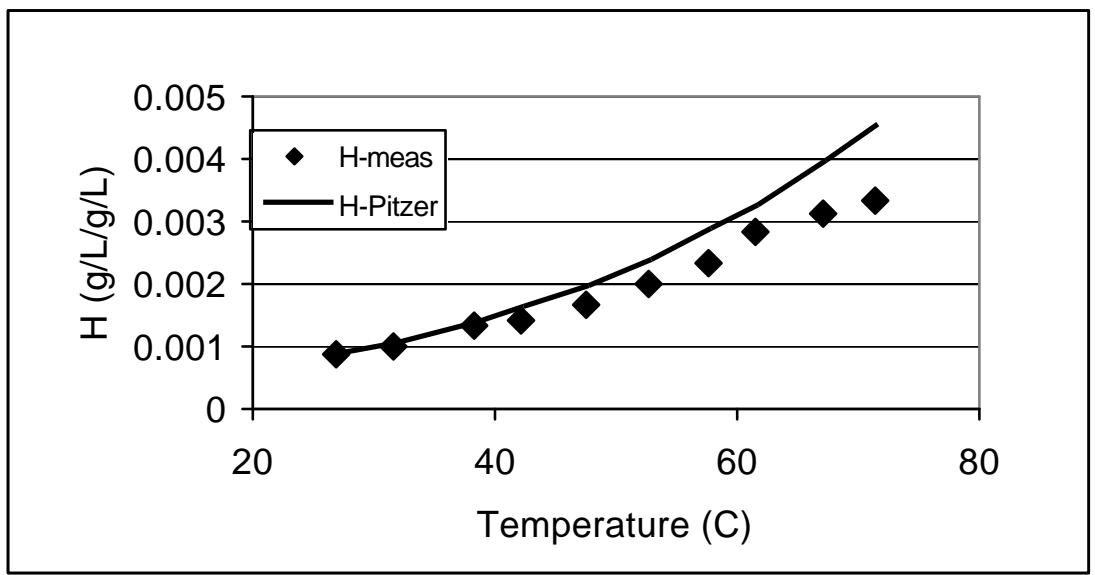


Figure 13. Comparison of Model and Experimental Data (6.2 $\mathrm{M}$ Na and $0.859 \mathrm{~m} \mathrm{NH}_{3}(\mathrm{PNNL})$ )

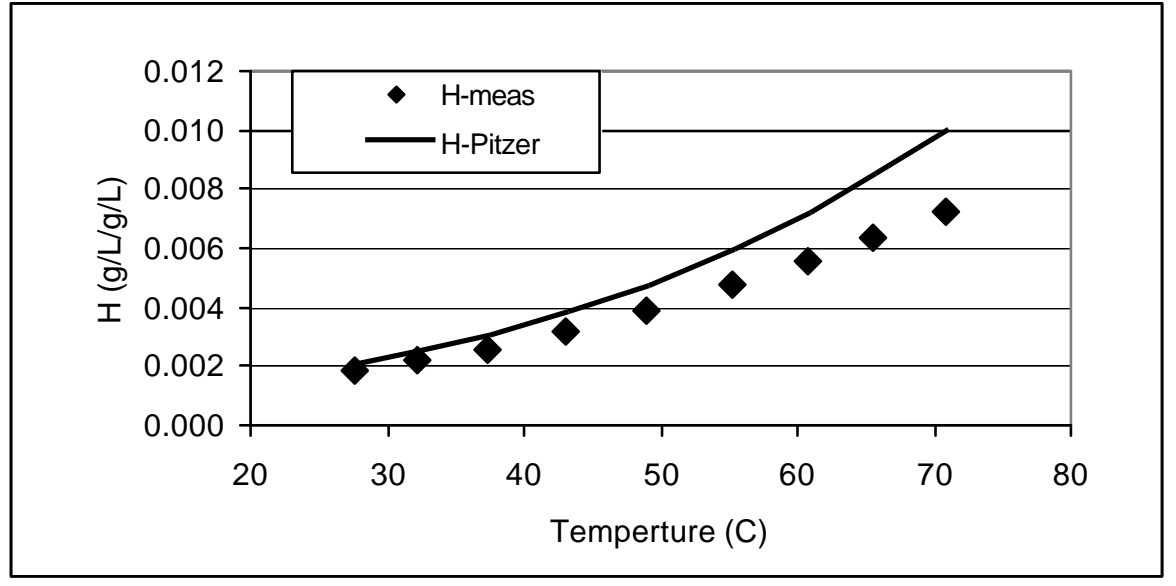

Figure 14. Comparison of Model and Experimental Data (Salt Solution \#1)

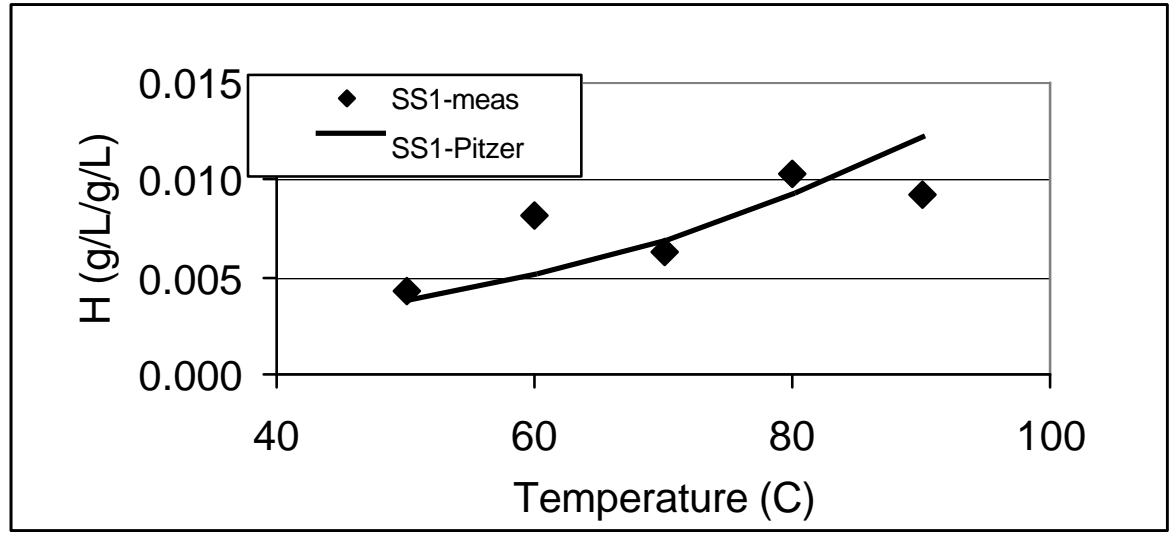

Figure 15. Comparison of Model and Experimental Data (Salt Solution \#2)

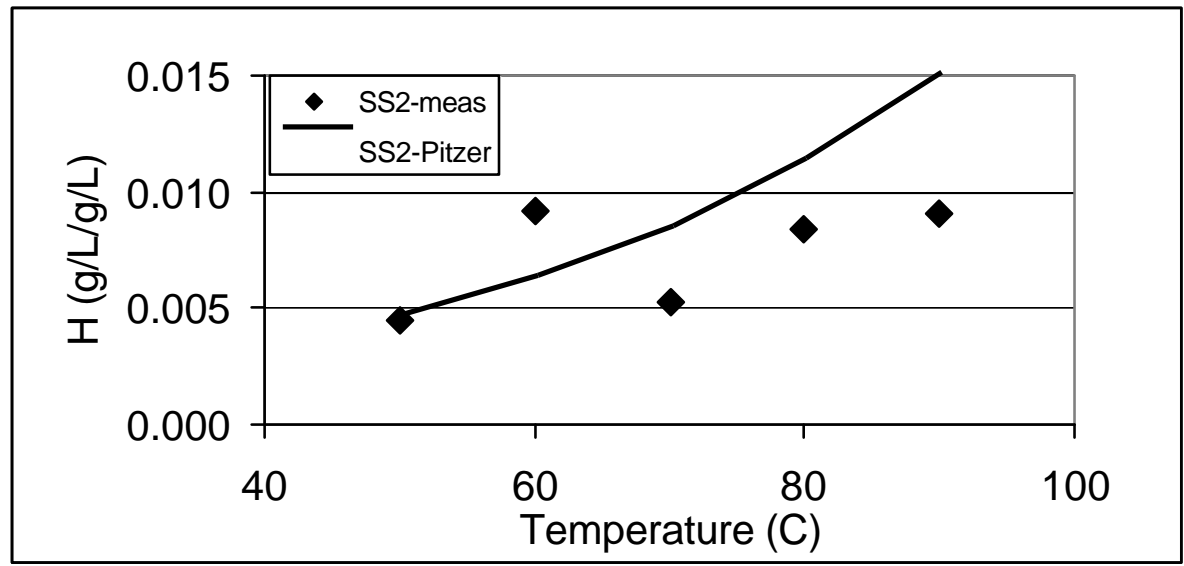


WSRC-TR-2000-00226

Revision 0

Page 16 of 18

January 4, 2001

The Pitzer model is a tool that SRS can use to predict ammonia VLE for new waste streams identified over a concentration range of $0-6.2 \mathrm{M}$ sodium and a temperature range of $25-90{ }^{\circ} \mathrm{C}$.

\section{Allowable Pump Tank Ammonia Concentrations}

Current ammonia concentration limits in Tank Farm receipts from the canyons had been based on the

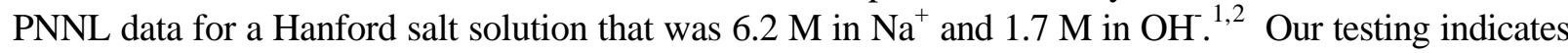
that this data is satisfactory for continued use in SRS High Level Waste Pump Tanks with salt concentrations similar to or less than those shown in Table 1. PNNL's original tests only went to temperatures as high as $70{ }^{\circ} \mathrm{C}$. Our data indicate that extrapolation of PNNL's data fit to $90{ }^{\circ} \mathrm{C}$ is acceptable.

Equation [17] gives the expression for the Henry's Law derived from data obtained with $6.2 \mathrm{M} \mathrm{Na}^{+}, 1.7$ $\mathrm{M} \mathrm{OH}^{-}$salt solution. Equation [18] gives the Henry's Law expression for a $12.6 \mathrm{M} \mathrm{Na}^{+}, 2.3 \mathrm{M} \mathrm{OH}^{-}$salt solution. $^{1}$

$$
\begin{aligned}
& \ln K_{H}=-7.357+3330.1 *(1 / T)-0.002139 * T \\
& \ln K_{H}=-7.577+2571.3 *(1 / T)+0.003076 * T
\end{aligned}
$$

In both equations the $K_{H}$ represents the Henry's Law Constant in units of moles/(kg*atm) and $T$ represents the system temperature in Kelvin. Equation [17] is acceptable for use in the pump tanks because the salt solution concentrations found in the pump tanks are bounded by the salt solution concentrations used to derive Equation [17] and the salt solutions tested by SRTC and found to give results similar to those used to derive Equation [17]. Several waste tanks have salt concentrations higher than those used to derive Equation [17], and therefore Equation [17] is not appropriate for calculating ammonia vapor pressures in those tanks. Equation [18] extends the number of waste tanks for which data are available. It should be noted that the actual dissolved $\mathrm{Na}^{+}$concentration in the solution used to derive Equation [18] is actually lower than the nominal 12.6 M given by PNNL due to the presence of some undissolved salts. The undissolved salts likely had no effect on the ammonia Henry's Law Constant, so the equation was actually derived for a lower salt concentration than $12.6 \mathrm{M} \mathrm{Na}^{+}$. Therefore, Equation [18] may be used to estimate the Henry's Law Constants for tanks containing supernate at salt concentrations above $6.2 \mathrm{M} \mathrm{Na}^{+}\left(6-9 \mathrm{M} \mathrm{Na}^{+}\right)$. Alternatively, the Pitzer model may be used to calculate Henry's Law Constants for salt solutions with $\mathrm{Na}^{+}$concentrations slightly above $6.2 \mathrm{M}$. The model has been compared to data from salt solutions in these ranges and found to agree well at low temperatures and to produce results which deviate conservatively from the actual data at higher temperatures (Figures 12 and 13).

Equations [17] and [18] were used to calculate ammonia partial pressures for a range of liquid phase ammonia concentrations. Tables 4 and 5 give data for $6.2 \mathrm{M} \mathrm{Na}^{+}, 1.7 \mathrm{M} \mathrm{OH}^{-}$salt solution and $12.6 \mathrm{M}$ $\mathrm{Na}^{+}, 2.3 \mathrm{M}$ in $\mathrm{OH}$ salt solution, respectively. PNNL's original data covered the range from $\sim 25$ to $\sim 70$ ${ }^{\circ} \mathrm{C}$. Testing completed by SRTC, while showing a high degree of variability, indicates that equation [17] may safely be extrapolated to $90{ }^{\circ} \mathrm{C}$. 
WSRC-TR-2000-00226

Revision 0

Page 17 of 18

January 4, 2001

Table 4: Calculated Partial Pressures of Ammonia in Equilibrium with 6.2 $\mathrm{M} \mathrm{Na}^{+}, 1.7 \mathrm{M} \mathrm{OH}^{-}$ Salt Solution Containing Varying Amounts of Ammonia

\begin{tabular}{|c|c|c|c|c|c|c|}
\hline \multirow{2}{*}{\multicolumn{2}{|c|}{$\begin{array}{l}\text { Ammonia Concentration } \\
(\mathrm{wt} \%)\end{array}$}} & \multicolumn{5}{|c|}{ Liquid Phase Ammonia Concentrations } \\
\hline & & 1.27 & 0.843 & 0.630 & 0.4769 & 0.0426 \\
\hline \multicolumn{2}{|c|}{$\begin{array}{l}\text { Ammonia Concentration } \\
\text { (molality) }\end{array}$} & $1.14 \mathrm{E}+00$ & 7.51E-01 & $5.60 \mathrm{E}-01$ & 4.23E-01 & 3.77E-02 \\
\hline Temp. $\left({ }^{\circ} \mathrm{C}\right)$ & $\underline{\mathbf{K}}_{\mathbf{H}}$ & \multicolumn{5}{|c|}{ Partial Pressure of Ammonia in Vapor Phase (atm) } \\
\hline 25 & 23.91 & $4.75 \mathrm{E}-0 \overline{2}$ & $3.14 \mathrm{E}-02$ & $2.34 \mathrm{E}-02$ & $1.77 \mathrm{E}-02$ & $1.57 \mathrm{E}-03$ \\
\hline 30 & 19.68 & $5.77 \mathrm{E}-02$ & 3.81E-02 & $2.85 \mathrm{E}-02$ & 2.15E-02 & $1.91 \mathrm{E}-03$ \\
\hline 40 & 13.56 & 8.38E-02 & 5.53E-02 & 4.13E-02 & $3.12 \mathrm{E}-02$ & $2.78 \mathrm{E}-03$ \\
\hline 50 & 9.55 & $1.19 \mathrm{E}-01$ & $7.86 \mathrm{E}-02$ & $5.86 \mathrm{E}-02$ & 4.43E-02 & $3.94 \mathrm{E}-03$ \\
\hline 57 & 7.56 & $1.50 \mathrm{E}-01$ & 9.92E-02 & 7.40E-02 & $5.60 \mathrm{E}-02$ & 4.98E-03 \\
\hline 70 & 5.02 & $2.26 \mathrm{E}-01$ & $1.49 \mathrm{E}-01$ & $1.12 \mathrm{E}-01$ & 8.43E-02 & $7.50 \mathrm{E}-03$ \\
\hline 80 & 3.73 & $3.04 \mathrm{E}-01$ & $2.01 \mathrm{E}-01$ & $1.50 \mathrm{E}-01$ & $1.13 \mathrm{E}-01$ & $1.01 \mathrm{E}-02$ \\
\hline 90 & 2.82 & 4.03E-01 & $2.66 \mathrm{E}-01$ & $1.99 \mathrm{E}-01$ & $1.50 \mathrm{E}-01$ & $1.34 \mathrm{E}-02$ \\
\hline
\end{tabular}

Table 5: Calculated Partial Pressures of Ammonia in Equilibrium with 12.6 $\mathrm{M} \mathrm{Na}^{+}, 2.3 \mathrm{M} \mathrm{OH}^{-}$ Salt Solution Containing Varying Amounts of Ammonia

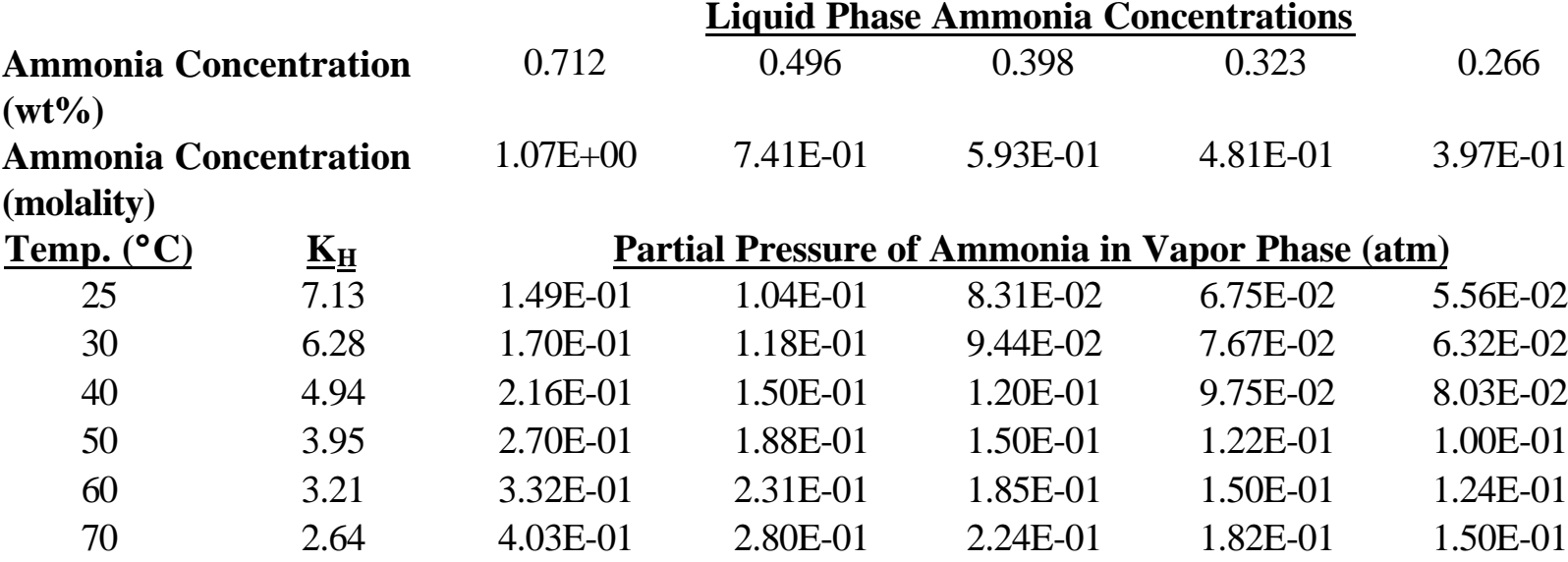

The corrected allowable ammonia concentration (15\% LFL) is $0.095 \mathrm{wt} \%$ in $6.2 \mathrm{M} \mathrm{Na}^{+}, 1.7 \mathrm{M} \mathrm{OH}^{-}$salt solution and 0.026 wt $\%$ in $12.6 \mathrm{M}^{\text {in } \mathrm{Na}^{+}}$and $2.3 \mathrm{M} \mathrm{OH}^{-}$salt solution over a temperature range of $25-70$ ${ }^{\circ} \mathrm{C}$. A flammable ammonia concentration cannot exist over a salt solution as long as the ammonia concentration is kept below these values. The Tank Farm WAC ${ }^{6}$ currently restricts the ammonia concentration to an composite lower flammability limit (CLFL) contribution of $\leq 15 \%$ for tanks designated as "organic" and a CLFL contribution of $\leq 5 \%$ for those tanks designated as non-organic.

To maintain the equilibrium vapor phase ammonia concentration at $\leq 15 \%$ of the LFL (i.e., 1.49 vol $\%$ or $0.0149 \mathrm{~atm}$ ) over the temperature range of 25 to $70{ }^{\circ} \mathrm{C}$, the liquid phase ammonia concentration must not exceed $0.095 \mathrm{wt} \%(1,240 \mathrm{mg} / \mathrm{L}$ assuming a solution density of $1.3 \mathrm{~g} / \mathrm{mL})$. If the temperature is allowed 
to be increased to $90{ }^{\circ} \mathrm{C}$, the allowable concentration for $15 \%$ CLFL in a $6.2 \mathrm{M} \mathrm{Na}^{+}, 1.7 \mathrm{M} \mathrm{OH}^{-}$salt solution becomes $0.0715 \mathrm{wt} \%$, or $930 \mathrm{mg} / \mathrm{L}$. Since virtually all of the salt solution that comes into the Tank Farm would have a concentration below $6.2 \mathrm{M} \mathrm{Na}^{+}, 1.7 \mathrm{M} \mathrm{OH}^{-}$it is conservative to use the ammonia concentration for that salt solution as the basis for the WAC. These sodium and hydroxide concentration restrictions should be placed on incoming transfers. However, if the Tank Farm accepts waste with a significantly higher salt concentration, a reduction in the allowable ammonia concentration to the values given for $12.6 \mathrm{M} \mathrm{Na}^{+}$and $2.3 \mathrm{M} \mathrm{OH}^{-}$should be considered. Note that the $15 \% \mathrm{LFL}$ limit is arbitrary and should not be considered immutable for all cases. If the need arises to receive material that has a higher concentration and analysis of the transfer indicates no problem then the transfer might be allowed with proper precautions.

\section{CONCLUSIONS}

SRTC has performed tests to determine ammonia vapor pressure over salt solutions with compositions believed to be similar to those expected in SRS Tank Farm pump tanks. These tests were performed to expand the range of temperature conditions for which data was available (up to $90{ }^{\circ} \mathrm{C}$ ), and to provide data for salt solutions which were expected to mimic those found in SRS Tank Farm pump tanks. The researchers were also concerned about the possibility of error being introduced into the PNNL tests by dilution with the ammonium hydroxide solution. A promising new method was found in the literature, multiple headspace extraction gas chromatography (MHE-GC). This method, as described in the literature, seemed to be an excellent method for deriving vapor-liquid equilibrium data.

The MHE-GC method was tested using the ammonia-salt solution systems described herein. The results of the tests showed considerable variability, enough that it would be difficult to use them directly to derive allowable salt solution ammonia concentrations for comparison with WAC compliance. However, the data were found to average very close to the data determined by PNNL for their 6.2 M Na Simulant B. Therefore, while the data may not be useful in and of themselves, they do add confidence for the continued use of the PNNL Simulant B data. Additionally, they provide some level of confidence for the extrapolation of PNNL's fit to their data up to $90{ }^{\circ} \mathrm{C}$.

While the MHE-GC method did not appear to provide the quick and easy method for evaluation of vaporliquid equilibrium that was originally expected, it did at times show promise. Unfortunately, the ammonia system was probably not the best system with which to develop this method. Ammonia is difficult to detect by gas chromatography. Further development work on this method might better be done with butanol or some other more appropriate system of interest to the Tank Farm.

\section{QUALITY ASSURANCE}

The test result data are documented in laboratory notebooks WSRC-NB-2000-00032 and WSRC-NB2000-00095.

\section{REFERENCES}


${ }^{1}$ J. D. Norton and L. R. Pederson, "Ammonia in Simulated Hanford Double-Shell Tank Wastes:Solubility and Effects on Surface Tension,” PNL-10173, September 1994.

${ }^{2}$ R. F. Swingle, II and T. L. White, "Contribution of Ammonia and Defoamers to Lower Flammability Limit in SRS High Level Waste Tanks,” WSRC-TR-99-00189, Revision 0, June 18, 1999.

${ }^{3}$ C. S. Boley, "High Temperature Ammonia Testing,” HLE-TTR-1999-060, Revision 0, August 10, 1999.

${ }^{4}$ R. F. Swingle, “Task Technical and Quality Assurance Plan for the Determination of Ammonia Partial Pressure in Pump Tank Salt Solutions," WSRC-RP-99-01118, Revision 0, December 21, 1999.

${ }^{5}$ X. S. Chai and J. Y. Zhu, "Simultaneous Measurements of Solute Concentration and Henry's Constant Using Multiple Headspace Extraction Gas Chromatography," Analytical Chemistry, Volume 70, Number 16, August 15, 1998.

${ }^{6}$ C. I. Aponte, "Waste Acceptance Criteria for High Level Liquid Waste Transfers to the 241-F/H Tank Farms (U),” X-SD-G-00001, Revision 4, April, 2000.

${ }^{7}$ K. S. Pitzer, “A Thermodynamic Model for Aqueous Solutions of Liquid-Like Density”, Reviews in Mineralogy, vol. 17, pp. 97-142, 1987.

${ }^{8}$ S. L. Clegg and P. Brimblecombe, "Solubility of Ammonia in Pure Aqueous and Multicomponent Solutions”, J. Phys. Chem. Vol 93, pp. 7237-7248, 1989. 
WSRC-TR-2000-00226

Revision 0

Page 20 of 18

January 4, 2001

\section{APPROVALS}

Authors

R. F. Swingle, Waste Processing Technology Date

M. R. Poirier, Waste Processing Technology Date

C. J. Berry, Environmental Biotechnology Date

T. B. Edwards, Statistical Consulting Date

J. E. Young, Analytical Development Date

Design Check

D. T. Hobbs, Design Check per Manual E7, Procedure 2.40

Waste Processing Technology

Management

W. B. Van Pelt, Level 4 Manager,

Date

Waste Processing Technology

B. L. Lewis, Manager

Date

CST Engineering 
WSRC-TR-2000-00226

Revision 0

Page 21 of 18

January 4, 2001

W. L. Tamosaitis, Level 3 Manager,

Waste Processing Technology 


\section{OVERVIEW OF APPENDICES}

The data generated to support the determination of ammonia partial pressure in pump tank salt solutions are presented and discussed in Appendix A and Appendix B. Details of the statistical analyses conducted in support of this study are also provided in these appendices. These statistical analyses were performed using JMP® Version 3.2.2, a statistical software package by SAS Institute, Inc. [1].

\section{OVERVIEW OF STATISTICALAPPROACH}

In this section, an overview of the statistical approach used to analyze these data is provided. The analysis of these data follows the approach proffered by Chai and Zhu [2] and outlined in the task technical and quality assurance plan [3]. Experiments using methanol at pressures of 1 atmosphere (atm), 19 psig, and $2 \mathrm{~atm}$ were conducted at temperatures of 50,60, 70, 80, and 90 degrees Celsius $\left({ }^{\circ} \mathrm{C}\right)$ following the protocol of [2]. The gas chromatograph (GC) peak area (A) values for the methanol tests are presented in Table B1 of Appendix B. This table also provides the vial pressure, the common logarithm $(\log )$ of $\mathrm{A}$, and values for $\mathrm{x}$ and $\mathrm{y}$ where

$$
y=\sum_{i=1}^{n-1} A_{i}=a+b A_{n}=a+b x
$$

The values of $\mathrm{x}$ and $\mathrm{y}$ for each test were used to determine an estimate of the slope, $\mathrm{b}$, in equation (1). For a given temperature, this slope is related to $\mathrm{H}_{\mathrm{c}}$ by the equation

$$
\mathrm{b}=-\frac{1}{\mathrm{f}}\left(1+\frac{1}{\beta \mathrm{H}_{\mathrm{c}}}\right)
$$

where $B$ is the phase ratio (with value of 213.7 for the tests conducted here) and $f$ is an unknown constant. The objective of the methanol studies is to estimate the unknown constant $f$ at each of the temperatures $50,60,70,80$, and $90^{\circ} \mathrm{C}$. A model was fit to known values of Henry's constant $\left(\mathrm{H}_{\mathrm{c}}\right)$ for methanol at temperatures of $50,60,70$, and $80{ }^{\circ} \mathrm{C}$. This model was used to predict an $\mathrm{H}_{\mathrm{c}}$ value for $90^{\circ} \mathrm{C}$. From these values, estimates of the f's for each vial pressure were determined using equation (2). The f's determined for a vial pressure of 19 psig correspond to those presented in [2]. Comparisons between the two sets of results are discussed below.

Experiments were conducted with ammonia at temperatures from 50 to $90^{\circ} \mathrm{C}$ and ammonia $\left(\mathrm{NH}_{3}\right)$ molal concentrations ranging from 0.5 to 3.5 for the three salt solutions of interest, and the data from these tests are presented in Table B2 in Appendix B. ${ }^{1}$ Using the f's determined from the methanol, $\mathrm{H}_{\mathrm{c}}$ values were determined for the ammonia tests, again using equation (2). These values were computed for each of the three salt solutions. Comparisons between these results and those from an earlier PNNL study [4] are discussed below.

Special experiments were also conducted with one-component salt solutions. These test results are presented in Table B3 of Appendix B. Using the f's determined from the methanol, $\mathrm{H}_{6}$ values were determined for each of these salt solutions, again using equation (2).

One other statistical analysis that was conducted on the results of each of these GC tests was an investigation into the relationship between the sampling sequence and the common logarithm of

A limited amount of testing was also conducted at 100 and $110^{\circ} \mathrm{C}$. These data are also presented in Table B2. 
the GC peak area, $\log (\mathrm{A})$. From [2], this relationship is expected to be linear, and it is explored for each set of results in the discussion that follows.

\section{METHANOL $H_{C}$ VALUES FROM THE LITERATURE}

Methanol $\mathrm{H}_{\mathrm{c}}$ values for temperatures from 40 to $80 \mathrm{oC}$, which were found in the literature, are provided in Table A1.

Table A1: Methanol $H_{c}$ Values

\begin{tabular}{|cc|}
\hline Temperature & $\mathbf{H}_{\mathbf{c}}$ \\
$40^{\circ} \mathrm{C}$ & 0.00044 \\
$50^{\circ} \mathrm{C}$ & 0.00071 \\
$60^{\circ} \mathrm{C}$ & 0.00112 \\
$70^{\circ} \mathrm{C}$ & 0.00170 \\
$80^{\circ} \mathrm{C}$ & 0.00260 \\
\hline
\end{tabular}

Exhibit A1 provides a polynomial (of degree 2) fit of these $\mathrm{H}_{t}$ values to temperature. This quadratic model was used to predict methanol $\mathrm{H}_{\mathrm{c}}$ values for other temperature of interest.

Exhibit A1. $\mathbf{H}_{\mathrm{c}}$ By Temperature, T
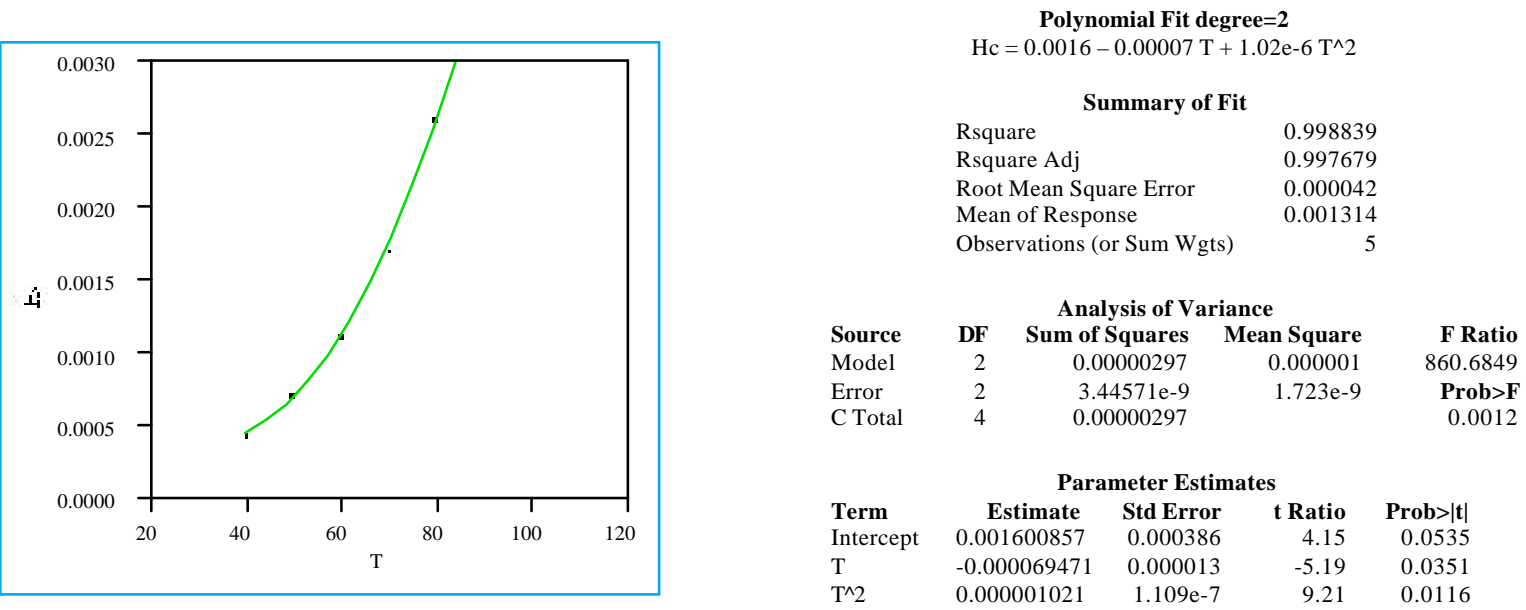

\section{METHANOL RESULTS}

Once again, the results from the methanol testing are presented in Table B1. The results are presented in the order that the data were generated. Two identifiers presented in that table will be used to label these tests: "Study" and "Test Sequence." Using this label, the Exhibits B1 through B12 in Appendix B provide a regression of $\log (\mathrm{A})$ on sampling sequence. Some of the experimental results demonstrate strong linear correlations between these two sets of values (with $\mathrm{R}^{2}$ values greater than $\left.99 \%\right)^{2}$. Other results yield plots for $\log (\mathrm{A})$ versus the sampling sequence that are not as highly correlated and that may indicate anomalies in the data.

For each test, equation (1) was fit to the peak area data (i.e., y was regressed on $\mathrm{x}$ ) to determine an estimate for the corresponding slope, b. Exhibits B13 through B24 provide the results from these regressions. Once again, the $\mathrm{R}^{2}$ values for many of these regressions are greater than $99 \%$ (as expected from the discussion presented in [2]). However, some of these fits generate $\mathrm{R}^{2}$ values that are much lower. Table A1 provides a summary of the results from the methanol tests, indexed by "Study" and "Test Sequence."

\footnotetext{
$2 \quad \mathrm{R}^{2}$ is called the coefficient of determination, and when expressed as a percentage, it is a measure of the percentage of the variation of the response values, the y's, explained by the model.
} 
Table A2: Summary of Methanol Results

\begin{tabular}{|c|c|c|c|c|c|c|c|c|}
\hline Study & $\begin{array}{c}\text { Test } \\
\text { Sequence }\end{array}$ & $\begin{array}{c}\text { Temperature } \\
\left({ }^{\circ} \mathrm{C}\right)\end{array}$ & Pressure & Test & $\begin{array}{c}\mathbf{R}^{2} \\
\text { Sampling } \\
\text { Sequence }\end{array}$ & $\begin{array}{c}\mathbf{R}^{2} \\
\mathbf{y} \mathbf{v s} \\
\mathbf{x}\end{array}$ & $\begin{array}{c}\text { slope, b } \\
\text { y vs } \\
\text { x }\end{array}$ & $\begin{array}{c}\text { phi, f } \\
\text { computed } \\
\text { from eqn }(2)\end{array}$ \\
\hline 1 & 1 & 50 & $1 \mathrm{~atm}$ & 1 & 0.938 & 0.979 & -38.7892 & 0.1957 \\
\hline 1 & 2 & 50 & $1 \mathrm{~atm}$ & 2 & 0.990 & 0.993 & -31.2688 & 0.2428 \\
\hline 1 & 3 & 50 & $1 \mathrm{~atm}$ & 3 & 0.693 & 0.671 & -11.5478 & 0.6573 \\
\hline 1 & 4 & 50 & 19 psig & 1 & 0.853 & 0.999 & -27.4751 & 0.2763 \\
\hline 1 & 5 & 50 & 19 psig & 2 & 0.855 & 0.967 & -19.9023 & 0.3814 \\
\hline 1 & 6 & 50 & 19 psig & 3 & 0.813 & 0.863 & -15.7763 & 0.4812 \\
\hline 1 & 7 & 50 & $2 \mathrm{~atm}$ & 1 & 0.833 & 0.886 & -11.4862 & 0.6609 \\
\hline 1 & 8 & 50 & $2 \mathrm{~atm}$ & 2 & 0.858 & 0.900 & -10.7259 & 0.7077 \\
\hline 1 & 9 & 50 & $2 \mathrm{~atm}$ & 3 & 0.845 & 0.891 & -10.8366 & 0.7005 \\
\hline 1 & 10 & 60 & $1 \mathrm{~atm}$ & 1 & 0.984 & 0.997 & -19.7078 & 0.2627 \\
\hline 1 & 11 & 60 & $1 \mathrm{~atm}$ & 2 & 0.986 & 0.998 & -21.1954 & 0.2443 \\
\hline 1 & 12 & 60 & $1 \mathrm{~atm}$ & 3 & 0.993 & 0.999 & -21.1828 & 0.2444 \\
\hline 1 & 13 & 60 & 19 psig & 1 & 0.989 & 0.997 & -16.5578 & 0.3127 \\
\hline 1 & 14 & 60 & 19 psig & 2 & 0.992 & 0.996 & -16.7621 & 0.3089 \\
\hline 1 & 15 & 60 & 19 psig & 3 & 0.984 & 0.998 & -16.642 & 0.3111 \\
\hline 1 & 16 & 60 & $2 \mathrm{~atm}$ & 1 & 0.950 & 0.999 & -12.1395 & 0.4265 \\
\hline 1 & 17 & 60 & $2 \mathrm{~atm}$ & 2 & 0.959 & 0.998 & -11.6707 & 0.4437 \\
\hline 1 & 18 & 60 & $2 \mathrm{~atm}$ & 3 & 0.971 & 0.994 & -9.9542 & 0.5202 \\
\hline 1 & 19 & 70 & $1 \mathrm{~atm}$ & 1 & 0.998 & 0.998 & -4.58921 & 0.8177 \\
\hline 1 & 20 & 70 & $1 \mathrm{~atm}$ & 2 & & & & \\
\hline 1 & 21 & 70 & 19 psig & 1 & 0.992 & 0.994 & -11.4062 & 0.3290 \\
\hline 1 & 22 & 70 & 19 psig & 2 & 0.988 & 0.981 & -13.0882 & 0.2867 \\
\hline 1 & 23 & 70 & 19 psig & 3 & 0.991 & 0.985 & -12.5826 & 0.2982 \\
\hline 1 & 24 & 70 & $2 \mathrm{~atm}$ & 1 & 0.981 & 0.978 & -8.88918 & 0.4222 \\
\hline 1 & 25 & 70 & $2 \mathrm{~atm}$ & 2 & 0.992 & 0.990 & -8.67823 & 0.4324 \\
\hline 1 & 26 & 70 & $2 \mathrm{~atm}$ & 3 & 0.990 & 0.985 & -8.51875 & 0.4405 \\
\hline 1 & 27 & 80 & $1 \mathrm{~atm}$ & 1 & 0.994 & 0.995 & -9.39544 & 0.2980 \\
\hline 1 & 28 & 80 & $1 \mathrm{~atm}$ & 2 & 0.948 & 0.948 & -8.96445 & 0.3123 \\
\hline 1 & 29 & 80 & $1 \mathrm{~atm}$ & 3 & 0.979 & 0.977 & -8.69785 & 0.3219 \\
\hline 1 & 30 & 80 & 19 psig & 1 & 0.993 & 0.994 & -7.31383 & 0.3828 \\
\hline 1 & 31 & 80 & 19 psig & 2 & 0.955 & 0.973 & -8.99484 & 0.3113 \\
\hline 1 & 32 & 80 & 19 psig & 3 & 0.924 & 0.923 & -8.97846 & 0.3118 \\
\hline 1 & 33 & 80 & $2 \mathrm{~atm}$ & 1 & 0.924 & 0.971 & -6.16336 & 0.4543 \\
\hline 1 & 34 & 80 & $2 \mathrm{~atm}$ & 2 & 0.988 & 0.983 & -6.0094 & 0.4659 \\
\hline 1 & 35 & 80 & $2 \mathrm{~atm}$ & 3 & 0.956 & 0.965 & -7.54973 & 0.3708 \\
\hline 1 & 36 & 90 & $1 \mathrm{~atm}$ & 1 & 0.994 & 0.992 & -5.72815 & 0.4001 \\
\hline 1 & 37 & 90 & $1 \mathrm{~atm}$ & 2 & 0.995 & 0.996 & -5.43936 & 0.4214 \\
\hline 1 & 38 & 90 & $1 \mathrm{~atm}$ & 3 & 0.980 & 0.977 & -5.79524 & 0.3955 \\
\hline 1 & 39 & 90 & 19 psig & 1 & 0.986 & 0.987 & -5.08625 & 0.4506 \\
\hline 1 & 40 & 90 & 19 psig & 2 & 0.999 & 0.999 & -4.54679 & 0.5041 \\
\hline 1 & 41 & 90 & 19 psig & 3 & 0.991 & 0.994 & -4.10972 & 0.5577 \\
\hline 1 & 42 & 90 & $2 \mathrm{~atm}$ & 1 & 0.980 & 0.952 & -3.5681 & 0.6423 \\
\hline 1 & 43 & 90 & $2 \mathrm{~atm}$ & 2 & 0.993 & 0.983 & -3.62845 & 0.6317 \\
\hline 1 & 44 & 90 & $2 \mathrm{~atm}$ & 3 & 0.964 & 0.977 & -3.67306 & 0.6240 \\
\hline 2 & 1 & 50 & $1 \mathrm{~atm}$ & 1 & 0.960 & 0.998 & -26.1605 & 0.2902 \\
\hline 2 & 2 & 50 & $1 \mathrm{~atm}$ & 2 & 0.927 & 0.997 & -27.6427 & 0.2746 \\
\hline 2 & 3 & 50 & $1 \mathrm{~atm}$ & 3 & 0.899 & 0.992 & -25.6753 & 0.2956 \\
\hline 2 & 4 & 60 & $1 \mathrm{~atm}$ & 1 & 0.960 & 0.981 & -19.0502 & 0.2718 \\
\hline 2 & 5 & 60 & $1 \mathrm{~atm}$ & 2 & 0.952 & 0.975 & -18.4794 & 0.2802 \\
\hline 2 & 6 & 60 & $1 \mathrm{~atm}$ & 3 & 0.946 & 0.974 & -18.0484 & 0.2869 \\
\hline 2 & 7 & 70 & $1 \mathrm{~atm}$ & 1 & 0.985 & 0.991 & -13.8382 & 0.2712 \\
\hline 2 & 8 & 70 & $1 \mathrm{~atm}$ & 2 & 0.986 & 0.990 & -14.8317 & 0.2530 \\
\hline 2 & 9 & 70 & $1 \mathrm{~atm}$ & 3 & 0.993 & 0.994 & -14.1948 & 0.2644 \\
\hline 2 & 10 & 80 & $1 \mathrm{~atm}$ & 1 & 0.986 & 0.990 & -8.88866 & 0.3150 \\
\hline 2 & 11 & 80 & $1 \mathrm{~atm}$ & 2 & 0.999 & 1.000 & -8.24197 & 0.3397 \\
\hline 2 & 12 & 80 & $1 \mathrm{~atm}$ & 3 & 0.992 & 0.994 & -8.73874 & 0.3204 \\
\hline 2 & 13 & 90 & $1 \mathrm{~atm}$ & 1 & 0.995 & 0.997 & -5.38691 & 0.4255 \\
\hline 2 & 14 & 90 & $1 \mathrm{~atm}$ & 2 & 0.996 & 0.997 & -5.53406 & 0.4142 \\
\hline 2 & 15 & 90 & $1 \mathrm{~atm}$ & 3 & 0.990 & 0.990 & -5.76699 & 0.3974 \\
\hline
\end{tabular}


Table A2: Summary of Methanol Results

(continued)

\begin{tabular}{|c|c|c|c|c|c|c|c|c|}
\hline Study & $\begin{array}{c}\text { Test } \\
\text { Sequence }\end{array}$ & $\begin{array}{c}\text { Temperature } \\
\left({ }^{\circ} \mathrm{C}\right)\end{array}$ & Pressure & Test & $\begin{array}{c}\mathbf{R}^{2} \\
\text { Sampling } \\
\text { Sequence }\end{array}$ & $\begin{array}{c}\mathbf{R}^{2} \\
\mathbf{y} \mathbf{v s} \\
\mathbf{x}\end{array}$ & $\begin{array}{c}\text { slope, b } \\
\text { y vs } \\
\text { x }\end{array}$ & $\begin{array}{c}\text { phi, f } \\
\text { computed } \\
\text { from eqn }(2)\end{array}$ \\
\hline 3 & 1 & 50 & $1 \mathrm{~atm}$ & 1 & 0.876 & 0.918 & -18.2158 & 0.4167 \\
\hline 3 & 2 & 50 & $1 \mathrm{~atm}$ & 2 & 0.865 & 0.901 & -15.4758 & 0.4905 \\
\hline 3 & 3 & 50 & $1 \mathrm{~atm}$ & 3 & 0.874 & 0.883 & -14.669 & 0.5175 \\
\hline 3 & 4 & 60 & $1 \mathrm{~atm}$ & 1 & 0.907 & 0.963 & -16.6183 & 0.3116 \\
\hline 3 & 5 & 60 & $1 \mathrm{~atm}$ & 2 & 0.937 & 0.964 & -16.2718 & 0.3182 \\
\hline 3 & 6 & 60 & $1 \mathrm{~atm}$ & 3 & 0.929 & 0.958 & -17.0034 & 0.3045 \\
\hline 3 & 7 & 70 & $1 \mathrm{~atm}$ & 1 & 0.969 & 0.978 & -13.2756 & 0.2827 \\
\hline 3 & 8 & 70 & $1 \mathrm{~atm}$ & 2 & 0.960 & 0.977 & -13.104 & 0.2864 \\
\hline 3 & 9 & 70 & $1 \mathrm{~atm}$ & 3 & 0.974 & 0.978 & -12.7842 & 0.2935 \\
\hline 3 & 10 & 80 & $1 \mathrm{~atm}$ & 1 & 0.983 & 0.987 & -8.75015 & 0.3200 \\
\hline 3 & 11 & 80 & $1 \mathrm{~atm}$ & 2 & 0.992 & 0.990 & -9.14224 & 0.3062 \\
\hline 3 & 12 & 80 & $1 \mathrm{~atm}$ & 3 & 0.990 & 0.990 & -8.93182 & 0.3135 \\
\hline 3 & 13 & 90 & $1 \mathrm{~atm}$ & 1 & 0.992 & 0.994 & -5.76836 & 0.3973 \\
\hline 3 & 14 & 90 & $1 \mathrm{~atm}$ & 2 & 0.996 & 0.998 & -5.18471 & 0.4421 \\
\hline 3 & 15 & 90 & $1 \mathrm{~atm}$ & 3 & 0.990 & 0.989 & -5.4165 & 0.4231 \\
\hline 4 & 1 & 70 & $1 \mathrm{~atm}$ & 1 & 0.963 & 0.997 & -15.832 & 0.2370 \\
\hline 4 & 2 & 70 & $1 \mathrm{~atm}$ & 2 & 0.990 & 0.995 & -16.8247 & 0.2230 \\
\hline 4 & 3 & 70 & $1 \mathrm{~atm}$ & 1 & 0.989 & 0.986 & -18.4655 & 0.2032 \\
\hline 4 & 4 & 70 & $1 \mathrm{~atm}$ & 2 & 0.993 & 0.988 & -17.2813 & 0.2171 \\
\hline 4 & 5 & 50 & $1 \mathrm{~atm}$ & 1 & 0.996 & 0.999 & -33.1103 & 0.2293 \\
\hline 4 & 6 & 50 & $1 \mathrm{~atm}$ & 2 & 0.786 & 0.898 & -27.1689 & 0.2794 \\
\hline 4 & 7 & 50 & $1 \mathrm{~atm}$ & 3 & 0.890 & 0.931 & -19.3215 & 0.3929 \\
\hline 4 & 8 & 50 & $1 \mathrm{~atm}$ & 1 & 0.867 & 0.995 & -30.3628 & 0.2500 \\
\hline 4 & 9 & 50 & $1 \mathrm{~atm}$ & 2 & 0.866 & 0.982 & -28.3509 & 0.2677 \\
\hline 4 & 10 & 50 & $1 \mathrm{~atm}$ & 3 & 0.830 & 0.936 & -24.9589 & 0.3041 \\
\hline 4 & 11 & 60 & $1 \mathrm{~atm}$ & 1 & 0.952 & 0.983 & -20.1916 & 0.2564 \\
\hline 4 & 12 & 60 & $1 \mathrm{~atm}$ & 2 & 0.926 & 0.957 & -18.6262 & 0.2780 \\
\hline 4 & 13 & 60 & $1 \mathrm{~atm}$ & 3 & 0.958 & 0.987 & -21.9587 & 0.2358 \\
\hline 4 & 14 & 60 & $1 \mathrm{~atm}$ & 1 & 0.917 & 0.921 & -21.6565 & 0.2391 \\
\hline 4 & 15 & 60 & $1 \mathrm{~atm}$ & 2 & 0.964 & 0.968 & -17.2716 & 0.2998 \\
\hline 4 & 16 & 60 & $1 \mathrm{~atm}$ & 3 & 0.999 & 0.999 & -4.83758 & 1.0704 \\
\hline
\end{tabular}

Plots of the estimated slopes, the b's, computed from the regression of $\mathrm{y}$ on $\mathrm{x}$ by temperature for the three vial pressures are provided in Exhibit A.2, and plots of the phi's (f's) computed from equation (2) by temperature are provided for the three vial pressures in Exhibit A3.

Exhibit A3: Slope, b, by Temperature (oC) for Each Vial Pressure

Vial Pressure $=1 \mathrm{~atm}$

Vial Pressure $=2 \mathrm{~atm}$

Vial Pressure $=19 \mathrm{psig}$

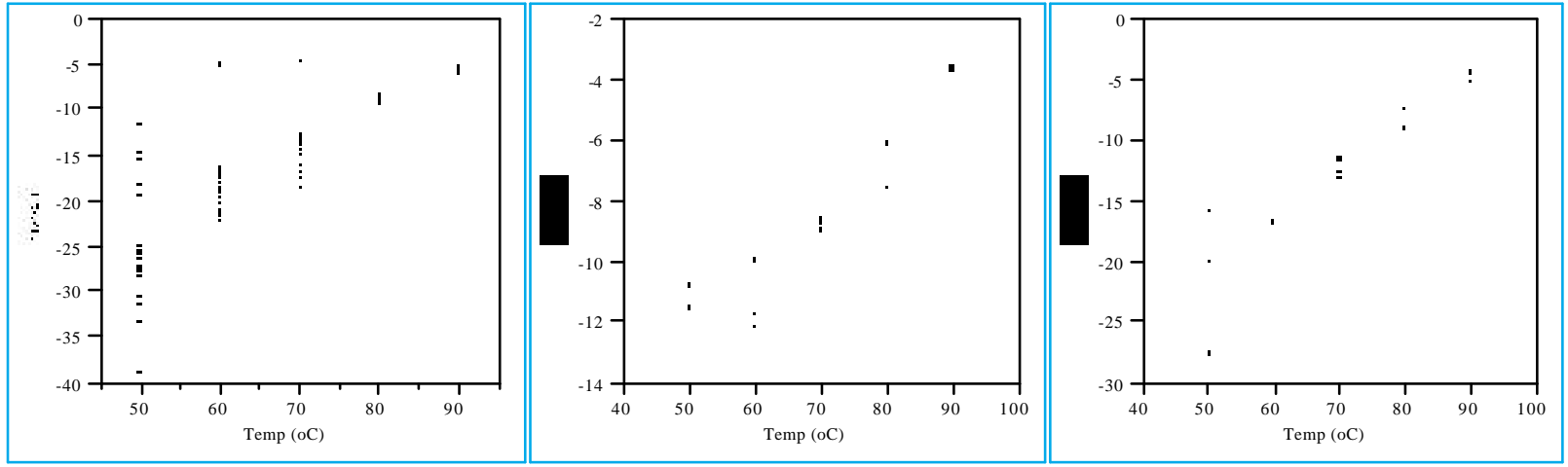


Exhibit A3: phi by Temperature (oC) for Each Vial Pressure

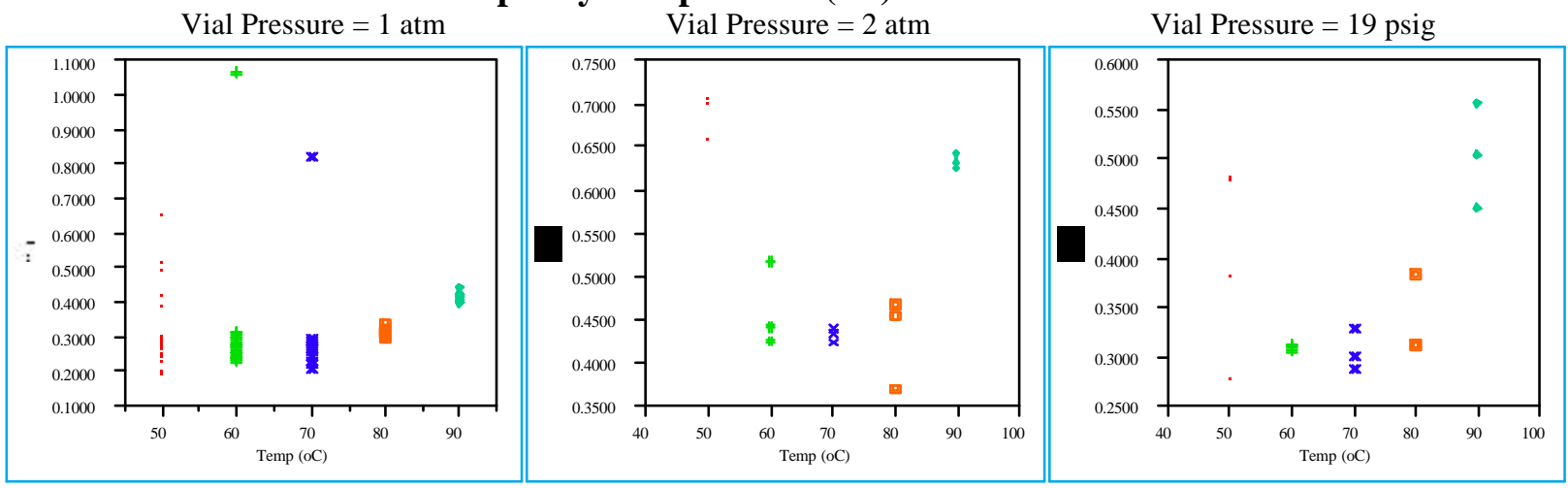

Note that there is a great deal of scatter revealed in the plots of the values of the slopes versus temperature for these tests and that this scatter is reflected in the plots of the phi values as well. Recall that the preliminary regressions $(\log (\mathrm{A})$ versus sampling sequence and y versus $\mathrm{x}$ ) for some of the experimental results yielded $\mathrm{R}^{2}$ values much lower than expected. Screening from Table A1 all results whose $\mathrm{R}^{2}$ values for either of these two regressions were less than $99 \%$ yields Exhibits A4 and A5. (This screening process eliminates all of the results from the testing conducted with vial pressure at 2 atm.)

Exhibit A4. Screened Slope (b) Values by Temperature (oC) for Each Vial Pressure

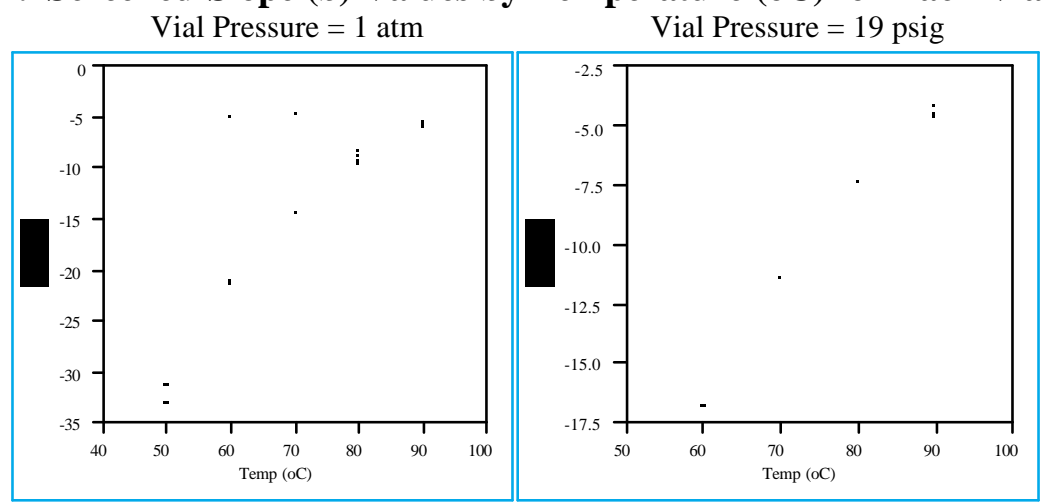

Exhibit A5. Screened Phi (f) Values by Temperature (oC) for Each Vial Pressure

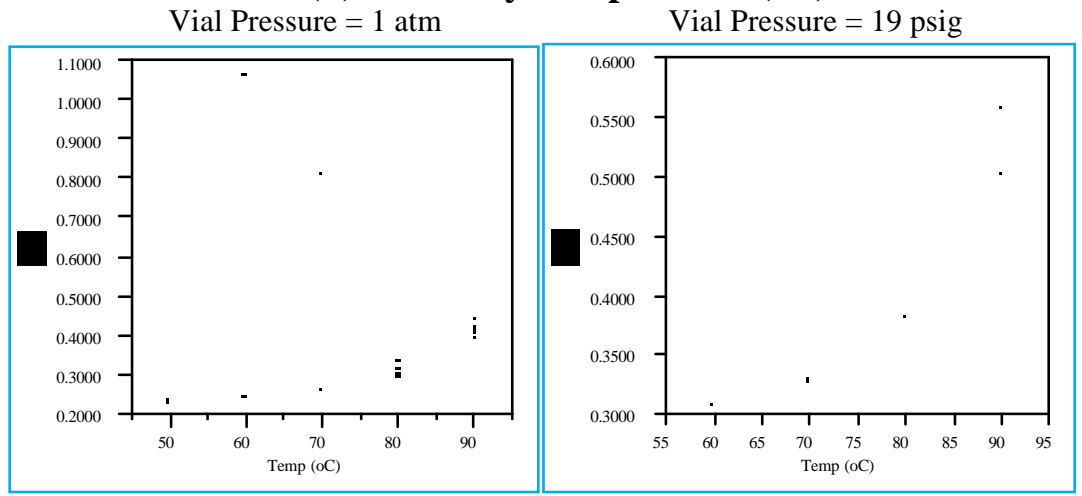

As seen in Exhibits A4 and A5, there are still 2 points that appear to be outliers for the 1 atm results: Study 1 Test Sequence 19 with a b value of -4.58921 and a phi value of 0.8177 and Study 4 Test Sequence 16 with a $b$ value of -4.83758 and a phi value of 1.0704 . Eliminating 
these two points from consideration and fitting these $\mathrm{f}$ 's to a quadratic function of temperature for each of the two remaining vial pressures yields Exhibit A6.

Exhibit A6. Screened Phi's versus Temperature by Vial Pressure

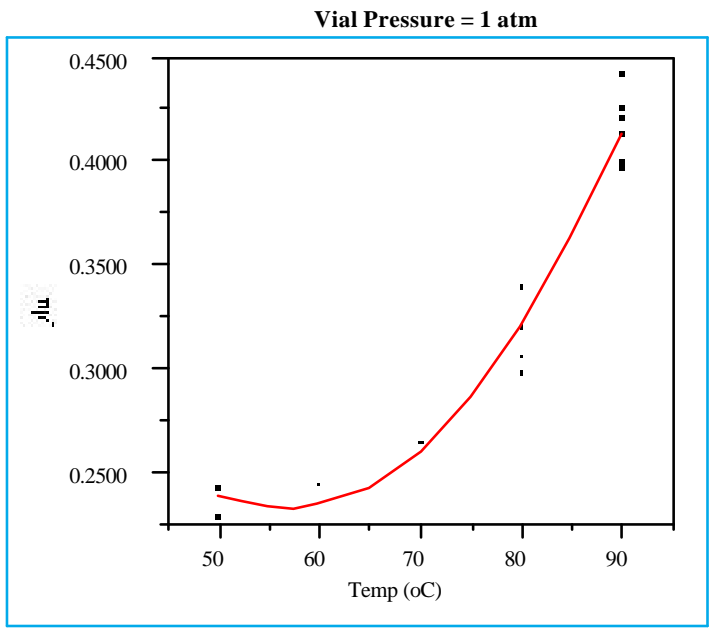

Polynomial Fit degree $=2$

phi $=0.76073-0.01864 \mathrm{Temp}(\mathrm{oC})+0.00016 \mathrm{Temp}(\mathrm{oC})^{\wedge} 2$ Summary of Fit

$$
\begin{array}{lr}
\text { RSquare } & 0.961825 \\
\text { RSquare Adj } & 0.955463 \\
\text { Root Mean Square Error } & 0.01593 \\
\text { Mean of Response } & 0.342872 \\
\text { Observations (or Sum Wgts) } & 15
\end{array}
$$

\begin{tabular}{|c|c|c|c|c|}
\hline \multicolumn{5}{|c|}{ Analysis of Variance } \\
\hline Sourc & DF & Sum of Squares & Mean Square & F Ratio \\
\hline Mod & 2 & 0.07672902 & 0.038365 & 151.172 \\
\hline Error & 12 & 0.00304535 & 0.000254 & Prob $>$ F \\
\hline
\end{tabular}

$\begin{array}{lllr}\text { C Total } & 14 & 0.07977436 & \text { Prob }>\text { } \\ \end{array}$

\begin{tabular}{lrrrr}
\multicolumn{4}{c}{ Parameter Estimates } & \\
Term & Estimate & Std Error & t Ratio & Prob $>|\mathbf{t}|$ \\
Intercept & 0.7607308 & 0.131879 & 5.77 & $<.0001$ \\
Temp $(\mathrm{oC})$ & -0.018641 & 0.003845 & -4.85 & 0.0004 \\
Temp $(\mathrm{oC})^{\wedge} 2$ & 0.0001642 & 0.000027 & 6.10 & $<.0001$
\end{tabular}

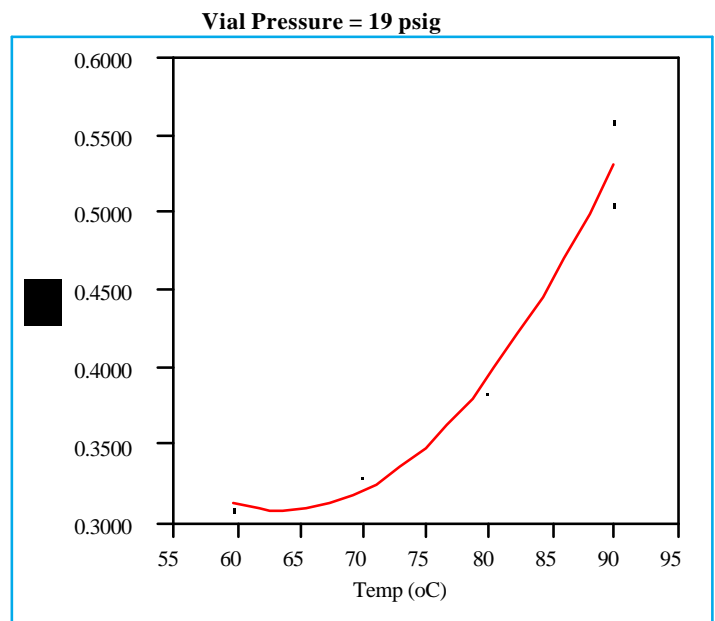

Polynomial Fit degree=2

phi $=1.62631-0.04134 \mathrm{Temp}(\mathrm{oC})+0.00032 \mathrm{Temp}(\mathrm{oC})^{\wedge} 2$ Summary of Fit

$$
\begin{array}{lr}
\text { RSquare } & 0.966128 \\
\text { RSquare Adj } & 0.932255 \\
\text { Root Mean Square Error } & 0.028504 \\
\text { Mean of Response } & 0.416499 \\
\text { Observations (or Sum Wgts) } & 5
\end{array}
$$

\begin{tabular}{lrrrr}
\multicolumn{5}{c}{ Analysis of Variance } \\
Source & DF & Sum of Squares & Mean Square & F Ratio \\
Model & 2 & 0.04634656 & 0.023173 & 28.5225 \\
Error & 2 & 0.00162491 & 0.000812 & Prob $>$ F \\
C Total & 4 & 0.04797147 & & 0.0339
\end{tabular}

\begin{tabular}{lrrrr}
\multicolumn{5}{c}{ Parameter Estimates } \\
Term & Estimate & Std Error & t Ratio & Prob $>|\mathbf{t}|$ \\
Intercept & 1.6263083 & 0.75249 & 2.16 & 0.1632 \\
Temp $(\mathrm{oC})$ & -0.041337 & 0.020225 & -2.04 & 0.1777 \\
Temp $(\mathrm{oC})^{\wedge} 2$ & 0.0003239 & 0.000133 & 2.43 & 0.1354
\end{tabular}

\section{COMPARISON OF PHI'S}

Since methanol was one of the chemicals considered in [2], phi values were determined and reported as part of that study. These values are given in the second column of Table A3. The third column of that table presents the f 's predicted using the equation from Exhibit A4 for vial pressure equal to 19 psig:

$$
\varphi_{19 \text { psig }}=1.62631-0.04134 \cdot \text { Temperature }+0.00032 \cdot \text { Temperature }^{2}
$$

Table A3: Methanol Phi Values

\begin{tabular}{ccc} 
Temperature & $\mathbf{F ~ [ 2 ] ~}$ & $\mathbf{F ~ ( 3 )}$ \\
$40^{\circ} \mathrm{C}$ & 0.271 & 0.4847 \\
$50^{\circ} \mathrm{C}$ & 0.286 & 0.3593 \\
$60^{\circ} \mathrm{C}$ & 0.313 & 0.2979 \\
$70^{\circ} \mathrm{C}$ & 0.354 & 0.3005 \\
$80^{\circ} \mathrm{C}$ & 0.416 & 0.3671 \\
\hline
\end{tabular}


Note that the values for 40 and 50 degrees Celsius appearing in the last column of Table 3 are extrapolations beyond the range of the data used to derive equation (3). Differences between these two sets of F's may be due to the fact that these unknown constants are influenced by the instrumentation and setup used and by the phase ratios of the experiments, which were different between the study conducted here and that of the article.

\section{AMMONIA RESULTS}

Once again, the results from the ammonia testing are presented in Table B2. The results are presented in the order that the data were generated. Two identifiers presented in that table will be used to label these tests: "Study" and "Test Sequence." Using this label, the Exhibits B25 through B38 in Appendix B provide a regression of $\log (\mathrm{A})$ on sampling sequence. Some of the experimental results demonstrate strong linear correlations between these two sets of values (with $\mathrm{R}^{2}$ values greater than $\left.99 \%\right)^{3}$. Other results yield plots for $\log (\mathrm{A})$ versus the sampling sequence that are not as highly correlated and that may indicate anomalies in the data.

For each test, equation (1) was fit to the peak area data (i.e., y was regressed on $\mathrm{x}$ ) to determine an estimate for the corresponding slope, $\mathrm{b}$. Exhibits B39 through B52 provide the results from these regressions. Once again, the $\mathrm{R}^{2}$ values for many of these regressions are greater than $99 \%$ (as expected from the discussion presented in [2]). However, some of these fits generate $\mathrm{R}^{2}$ values that are much lower. Table A4 provides a summary of the results from the ammonia tests, indexed by "Study" and "Test Sequence."

$\mathrm{R}^{2}$ is called the coefficient of determination, and when expressed as a percentage, it is a measure of the percentage of the variation of the response values, the y's, explained by the model. 
Table A4: Summary of Ammonia Results

\begin{tabular}{|c|c|c|c|c|c|c|c|c|c|}
\hline Study & $\begin{array}{c}\text { Test } \\
\text { Sequence }\end{array}$ & $\begin{array}{c}\text { Salt } \\
\text { Solution }\end{array}$ & $\begin{array}{c}\text { NH3 Conc } \\
\text { (molal) }\end{array}$ & $\underset{{ }^{\circ} \mathrm{C}}{\text { Temperature }}$ & $\begin{array}{c}\mathbf{R}^{2} \\
\text { Sampling } \\
\text { Sequence }\end{array}$ & $\begin{array}{c}\mathbf{R}^{2} \\
\mathbf{y} v \mathbf{v s} \\
\mathbf{x}\end{array}$ & $\begin{array}{l}\text { slope, b } \\
\text { y vs x }\end{array}$ & $\begin{array}{c}\text { phi } \\
\text { (predicted) }\end{array}$ & $\begin{array}{c}\mathbf{H}_{\mathbf{c}} \\
\text { Henry's } \\
\text { Constant }\end{array}$ \\
\hline 1 & 1 & 1 & 0.5 & 50 & 0.992825 & 0.991448 & -10.438 & 0.239181 & 0.003075 \\
\hline 1 & 2 & 1 & 2 & 50 & 0.998653 & 0.99987 & -8.28219 & 0.239181 & 0.004691 \\
\hline 1 & 3 & 1 & 3.5 & 50 & 0.998569 & 0.999892 & -7.97414 & 0.239181 & 0.005072 \\
\hline 1 & 4 & 2 & 0.5 & 50 & 0.996247 & 0.99512 & -9.32955 & 0.239181 & 0.003737 \\
\hline 1 & 5 & 2 & 2 & 50 & 0.996734 & 0.999679 & -8.27981 & 0.239181 & 0.004694 \\
\hline 1 & 6 & 2 & 2 & 50 & 0.996719 & 0.998326 & -8.3207 & 0.239181 & 0.004648 \\
\hline 1 & 7 & 2 & 3.5 & 50 & 0.998395 & 0.999829 & -8.24171 & 0.239181 & 0.004738 \\
\hline 1 & 8 & 3 & 0.5 & 50 & 0.885361 & 0.921936 & -7.47962 & 0.239181 & 0.005833 \\
\hline 1 & 9 & 3 & 2 & 50 & 0.856632 & 0.931209 & -7.72844 & 0.239181 & 0.005424 \\
\hline 1 & 10 & 3 & 3.5 & 50 & 0.951042 & 0.978783 & -9.82141 & 0.239181 & 0.003411 \\
\hline 1 & 11 & 1 & 0.5 & 60 & 0.994514 & 0.998608 & -7.65422 & 0.233391 & 0.005852 \\
\hline 1 & 12 & 1 & 2 & 60 & 0.998721 & 0.999959 & -6.63316 & 0.233391 & 0.008396 \\
\hline 1 & 13 & 1 & 3.5 & 60 & 0.998784 & 0.999942 & -6.18857 & 0.233391 & 0.010356 \\
\hline 1 & 14 & 2 & 0.5 & 60 & 0.996906 & 0.998013 & -7.17568 & 0.233391 & 0.006820 \\
\hline 1 & 15 & 2 & 2 & 60 & 0.997494 & 0.999648 & -6.11188 & 0.233391 & 0.010791 \\
\hline 1 & 16 & 2 & 2 & 60 & 0.999008 & 0.999719 & -6.34671 & 0.233391 & 0.009562 \\
\hline 1 & 17 & 2 & 3.5 & 60 & 0.998529 & 0.999964 & -6.31698 & 0.233391 & 0.009702 \\
\hline 1 & 18 & 3 & 0.5 & 60 & 0.806196 & 0.863186 & -6.4377 & 0.233391 & 0.009158 \\
\hline 1 & 19 & 3 & 2 & 60 & 0.76985 & 0.873326 & -11.1887 & 0.233391 & 0.002856 \\
\hline 1 & 20 & 3 & 3.5 & 60 & 0.958971 & 0.980959 & -8.44087 & 0.233391 & 0.004744 \\
\hline 1 & 21 & 1 & 0.5 & 70 & 0.997278 & 0.999084 & -3.90572 & 0.260441 & 0.267417 \\
\hline 1 & 22 & 1 & 2 & 70 & 0.999155 & 0.999941 & -3.51937 & 0.260441 & -0.055170 \\
\hline 1 & 23 & 1 & 3.5 & 70 & 0.999605 & 0.999966 & -3.45295 & 0.260441 & -0.045690 \\
\hline 1 & 24 & 2 & 0.5 & 70 & 0.997711 & 0.999731 & -4.20402 & 0.260441 & 0.048494 \\
\hline 1 & 25 & 2 & 2 & 70 & 0.997986 & 0.999697 & -3.64293 & 0.260441 & -0.089820 \\
\hline 1 & 26 & 2 & 2 & 70 & 0.998646 & 0.999574 & -3.62898 & 0.260441 & -0.083880 \\
\hline 1 & 27 & 2 & 3.5 & 70 & 0.999098 & 0.999905 & -3.55503 & 0.260441 & -0.062080 \\
\hline 1 & 28 & 3 & 0.5 & 70 & 0.547734 & 0.403505 & -9.92048 & 0.260441 & 0.002906 \\
\hline 1 & 29 & 3 & 2 & 70 & 0.911284 & 0.943151 & -5.53553 & 0.260441 & 0.010419 \\
\hline 1 & 30 & 3 & 3.5 & 70 & 0.981872 & 0.991626 & -4.93163 & 0.260441 & 0.016181 \\
\hline 1 & 31 & 1 & 0.5 & 80 & 0.995972 & 0.998779 & -4.96241 & 0.320331 & 0.007805 \\
\hline 1 & 32 & 1 & 2 & 80 & 0.999417 & 0.999966 & -4.46917 & 0.320331 & 0.010662 \\
\hline 1 & 33 & 1 & 3.5 & 80 & 0.9995 & 0.999965 & -4.26177 & 0.320331 & 0.012602 \\
\hline 1 & 34 & 2 & 0.5 & 80 & 0.995833 & 0.99574 & -4.98034 & 0.320331 & 0.007730 \\
\hline 1 & 35 & 2 & 2 & 80 & 0.954584 & 0.09366 & -0.93719 & 0.320331 & -0.006580 \\
\hline 1 & 36 & 2 & 2 & 80 & 0.998364 & 0.999833 & -4.47606 & 0.320331 & 0.010608 \\
\hline 1 & 37 & 2 & 3.5 & 80 & 0.99903 & 0.999929 & -4.36324 & 0.320331 & 0.011572 \\
\hline 1 & 38 & 3 & 0.5 & 80 & 0.766294 & 0.787446 & -8.57298 & 0.320331 & 0.002635 \\
\hline 1 & 39 & 3 & 2 & 80 & 0.884411 & 0.977028 & -8.20431 & 0.320331 & 0.002827 \\
\hline 1 & 40 & 3 & 3.5 & 80 & 0.98174 & 0.996567 & -7.32688 & 0.320331 & 0.003416 \\
\hline 1 & 41 & 1 & 0.5 & 90 & 0.989985 & 0.994251 & -4.14381 & 0.413061 & 0.006467 \\
\hline 1 & 42 & 1 & 2 & 90 & 0.999626 & 0.999953 & -3.66816 & 0.413061 & 0.008933 \\
\hline 1 & 43 & 1 & 3.5 & 90 & 0.999777 & 0.999987 & -3.5721 & 0.413061 & 0.009678 \\
\hline 1 & 44 & 2 & 0.5 & 90 & 0.987103 & 0.993736 & -5.1137 & 0.413061 & 0.004137 \\
\hline 1 & 45 & 2 & 2 & 90 & 0.995969 & 0.999074 & -3.69887 & 0.413061 & 0.008718 \\
\hline 1 & 46 & 2 & 2 & 90 & 0.994884 & 0.998988 & -3.70178 & 0.413061 & 0.008698 \\
\hline 1 & 47 & 2 & 3.5 & 90 & 0.998492 & 0.999846 & -3.55772 & 0.413061 & 0.009801 \\
\hline 1 & 48 & 3 & 0.5 & 90 & 0.502232 & 0.41638 & -8.60321 & 0.413061 & 0.001802 \\
\hline 1 & 49 & 3 & 2 & 90 & 0.928177 & 0.966369 & -7.38201 & 0.413061 & 0.002246 \\
\hline 1 & 50 & 3 & 3.5 & 90 & 0.990991 & 0.995328 & -5.94843 & 0.413061 & 0.003158 \\
\hline 2 & 1 & 3 & 0.5 & 50 & 0.921918 & 0.933698 & -9.855 & 0.239181 & 0.003391 \\
\hline 2 & 2 & 3 & 2 & 50 & 0.929887 & 0.919007 & -16.3473 & 0.239181 & 0.001581 \\
\hline 2 & 3 & 3 & 3.5 & 50 & 0.695513 & 0.648641 & -12.2048 & 0.239181 & 0.002398 \\
\hline 2 & 4 & 3 & 0.5 & 60 & 0.956715 & 0.973052 & -12.5515 & 0.233391 & 0.002385 \\
\hline 2 & 5 & 3 & 2 & 60 & 0.844818 & 0.844179 & -12.2645 & 0.233391 & 0.002471 \\
\hline 2 & 6 & 3 & 3.5 & 60 & 0.501978 & 0.735473 & -12.3061 & 0.233391 & 0.002458 \\
\hline 2 & 7 & 3 & 0.5 & 70 & 0.952834 & 0.991498 & -9.60856 & 0.260441 & 0.003063 \\
\hline 2 & 8 & 3 & 2 & 70 & 0.9595 & 0.994012 & -10.403 & 0.260441 & 0.002692 \\
\hline 2 & 9 & 2 & 2 & 80 & 0.996122 & 0.99895 & -6.30834 & 0.320331 & 0.004508 \\
\hline 2 & 10 & 3 & 0.5 & 80 & 0.995335 & 0.998593 & -6.61753 & 0.320331 & 0.004110 \\
\hline 2 & 11 & 3 & 2 & 80 & 0.952335 & 0.932371 & -6.47385 & 0.320331 & 0.004286 \\
\hline 2 & 12 & 3 & 0.5 & 90 & 0.995874 & 0.99774 & -5.52918 & 0.413061 & 0.003584 \\
\hline 2 & 13 & 3 & 2 & 90 & 0.944412 & 0.993111 & -4.92812 & 0.413061 & 0.004444 \\
\hline
\end{tabular}


Table A4: Summary of Ammonia Results

\begin{tabular}{|c|c|c|c|c|c|c|c|c|c|}
\hline$\underset{3}{\text { Study }}$ & $\begin{array}{c}\text { Test } \\
\text { Sequence } \\
1\end{array}$ & $\begin{array}{c}\text { Salt } \\
\text { Solution } \\
2\end{array}$ & $\begin{array}{c}\text { NH3 Conc } \\
\text { (molal) } \\
2\end{array}$ & $\begin{array}{c}\text { Temperature } \\
{ }^{\mathbf{0}} \mathbf{C} \\
80\end{array}$ & $\begin{array}{c}\mathbf{R}^{2} \\
\text { Sampling } \\
\text { Sequence } \\
0.990992\end{array}$ & $\begin{array}{c}\mathbf{R}^{2} \\
\mathbf{y} \mathbf{~ v s} \\
\mathbf{x} \\
0.998536\end{array}$ & $\begin{array}{c}\text { slope, b } \\
\text { y vs x } \\
-8.88279\end{array}$ & $\begin{array}{c}\text { phi } \\
\text { (predicted) } \\
0.320331\end{array}$ & $\begin{array}{c}\mathbf{H}_{\mathbf{c}} \\
\text { Henry's } \\
\text { Constant } \\
0.002494\end{array}$ \\
\hline 3 & 2 & 3 & 0.5 & 80 & 0.996456 & 0.995446 & -6.20411 & 0.320331 & 0.004661 \\
\hline 3 & 3 & 3 & 2 & 80 & 0.995335 & 0.998593 & -6.61753 & 0.320331 & 0.004110 \\
\hline 3 & 4 & 3 & 0.5 & 90 & 0.952335 & 0.932371 & -6.47385 & 0.413061 & 0.002749 \\
\hline 3 & 5 & 3 & 2 & 90 & 0.998478 & 0.999084 & -7.20821 & 0.413061 & 0.002327 \\
\hline 3 & 6 & 2 & 2 & 80 & 0.958303 & 0.979068 & -5.80659 & 0.320331 & 0.005351 \\
\hline 3 & 7 & 3 & 2 & 90 & 0.989092 & 0.993884 & -6.00148 & 0.413061 & 0.003112 \\
\hline 3 & 8 & 1 & 0.5 & 90 & 0.972446 & 0.985086 & -5.6646 & 0.413061 & 0.003435 \\
\hline 3 & 9 & 2 & 0.5 & 90 & 0.988202 & 0.994488 & -6.85374 & 0.413061 & 0.002513 \\
\hline 4 & 1 & 1 & 0.5 & 70 & 0.991019 & 0.998972 & -7.17056 & 0.260441 & 0.005305 \\
\hline 4 & 2 & 1 & 2 & 70 & 0.999077 & 0.999698 & -6.366 & 0.260441 & 0.006994 \\
\hline 4 & 3 & 1 & 3.5 & 70 & 0.999447 & 0.999684 & -6.57471 & 0.260441 & 0.006460 \\
\hline 4 & 4 & 2 & 0.5 & 70 & 0.998153 & 0.998507 & -7.12812 & 0.260441 & 0.005373 \\
\hline 4 & 5 & 2 & 2 & 70 & 0.998319 & 0.999512 & -7.17319 & 0.260441 & 0.005301 \\
\hline 4 & 6 & 2 & 3.5 & 70 & 0.999062 & 0.999427 & -7.23884 & 0.260441 & 0.005198 \\
\hline 4 & 7 & 3 & 0.5 & 70 & 0.923557 & 0.934807 & -6.3919 & 0.260441 & 0.006923 \\
\hline 4 & 8 & 3 & 2 & 70 & 0.920728 & 0.985931 & -8.01572 & 0.260441 & 0.004231 \\
\hline 4 & 9 & 3 & 3.5 & 70 & 0.98253 & 0.991706 & -6.92072 & 0.260441 & 0.005735 \\
\hline 4 & 10 & 3 & 0.5 & 60 & 0.747275 & 0.693194 & -9.21351 & 0.233391 & 0.004000 \\
\hline 4 & 11 & 3 & 2 & 60 & 0.8918 & 0.941938 & -7.52707 & 0.233391 & 0.006081 \\
\hline 4 & 12 & 3 & 3.5 & 60 & 0.9748 & 0.981284 & -8.63346 & 0.233391 & 0.004534 \\
\hline 4 & 13 & 3 & 0.5 & 50 & 0.690551 & 0.539655 & -5.66432 & 0.239181 & 0.012971 \\
\hline 5 & 1 & 3 & 2 & 50 & 0.826948 & 0.924804 & -8.83198 & 0.239181 & 0.004137 \\
\hline 5 & 2 & 3 & 3.5 & 50 & 0.9603 & 0.953535 & -11.0334 & 0.239181 & 0.002808 \\
\hline 5 & 3 & 1 & 0.5 & 100 & 0.994323 & 0.998176 & -4.18498 & 0.538631 & 0.003669 \\
\hline 5 & 4 & 1 & 2 & 100 & 0.99546 & 0.999055 & -3.97826 & 0.538631 & 0.004027 \\
\hline 5 & 5 & 1 & 3.5 & 100 & 0.99922 & 0.999643 & -3.94391 & 0.538631 & 0.004093 \\
\hline 5 & 6 & 2 & 0.5 & 100 & 0.993864 & 0.999205 & -3.86517 & 0.538631 & 0.004254 \\
\hline 5 & 7 & 2 & 2 & 100 & 0.997379 & 0.999164 & -4.01547 & 0.538631 & 0.003957 \\
\hline 5 & 8 & 2 & 2 & 100 & 0.976805 & 0.93872 & -3.8325 & 0.538631 & 0.004324 \\
\hline 5 & 9 & 2 & 3.5 & 100 & 0.996732 & 0.998834 & -3.75649 & 0.538631 & 0.004497 \\
\hline 5 & 10 & 3 & 0.5 & 100 & 0.99586 & 0.925705 & -1.55738 & 0.538631 & -0.028560 \\
\hline 5 & 11 & 3 & 2 & 100 & 0.011498 & 0.525979 & -1.83634 & 0.538631 & -0.422640 \\
\hline 5 & 12 & 3 & 3.5 & 100 & 0.979579 & 0.974427 & -3.04784 & 0.538631 & 0.007172 \\
\hline 5 & 13 & 1 & 0.5 & 110 & 0.785614 & 0.924383 & -3.99127 & 0.697041 & 0.002582 \\
\hline 5 & 14 & 1 & 2 & 110 & 0.970096 & 0.990432 & -2.85442 & 0.697041 & 0.004650 \\
\hline 5 & 15 & 1 & 3.5 & 110 & 0.999723 & 0.999954 & -3.05525 & 0.697041 & 0.004074 \\
\hline 5 & 16 & 2 & 0.5 & 110 & 0.356063 & 0.760817 & -4.47456 & 0.697041 & 0.002172 \\
\hline 6 & 1 & 1 & 3.5 & 110 & 0.999729 & 0.999958 & -3.05601 & 0.697041 & 0.004072 \\
\hline 6 & 2 & 2 & 0.5 & 110 & 0.732103 & 0.853523 & -5.5938 & 0.697041 & 0.001587 \\
\hline 6 & 3 & 2 & 2 & 110 & 0.964694 & 0.980045 & -3.51423 & 0.697041 & 0.003175 \\
\hline 6 & 4 & 2 & 2 & 110 & 0.960364 & 0.94916 & -3.25934 & 0.697041 & 0.003618 \\
\hline 6 & 5 & 2 & 3.5 & 110 & 0.952216 & 0.980189 & -2.95224 & 0.697041 & 0.004350 \\
\hline 7 & 1 & 3 & 0.5 & 50 & 0.969892 & 0.984779 & -3.60627 & 0.239181 & -0.033480 \\
\hline 7 & 2 & 3 & 2 & 50 & 0.999114 & 0.998939 & -4.87737 & 0.239181 & 0.027627 \\
\hline 7 & 3 & 3 & 3.5 & 50 & 0.994769 & 0.997884 & -4.84632 & 0.239181 & 0.028916 \\
\hline 7 & 4 & 3 & 0.5 & 60 & 0.815104 & 0.966785 & -2.94125 & 0.233391 & -0.014680 \\
\hline 7 & 5 & 3 & 2 & 60 & 0.999637 & 0.996914 & -2.7196 & 0.233391 & -0.012600 \\
\hline 8 & 1 & 3 & 0.5 & 60 & 0.978118 & 0.961543 & -4.5989 & 0.233391 & 0.062746 \\
\hline 8 & 2 & 3 & 2 & 60 & 0.993937 & 0.998851 & -3.82081 & 0.233391 & -0.04251 \\
\hline 8 & 3 & 3 & 3.5 & 60 & 0.999032 & 0.99914 & -4.10526 & 0.233391 & -0.10991 \\
\hline 8 & 4 & 3 & 0.5 & 70 & 0.974833 & 0.988533 & -4.20831 & 0.260441 & 0.047928 \\
\hline 8 & 5 & 3 & 2 & 70 & 0.975969 & 0.99743 & -3.72719 & 0.260441 & -0.15712 \\
\hline 8 & 6 & 3 & 3.5 & 70 & 0.997326 & 0.999116 & -3.73823 & 0.260441 & -0.17424 \\
\hline 8 & 7 & 3 & 0.5 & 80 & 0.918447 & 0.983184 & -2.89107 & 0.320331 & -0.06227 \\
\hline 8 & 8 & 3 & 2 & 80 & 0.994238 & 0.998289 & -3.49811 & 0.320331 & 0.038173 \\
\hline 8 & 9 & 3 & 3.5 & 80 & 0.99614 & 0.999643 & -3.33024 & 0.320331 & 0.068914 \\
\hline 8 & 10 & 3 & 0.5 & 90 & 0.99221 & 0.976144 & -2.09117 & 0.413061 & -0.03378 \\
\hline 8 & 11 & 3 & 2 & 90 & 0.993309 & 0.996396 & -3.13192 & 0.413061 & 0.01567 \\
\hline 8 & 12 & 3 & 3.5 & 90 & 0.997705 & 0.999845 & -3.36244 & 0.413061 & 0.011833 \\
\hline
\end{tabular}


Equation (2) along with the phi's derived from the methanol results can be used to determine a value for $\mathrm{H}_{\mathrm{c}}$ at each temperature of interest for each salt solution and each $\mathrm{NH}_{3}$ concentration. These values are provided in the last column of Table A4.

Exhibits A7 and A8 provides plots of the slopes, the b's, and the $\mathrm{H}_{\mathrm{c}}$ values from the ammonia data regressions for each of the three salt solutions. The small squares represent test results for $\mathrm{NH}_{3}$ concentrations of 0.5 molal, the " + ' $\mathrm{s}$ " represent $\mathrm{NH}_{3}$ concentrations of 2.0 molal, and the " $\mathrm{x}$ ' $\mathrm{s}$ " represent test results for $\mathrm{NH}_{3}$ concentrations of 3.5 molal.

Salt Solution 1

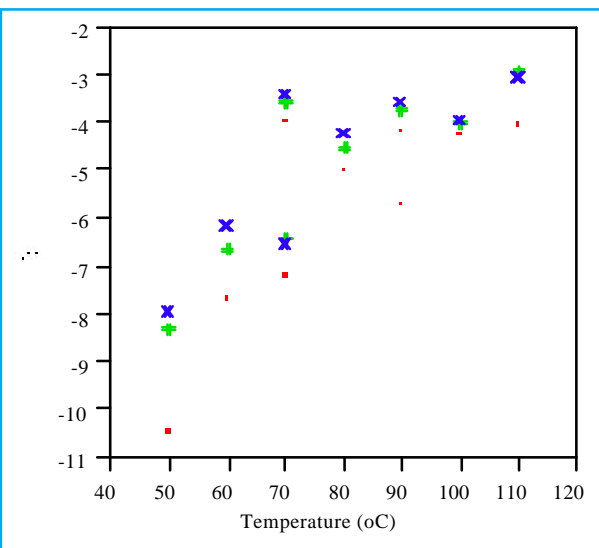

Exhibit A7: Slope, b, by Temperature $\left({ }^{\circ} \mathrm{C}\right)$ for Each Salt Solution Salt Solution 2

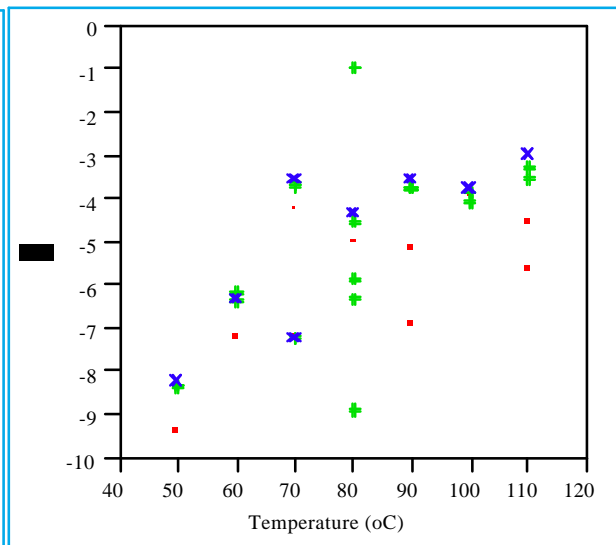

Salt Solution 3

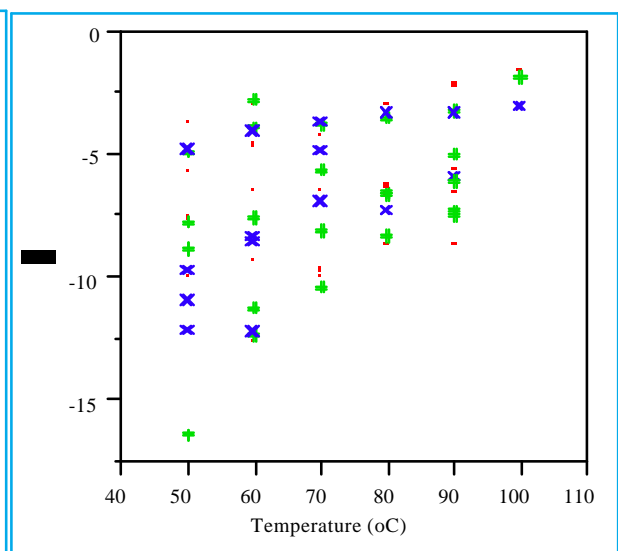

Exhibit A8. Plots of $\mathrm{H}_{\mathrm{c}}$ versus Temperature by Salt Solution/ $\mathrm{NH}_{3}$ Concentration

Salt Solution 1

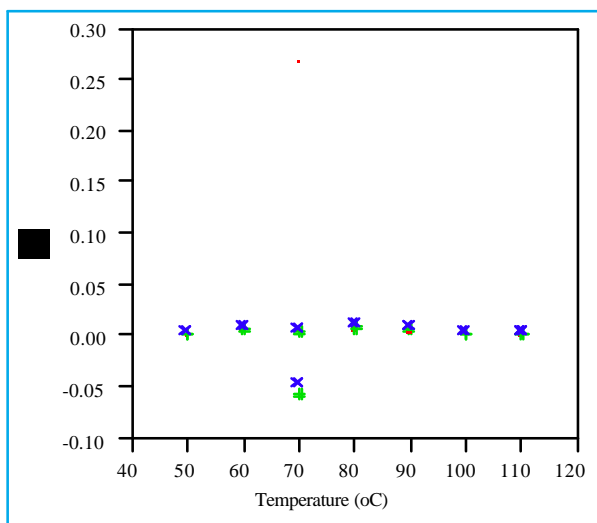

Salt Solution 2

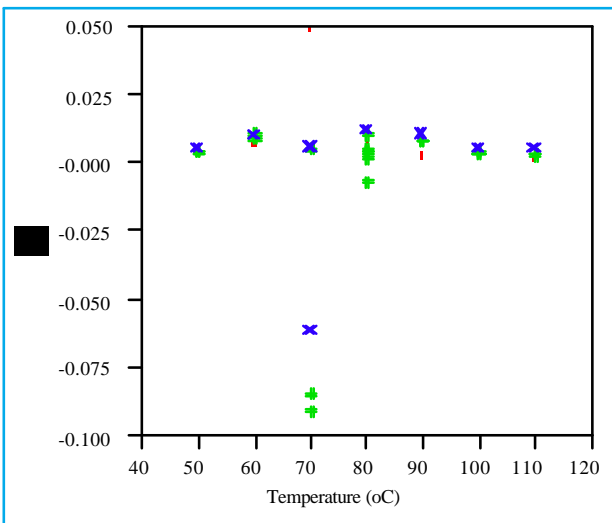

Salt Solution 3

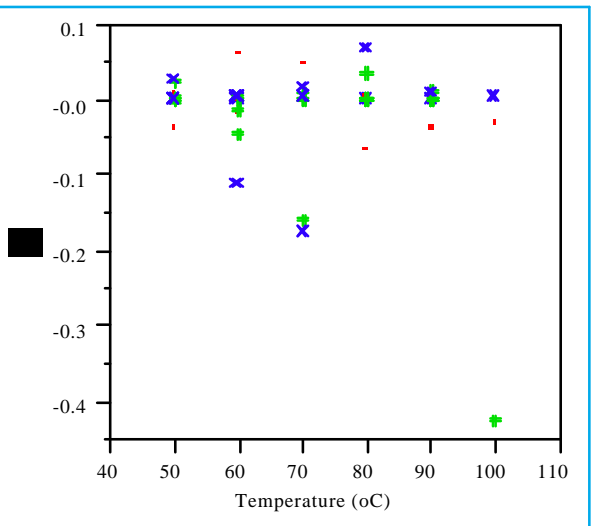

As seen in the data for both of these exhibits there are several probable outliers as well as some infeasible points (i.e., results with negative $\mathrm{H}_{\mathrm{c}}$ values). Seventeen tests yielded estimates of Hc that were negative. Fifity-one tests yielded $\mathrm{R}^{2}$ values (for one or both of the regressions conducted on the raw data) that were less than 99\%. In addition, one test (Study 1 Test Sequence 21, a $70{ }^{\circ} \mathrm{C}$ test) yielded an $\mathrm{H}_{\mathrm{c}}$ value of 0.267 and another test (Study 1 Test Sequence 24 , a $70{ }^{\circ} \mathrm{C}$ test) yielded an $\mathrm{H}_{\mathrm{c}}$ value of 0.044 . These values were much greater than the results from other similar tests. These questionable data values were removed, and the remaining $\mathrm{H}_{\mathrm{c}}$ results were plotted in Exhibit A9. 
Exhibit A9. Plots of Screened $H_{c}$ versus Temperature by Salt Solution

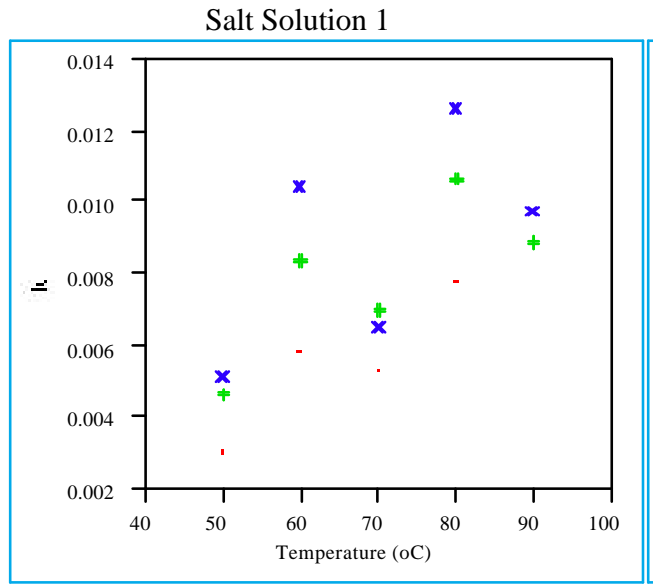

Salt Solution 2

Salt Solution 3

Exhibits A10 and A11 provide an Analysis of Variance (ANOVA) from a statistical investigation for a significant influence on the $\mathrm{H}_{c}$ values due to the $\mathrm{NH}_{3}$ concentration as well as temperature for each salt solution.

Exhibit A10. ANOVA of Statistical Model Relating $\mathrm{H}_{\mathrm{c}}$ to $\mathrm{NH}_{3}$ Concentration and Temperature For Salt Solutions 1 and 2

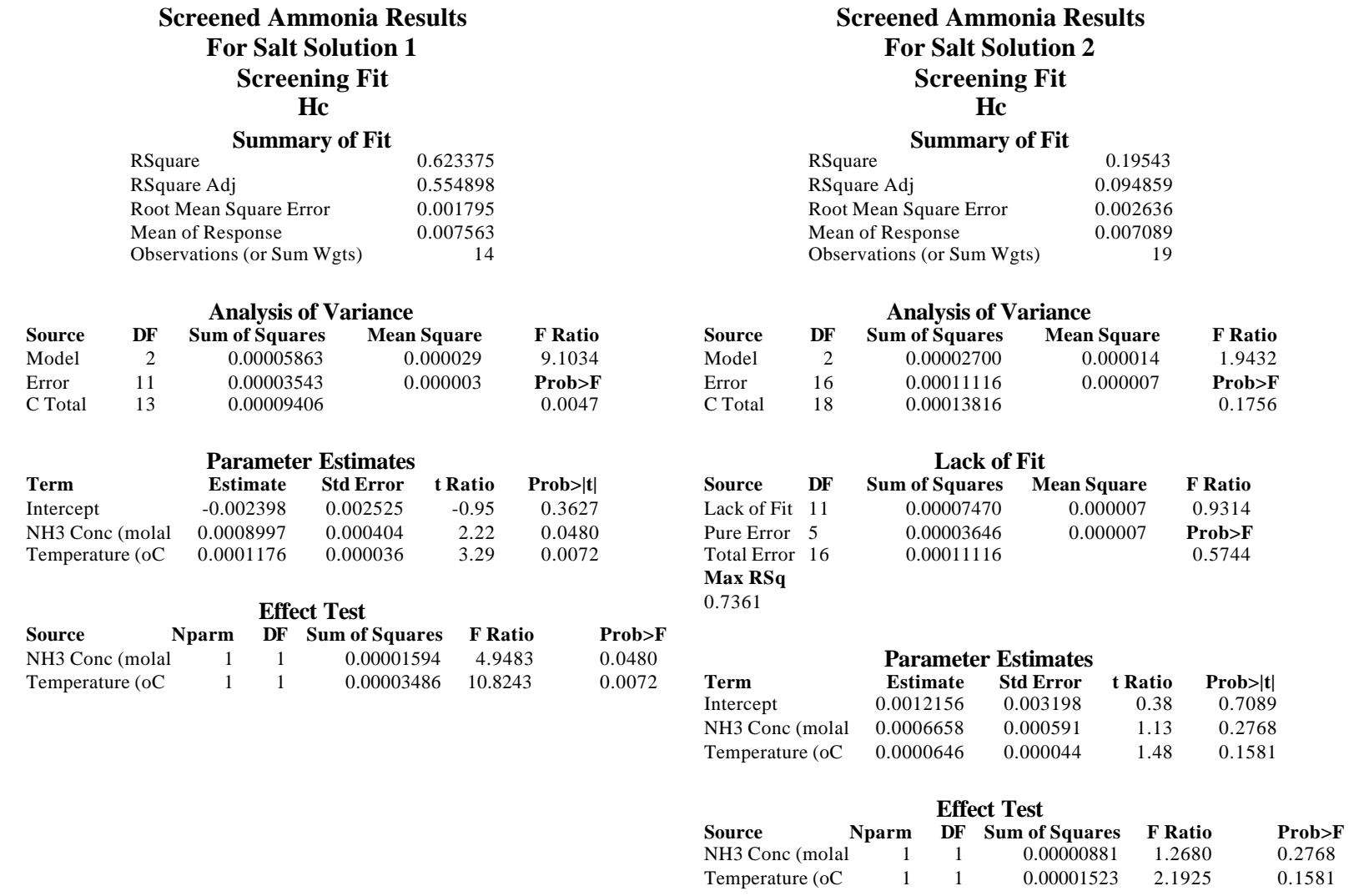




\section{Exhibit A11. ANOVA of Statistical Model Relating $\mathrm{H}_{\mathrm{c}}$ to $\mathrm{NH}_{3}$ Concentration and Temperature For Salt Solution 3}

\begin{tabular}{|c|c|c|c|c|}
\hline \multicolumn{5}{|c|}{ Screened Ammonia Results } \\
\hline \multicolumn{5}{|c|}{ Hc } \\
\hline \multicolumn{5}{|c|}{ Summary of Fit } \\
\hline & $\mathrm{RSq}$ & & 0.305094 & \\
\hline & $\mathrm{RSq}$ & Ire Adj & 0.15067 & \\
\hline & Roo & Mean Square Error & 0.018622 & \\
\hline & Mea & of Response & 0.017757 & \\
\hline & Obs & vations (or Sum W & 12 & \\
\hline \multicolumn{5}{|c|}{ Analysis of Variance } \\
\hline Source & DF & Sum of Squares & Mean Square & F Ratio \\
\hline Model & 2 & 0.00137028 & 0.000685 & 1.9757 \\
\hline Error & 9 & 0.00312105 & 0.000347 & Prob $>$ F \\
\hline C Total & 11 & 0.00449133 & & 0.1944 \\
\hline
\end{tabular}

\begin{tabular}{llrrr}
\multicolumn{7}{c}{ Lack of Fit } \\
Source & DF & Sum of Squares & Mean Square & F Ratio \\
Lack of Fit & 5 & 0.00241409 & 0.000483 & 2.7318 \\
Pure Error & 4 & 0.00070697 & 0.000177 & Prob>F \\
Total Error & 9 & 0.00312105 & & 0.1759 \\
Max RSq & & & & \\
0.8426 & & & & \\
& & & & \\
& & & & \\
Term & Parameter Estimates & & \\
Intercept & Estimate & Std Error & t Ratio & Prob>|t| \\
NH3 Conc (molal & 0.0351062 & 0.034628 & 1.01 & 0.3372 \\
Temperature (oC & 0.0072097 & 0.004776 & 1.51 & 0.1654 \\
& -0.000413 & 0.000394 & -1.05 & 0.3218
\end{tabular}

\begin{tabular}{lrrrrl} 
& \multicolumn{2}{c}{ Effect Test } & & \\
Source & Nparm & DF & Sum of Squares & F Ratio & Prob>F \\
NH3 Conc (molal & 1 & 1 & 0.00079019 & 2.2786 & 0.1654 \\
Temperature (oC & 1 & 1 & 0.00038122 & 1.0993 & 0.3218
\end{tabular}

There is some indication that the $\mathrm{NH}_{3}$ concentration affects the $\mathrm{H}_{\mathrm{c}}$ values for Salt Solution 1 but not for the other two salt solutions. However, there is a great deal of noise in these results. If $\mathrm{NH}_{3}$ is ignored, then temperature alone can be used to model the $\mathrm{H}_{\mathrm{c}}$ responses for each Salt Solution. Exhibits A12, A13, and A14 provide the results of fitting such a model to the average $\mathrm{H}_{\mathrm{c}}$ response at each temperature for Salt Solutions 12 and 3, respectively.

Exhibit A12: Average $H_{c}$ versus Temperature for Salt Solution 1

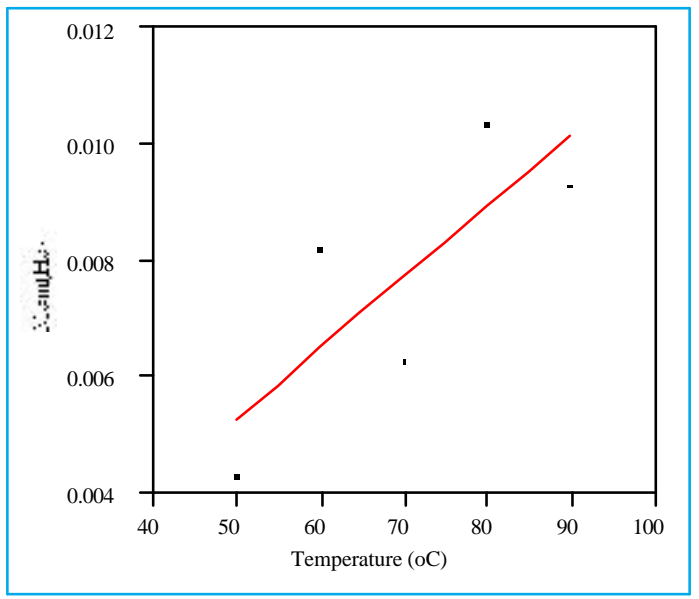

\begin{tabular}{|c|c|c|c|c|c|}
\hline \\
\hline \multicolumn{6}{|c|}{$\begin{array}{c}\text { Mean }(\mathrm{Hc})=-0.0009+0.00012 \text { Temperature } \\
(\mathrm{oC})\end{array}$} \\
\hline \multicolumn{6}{|c|}{ Summary of Fit } \\
\hline \multicolumn{6}{|c|}{0.629366} \\
\hline \multicolumn{6}{|c|}{ RSquare Adj } \\
\hline \multicolumn{6}{|c|}{ Root Mean Square Error } \\
\hline \multicolumn{6}{|c|}{ Mean of Response } \\
\hline \multicolumn{6}{|c|}{ Observations (or Sum Wgts) } \\
\hline \multicolumn{6}{|c|}{ Analysis of Variance } \\
\hline Source & DF & Sum of Squares & Mean & are & F Ratio \\
\hline Model & 1 & 0.00001490 & & 015 & 5.0942 \\
\hline Error & 3 & 0.00000878 & & 003 & Prob $>$ F \\
\hline C Total & 4 & 0.00002368 & & & 0.1092 \\
\hline \multicolumn{6}{|c|}{ Parameter Estimates } \\
\hline Term & & Estimate & Error & t Ratio & Prob $>|t|$ \\
\hline Intercept & & -0.000866 & 03862 & -0.22 & 0.8371 \\
\hline Temperatu & $(\mathrm{oC})$ & 0.0001221 & 00054 & 2.26 & 0.1092 \\
\hline
\end{tabular}




\section{Exhibit A13: Average $H_{c}$ versus Temperature for Salt Solution 2}

$\operatorname{Mean}(\mathrm{Hc})$ By Temperature (oC)

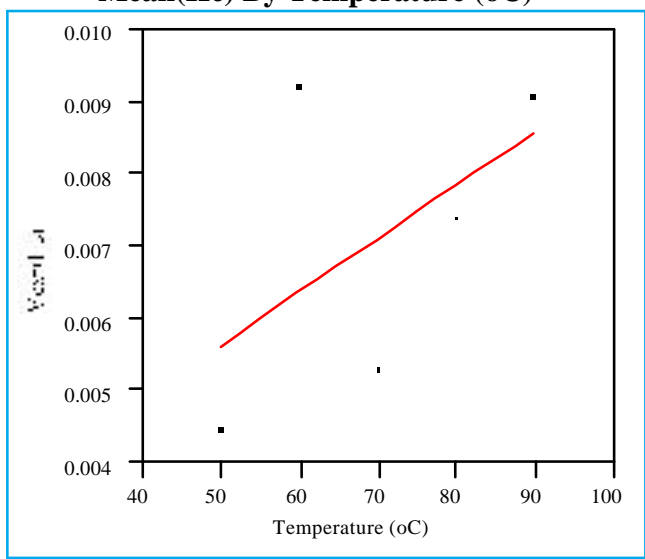

Linear Fit

$\operatorname{Mean}(\mathrm{Hc})=0.0019+0.00007$ Temperature $(\mathrm{oC})$

$\begin{array}{lr}\text { RSquare Summary of Fit } & \\ \text { RSquare Adj } & 0.292311 \\ \text { Root Mean Square Error } & 0.056414 \\ \text { Mean of Response } & 0.002102 \\ \text { Observations (or Sum Wgts) } & 0.007084 \\ & 5\end{array}$

Analysis of Variance

$\begin{array}{llllr}\text { Error } & 3 & 0.00000548 & 0.000005 & 1.2391 \\ \text { C } & 0.00001326 & 0.000004 & \text { Prob }>\mathbf{F}\end{array}$

$\begin{array}{llll}\text { C Total } & 4 & 0.00001873 & 0.3468\end{array}$

\begin{tabular}{lrcrr} 
& \multicolumn{2}{c}{ Parameter Estimates } & & \\
Term & $\begin{array}{r}\text { Estimate } \\
\text { Std Error }\end{array}$ & t Ratio & Prob $>|\mathbf{t}|$ \\
Intercept & 0.0019039 & 0.004747 & 0.40 & 0.7152 \\
Temperature (oC) & 0.000074 & 0.000066 & 1.11 & 0.3468
\end{tabular}

Exhibit A14: Average $H_{c}$ versus Temperature for Salt Solution 3

$\operatorname{Mean}(\mathrm{Hc})$ By Temperature (oC)

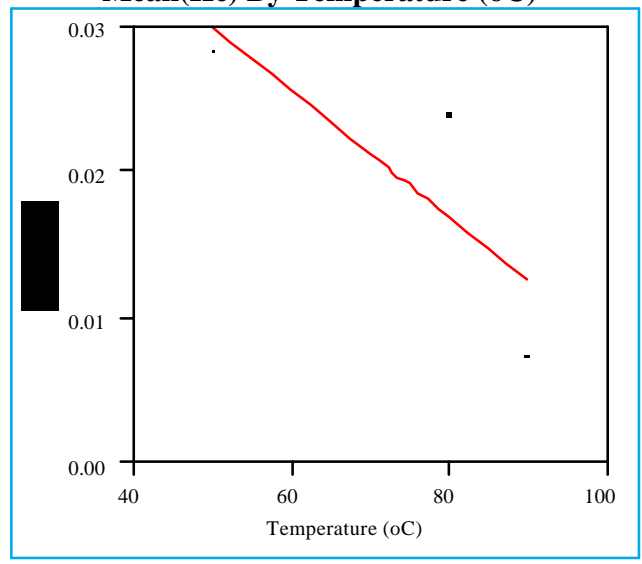

Linear Fit

$\operatorname{Mean}(\mathrm{Hc})=0.05183-0.00044$ Temperature

(oC)

\begin{tabular}{lr}
\multicolumn{1}{c}{ Summary of Fit } \\
RSquare & 0.6716 \\
RSquare Adj & 0.3432 \\
Root Mean Square Error & 0.008974 \\
Mean of Response & 0.01986 \\
Observations (or Sum Wgts) & 3
\end{tabular}

Analysis of Variance

Source DF Sum of Squares Mean Square F Ratio

$\begin{array}{lrrrr}\text { Model } & 1 & 0.00016469 & 0.000165 & 2.0451 \\ \text { Error } & 1 & 0.00008053 & 0.000081 & \text { Prob }>\text { F }\end{array}$

$\begin{array}{llll}\text { C Total } & 2 & 0.00024522 & 0.3885\end{array}$

\begin{tabular}{lrcrr}
\multicolumn{2}{c}{ Parameter Estimates } & & \\
Term & Estimate & Std Error & t Ratio & Prob $>|\mathbf{t}|$ \\
Intercept & 0.0518276 & 0.022947 & 2.26 & 0.2653 \\
Temperature (oC) & -0.000436 & 0.000305 & -1.43 & 0.3885
\end{tabular}

These results indicate very poor predictability of these $\mathrm{H}_{\mathrm{c}}$ models developed using temperature alone. The results for Salt Solution 3 show little (if any) temperature effect. The average value at $60{ }^{\circ} \mathrm{C}$ for Salt Solution 2 prevents a statistically significant relationship between these $\mathrm{H}_{\mathrm{c}}$ values and temperature. Even the Salt Solution 1 results show a great deal of scatter, and the fit of the Hc values to temperature is not statistically significant at a $95 \%$ confidence level.

The model of $\mathrm{H}_{\mathrm{c}}$ as a function of both temperature and $\mathrm{NH}_{3}$ molal concentration for Salt Solution 1 is investigated in Exhibit A15 through Exhibit A17. These exhibits provide (for each NH3 molal concentration) plots of the experimental $H_{c}$ values, the model predictions for the $H_{c}$ values, the upper and lower prediction limits for the average $\mathrm{H}_{\mathrm{c}}$ values, and predictions based upon the PNNL model. 
Exhibit A15: Salt Soln 1 Hc Plots for $\mathrm{NH}_{3}$ Concentration at 0.5 molal

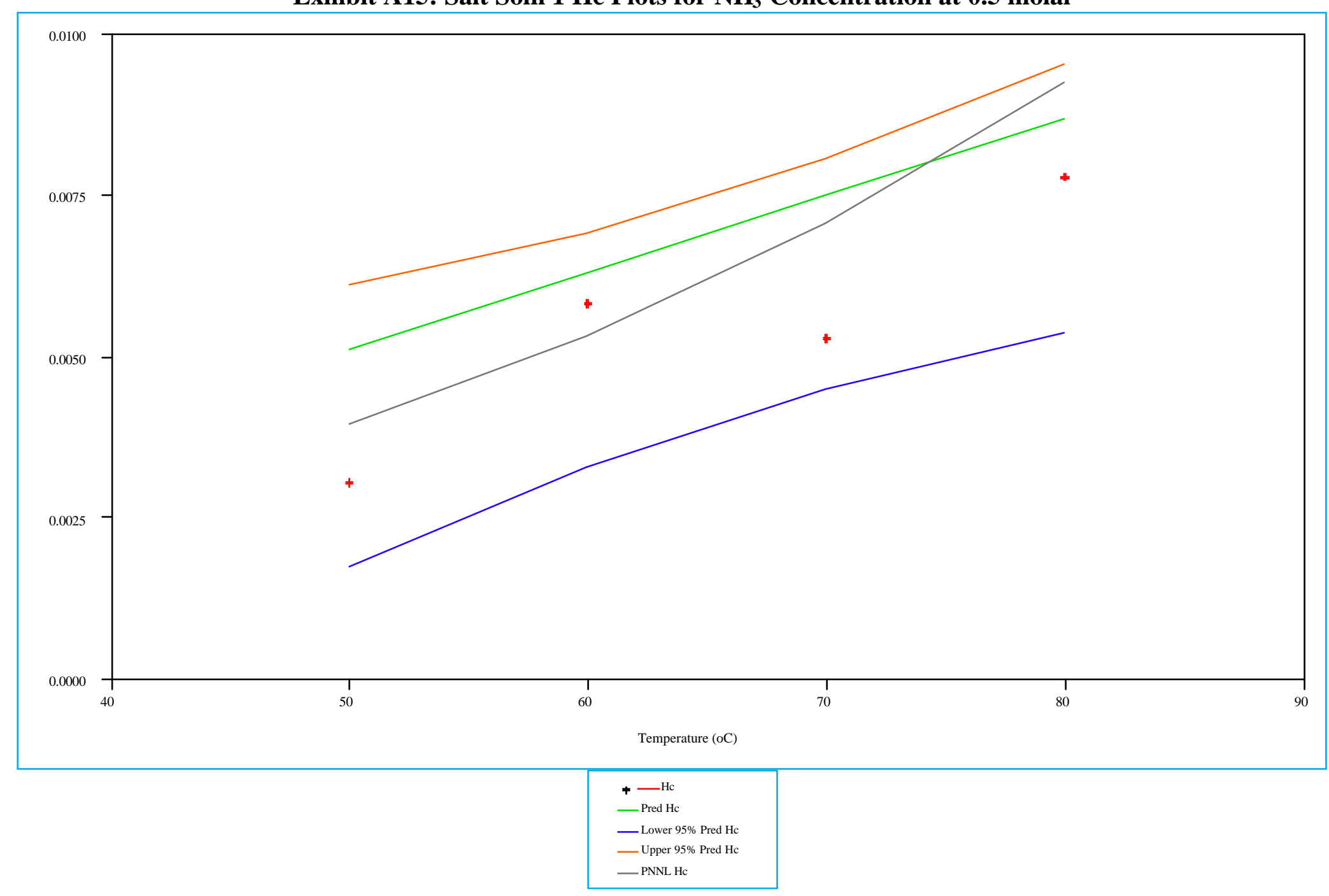

Page A14 of A22 
Exhibit A16: Salt Soln 1 Hc Plots for $\mathrm{NH}_{3}$ Concentration at 2.0 molal

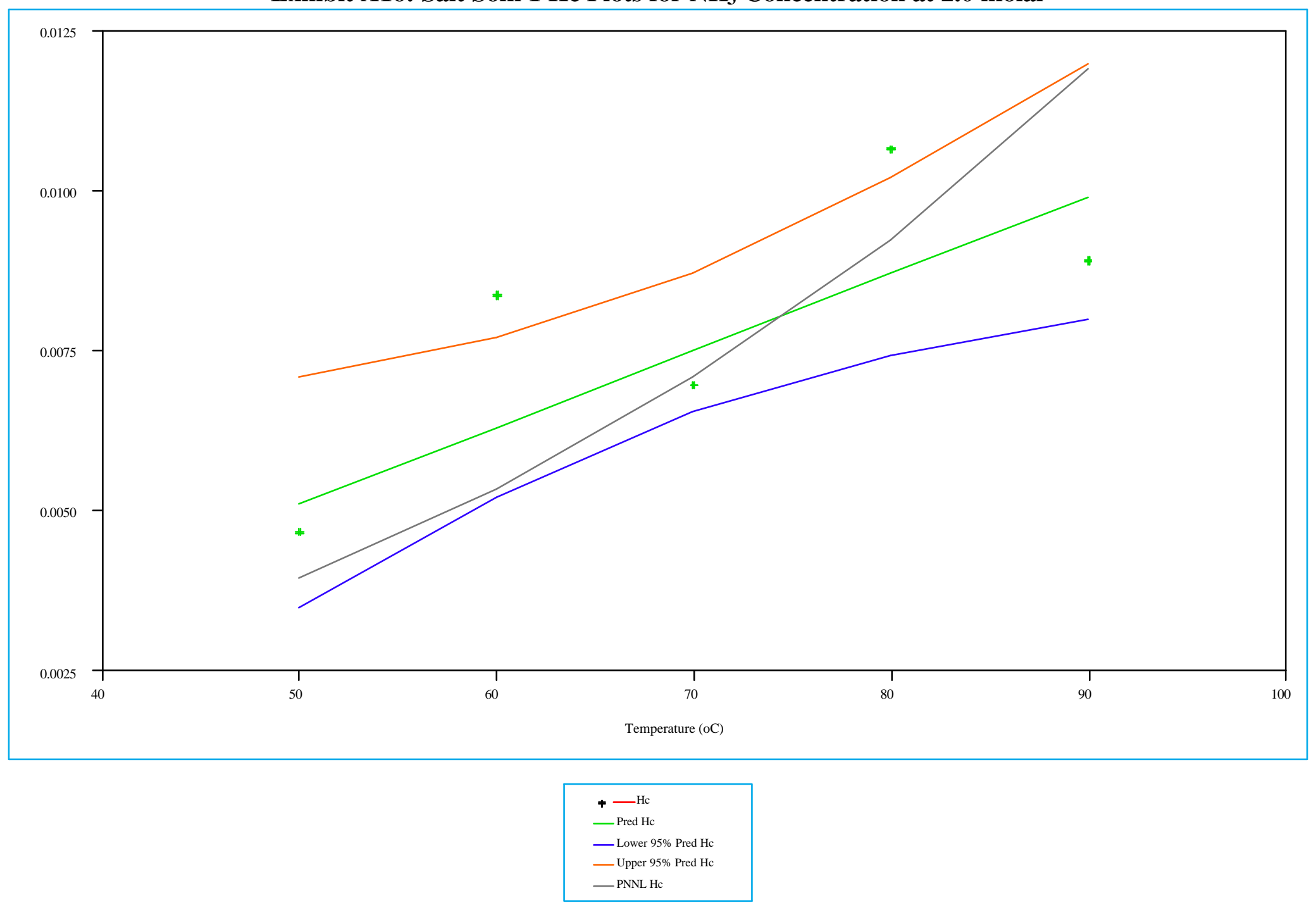

Page A15 of A22 
Exhibit A17: Salt Soln 1 Hc Plots for $\mathrm{NH}_{3}$ Concentration at 3.5 molal

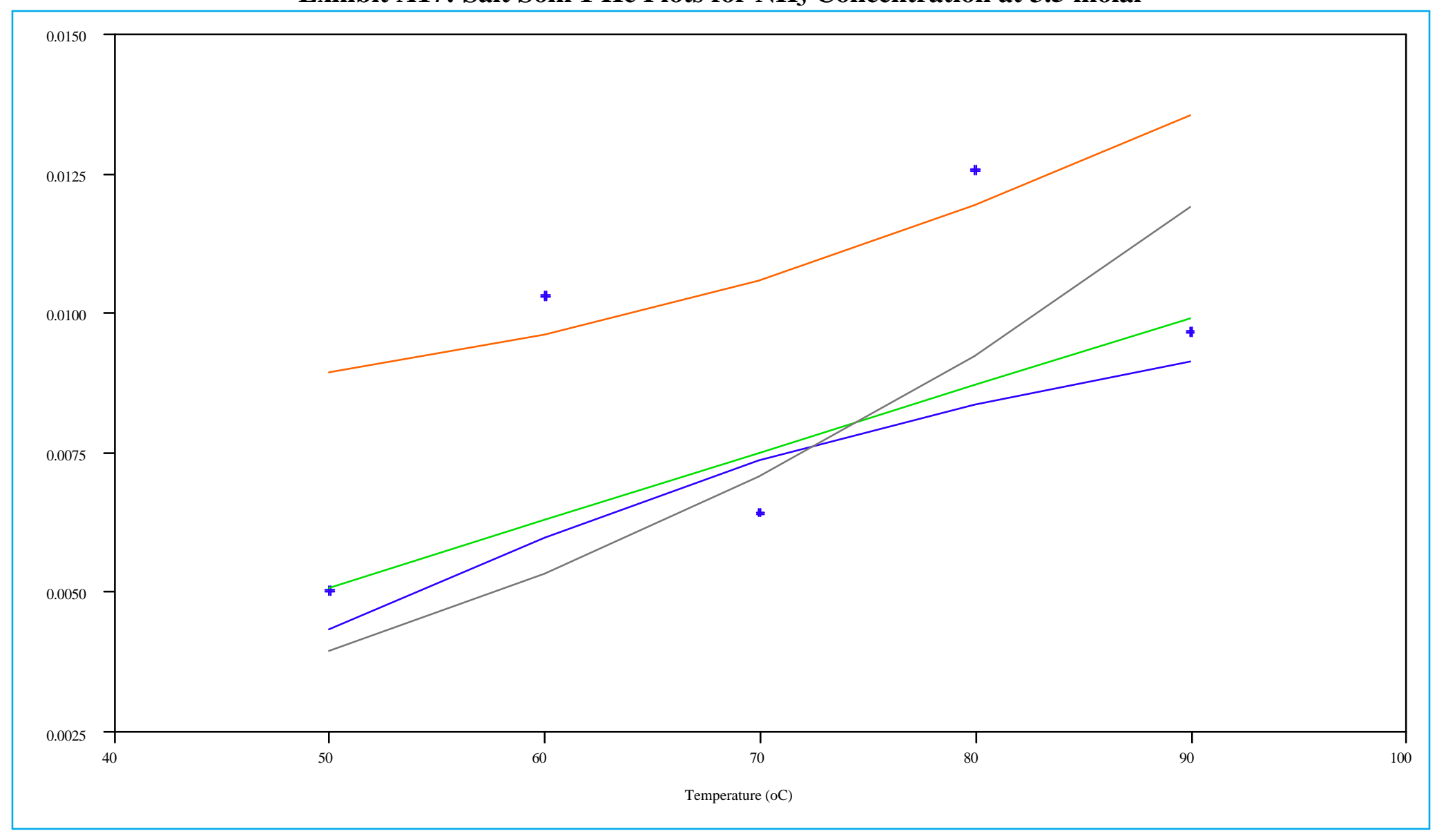

$+-\mathrm{Hc}$

- Lower 95\% Pred Hc

— Upper $95 \%$ Pred Hc

- $\mathrm{PNNL}$ Hc 
These plots indicate reasonable (based upon the uncertainties of the SRTC fitted model) agreement between the experimental results generated as part of this study and the results obtained with the PNNL model. This is true over the entire temperature range (50 to $90{ }^{\circ} \mathrm{C}$ ) considered in this investigation.

Exhibit A18 provides an additional plot of the $\mathrm{H}_{\mathrm{c}}$ values from these tests. In this plot, the average $\mathrm{H}_{\mathrm{c}}$ values at each temperature tested are plotted for Salt Solutions 2 and 3. The results from the Salt Solution 1 tests are broken down by $\mathrm{NH}_{3}$ molal concentration as well. Values determined by the PNNL model at each of these temperatures is also plotted as part of this exhibit.

Exhibit A18: Summary Plot of the $H_{c}$ Values from the Ammonia Tests

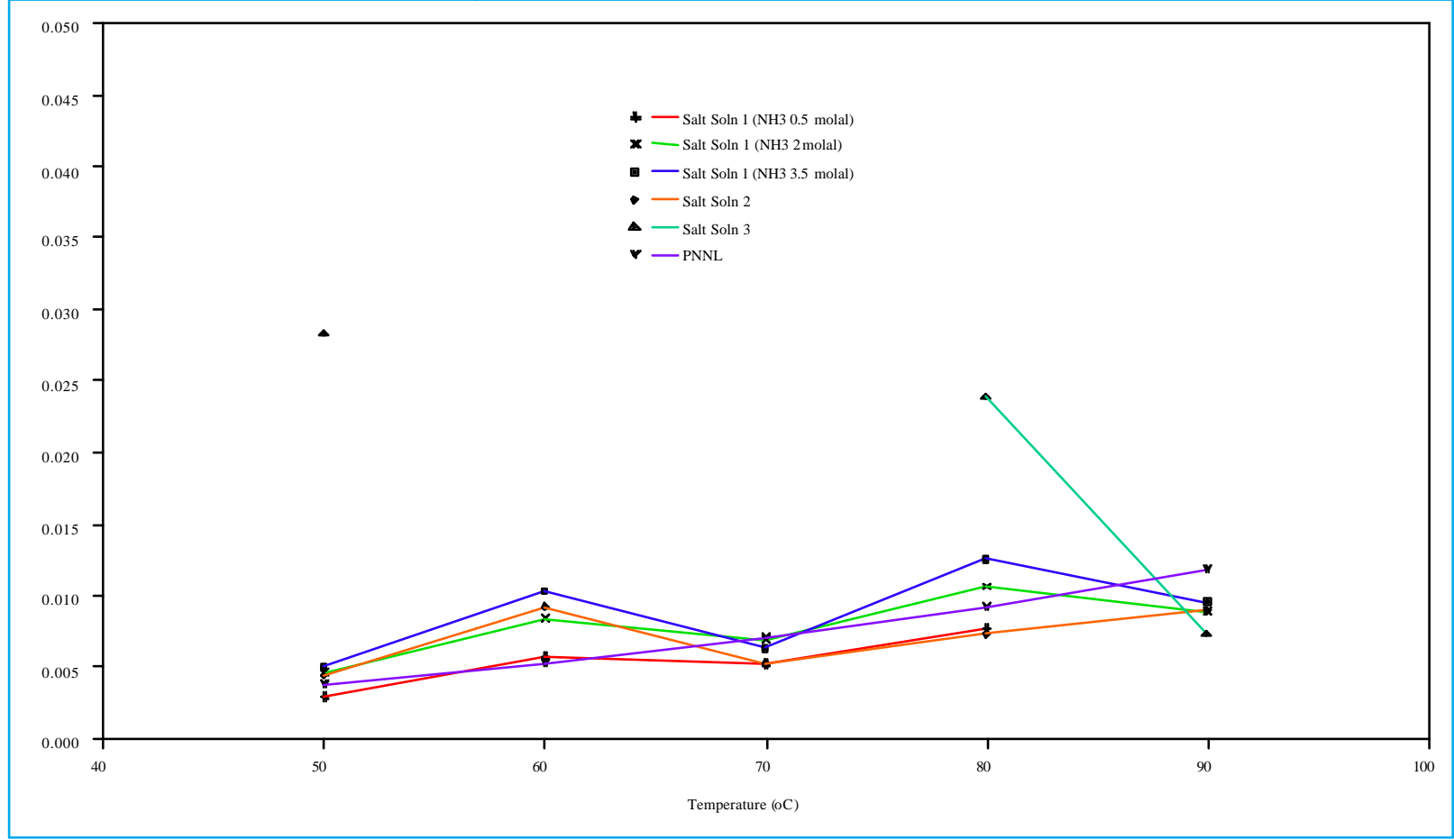




\section{SPECIAL SALT SOLUTION RESULTS}

The results from the testing of these special salt solutions are provided in Table B3. The results are presented in the order that the data were generated. The "Test Sequence" column of Table B3 will be used to identify these tests in the discussion that follows. Using this identifier, the Exhibits B63 through B70 in Appendix B provide a regression of $\log (\mathrm{A})$ versus the sampling sequence for these tests. All of these experimental results demonstrate strong linear correlations between these two sets of values $\left(\mathrm{R}^{2}\right.$ values are greater than $99 \%$ for all of the tests except for 1 , $10,11,17,18,49$, and 51).

For each test, equation (1) was fit to the peak area data (i.e., y was regressed on $\mathrm{x}$ ) to determine an estimate for the corresponding slope, b. Exhibits B17 through B78 in Appendix B provide the results from these regressions. Once again, the $\mathrm{R}^{2}$ values for almost all of these regressions are greater than $99 \%$ (only tests $17,36,49$, and 51 yielded $\mathrm{R}^{2}$ values less than $99 \%$ ). Table A5 provides a summary of the results from these special salt solution tests (indexed by "Test Sequence").

Table A5: Summary of Results for Special Salt Solutions

\begin{tabular}{|c|c|c|c|c|c|c|}
\hline $\begin{array}{c}\text { Test } \\
\text { Sequence }\end{array}$ & $\begin{array}{c}\text { Special } \\
\text { Salt } \\
\text { Solution }\end{array}$ & $\begin{array}{c}\text { Temperature } \\
\left({ }^{\circ} \mathrm{C}\right)\end{array}$ & $\begin{array}{c}\mathbf{R}^{2} \\
\text { Sampling } \\
\text { Sequence }\end{array}$ & $\begin{array}{c}R^{2} \\
\mathbf{y} v \mathbf{v S}\end{array}$ & $\begin{array}{l}\text { slope, } b \\
\text { y vS } x\end{array}$ & $\begin{array}{c}\mathbf{H}_{\mathbf{c}} \\
\text { Henry's } \\
\text { Constant }\end{array}$ \\
\hline 2 & $\mathrm{NaAlO} 2 \# 1$ & 50 & $\begin{array}{l}1.7000 \\
1.000\end{array}$ & $\begin{array}{l}0.999 \\
1.000\end{array}$ & $\begin{array}{l}-12.5263 \\
-12.8765\end{array}$ & $\begin{array}{l}0.00231 \\
0.00221\end{array}$ \\
\hline 3 & $\mathrm{NaAlO} 2 \# 2$ & 50 & 0.998 & 0.999 & -14.0997 & 0.00194 \\
\hline 4 & $\mathrm{NaAlO} 2 \# 2$ & 50 & 0.997 & 1.000 & -13.9694 & 0.00197 \\
\hline 5 & $\mathrm{NaAlO} 2 \# 2$ & 50 & 0.997 & 0.999 & -13.3721 & 0.00209 \\
\hline 6 & NaNO3 & 50 & 0.999 & 0.999 & -14.4218 & 0.00188 \\
\hline 7 & NaNO3 & 50 & 0.993 & 0.998 & -13.9127 & 0.00198 \\
\hline 8 & NaNO3 & 50 & 0.991 & 0.997 & -13.8142 & 0.00200 \\
\hline 9 & $\mathrm{NaNO}_{2}$ & 50 & 0.994 & 0.991 & -13.5615 & 0.00205 \\
\hline 10 & $\mathrm{NaNO} 2$ & 50 & 0.988 & 0.992 & -13.4761 & 0.00207 \\
\hline 11 & $\mathrm{NaNO} 2$ & 50 & 0.985 & 0.992 & -13.5753 & 0.00205 \\
\hline 12 & $\mathrm{Na} 2 \mathrm{CO} 3$ & 50 & 0.998 & 0.996 & -10.6715 & 0.00296 \\
\hline 13 & $\mathrm{Na} 2 \mathrm{CO} 3$ & 50 & 0.996 & 0.996 & -10.4139 & 0.00309 \\
\hline 14 & $\mathrm{Na} 2 \mathrm{CO} 3$ & 50 & 0.992 & 0.994 & -9.9661 & 0.00333 \\
\hline 15 & $\mathrm{Na} 2 \mathrm{SO} 4$ & 50 & 0.995 & 0.995 & -10.6832 & 0.00296 \\
\hline 16 & $\mathrm{Na} 2 \mathrm{SO} 4$ & 50 & 0.994 & 0.995 & -10.6505 & 0.00297 \\
\hline 17 & $\mathrm{Na} 2 \mathrm{SO} 4$ & 50 & 0.988 & 0.988 & -10.1814 & 0.00321 \\
\hline 18 & $\mathrm{Na} 2 \mathrm{CO} 3$ & 60 & 0.989 & 1.000 & -8.4451 & 0.00474 \\
\hline 19 & $\mathrm{Na} 2 \mathrm{CO} 3$ & 60 & 0.997 & 1.000 & -8.5305 & 0.00464 \\
\hline 20 & $\mathrm{Na} 2 \mathrm{CO} 3$ & 60 & 0.997 & 0.999 & -8.8055 & 0.00436 \\
\hline 21 & $\mathrm{Na} 2 \mathrm{SO} 4$ & 60 & 0.999 & 0.999 & -8.3378 & 0.00487 \\
\hline 22 & $\mathrm{Na} 2 \mathrm{SO} 4$ & 60 & 0.998 & 0.998 & -9.3809 & 0.00387 \\
\hline 23 & $\mathrm{Na} 2 \mathrm{SO} 4$ & 60 & 0.998 & 0.998 & -10.9314 & 0.00297 \\
\hline 24 & $\mathrm{NaNO} 2$ & 60 & 0.997 & 0.997 & -13.1221 & 0.00223 \\
\hline 25 & $\mathrm{NaNO} 2$ & 60 & 0.996 & 0.996 & -12.7955 & 0.00232 \\
\hline 26 & $\mathrm{NaNO} 2$ & 60 & 0.997 & 0.998 & -12.7631 & 0.00233 \\
\hline 27 & NaNO3 & 60 & 0.996 & 0.995 & -12.0351 & 0.00254 \\
\hline 28 & NaNO3 & 60 & 0.996 & 0.996 & -12.1407 & 0.00251 \\
\hline 29 & NaNO3 & 60 & 0.995 & 0.993 & -11.8083 & 0.00262 \\
\hline 30 & $\mathrm{Na} 2 \mathrm{CO} 3$ & 70 & 0.997 & 0.996 & -7.5204 & 0.00480 \\
\hline 31 & $\mathrm{Na} 2 \mathrm{CO} 3$ & 70 & 0.994 & 0.993 & -7.1750 & 0.00530 \\
\hline 32 & $\mathrm{Na} 2 \mathrm{CO} 3$ & 70 & 0.997 & 0.998 & -6.8650 & 0.00584 \\
\hline 33 & $\mathrm{Na} 2 \mathrm{SO} 4$ & 70 & 0.998 & 0.999 & -7.2396 & 0.00520 \\
\hline
\end{tabular}


Table A5: Summary of Results for Special Salt Solutions

\begin{tabular}{|c|c|c|c|c|c|c|}
\hline Test & $\begin{array}{c}\text { Special } \\
\text { Salt }\end{array}$ & Temperature & $\begin{array}{c}\mathbf{R}^{2} \\
\text { Sampling }\end{array}$ & $\mathbf{R}^{2}$ & slope, b & $\begin{array}{c}\mathbf{H}_{\mathrm{c}} \\
\text { Henry's }\end{array}$ \\
\hline Sequence & Solution & $\left({ }^{\circ} \mathrm{C}\right)$ & Sequence & y vs x & y vs x & Constant \\
\hline 34 & $\mathrm{Na} 2 \mathrm{SO} 4$ & 70 & 0.993 & 0.994 & -7.8328 & 0.00443 \\
\hline 35 & $\mathrm{Na} 2 \mathrm{SO} 4$ & 70 & 0.998 & 0.999 & -7.8729 & 0.00438 \\
\hline 36 & NaNO2 & 70 & 0.993 & 0.990 & -9.1854 & 0.00331 \\
\hline 37 & NaNO2 & 70 & 0.993 & 0.992 & -9.6993 & 0.00302 \\
\hline 38 & NaNO2 & 70 & 0.992 & 0.996 & -9.5843 & 0.00308 \\
\hline 39 & NaNO3 & 70 & 0.998 & 0.996 & -9.0433 & 0.00340 \\
\hline 40 & NaNO3 & 70 & 0.997 & 0.995 & -9.3979 & 0.00318 \\
\hline 41 & NaNO3 & 70 & 0.995 & 0.993 & -9.0050 & 0.00342 \\
\hline 42 & $\mathrm{Na} 2 \mathrm{CO} 3$ & 80 & 0.998 & 0.999 & -5.9215 & 0.00513 \\
\hline 43 & $\mathrm{Na} 2 \mathrm{CO} 3$ & 80 & 0.997 & 0.997 & -5.9438 & 0.00509 \\
\hline 44 & $\mathrm{Na} 2 \mathrm{CO} 3$ & 80 & 0.999 & 0.999 & -5.6101 & 0.00577 \\
\hline 45 & $\mathrm{Na} 2 \mathrm{SO} 4$ & 80 & 0.996 & 0.998 & -6.1327 & 0.00477 \\
\hline 46 & $\mathrm{Na} 2 \mathrm{SO} 4$ & 80 & 0.996 & 0.996 & -6.1171 & 0.00480 \\
\hline 47 & $\mathrm{Na} 2 \mathrm{SO} 4$ & 80 & 0.995 & 0.996 & -5.9858 & 0.00502 \\
\hline 48 & NaNO2 & 80 & 0.996 & 0.997 & -6.6433 & 0.00408 \\
\hline 49 & NaNO2 & 80 & 0.987 & 0.974 & -7.0474 & 0.00366 \\
\hline 50 & NaNO2 & 80 & 0.994 & 0.992 & -6.9935 & 0.00371 \\
\hline 51 & NaNO3 & 80 & 0.986 & 0.989 & -6.7806 & 0.00393 \\
\hline 52 & NaNO3 & 80 & 0.996 & 0.997 & -6.5377 & 0.00421 \\
\hline 53 & $\mathrm{NaOH} 1 \mathrm{M}$ & 90 & 0.992 & 0.996 & -5.1701 & 0.00405 \\
\hline 54 & $\mathrm{NaOH} 1 \mathrm{M}$ & 80 & 0.993 & 0.997 & -5.8403 & 0.00529 \\
\hline 55 & $\mathrm{NaOH} 1 \mathrm{M}$ & 80 & 0.997 & 1.000 & -5.9938 & 0.00500 \\
\hline 56 & $\mathrm{NaOH} 1 \mathrm{M}$ & 70 & 0.997 & 0.995 & -6.6963 & 0.00619 \\
\hline 57 & $\mathrm{NaOH} 1 \mathrm{M}$ & 70 & 0.999 & 1.000 & -6.7450 & 0.00608 \\
\hline 58 & $\mathrm{NaOH} 1 \mathrm{M}$ & 60 & 0.998 & 0.998 & -7.0420 & 0.00715 \\
\hline 59 & $\mathrm{NaOH} 1 \mathrm{M}$ & 60 & 0.999 & 0.998 & -6.9527 & 0.00739 \\
\hline 60 & $\mathrm{NaOH} 1 \mathrm{M}$ & 50 & 0.996 & 0.995 & -7.0162 & 0.00679 \\
\hline 61 & $\mathrm{NaOH} 1 \mathrm{M}$ & 50 & 0.998 & 0.998 & -6.3485 & 0.00888 \\
\hline
\end{tabular}

Equation (2) along with the phi's derived from the methanol results can be used to determine a value for $\mathrm{H}_{\mathrm{c}}$ at each temperature of interest for each salt solution and each $\mathrm{NH}_{3}$ concentration. These $\mathrm{H}_{\mathrm{c}}$ values are provided in the last column of Table A5.

Exhibits A19 and A20 provides plots of the slopes, the b's, and the $\mathrm{H}_{\mathrm{c}}$ values from the ammonia data regressions for each of the three salt solutions. The small squares represent test results for $\mathrm{NH}_{3}$ concentrations of 0.5 molal, the " + ' $\mathrm{s}$ " represent $\mathrm{NH}_{3}$ concentrations of 2.0 molal, and the "x's" represent test results for $\mathrm{NH}_{3}$ concentrations of 3.5 molal.

Exhibit A19: Slope, b, by Temperature $\left({ }^{\circ} \mathrm{C}\right)$ for Each Special Salt Solution

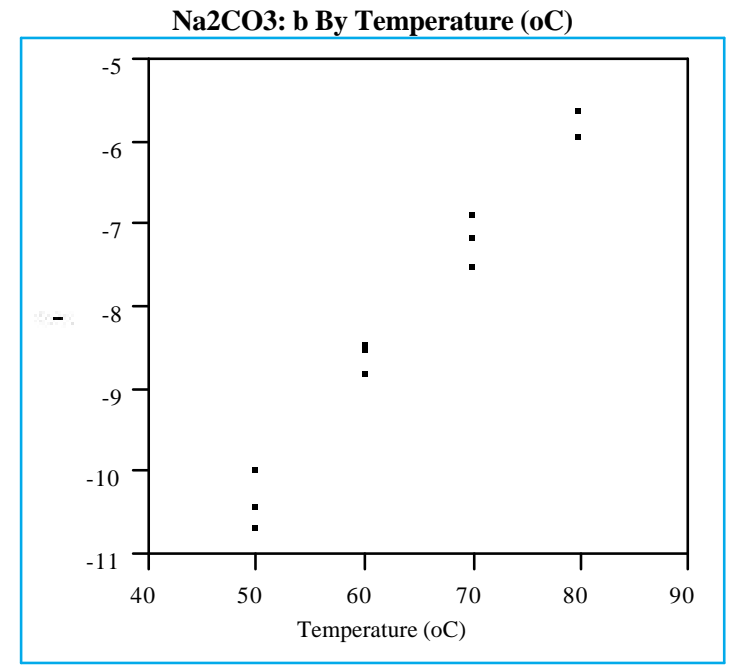




\section{Exhibit A19: Slope, b, by Temperature $\left({ }^{\circ} \mathrm{C}\right)$ for Each Special Salt Solution (continued)}

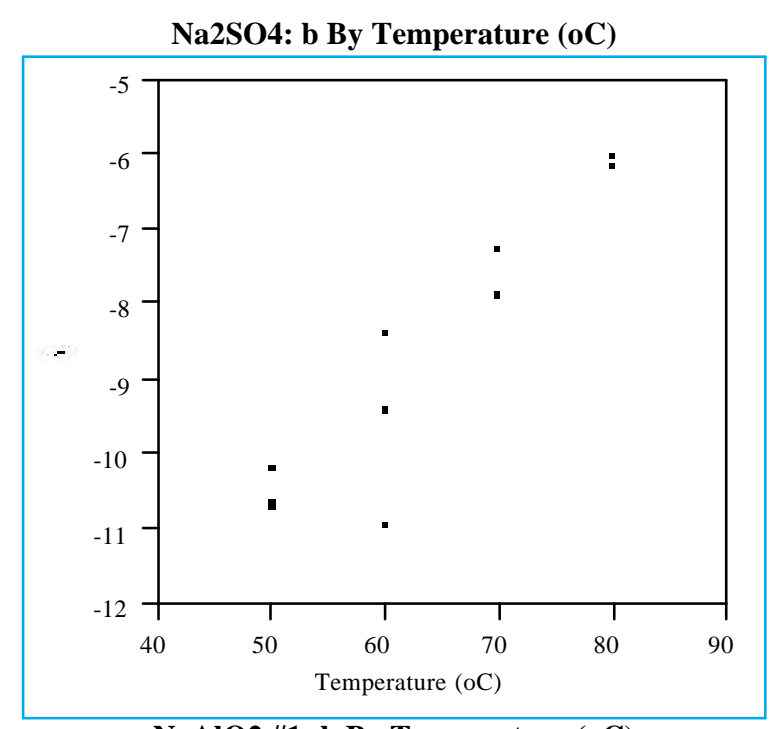

NaAIO2 \#1: b By Temperature (oC)
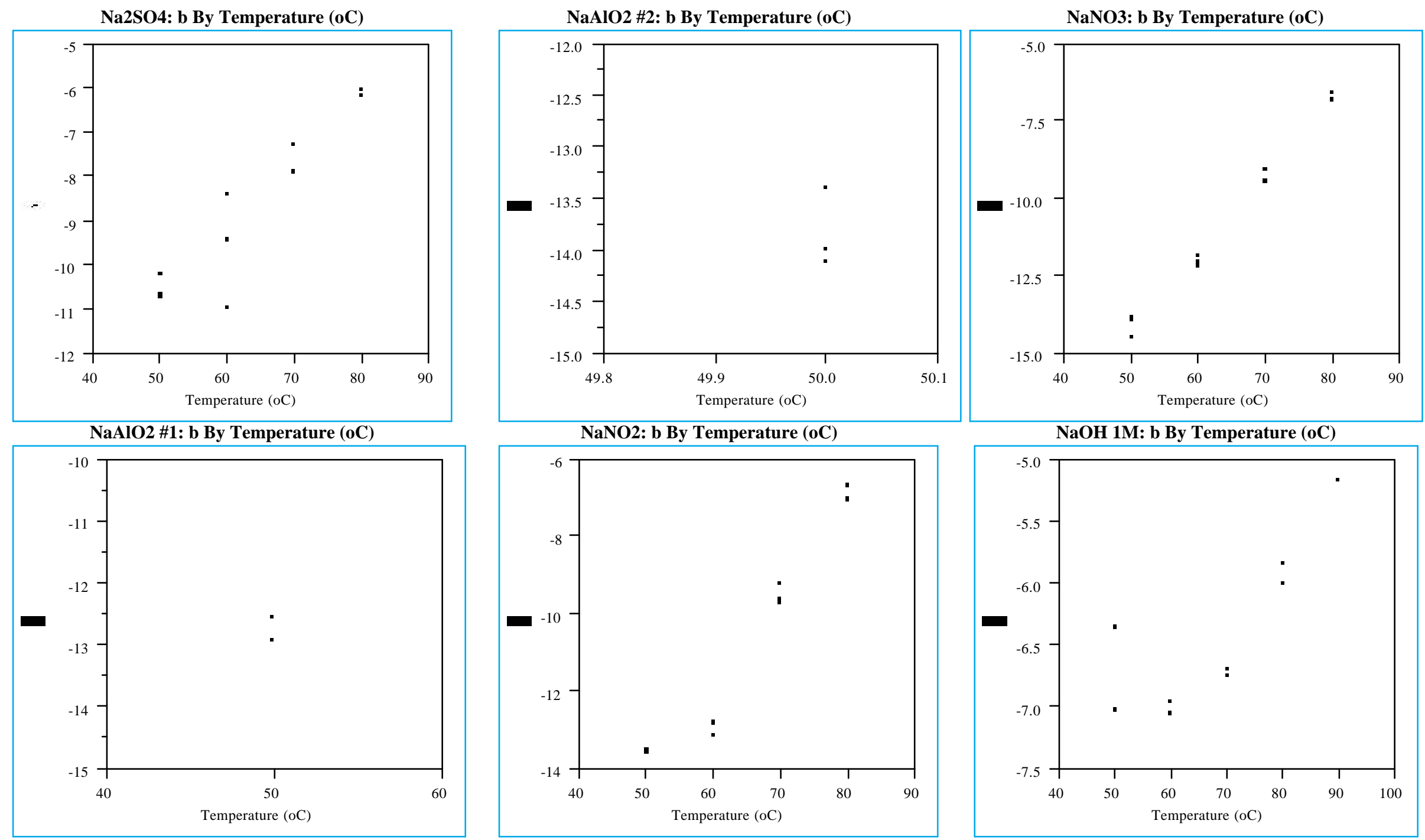

Page A 20 of A22 
Exhibit A20: Slope, b, by Temperature $\left({ }^{\circ} \mathrm{C}\right)$ for Each Special Salt Solution

Na2CO3: Hc By Temperature (oC)

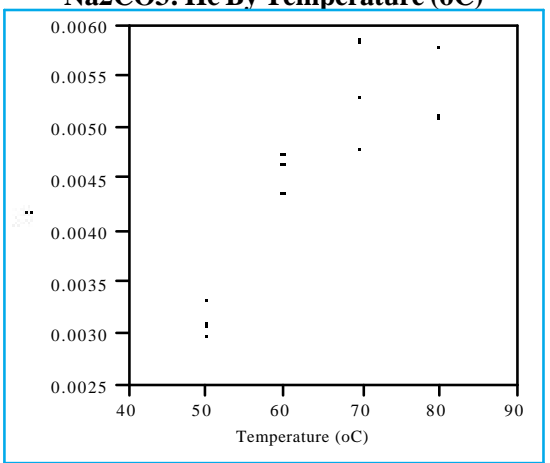

Na2SO4: Hc By Temperature (oC)

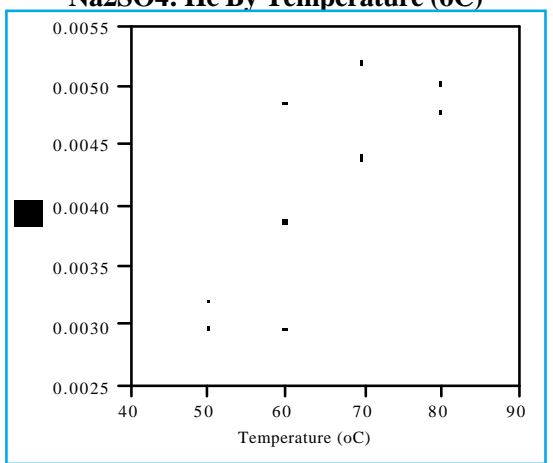

NaAlO2 \#1: Hc By Temperature (oC)

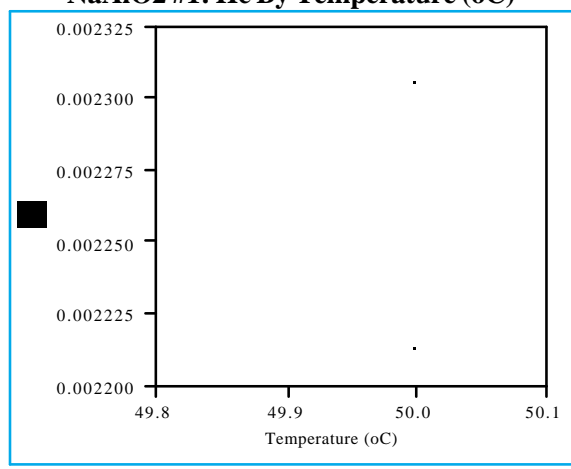

NaAlO2 \#2: Hc By Temperature (oC)

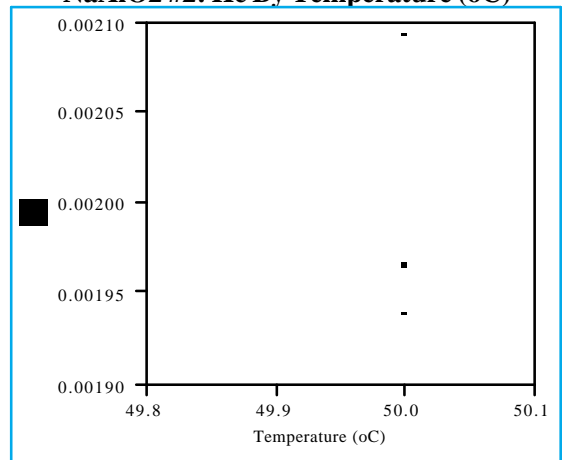

NaNO2: Hc By Temperature (oC)

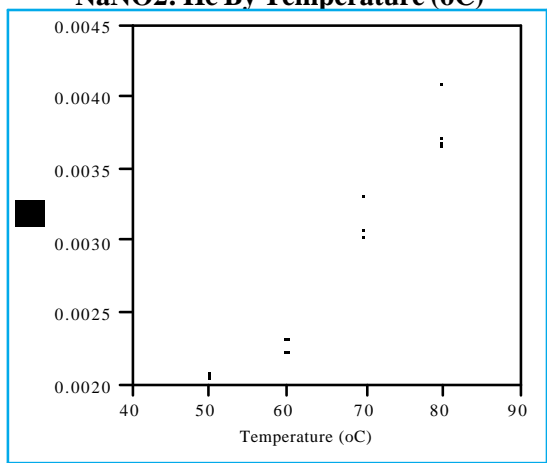

NaNO3: Hc By Temperature (oC)

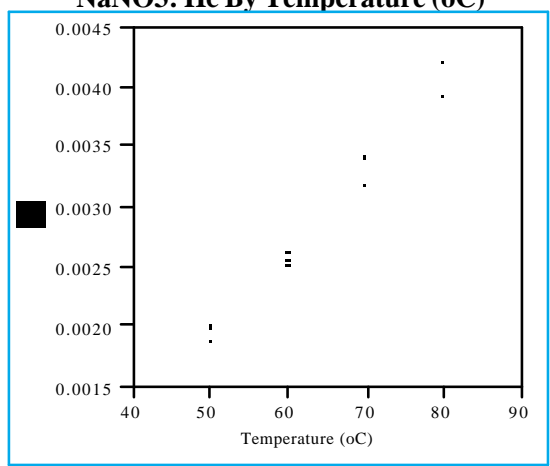

NaOH 1M: Hc By Temperature (oC)

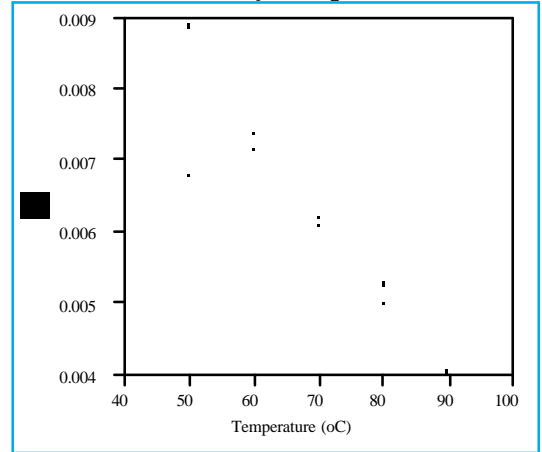


Summary information for the $\mathrm{H}_{\mathrm{c}}$ values that were generated from these special salt solutions is provided in Table A6.

Table A6: Henry's Constant Values for Special Salt Solutions by Temperature

\begin{tabular}{|c|c|c|c|c|}
\hline $\begin{array}{c}\text { Salt } \\
\text { Solution }\end{array}$ & $\begin{array}{c}\text { Temperature } \\
{ }^{\circ} \mathrm{C} \\
\end{array}$ & $\begin{array}{c}\text { Average } \\
\text { H}_{c} \\
\text { Henry's } \\
\text { Constant }\end{array}$ & $\begin{array}{c}\text { Standard } \\
\text { Deviation } \\
\text { of Hc } \\
\text { Values } \\
\end{array}$ & $\begin{array}{c}\% \text { Relative } \\
\text { Standard } \\
\text { Deviation } \\
\text { of } \mathrm{H}_{\mathrm{c}} \text { Values }\end{array}$ \\
\hline $\mathrm{Na} 2 \mathrm{CO} 3$ & 50 & 0.00313 & 0.00018 & $5.9 \%$ \\
\hline $\mathrm{Na} 2 \mathrm{CO} 3$ & 60 & 0.00458 & 0.00020 & $4.3 \%$ \\
\hline $\mathrm{Na} 2 \mathrm{CO} 3$ & 70 & 0.00531 & 0.00052 & $9.8 \%$ \\
\hline $\mathrm{Na} 2 \mathrm{CO} 3$ & 80 & 0.00533 & 0.00038 & $7.2 \%$ \\
\hline $\mathrm{Na} 2 \mathrm{SO} 4$ & 50 & 0.00305 & 0.00014 & $4.6 \%$ \\
\hline $\mathrm{Na} 2 \mathrm{SO} 4$ & 60 & 0.00390 & 0.00095 & $24.4 \%$ \\
\hline $\mathrm{Na} 2 \mathrm{SO} 4$ & 70 & 0.00467 & 0.00046 & $9.8 \%$ \\
\hline $\mathrm{Na} 2 \mathrm{SO} 4$ & 80 & 0.00486 & 0.00014 & $2.8 \%$ \\
\hline NaAlO2\#1 & 50 & 0.00226 & 0.00007 & $2.9 \%$ \\
\hline $\mathrm{NaAlO} 2 \# 2$ & 50 & 0.00200 & 0.00008 & $4.1 \%$ \\
\hline NaNO2 & 50 & 0.00206 & 0.00001 & $0.6 \%$ \\
\hline $\mathrm{NaNO} 2$ & 60 & 0.00229 & 0.00005 & $2.3 \%$ \\
\hline $\mathrm{NaNO} 2$ & 70 & 0.00313 & 0.00015 & $4.9 \%$ \\
\hline $\mathrm{NaNO} 2$ & 80 & 0.00382 & 0.00023 & $6.0 \%$ \\
\hline NaNO3 & 50 & 0.00195 & 0.00006 & $3.2 \%$ \\
\hline NaNO3 & 60 & 0.00256 & 0.00006 & $2.2 \%$ \\
\hline NaNO3 & 70 & 0.00333 & 0.00013 & $4.0 \%$ \\
\hline NaNO3 & 80 & 0.00407 & 0.00020 & $4.8 \%$ \\
\hline $\mathrm{NaOH} 1 \mathrm{M}$ & 50 & 0.00783 & 0.00148 & $18.9 \%$ \\
\hline $\mathrm{NaOH} 1 \mathrm{M}$ & 60 & 0.00727 & 0.00017 & $2.3 \%$ \\
\hline $\mathrm{NaOH} 1 \mathrm{M}$ & 70 & 0.00613 & 0.00007 & $1.2 \%$ \\
\hline $\mathrm{NaOH} 1 \mathrm{M}$ & 80 & 0.00514 & 0.00020 & $3.9 \%$ \\
\hline $\mathrm{NaOH} 1 \mathrm{M}$ & 90 & 0.00405 & & \\
\hline
\end{tabular}

\section{CONCLUSIONS}

The results from this testing were more varied than suggested by the results discussed in reference [2]. Screening mechanisms were incorporated in the review of these data to help reduce this scatter. However, anomalies remained even after this screening process was completed. Attempts to model the resulting $\mathrm{H}_{\mathrm{c}}$ values as a function of temperature (and possibly $\mathrm{NH}_{3}$ molal concentration) yielded models with large uncertainties. The Salt Solution 3 results show little (if any) temperature effect at all.

Comparisons between the Salt Solution 1 results (including a model of $\mathrm{Hc}$ as a function of temperature and NH3 molal concentration) and those predicted by a model developed by PNNL show reasonably good agreement (within the uncertainties of the model developed here).

\section{REFERENCES}

[1] SAS Institute, Inc., JMP® Statistics and Graphics Guide, Version 3, SAS Institute, Inc., Cary, NC, 1994.

[2] Chai, X. S. and J. Y. Zhu, "Simultaneous Measurements of Solute Concentration and Henry's Constant Using Multiple Headspace Extraction Gas Chromatography," Analytical Chemistry, Vol. 70, No. 16, August 15, 1998, pages 3481-3487.

[3] Swingle, R. F., M. R. Poirier, C. J. Berry, "Task Technical and Quality Assurance Plan for the Determination of Ammonia Partial Pressure in Pump Tank Salt Solutions," WSRC-RP-99-01118, Revision 0, December 21, 1999.

[4] J. D. Norton and L. R. Pederson, "Ammonia in Simulated Hanford Double-Shell Tank Wastes: Solubility and Effects on Surface Tension," PNL-10173, September 1994. 
Table B1. Methanol Test Results

\begin{tabular}{|c|c|c|}
\hline & Test & Test \\
\hline Study & Sequence & Identifier \\
\hline 1 & 1 & $50 \mathrm{C}-1-1-1$ \\
\hline 1 & 1 & $50 \mathrm{C}-1-1-2$ \\
\hline 1 & 1 & $50 \mathrm{C}-1-1-3$ \\
\hline 1 & 1 & $50 \mathrm{C}-1-1-4$ \\
\hline 1 & 1 & $50 \mathrm{C}-1-1-5$ \\
\hline 1 & 1 & $50 \mathrm{C}-1-1-6$ \\
\hline 1 & 1 & $50 C-1-1-7$ \\
\hline 1 & 1 & $50 \mathrm{C}-1-1-8$ \\
\hline 1 & 1 & $50 \mathrm{C}-1-1-9$ \\
\hline 1 & 1 & $50 \mathrm{C}-1-1-10$ \\
\hline 1 & 2 & $50 \mathrm{C}-1-2-1$ \\
\hline 1 & 2 & $50 \mathrm{C}-1-2-2$ \\
\hline 1 & 2 & $50 \mathrm{C}-1-2-3$ \\
\hline 1 & 2 & $50 \mathrm{C}-1-2-4$ \\
\hline 1 & 2 & $50 \mathrm{C}-1-2-5$ \\
\hline 1 & 2 & $50 \mathrm{C}-1-2-6$ \\
\hline 1 & 2 & $50 \mathrm{C}-1-2-7$ \\
\hline 1 & 2 & $50 \mathrm{C}-1-2-8$ \\
\hline 1 & 2 & $50 \mathrm{C}-1-2-9$ \\
\hline 1 & 2 & $50 \mathrm{C}-1-2-10$ \\
\hline 1 & 3 & $50 C-1-3-1$ \\
\hline 1 & 3 & $50 \mathrm{C}-1-3-2$ \\
\hline 1 & 3 & $50 \mathrm{C}-1-3-3$ \\
\hline 1 & 3 & $50 \mathrm{C}-1-3-4$ \\
\hline 1 & 3 & $50 C-1-3-5$ \\
\hline 1 & 3 & $50 \mathrm{C}-1-3-6$ \\
\hline 1 & 3 & $50 \mathrm{C}-1-3-7$ \\
\hline 1 & 3 & $50 C-1-3-8$ \\
\hline 1 & 3 & $50 C-1-3-9$ \\
\hline 1 & 3 & $50 \mathrm{C}-1-3-10$ \\
\hline 1 & 4 & $50 \mathrm{C}-19-1-1$ \\
\hline 1 & 4 & $50 \mathrm{C}-19-1-2$ \\
\hline 1 & 4 & $50 \mathrm{C}-19-1-3$ \\
\hline 1 & 4 & $50 \mathrm{C}-19-1-4$ \\
\hline 1 & 4 & $50 \mathrm{C}-19-1-5$ \\
\hline 1 & 4 & $50 \mathrm{C}-19-1-6$ \\
\hline 1 & 4 & $50 \mathrm{C}-19-1-7$ \\
\hline 1 & 4 & $50 \mathrm{C}-19-1-8$ \\
\hline 1 & 4 & $50 \mathrm{C}-19-1-9$ \\
\hline 1 & 4 & $50 \mathrm{C}-19-1-10$ \\
\hline 1 & 5 & $50 \mathrm{C}-19-2-1$ \\
\hline 1 & 5 & $50 \mathrm{C}-19-2-2$ \\
\hline 1 & 5 & $50 \mathrm{C}-19-2-3$ \\
\hline 1 & 5 & $50 \mathrm{C}-19-2-4$ \\
\hline 1 & 5 & $50 \mathrm{C}-19-2-5$ \\
\hline 1 & 5 & $50 \mathrm{C}-19-2-6$ \\
\hline 1 & 5 & $50 \mathrm{C}-19-2-7$ \\
\hline 1 & 5 & $50 \mathrm{C}-19-2-8$ \\
\hline 1 & 5 & $50 \mathrm{C}-19-2-9$ \\
\hline 1 & 5 & $50 \mathrm{C}-19-2-10$ \\
\hline 1 & 6 & $50 \mathrm{C}-19-3-1$ \\
\hline 1 & 6 & $50 C-19-3-2$ \\
\hline 1 & 6 & $50 \mathrm{C}-19-3-3$ \\
\hline 1 & 6 & $50 \mathrm{C}-19-3-4$ \\
\hline 1 & 6 & $50 \mathrm{C}-19-3-5$ \\
\hline 1 & 6 & $50 C-19-3-6$ \\
\hline 1 & 6 & $50 \mathrm{C}-19-3-7$ \\
\hline 1 & 6 & $50 \mathrm{C}-19-3-8$ \\
\hline 1 & 6 & $50 \mathrm{C}-19-3-9$ \\
\hline 1 & 6 & $50 \mathrm{C}-19-3-10$ \\
\hline 1 & 7 & $50 \mathrm{C}-2-1-1$ \\
\hline 1 & 7 & $50 \mathrm{C}-2-1-2$ \\
\hline 1 & 7 & $50 \mathrm{C}-2-1-3$ \\
\hline 1 & 7 & $50 \mathrm{C}-2-1-4$ \\
\hline 1 & 7 & $50 C-2-1-5$ \\
\hline
\end{tabular}

\begin{tabular}{|c|c|c|c|c|c|c|c|}
\hline Temperature & Vial & Test & Sampling & A & & & \\
\hline$\left({ }^{\circ} \mathrm{C}\right)$ & Pressusre & Run & Sequence & (peak area) & $\mathrm{y}$ & $\mathrm{x}$ & $\log (\mathrm{A})$ \\
\hline 50 & $1 \mathrm{~atm}$ & 1 & 1 & 4713.83 & 4713.83 & 4840.51 & 3.6734 \\
\hline 50 & $1 \mathrm{~atm}$ & 1 & 2 & 4840.51 & 9554.34 & 4734.11 & 3.6849 \\
\hline 50 & $1 \mathrm{~atm}$ & 1 & 3 & 4734.11 & 14288.45 & 4621.69 & 3.6752 \\
\hline 50 & $1 \mathrm{~atm}$ & 1 & 4 & 4621.69 & 18910.13 & 4506.92 & 3.6648 \\
\hline 50 & $1 \mathrm{~atm}$ & 1 & 5 & 4506.92 & 23417.05 & 4354.04 & 3.6539 \\
\hline 50 & $1 \mathrm{~atm}$ & 1 & 6 & 4354.04 & 27771.09 & 4245.94 & 3.6389 \\
\hline 50 & $1 \mathrm{~atm}$ & 1 & 7 & 4245.94 & 32017.03 & 4096.29 & 3.6280 \\
\hline 50 & $1 \mathrm{~atm}$ & 1 & 8 & 4096.29 & 36113.31 & 4003.54 & 3.6124 \\
\hline 50 & $1 \mathrm{~atm}$ & 1 & 9 & 4003.54 & 40116.86 & 4043.60 & 3.6024 \\
\hline 50 & $1 \mathrm{~atm}$ & 1 & 10 & 4043.60 & 44160.46 & & 3.6068 \\
\hline 50 & $1 \mathrm{~atm}$ & 2 & 1 & 5301.99 & 5301.99 & 5061.61 & 3.7244 \\
\hline 50 & $1 \mathrm{~atm}$ & 2 & 2 & 5061.61 & 10363.60 & 4818.75 & 3.7043 \\
\hline 50 & $1 \mathrm{~atm}$ & 2 & 3 & 4818.75 & 15182.36 & 4633.92 & 3.6829 \\
\hline 50 & $1 \mathrm{~atm}$ & 2 & 4 & 4633.92 & 19816.28 & 4518.79 & 3.6659 \\
\hline 50 & $1 \mathrm{~atm}$ & 2 & 5 & 4518.79 & 24335.07 & 4391.15 & 3.6550 \\
\hline 50 & $1 \mathrm{~atm}$ & 2 & 6 & 4391.15 & 28726.22 & 4242.09 & 3.6426 \\
\hline 50 & $1 \mathrm{~atm}$ & 2 & 7 & 4242.09 & 32968.31 & 4131.79 & 3.6276 \\
\hline 50 & $1 \mathrm{~atm}$ & 2 & 8 & 4131.79 & 37100.10 & 4018.81 & 3.6161 \\
\hline 50 & $1 \mathrm{~atm}$ & 2 & 9 & 4018.81 & 41118.91 & 3858.32 & 3.6041 \\
\hline 50 & $1 \mathrm{~atm}$ & 2 & 10 & 3858.32 & 44977.23 & & 3.5864 \\
\hline 50 & $1 \mathrm{~atm}$ & 3 & 1 & 6116.60 & 6116.60 & 7846.68 & 3.7865 \\
\hline 50 & $1 \mathrm{~atm}$ & 3 & 2 & 7846.68 & 13963.29 & 5416.69 & 3.8947 \\
\hline 50 & $1 \mathrm{~atm}$ & 3 & 3 & 5416.69 & 19379.98 & 5298.98 & 3.7337 \\
\hline 50 & $1 \mathrm{~atm}$ & 3 & 4 & 5298.98 & 24678.96 & 5148.20 & 3.7242 \\
\hline 50 & $1 \mathrm{~atm}$ & 3 & 5 & 5148.20 & 29827.16 & 5021.56 & 3.7117 \\
\hline 50 & $1 \mathrm{~atm}$ & 3 & 6 & 5021.56 & 34848.72 & 4850.27 & 3.7008 \\
\hline 50 & $1 \mathrm{~atm}$ & 3 & 7 & 4850.27 & 39699.00 & 4742.96 & 3.6858 \\
\hline 50 & $1 \mathrm{~atm}$ & 3 & 8 & 4742.96 & 44441.95 & 4606.10 & 3.6760 \\
\hline 50 & $1 \mathrm{~atm}$ & 3 & 9 & 4606.10 & 49048.06 & 4451.51 & 3.6633 \\
\hline 50 & $1 \mathrm{~atm}$ & 3 & 10 & 4451.51 & 53499.56 & & 3.6485 \\
\hline 50 & 19 psig & 1 & 1 & 6370.98 & 6370.98 & 4898.33 & 3.8042 \\
\hline 50 & 19 psig & 1 & 2 & 4898.33 & 11269.30 & 4695.96 & 3.6900 \\
\hline 50 & 19 psig & 1 & 3 & 4695.96 & 15965.26 & 4509.57 & 3.6717 \\
\hline 50 & 19 psig & 1 & 4 & 4509.57 & 20474.83 & 4343.54 & 3.6541 \\
\hline 50 & 19 psig & 1 & 5 & 4343.54 & 24818.38 & 4205.17 & 3.6378 \\
\hline 50 & 19 psig & 1 & 6 & 4205.17 & 29023.54 & 4055.12 & 3.6238 \\
\hline 50 & 19 psig & 1 & 7 & 4055.12 & 33078.66 & 3911.61 & 3.6080 \\
\hline 50 & 19 psig & 1 & 8 & 3911.61 & 36990.28 & 3765.71 & 3.5924 \\
\hline 50 & 19 psig & 1 & 9 & 3765.71 & 40755.98 & 3628.03 & 3.5758 \\
\hline 50 & 19 psig & 1 & 10 & 3628.03 & 44384.02 & & 3.5597 \\
\hline 50 & 19 psig & 2 & 1 & 6813.08 & 6813.08 & 5104.41 & 3.8333 \\
\hline 50 & 19 psig & 2 & 2 & 5104.41 & 11917.50 & 4510.72 & 3.7079 \\
\hline 50 & 19 psig & 2 & 3 & 4510.72 & 16428.22 & 4326.60 & 3.6542 \\
\hline 50 & 19 psig & 2 & 4 & 4326.60 & 20754.82 & 4155.04 & 3.6361 \\
\hline 50 & 19 psig & 2 & 5 & 4155.04 & 24909.86 & 3974.46 & 3.6186 \\
\hline 50 & 19 psig & 2 & 6 & 3974.46 & 28884.32 & 3820.32 & 3.5993 \\
\hline 50 & 19 psig & 2 & 7 & 3820.32 & 32704.65 & 3670.14 & 3.5821 \\
\hline 50 & 19 psig & 2 & 8 & 3670.14 & 36374.79 & 3496.54 & 3.5647 \\
\hline 50 & 19 psig & 2 & 9 & 3496.54 & 39871.33 & 3304.03 & 3.5436 \\
\hline 50 & 19 psig & 2 & 10 & 3304.03 & 43175.35 & & 3.5190 \\
\hline 50 & 19 psig & 3 & 1 & 7531.48 & 7531.48 & 5740.20 & 3.8769 \\
\hline 50 & 19 psig & 3 & 2 & 5740.20 & 13271.67 & 4531.20 & 3.7589 \\
\hline 50 & 19 psig & 3 & 3 & 4531.20 & 17802.87 & 4425.63 & 3.6562 \\
\hline 50 & 19 psig & 3 & 4 & 4425.63 & 22228.50 & 4257.58 & 3.6460 \\
\hline 50 & 19 psig & 3 & 5 & 4257.58 & 26486.08 & 4083.11 & 3.6292 \\
\hline 50 & 19 psig & 3 & 6 & 4083.11 & 30569.19 & 3918.35 & 3.6110 \\
\hline 50 & 19 psig & 3 & 7 & 3918.35 & 34487.54 & 3770.76 & 3.5931 \\
\hline 50 & 19 psig & 3 & 8 & 3770.76 & 38258.29 & 3625.96 & 3.5764 \\
\hline 50 & 19 psig & 3 & 9 & 3625.96 & 41884.25 & 3424.13 & 3.5594 \\
\hline 50 & 19 psig & 3 & 10 & 3424.13 & 45308.38 & & 3.5346 \\
\hline 50 & $2 \mathrm{~atm}$ & 1 & 1 & 8258.80 & 8258.80 & 5794.81 & 3.9169 \\
\hline 50 & $2 \mathrm{~atm}$ & 1 & 2 & 5794.81 & 14053.61 & 4487.95 & 3.7630 \\
\hline 50 & $2 \mathrm{~atm}$ & 1 & 3 & 4487.95 & 18541.56 & 4038.38 & 3.6520 \\
\hline 50 & $2 \mathrm{~atm}$ & 1 & 4 & 4038.38 & 22579.94 & 3820.12 & 3.6062 \\
\hline 50 & $2 \mathrm{~atm}$ & 1 & 5 & 3820.12 & 26400.06 & 3662.59 & 3.5821 \\
\hline
\end{tabular}


Table B1. Methanol Test Results

(continued)

\begin{tabular}{|c|c|c|}
\hline & Test & Test \\
\hline Study & Sequence & Identifier \\
\hline 1 & 7 & $50 C-2-1-6$ \\
\hline 1 & 7 & $50 \mathrm{C}-2-1-7$ \\
\hline 1 & 7 & $50 \mathrm{C}-2-1-8$ \\
\hline 1 & 7 & $50 \mathrm{C}-2-1-9$ \\
\hline 1 & 7 & $50 \mathrm{C}-2-1-10$ \\
\hline 1 & 8 & $50 \mathrm{C}-2-2-1$ \\
\hline 1 & 8 & $50 \mathrm{C}-2-2-2$ \\
\hline 1 & 8 & $50 \mathrm{C}-2-2-3$ \\
\hline 1 & 8 & $50 \mathrm{C}-2-2-4$ \\
\hline 1 & 8 & $50 \mathrm{C}-2-2-5$ \\
\hline 1 & 8 & $50 C-2-2-6$ \\
\hline 1 & 8 & $50 \mathrm{C}-2-2-7$ \\
\hline 1 & 8 & $50 \mathrm{C}-2-2-8$ \\
\hline 1 & 8 & $50 \mathrm{C}-2-2-9$ \\
\hline 1 & 8 & $50 \mathrm{C}-2-2-10$ \\
\hline 1 & 9 & $50 \mathrm{C}-2-3-1$ \\
\hline 1 & 9 & $50 \mathrm{C}-2-3-2$ \\
\hline 1 & 9 & $50 \mathrm{C}-2-3-3$ \\
\hline 1 & 9 & $50 \mathrm{C}-2-3-4$ \\
\hline 1 & 9 & $50 \mathrm{C}-2-3-5$ \\
\hline 1 & 9 & $50 \mathrm{C}-2-3-6$ \\
\hline 1 & 9 & $50 C-2-3-7$ \\
\hline 1 & 9 & $50 \mathrm{C}-2-3-8$ \\
\hline 1 & 9 & $50 C-2-3-9$ \\
\hline 1 & 9 & $50 \mathrm{C}-2-3-10$ \\
\hline 1 & 10 & $60 C-1-1-1$ \\
\hline 1 & 10 & $60 C-1-1-2$ \\
\hline 1 & 10 & $60 C-1-1-3$ \\
\hline 1 & 10 & $60 C-1-1-4$ \\
\hline 1 & 10 & $60 \mathrm{C}-1-1-5$ \\
\hline 1 & 10 & $60 C-1-1-6$ \\
\hline 1 & 10 & $60 C-1-1-7$ \\
\hline 1 & 10 & $60 C-1-1-8$ \\
\hline 1 & 10 & $60 C-1-1-9$ \\
\hline 1 & 10 & $60 \mathrm{C}-1-1-10$ \\
\hline 1 & 11 & $60 C-1-2-1$ \\
\hline 1 & 11 & $60 \mathrm{C}-1-2-2$ \\
\hline 1 & 11 & $60 \mathrm{C}-1-2-3$ \\
\hline 1 & 11 & $60 C-1-2-4$ \\
\hline 1 & 11 & $60 C-1-2-5$ \\
\hline 1 & 11 & $60 C-1-2-6$ \\
\hline 1 & 11 & $60 C-1-2-7$ \\
\hline 1 & 11 & $60 C-1-2-8$ \\
\hline 1 & 11 & $60 C-1-2-9$ \\
\hline 1 & 11 & $60 \mathrm{C}-1-2-10$ \\
\hline 1 & 12 & $60 C-1-3-1$ \\
\hline 1 & 12 & $60 C-1-3-2$ \\
\hline 1 & 12 & $60 C-1-3-3$ \\
\hline 1 & 12 & $60 C-1-3-4$ \\
\hline 1 & 12 & $60 C-1-3-5$ \\
\hline 1 & 12 & $60 C-1-3-6$ \\
\hline 1 & 12 & $60 C-1-3-7$ \\
\hline 1 & 12 & $60 C-1-3-8$ \\
\hline 1 & 12 & $60 C-1-3-9$ \\
\hline 1 & 12 & $60 C-1-3-10$ \\
\hline 1 & 13 & $60 \mathrm{C}-19-1-1$ \\
\hline 1 & 13 & $60 \mathrm{C}-19-1-2$ \\
\hline 1 & 13 & $60 \mathrm{C}-19-1-3$ \\
\hline 1 & 13 & $60 \mathrm{C}-19-1-4$ \\
\hline 1 & 13 & $60 C-19-1-5$ \\
\hline 1 & 13 & $60 C-19-1-6$ \\
\hline 1 & 13 & $60 \mathrm{C}-19-1-7$ \\
\hline 1 & 13 & $60 C-19-1-8$ \\
\hline 1 & 13 & $60 C-19-1-9$ \\
\hline 1 & 13 & $60 \mathrm{C}-19-1-10$ \\
\hline
\end{tabular}

\begin{tabular}{|c|c|c|c|c|c|c|c|}
\hline $\begin{array}{c}\text { Temperature } \\
\left({ }^{\circ} \mathrm{C}\right)\end{array}$ & $\begin{array}{c}\text { Vial } \\
\text { Pressusre }\end{array}$ & $\begin{array}{l}\text { Test } \\
\text { Run }\end{array}$ & $\begin{array}{l}\text { Sampling } \\
\text { Sequence }\end{array}$ & $\begin{array}{c}\text { A } \\
\text { (peak area) }\end{array}$ & & & $\log (\mathrm{A})$ \\
\hline 50 & $2 \mathrm{~atm}$ & 1 & $\begin{array}{c}\text { Sequence } \\
6\end{array}$ & $\begin{array}{c}\text { (peak area) } \\
3662.59\end{array}$ & $\begin{array}{c}\mathrm{y} \\
30062.65\end{array}$ & $\begin{array}{c}\mathrm{x} \\
3482.92\end{array}$ & $\begin{array}{l}\log (\mathrm{A}) \\
3.5638\end{array}$ \\
\hline 50 & $2 \mathrm{~atm}$ & 1 & 7 & 3482.92 & 33545.57 & 3312.32 & 3.5419 \\
\hline 50 & $2 \mathrm{~atm}$ & 1 & 8 & 3312.32 & 36857.89 & 3107.07 & 3.5201 \\
\hline 50 & $2 \mathrm{~atm}$ & 1 & 9 & 3107.07 & 39964.96 & 2915.30 & 3.4924 \\
\hline 50 & $2 \mathrm{~atm}$ & 1 & 10 & 2915.30 & 42880.26 & & 3.4647 \\
\hline 50 & $2 \mathrm{~atm}$ & 2 & 1 & 7886.45 & 7886.45 & 5628.16 & 3.8969 \\
\hline 50 & $2 \mathrm{~atm}$ & 2 & 2 & 5628.16 & 13514.61 & 4324.79 & 3.7504 \\
\hline 50 & $2 \mathrm{~atm}$ & 2 & 3 & 4324.79 & 17839.40 & 3857.71 & 3.6360 \\
\hline 50 & $2 \mathrm{~atm}$ & 2 & 4 & 3857.71 & 21697.11 & 3655.55 & 3.5863 \\
\hline 50 & $2 \mathrm{~atm}$ & 2 & 5 & 3655.55 & 25352.66 & 3450.83 & 3.5630 \\
\hline 50 & $2 \mathrm{~atm}$ & 2 & 6 & 3450.83 & 28803.49 & 3273.24 & 3.5379 \\
\hline 50 & $2 \mathrm{~atm}$ & 2 & 7 & 3273.24 & 32076.74 & 3074.53 & 3.5150 \\
\hline 50 & $2 \mathrm{~atm}$ & 2 & 8 & 3074.53 & 35151.26 & 2900.53 & 3.4878 \\
\hline 50 & $2 \mathrm{~atm}$ & 2 & 9 & 2900.53 & 38051.79 & 2677.23 & 3.4625 \\
\hline 50 & $2 \mathrm{~atm}$ & 2 & 10 & 2677.23 & 40729.03 & & 3.4277 \\
\hline 50 & $2 \mathrm{~atm}$ & 3 & 1 & 7907.73 & 7907.73 & 5537.53 & 3.8981 \\
\hline 50 & $2 \mathrm{~atm}$ & 3 & 2 & 5537.53 & 13445.26 & 4217.00 & 3.7433 \\
\hline 50 & $2 \mathrm{~atm}$ & 3 & 3 & 4217.00 & 17662.26 & 3782.28 & 3.6250 \\
\hline 50 & $2 \mathrm{~atm}$ & 3 & 4 & 3782.28 & 21444.54 & 3600.09 & 3.5778 \\
\hline 50 & $2 \mathrm{~atm}$ & 3 & 5 & 3600.09 & 25044.63 & 3446.65 & 3.5563 \\
\hline 50 & $2 \mathrm{~atm}$ & 3 & 6 & 3446.65 & 28491.29 & 3238.88 & 3.5374 \\
\hline 50 & $2 \mathrm{~atm}$ & 3 & 7 & 3238.88 & 31730.17 & 3041.24 & 3.5104 \\
\hline 50 & $2 \mathrm{~atm}$ & 3 & 8 & 3041.24 & 34771.41 & 2866.76 & 3.4831 \\
\hline 50 & $2 \mathrm{~atm}$ & 3 & 9 & 2866.76 & 37638.17 & 2653.56 & 3.4574 \\
\hline 50 & $2 \mathrm{~atm}$ & 3 & 10 & 2653.56 & 40291.73 & & 3.4238 \\
\hline 60 & $1 \mathrm{~atm}$ & 1 & 1 & 13661.36 & 13661.36 & 13713.54 & 4.1355 \\
\hline 60 & $1 \mathrm{~atm}$ & 1 & 2 & 13713.54 & 27374.90 & 13069.40 & 4.1372 \\
\hline 60 & $1 \mathrm{~atm}$ & 1 & 3 & 13069.40 & 40444.29 & 12394.15 & 4.1163 \\
\hline 60 & $1 \mathrm{~atm}$ & 1 & 4 & 12394.15 & 52838.45 & 11805.24 & 4.0932 \\
\hline 60 & $1 \mathrm{~atm}$ & 1 & 5 & 11805.24 & 64643.69 & 11253.56 & 4.0721 \\
\hline 60 & $1 \mathrm{~atm}$ & 1 & 6 & 11253.56 & 75897.25 & 10741.44 & 4.0513 \\
\hline 60 & $1 \mathrm{~atm}$ & 1 & 7 & 10741.44 & 86638.70 & 10184.17 & 4.0311 \\
\hline 60 & $1 \mathrm{~atm}$ & 1 & 8 & 10184.17 & 96822.86 & 9581.08 & 4.0079 \\
\hline 60 & $1 \mathrm{~atm}$ & 1 & 9 & 9581.08 & 106403.90 & 8893.84 & 3.9814 \\
\hline 60 & $1 \mathrm{~atm}$ & 1 & 10 & 8893.84 & 115297.80 & & 3.9491 \\
\hline 60 & $1 \mathrm{~atm}$ & 2 & 1 & 7894.89 & 7894.89 & 7937.89 & 3.8973 \\
\hline 60 & $1 \mathrm{~atm}$ & 2 & 2 & 7937.89 & 15832.78 & 7542.26 & 3.8997 \\
\hline 60 & $1 \mathrm{~atm}$ & 2 & 3 & 7542.26 & 23375.04 & 7197.91 & 3.8775 \\
\hline 60 & $1 \mathrm{~atm}$ & 2 & 4 & 7197.91 & 30572.95 & 6882.75 & 3.8572 \\
\hline 60 & $1 \mathrm{~atm}$ & 2 & 5 & 6882.75 & 37455.69 & 6579.11 & 3.8378 \\
\hline 60 & $1 \mathrm{~atm}$ & 2 & 6 & 6579.11 & 44034.80 & 6257.32 & 3.8182 \\
\hline 60 & $1 \mathrm{~atm}$ & 2 & 7 & 6257.32 & 50292.12 & 5995.07 & 3.7964 \\
\hline 60 & $1 \mathrm{~atm}$ & 2 & 8 & 5995.07 & 56287.19 & 5690.14 & 3.7778 \\
\hline 60 & $1 \mathrm{~atm}$ & 2 & 9 & 5690.14 & 61977.33 & 5310.62 & 3.7551 \\
\hline 60 & $1 \mathrm{~atm}$ & 2 & 10 & 5310.62 & 67287.94 & & 3.7251 \\
\hline 60 & $1 \mathrm{~atm}$ & 3 & 1 & 7947.11 & 7947.11 & 7857.56 & 3.9002 \\
\hline 60 & $1 \mathrm{~atm}$ & 3 & 2 & 7857.56 & 15804.66 & 7469.09 & 3.8953 \\
\hline 60 & $1 \mathrm{~atm}$ & 3 & 3 & 7469.09 & 23273.76 & 7129.83 & 3.8733 \\
\hline 60 & $1 \mathrm{~atm}$ & 3 & 4 & 7129.83 & 30403.58 & 6817.23 & 3.8531 \\
\hline 60 & $1 \mathrm{~atm}$ & 3 & 5 & 6817.23 & 37220.81 & 6490.30 & 3.8336 \\
\hline 60 & $1 \mathrm{~atm}$ & 3 & 6 & 6490.30 & 43711.10 & 6194.28 & 3.8123 \\
\hline 60 & $1 \mathrm{~atm}$ & 3 & 7 & 6194.28 & 49905.39 & 5919.81 & 3.7920 \\
\hline 60 & $1 \mathrm{~atm}$ & 3 & 8 & 5919.81 & 55825.20 & 5622.93 & 3.7723 \\
\hline 60 & $1 \mathrm{~atm}$ & 3 & 9 & 5622.93 & 61448.12 & 5274.69 & 3.7500 \\
\hline 60 & $1 \mathrm{~atm}$ & 3 & 10 & 5274.69 & 66722.81 & & 3.7222 \\
\hline 60 & 19 psig & 1 & 1 & 8449.79 & 8449.79 & 7367.79 & 3.9268 \\
\hline 60 & 19 psig & 1 & 2 & 7367.79 & 15817.57 & 7028.60 & 3.8673 \\
\hline 60 & 19 psig & 1 & 3 & 7028.60 & 22846.17 & 6514.84 & 3.8469 \\
\hline 60 & 19 psig & 1 & 4 & 6514.84 & 29361.01 & 6112.44 & 3.8139 \\
\hline 60 & 19 psig & 1 & 5 & 6112.44 & 35473.46 & 5751.14 & 3.7862 \\
\hline 60 & 19 psig & 1 & 6 & 5751.14 & 41224.59 & 5442.13 & 3.7598 \\
\hline 60 & 19 psig & 1 & 7 & 5442.13 & 46666.72 & 5147.14 & 3.7358 \\
\hline 60 & 19 psig & 1 & 8 & 5147.14 & 51813.86 & 4851.27 & 3.7116 \\
\hline 60 & 19 psig & 1 & 9 & 4851.27 & 56665.13 & 4421.51 & 3.6859 \\
\hline 60 & 19 psig & 1 & 10 & 4421.51 & 61086.63 & & 3.6456 \\
\hline
\end{tabular}


Table B1. Methanol Test Results

(continued)

\begin{tabular}{|c|c|}
\hline & Test \\
\hline Study & Sequence \\
\hline 1 & 14 \\
\hline 1 & 14 \\
\hline 1 & 14 \\
\hline 1 & 14 \\
\hline 1 & 14 \\
\hline 1 & 14 \\
\hline 1 & 14 \\
\hline 1 & 14 \\
\hline 1 & 14 \\
\hline 1 & 14 \\
\hline 1 & 15 \\
\hline 1 & 15 \\
\hline 1 & 15 \\
\hline 1 & 15 \\
\hline 1 & 15 \\
\hline 1 & 15 \\
\hline 1 & 15 \\
\hline 1 & 15 \\
\hline 1 & 15 \\
\hline 1 & 15 \\
\hline 1 & 16 \\
\hline 1 & 16 \\
\hline 1 & 16 \\
\hline 1 & 16 \\
\hline 1 & 16 \\
\hline 1 & 16 \\
\hline 1 & 16 \\
\hline 1 & 16 \\
\hline 1 & 16 \\
\hline 1 & 16 \\
\hline 1 & 17 \\
\hline 1 & 17 \\
\hline 1 & 17 \\
\hline 1 & 17 \\
\hline 1 & 17 \\
\hline 1 & 17 \\
\hline 1 & 17 \\
\hline 1 & 17 \\
\hline 1 & 17 \\
\hline 1 & 17 \\
\hline 1 & 18 \\
\hline 1 & 18 \\
\hline 1 & 18 \\
\hline 1 & 18 \\
\hline 1 & 18 \\
\hline 1 & 18 \\
\hline 1 & 18 \\
\hline 1 & 18 \\
\hline 1 & 18 \\
\hline 1 & 18 \\
\hline 1 & 19 \\
\hline 1 & 19 \\
\hline 1 & 19 \\
\hline 1 & 19 \\
\hline 1 & 19 \\
\hline 1 & 19 \\
\hline 1 & 19 \\
\hline 1 & 19 \\
\hline 1 & 19 \\
\hline 1 & 19 \\
\hline 1 & 20 \\
\hline 1 & 20 \\
\hline 1 & 20 \\
\hline 1 & 20 \\
\hline 1 & 20 \\
\hline 1 & 21 \\
\hline
\end{tabular}

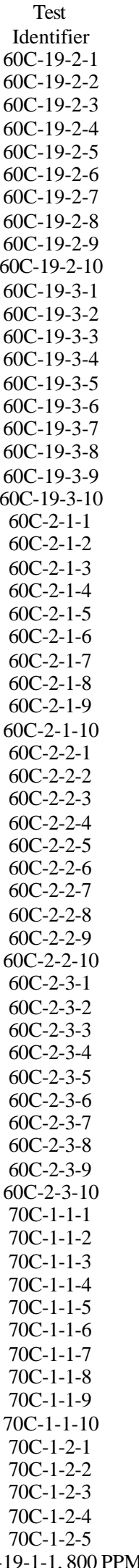

\begin{tabular}{|c|c|c|c|c|}
\hline $\begin{array}{c}\text { Temperature } \\
\left({ }^{\circ} \mathrm{C}\right)\end{array}$ & $\begin{array}{c}\text { Vial } \\
\text { Pressusre }\end{array}$ & $\begin{array}{l}\text { Test } \\
\text { Run }\end{array}$ & $\begin{array}{l}\text { Sampling } \\
\text { Sequence }\end{array}$ & $\begin{array}{c}\mathrm{A} \\
\text { (peak area) }\end{array}$ \\
\hline 60 & 19 psig & 2 & 1 & 8084.34 \\
\hline 60 & $19 \mathrm{psig}$ & 2 & 2 & 7095.81 \\
\hline 60 & 19 psig & 2 & 3 & 6876.61 \\
\hline 60 & $19 \mathrm{psig}$ & 2 & 4 & 6311.25 \\
\hline 60 & $19 \mathrm{psig}$ & 2 & 5 & 5978.95 \\
\hline 60 & 19 psig & 2 & 6 & 5658.95 \\
\hline 60 & 19 psig & 2 & 7 & 5329.56 \\
\hline 60 & 19 psig & 2 & 8 & 4984.22 \\
\hline 60 & $19 \mathrm{psig}$ & 2 & 9 & 4683.93 \\
\hline 60 & 19 psig & 2 & 10 & 4337.07 \\
\hline 60 & 19 psig & 3 & 1 & 8272.10 \\
\hline 60 & $19 \mathrm{psig}$ & 3 & 2 & 7013.12 \\
\hline 60 & 19 psig & 3 & 3 & 6709.23 \\
\hline 60 & 19 psig & 3 & 4 & 6217.09 \\
\hline 60 & 19 psig & 3 & 5 & 5910.68 \\
\hline 60 & 19 psig & 3 & 6 & 5551.14 \\
\hline 60 & 19 psig & 3 & 7 & 5229.67 \\
\hline 60 & 19 psig & 3 & 8 & 4903.83 \\
\hline 60 & 19 psig & 3 & 9 & 4590.78 \\
\hline 60 & 19 psig & 3 & 10 & 4240.85 \\
\hline 60 & $2 \mathrm{~atm}$ & 1 & 1 & 9298.16 \\
\hline 60 & $2 \mathrm{~atm}$ & 1 & 2 & 6535.22 \\
\hline 60 & $2 \mathrm{~atm}$ & 1 & 3 & 5989.82 \\
\hline 60 & $2 \mathrm{~atm}$ & 1 & 4 & 5503.16 \\
\hline 60 & $2 \mathrm{~atm}$ & 1 & 5 & 5099.80 \\
\hline 60 & $2 \mathrm{~atm}$ & 1 & 6 & 4699.35 \\
\hline 60 & $2 \mathrm{~atm}$ & 1 & 7 & 4308.52 \\
\hline 60 & $2 \mathrm{~atm}$ & 1 & 8 & 3927.55 \\
\hline 60 & $2 \mathrm{~atm}$ & 1 & 9 & 3584.50 \\
\hline 60 & $2 \mathrm{~atm}$ & 1 & 10 & 3215.58 \\
\hline 60 & $2 \mathrm{~atm}$ & 2 & 1 & 8940.90 \\
\hline 60 & $2 \mathrm{~atm}$ & 2 & 2 & 6467.62 \\
\hline 60 & $2 \mathrm{~atm}$ & 2 & 3 & 5863.37 \\
\hline 60 & $2 \mathrm{~atm}$ & 2 & 4 & 5313.62 \\
\hline 60 & $2 \mathrm{~atm}$ & 2 & 5 & 4955.32 \\
\hline 60 & $2 \mathrm{~atm}$ & 2 & 6 & 4558.96 \\
\hline 60 & $2 \mathrm{~atm}$ & 2 & 7 & 4157.80 \\
\hline 60 & $2 \mathrm{~atm}$ & 2 & 8 & 3797.11 \\
\hline 60 & $2 \mathrm{~atm}$ & 2 & 9 & 3443.59 \\
\hline 60 & $2 \mathrm{~atm}$ & 2 & 10 & 3078.84 \\
\hline 60 & $2 \mathrm{~atm}$ & 3 & 1 & 8966.50 \\
\hline 60 & $2 \mathrm{~atm}$ & 3 & 2 & 6560.39 \\
\hline 60 & $2 \mathrm{~atm}$ & 3 & 3 & 5695.11 \\
\hline 60 & $2 \mathrm{~atm}$ & 3 & 4 & 5120.63 \\
\hline 60 & $2 \mathrm{~atm}$ & 3 & 5 & 4799.98 \\
\hline 60 & $2 \mathrm{~atm}$ & 3 & 6 & 4387.23 \\
\hline 60 & $2 \mathrm{~atm}$ & 3 & 7 & 3923.80 \\
\hline 60 & $2 \mathrm{~atm}$ & 3 & 8 & 3464.53 \\
\hline 60 & $2 \mathrm{~atm}$ & 3 & 9 & 3070.15 \\
\hline 60 & $2 \mathrm{~atm}$ & 3 & 10 & 2669.96 \\
\hline 70 & $1 \mathrm{~atm}$ & 1 & 1 & 8938.14 \\
\hline 70 & $1 \mathrm{~atm}$ & 1 & 2 & 6799.07 \\
\hline 70 & $1 \mathrm{~atm}$ & 1 & 3 & 4995.69 \\
\hline 70 & $1 \mathrm{~atm}$ & 1 & 4 & 4074.60 \\
\hline 70 & $1 \mathrm{~atm}$ & 1 & 5 & 3238.00 \\
\hline 70 & $1 \mathrm{~atm}$ & 1 & 6 & 2498.06 \\
\hline 70 & $1 \mathrm{~atm}$ & 1 & 7 & 1967.55 \\
\hline 70 & $1 \mathrm{~atm}$ & 1 & 8 & 1517.49 \\
\hline 70 & $1 \mathrm{~atm}$ & 1 & 9 & 1186.82 \\
\hline 70 & $1 \mathrm{~atm}$ & 1 & 10 & 1012.85 \\
\hline 70 & $1 \mathrm{~atm}$ & 2 & 1 & 1629.40 \\
\hline 70 & $1 \mathrm{~atm}$ & 2 & 2 & 1183.56 \\
\hline 70 & $1 \mathrm{~atm}$ & 2 & 3 & 864.08 \\
\hline 70 & $1 \mathrm{~atm}$ & 2 & 4 & 728.83 \\
\hline 70 & $1 \mathrm{~atm}$ & 2 & 5 & 545.31 \\
\hline 70 & 19 psig & 1 & 1 & 1084.29 \\
\hline
\end{tabular}

\begin{tabular}{ccc}
$\mathrm{y}$ & $\mathrm{x}$ & $\log (\mathrm{A})$ \\
8084.34 & 7095.81 & 3.9076 \\
15180.14 & 6876.61 & 3.8510 \\
22056.75 & 6311.25 & 3.8374 \\
28368.00 & 5978.95 & 3.8001 \\
34346.95 & 5658.95 & 3.7766 \\
40005.90 & 5329.56 & 3.7527 \\
45335.46 & 4984.22 & 3.7267 \\
50319.68 & 4683.93 & 3.6976 \\
55003.61 & 4337.07 & 3.6706 \\
59340.68 &. & 3.6372 \\
8272.10 & 7013.12 & 3.9176 \\
15285.22 & 6709.23 & 3.8459 \\
21994.45 & 6217.09 & 3.8267 \\
28211.54 & 5910.68 & 3.7936 \\
34122.22 & 5551.14 & 3.7716 \\
39673.36 & 5229.67 & 3.7444 \\
44903.03 & 4903.83 & 3.7185 \\
49806.87 & 4590.78 & 3.6905 \\
54397.65 & 4240.85 & 3.6619 \\
58638.50 &. & 3.6275 \\
9298.16 & 6535.22 & 3.9684 \\
15833.38 & 5989.82 & 3.8153 \\
21823.20 & 5503.16 & 3.7774 \\
27326.36 & 5099.80 & 3.7406 \\
32426.17 & 4699.35 & 3.7076 \\
37125.52 & 4308.52 & 3.6720 \\
41434.04 & 3927.55 & 3.6343 \\
45361.59 & 3584.50 & 3.5941 \\
48946.08 & 3215.58 & 3.5544 \\
52161.66 &. & 3.5073 \\
8940.90 & 6467.62 & 3.9514 \\
15408.52 & 5863.37 & 3.8107 \\
21271.89 & 5313.62 & 3.7681 \\
26585.51 & 4955.32 & 3.7254 \\
31540.83 & 4558.96 & 3.6951 \\
36099.79 & 4157.80 & 3.6589 \\
40257.58 & 3797.11 & 3.6189 \\
44054.69 & 3443.59 & 3.5795 \\
47498.29 & 3078.84 & 3.5370 \\
50577.13 &. & 3.4884 \\
8966.50 & 6560.39 & 3.9526 \\
15526.89 & 5695.11 & 3.8169 \\
21221.99 & 5120.63 & 3.7555 \\
26342.62 & 4799.98 & 3.7093 \\
31142.60 & 4387.23 & 3.6812 \\
35529.83 & 3923.80 & 3.6422 \\
39453.63 & 3464.53 & 3.5937 \\
42918.17 & 3070.15 & 3.5396 \\
45988.31 & 2669.96 & 3.4872 \\
48658.27 &. & 3.4265 \\
8938.14 & 6799.07 & 3.9512 \\
15737.21 & 4995.69 & 3.8325 \\
20732.90 & 4074.60 & 3.6986 \\
24807.51 & 3238.00 & 3.6101 \\
28045.50 & 2498.06 & 3.5103 \\
30543.56 & 1967.55 & 3.3976 \\
32511.11 & 1517.49 & 3.2939 \\
34028.61 & 1186.82 & 3.1811 \\
35215.43 & 1012.85 & 3.0744 \\
36228.28 &. & 3.0055 \\
1629.40 & 1183.56 & 3.2120 \\
2812.96 & 864.08 & 3.0732 \\
3677.04 & 728.83 & 2.9366 \\
4405.87 & 545.31 & 2.8626 \\
4951.18 & 1084.29 & 2.7366 \\
1084.29 & 1024.87 & 3.0351 \\
& & \\
\hline
\end{tabular}


Table B1. Methanol Test Results

(continued)

\begin{tabular}{|c|c|c|}
\hline & Test & Test \\
\hline Study & Sequence & Identifier \\
\hline 1 & 21 & 70C-19-1-2, 800 PPM \\
\hline 1 & 21 & 70C-19-1-3, 800 PPM \\
\hline 1 & 21 & $70 \mathrm{C}-19-1-4,800$ PPM \\
\hline 1 & 21 & 70C-19-1-5, 800 PPM \\
\hline 1 & 21 & $70 \mathrm{C}-19-1-6,800 \mathrm{PPM}$ \\
\hline 1 & 21 & 70C-19-1-7, 800 PPM \\
\hline 1 & 21 & 70C-19-1-8, 800 PPM \\
\hline 1 & 21 & 70C-19-1-9, 800 PPM \\
\hline 1 & 21 & 70C-19-1-10, 800 PPM \\
\hline 1 & 22 & 70C-19-2-1, 800 PPM \\
\hline 1 & 22 & 70C-19-2-2, 800 PPM \\
\hline 1 & 22 & 70C-19-2-3, 800 PPM \\
\hline 1 & 22 & 70C-19-2-4, 800 PPM \\
\hline 1 & 22 & 70C-19-2-5, 800 PPM \\
\hline 1 & 22 & 70C-19-2-6, 800 PPM \\
\hline 1 & 22 & 70C-19-2-7, 800 PPM \\
\hline 1 & 22 & $70 \mathrm{C}-19-2-8,800 \mathrm{PPM}$ \\
\hline 1 & 22 & 70C-19-2-9, 800 PPM \\
\hline 1 & 22 & 70C-19-2-10, 800 PPM \\
\hline 1 & 23 & 70C-19-3-1, 800 PPM \\
\hline 1 & 23 & 70C-19-3-2, 800 PPM \\
\hline 1 & 23 & 70C-19-3-3, $800 \mathrm{PPM}$ \\
\hline 1 & 23 & 70C-19-3-4, $800 \mathrm{PPM}$ \\
\hline 1 & 23 & 70C-19-3-5, 800 PPM \\
\hline 1 & 23 & 70C-19-3-6, 800 PPM \\
\hline 1 & 23 & 70C-19-3-7, 800 PPM \\
\hline 1 & 23 & 70C-19-3-8, 800 PPM \\
\hline 1 & 23 & 70С-19-3-9, 800 PPM \\
\hline 1 & 23 & 70C-19-3-10, 800 PPM \\
\hline 1 & 24 & 70C-2-1-1, 800 PPM \\
\hline 1 & 24 & $70 \mathrm{C}-2-1-2,800 \mathrm{PPM}$ \\
\hline 1 & 24 & 70C-2-1-3, 800 PPM \\
\hline 1 & 24 & 70C-2-1-4, 800 PPM \\
\hline 1 & 24 & 70C-2-1-5, 800 PPM \\
\hline 1 & 24 & 70C-2-1-6, 800 PPM \\
\hline 1 & 24 & 70C-2-1-7, 800 PPM \\
\hline 1 & 24 & 70C-2-1-8, 800 PPM \\
\hline 1 & 24 & 70C-2-1-9, 800 PPM \\
\hline 1 & 24 & $70 \mathrm{C}-2-1-10,800$ PPM \\
\hline 1 & 25 & 70C-2-2-1, 800 PPM \\
\hline 1 & 25 & 70C-2-2-2, 800 PPM \\
\hline 1 & 25 & $70 \mathrm{C}-2-2-3,800 \mathrm{PPM}$ \\
\hline 1 & 25 & 70C-2-2-4, 800 PPM \\
\hline 1 & 25 & 70C-2-2-5, 800 PPM \\
\hline 1 & 25 & $70 \mathrm{C}-2-2-6,800 \mathrm{PPM}$ \\
\hline 1 & 25 & 70C-2-2-7, 800 PPM \\
\hline 1 & 25 & 70C-2-2-8, 800 PPM \\
\hline 1 & 25 & 70C-2-2-9, 800 PPM \\
\hline 1 & 25 & 70C-2-2-10, 800 PPM \\
\hline 1 & 26 & $70 \mathrm{C}-2-3-1,800 \mathrm{PPM}$ \\
\hline 1 & 26 & $70 \mathrm{C}-2-3-2,800 \mathrm{PPM}$ \\
\hline 1 & 26 & 70C-2-3-3, 800 PPM \\
\hline 1 & 26 & $70 \mathrm{C}-2-3-4,800 \mathrm{PPM}$ \\
\hline 1 & 26 & 70C-2-3-5, 800 PPM \\
\hline 1 & 26 & 70C-2-3-6, 800 PPM \\
\hline 1 & 26 & 70C-2-3-7, 800 PPM \\
\hline 1 & 26 & $70 \mathrm{C}-2-3-8,800 \mathrm{PPM}$ \\
\hline 1 & 26 & $70 \mathrm{C}-2-3-9,800 \mathrm{PPM}$ \\
\hline 1 & 26 & $70 \mathrm{C}-2-3-10,800 \mathrm{PPM}$ \\
\hline 1 & 27 & $80 C-1-1-1$ \\
\hline 1 & 27 & $80 \mathrm{C}-1-1-2$ \\
\hline 1 & 27 & $80 C-1-1-3$ \\
\hline 1 & 27 & $80 C-1-1-4$ \\
\hline 1 & 27 & $80 C-1-1-5$ \\
\hline 1 & 27 & $80 C-1-1-6$ \\
\hline 1 & 27 & $80 \mathrm{C}-1-1-7$ \\
\hline 1 & 27 & $80 C-1-1-8$ \\
\hline
\end{tabular}

\begin{tabular}{|c|c|c|c|c|c|c|c|}
\hline Temperature & Vial & Test & Sampling & A & & & \\
\hline$\left({ }^{\circ} \mathrm{C}\right)$ & Pressusre & Run & Sequence & (peak area) & $\mathrm{y}$ & $\mathrm{x}$ & $\log (\mathrm{A})$ \\
\hline 70 & 19 psig & 1 & 2 & 1024.87 & 2109.15 & 937.88 & 3.0107 \\
\hline 70 & 19 psig & 1 & 3 & 937.88 & 3047.04 & 888.64 & 2.9721 \\
\hline 70 & 19 psig & 1 & 4 & 888.64 & 3935.67 & 799.31 & 2.9487 \\
\hline 70 & 19 psig & 1 & 5 & 799.31 & 4734.98 & 736.56 & 2.9027 \\
\hline 70 & 19 psig & 1 & 6 & 736.56 & 5471.54 & 652.39 & 2.8672 \\
\hline 70 & 19 psig & 1 & 7 & 652.39 & 6123.93 & 603.26 & 2.8145 \\
\hline 70 & 19 psig & 1 & 8 & 603.26 & 6727.19 & 527.36 & 2.7805 \\
\hline 70 & 19 psig & 1 & 9 & 527.36 & 7254.55 & 499.90 & 2.7221 \\
\hline 70 & 19 psig & 1 & 10 & 499.90 & 7754.44 & & 2.6989 \\
\hline 70 & 19 psig & 2 & 1 & 1093.66 & 1093.66 & 949.96 & 3.0389 \\
\hline 70 & 19 psig & 2 & 2 & 949.96 & 2043.62 & 911.61 & 2.9777 \\
\hline 70 & 19 psig & 2 & 3 & 911.61 & 2955.23 & 882.91 & 2.9598 \\
\hline 70 & 19 psig & 2 & 4 & 882.91 & 3838.13 & 794.46 & 2.9459 \\
\hline 70 & 19 psig & 2 & 5 & 794.46 & 4632.60 & 738.87 & 2.9001 \\
\hline 70 & 19 psig & 2 & 6 & 738.87 & 5371.46 & 662.78 & 2.8686 \\
\hline 70 & 19 psig & 2 & 7 & 662.78 & 6034.24 & 611.43 & 2.8214 \\
\hline 70 & 19 psig & 2 & 8 & 611.43 & 6645.67 & 572.27 & 2.7863 \\
\hline 70 & 19 psig & 2 & 9 & 572.27 & 7217.94 & 503.01 & 2.7576 \\
\hline 70 & 19 psig & 2 & 10 & 503.01 & 7720.95 & & 2.7016 \\
\hline 70 & 19 psig & 3 & 1 & 1191.24 & 1191.24 & 1026.13 & 3.0760 \\
\hline 70 & 19 psig & 3 & 2 & 1026.13 & 2217.36 & 977.06 & 3.0112 \\
\hline 70 & 19 psig & 3 & 3 & 977.06 & 3194.42 & 936.46 & 2.9899 \\
\hline 70 & 19 psig & 3 & 4 & 936.46 & 4130.88 & 847.87 & 2.9715 \\
\hline 70 & 19 psig & 3 & 5 & 847.87 & 4978.75 & 785.74 & 2.9283 \\
\hline 70 & 19 psig & 3 & 6 & 785.74 & 5764.50 & 717.46 & 2.8953 \\
\hline 70 & 19 psig & 3 & 7 & 717.46 & 6481.96 & 639.99 & 2.8558 \\
\hline 70 & 19 psig & 3 & 8 & 639.99 & 7121.95 & 586.77 & 2.8062 \\
\hline 70 & 19 psig & 3 & 9 & 586.77 & 7708.72 & 531.51 & 2.7685 \\
\hline 70 & 19 psig & 3 & 10 & 531.51 & 8240.23 & & 2.7255 \\
\hline 70 & $2 \mathrm{~atm}$ & 1 & 1 & 1337.09 & 1337.09 & 995.92 & 3.1262 \\
\hline 70 & $2 \mathrm{~atm}$ & 1 & 2 & 995.92 & 2333.00 & 906.16 & 2.9982 \\
\hline 70 & $2 \mathrm{~atm}$ & 1 & 3 & 906.16 & 3239.16 & 861.62 & 2.9572 \\
\hline 70 & $2 \mathrm{~atm}$ & 1 & 4 & 861.62 & 4100.78 & 759.59 & 2.9353 \\
\hline 70 & $2 \mathrm{~atm}$ & 1 & 5 & 759.59 & 4860.38 & 687.26 & 2.8806 \\
\hline 70 & $2 \mathrm{~atm}$ & 1 & 6 & 687.26 & 5547.64 & 597.96 & 2.8371 \\
\hline 70 & $2 \mathrm{~atm}$ & 1 & 7 & 597.96 & 6145.60 & 507.10 & 2.7767 \\
\hline 70 & $2 \mathrm{~atm}$ & 1 & 8 & 507.10 & 6652.70 & 427.00 & 2.7051 \\
\hline 70 & $2 \mathrm{~atm}$ & 1 & 9 & 427.00 & 7079.70 & 363.13 & 2.6304 \\
\hline 70 & $2 \mathrm{~atm}$ & 1 & 10 & 363.13 & 7442.83 & & 2.5601 \\
\hline 70 & $2 \mathrm{~atm}$ & 2 & 1 & 1310.35 & 1310.35 & 1046.58 & 3.1174 \\
\hline 70 & $2 \mathrm{~atm}$ & 2 & 2 & 1046.58 & 2356.93 & 970.38 & 3.0198 \\
\hline 70 & $2 \mathrm{~atm}$ & 2 & 3 & 970.38 & 3327.31 & 873.55 & 2.9869 \\
\hline 70 & $2 \mathrm{~atm}$ & 2 & 4 & 873.55 & 4200.86 & 784.14 & 2.9413 \\
\hline 70 & $2 \mathrm{~atm}$ & 2 & 5 & 784.14 & 4985.00 & 688.26 & 2.8944 \\
\hline 70 & $2 \mathrm{~atm}$ & 2 & 6 & 688.26 & 5673.26 & 607.07 & 2.8378 \\
\hline 70 & $2 \mathrm{~atm}$ & 2 & 7 & 607.07 & 6280.33 & 514.83 & 2.7832 \\
\hline 70 & $2 \mathrm{~atm}$ & 2 & 8 & 514.83 & 6795.16 & 447.63 & 2.7117 \\
\hline 70 & $2 \mathrm{~atm}$ & 2 & 9 & 447.63 & 7242.79 & 379.13 & 2.6509 \\
\hline 70 & $2 \mathrm{~atm}$ & 2 & 10 & 379.13 & 7621.92 & & 2.5788 \\
\hline 70 & $2 \mathrm{~atm}$ & 3 & 1 & 1374.11 & 1374.11 & 1082.77 & 3.1380 \\
\hline 70 & $2 \mathrm{~atm}$ & 3 & 2 & 1082.77 & 2456.88 & 984.29 & 3.0345 \\
\hline 70 & $2 \mathrm{~atm}$ & 3 & 3 & 984.29 & 3441.17 & 933.16 & 2.9931 \\
\hline 70 & $2 \mathrm{~atm}$ & 3 & 4 & 933.16 & 4374.33 & 794.88 & 2.9700 \\
\hline 70 & $2 \mathrm{~atm}$ & 3 & 5 & 794.88 & 5169.22 & 724.37 & 2.9003 \\
\hline 70 & $2 \mathrm{~atm}$ & 3 & 6 & 724.37 & 5893.58 & 612.12 & 2.8600 \\
\hline 70 & $2 \mathrm{~atm}$ & 3 & 7 & 612.12 & 6505.70 & 528.49 & 2.7868 \\
\hline 70 & $2 \mathrm{~atm}$ & 3 & 8 & 528.49 & 7034.20 & 445.47 & 2.7230 \\
\hline 70 & $2 \mathrm{~atm}$ & 3 & 9 & 445.47 & 7479.67 & 392.33 & 2.6488 \\
\hline 70 & $2 \mathrm{~atm}$ & 3 & 10 & 392.33 & 7871.99 & & 2.5936 \\
\hline 80 & $1 \mathrm{~atm}$ & 1 & 1 & 563.01 & 563.01 & 491.73 & 2.7505 \\
\hline 80 & $1 \mathrm{~atm}$ & 1 & 2 & 491.73 & 1054.75 & 425.77 & 2.6917 \\
\hline 80 & $1 \mathrm{~atm}$ & 1 & 3 & 425.77 & 1480.52 & 384.55 & 2.6292 \\
\hline 80 & $1 \mathrm{~atm}$ & 1 & 4 & 384.55 & 1865.07 & 343.10 & 2.5850 \\
\hline 80 & $1 \mathrm{~atm}$ & 1 & 5 & 343.10 & 2208.17 & 305.89 & 2.5354 \\
\hline 80 & $1 \mathrm{~atm}$ & 1 & 6 & 305.89 & 2514.05 & 287.07 & 2.4856 \\
\hline 80 & $1 \mathrm{~atm}$ & 1 & 7 & 287.07 & 2801.12 & 251.83 & 2.4580 \\
\hline 80 & $1 \mathrm{~atm}$ & 1 & 8 & 251.83 & 3052.95 & 210.35 & 2.4011 \\
\hline
\end{tabular}


Table B1. Methanol Test Results

(continued)

\begin{tabular}{|c|c|c|}
\hline & Test & Test \\
\hline Study & Sequence & Identifier \\
\hline 1 & 27 & $80 C-1-1-9$ \\
\hline 1 & 27 & $80 \mathrm{C}-1-1-10$ \\
\hline 1 & 28 & $80 C-1-2-1$ \\
\hline 1 & 28 & $80 \mathrm{C}-1-2-2$ \\
\hline 1 & 28 & $80 \mathrm{C}-1-2-3$ \\
\hline 1 & 28 & $80 C-1-2-4$ \\
\hline 1 & 28 & $80 C-1-2-5$ \\
\hline 1 & 28 & $80 C-1-2-6$ \\
\hline 1 & 28 & $80 C-1-2-7$ \\
\hline 1 & 28 & $80 C-1-2-8$ \\
\hline 1 & 28 & $80 C-1-2-9$ \\
\hline 1 & 28 & $80 \mathrm{C}-1-2-10$ \\
\hline 1 & 29 & $80 C-1-3-1$ \\
\hline 1 & 29 & $80 C-1-3-2$ \\
\hline 1 & 29 & $80 \mathrm{C}-1-3-3$ \\
\hline 1 & 29 & $80 C-1-3-4$ \\
\hline 1 & 29 & $80 C-1-3-5$ \\
\hline 1 & 29 & $80 C-1-3-6$ \\
\hline 1 & 29 & $80 C-1-3-7$ \\
\hline 1 & 29 & $80 C-1-3-8$ \\
\hline 1 & 29 & $80 C-1-3-9$ \\
\hline 1 & 29 & $80 \mathrm{C}-1-3-10$ \\
\hline 1 & 30 & $80 C-19-1-1$ \\
\hline 1 & 30 & $80 \mathrm{C}-19-1-2$ \\
\hline 1 & 30 & $80 \mathrm{C}-19-1-3$ \\
\hline 1 & 30 & $80 C-19-1-4$ \\
\hline 1 & 30 & $80 C-19-1-5$ \\
\hline 1 & 30 & $80 C-19-1-6$ \\
\hline 1 & 30 & $80 \mathrm{C}-19-1-7$ \\
\hline 1 & 30 & $80 C-19-1-8$ \\
\hline 1 & 30 & $80 \mathrm{C}-19-1-9$ \\
\hline 1 & 30 & $80 \mathrm{C}-19-1-10$ \\
\hline 1 & 31 & $80 \mathrm{C}-19-2-1$ \\
\hline 1 & 31 & $80 \mathrm{C}-19-2-2$ \\
\hline 1 & 31 & $80 \mathrm{C}-19-2-3$ \\
\hline 1 & 31 & $80 \mathrm{C}-19-2-4$ \\
\hline 1 & 31 & $80 \mathrm{C}-19-2-5$ \\
\hline 1 & 31 & $80 \mathrm{C}-19-2-6$ \\
\hline 1 & 31 & $80 \mathrm{C}-19-2-7$ \\
\hline 1 & 31 & $80 \mathrm{C}-19-2-8$ \\
\hline 1 & 31 & $80 C-19-2-9$ \\
\hline 1 & 31 & $80 \mathrm{C}-19-2-10$ \\
\hline 1 & 32 & $80 \mathrm{C}-19-3-1$ \\
\hline 1 & 32 & $80 \mathrm{C}-19-3-2$ \\
\hline 1 & 32 & $80 \mathrm{C}-19-3-3$ \\
\hline 1 & 32 & $80 \mathrm{C}-19-3-4$ \\
\hline 1 & 32 & $80 C-19-3-5$ \\
\hline 1 & 32 & $80 C-19-3-6$ \\
\hline 1 & 32 & $80 \mathrm{C}-19-3-7$ \\
\hline 1 & 32 & $80 C-19-3-8$ \\
\hline 1 & 32 & $80 C-19-3-9$ \\
\hline 1 & 32 & $80 \mathrm{C}-19-3-10$ \\
\hline 1 & 33 & $80 \mathrm{C}-2-1-1$ \\
\hline 1 & 33 & $80 C-2-1-2$ \\
\hline 1 & 33 & $80 C-2-1-3$ \\
\hline 1 & 33 & $80 C-2-1-4$ \\
\hline 1 & 33 & $80 C-2-1-5$ \\
\hline 1 & 33 & $80 C-2-1-6$ \\
\hline 1 & 33 & $80 C-2-1-7$ \\
\hline 1 & 33 & $80 \mathrm{C}-2-1-8$ \\
\hline 1 & 33 & $80 C-2-1-9$ \\
\hline 1 & 33 & $80 \mathrm{C}-2-1-10$ \\
\hline 1 & 34 & $80 C-2-2-1$ \\
\hline 1 & 34 & $80 \mathrm{C}-2-2-2$ \\
\hline 1 & 34 & $80 \mathrm{C}-2-2-3$ \\
\hline 1 & 34 & $80 \mathrm{C}-2-2-4$ \\
\hline
\end{tabular}

\begin{tabular}{|c|c|c|c|c|c|c|c|}
\hline Temperature & Vial & Test & Sampling & A & & & \\
\hline$\left({ }^{\circ} \mathrm{C}\right)$ & Pressusre & Run & Sequence & (peak area) & $\mathrm{y}$ & $\mathrm{x}$ & $\log (\mathrm{A})$ \\
\hline 80 & $1 \mathrm{~atm}$ & 1 & 9 & 210.35 & 3263.30 & 202.88 & 2.3229 \\
\hline 80 & $1 \mathrm{~atm}$ & 1 & 10 & 202.88 & 3466.18 & & 2.3072 \\
\hline 80 & $1 \mathrm{~atm}$ & 2 & 1 & 543.13 & 543.13 & 488.82 & 2.7349 \\
\hline 80 & $1 \mathrm{~atm}$ & 2 & 2 & 488.82 & 1031.95 & 404.57 & 2.6891 \\
\hline 80 & $1 \mathrm{~atm}$ & 2 & 3 & 404.57 & 1436.52 & 370.32 & 2.6070 \\
\hline 80 & $1 \mathrm{~atm}$ & 2 & 4 & 370.32 & 1806.83 & 354.36 & 2.5686 \\
\hline 80 & $1 \mathrm{~atm}$ & 2 & 5 & 354.36 & 2161.19 & 279.79 & 2.5494 \\
\hline 80 & $1 \mathrm{~atm}$ & 2 & 6 & 279.79 & 2440.98 & 274.50 & 2.4468 \\
\hline 80 & $1 \mathrm{~atm}$ & 2 & 7 & 274.50 & 2715.48 & 292.68 & 2.4385 \\
\hline 80 & $1 \mathrm{~atm}$ & 2 & 8 & 292.68 & 3008.16 & 200.61 & 2.4664 \\
\hline 80 & $1 \mathrm{~atm}$ & 2 & 9 & 200.61 & 3208.77 & 178.28 & 2.3023 \\
\hline 80 & $1 \mathrm{~atm}$ & 2 & 10 & 178.28 & 3387.05 & & 2.2511 \\
\hline 80 & $1 \mathrm{~atm}$ & 3 & 1 & 547.97 & 547.97 & 491.76 & 2.7388 \\
\hline 80 & $1 \mathrm{~atm}$ & 3 & 2 & 491.76 & 1039.73 & 409.74 & 2.6918 \\
\hline 80 & $1 \mathrm{~atm}$ & 3 & 3 & 409.74 & 1449.47 & 366.27 & 2.6125 \\
\hline 80 & $1 \mathrm{~atm}$ & 3 & 4 & 366.27 & 1815.74 & 315.64 & 2.5638 \\
\hline 80 & $1 \mathrm{~atm}$ & 3 & 5 & 315.64 & 2131.38 & 322.46 & 2.4992 \\
\hline 80 & $1 \mathrm{~atm}$ & 3 & 6 & 322.46 & 2453.84 & 247.53 & 2.5085 \\
\hline 80 & $1 \mathrm{~atm}$ & 3 & 7 & 247.53 & 2701.37 & 245.37 & 2.3936 \\
\hline 80 & $1 \mathrm{~atm}$ & 3 & 8 & 245.37 & 2946.74 & 217.19 & 2.3898 \\
\hline 80 & $1 \mathrm{~atm}$ & 3 & 9 & 217.19 & 3163.93 & 173.34 & 2.3368 \\
\hline 80 & $1 \mathrm{~atm}$ & 3 & 10 & 173.34 & 3337.27 & & 2.2389 \\
\hline 80 & 19 psig & 1 & 1 & 515.07 & 515.07 & 442.78 & 2.7119 \\
\hline 80 & 19 psig & 1 & 2 & 442.78 & 957.84 & 386.32 & 2.6462 \\
\hline 80 & 19 psig & 1 & 3 & 386.32 & 1344.17 & 323.71 & 2.5870 \\
\hline 80 & 19 psig & 1 & 4 & 323.71 & 1667.87 & 276.95 & 2.5101 \\
\hline 80 & 19 psig & 1 & 5 & 276.95 & 1944.82 & 246.77 & 2.4424 \\
\hline 80 & $19 \mathrm{psig}$ & 1 & 6 & 246.77 & 2191.59 & 199.96 & 2.3923 \\
\hline 80 & 19 psig & 1 & 7 & 199.96 & 2391.55 & 199.53 & 2.3009 \\
\hline 80 & 19 psig & 1 & 8 & 199.53 & 2591.08 & 161.97 & 2.3000 \\
\hline 80 & 19 psig & 1 & 9 & 161.97 & 2753.04 & 134.93 & 2.2094 \\
\hline 80 & 19 psig & 1 & 10 & 134.93 & 2887.97 & & 2.1301 \\
\hline 80 & 19 psig & 2 & 1 & 564.49 & 564.49 & 452.22 & 2.7517 \\
\hline 80 & 19 psig & 2 & 2 & 452.22 & 1016.70 & 382.58 & 2.6553 \\
\hline 80 & $19 \mathrm{psig}$ & 2 & 3 & 382.58 & 1399.28 & 335.47 & 2.5827 \\
\hline 80 & 19 psig & 2 & 4 & 335.47 & 1734.75 & 293.10 & 2.5257 \\
\hline 80 & 19 psig & 2 & 5 & 293.10 & 2027.85 & 272.02 & 2.4670 \\
\hline 80 & 19 psig & 2 & 6 & 272.02 & 2299.88 & 229.93 & 2.4346 \\
\hline 80 & 19 psig & 2 & 7 & 229.93 & 2529.80 & 237.09 & 2.3616 \\
\hline 80 & 19 psig & 2 & 8 & 237.09 & 2766.90 & 184.55 & 2.3749 \\
\hline 80 & $19 \mathrm{psig}$ & 2 & 9 & 184.55 & 2951.45 & 197.27 & 2.2661 \\
\hline 80 & $19 \mathrm{psig}$ & 2 & 10 & 197.27 & 3148.72 & . & 2.2951 \\
\hline 80 & 19 psig & 3 & 1 & 554.82 & 554.82 & 481.31 & 2.7442 \\
\hline 80 & 19 psig & 3 & 2 & 481.31 & 1036.13 & 387.85 & 2.6824 \\
\hline 80 & $19 \mathrm{psig}$ & 3 & 3 & 387.85 & 1423.98 & 356.03 & 2.5887 \\
\hline 80 & 19 psig & 3 & 4 & 356.03 & 1780.01 & 320.01 & 2.5515 \\
\hline 80 & 19 psig & 3 & 5 & 320.01 & 2100.02 & 301.21 & 2.5052 \\
\hline 80 & 19 psig & 3 & 6 & 301.21 & 2401.22 & 321.49 & 2.4789 \\
\hline 80 & 19 psig & 3 & 7 & 321.49 & 2722.72 & 202.52 & 2.5072 \\
\hline 80 & 19 psig & 3 & 8 & 202.52 & 2925.24 & 203.36 & 2.3065 \\
\hline 80 & 19 psig & 3 & 9 & 203.36 & 3128.60 & 207.83 & 2.3083 \\
\hline 80 & 19 psig & 3 & 10 & 207.83 & 3336.43 & & 2.3177 \\
\hline 80 & $2 \mathrm{~atm}$ & 1 & 1 & 637.25 & 637.25 & 472.13 & 2.8043 \\
\hline 80 & $2 \mathrm{~atm}$ & 1 & 2 & 472.13 & 1109.38 & 404.21 & 2.6741 \\
\hline 80 & $2 \mathrm{~atm}$ & 1 & 3 & 404.21 & 1513.59 & 325.20 & 2.6066 \\
\hline 80 & $2 \mathrm{~atm}$ & 1 & 4 & 325.20 & 1838.79 & 291.73 & 2.5121 \\
\hline 80 & $2 \mathrm{~atm}$ & 1 & 5 & 291.73 & 2130.51 & 230.04 & 2.4650 \\
\hline 80 & $2 \mathrm{~atm}$ & 1 & 6 & 230.04 & 2360.55 & 197.43 & 2.3618 \\
\hline 80 & $2 \mathrm{~atm}$ & 1 & 7 & 197.43 & 2557.99 & 152.23 & 2.2954 \\
\hline 80 & $2 \mathrm{~atm}$ & 1 & 8 & 152.23 & 2710.22 & 115.44 & 2.1825 \\
\hline 80 & $2 \mathrm{~atm}$ & 1 & 9 & 115.44 & 2825.65 & 171.43 & 2.0623 \\
\hline 80 & $2 \mathrm{~atm}$ & 1 & 10 & 171.43 & 2997.08 & & 2.2341 \\
\hline 80 & $2 \mathrm{~atm}$ & 2 & 1 & 615.00 & 615.00 & 478.06 & 2.7889 \\
\hline 80 & $2 \mathrm{~atm}$ & 2 & 2 & 478.06 & 1093.07 & 393.94 & 2.6795 \\
\hline 80 & $2 \mathrm{~atm}$ & 2 & 3 & 393.94 & 1487.00 & 349.92 & 2.5954 \\
\hline 80 & $2 \mathrm{~atm}$ & 2 & 4 & 349.92 & 1836.92 & 328.14 & 2.5440 \\
\hline
\end{tabular}


Table B1. Methanol Test Results

(continued)

\begin{tabular}{|c|c|c|}
\hline & Test & Test \\
\hline Study & Sequence & Identifier \\
\hline 1 & 34 & $80 C-2-2-5$ \\
\hline 1 & 34 & $80 C-2-2-6$ \\
\hline 1 & 34 & $80 \mathrm{C}-2-2-7$ \\
\hline 1 & 34 & $80 \mathrm{C}-2-2-8$ \\
\hline 1 & 34 & $80 C-2-2-9$ \\
\hline 1 & 34 & $80 \mathrm{C}-2-2-10$ \\
\hline 1 & 35 & $80 \mathrm{C}-2-3-1$ \\
\hline 1 & 35 & $80 \mathrm{C}-2-3-2$ \\
\hline 1 & 35 & $80 \mathrm{C}-2-3-3$ \\
\hline 1 & 35 & $80 \mathrm{C}-2-3-4$ \\
\hline 1 & 35 & $80 C-2-3-5$ \\
\hline 1 & 35 & $80 C-2-3-6$ \\
\hline 1 & 35 & $80 C-2-3-7$ \\
\hline 1 & 35 & $80 \mathrm{C}-2-3-8$ \\
\hline 1 & 35 & $80 C-2-3-9$ \\
\hline 1 & 35 & $80 C-2-3-10$ \\
\hline 1 & 36 & $90 \mathrm{C}-1-1-1,800 \mathrm{PPM}$ \\
\hline 1 & 36 & $90 \mathrm{C}-1-1-2,800 \mathrm{PPM}$ \\
\hline 1 & 36 & $90 \mathrm{C}-1-1-3,800 \mathrm{PPM}$ \\
\hline 1 & 36 & $90 \mathrm{C}-1-1-4,800 \mathrm{PPM}$ \\
\hline 1 & 36 & $90 \mathrm{C}-1-1-5,800 \mathrm{PPM}$ \\
\hline 1 & 36 & $90 \mathrm{C}-1-1-6,800 \mathrm{PPM}$ \\
\hline 1 & 36 & $90 \mathrm{C}-1-1-7,800 \mathrm{PPM}$ \\
\hline 1 & 36 & $90 \mathrm{C}-1-1-8,800 \mathrm{PPM}$ \\
\hline 1 & 36 & $90 \mathrm{C}-1-1-9,800 \mathrm{PPM}$ \\
\hline 1 & 36 & $90 \mathrm{C}-1-1-10,800 \mathrm{PPM}$ \\
\hline 1 & 37 & $90 \mathrm{C}-1-2-1,800 \mathrm{PPM}$ \\
\hline 1 & 37 & $90 \mathrm{C}-1-2-2,800 \mathrm{PPM}$ \\
\hline 1 & 37 & $90 \mathrm{C}-1-2-3,800 \mathrm{PPM}$ \\
\hline 1 & 37 & $90 \mathrm{C}-1-2-4,800 \mathrm{PPM}$ \\
\hline 1 & 37 & $90 \mathrm{C}-1-2-5,800$ PPM \\
\hline 1 & 37 & $90 \mathrm{C}-1-2-6,800 \mathrm{PPM}$ \\
\hline 1 & 37 & $90 \mathrm{C}-1-2-7,800 \mathrm{PPM}$ \\
\hline 1 & 37 & $90 \mathrm{C}-1-2-8,800 \mathrm{PPM}$ \\
\hline 1 & 37 & $90 \mathrm{C}-1-2-9,800 \mathrm{PPM}$ \\
\hline 1 & 37 & $90 \mathrm{C}-1-2-10,800 \mathrm{PPM}$ \\
\hline 1 & 38 & $90 \mathrm{C}-1-3-1,800 \mathrm{PPM}$ \\
\hline 1 & 38 & $90 \mathrm{C}-1-3-2,800 \mathrm{PPM}$ \\
\hline 1 & 38 & $90 \mathrm{C}-1-3-3,800 \mathrm{PPM}$ \\
\hline 1 & 38 & $90 \mathrm{C}-1-3-4,800 \mathrm{PPM}$ \\
\hline 1 & 38 & $90 \mathrm{C}-1-3-5,800 \mathrm{PPM}$ \\
\hline 1 & 38 & $90 \mathrm{C}-1-3-6,800 \mathrm{PPM}$ \\
\hline 1 & 38 & $90 \mathrm{C}-1-3-7,800 \mathrm{PPM}$ \\
\hline 1 & 38 & $90 \mathrm{C}-1-3-8,800$ PPM \\
\hline 1 & 38 & $90 \mathrm{C}-1-3-9,800 \mathrm{PPM}$ \\
\hline 1 & 38 & $90 \mathrm{C}-1-3-10,800 \mathrm{PPM}$ \\
\hline 1 & 39 & 90C-19-1-1, 800 PPM \\
\hline 1 & 39 & $90 \mathrm{C}-19-1-2,800 \mathrm{PPM}$ \\
\hline 1 & 39 & 90C-19-1-3, 800 PPM \\
\hline 1 & 39 & $90 \mathrm{C}-19-1-4,800 \mathrm{PPM}$ \\
\hline 1 & 39 & $90 \mathrm{C}-19-1-5,800$ PPM \\
\hline 1 & 39 & 90C-19-1-6, 800 PPM \\
\hline 1 & 39 & 90C-19-1-7, 800 PPM \\
\hline 1 & 39 & 90C-19-1-8, 800 PPM \\
\hline 1 & 39 & 90С-19-1-9, 800 PPM \\
\hline 1 & 39 & $90 \mathrm{C}-19-1-10,800 \mathrm{PPM}$ \\
\hline 1 & 40 & 90C-19-2-1,800 PPM \\
\hline 1 & 40 & $90 \mathrm{C}-19-2-2,800$ PPM \\
\hline 1 & 40 & 90C-19-2-3, 800 PPM \\
\hline 1 & 40 & 90C-19-2-4, 800 PPM \\
\hline 1 & 40 & $90 \mathrm{C}-19-2-5,800 \mathrm{PPM}$ \\
\hline 1 & 40 & 90C-19-2-6, 800 PPM \\
\hline 1 & 40 & $90 \mathrm{C}-19-2-7,800 \mathrm{PPM}$ \\
\hline 1 & 40 & 90C-19-2-8, 800 PPM \\
\hline 1 & 40 & $90 \mathrm{C}-19-2-9,800$ PPM \\
\hline 1 & 40 & 90C-19-2-10, 800 PPM \\
\hline
\end{tabular}

\begin{tabular}{|c|c|c|c|c|c|c|c|}
\hline Temperature & Vial & Test & Sampling & A & & & \\
\hline$\left({ }^{\circ} \mathrm{C}\right)$ & Pressusre & Run & Sequence & (peak area) & $\mathrm{y}$ & $\mathrm{x}$ & $\log (\mathrm{A})$ \\
\hline 80 & $2 \mathrm{~atm}$ & 2 & 5 & 328.14 & 2165.07 & 235.15 & 2.5161 \\
\hline 80 & $2 \mathrm{~atm}$ & 2 & 6 & 235.15 & 2400.22 & 191.63 & 2.3713 \\
\hline 80 & $2 \mathrm{~atm}$ & 2 & 7 & 191.63 & 2591.85 & 169.63 & 2.2825 \\
\hline 80 & $2 \mathrm{~atm}$ & 2 & 8 & 169.63 & 2761.48 & 123.25 & 2.2295 \\
\hline 80 & $2 \mathrm{~atm}$ & 2 & 9 & 123.25 & 2884.73 & 105.57 & 2.0908 \\
\hline 80 & $2 \mathrm{~atm}$ & 2 & 10 & 105.57 & 2990.30 & & 2.0235 \\
\hline 80 & $2 \mathrm{~atm}$ & 3 & 1 & 700.64 & 700.64 & 510.02 & 2.8455 \\
\hline 80 & $2 \mathrm{~atm}$ & 3 & 2 & 510.02 & 1210.66 & 480.14 & 2.7076 \\
\hline 80 & $2 \mathrm{~atm}$ & 3 & 3 & 480.14 & 1690.80 & 362.28 & 2.6814 \\
\hline 80 & $2 \mathrm{~atm}$ & 3 & 4 & 362.28 & 2053.08 & 353.56 & 2.5590 \\
\hline 80 & $2 \mathrm{~atm}$ & 3 & 5 & 353.56 & 2406.64 & 259.55 & 2.5485 \\
\hline 80 & $2 \mathrm{~atm}$ & 3 & 6 & 259.55 & 2666.19 & 256.29 & 2.4142 \\
\hline 80 & $2 \mathrm{~atm}$ & 3 & 7 & 256.29 & 2922.48 & 227.16 & 2.4087 \\
\hline 80 & $2 \mathrm{~atm}$ & 3 & 8 & 227.16 & 3149.64 & 227.98 & 2.3563 \\
\hline 80 & $2 \mathrm{~atm}$ & 3 & 9 & 227.98 & 3377.62 & 172.54 & 2.3579 \\
\hline 80 & $2 \mathrm{~atm}$ & 3 & 10 & 172.54 & 3550.16 & & 2.2369 \\
\hline 90 & $1 \mathrm{~atm}$ & 1 & 1 & 1616.04 & 1616.04 & 1194.55 & 3.2085 \\
\hline 90 & $1 \mathrm{~atm}$ & 1 & 2 & 1194.55 & 2810.59 & 938.67 & 3.0772 \\
\hline 90 & $1 \mathrm{~atm}$ & 1 & 3 & 938.67 & 3749.26 & 830.08 & 2.9725 \\
\hline 90 & $1 \mathrm{~atm}$ & 1 & 4 & 830.08 & 4579.34 & 729.51 & 2.9191 \\
\hline 90 & $1 \mathrm{~atm}$ & 1 & 5 & 729.51 & 5308.84 & 578.23 & 2.8630 \\
\hline 90 & $1 \mathrm{~atm}$ & 1 & 6 & 578.23 & 5887.07 & 454.68 & 2.7621 \\
\hline 90 & $1 \mathrm{~atm}$ & 1 & 7 & 454.68 & 6341.75 & 350.01 & 2.6577 \\
\hline 90 & $1 \mathrm{~atm}$ & 1 & 8 & 350.01 & 6691.76 & 304.04 & 2.5441 \\
\hline 90 & $1 \mathrm{~atm}$ & 1 & 9 & 304.04 & 6995.80 & 247.08 & 2.4829 \\
\hline 90 & $1 \mathrm{~atm}$ & 1 & 10 & 247.08 & 7242.88 & & 2.3928 \\
\hline 90 & $1 \mathrm{~atm}$ & 2 & 1 & 1450.10 & 1450.10 & 1182.98 & 3.1614 \\
\hline 90 & $1 \mathrm{~atm}$ & 2 & 2 & 1182.98 & 2633.08 & 999.15 & 3.0730 \\
\hline 90 & $1 \mathrm{~atm}$ & 2 & 3 & 999.15 & 3632.23 & 837.73 & 2.9996 \\
\hline 90 & $1 \mathrm{~atm}$ & 2 & 4 & 837.73 & 4469.96 & 690.38 & 2.9231 \\
\hline 90 & $1 \mathrm{~atm}$ & 2 & 5 & 690.38 & 5160.34 & 547.65 & 2.8391 \\
\hline 90 & $1 \mathrm{~atm}$ & 2 & 6 & 547.65 & 5707.99 & 449.47 & 2.7385 \\
\hline 90 & $1 \mathrm{~atm}$ & 2 & 7 & 449.47 & 6157.46 & 336.60 & 2.6527 \\
\hline 90 & $1 \mathrm{~atm}$ & 2 & 8 & 336.60 & 6494.06 & 262.81 & 2.5271 \\
\hline 90 & $1 \mathrm{~atm}$ & 2 & 9 & 262.81 & 6756.87 & 235.63 & 2.4196 \\
\hline 90 & $1 \mathrm{~atm}$ & 2 & 10 & 235.63 & 6992.50 & & 2.3722 \\
\hline 90 & $1 \mathrm{~atm}$ & 3 & 1 & 1336.37 & 1336.37 & 1208.31 & 3.1259 \\
\hline 90 & $1 \mathrm{~atm}$ & 3 & 2 & 1208.31 & 2544.68 & 1044.35 & 3.0822 \\
\hline 90 & $1 \mathrm{~atm}$ & 3 & 3 & 1044.35 & 3589.03 & 833.48 & 3.0188 \\
\hline 90 & $1 \mathrm{~atm}$ & 3 & 4 & 833.48 & 4422.51 & 857.56 & 2.9209 \\
\hline 90 & $1 \mathrm{~atm}$ & 3 & 5 & 857.56 & 5280.07 & 563.64 & 2.9333 \\
\hline 90 & $1 \mathrm{~atm}$ & 3 & 6 & 563.64 & 5843.71 & 485.39 & 2.7510 \\
\hline 90 & $1 \mathrm{~atm}$ & 3 & 7 & 485.39 & 6329.10 & 395.27 & 2.6861 \\
\hline 90 & $1 \mathrm{~atm}$ & 3 & 8 & 395.27 & 6724.37 & 315.52 & 2.5969 \\
\hline 90 & $1 \mathrm{~atm}$ & 3 & 9 & 315.52 & 7039.89 & 263.19 & 2.4990 \\
\hline 90 & $1 \mathrm{~atm}$ & 3 & 10 & 263.19 & 7303.08 & & 2.4203 \\
\hline 90 & $19 \mathrm{psig}$ & 1 & 1 & 1479.29 & 1479.29 & 1012.14 & 3.1701 \\
\hline 90 & 19 psig & 1 & 2 & 1012.14 & 2491.43 & 843.60 & 3.0052 \\
\hline 90 & $19 \mathrm{psig}$ & 1 & 3 & 843.60 & 3335.03 & 722.17 & 2.9261 \\
\hline 90 & $19 \mathrm{psig}$ & 1 & 4 & 722.17 & 4057.20 & 592.69 & 2.8586 \\
\hline 90 & 19 psig & 1 & 5 & 592.69 & 4649.89 & 453.40 & 2.7728 \\
\hline 90 & 19 psig & 1 & 6 & 453.40 & 5103.29 & 394.38 & 2.6565 \\
\hline 90 & 19 psig & 1 & 7 & 394.38 & 5497.66 & 249.02 & 2.5959 \\
\hline 90 & 19 psig & 1 & 8 & 249.02 & 5746.68 & 188.33 & 2.3962 \\
\hline 90 & $19 \mathrm{psig}$ & 1 & 9 & 188.33 & 5935.01 & 152.64 & 2.2749 \\
\hline 90 & $19 \mathrm{psig}$ & 1 & 10 & 152.64 & 6087.65 & & 2.1837 \\
\hline 90 & $19 \mathrm{psig}$ & 2 & 1 & 1531.86 & 1531.86 & 1171.08 & 3.1852 \\
\hline 90 & 19 psig & 2 & 2 & 1171.08 & 2702.95 & 945.92 & 3.0686 \\
\hline 90 & 19 psig & 2 & 3 & 945.92 & 3648.86 & 728.25 & 2.9759 \\
\hline 90 & 19 psig & 2 & 4 & 728.25 & 4377.11 & 589.20 & 2.8623 \\
\hline 90 & 19 psig & 2 & 5 & 589.20 & 4966.31 & 435.32 & 2.7703 \\
\hline 90 & 19 psig & 2 & 6 & 435.32 & 5401.63 & 333.39 & 2.6388 \\
\hline 90 & $19 \mathrm{psig}$ & 2 & 7 & 333.39 & 5735.02 & 259.37 & 2.5230 \\
\hline 90 & 19 psig & 2 & 8 & 259.37 & 5994.39 & 201.17 & 2.4139 \\
\hline 90 & 19 psig & 2 & 9 & 201.17 & 6195.56 & 166.46 & 2.3036 \\
\hline 90 & 19 psig & 2 & 10 & 166.46 & 6362.03 & 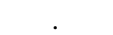 & 2.2213 \\
\hline
\end{tabular}


Table B1. Methanol Test Results

(continued)

\begin{tabular}{|c|c|c|}
\hline & Test & Test \\
\hline Study & Sequence & Identifier \\
\hline 1 & 41 & 90C-19-3-1, 800 PPM \\
\hline 1 & 41 & $90 \mathrm{C}-19-3-2,800$ PPM \\
\hline 1 & 41 & 90C-19-3-3, 800 PPM \\
\hline 1 & 41 & 90С-19-3-4, 800 PPM \\
\hline 1 & 41 & $90 \mathrm{C}-19-3-5,800$ PPM \\
\hline 1 & 41 & $90 \mathrm{C}-19-3-6,800$ PРM \\
\hline 1 & 41 & 90С-19-3-7, 800 PPM \\
\hline 1 & 41 & 90С-19-3-8, 800 PPM \\
\hline 1 & 41 & 90C-19-3-9, 800 PPM \\
\hline 1 & 41 & 90C-19-3-10, 800 PPM \\
\hline 1 & 42 & $90 \mathrm{C}-2-1-1,800 \mathrm{PPM}$ \\
\hline 1 & 42 & $90 \mathrm{C}-2-1-2,800 \mathrm{PPM}$ \\
\hline 1 & 42 & $90 \mathrm{C}-2-1-3,800 \mathrm{PPM}$ \\
\hline 1 & 42 & $90 \mathrm{C}-2-1-4,800 \mathrm{PPM}$ \\
\hline 1 & 42 & $90 \mathrm{C}-2-1-5,800 \mathrm{PPM}$ \\
\hline 1 & 42 & $90 \mathrm{C}-2-1-6,800 \mathrm{PPM}$ \\
\hline 1 & 42 & $90 \mathrm{C}-2-1-7,800 \mathrm{PPM}$ \\
\hline 1 & 42 & $90 \mathrm{C}-2-1-8,800 \mathrm{PPM}$ \\
\hline 1 & 42 & $90 \mathrm{C}-2-1-9,800 \mathrm{PPM}$ \\
\hline 1 & 42 & $90 \mathrm{C}-2-1-10,800 \mathrm{PPM}$ \\
\hline 1 & 43 & $90 \mathrm{C}-2-2-1,800 \mathrm{PPM}$ \\
\hline 1 & 43 & $90 \mathrm{C}-2-2-2,800 \mathrm{PPM}$ \\
\hline 1 & 43 & $90 \mathrm{C}-2-2-3,800$ PPM \\
\hline 1 & 43 & $90 \mathrm{C}-2-2-4,800 \mathrm{PPM}$ \\
\hline 1 & 43 & $90 \mathrm{C}-2-2-5,800 \mathrm{PPM}$ \\
\hline 1 & 43 & 90C-2-2-6, 800 PPM \\
\hline 1 & 43 & $90 \mathrm{C}-2-2-7,800 \mathrm{PPM}$ \\
\hline 1 & 43 & 90C-2-2-8, 800 PPM \\
\hline 1 & 43 & $90 \mathrm{C}-2-2-9,800$ PPM \\
\hline 1 & 43 & 90C-2-2-10, 800 PPM \\
\hline 1 & 44 & $90 \mathrm{C}-2-3-1,800$ PPM \\
\hline 1 & 44 & $90 \mathrm{C}-2-3-2,800 \mathrm{PPM}$ \\
\hline 1 & 44 & 90C-2-3-3, 800 PPM \\
\hline 1 & 44 & $90 \mathrm{C}-2-3-4,800 \mathrm{PPM}$ \\
\hline 1 & 44 & $90 \mathrm{C}-2-3-5,800 \mathrm{PPM}$ \\
\hline 1 & 44 & $90 \mathrm{C}-2-3-6,800$ PPM \\
\hline 1 & 44 & $90 \mathrm{C}-2-3-7,800 \mathrm{PPM}$ \\
\hline 1 & 44 & 90C-2-3-8, 800 PPM \\
\hline 1 & 44 & $90 \mathrm{C}-2-3-9,800 \mathrm{PPM}$ \\
\hline 1 & 44 & $90 \mathrm{C}-2-3-10,800 \mathrm{PPM}$ \\
\hline 2 & 1 & $50 \mathrm{C}-1-1-1,1600 \mathrm{PPM}$ \\
\hline 2 & 1 & $50 \mathrm{C}-1-1-1,1600 \mathrm{PPM}$ \\
\hline 2 & 1 & $50 \mathrm{C}-1-1-1,1600 \mathrm{PPM}$ \\
\hline 2 & 1 & $50 \mathrm{C}-1-1-1,1600$ PPM \\
\hline 2 & 1 & $50 \mathrm{C}-1-1-1,1600 \mathrm{PPM}$ \\
\hline 2 & 1 & $50 \mathrm{C}-1-1-1,1600 \mathrm{PPM}$ \\
\hline 2 & 1 & $50 \mathrm{C}-1-1-1,1600 \mathrm{PPM}$ \\
\hline 2 & 1 & $50 \mathrm{C}-1-1-1,1600 \mathrm{PPM}$ \\
\hline 2 & 1 & $50 \mathrm{C}-1-1-1,1600 \mathrm{PPM}$ \\
\hline 2 & 1 & $50 \mathrm{C}-1-1-10,1600 \mathrm{PPM}$ \\
\hline 2 & 2 & $50 \mathrm{C}-1-2-1,1600$ PPM \\
\hline 2 & 2 & $50 \mathrm{C}-1-2-1,1600 \mathrm{PPM}$ \\
\hline 2 & 2 & $50 \mathrm{C}-1-2-1,1600 \mathrm{PPM}$ \\
\hline 2 & 2 & $50 \mathrm{C}-1-2-1,1600 \mathrm{PPM}$ \\
\hline 2 & 2 & $50 \mathrm{C}-1-2-1,1600$ PPM \\
\hline 2 & 2 & $50 \mathrm{C}-1-2-1,1600 \mathrm{PPM}$ \\
\hline 2 & 2 & $50 \mathrm{C}-1-2-1,1600 \mathrm{PPM}$ \\
\hline 2 & 2 & $50 \mathrm{C}-1-2-1,1600 \mathrm{PPM}$ \\
\hline 2 & 2 & $50 \mathrm{C}-1-2-1,1600$ PPM \\
\hline 2 & 2 & $50 \mathrm{C}-1-2-10,1600 \mathrm{PPM}$ \\
\hline 2 & 3 & $50 \mathrm{C}-1-3-1,1600 \mathrm{PPM}$ \\
\hline 2 & 3 & $50 \mathrm{C}-1-3-1,1600 \mathrm{PPM}$ \\
\hline 2 & 3 & $50 \mathrm{C}-1-3-1,1600 \mathrm{PPM}$ \\
\hline 2 & 3 & $50 \mathrm{C}-1-3-1,1600 \mathrm{PPM}$ \\
\hline 2 & 3 & $50 \mathrm{C}-1-3-1,1600 \mathrm{PPM}$ \\
\hline 2 & 3 & 50C-1-3-1, 1600 PPM \\
\hline
\end{tabular}

\begin{tabular}{|c|c|c|c|c|c|c|c|}
\hline Temperature & Vial & Test & Sampling & A & & & \\
\hline$\left({ }^{\circ} \mathrm{C}\right)$ & Pressusre & Run & Sequence & (peak area) & $\mathrm{y}$ & $\mathrm{x}$ & $\log (\mathrm{A})$ \\
\hline 90 & 19 psig & 3 & 1 & 1296.60 & 1296.60 & 914.75 & 3.1128 \\
\hline 90 & $19 \mathrm{psig}$ & 3 & 2 & 914.75 & 2211.35 & 729.07 & 2.9613 \\
\hline 90 & $19 \mathrm{psig}$ & 3 & 3 & 729.07 & 2940.42 & 560.31 & 2.8628 \\
\hline 90 & 19 psig & 3 & 4 & 560.31 & 3500.73 & 406.65 & 2.7484 \\
\hline 90 & $19 \mathrm{psig}$ & 3 & 5 & 406.65 & 3907.38 & 335.35 & 2.6092 \\
\hline 90 & $19 \mathrm{psig}$ & 3 & 6 & 335.35 & 4242.73 & 233.02 & 2.5255 \\
\hline 90 & $19 \mathrm{psig}$ & 3 & 7 & 233.02 & 4475.75 & 138.44 & 2.3674 \\
\hline 90 & 19 psig & 3 & 8 & 138.44 & 4614.19 & 99.34 & 2.1413 \\
\hline 90 & $19 \mathrm{psig}$ & 3 & 9 & 99.34 & 4713.53 & 84.44 & 1.9971 \\
\hline 90 & $19 \mathrm{psig}$ & 3 & 10 & 84.44 & 4797.96 & 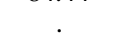 & 1.9265 \\
\hline 90 & $2 \mathrm{~atm}$ & 1 & 1 & 1558.58 & 1558.58 & 1203.10 & 3.1927 \\
\hline 90 & $2 \mathrm{~atm}$ & 1 & 2 & 1203.10 & 2761.69 & 1243.22 & 3.0803 \\
\hline 90 & $2 \mathrm{~atm}$ & 1 & 3 & 1243.22 & 4004.91 & 831.68 & 3.0945 \\
\hline 90 & $2 \mathrm{~atm}$ & 1 & 4 & 831.68 & 4836.58 & 547.41 & 2.9200 \\
\hline 90 & $2 \mathrm{~atm}$ & 1 & 5 & 547.41 & 5383.99 & 363.29 & 2.7383 \\
\hline 90 & $2 \mathrm{~atm}$ & 1 & 6 & 363.29 & 5747.28 & 247.27 & 2.5603 \\
\hline 90 & $2 \mathrm{~atm}$ & 1 & 7 & 247.27 & 5994.55 & 191.26 & 2.3932 \\
\hline 90 & $2 \mathrm{~atm}$ & 1 & 8 & 191.26 & 6185.81 & 110.26 & 2.2816 \\
\hline 90 & $2 \mathrm{~atm}$ & 1 & 9 & 110.26 & 6296.07 & 82.50 & 2.0424 \\
\hline 90 & $2 \mathrm{~atm}$ & 1 & 10 & 82.50 & 6378.57 & & 1.9164 \\
\hline 90 & $2 \mathrm{~atm}$ & 2 & 1 & 1540.41 & 1540.41 & 1085.27 & 3.1876 \\
\hline 90 & $2 \mathrm{~atm}$ & 2 & 2 & 1085.27 & 2625.68 & 930.65 & 3.0355 \\
\hline 90 & $2 \mathrm{~atm}$ & 2 & 3 & 930.65 & 3556.32 & 697.52 & 2.9688 \\
\hline 90 & $2 \mathrm{~atm}$ & 2 & 4 & 697.52 & 4253.84 & 466.66 & 2.8436 \\
\hline 90 & $2 \mathrm{~atm}$ & 2 & 5 & 466.66 & 4720.51 & 310.66 & 2.6690 \\
\hline 90 & $2 \mathrm{~atm}$ & 2 & 6 & 310.66 & 5031.17 & 198.12 & 2.4923 \\
\hline 90 & $2 \mathrm{~atm}$ & 2 & 7 & 198.12 & 5229.29 & 160.42 & 2.2969 \\
\hline 90 & $2 \mathrm{~atm}$ & 2 & 8 & 160.42 & 5389.71 & 103.92 & 2.2053 \\
\hline 90 & $2 \mathrm{~atm}$ & 2 & 9 & 103.92 & 5493.63 & 70.61 & 2.0167 \\
\hline 90 & $2 \mathrm{~atm}$ & 2 & 10 & 70.61 & 5564.25 & & 1.8489 \\
\hline 90 & $2 \mathrm{~atm}$ & 3 & 1 & 1746.87 & 1746.87 & 1260.76 & 3.2423 \\
\hline 90 & $2 \mathrm{~atm}$ & 3 & 2 & 1260.76 & 3007.63 & 877.76 & 3.1006 \\
\hline 90 & $2 \mathrm{~atm}$ & 3 & 3 & 877.76 & 3885.39 & 832.52 & 2.9434 \\
\hline 90 & $2 \mathrm{~atm}$ & 3 & 4 & 832.52 & 4717.91 & 567.71 & 2.9204 \\
\hline 90 & $2 \mathrm{~atm}$ & 3 & 5 & 567.71 & 5285.61 & 331.15 & 2.7541 \\
\hline 90 & $2 \mathrm{~atm}$ & 3 & 6 & 331.15 & 5616.77 & 209.58 & 2.5200 \\
\hline 90 & $2 \mathrm{~atm}$ & 3 & 7 & 209.58 & 5826.34 & 155.26 & 2.3213 \\
\hline 90 & $2 \mathrm{~atm}$ & 3 & 8 & 155.26 & 5981.60 & 194.06 & 2.1911 \\
\hline 90 & $2 \mathrm{~atm}$ & 3 & 9 & 194.06 & 6175.66 & 76.81 & 2.2879 \\
\hline 90 & $2 \mathrm{~atm}$ & 3 & 10 & 76.81 & 6252.48 & & 1.8854 \\
\hline 50 & $1 \mathrm{~atm}$ & 1 & 1 & 8628.37 & 8628.37 & 7531.39 & 3.9359 \\
\hline 50 & $1 \mathrm{~atm}$ & 1 & 2 & 7531.39 & 16159.75 & 7194.30 & 3.8769 \\
\hline 50 & $1 \mathrm{~atm}$ & 1 & 3 & 7194.30 & 23354.06 & 6911.16 & 3.8570 \\
\hline 50 & $1 \mathrm{~atm}$ & 1 & 4 & 6911.16 & 30265.21 & 6668.30 & 3.8396 \\
\hline 50 & $1 \mathrm{~atm}$ & 1 & 5 & 6668.30 & 36933.51 & 6452.92 & 3.8240 \\
\hline 50 & $1 \mathrm{~atm}$ & 1 & 6 & 6452.92 & 43386.43 & 6209.50 & 3.8098 \\
\hline 50 & $1 \mathrm{~atm}$ & 1 & 7 & 6209.50 & 49595.93 & 5970.37 & 3.7931 \\
\hline 50 & $1 \mathrm{~atm}$ & 1 & 8 & 5970.37 & 55566.30 & 5735.35 & 3.7760 \\
\hline 50 & $1 \mathrm{~atm}$ & 1 & 9 & 5735.35 & 61301.65 & 5446.13 & 3.7586 \\
\hline 50 & $1 \mathrm{~atm}$ & 1 & 10 & 5446.13 & 66747.77 & & 3.7361 \\
\hline 50 & $1 \mathrm{~atm}$ & 2 & 1 & 8721.60 & 8721.60 & 7390.71 & 3.9406 \\
\hline 50 & $1 \mathrm{~atm}$ & 2 & 2 & 7390.71 & 16112.31 & 7063.57 & 3.8687 \\
\hline 50 & $1 \mathrm{~atm}$ & 2 & 3 & 7063.57 & 23175.88 & 6806.32 & 3.8490 \\
\hline 50 & $1 \mathrm{~atm}$ & 2 & 4 & 6806.32 & 29982.19 & 6529.19 & 3.8329 \\
\hline 50 & $1 \mathrm{~atm}$ & 2 & 5 & 6529.19 & 36511.38 & 6309.63 & 3.8149 \\
\hline 50 & $1 \mathrm{~atm}$ & 2 & 6 & 6309.63 & 42821.01 & 6068.27 & 3.8000 \\
\hline 50 & $1 \mathrm{~atm}$ & 2 & 7 & 6068.27 & 48889.28 & 5910.12 & 3.7831 \\
\hline 50 & $1 \mathrm{~atm}$ & 2 & 8 & 5910.12 & 54799.40 & 5738.33 & 3.7716 \\
\hline 50 & $1 \mathrm{~atm}$ & 2 & 9 & 5738.33 & 60537.73 & 5460.37 & 3.7588 \\
\hline 50 & $1 \mathrm{~atm}$ & 2 & 10 & 5460.37 & 65998.09 & & 3.7372 \\
\hline 50 & $1 \mathrm{~atm}$ & 3 & 1 & 9201.38 & 9201.38 & 7512.01 & 3.9639 \\
\hline 50 & $1 \mathrm{~atm}$ & 3 & 2 & 7512.01 & 16713.39 & 7054.94 & 3.8758 \\
\hline 50 & $1 \mathrm{~atm}$ & 3 & 3 & 7054.94 & 23768.33 & 6761.43 & 3.8485 \\
\hline 50 & $1 \mathrm{~atm}$ & 3 & 4 & 6761.43 & 30529.76 & 6506.13 & 3.8300 \\
\hline 50 & $1 \mathrm{~atm}$ & 3 & 5 & 6506.13 & 37035.89 & 6275.28 & 3.8133 \\
\hline 50 & $1 \mathrm{~atm}$ & 3 & 6 & 6275.28 & 43311.17 & 6074.76 & 3.7976 \\
\hline
\end{tabular}


Table B1. Methanol Test Results

(continued)

\begin{tabular}{|c|c|c|}
\hline & Test & Test \\
\hline Study & Sequence & Identifier \\
\hline 2 & 3 & 50C-1-3-1, 1600 PPM \\
\hline 2 & 3 & $50 \mathrm{C}-1-3-1,1600 \mathrm{PPM}$ \\
\hline 2 & 3 & 50C-1-3-1, 1600 PPM \\
\hline 2 & 3 & $50 \mathrm{C}-1-3-10,1600 \mathrm{PPM}$ \\
\hline 2 & 4 & $60 \mathrm{C}-1-1-1,1600$ PPM \\
\hline 2 & 4 & $60 \mathrm{C}-1-1-1,1600$ PPM \\
\hline 2 & 4 & 60C-1-1-1, 1600 PPM \\
\hline 2 & 4 & $60 \mathrm{C}-1-1-1,1600$ PPM \\
\hline 2 & 4 & $60 \mathrm{C}-1-1-1,1600 \mathrm{PPM}$ \\
\hline 2 & 4 & $60 \mathrm{C}-1-1-1,1600 \mathrm{PPM}$ \\
\hline 2 & 4 & $60 \mathrm{C}-1-1-1,1600 \mathrm{PPM}$ \\
\hline 2 & 4 & $60 \mathrm{C}-1-1-1,1600 \mathrm{PPM}$ \\
\hline 2 & 4 & $60 \mathrm{C}-1-1-1,1600 \mathrm{PPM}$ \\
\hline 2 & 4 & $60 \mathrm{C}-1-1-10,1600 \mathrm{PPM}$ \\
\hline 2 & 5 & $60 \mathrm{C}-1-2-1,1600$ PPM \\
\hline 2 & 5 & $60 \mathrm{C}-1-2-1,1600 \mathrm{PPM}$ \\
\hline 2 & 5 & $60 \mathrm{C}-1-2-1,1600 \mathrm{PPM}$ \\
\hline 2 & 5 & $60 \mathrm{C}-1-2-1,1600$ PPM \\
\hline 2 & 5 & $60 \mathrm{C}-1-2-1,1600 \mathrm{PPM}$ \\
\hline 2 & 5 & $60 \mathrm{C}-1-2-1,1600$ PPM \\
\hline 2 & 5 & $60 \mathrm{C}-1-2-1,1600$ PPM \\
\hline 2 & 5 & $60 \mathrm{C}-1-2-1,1600$ PPM \\
\hline 2 & 5 & $60 \mathrm{C}-1-2-1,1600 \mathrm{PPM}$ \\
\hline 2 & 5 & $60 \mathrm{C}-1-2-10,1600 \mathrm{PPM}$ \\
\hline 2 & 6 & $60 \mathrm{C}-1-3-1,1600 \mathrm{PPM}$ \\
\hline 2 & 6 & $60 \mathrm{C}-1-3-1,1600$ PPM \\
\hline 2 & 6 & $60 \mathrm{C}-1-3-1,1600 \mathrm{PPM}$ \\
\hline 2 & 6 & $60 \mathrm{C}-1-3-1,1600$ PPM \\
\hline 2 & 6 & $60 \mathrm{C}-1-3-1,1600 \mathrm{PPM}$ \\
\hline 2 & 6 & $60 \mathrm{C}-1-3-1,1600 \mathrm{PPM}$ \\
\hline 2 & 6 & $60 \mathrm{C}-1-3-1,1600$ PPM \\
\hline 2 & 6 & $60 \mathrm{C}-1-3-1,1600 \mathrm{PPM}$ \\
\hline 2 & 6 & $60 \mathrm{C}-1-3-1,1600 \mathrm{PPM}$ \\
\hline 2 & 6 & $60 \mathrm{C}-1-3-10,1600 \mathrm{PPM}$ \\
\hline 2 & 7 & 70C-1-1-1, 1600 PPM \\
\hline 2 & 7 & 70C-1-1-1, 1600 PPM \\
\hline 2 & 7 & 70C-1-1-1, 1600 PPM \\
\hline 2 & 7 & 70C-1-1-1, 1600 PPM \\
\hline 2 & 7 & $70 \mathrm{C}-1-1-1,1600$ PPM \\
\hline 2 & 7 & 70C-1-1-1, 1600 PPM \\
\hline 2 & 7 & 70C-1-1-1, 1600 PPM \\
\hline 2 & 7 & 70C-1-1-1, 1600 PPM \\
\hline 2 & 7 & 70C-1-1-1, 1600 PPM \\
\hline 2 & 7 & $70 \mathrm{C}-1-1-10,1600 \mathrm{PPM}$ \\
\hline 2 & 8 & 70C-1-2-1, 1600 PPM \\
\hline 2 & 8 & 70C-1-2-1, 1600 PPM \\
\hline 2 & 8 & $70 \mathrm{C}-1-2-1,1600 \mathrm{PPM}$ \\
\hline 2 & 8 & 70C-1-2-1, 1600 PPM \\
\hline 2 & 8 & 70C-1-2-1, 1600 PPM \\
\hline 2 & 8 & 70C-1-2-1, 1600 PPM \\
\hline 2 & 8 & 70C-1-2-1, 1600 PPM \\
\hline 2 & 8 & 70C-1-2-1, 1600 PPM \\
\hline 2 & 8 & 70C-1-2-1, 1600 PPM \\
\hline 2 & 8 & $70 \mathrm{C}-1-2-10,1600 \mathrm{PPM}$ \\
\hline 2 & 9 & 70C-1-3-1, 1600 PPM \\
\hline 2 & 9 & 70C-1-3-1, 1600 PPM \\
\hline 2 & 9 & 70C-1-3-1, 1600 PPM \\
\hline 2 & 9 & 70C-1-3-1, 1600 PPM \\
\hline 2 & 9 & 70C-1-3-1, 1600 PPM \\
\hline 2 & 9 & $70 \mathrm{C}-1-3-1,1600 \mathrm{PPM}$ \\
\hline 2 & 9 & 70C-1-3-1, 1600 PPM \\
\hline 2 & 9 & 70C-1-3-1, 1600 PPM \\
\hline 2 & 9 & 70C-1-3-1, 1600 PPM \\
\hline 2 & 9 & $70 \mathrm{C}-1-3-10,1600 \mathrm{PPM}$ \\
\hline 2 & 10 & $80 \mathrm{C}-1-1-1,1600 \mathrm{PPM}$ \\
\hline 2 & 10 & 80C-1-1-1, 1600 PPM \\
\hline
\end{tabular}

\begin{tabular}{|c|c|c|c|c|c|c|c|}
\hline Temperature & Vial & Test & Sampling & A & & & \\
\hline$\left({ }^{\circ} \mathrm{C}\right)$ & Pressusre & Run & Sequence & (peak area) & $\mathrm{y}$ & $\mathrm{x}$ & $\log (\mathrm{A})$ \\
\hline 50 & $1 \mathrm{~atm}$ & 3 & 7 & 6074.76 & 49385.93 & 5864.18 & 3.7835 \\
\hline 50 & $1 \mathrm{~atm}$ & 3 & 8 & 5864.18 & 55250.11 & 5644.20 & 3.7682 \\
\hline 50 & $1 \mathrm{~atm}$ & 3 & 9 & 5644.20 & 60894.31 & 5402.62 & 3.7516 \\
\hline 50 & $1 \mathrm{~atm}$ & 3 & 10 & 5402.62 & 66296.93 & & 3.7326 \\
\hline 60 & $1 \mathrm{~atm}$ & 1 & 1 & 13283.10 & 13283.10 & 11588.69 & 4.1233 \\
\hline 60 & $1 \mathrm{~atm}$ & 1 & 2 & 11588.69 & 24871.78 & 10300.88 & 4.0640 \\
\hline 60 & $1 \mathrm{~atm}$ & 1 & 3 & 10300.88 & 35172.67 & 9962.88 & 4.0129 \\
\hline 60 & $1 \mathrm{~atm}$ & 1 & 4 & 9962.88 & 45135.54 & 9488.90 & 3.9984 \\
\hline 60 & $1 \mathrm{~atm}$ & 1 & 5 & 9488.90 & 54624.44 & 9084.31 & 3.9772 \\
\hline 60 & $1 \mathrm{~atm}$ & 1 & 6 & 9084.31 & 63708.76 & 8648.56 & 3.9583 \\
\hline 60 & $1 \mathrm{~atm}$ & 1 & 7 & 8648.56 & 72357.32 & 8225.93 & 3.9369 \\
\hline 60 & $1 \mathrm{~atm}$ & 1 & 8 & 8225.93 & 80583.25 & 7820.30 & 3.9152 \\
\hline 60 & $1 \mathrm{~atm}$ & 1 & 9 & 7820.30 & 88403.54 & 7339.66 & 3.8932 \\
\hline 60 & $1 \mathrm{~atm}$ & 1 & 10 & 7339.66 & 95743.21 & & 3.8657 \\
\hline 60 & $1 \mathrm{~atm}$ & 2 & 1 & 13282.24 & 13282.24 & 11513.78 & 4.1233 \\
\hline 60 & $1 \mathrm{~atm}$ & 2 & 2 & 11513.78 & 24796.02 & 10118.13 & 4.0612 \\
\hline 60 & $1 \mathrm{~atm}$ & 2 & 3 & 10118.13 & 34914.15 & 9765.22 & 4.0051 \\
\hline 60 & $1 \mathrm{~atm}$ & 2 & 4 & 9765.22 & 44679.37 & 9328.14 & 3.9897 \\
\hline 60 & $1 \mathrm{~atm}$ & 2 & 5 & 9328.14 & 54007.50 & 8872.78 & 3.9698 \\
\hline 60 & $1 \mathrm{~atm}$ & 2 & 6 & 8872.78 & 62880.28 & 8459.70 & 3.9481 \\
\hline 60 & $1 \mathrm{~atm}$ & 2 & 7 & 8459.70 & 71339.98 & 8086.00 & 3.9274 \\
\hline 60 & $1 \mathrm{~atm}$ & 2 & 8 & 8086.00 & 79425.98 & 7677.68 & 3.9077 \\
\hline 60 & $1 \mathrm{~atm}$ & 2 & 9 & 7677.68 & 87103.66 & 7184.70 & 3.8852 \\
\hline 60 & $1 \mathrm{~atm}$ & 2 & 10 & 7184.70 & 94288.36 & & 3.8564 \\
\hline 60 & $1 \mathrm{~atm}$ & 3 & 1 & 13548.69 & 13548.69 & 11566.54 & 4.1319 \\
\hline 60 & $1 \mathrm{~atm}$ & 3 & 2 & 11566.54 & 25115.23 & 10139.14 & 4.0632 \\
\hline 60 & $1 \mathrm{~atm}$ & 3 & 3 & 10139.14 & 35254.38 & 9753.54 & 4.0060 \\
\hline 60 & $1 \mathrm{~atm}$ & 3 & 4 & 9753.54 & 45007.92 & 9287.95 & 3.9892 \\
\hline 60 & $1 \mathrm{~atm}$ & 3 & 5 & 9287.95 & 54295.87 & 8866.47 & 3.9679 \\
\hline 60 & $1 \mathrm{~atm}$ & 3 & 6 & 8866.47 & 63162.34 & 8424.91 & 3.9478 \\
\hline 60 & $1 \mathrm{~atm}$ & 3 & 7 & 8424.91 & 71587.25 & 8037.42 & 3.9256 \\
\hline 60 & $1 \mathrm{~atm}$ & 3 & 8 & 8037.42 & 79624.67 & 7632.27 & 3.9051 \\
\hline 60 & $1 \mathrm{~atm}$ & 3 & 9 & 7632.27 & 87256.94 & 7154.28 & 3.8827 \\
\hline 60 & $1 \mathrm{~atm}$ & 3 & 10 & 7154.28 & 94411.22 & & 3.8546 \\
\hline 70 & $1 \mathrm{~atm}$ & 1 & 1 & 15012.36 & 15012.36 & 13175.28 & 4.1764 \\
\hline 70 & $1 \mathrm{~atm}$ & 1 & 2 & 13175.28 & 28187.64 & 11731.89 & 4.1198 \\
\hline 70 & $1 \mathrm{~atm}$ & 1 & 3 & 11731.89 & 39919.53 & 10798.25 & 4.0694 \\
\hline 70 & $1 \mathrm{~atm}$ & 1 & 4 & 10798.25 & 50717.78 & 10072.85 & 4.0334 \\
\hline 70 & $1 \mathrm{~atm}$ & 1 & 5 & 10072.85 & 60790.63 & 9481.13 & 4.0032 \\
\hline 70 & $1 \mathrm{~atm}$ & 1 & 6 & 9481.13 & 70271.77 & 8978.88 & 3.9769 \\
\hline 70 & $1 \mathrm{~atm}$ & 1 & 7 & 8978.88 & 79250.65 & 8337.43 & 3.9532 \\
\hline 70 & $1 \mathrm{~atm}$ & 1 & 8 & 8337.43 & 87588.07 & 7735.36 & 3.9210 \\
\hline 70 & $1 \mathrm{~atm}$ & 1 & 9 & 7735.36 & 95323.43 & 7022.66 & 3.8885 \\
\hline 70 & $1 \mathrm{~atm}$ & 1 & 10 & 7022.66 & 102346.10 & & 3.8465 \\
\hline 70 & $1 \mathrm{~atm}$ & 2 & 1 & 15228.11 & 15228.11 & 13426.20 & 4.1826 \\
\hline 70 & $1 \mathrm{~atm}$ & 2 & 2 & 13426.20 & 28654.31 & 11840.79 & 4.1280 \\
\hline 70 & $1 \mathrm{~atm}$ & 2 & 3 & 11840.79 & 40495.09 & 11348.19 & 4.0734 \\
\hline 70 & $1 \mathrm{~atm}$ & 2 & 4 & 11348.19 & 51843.28 & 10679.80 & 4.0549 \\
\hline 70 & $1 \mathrm{~atm}$ & 2 & 5 & 10679.80 & 62523.08 & 10072.99 & 4.0286 \\
\hline 70 & $1 \mathrm{~atm}$ & 2 & 6 & 10072.99 & 72596.07 & 9409.74 & 4.0032 \\
\hline 70 & $1 \mathrm{~atm}$ & 2 & 7 & 9409.74 & 82005.81 & 8745.97 & 3.9736 \\
\hline 70 & $1 \mathrm{~atm}$ & 2 & 8 & 8745.97 & 90751.79 & 8131.47 & 3.9418 \\
\hline 70 & $1 \mathrm{~atm}$ & 2 & 9 & 8131.47 & 98883.26 & 7406.35 & 3.9102 \\
\hline 70 & $1 \mathrm{~atm}$ & 2 & 10 & 7406.35 & 106289.60 & & 3.8696 \\
\hline 70 & $1 \mathrm{~atm}$ & 3 & 1 & 15328.94 & 15328.94 & 13799.00 & 4.1855 \\
\hline 70 & $1 \mathrm{~atm}$ & 3 & 2 & 13799.00 & 29127.93 & 12334.48 & 4.1398 \\
\hline 70 & $1 \mathrm{~atm}$ & 3 & 3 & 12334.48 & 41462.41 & 11590.25 & 4.0911 \\
\hline 70 & $1 \mathrm{~atm}$ & 3 & 4 & 11590.25 & 53052.66 & 10918.28 & 4.0641 \\
\hline 70 & $1 \mathrm{~atm}$ & 3 & 5 & 10918.28 & 63970.94 & 10293.86 & 4.0382 \\
\hline 70 & $1 \mathrm{~atm}$ & 3 & 6 & 10293.86 & 74264.79 & 9567.80 & 4.0126 \\
\hline 70 & $1 \mathrm{~atm}$ & 3 & 7 & 9567.80 & 83832.59 & 8855.91 & 3.9808 \\
\hline 70 & $1 \mathrm{~atm}$ & 3 & 8 & 8855.91 & 92688.51 & 8143.26 & 3.9472 \\
\hline 70 & $1 \mathrm{~atm}$ & 3 & 9 & 8143.26 & 100831.80 & 7462.78 & 3.9108 \\
\hline 70 & $1 \mathrm{~atm}$ & 3 & 10 & 7462.78 & 108294.60 & & 3.8729 \\
\hline 80 & $1 \mathrm{~atm}$ & 1 & 1 & 12623.84 & 12623.84 & 10190.29 & 4.1012 \\
\hline 80 & $1 \mathrm{~atm}$ & 1 & 2 & 10190.29 & 22814.13 & 8590.72 & 4.0082 \\
\hline
\end{tabular}


Table B1. Methanol Test Results

(continued)

\begin{tabular}{|c|c|}
\hline Study & $\begin{array}{c}\text { Test } \\
\text { Sequence }\end{array}$ \\
\hline 2 & 10 \\
\hline 2 & 10 \\
\hline 2 & 10 \\
\hline 2 & 10 \\
\hline 2 & 10 \\
\hline 2 & 10 \\
\hline 2 & 10 \\
\hline 2 & 10 \\
\hline 2 & 11 \\
\hline 2 & 11 \\
\hline 2 & 11 \\
\hline 2 & 11 \\
\hline 2 & 11 \\
\hline 2 & 11 \\
\hline 2 & 11 \\
\hline 2 & 11 \\
\hline 2 & 11 \\
\hline 2 & 11 \\
\hline 2 & 12 \\
\hline 2 & 12 \\
\hline 2 & 12 \\
\hline 2 & 12 \\
\hline 2 & 12 \\
\hline 2 & 12 \\
\hline 2 & 12 \\
\hline 2 & 12 \\
\hline 2 & 12 \\
\hline 2 & 12 \\
\hline 2 & 13 \\
\hline 2 & 13 \\
\hline 2 & 13 \\
\hline 2 & 13 \\
\hline 2 & 13 \\
\hline 2 & 13 \\
\hline 2 & 13 \\
\hline 2 & 13 \\
\hline 2 & 13 \\
\hline 2 & 13 \\
\hline 2 & 14 \\
\hline 2 & 14 \\
\hline 2 & 14 \\
\hline 2 & 14 \\
\hline 2 & 14 \\
\hline 2 & 14 \\
\hline 2 & 14 \\
\hline 2 & 14 \\
\hline 2 & 14 \\
\hline 2 & 14 \\
\hline 2 & 15 \\
\hline 2 & 15 \\
\hline 2 & 15 \\
\hline 2 & 15 \\
\hline 2 & 15 \\
\hline 2 & 15 \\
\hline 2 & 15 \\
\hline 2 & 15 \\
\hline 2 & 15 \\
\hline 2 & 15 \\
\hline 3 & 1 \\
\hline 3 & 1 \\
\hline 3 & 1 \\
\hline 3 & 1 \\
\hline 3 & 1 \\
\hline 3 & 1 \\
\hline 3 & 1 \\
\hline 3 & 1 \\
\hline
\end{tabular}

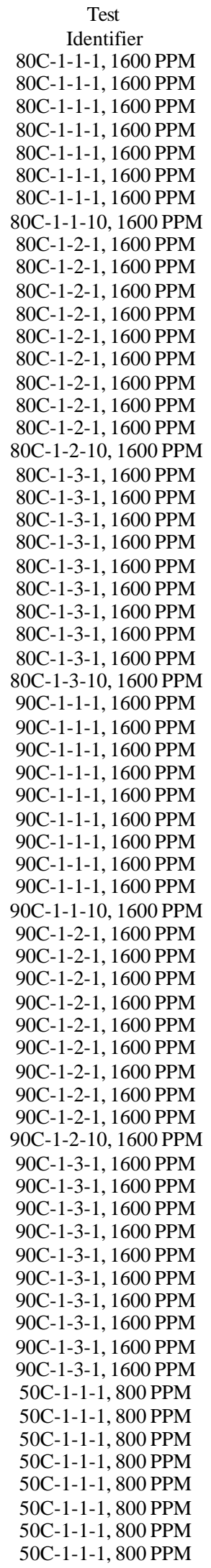

$\begin{array}{ccccc}\text { Temperature } & \text { Vial } & \text { Test } & \text { Sampling } & \text { A } \\ \left({ }^{\circ} \mathrm{C}\right) & \text { Pressusre } & \text { Run } & \text { Sequence } & \text { (peak area) } \\ 80 & 1 \mathrm{~atm} & 1 & 3 & 8590.72 \\ 80 & 1 \mathrm{~atm} & 1 & 4 & 7428.67 \\ 80 & 1 \mathrm{~atm} & 1 & 5 & 6817.07 \\ 80 & 1 \mathrm{~atm} & 1 & 6 & 6372.10 \\ 80 & 1 \mathrm{~atm} & 1 & 7 & 5662.79 \\ 80 & 1 \mathrm{~atm} & 1 & 8 & 4961.03 \\ 80 & 1 \mathrm{~atm} & 1 & 9 & 4353.18 \\ 80 & 1 \mathrm{~atm} & 1 & 10 & 3711.04 \\ 80 & 1 \mathrm{~atm} & 2 & 1 & 11056.58 \\ 80 & 1 \mathrm{~atm} & 2 & 2 & 9863.13 \\ 80 & 1 \mathrm{~atm} & 2 & 3 & 8711.02 \\ 80 & 1 \mathrm{~atm} & 2 & 4 & 7685.18 \\ 80 & 1 \mathrm{~atm} & 2 & 5 & 6681.07 \\ 80 & 1 \mathrm{~atm} & 2 & 6 & 5863.43 \\ 80 & 1 \mathrm{~atm} & 2 & 7 & 5185.42 \\ 80 & 1 \mathrm{~atm} & 2 & 8 & 4643.49 \\ 80 & 1 \mathrm{~atm} & 2 & 9 & 4020.23 \\ 80 & 1 \mathrm{~atm} & 2 & 10 & 3430.93 \\ 80 & 1 \mathrm{~atm} & 3 & 1 & 12412.37 \\ 80 & 1 \mathrm{~atm} & 3 & 2 & 10291.99 \\ 80 & 1 \mathrm{~atm} & 3 & 3 & 8718.68 \\ 80 & 1 \mathrm{~atm} & 3 & 4 & 7636.17 \\ 80 & 1 \mathrm{~atm} & 3 & 5 & 6798.13 \\ 80 & 1 \mathrm{~atm} & 3 & 6 & 6269.31 \\ 80 & 1 \mathrm{~atm} & 3 & 7 & 5657.41 \\ 80 & 1 \mathrm{~atm} & 3 & 8 & 4953.51 \\ 80 & 1 \mathrm{~atm} & 3 & 9 & 4332.92 \\ 80 & 1 \mathrm{~atm} & 3 & 10 & 3747.31 \\ 90 & 1 \mathrm{~atm} & 1 & 1 & 22761.29 \\ 90 & 1 \mathrm{~atm} & 1 & 2 & 17932.13 \\ 90 & 1 \mathrm{~atm} & 1 & 3 & 14294.05 \\ 90 & 1 \mathrm{~atm} & 1 & 4 & 11833.02 \\ 90 & 1 \mathrm{~atm} & 1 & 5 & 9850.72 \\ 90 & 1 \mathrm{~atm} & 1 & 6 & 8274.10 \\ 90 & 1 \mathrm{~atm} & 1 & 7 & 6675.24 \\ 90 & 1 \mathrm{~atm} & 1 & 8 & 5218.02 \\ 90 & 1 \mathrm{~atm} & 1 & 9 & 4039.65 \\ 90 & 1 \mathrm{~atm} & 3 & 9 & 4281.22 \\ 90 & 1 \mathrm{~atm} & 3 & 10 & 3298.25 \\ 50 & 1 \mathrm{~atm} & 1 & 1 & 5728.88 \\ 50 & 1 \mathrm{~atm} & 1 & 2 & 4726.69 \\ 50 & 1 \mathrm{~atm} & 1 & 3 & 4006.16 \\ 50 & 1 \mathrm{~atm} & 1 & 4 & 3812.10 \\ 90 & 1 \mathrm{~atm} & 1 & 5 & 3662.28 \\ 90 & 1 \mathrm{~atm} & 1 & 10 & 3034.96 \\ 90 & 1 \mathrm{~atm} & 3 & 5 & 10769.55 \\ 90 & 1 \mathrm{~atm} & 2 & 1 & 22104.26 \\ 90 & 1 \mathrm{~atm} & 2 & 2 & 17489.83 \\ 90 & 1 \mathrm{~atm} & 2 & 3 & 14111.05 \\ 90 & 1 \mathrm{~atm} & 2 & 4 & 11858.99 \\ 90 & 1 \mathrm{~atm} & 2 & 5 & 10054.42 \\ 90 & 1 \mathrm{~atm} & 2 & 6 & 8265.54 \\ 90 & 1 \mathrm{~atm} & 2 & 7 & 6661.87 \\ 90 & 1 \mathrm{~atm} & 2 & 8 & 5255.36 \\ 90 & 1 \mathrm{~atm} & 2 & 9 & 4088.46 \\ 90 & 1 \mathrm{~atm} & 2 & 10 & 3153.83 \\ 90 & 1 \mathrm{~atm} & 3 & 1 & 21545.09 \\ 90 & 1 \mathrm{~atm} & 3 & 2 & 17125.90 \\ & 1 & 7 & 3 & 3 \\ 90 & 1 & 8 & 3263.15\end{array}$

$\mathrm{y}$
31404.86
38833.52
45650.59
52022.69
57685.47
62646.50
66999.68
70710.72
11056.58

20919.70

29630.73

37315.91

43996.98

49860.40

55045.82

59689.30

63709.54

67140.47

12412.37

22704.36

31423.04

39059.21

45857.35

52126.66

57784.06

62737.57

67070.49

70817.80

22761.29

40693.42

54987.47

66820.49

76671.21

84945.31

91620.55

96838.56

100878.20

103913.20

22104.26

39594.09

53705.13

65564.13

75618.55

83884.09

90545.95

95801.32

99889.77

103043.60

21545.09

38670.99

53793.24

66186.33

76955.87

85837.58

92875.28

98399.95

102681.20

105979.40

5728.88

10455.57

14461.73

18273.83

21936.11

25464.15

28843.74

32106.89

$\begin{array}{cc}\mathrm{x} & \log (\mathrm{A}) \\ 7428.67 & 3.9340 \\ 6817.07 & 3.8709 \\ 6372.10 & 3.8336 \\ 5662.79 & 3.8043 \\ 4961.03 & 3.7530 \\ 4353.18 & 3.6956 \\ 3711.04 & 3.6388 \\ . & 3.5695 \\ 9863.13 & 4.0436 \\ 8711.02 & 3.9940 \\ 7685.18 & 3.9401 \\ 6681.07 & 3.8857 \\ 5863.43 & 3.8248 \\ 5185.42 & 3.7682 \\ 4643.49 & 3.7148 \\ 4020.23 & 3.6668 \\ 3430.93 & 3.6043 \\ . & 3.5354 \\ 10291.99 & 4.0939 \\ 8718.68 & 4.0125 \\ 7636.17 & 3.9405 \\ 6798.13 & 3.8829 \\ 6269.31 & 3.8324 \\ 5657.41 & 3.7972 \\ 4953.51 & 3.7526 \\ 4332.92 & 3.6949 \\ 3747.31 & 3.6368 \\ & 3.5737 \\ 17932.13 & 4.3572 \\ 14294.05 & 4.2536 \\ 11833.02 & 4.1552 \\ 9850.72 & 4.0731 \\ 8274.10 & 3.9935 \\ 6675.24 & 3.9177 \\ 5218.02 & 3.8245 \\ 4039.65 & 3.7175 \\ 3034.96 & 3.6063 \\ . & 3.4822 \\ 17489.83 & 4.3445 \\ 14111.05 & 4.2428 \\ 11858.99 & 4.1496 \\ 10054.42 & 4.0740 \\ 8265.54 & 4.0024 \\ 6661.87 & 3.9173 \\ 5255.36 & 3.8236 \\ 4088.46 & 3.7206 \\ 3153.83 & 3.6116 \\ . & 3.4988 \\ 17125.90 & 4.3333 \\ 15122.24 & 4.2337 \\ 12393.09 & 4.1796 \\ 10769.55 & 4.0932 \\ 8881.71 & 4.0322 \\ 7037.69 & 3.9485 \\ 5524.68 & 3.8474 \\ 4281.22 & 3.7423 \\ 3298.25 & 3.6316 \\ 4726.69 & 3.5183 \\ 3806.16 & 3.7581 \\ 3662.10 & 3.6027 \\ 3528.05 & 3.5812 \\ 3379.59 & 3.5638 \\ 3263.15 & 3.5475 \\ 315289 \\ & 3.5136 \\ & \end{array}$

$3152.35 \quad 3.5136$ 
Table B1. Methanol Test Results

(continued)

\begin{tabular}{|c|c|}
\hline Study & $\begin{array}{c}\text { Test } \\
\text { Sequence }\end{array}$ \\
\hline 3 & 1 \\
\hline 3 & 1 \\
\hline 3 & 2 \\
\hline 3 & 2 \\
\hline 3 & 2 \\
\hline 3 & 2 \\
\hline 3 & 2 \\
\hline 3 & 2 \\
\hline 3 & 2 \\
\hline 3 & 2 \\
\hline 3 & 2 \\
\hline 3 & 2 \\
\hline 3 & 3 \\
\hline 3 & 3 \\
\hline 3 & 3 \\
\hline 3 & 3 \\
\hline 3 & 3 \\
\hline 3 & 3 \\
\hline 3 & 3 \\
\hline 3 & 3 \\
\hline 3 & 3 \\
\hline 3 & 3 \\
\hline 3 & 4 \\
\hline 3 & 4 \\
\hline 3 & 4 \\
\hline 3 & 4 \\
\hline 3 & 4 \\
\hline 3 & 4 \\
\hline 3 & 4 \\
\hline 3 & 4 \\
\hline 3 & 4 \\
\hline 3 & 4 \\
\hline 3 & 5 \\
\hline 3 & 5 \\
\hline 3 & 5 \\
\hline 3 & 5 \\
\hline 3 & 5 \\
\hline 3 & 5 \\
\hline 3 & 5 \\
\hline 3 & 5 \\
\hline 3 & 5 \\
\hline 3 & 5 \\
\hline 3 & 6 \\
\hline 3 & 6 \\
\hline 3 & 6 \\
\hline 3 & 6 \\
\hline 3 & 6 \\
\hline 3 & 6 \\
\hline 3 & 6 \\
\hline 3 & 6 \\
\hline 3 & 6 \\
\hline 3 & 6 \\
\hline 3 & 7 \\
\hline 3 & 7 \\
\hline 3 & 7 \\
\hline 3 & 7 \\
\hline 3 & 7 \\
\hline 3 & 7 \\
\hline 3 & 7 \\
\hline 3 & 7 \\
\hline 3 & 7 \\
\hline 3 & 7 \\
\hline 3 & 8 \\
\hline 3 & 8 \\
\hline 3 & 8 \\
\hline 3 & 8 \\
\hline
\end{tabular}

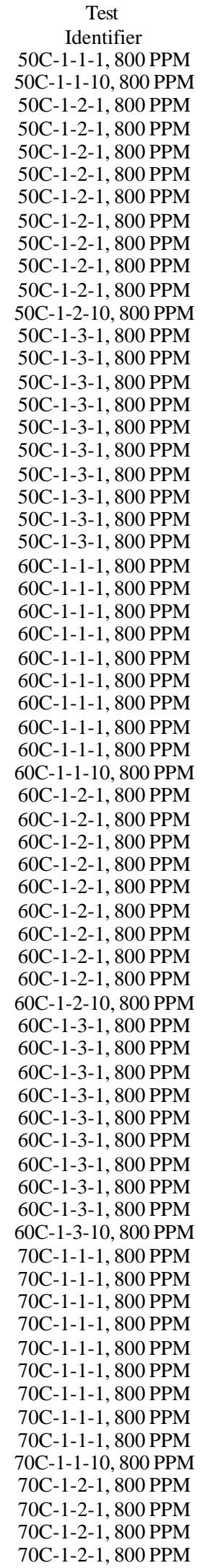

Temperature Vial Test Sampling

A $\left({ }^{\circ} \mathrm{C}\right) \quad$ Pressusre Run Sequence (peak area)

$\begin{array}{ccccc}50 & 1 \mathrm{~atm} & 1 & 9 & 3 \\ 50 & 1 \mathrm{~atm} & 1 & 10 & 2 \\ 50 & 1 \mathrm{~atm} & 2 & 1 & 608 \\ 50 & 1 \mathrm{~atm} & 2 & 2 & 488\end{array}$

3152.35
2990.91

$\begin{array}{ccc}\mathrm{y} & \mathrm{x} & \log (\mathrm{A}) \\ 35259.24 & 2990.91 & 3.4986 \\ 38250.15 & . & 3.4758 \\ 6081.62 & 4889.24 & 3.7840 \\ 10970.86 & 4018.14 & 3.6892 \\ 14989.00 & 3778.23 & 3.6040 \\ 18767.22 & 3599.73 & 3.5773 \\ 22366.96 & 3423.67 & 3.5563 \\ 25790.62 & 3305.11 & 3.5345 \\ 29095.73 & 3201.58 & 3.5192 \\ 32297.31 & 3040.98 & 3.5054 \\ 35338.29 & 2896.33 & 3.4830\end{array}$

$\begin{array}{lll}35338.29 & 2896.33 & 3.4830 \\ 38234.62 & & 3.4618\end{array}$

$\begin{array}{llllll}50 & 1 \mathrm{~atm} & 2 & 10 & 2896.33 & 38234.62\end{array}$

50

50

50

50

50

50

60

60

60

6081.62
4889.24

4018.14

3778.23

3599.73

3423.67

3305.11

3201.58

6166.27

5196.04

4199.73

11362.31

5196.04

3.7900

3938.29

3743.65

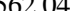

19500.33

3561.97

3295.56

3174.85

26805.95

30227.93

33523.49

36698.34

3055.87

39754.22

8366.54

6633.85

5787.19

5384.72

5152.61

15000.39
20787.58

26172.30

4897.60

4673.37

4478.72

4256.00

3958.26

7624.50

6413.28

5530.41

5240.91

4936.13

4724.96

4472.30

4278.61

4054.49

3796.29

7542.81

6362.82

5455.51

5204.64

4967.57

4728.77

4513.25

4292.08

4082.32

3857.19

9116.05

7652.50

6455.80

6199.30

5845.87

5513.65

5109.70

4687.96

4322.71

31324.91

$4199.73 \quad 3.7157$

$3938.29 \quad 3.6232$

$3743.65 \quad 3.5953$

$3561.97 \quad 3.5733$

$3421.98 \quad 3.5517$

$3295.56 \quad 3.5343$

$3174.85 \quad 3.5179$

$3055.87 \quad 3.5017$

$6633.85 \quad 3.9225$

$5787.19 \quad 3.8218$

$5384.72 \quad 3.7625$

36222.51

5152.61

3.7312

40895.88

4897.60

3.7120

45374.60

3.6900

49630.59

53588.85

7624.50

14037.79

19568.19

24809.11

29745.24

34470.19

38942.49

43221.10

47275.59

51071.88

7542.81
13905.63

19361.13

24565.77

4478.72

3.6696

$4256.00 \quad 3.6512$

$3958.26 \quad 3.6290$

$6413.28 \quad 3.8822$

$5530.41 \quad 3.8071$

$5240.91 \quad 3.7428$

$4936.13 \quad 3.7194$

$4724.96 \quad 3.6934$

$4472.30 \quad 3.6744$

$4278.61 \quad 3.6505$

$4054.49 \quad 3.6313$

$3796.29 \quad 3.6079$

$\begin{array}{ll}6362.82 & 3.5794 \\ 5455.51 & 3.8036\end{array}$

(9533.34

34262.11

38775.35

43067.44

47149.75

51006.95

9116.05

16768.55

23224.35

29423.64

35269.51

40783.16

45892.86

50580.82

3919.49

54903.53

9218.45

58823.02

$\begin{array}{llll}1 \mathrm{~atm} & 2 & 2 & 7629.00 \\ 1 \mathrm{~atm} & 2 & 3 & 6578.52\end{array}$

9218.45

16847.46
23425.97

5985.12

29411.09

$\begin{array}{ll}5455.51 & 3.8036\end{array}$

$5204.64 \quad 3.7368$

$4967.57 \quad 3.7164$

$4728.77 \quad 3.6961$

$4513.25 \quad 3.6747$

$4292.08 \quad 3.6545$

$4082.32 \quad 3.6327$

$3857.19 \quad 3.6109$

$7652.50-3.5863$

$6455.80 \quad 3.8838$

$6199.30 \quad 3.8100$

$5845.87 \quad 3.7923$

$5513.65 \quad 3.7668$

$5109.70 \quad 3.7414$

$4687.96 \quad 3.7084$

$4322.71 \quad 3.6710$

$3919.49 \quad 3.6358$

$\begin{array}{ll}7629.00 & 3.9647\end{array}$

$6578.52 \quad 3.8825$

$\begin{array}{ll}5985.12 & 3.8181 \\ 5598.52 & 3.7771\end{array}$ 
Table B1. Methanol Test Results

(continued)

\begin{tabular}{|c|c|}
\hline & Test \\
\hline Study & Sequence \\
\hline 3 & 8 \\
\hline 3 & 8 \\
\hline 3 & 8 \\
\hline 3 & 8 \\
\hline 3 & 8 \\
\hline 3 & 8 \\
\hline 3 & 9 \\
\hline 3 & 9 \\
\hline 3 & 9 \\
\hline 3 & 9 \\
\hline 3 & 9 \\
\hline 3 & 9 \\
\hline 3 & 9 \\
\hline 3 & 9 \\
\hline 3 & 9 \\
\hline 3 & 9 \\
\hline 3 & 10 \\
\hline 3 & 10 \\
\hline 3 & 10 \\
\hline 3 & 10 \\
\hline 3 & 10 \\
\hline 3 & 10 \\
\hline 3 & 10 \\
\hline 3 & 10 \\
\hline 3 & 10 \\
\hline 3 & 10 \\
\hline 3 & 11 \\
\hline 3 & 11 \\
\hline 3 & 11 \\
\hline 3 & 11 \\
\hline 3 & 11 \\
\hline 3 & 11 \\
\hline 3 & 11 \\
\hline 3 & 11 \\
\hline 3 & 11 \\
\hline 3 & 11 \\
\hline 3 & 12 \\
\hline 3 & 12 \\
\hline 3 & 12 \\
\hline 3 & 12 \\
\hline 3 & 12 \\
\hline 3 & 12 \\
\hline 3 & 12 \\
\hline 3 & 12 \\
\hline 3 & 12 \\
\hline 3 & 12 \\
\hline 3 & 13 \\
\hline 3 & 13 \\
\hline 3 & 13 \\
\hline 3 & 13 \\
\hline 3 & 13 \\
\hline 3 & 13 \\
\hline 3 & 13 \\
\hline 3 & 13 \\
\hline 3 & 13 \\
\hline 3 & 13 \\
\hline 3 & 14 \\
\hline 3 & 14 \\
\hline 3 & 14 \\
\hline 3 & 14 \\
\hline 3 & 14 \\
\hline 3 & 14 \\
\hline 3 & 14 \\
\hline 3 & 14 \\
\hline 3 & 14 \\
\hline 3 & 14 \\
\hline
\end{tabular}

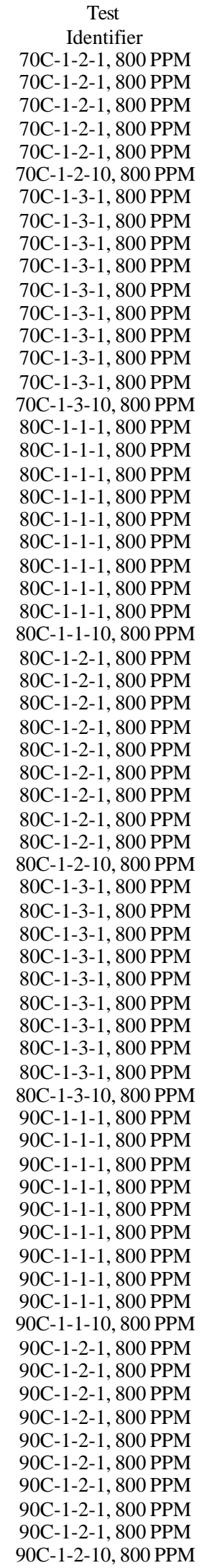

Temperature Vial Test Sampling

$\left({ }^{\circ} \mathrm{C}\right) \quad$ Pressusre Run Sequence (peak area)

$\begin{array}{lllll}70 & 1 \mathrm{~atm} & 2 & 5 & 5 \\ 70 & 1 \mathrm{~atm} & 2 & 6 & 5 \\ 70 & 1 \mathrm{~atm} & 2 & 7 & 5 \\ 70 & 1 \mathrm{~atm} & 2 & 8 & 467\end{array}$

70

70

70

70

70

70

70
70

70

70

70

70
80

80

80

80

80

80

80

80

80
80

80

80

80

80

80

80

80

80

80

80

80

80

80

80

80

80

80

80

80

90
90

90

90

90

90

90

90

90

90

90

90

90

90

90

90

90

90
90

90
90

$\begin{array}{ll}1 \mathrm{~atm} & 2 \\ 1 \mathrm{~atm} & 2\end{array}$

$1 \mathrm{~atm} \quad 3$

$1 \mathrm{~atm} \quad 3$

$1 \mathrm{~atm}$
$1 \mathrm{~atm}$

$1 \mathrm{~atm}$

$1 \mathrm{~atm} \quad 3$

$\begin{array}{ll}1 \mathrm{~atm} & 3 \\ 1 \mathrm{~atm} & 3\end{array}$

$1 \mathrm{~atm}$

$1 \mathrm{~atm}$

$1 \mathrm{~atm}$

$1 \mathrm{~atm}$

$1 \mathrm{~atm}$

$1 \mathrm{~atm}$

$1 \mathrm{~atm}$

$1 \mathrm{~atm}$

$1 \mathrm{~atm}$

$1 \mathrm{~atm}$

$1 \mathrm{~atm}$

$1 \mathrm{~atm}$

$1 \mathrm{~atm}$

$1 \mathrm{~atm}$

$1 \mathrm{~atm}$

$1 \mathrm{~atm}$

$1 \mathrm{~atm}$

$1 \mathrm{~atm}$

$1 \mathrm{~atm}$

$1 \mathrm{~atm}$

$1 \mathrm{~atm}$

$1 \mathrm{~atm}$

$1 \mathrm{~atm}$

$1 \mathrm{~atm}$

$1 \mathrm{~atm}$

$1 \mathrm{~atm}$

$1 \mathrm{~atm}$

$1 \mathrm{~atm}$

$1 \mathrm{~atm}$

$1 \mathrm{~atm} \quad 3$

$\begin{array}{ll}1 \mathrm{~atm} & 1 \\ 1 \mathrm{~atm} & 1\end{array}$

$1 \mathrm{~atm}$

$1 \mathrm{~atm}$

$1 \mathrm{~atm}$

$1 \mathrm{~atm}$

$1 \mathrm{~atm}$

$1 \mathrm{~atm}$

$1 \mathrm{~atm}$

$1 \mathrm{~atm}$

$1 \mathrm{~atm}$

$1 \mathrm{~atm}$

$1 \mathrm{~atm}$

$1 \mathrm{~atm}$

$1 \mathrm{~atm}$

$1 \mathrm{~atm}$

$1 \mathrm{~atm}$

$1 \mathrm{~atm}$

$1 \mathrm{~atm}$

$1 \mathrm{~atm}$
5598.52

5042.86

4672.34

4310.18

3926.75

8800.14

7641.65

6664.71

5952.73

5550.57

5301.87

5004.98

4653.12

4271.24

3894.42

12102.47

9703.64

8171.76

6911.41

6465.08

6008.92

5328.45

4673.99

4093.64

3491.12

11538.48

9988.24

8369.02

7640.55

7127.87

6416.89

5667.84

4951.84

4332.29

3695.23

12037.99

10022.74

8335.79

7390.74

6904.67

6318.65

5559.87

4872.31

4247.11

3654.17

14411.69

10354.32

8683.20

7279.36

6358.74

5173.02

4202.75

3303.03

2572.07

1972.51

14181.60

10203.62

8299.99

6654.82

5467.74

4532.66

3657.03

2837.97

2172.60

1623.67

$\mathrm{y}$
35009.61
40356.82
45399.68
50072.02
54382.19
58308.94
8800.14
16441.79
23106.50
29059.23
34609.80
39911.67
44916.65
49569.77
53841.01
57735.43
12102.47

21806.12

29977.87

36889.28

43354.36

49363.27

54691.72

59365.71

63459.35

66950.47

11538.48

21526.71

29895.73

37536.27

44664.14

51081.03

56748.87

61700.71

66033.00

69728.23

12037.99

22060.73

30396.52

37787.25

44691.92

51010.57

56570.44

61442.75

65689.86

69344.04

14411.69

24766.01

33449.20

40728.56

47087.29

52260.31

56463.06

59766.09

62338.16

64310.67

14181.60

24385.22

32685.20

39340.03

44807.76

49340.43

52997.46

55835.42

58008.02

59631.69

$\mathrm{x} \quad \log (\mathrm{A})$

$\begin{array}{ll}5347.21 & 3.7481 \\ 5042.86 & 3.7281\end{array}$

$4672.34 \quad 3.7027$

$4310.18 \quad 3.6695$

$3926.75 \quad 3.6345$

$7641.65-3.5940$

$6664.71 \quad 3.8832$

$5952.73 \quad 3.8238$

$\begin{array}{ll}5550.57 & 3.7747\end{array}$

$5301.87 \quad 3.7443$

$5004.98 \quad 3.7244$

$4653.12 \quad 3.6994$

$4271.24 \quad 3.6677$

$3894.42 \quad 3.6306$

$\begin{array}{ll} & 3.5904 \\ 9703.64 & 4.0829\end{array}$

$8171.76 \quad 3.9869$

$6911.41 \quad 3.9123$

$6465.08 \quad 3.8396$

$6008.92 \quad 3.8106$

$5328.45 \quad 3.7788$

$4673.99 \quad 3.7266$

$4093.64 \quad 3.6697$

$3491.12 \quad 3.6121$

$9988.24 \quad 4.0621$

$8369.02 \quad 3.9995$

$\begin{array}{ll}7640.55 & 3.9227\end{array}$

$7127.87 \quad 3.8831$

\begin{tabular}{l}
$6416.89 \quad 3.8530$ \\
\hline
\end{tabular}

$5667.84 \quad 3.8073$

$4951.84 \quad 3.7534$

$4332.29 \quad 3.6948$

$3695.23 \quad 3.6367$

$1002274 \quad 3.5676$

$8335.79 \quad 4.0010$

$\begin{array}{ll}7390.74 & 3.9209\end{array}$

$6904.67 \quad 3.8687$

$6318.65 \quad 3.8391$

$5559.87 \quad 3.8006$

$4872.31 \quad 3.7451$

$4247.11 \quad 3.6877$

$3654.17 \quad 3.6281$

$10354.32 \quad 4.1587$

$8683.20 \quad 4.0151$

$7279.36 \quad 3.9387$

$6358.74 \quad 3.8621$

$5173.02 \quad 3.8034$

$4202.75 \quad 3.7137$

$3303.03 \quad 3.6235$

$2572.07 \quad 3.5189$

$1972.51 \quad 3.4103$

$10203.62-4.1517$

$8299.99 \quad 4.0088$

$6654.82 \quad 3.9191$ 
Table B1. Methanol Test Results

(continued)

\begin{tabular}{|c|c|}
\hline & Test \\
\hline Study & Sequence \\
\hline 3 & 15 \\
\hline 3 & 15 \\
\hline 3 & 15 \\
\hline 3 & 15 \\
\hline 3 & 15 \\
\hline 3 & 15 \\
\hline 3 & 15 \\
\hline 3 & 15 \\
\hline 3 & 15 \\
\hline 3 & 15 \\
\hline 4 & 1 \\
\hline 4 & 1 \\
\hline 4 & 1 \\
\hline 4 & 1 \\
\hline 4 & 1 \\
\hline 4 & 1 \\
\hline 4 & 1 \\
\hline 4 & 1 \\
\hline 4 & 1 \\
\hline 4 & 1 \\
\hline 4 & 2 \\
\hline 4 & 2 \\
\hline 4 & 2 \\
\hline 4 & 2 \\
\hline 4 & 2 \\
\hline 4 & 2 \\
\hline 4 & 2 \\
\hline 4 & 2 \\
\hline 4 & 2 \\
\hline 4 & 2 \\
\hline 4 & 3 \\
\hline 4 & 3 \\
\hline 4 & 3 \\
\hline 4 & 3 \\
\hline 4 & 3 \\
\hline 4 & 3 \\
\hline 4 & 3 \\
\hline 4 & 3 \\
\hline 4 & 3 \\
\hline 4 & 3 \\
\hline 4 & 4 \\
\hline 4 & 4 \\
\hline 4 & 4 \\
\hline 4 & 4 \\
\hline 4 & 4 \\
\hline 4 & 4 \\
\hline 4 & 4 \\
\hline 4 & 4 \\
\hline 4 & 4 \\
\hline 4 & 4 \\
\hline 4 & 5 \\
\hline 4 & 5 \\
\hline 4 & 5 \\
\hline 4 & 5 \\
\hline 4 & 5 \\
\hline 4 & 5 \\
\hline 4 & 5 \\
\hline 4 & 5 \\
\hline 4 & 5 \\
\hline 4 & 5 \\
\hline 4 & 6 \\
\hline 4 & 6 \\
\hline 4 & 6 \\
\hline 4 & 6 \\
\hline 4 & 6 \\
\hline 4 & 6 \\
\hline
\end{tabular}

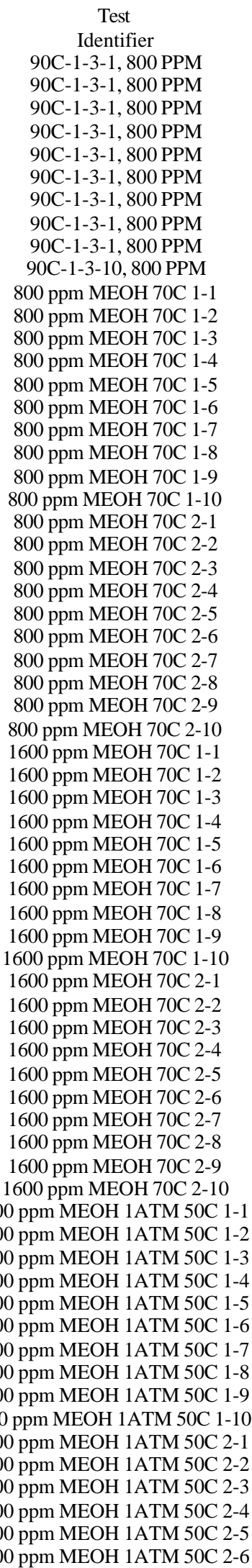

\begin{tabular}{|c|c|c|c|c|}
\hline Temperature & Vial & Test & Sampling & A \\
\hline$\left({ }^{\circ} \mathrm{C}\right)$ & Pressusre & Run & Sequence & (peak area) \\
\hline 90 & $1 \mathrm{~atm}$ & 3 & 1 & 13176.43 \\
\hline 90 & $1 \mathrm{~atm}$ & 3 & 2 & 10986.73 \\
\hline 90 & $1 \mathrm{~atm}$ & 3 & 3 & 9827.44 \\
\hline 90 & $1 \mathrm{~atm}$ & 3 & 4 & 8203.01 \\
\hline 90 & $1 \mathrm{~atm}$ & 3 & 5 & 6645.00 \\
\hline 90 & $1 \mathrm{~atm}$ & 3 & 6 & 5307.12 \\
\hline 90 & $1 \mathrm{~atm}$ & 3 & 7 & 4192.14 \\
\hline 90 & $1 \mathrm{~atm}$ & 3 & 8 & 3293.64 \\
\hline 90 & $1 \mathrm{~atm}$ & 3 & 9 & 2553.87 \\
\hline 90 & $1 \mathrm{~atm}$ & 3 & 10 & 1939.49 \\
\hline 70 & $1 \mathrm{~atm}$ & 1 & 1 & 7758.64 \\
\hline 70 & $1 \mathrm{~atm}$ & 1 & 2 & 8095.53 \\
\hline 70 & $1 \mathrm{~atm}$ & 1 & 3 & 7677.50 \\
\hline 70 & $1 \mathrm{~atm}$ & 1 & 4 & 7294.18 \\
\hline 70 & $1 \mathrm{~atm}$ & 1 & 5 & 6827.97 \\
\hline 70 & $1 \mathrm{~atm}$ & 1 & 6 & 6378.22 \\
\hline 70 & $1 \mathrm{~atm}$ & 1 & 7 & 5929.58 \\
\hline 70 & $1 \mathrm{~atm}$ & 1 & 8 & 5536.61 \\
\hline 70 & $1 \mathrm{~atm}$ & 1 & 9 & 5168.10 \\
\hline 70 & $1 \mathrm{~atm}$ & 1 & 10 & 4815.16 \\
\hline 70 & $1 \mathrm{~atm}$ & 2 & 1 & 7968.98 \\
\hline 70 & $1 \mathrm{~atm}$ & 2 & 2 & 7733.84 \\
\hline 70 & $1 \mathrm{~atm}$ & 2 & 3 & 7455.15 \\
\hline 70 & $1 \mathrm{~atm}$ & 2 & 4 & 7063.29 \\
\hline 70 & $1 \mathrm{~atm}$ & 2 & 5 & 6595.27 \\
\hline 70 & $1 \mathrm{~atm}$ & 2 & 6 & 6198.11 \\
\hline 70 & $1 \mathrm{~atm}$ & 2 & 7 & 5843.49 \\
\hline 70 & $1 \mathrm{~atm}$ & 2 & 8 & 5496.97 \\
\hline 70 & $1 \mathrm{~atm}$ & 2 & 9 & 5110.93 \\
\hline 70 & $1 \mathrm{~atm}$ & 2 & 10 & 4730.85 \\
\hline 70 & $1 \mathrm{~atm}$ & 1 & 1 & 12998.90 \\
\hline 70 & $1 \mathrm{~atm}$ & 1 & 2 & 12175.33 \\
\hline 70 & $1 \mathrm{~atm}$ & 1 & 3 & 11934.03 \\
\hline 70 & $1 \mathrm{~atm}$ & 1 & 4 & 11417.57 \\
\hline 70 & $1 \mathrm{~atm}$ & 1 & 5 & 10812.09 \\
\hline 70 & $1 \mathrm{~atm}$ & 1 & 6 & 10208.69 \\
\hline 70 & $1 \mathrm{~atm}$ & 1 & 7 & 9584.39 \\
\hline 70 & $1 \mathrm{~atm}$ & 1 & 8 & 8991.87 \\
\hline 70 & $1 \mathrm{~atm}$ & 1 & 9 & 8433.99 \\
\hline 70 & $1 \mathrm{~atm}$ & 1 & 10 & 7879.63 \\
\hline 70 & $1 \mathrm{~atm}$ & 2 & 1 & 13891.17 \\
\hline 70 & $1 \mathrm{~atm}$ & 2 & 2 & 12537.30 \\
\hline 70 & $1 \mathrm{~atm}$ & 2 & 3 & 12309.27 \\
\hline 70 & $1 \mathrm{~atm}$ & 2 & 4 & 11606.89 \\
\hline 70 & $1 \mathrm{~atm}$ & 2 & 5 & 11017.47 \\
\hline 70 & $1 \mathrm{~atm}$ & 2 & 6 & 10324.18 \\
\hline 70 & $1 \mathrm{~atm}$ & 2 & 7 & 9689.40 \\
\hline 70 & $1 \mathrm{~atm}$ & 2 & 8 & 8995.52 \\
\hline 70 & $1 \mathrm{~atm}$ & 2 & 9 & 8455.53 \\
\hline 70 & $1 \mathrm{~atm}$ & 2 & 10 & 7886.97 \\
\hline 50 & $1 \mathrm{~atm}$ & 1 & 1 & 53364.28 \\
\hline 50 & $1 \mathrm{~atm}$ & 1 & 2 & 52791.73 \\
\hline 50 & $1 \mathrm{~atm}$ & 1 & 3 & 51435.20 \\
\hline 50 & $1 \mathrm{~atm}$ & 1 & 4 & 49654.22 \\
\hline 50 & $1 \mathrm{~atm}$ & 1 & 5 & 48097.08 \\
\hline 50 & $1 \mathrm{~atm}$ & 1 & 6 & 46738.55 \\
\hline 50 & $1 \mathrm{~atm}$ & 1 & 7 & 45238.79 \\
\hline 50 & $1 \mathrm{~atm}$ & 1 & 8 & 43863.28 \\
\hline 50 & $1 \mathrm{~atm}$ & 1 & 9 & 42724.62 \\
\hline 50 & $1 \mathrm{~atm}$ & 1 & 10 & 41427.21 \\
\hline 50 & $1 \mathrm{~atm}$ & 2 & 1 & 7893.66 \\
\hline 50 & $1 \mathrm{~atm}$ & 2 & 2 & 6302.22 \\
\hline 50 & $1 \mathrm{~atm}$ & 2 & 3 & 5876.38 \\
\hline 50 & $1 \mathrm{~atm}$ & 2 & 4 & 5566.88 \\
\hline 50 & $1 \mathrm{~atm}$ & 2 & 5 & 5325.62 \\
\hline 50 & $1 \mathrm{~atm}$ & 2 & 6 & 5146.83 \\
\hline
\end{tabular}

$\mathrm{y}$
13176.43
24163.15
33990.59
42193.61
48838.61
54145.73
58337.87
61631.51
64185.37
66124.86
7758.64
15854.17
23531.67
30825.85
37653.82
44032.04
49961.62
55498.23
60666.33
65481.49
7968.98
15702.82

$\begin{array}{ll}\mathrm{x} & \log (\mathrm{A})\end{array}$

$\begin{array}{ll}10986.73 & 4.1198 \\ 9827.44 & 4.0409\end{array}$

$8203.01 \quad 3.9924$

$6645.00 \quad 3.9140$

$5307.12 \quad 3.8225$

$\begin{array}{ll}4192.14 & 3.7249\end{array}$

$3293.64 \quad 3.6224$

$2553.87 \quad 3.5177$

$1939.49 \quad 3.4072$

$\begin{array}{ll}. & 3.2877 \\ 8095.53 & 3.8898\end{array}$

$7677.50 \quad 3.9082$

$7294.18 \quad 3.8852$

$6827.97 \quad 3.8630$

$6378.22 \quad 3.8343$

$5929.58 \quad 3.8047$

$5536.61 \quad 3.7730$

$5168.10 \quad 3.7432$

$4815.16 \quad 3.7133$

$\begin{array}{ll}7733.84 & 3.9014\end{array}$

$\begin{array}{lll}15702.82 & 7455.15 & 3.8884\end{array}$

$\begin{array}{lll}23157.97 & 7063.29 & 3.8725\end{array}$

$\begin{array}{lll}30221.26 & 6595.27 & 3.8490\end{array}$

$\begin{array}{lll}36816.52 & 6198.11 & 3.8192\end{array}$

$43014.63 \quad 5843.49 \quad 3.7923$

$\begin{array}{lll}48858.12 & 5496.97 & 3.7667\end{array}$

$\begin{array}{lll}54355.09 & 5110.93 & 3.7401\end{array}$

$\begin{array}{lll}59466.02 & 4730.85 & 3.7085\end{array}$

64196.87

12998.90

25174.23

37108.26

48525.83

59337.92

69546.62

79131.01

88122.87

96556.86

104436.50

13891.17

26428.47

38737.75

50344.63

61362.10

71686.28

81375.69

90371.21

98826.74

106713.70

53364.28

106156.00

157591.20

207245.40

255342.50

302081.10

347319.80

391183.10

433907.70

475334.90

7893.66

14195.88

20072.26

25639.14

30964.75

12175.33

3.6749

$11934.03-4.0855$

$11417.57 \quad 4.0768$

$10812.09 \quad 4.0576$

$10208.69 \quad 4.0339$

$9584.39 \quad 4.0090$

$8991.87 \quad 3.9816$

$8433.99 \quad 3.9539$

$7879.63 \quad 3.9260$

$12537.30-\quad 3.8965$

$12309.27 \quad 4.0982$

$11606.89 \quad 4.0902$

$11017.47 \quad 4.0647$

$10324.18 \quad 4.0421$

$9689.40 \quad 4.0139$

$8995.52 \quad 3.9863$

$8455.53 \quad 3.9540$

$7886.97 \quad 3.9271$

52791.73

$51435.20 \quad 4.7226$

$49654.22 \quad 4.7113$

$48097.08 \quad 4.6960$

$46738.55 \quad 4.6821$

$45238.79 \quad 4.6697$

$43863.28 \quad 4.6555$

$42724.62 \quad 4.6421$

$41427.21 \quad 4.6307$

$\begin{array}{ll}6302.22 & 3.8973 \\ 5876.38 & 3.7995\end{array}$

$5876.38 \quad 3.7995$

$5566.88 \quad 3.7691$

$5325.62 \quad 3.7456$

$\begin{array}{lll}36111.58 & 4986.92 & 3.7115\end{array}$ 
Table B1. Methanol Test Results

(continued)

\begin{tabular}{|c|c|}
\hline & Test \\
\hline Study & Sequence \\
\hline 4 & 6 \\
\hline 4 & 6 \\
\hline 4 & 6 \\
\hline 4 & 6 \\
\hline 4 & 7 \\
\hline 4 & 7 \\
\hline 4 & 7 \\
\hline 4 & 7 \\
\hline 4 & 7 \\
\hline 4 & 7 \\
\hline 4 & 7 \\
\hline 4 & 7 \\
\hline 4 & 7 \\
\hline 4 & 7 \\
\hline 4 & 8 \\
\hline 4 & 8 \\
\hline 4 & 8 \\
\hline 4 & 8 \\
\hline 4 & 8 \\
\hline 4 & 8 \\
\hline 4 & 8 \\
\hline 4 & 8 \\
\hline 4 & 8 \\
\hline 4 & 8 \\
\hline 4 & 9 \\
\hline 4 & 9 \\
\hline 4 & 9 \\
\hline 4 & 9 \\
\hline 4 & 9 \\
\hline 4 & 9 \\
\hline 4 & 9 \\
\hline 4 & 9 \\
\hline 4 & 9 \\
\hline 4 & 9 \\
\hline 4 & 10 \\
\hline 4 & 10 \\
\hline 4 & 10 \\
\hline 4 & 10 \\
\hline 4 & 10 \\
\hline 4 & 10 \\
\hline 4 & 10 \\
\hline 4 & 10 \\
\hline 4 & 10 \\
\hline 4 & 10 \\
\hline 4 & 11 \\
\hline 4 & 11 \\
\hline 4 & 11 \\
\hline 4 & 11 \\
\hline 4 & 11 \\
\hline 4 & 11 \\
\hline 4 & 11 \\
\hline 4 & 11 \\
\hline 4 & 11 \\
\hline 4 & 11 \\
\hline 4 & 12 \\
\hline 4 & 12 \\
\hline 4 & 12 \\
\hline 4 & 12 \\
\hline 4 & 12 \\
\hline 4 & 12 \\
\hline 4 & 12 \\
\hline 4 & 12 \\
\hline 4 & 12 \\
\hline 4 & 12 \\
\hline 4 & 13 \\
\hline 4 & 13 \\
\hline
\end{tabular}

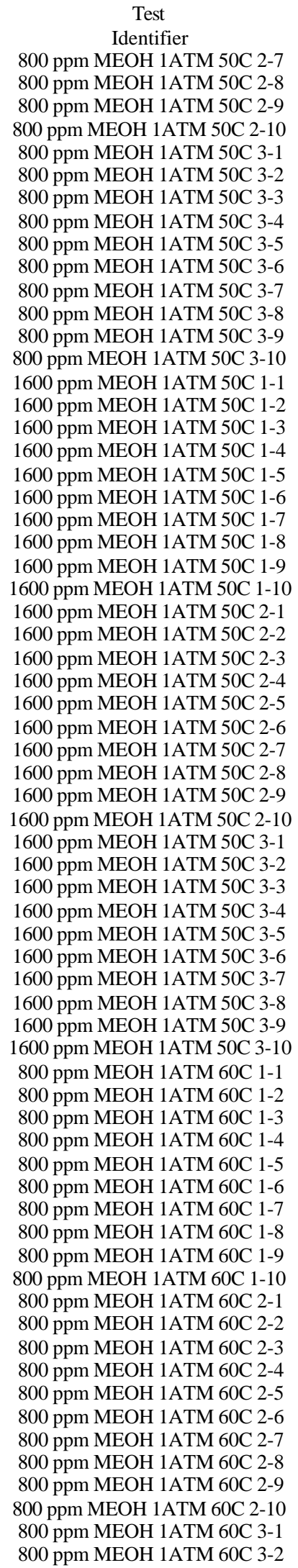

\begin{tabular}{|c|c|c|c|c|}
\hline Temperature & Vial & Test & Sampling & A \\
\hline & Pressusre & Run & Sequence & (peak area \\
\hline 50 & $1 \mathrm{~atm}$ & 2 & 7 & 4986.92 \\
\hline 50 & $1 \mathrm{~atm}$ & 2 & 8 & 4833.04 \\
\hline 50 & $1 \mathrm{~atm}$ & 2 & 9 & 4964.67 \\
\hline 50 & $1 \mathrm{~atm}$ & 2 & 10 & 4833.93 \\
\hline 50 & $1 \mathrm{~atm}$ & 3 & 1 & 7597.01 \\
\hline 50 & $1 \mathrm{~atm}$ & 3 & 2 & 6396.23 \\
\hline 50 & $1 \mathrm{~atm}$ & 3 & 3 & 5609.99 \\
\hline 50 & $1 \mathrm{~atm}$ & 3 & 4 & 5257.33 \\
\hline 50 & $1 \mathrm{~atm}$ & 3 & 5 & 5017.96 \\
\hline 50 & $1 \mathrm{~atm}$ & 3 & 6 & 4843.09 \\
\hline 50 & $1 \mathrm{~atm}$ & 3 & 7 & 4645.25 \\
\hline 50 & $1 \mathrm{~atm}$ & 3 & 8 & 4521.94 \\
\hline 50 & $1 \mathrm{~atm}$ & 3 & 9 & 4386.65 \\
\hline 50 & $1 \mathrm{~atm}$ & 3 & 10 & 4189.31 \\
\hline 50 & $1 \mathrm{~atm}$ & 1 & 1 & 10163.37 \\
\hline 50 & $1 \mathrm{~atm}$ & 1 & 2 & 8212.66 \\
\hline 50 & $1 \mathrm{~atm}$ & 1 & 3 & 7836.51 \\
\hline 50 & $1 \mathrm{~atm}$ & 1 & 4 & 7568.10 \\
\hline 50 & $1 \mathrm{~atm}$ & 1 & 5 & 7307.14 \\
\hline 50 & $1 \mathrm{~atm}$ & 1 & 6 & 7099.57 \\
\hline 50 & $1 \mathrm{~atm}$ & 1 & 7 & 6890.75 \\
\hline 50 & $1 \mathrm{~atm}$ & 1 & 8 & 6706.88 \\
\hline 50 & $1 \mathrm{~atm}$ & 1 & 9 & 6482.76 \\
\hline 50 & $1 \mathrm{~atm}$ & 1 & 10 & 6204.09 \\
\hline 50 & $1 \mathrm{~atm}$ & 2 & 1 & 10222.54 \\
\hline 50 & $1 \mathrm{~atm}$ & 2 & 2 & 8322.90 \\
\hline 50 & $1 \mathrm{~atm}$ & 2 & 3 & 7691.36 \\
\hline 50 & $1 \mathrm{~atm}$ & 2 & 4 & 7518.70 \\
\hline 50 & $1 \mathrm{~atm}$ & 2 & 5 & 7255.51 \\
\hline 50 & $1 \mathrm{~atm}$ & 2 & 6 & 7024.35 \\
\hline 50 & $1 \mathrm{~atm}$ & 2 & 7 & 6809.04 \\
\hline 50 & $1 \mathrm{~atm}$ & 2 & 8 & 6604.87 \\
\hline 50 & $1 \mathrm{~atm}$ & 2 & 9 & 6388.58 \\
\hline 50 & $1 \mathrm{~atm}$ & 2 & 10 & 6153.28 \\
\hline 50 & $1 \mathrm{~atm}$ & 3 & 1 & 10659.04 \\
\hline 50 & $1 \mathrm{~atm}$ & 3 & 2 & 8552.97 \\
\hline 50 & $1 \mathrm{~atm}$ & 3 & 3 & 7683.28 \\
\hline 50 & $1 \mathrm{~atm}$ & 3 & 4 & 7367.68 \\
\hline 50 & $1 \mathrm{~atm}$ & 3 & 5 & 7075.52 \\
\hline 50 & $1 \mathrm{~atm}$ & 3 & 6 & 6861.58 \\
\hline 50 & $1 \mathrm{~atm}$ & 3 & 7 & 6749.72 \\
\hline 50 & $1 \mathrm{~atm}$ & 3 & 8 & 6533.07 \\
\hline 50 & $1 \mathrm{~atm}$ & 3 & 9 & 6313.58 \\
\hline 50 & $1 \mathrm{~atm}$ & 3 & 10 & 6129.32 \\
\hline 60 & $1 \mathrm{~atm}$ & 1 & 1 & 9220.88 \\
\hline 60 & $1 \mathrm{~atm}$ & 1 & 2 & 7952.08 \\
\hline 60 & $1 \mathrm{~atm}$ & 1 & 3 & 7135.37 \\
\hline 60 & $1 \mathrm{~atm}$ & 1 & 4 & 7016.00 \\
\hline 60 & $1 \mathrm{~atm}$ & 1 & 5 & 6622.65 \\
\hline 60 & $1 \mathrm{~atm}$ & 1 & 6 & 6296.31 \\
\hline 60 & $1 \mathrm{~atm}$ & 1 & 7 & 5982.77 \\
\hline 60 & $1 \mathrm{~atm}$ & 1 & 8 & 5736.06 \\
\hline 60 & $1 \mathrm{~atm}$ & 1 & 9 & 5491.33 \\
\hline 60 & $1 \mathrm{~atm}$ & 1 & 10 & 5257.24 \\
\hline 60 & $1 \mathrm{~atm}$ & 2 & 1 & 9657.61 \\
\hline 60 & $1 \mathrm{~atm}$ & 2 & 2 & 8223.15 \\
\hline 60 & $1 \mathrm{~atm}$ & 2 & 3 & 7126.30 \\
\hline 60 & $1 \mathrm{~atm}$ & 2 & 4 & 6835.13 \\
\hline 60 & $1 \mathrm{~atm}$ & 2 & 5 & 6558.25 \\
\hline 60 & $1 \mathrm{~atm}$ & 2 & 6 & 6296.73 \\
\hline 60 & $1 \mathrm{~atm}$ & 2 & 7 & 6011.63 \\
\hline 60 & $1 \mathrm{~atm}$ & 2 & 8 & 5763.26 \\
\hline 60 & $1 \mathrm{~atm}$ & 2 & 9 & 5501.69 \\
\hline 60 & $1 \mathrm{~atm}$ & 2 & 10 & 5154.60 \\
\hline 60 & $1 \mathrm{~atm}$ & 3 & 1 & 8661.65 \\
\hline 60 & $1 \mathrm{~atm}$ & 3 & 2 & 7575.23 \\
\hline
\end{tabular}

$y$
41098.51
45931.54
50896.21
55730.14
7597.01
13993.25
19603.23
24860.56
29878.52
34721.61
39366.85
43888.79
48275.44
52464.75
10163.37

18376.03

26212.53

33780.63

41087.77

48187.34

55078.09

61784.97

68267.73

74471.82

10222.54

18545.44

26236.79

33755.49

41011.00

48035.35

54844.39

61449.26

67837.84

73991.12

10659.04

19212.01

26895.28

34262.96

41338.48

48200.05

54949.77

61482.84

67796.42

73925.74

9220.88

17172.95

24308.32

31324.32

37946.97

44243.28

50226.06

55962.12

61453.45

66710.69

9657.61

17880.76

25007.06

31842.19

38400.44

44697.17

50708.80

56472.06

61973.75

67128.35

8661.65

16236.88

$\begin{array}{cc}\mathrm{x} & \log (\mathrm{A}) \\ 4833.04 & 3.6978 \\ 4964.67 & 3.6842 \\ 4833.93 & 3.6959 \\ . & 3.6843 \\ 6396.23 & 3.8806 \\ 5609.99 & 3.8059 \\ 5257.33 & 3.7490 \\ 5017.96 & 3.7208 \\ 4843.09 & 3.7005 \\ 4645.25 & 3.6851 \\ 4521.94 & 3.6670 \\ 4386.65 & 3.6553 \\ 4189.31 & 3.6421 \\ & 3.6221 \\ 8212.66 & 4.0070 \\ 7836.51 & 3.9145 \\ 7568.10 & 3.8941 \\ 7307.14 & 3.8790 \\ 7099.57 & 3.8637 \\ 6890.75 & 3.8512 \\ 6706.88 & 3.8383 \\ 6482.76 & 3.8265 \\ 6204.09 & 3.8118 \\ & 3.7927 \\ 8322.90 & 4.0096 \\ 7691.36 & 3.9203 \\ 7518.70 & 3.8860 \\ 7255.51 & 3.8761 \\ 7024.35 & 3.8607 \\ 6809.04 & 3.8466 \\ 6604.87 & 3.8331 \\ 6388.58 & 3.8199 \\ 6153.28 & 3.8054 \\ . & 3.7891 \\ 8552.97 & 4.0277 \\ 7683.28 & 3.9321 \\ 7367.68 & 3.8855 \\ 7075.52 & 3.8673 \\ 6861.58 & 3.8498 \\ 6749.72 & 3.8364 \\ 6533.07 & 3.8293 \\ 6313.58 & 3.8151 \\ 6129.32 & 3.8003 \\ . & 3.7874 \\ 7952.08 & 3.9648 \\ 7135.37 & 3.9005 \\ 7016.00 & 3.8534 \\ 6622.65 & 3.8461 \\ 6296.31 & 3.8210 \\ 5982.77 & 3.7991 \\ 5736.06 & 3.7769 \\ 5491.33 & 3.7586 \\ 5257.24 & 3.7397 \\ . & 3.7208 \\ 8223.15 & 3.9849 \\ 7126.30 & 3.9150 \\ 6835.13 & 3.8529 \\ 6558.25 & 3.8347 \\ 6296.73 & 3.8168 \\ 6011.63 & 3.7991 \\ 5763.26 & 3.7790 \\ 5501.69 & 3.7607 \\ 5154.60 & 3.7405 \\ . & 3.7122 \\ 7575.23 & 3.9376 \\ 6902.68 & 3.8794 \\ & \end{array}$

$6902.68 \quad 3.8794$ 
Table B1. Methanol Test Results

(continued)

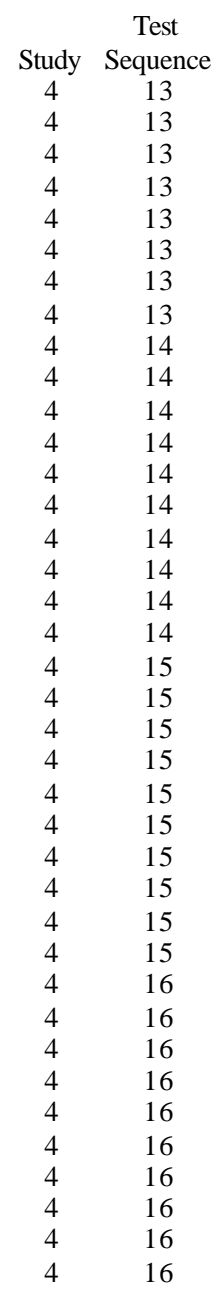

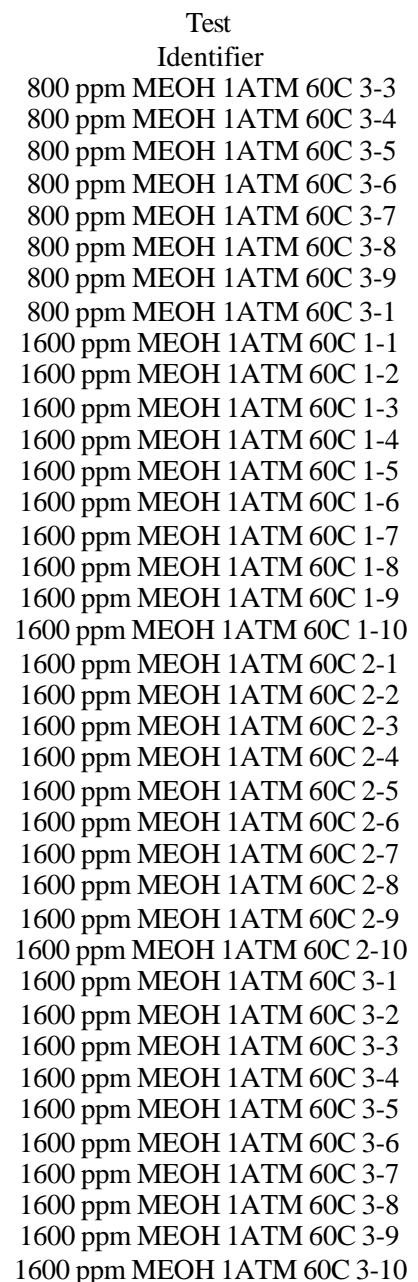

$\begin{array}{ccccc}\begin{array}{c}\text { Temperature } \\ \left({ }^{\circ} \mathrm{C}\right)\end{array} & \begin{array}{c}\text { Vial } \\ \text { Pressusre }\end{array} & \begin{array}{c}\text { Test } \\ \text { Run }\end{array} & \begin{array}{c}\text { Sampling } \\ \text { Sequence }\end{array} & \begin{array}{c}\text { A } \\ \text { (peak area) }\end{array} \\ 60 & 1 \mathrm{~atm} & 3 & 3 & 6902.68 \\ 60 & 1 \mathrm{~atm} & 3 & 4 & 6760.47 \\ 60 & 1 \mathrm{~atm} & 3 & 5 & 6427.27 \\ 60 & 1 \mathrm{~atm} & 3 & 6 & 6132.79 \\ 60 & 1 \mathrm{~atm} & 3 & 7 & 5851.34 \\ 60 & 1 \mathrm{~atm} & 3 & 8 & 5669.88 \\ 60 & 1 \mathrm{~atm} & 3 & 9 & 5408.27 \\ 60 & 1 \mathrm{~atm} & 3 & 10 & 5135.80 \\ 60 & 1 \mathrm{~atm} & 1 & 1 & 14051.44 \\ 60 & 1 \mathrm{~atm} & 1 & 2 & 12466.06 \\ 60 & 1 \mathrm{~atm} & 1 & 3 & 10991.72 \\ 60 & 1 \mathrm{~atm} & 1 & 4 & 10599.05 \\ 60 & 1 \mathrm{~atm} & 1 & 5 & 10094.39 \\ 60 & 1 \mathrm{~atm} & 1 & 6 & 10438.04 \\ 60 & 1 \mathrm{~atm} & 1 & 7 & 9863.13 \\ 60 & 1 \mathrm{~atm} & 1 & 8 & 9337.67 \\ 60 & 1 \mathrm{~atm} & 1 & 9 & 8850.60 \\ 60 & 1 \mathrm{~atm} & 1 & 10 & 8222.66 \\ 60 & 1 \mathrm{~atm} & 2 & 1 & 13956.97 \\ 60 & 1 \mathrm{~atm} & 2 & 2 & 12239.42 \\ 60 & 1 \mathrm{~atm} & 2 & 3 & 10733.53 \\ 60 & 1 \mathrm{~atm} & 2 & 4 & 10850.34 \\ 60 & 1 \mathrm{~atm} & 2 & 5 & 10154.16 \\ 60 & 1 \mathrm{~atm} & 2 & 6 & 9477.46 \\ 60 & 1 \mathrm{~atm} & 2 & 7 & 8960.37 \\ 60 & 1 \mathrm{~atm} & 2 & 8 & 8829.63 \\ 60 & 1 \mathrm{~atm} & 2 & 9 & 8037.51 \\ 60 & 1 \mathrm{~atm} & 2 & 10 & 7258.41 \\ 60 & 1 \mathrm{~atm} & 3 & 1 & 11319.76 \\ 60 & 1 \mathrm{~atm} & 3 & 2 & 8730.57 \\ 60 & 1 \mathrm{~atm} & 3 & 3 & 6735.21 \\ 60 & 1 \mathrm{~atm} & 3 & 4 & 5288.18 \\ 60 & 1 \mathrm{~atm} & 3 & 5 & 4273.71 \\ 60 & 1 \mathrm{~atm} & 3 & 6 & 3401.54 \\ 60 & 1 \mathrm{~atm} & 3 & 7 & 2733.39 \\ 60 & 1 \mathrm{~atm} & 3 & 8 & 2179.00 \\ 60 & 1 \mathrm{~atm} & 3 & 9 & 1749.85 \\ 60 & 1 \mathrm{~atm} & 3 & 10 & 1409.20\end{array}$

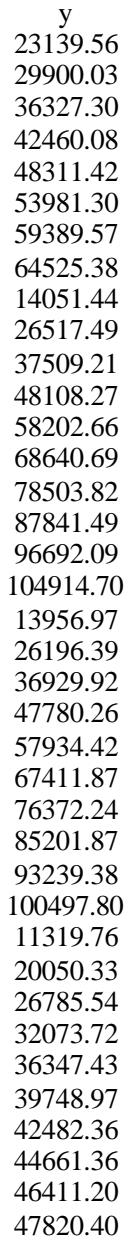

$x$

6760.47

6427.27

6132.79

5851.34

5669.88

5408.27

5135.80

12466.06

10991.72

10599.05

10094.39

10438.04

9863.13

9337.67

8850.60

8222.66

12239.4

10733.53

10850.34

10154.16

9477.46

8960.37

8829.63

8037.51

7258.41

8730.57

6735.21

5288.18

4273.71

3401.54

2733.39

2179.00

1749.85

1409.20 $\log (\mathrm{A})$

3.8390

3.8300

3.8080

3.7877

3.7673

3.7536

3.7331

3.7106

4.1477

4.0957

4.0411

4.0253

4.0041

4.0186

3.9940

3.9702

3.9470

3.9150

4.1448

4.0878

4.0307

4.0354

4.0066

3.9767

3.9523

3.9459

3.9051

3.8608

4.0538

3.9410

3.8284

3.7233

3.6308

3.5317

3.4367

3.3383

3.2430
3.1490 
Table B2. Ammonia Test Results

\begin{tabular}{|c|c|}
\hline Study & $\begin{array}{c}\text { Test } \\
\text { Sequence }\end{array}$ \\
\hline 1 & 1 \\
\hline 1 & 1 \\
\hline 1 & 1 \\
\hline 1 & 1 \\
\hline 1 & 1 \\
\hline 1 & 1 \\
\hline 1 & 1 \\
\hline 1 & 1 \\
\hline 1 & 1 \\
\hline 1 & 1 \\
\hline 1 & 2 \\
\hline 1 & 2 \\
\hline 1 & 2 \\
\hline 1 & 2 \\
\hline 1 & 2 \\
\hline 1 & 2 \\
\hline 1 & 2 \\
\hline 1 & 2 \\
\hline 1 & 2 \\
\hline 1 & 2 \\
\hline 1 & 3 \\
\hline 1 & 3 \\
\hline 1 & 3 \\
\hline 1 & 3 \\
\hline 1 & 3 \\
\hline 1 & 3 \\
\hline 1 & 3 \\
\hline 1 & 3 \\
\hline 1 & 3 \\
\hline 1 & 3 \\
\hline 1 & 4 \\
\hline 1 & 4 \\
\hline 1 & 4 \\
\hline 1 & 4 \\
\hline 1 & 4 \\
\hline 1 & 4 \\
\hline 1 & 4 \\
\hline 1 & 4 \\
\hline 1 & 4 \\
\hline 1 & 4 \\
\hline 1 & 5 \\
\hline 1 & 5 \\
\hline 1 & 5 \\
\hline 1 & 5 \\
\hline 1 & 5 \\
\hline 1 & 5 \\
\hline 1 & 5 \\
\hline 1 & 5 \\
\hline 1 & 5 \\
\hline 1 & 5 \\
\hline 1 & 6 \\
\hline 1 & 6 \\
\hline 1 & 6 \\
\hline 1 & 6 \\
\hline 1 & 6 \\
\hline 1 & 6 \\
\hline 1 & 6 \\
\hline 1 & 6 \\
\hline 1 & 6 \\
\hline 1 & 6 \\
\hline 1 & 7 \\
\hline 1 & 7 \\
\hline 1 & 7 \\
\hline 1 & 7 \\
\hline 1 & 7 \\
\hline 1 & 7 \\
\hline
\end{tabular}

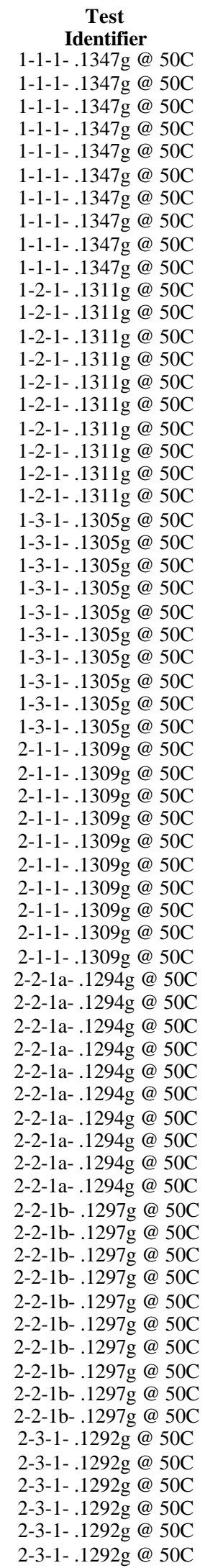

\begin{tabular}{|c|c|c|c|c|c|c|c|}
\hline $\begin{array}{c}\text { Salt } \\
\text { Solution }\end{array}$ & $\begin{array}{c}\mathrm{NH}_{3} \text { Conc } \\
\text { (molal) }\end{array}$ & $\begin{array}{c}\text { Temperature } \\
\left({ }^{\circ} \mathbf{C}\right)\end{array}$ & $\begin{array}{l}\text { Sampling } \\
\text { Sequence }\end{array}$ & $\begin{array}{c}\mathbf{A} \\
\text { (peak area) }\end{array}$ & $\mathbf{y}$ & $\mathbf{x}$ & $\log (A)$ \\
\hline 1 & 0.5 & 50 & 1 & 80.01 & 80.01 & 70.29 & 1.9031 \\
\hline 1 & 0.5 & 50 & 2 & 70.29 & 150.30 & 62.51 & 1.8469 \\
\hline 1 & 0.5 & 50 & 3 & 62.51 & 212.81 & 59.20 & 1.7960 \\
\hline 1 & 0.5 & 50 & 4 & 59.20 & 272.01 & 54.02 & 1.7723 \\
\hline 1 & 0.5 & 50 & 5 & 54.02 & 326.03 & 48.63 & 1.7326 \\
\hline 1 & 0.5 & 50 & 6 & 48.63 & 374.66 & 41.94 & 1.6869 \\
\hline 1 & 0.5 & 50 & 7 & 41.94 & 416.60 & 40.23 & 1.6226 \\
\hline 1 & 0.5 & 50 & 8 & 40.23 & 456.83 & 33.78 & 1.6045 \\
\hline 1 & 0.5 & 50 & 9 & 33.78 & 490.61 & 30.95 & 1.5287 \\
\hline 1 & 0.5 & 50 & 10 & 30.95 & 521.56 & & 1.4907 \\
\hline 1 & 2 & 50 & 1 & 539.52 & 539.52 & 500.51 & 2.7320 \\
\hline 1 & 2 & 50 & 2 & 500.51 & 1040.02 & 439.88 & 2.6994 \\
\hline 1 & 2 & 50 & 3 & 439.88 & 1479.90 & 386.20 & 2.6433 \\
\hline 1 & 2 & 50 & 4 & 386.20 & 1866.10 & 337.24 & 2.5868 \\
\hline 1 & 2 & 50 & 5 & 337.24 & 2203.33 & 298.42 & 2.5279 \\
\hline 1 & 2 & 50 & 6 & 298.42 & 2501.75 & 261.18 & 2.4748 \\
\hline 1 & 2 & 50 & 7 & 261.18 & 2762.93 & 232.07 & 2.4169 \\
\hline 1 & 2 & 50 & 8 & 232.07 & 2995.01 & 203.00 & 2.3656 \\
\hline 1 & 2 & 50 & 9 & 203.00 & 3198.00 & 180.40 & 2.3075 \\
\hline 1 & 2 & 50 & 10 & 180.40 & 3378.40 & 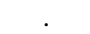 & 2.2562 \\
\hline 1 & 3.5 & 50 & 1 & 1012.66 & 1012.66 & 930.73 & 3.0055 \\
\hline 1 & 3.5 & 50 & 2 & 930.73 & 1943.39 & 816.79 & 2.9688 \\
\hline 1 & 3.5 & 50 & 3 & 816.79 & 2760.18 & 716.49 & 2.9121 \\
\hline 1 & 3.5 & 50 & 4 & 716.49 & 3476.67 & 629.15 & 2.8552 \\
\hline 1 & 3.5 & 50 & 5 & 629.15 & 4105.82 & 546.06 & 2.7988 \\
\hline 1 & 3.5 & 50 & 6 & 546.06 & 4651.88 & 475.44 & 2.7372 \\
\hline 1 & 3.5 & 50 & 7 & 475.44 & 5127.32 & 417.58 & 2.6771 \\
\hline 1 & 3.5 & 50 & 8 & 417.58 & 5544.90 & 363.90 & 2.6207 \\
\hline 1 & 3.5 & 50 & 9 & 363.90 & 5908.79 & 319.36 & 2.5610 \\
\hline 1 & 3.5 & 50 & 10 & 319.36 & 6228.16 & & 2.5043 \\
\hline 2 & 0.5 & 50 & 1 & 75.37 & 75.37 & 66.23 & 1.8772 \\
\hline 2 & 0.5 & 50 & 2 & 66.23 & 141.60 & 60.51 & 1.8211 \\
\hline 2 & 0.5 & 50 & 3 & 60.51 & 202.12 & 55.06 & 1.7818 \\
\hline 2 & 0.5 & 50 & 4 & 55.06 & 257.17 & 46.09 & 1.7408 \\
\hline 2 & 0.5 & 50 & 5 & 46.09 & 303.26 & 43.53 & 1.6636 \\
\hline 2 & 0.5 & 50 & 6 & 43.53 & 346.79 & 38.20 & 1.6388 \\
\hline 2 & 0.5 & 50 & 7 & 38.20 & 384.99 & 34.61 & 1.5820 \\
\hline 2 & 0.5 & 50 & 8 & 34.61 & 419.60 & 30.60 & 1.5392 \\
\hline 2 & 0.5 & 50 & 9 & 30.60 & 450.20 & 26.30 & 1.4858 \\
\hline 2 & 0.5 & 50 & 10 & 26.30 & 476.50 & & 1.4199 \\
\hline 2 & 2 & 51 & 1 & 269.36 & 269.36 & 254.05 & 2.4303 \\
\hline 2 & 2 & 51 & 2 & 254.05 & 523.42 & 227.09 & 2.4049 \\
\hline 2 & 2 & 51 & 3 & 227.09 & 750.50 & 197.31 & 2.3562 \\
\hline 2 & 2 & 51 & 4 & 197.31 & 947.81 & 173.72 & 2.2952 \\
\hline 2 & 2 & 51 & 5 & 173.72 & 1121.53 & 153.39 & 2.2398 \\
\hline 2 & 2 & 51 & 6 & 153.39 & 1274.92 & 133.78 & 2.1858 \\
\hline 2 & 2 & 51 & 7 & 133.78 & 1408.69 & 118.56 & 2.1264 \\
\hline 2 & 2 & 51 & 8 & 118.56 & 1527.26 & 103.33 & 2.0739 \\
\hline 2 & 2 & 51 & 9 & 103.33 & 1630.59 & 91.12 & 2.0142 \\
\hline 2 & 2 & 51 & 10 & 91.12 & 1721.70 & 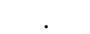 & 1.9596 \\
\hline 2 & 2 & 52 & 1 & 281.60 & 281.60 & 259.07 & 2.4496 \\
\hline 2 & 2 & 52 & 2 & 259.07 & 540.67 & 235.29 & 2.4134 \\
\hline 2 & 2 & 52 & 3 & 235.29 & 775.97 & 205.06 & 2.3716 \\
\hline 2 & 2 & 52 & 4 & 205.06 & 981.03 & 177.09 & 2.3119 \\
\hline 2 & 2 & 52 & 5 & 177.09 & 1158.11 & 157.58 & 2.2482 \\
\hline 2 & 2 & 52 & 6 & 157.58 & 1315.70 & 137.57 & 2.1975 \\
\hline 2 & 2 & 52 & 7 & 137.57 & 1453.26 & 124.81 & 2.1385 \\
\hline 2 & 2 & 52 & 8 & 124.81 & 1578.07 & 106.84 & 2.0962 \\
\hline 2 & 2 & 52 & 9 & 106.84 & 1684.91 & 92.38 & 2.0287 \\
\hline 2 & 2 & 52 & 10 & 92.38 & 1777.29 & & 1.9656 \\
\hline 2 & 3.5 & 50 & 1 & 458.43 & 458.43 & 426.93 & 2.6613 \\
\hline 2 & 3.5 & 50 & 2 & 426.93 & 885.36 & 374.42 & 2.6304 \\
\hline 2 & 3.5 & 50 & 3 & 374.42 & 1259.77 & 327.87 & 2.5734 \\
\hline 2 & 3.5 & 50 & 4 & 327.87 & 1587.65 & 287.01 & 2.5157 \\
\hline 2 & 3.5 & 50 & 5 & 287.01 & 1874.65 & 253.19 & 2.4579 \\
\hline 2 & 3.5 & 50 & 6 & 253.19 & 2127.84 & 225.35 & 2.4034 \\
\hline
\end{tabular}


Table B2. Ammonia Test Results

(continued)

Test

Study Sequence

\begin{tabular}{|c|c|}
\hline 1 & 7 \\
\hline 1 & 7 \\
\hline 1 & 7 \\
\hline 1 & 7 \\
\hline 1 & 8 \\
\hline 1 & 8 \\
\hline 1 & 8 \\
\hline 1 & 8 \\
\hline 1 & 8 \\
\hline 1 & 8 \\
\hline 1 & 8 \\
\hline 1 & 8 \\
\hline 1 & 8 \\
\hline 1 & 8 \\
\hline 1 & 9 \\
\hline 1 & 9 \\
\hline 1 & 9 \\
\hline 1 & 9 \\
\hline 1 & 9 \\
\hline 1 & 9 \\
\hline 1 & 9 \\
\hline 1 & 9 \\
\hline 1 & 9 \\
\hline 1 & 9 \\
\hline 1 & 10 \\
\hline 1 & 10 \\
\hline 1 & 10 \\
\hline 1 & 10 \\
\hline 1 & 10 \\
\hline 1 & 10 \\
\hline 1 & 10 \\
\hline 1 & 10 \\
\hline 1 & 10 \\
\hline 1 & 10 \\
\hline 1 & 11 \\
\hline 1 & 11 \\
\hline 1 & 11 \\
\hline 1 & 11 \\
\hline 1 & 11 \\
\hline 1 & 11 \\
\hline 1 & 11 \\
\hline 1 & 11 \\
\hline 1 & 11 \\
\hline 1 & 11 \\
\hline 1 & 12 \\
\hline 1 & 12 \\
\hline 1 & 12 \\
\hline 1 & 12 \\
\hline 1 & 12 \\
\hline 1 & 12 \\
\hline 1 & 12 \\
\hline 1 & 12 \\
\hline 1 & 12 \\
\hline 1 & 12 \\
\hline 1 & 13 \\
\hline 1 & 13 \\
\hline 1 & 13 \\
\hline 1 & 13 \\
\hline 1 & 13 \\
\hline 1 & 13 \\
\hline 1 & 13 \\
\hline 1 & 13 \\
\hline 1 & 13 \\
\hline 1 & 13 \\
\hline 1 & 14 \\
\hline 1 & 14 \\
\hline
\end{tabular}

Test

Identifier

2-3-1-.1292g @ 50C

2-3-1-.1292g@50C

2-3-1-.1292g@50C

2-3-1-.1292g@50C

3-1-1-.1330g@ 50C

3-1-1-.1330g@ @0C

3-1-1-.1330g @ 50C

3-1-1-.1330g@50C

3-1-1-.1330g@ 50C

3-1-1-.1330g@ 50C

3-1-1-.1330g@ $@ 50 \mathrm{C}$

3-1-1-.1330g@ @0C

3-1-1-.1330g@ 50C

3-1-1-.1330g@ $00 \mathrm{C}$

3-2-1-.1314g@50C

3-2-1-.1314g@ $@ 50 \mathrm{C}$

3-2-1-.1314g@50C

3-2-1-.1314g@ $@ 50 \mathrm{C}$

3-2-1-.1314g@ @ 50C

3-2-1-.1314g @ 50C

3-2-1-.1314g@50C

3-2-1-.1314g @ 50C

3-2-1-.1314g@ @0C

3-2-1-.1314g@ $@ 50 \mathrm{C}$

3-3-1-.1302g@ 50C

3-3-1-.1302g @ 50C

3-3-1-.1302g@ @ $0 \mathrm{C}$

3-3-1-.1302g@ 50C

3-3-1-.1302g@ $@ 50 \mathrm{C}$

3-3-1-.1302g@50C

3-3-1-.1302g@ @ $0 \mathrm{C}$

3-3-1-.1302g @ 50C

3-3-1-.1302g @ 50C

3-3-1-.1302g@50C

1-1-2-.1330g@60c

1-1-2-.1330g@60c

1-1-2-.1330g@60c

1-1-2-.1330g@60c

1-1-2-.1330g @ 60c

1-1-2-.1330g@60c

1-1-2-.1330g@60c

1-1-2-.1330g@60c

1-1-2-.1330g@60c

1-1-2-.1330g@60c

1-2-2-.1321g@60C

1-2-2-.1321g@60C

1-2-2-.1321g@60C

1-2-2-.1321g@60C

1-2-2-.1321g@60C

1-2-2-.1321g @ 60C

1-2-2-.1321g@60C

1-2-2-.1321g @ 60C

1-2-2-.1321g @ 60C

1-2-2-.1321g@60C

1-3-2-.1305g@60C

1-3-2-.1305g @ 60C

1-3-2-.1305g@60C

1-3-2-.1305g@60C

1-3-2-.1305g@60C

1-3-2-.1305g @ 60C

1-3-2-.1305g@60C

1-3-2-.1305g @ 60C

1-3-2-.1305g@60C

1-3-2-.1305g @60C

2-1-2-.1313g @ 60C

2-1-2-.1313g @ 60C

\begin{tabular}{|c|c|c|c|c|c|c|c|}
\hline $\begin{array}{c}\text { Salt } \\
\text { Solution }\end{array}$ & $\begin{array}{c}\mathrm{NH}_{3} \text { Conc } \\
(\text { molal })\end{array}$ & $\begin{array}{c}\text { Temperature } \\
\left({ }^{\circ} \mathbf{C}\right)\end{array}$ & $\begin{array}{l}\text { Sampling } \\
\text { Sequence }\end{array}$ & $\underset{\text { (peak area) }}{\mathbf{A}}$ & $\mathbf{y}$ & $\mathbf{x}$ & $\log (A)$ \\
\hline 2 & 3.5 & 50 & 7 & 225.35 & 2353.19 & 195.05 & 2.3529 \\
\hline 2 & 3.5 & 50 & 8 & 195.05 & 2548.24 & 172.28 & 2.2901 \\
\hline 2 & 3.5 & 50 & 9 & 172.28 & 2720.52 & 152.44 & 2.2362 \\
\hline 2 & 3.5 & 50 & 10 & 152.44 & 2872.96 & & 2.1831 \\
\hline 3 & 0.5 & 50 & 1 & 32.90 & 32.90 & 20.04 & 1.5172 \\
\hline 3 & 0.5 & 50 & 2 & 20.04 & 52.94 & 15.96 & 1.3019 \\
\hline 3 & 0.5 & 50 & 3 & 15.96 & 68.90 & 13.04 & 1.2031 \\
\hline 3 & 0.5 & 50 & 4 & 13.04 & 81.95 & 13.19 & 1.1154 \\
\hline 3 & 0.5 & 50 & 5 & 13.19 & 95.13 & 13.63 & 1.1201 \\
\hline 3 & 0.5 & 50 & 6 & 13.63 & 108.76 & 9.01 & 1.1344 \\
\hline 3 & 0.5 & 50 & 7 & 9.01 & 117.76 & 7.49 & 0.9545 \\
\hline 3 & 0.5 & 50 & 8 & 7.49 & 125.25 & 7.28 & 0.8744 \\
\hline 3 & 0.5 & 50 & 9 & 7.28 & 132.54 & 7.89 & 0.8623 \\
\hline 3 & 0.5 & 50 & 10 & 7.89 & 140.43 & & 0.8972 \\
\hline 3 & 2 & 50 & 1 & 17.22 & 17.22 & 20.70 & 1.2361 \\
\hline 3 & 2 & 50 & 2 & 20.70 & 37.93 & 20.98 & 1.3161 \\
\hline 3 & 2 & 50 & 3 & 20.98 & 58.91 & 19.98 & 1.3218 \\
\hline 3 & 2 & 50 & 4 & 19.98 & 78.88 & 13.31 & 1.3005 \\
\hline 3 & 2 & 50 & 5 & 13.31 & 92.19 & 13.06 & 1.1242 \\
\hline 3 & 2 & 50 & 6 & 13.06 & 105.25 & 11.96 & 1.1158 \\
\hline 3 & 2 & 50 & 7 & 11.96 & 117.21 & 10.80 & 1.0777 \\
\hline 3 & 2 & 50 & 8 & 10.80 & 128.01 & 8.29 & 1.0334 \\
\hline 3 & 2 & 50 & 9 & 8.29 & 136.30 & 8.26 & 0.9186 \\
\hline 3 & 2 & 50 & 10 & 8.26 & 144.56 & . & 0.9169 \\
\hline 3 & 3.5 & 50 & 1 & 30.46 & 30.46 & 31.40 & 1.4837 \\
\hline 3 & 3.5 & 50 & 2 & 31.40 & 61.86 & 29.65 & 1.4969 \\
\hline 3 & 3.5 & 50 & 3 & 29.65 & 91.51 & 25.82 & 1.4720 \\
\hline 3 & 3.5 & 50 & 4 & 25.82 & 117.34 & 24.01 & 1.4120 \\
\hline 3 & 3.5 & 50 & 5 & 24.01 & 141.35 & 22.34 & 1.3805 \\
\hline 3 & 3.5 & 50 & 6 & 22.34 & 163.69 & 20.19 & 1.3491 \\
\hline 3 & 3.5 & 50 & 7 & 20.19 & 183.88 & 15.51 & 1.3051 \\
\hline 3 & 3.5 & 50 & 8 & 15.51 & 199.39 & 14.61 & 1.1906 \\
\hline 3 & 3.5 & 50 & 9 & 14.61 & 214.00 & 14.25 & 1.1646 \\
\hline 3 & 3.5 & 50 & 10 & 14.25 & 228.25 & & 1.1539 \\
\hline 1 & 0.5 & 60 & 1 & 167.14 & 167.14 & 161.70 & 2.2231 \\
\hline 1 & 0.5 & 60 & 2 & 161.70 & 328.84 & 138.00 & 2.2087 \\
\hline 1 & 0.5 & 60 & 3 & 138.00 & 466.83 & 124.80 & 2.1399 \\
\hline 1 & 0.5 & 60 & 4 & 124.80 & 591.63 & 104.14 & 2.0962 \\
\hline 1 & 0.5 & 60 & 5 & 104.14 & 695.77 & 91.65 & 2.0176 \\
\hline 1 & 0.5 & 60 & 6 & 91.65 & 787.42 & 80.47 & 1.9621 \\
\hline 1 & 0.5 & 60 & 7 & 80.47 & 867.88 & 70.19 & 1.9056 \\
\hline 1 & 0.5 & 60 & 8 & 70.19 & 938.08 & 60.61 & 1.8463 \\
\hline 1 & 0.5 & 60 & 9 & 60.61 & 998.69 & 52.35 & 1.7826 \\
\hline 1 & 0.5 & 60 & 10 & 52.35 & 1051.04 & & 1.7189 \\
\hline 1 & 2 & 60 & 1 & 987.77 & 987.77 & 889.88 & 2.9947 \\
\hline 1 & 2 & 60 & 2 & 889.88 & 1877.65 & 760.53 & 2.9493 \\
\hline 1 & 2 & 60 & 3 & 760.53 & 2638.19 & 644.35 & 2.8811 \\
\hline 1 & 2 & 60 & 4 & 644.35 & 3282.53 & 547.65 & 2.8091 \\
\hline 1 & 2 & 60 & 5 & 547.65 & 3830.18 & 465.25 & 2.7385 \\
\hline 1 & 2 & 60 & 6 & 465.25 & 4295.43 & 393.20 & 2.6677 \\
\hline 1 & 2 & 60 & 7 & 393.20 & 4688.64 & 334.72 & 2.5946 \\
\hline 1 & 2 & 60 & 8 & 334.72 & 5023.36 & 283.61 & 2.5247 \\
\hline 1 & 2 & 60 & 9 & 283.61 & 5306.97 & 240.56 & 2.4527 \\
\hline 1 & 2 & 60 & 10 & 240.56 & 5547.53 & . & 2.3812 \\
\hline 1 & 3.5 & 60 & 1 & 1603.30 & 1603.30 & 1427.65 & 3.2050 \\
\hline 1 & 3.5 & 60 & 2 & 1427.65 & 3030.94 & 1198.57 & 3.1546 \\
\hline 1 & 3.5 & 60 & 3 & 1198.57 & 4229.52 & 1009.79 & 3.0787 \\
\hline 1 & 3.5 & 60 & 4 & 1009.79 & 5239.31 & 844.50 & 3.0042 \\
\hline 1 & 3.5 & 60 & 5 & 844.50 & 6083.80 & 710.94 & 2.9266 \\
\hline 1 & 3.5 & 60 & 6 & 710.94 & 6794.74 & 591.86 & 2.8518 \\
\hline 1 & 3.5 & 60 & 7 & 591.86 & 7386.60 & 493.86 & 2.7722 \\
\hline 1 & 3.5 & 60 & 8 & 493.86 & 7880.46 & 417.31 & 2.6936 \\
\hline 1 & 3.5 & 60 & 9 & 417.31 & 8297.76 & 345.13 & 2.6205 \\
\hline 1 & 3.5 & 60 & 10 & 345.13 & 8642.89 & & 2.5380 \\
\hline 2 & 0.5 & 60 & 1 & 111.90 & 111.90 & 97.18 & 2.0488 \\
\hline 2 & 0.5 & 60 & 2 & 97.18 & 209.08 & 84.62 & 1.9876 \\
\hline
\end{tabular}


Table B2. Ammonia Test Results

(continued)

Test

Study Sequence

1 14

$1142-1-2-.1313 \mathrm{~g} @ 60 \mathrm{C}$

$1142-1-2-.1313 \mathrm{~g} @ 60 \mathrm{C}$

$1 \quad 14 \quad 2-1-2-.1313 \mathrm{~g} @ 60 \mathrm{C}$

$\begin{array}{lll}1 & 14 & 2-1-2-.1313 \mathrm{~g} @ 60 \mathrm{C} \\ 1 & 14 & 2-1-2-.1313 \mathrm{~g} @ 60 \mathrm{C}\end{array}$

$1142-1-2-.1313 \mathrm{~g} @ 60 \mathrm{C}$

$1 \quad 152-2-2 \mathrm{a}-.1293 \mathrm{~g} @ 60 \mathrm{C}$

$1 \quad 15 \quad 2-2-2 \mathrm{a}-.1293 \mathrm{~g} @ 60 \mathrm{C}$

$1 \quad 15 \quad 2-2-2 \mathrm{a}-.1293 \mathrm{~g} @ 60 \mathrm{C}$

$1 \quad 15 \quad 2-2-2 \mathrm{a}-.1293 \mathrm{~g} @ 60 \mathrm{C}$

$115 \quad 2-2-2 \mathrm{a}-.1293 \mathrm{~g} @ 60 \mathrm{C}$

$115 \quad 2-2-2 \mathrm{a}-.1293 \mathrm{~g} @ 60 \mathrm{C}$

$1 \quad 15 \quad 2-2-2 \mathrm{a}-.1293 \mathrm{~g} @ 60 \mathrm{C}$

$1 \quad 15 \quad 2-2-2 \mathrm{a}-.1293 \mathrm{~g} @ 60 \mathrm{C}$

$1 \quad 15 \quad 2-2-2 \mathrm{a}-.1293 \mathrm{~g} @ 60 \mathrm{C}$

$1 \quad 152-2-2 \mathrm{a}-.1293 \mathrm{~g} @ 60 \mathrm{C}$

$1 \quad 162-2-2 b-.1291 \mathrm{~g} @ 60 \mathrm{C}$

$116 \quad 2-2-2 b-.1291 \mathrm{~g} @ 60 \mathrm{C}$

$\begin{array}{lll}1 & 16 & 2-2-2 \mathrm{~b}-.1291 \mathrm{~g} @ 60 \mathrm{C} \\ 1 & 16 & 2-2-2 \mathrm{~b}-.1291 \mathrm{~g} @ 60 \mathrm{C}\end{array}$

$1 \quad 16 \quad 2-2-2 \mathrm{~b}-.1291 \mathrm{~g} @ 60 \mathrm{C}$

$1162-2-2 \mathrm{~b}-.1291 \mathrm{~g} @ 60 \mathrm{C}$

$116 \quad 2-2-2 b-.1291 \mathrm{~g} @ 60 \mathrm{C}$

$1162-2-2 \mathrm{~b}-.1291 \mathrm{~g} @ 60 \mathrm{C}$

$1 \quad 16 \quad 2-2-2 \mathrm{~b}-.1291 \mathrm{~g} @ 60 \mathrm{C}$

$116 \quad 2-2-2 \mathrm{~b}-.1291 \mathrm{~g} @ 60 \mathrm{C}$
2-3-2-.1287g@60C 2-3-2-.1287g@60C

2-3-2-.1287g@60C

2-3-2-.1287g@60C

2-3-2-.1287g@60C

2-3-2-.1287g@60C

2-3-2-.1287g@60C

2-3-2-.1287g@60C

2-3-2-.1287g@60C

2-3-2-.1287g@60C

3-1-2-.1327g@60C

3-1-2-.1327g@60C

3-1-2-.1327g @ 60C

3-1-2-.1327g@60C

3-1-2-.1327g@60C

3-1-2-.1327g@60C

3-1-2-.1327g@60C

3-1-2-.1327g@60C

3-1-2-.1327g@60C

3-1-2-.1327g@60C

3-2-2-.1318g@60C

3-2-2-.1318g@60C

3-2-2-.1318g@60C

3-2-2-.1318g@60C

3-2-2-.1318g@60C

3-2-2-.1318g@60C

3-2-2-.1318g@60C

3-2-2-.1318g@60C

3-3-2-.1314g@60C

3-3-2-.1314g@60C

3-3-2-.1314g@60C

3-3-2-.1314g@60C

3-3-2-.1314g@60C

3-3-2-.1314g@60C

3-3-2-.1314g@60C

3-3-2-.1314g@60C
3-2-2-.1318g@60C

3-2-2-.1318g@60C

\begin{tabular}{|c|c|c|c|c|c|c|c|}
\hline $\begin{array}{c}\text { Salt } \\
\text { Solution } \\
2\end{array}$ & $\begin{array}{c}\mathrm{NH}_{3} \text { Conc } \\
\text { (molal) } \\
0.5\end{array}$ & $\begin{array}{c}\text { Temperature } \\
\left({ }^{\circ} \mathbf{C}\right) \\
60\end{array}$ & $\begin{array}{c}\text { Sampling } \\
\text { Sequence } \\
3\end{array}$ & $\begin{array}{c}\mathbf{A} \\
\text { (peak area) } \\
84.62\end{array}$ & $\begin{array}{c}\mathbf{y} \\
293.70\end{array}$ & $\begin{array}{c}\mathbf{x} \\
72.22\end{array}$ & $\begin{array}{l}\log (\mathbf{A}) \\
1.9275\end{array}$ \\
\hline 2 & 0.5 & 60 & 4 & 72.22 & 365.91 & 62.10 & 1.8586 \\
\hline 2 & 0.5 & 60 & 5 & 62.10 & 428.02 & 55.34 & 1.7931 \\
\hline 2 & 0.5 & 60 & 6 & 55.34 & 483.35 & 46.04 & 1.7430 \\
\hline 2 & 0.5 & 60 & 7 & 46.04 & 529.39 & 37.38 & 1.6632 \\
\hline 2 & 0.5 & 60 & 8 & 37.38 & 566.77 & 35.07 & 1.5726 \\
\hline 2 & 0.5 & 60 & 9 & 35.07 & 601.84 & 29.78 & 1.5449 \\
\hline 2 & 0.5 & 60 & 10 & 29.78 & 631.62 & & 1.4739 \\
\hline 2 & 2 & 61 & 1 & 326.42 & 326.42 & 295.06 & 2.5138 \\
\hline 2 & 2 & 61 & 2 & 295.06 & 621.48 & 248.05 & 2.4699 \\
\hline 2 & 2 & 61 & 3 & 248.05 & 869.53 & 209.19 & 2.3945 \\
\hline 2 & 2 & 61 & 4 & 209.19 & 1078.71 & 173.52 & 2.3205 \\
\hline 2 & 2 & 61 & 5 & 173.52 & 1252.24 & 146.24 & 2.2394 \\
\hline 2 & 2 & 61 & 6 & 146.24 & 1398.47 & 118.47 & 2.1651 \\
\hline 2 & 2 & 61 & 7 & 118.47 & 1516.94 & 102.59 & 2.0736 \\
\hline 2 & 2 & 61 & 8 & 102.59 & 1619.53 & 85.72 & 2.0111 \\
\hline 2 & 2 & 61 & 9 & 85.72 & 1705.26 & 69.26 & 1.9331 \\
\hline 2 & 2 & 61 & 10 & 69.26 & 1774.52 & & 1.8405 \\
\hline 2 & 2 & 62 & 1 & 281.84 & 281.84 & 249.75 & 2.4500 \\
\hline 2 & 2 & 62 & 2 & 249.75 & 531.59 & 211.19 & 2.3975 \\
\hline 2 & 2 & 62 & 3 & 211.19 & 742.79 & 178.95 & 2.3247 \\
\hline 2 & 2 & 62 & 4 & 178.95 & 921.74 & 147.52 & 2.2527 \\
\hline 2 & 2 & 62 & 5 & 147.52 & 1069.26 & 127.08 & 2.1689 \\
\hline 2 & 2 & 62 & 6 & 127.08 & 1196.34 & 104.97 & 2.1041 \\
\hline 2 & 2 & 62 & 7 & 104.97 & 1301.31 & 89.19 & 2.0211 \\
\hline 2 & 2 & 62 & 8 & 89.19 & 1390.50 & 75.27 & 1.9503 \\
\hline 2 & 2 & 62 & 9 & 75.27 & 1465.77 & 64.69 & 1.8766 \\
\hline 2 & 2 & 62 & 10 & 64.69 & 1530.46 & & 1.8109 \\
\hline 2 & 3.5 & 60 & 1 & 430.53 & 430.53 & 388.56 & 2.6340 \\
\hline 2 & 3.5 & 60 & 2 & 388.56 & 819.09 & 327.73 & 2.5895 \\
\hline 2 & 3.5 & 60 & 3 & 327.73 & 1146.82 & 276.84 & 2.5155 \\
\hline 2 & 3.5 & 60 & 4 & 276.84 & 1423.67 & 231.64 & 2.4422 \\
\hline 2 & 3.5 & 60 & 5 & 231.64 & 1655.31 & 195.56 & 2.3648 \\
\hline 2 & 3.5 & 60 & 6 & 195.56 & 1850.87 & 164.61 & 2.2913 \\
\hline 2 & 3.5 & 60 & 7 & 164.61 & 2015.48 & 137.35 & 2.2165 \\
\hline 2 & 3.5 & 60 & 8 & 137.35 & 2152.83 & 116.21 & 2.1378 \\
\hline 2 & 3.5 & 60 & 9 & 116.21 & 2269.04 & 98.52 & 2.0653 \\
\hline 2 & 3.5 & 60 & 10 & 98.52 & 2367.56 & & 1.9935 \\
\hline 3 & 0.5 & 60 & 1 & 23.47 & 23.47 & 14.06 & 1.3705 \\
\hline 3 & 0.5 & 60 & 2 & 14.06 & 37.53 & 10.09 & 1.1479 \\
\hline 3 & 0.5 & 60 & 3 & 10.09 & 47.61 & 8.44 & 1.0037 \\
\hline 3 & 0.5 & 60 & 4 & 8.44 & 56.06 & 9.53 & 0.9265 \\
\hline 3 & 0.5 & 60 & 5 & 9.53 & 65.58 & 5.47 & 0.9790 \\
\hline 3 & 0.5 & 60 & 6 & 5.47 & 71.05 & 6.06 & 0.7376 \\
\hline 3 & 0.5 & 60 & 7 & 6.06 & 77.11 & 4.61 & 0.7825 \\
\hline 3 & 0.5 & 60 & 8 & 4.61 & 81.72 & 5.82 & 0.6635 \\
\hline 3 & 0.5 & 60 & 9 & 5.82 & 87.54 & 5.28 & 0.7652 \\
\hline 3 & 0.5 & 60 & 10 & 5.28 & 92.82 & & 0.7226 \\
\hline 3 & 2 & 60 & 1 & 12.85 & 12.85 & 15.98 & 1.1089 \\
\hline 3 & 2 & 60 & 2 & 15.98 & 28.83 & 13.70 & 1.2035 \\
\hline 3 & 2 & 60 & 3 & 13.70 & 42.53 & 13.09 & 1.1368 \\
\hline 3 & 2 & 60 & 4 & 13.09 & 55.61 & 11.41 & 1.1168 \\
\hline 3 & 2 & 60 & 5 & 11.41 & 67.02 & 11.18 & 1.0573 \\
\hline 3 & 2 & 60 & 6 & 11.18 & 78.20 & 12.77 & 1.0482 \\
\hline 3 & 2 & 60 & 7 & 12.77 & 90.97 & 8.54 & 1.1061 \\
\hline 3 & 2 & 60 & 8 & 8.54 & 99.50 & 8.68 & 0.9313 \\
\hline 3 & 2 & 60 & 9 & 8.68 & 108.18 & 7.81 & 0.9383 \\
\hline 3 & 2 & 60 & 10 & 7.81 & 115.99 & & 0.8927 \\
\hline 3 & 3.5 & 60 & 1 & 25.14 & 25.14 & 26.03 & 1.4004 \\
\hline 3 & 3.5 & 60 & 2 & 26.03 & 51.17 & 23.51 & 1.4155 \\
\hline 3 & 3.5 & 60 & 3 & 23.51 & 74.68 & 21.70 & 1.3712 \\
\hline 3 & 3.5 & 60 & 4 & 21.70 & 96.37 & 17.75 & 1.3364 \\
\hline 3 & 3.5 & 60 & 5 & 17.75 & 114.13 & 17.67 & 1.2493 \\
\hline 3 & 3.5 & 60 & 6 & 17.67 & 131.80 & 13.25 & 1.2473 \\
\hline 3 & 3.5 & 60 & 7 & 13.25 & 145.05 & 13.10 & 1.1223 \\
\hline 3 & 3.5 & 60 & 8 & 13.10 & 158.15 & 10.35 & 1.1172 \\
\hline
\end{tabular}


Table B2. Ammonia Test Results

(continued)

Test

Study Sequence $1 \quad 20$

120

$\begin{array}{ll}1 & 21 \\ 1 & 21\end{array}$

$1 \quad 21$

$\begin{array}{ll}1 & 21 \\ 1 & 21\end{array}$

121

$1 \quad 21$

$1 \quad 21$

$1 \quad 21$

122

122

$\begin{array}{ll}1 & 22 \\ 1 & 22\end{array}$

$1 \quad 22$

122

122

122

$1 \quad 22$

123

$1 \quad 23$

$\begin{array}{ll}1 & 23 \\ 1 & 23\end{array}$

123

123

123

$\begin{array}{ll}1 & 23 \\ 1 & 23\end{array}$

123

124
Test

Identifier

3-3-2-.1314g @ 60C

.1314g@60C

1-1-3-.1326g @ 70C

1-1-3-.1326g @ 70C

1-1-3-.1326g @ 70C

1-1-3-.1326g@70C

1-1-3-.1326g @ 70C

1-1-3-.1326g @ 70C

1-1-3-.1326g@70C

1-1-3-.1326g@70C

1-1-3-.1326g @ 70C

1-1-3-.1326g @ 70C

1-2-3-.1315g @ 70C

1-2-3-.1315g@70C

1-2-3-.1315g @ 70C

1-2-3-.1315g @ 70C

1-2-3-.1315g @ 70C

1-2-3-.1315g@70C

1-2-3-.1315g@70C

1-2-3-.1315g@70C

1-2-3-.1315g@70C

1-2-3-.1315g@70C

1-3-3-.1310g@70C

1-3-3-.1310g@70C

1-3-3-.1310g@70C

1-3-3-.1310g@70C

1-3-3-.1310g@70C

1-3-3-.1310g @ 70C

1-3-3-.1310g@70C

1-3-3-.1310g @ 70C

1-3-3-.1310g@70C

1-3-3-.1310g@70C

2-1-3-.1308g @ 70C

2-1-3-.1308g@70C

2-1-3-.1308g@70C

2-1-3-.1308g@70C

2-1-3-.1308g@70C

2-1-3-.1308g@70C

2-1-3-.1308g@70C

2-1-3-.1308g@70C

2-1-3-.1308g@70C

2-1-3-.1308g@70C

2-2-3a.1299g@70C

2-2-3a.1299g@70C

2-2-3a.1299g@70C

2-2-3a.1299g@70C

2-2-3a.1299g@70C

2-2-3a.1299g@70C

2-2-3a.1299g@70C

2-2-3a.1299g@70C

2-2-3a.1299g@70C

2-2-3a.1299g@70C

2-2-3b.1293g@70C

2-2-3b.1293g@70C

2-2-3b.1293g@70C

2-2-3b.1293g @ 70C

2-2-3b.1293g@70C

2-2-3b.1293g @ 70C

2-2-3b.1293g @ 70C

2-2-3b.1293g @ 70C

2-2-3b.1293g@70C

2-2-3b.1293g@70C

2-3-3 - .1293g @ 70C

2-3-3 - .1293g @ 70C

2-3-3 - .1293g @ 70C

2-3-3 -.1293g@70C

\begin{tabular}{|c|c|c|c|c|c|c|c|}
\hline $\begin{array}{c}\text { Salt } \\
\text { Solution }\end{array}$ & $\begin{array}{c}\mathrm{NH}_{3} \text { Conc } \\
(\text { molal })\end{array}$ & $\begin{array}{c}\text { Temperature } \\
\left({ }^{\circ} \mathbf{C}\right)\end{array}$ & $\begin{array}{l}\text { Sampling } \\
\text { Sequence }\end{array}$ & $\underset{\text { (peak area) }}{\mathbf{A}}$ & $\mathbf{y}$ & $\mathbf{x}$ & $\log (\mathrm{A})$ \\
\hline 3 & 3.5 & 60 & 9 & 10.35 & 168.50 & 10.07 & 1.0151 \\
\hline 3 & 3.5 & 60 & 10 & 10.07 & 178.58 & & 1.0031 \\
\hline 1 & 0.5 & 70 & 1 & 145.58 & 145.58 & 118.73 & 2.1631 \\
\hline 1 & 0.5 & 70 & 2 & 118.73 & 264.31 & 87.47 & 2.0745 \\
\hline 1 & 0.5 & 70 & 3 & 87.47 & 351.78 & 64.94 & 1.9418 \\
\hline 1 & 0.5 & 70 & 4 & 64.94 & 416.71 & 46.81 & 1.8125 \\
\hline 1 & 0.5 & 70 & 5 & 46.81 & 463.52 & 35.77 & 1.6703 \\
\hline 1 & 0.5 & 70 & 6 & 35.77 & 499.29 & 26.17 & 1.5535 \\
\hline 1 & 0.5 & 70 & 7 & 26.17 & 525.46 & 20.54 & 1.4177 \\
\hline 1 & 0.5 & 70 & 8 & 20.54 & 546.00 & 16.32 & 1.3127 \\
\hline 1 & 0.5 & 70 & 9 & 16.32 & 562.32 & 13.01 & 1.2128 \\
\hline 1 & 0.5 & 70 & 10 & 13.01 & 575.33 & & 1.1143 \\
\hline 1 & 2 & 70 & 1 & 912.20 & 912.20 & 709.02 & 2.9601 \\
\hline 1 & 2 & 70 & 2 & 709.02 & 1621.21 & 508.68 & 2.8507 \\
\hline 1 & 2 & 70 & 3 & 508.68 & 2129.90 & 364.55 & 2.7064 \\
\hline 1 & 2 & 70 & 4 & 364.55 & 2494.44 & 258.75 & 2.5618 \\
\hline 1 & 2 & 70 & 5 & 258.75 & 2753.19 & 183.90 & 2.4129 \\
\hline 1 & 2 & 70 & 6 & 183.90 & 2937.09 & 132.50 & 2.2646 \\
\hline 1 & 2 & 70 & 7 & 132.50 & 3069.59 & 96.43 & 2.1222 \\
\hline 1 & 2 & 70 & 8 & 96.43 & 3166.02 & 68.01 & 1.9842 \\
\hline 1 & 2 & 70 & 9 & 68.01 & 3234.03 & 53.06 & 1.8326 \\
\hline 1 & 2 & 70 & 10 & 53.06 & 3287.09 & & 1.7247 \\
\hline 1 & 3.5 & 70 & 1 & 1594.14 & 1594.14 & 1202.37 & 3.2025 \\
\hline 1 & 3.5 & 70 & 2 & 1202.37 & 2796.52 & 856.51 & 3.0800 \\
\hline 1 & 3.5 & 70 & 3 & 856.51 & 3653.02 & 609.05 & 2.9327 \\
\hline 1 & 3.5 & 70 & 4 & 609.05 & 4262.07 & 429.31 & 2.7846 \\
\hline 1 & 3.5 & 70 & 5 & 429.31 & 4691.38 & 305.28 & 2.6328 \\
\hline 1 & 3.5 & 70 & 6 & 305.28 & 4996.66 & 214.00 & 2.4847 \\
\hline 1 & 3.5 & 70 & 7 & 214.00 & 5210.67 & 155.11 & 2.3304 \\
\hline 1 & 3.5 & 70 & 8 & 155.11 & 5365.77 & 111.46 & 2.1906 \\
\hline 1 & 3.5 & 70 & 9 & 111.46 & 5477.23 & 82.50 & 2.0471 \\
\hline 1 & 3.5 & 70 & 10 & 82.50 & 5559.73 & . & 1.9164 \\
\hline 2 & 0.5 & 70 & 1 & 101.54 & 101.54 & 80.72 & 2.0066 \\
\hline 2 & 0.5 & 70 & 2 & 80.72 & 182.26 & 61.52 & 1.9070 \\
\hline 2 & 0.5 & 70 & 3 & 61.52 & 243.78 & 47.31 & 1.7890 \\
\hline 2 & 0.5 & 70 & 4 & 47.31 & 291.09 & 35.57 & 1.6750 \\
\hline 2 & 0.5 & 70 & 5 & 35.57 & 326.66 & 27.46 & 1.5511 \\
\hline 2 & 0.5 & 70 & 6 & 27.46 & 354.12 & 21.14 & 1.4387 \\
\hline 2 & 0.5 & 70 & 7 & 21.14 & 375.26 & 15.46 & 1.3250 \\
\hline 2 & 0.5 & 70 & 8 & 15.46 & 390.72 & 12.64 & 1.1892 \\
\hline 2 & 0.5 & 70 & 9 & 12.64 & 403.36 & 8.31 & 1.1019 \\
\hline 2 & 0.5 & 70 & 10 & 8.31 & 411.67 & & 0.9198 \\
\hline 2 & 2 & 71 & 1 & 398.18 & 398.18 & 316.36 & 2.6001 \\
\hline 2 & 2 & 71 & 2 & 316.36 & 714.53 & 229.61 & 2.5002 \\
\hline 2 & 2 & 71 & 3 & 229.61 & 944.15 & 166.27 & 2.3610 \\
\hline 2 & 2 & 71 & 4 & 166.27 & 1110.42 & 118.53 & 2.2208 \\
\hline 2 & 2 & 71 & 5 & 118.53 & 1228.95 & 87.26 & 2.0738 \\
\hline 2 & 2 & 71 & 6 & 87.26 & 1316.21 & 61.38 & 1.9408 \\
\hline 2 & 2 & 71 & 7 & 61.38 & 1377.60 & 46.21 & 1.7881 \\
\hline 2 & 2 & 71 & 8 & 46.21 & 1423.80 & 35.34 & 1.6647 \\
\hline 2 & 2 & 71 & 9 & 35.34 & 1459.14 & 28.03 & 1.5483 \\
\hline 2 & 2 & 71 & 10 & 28.03 & 1487.18 & & 1.4476 \\
\hline 2 & 2 & 72 & 1 & 422.03 & 422.03 & 330.89 & 2.6253 \\
\hline 2 & 2 & 72 & 2 & 330.89 & 752.92 & 238.63 & 2.5197 \\
\hline 2 & 2 & 72 & 3 & 238.63 & 991.55 & 173.47 & 2.3777 \\
\hline 2 & 2 & 72 & 4 & 173.47 & 1165.01 & 121.45 & 2.2392 \\
\hline 2 & 2 & 72 & 5 & 121.45 & 1286.46 & 89.31 & 2.0844 \\
\hline 2 & 2 & 72 & 6 & 89.31 & 1375.77 & 65.06 & 1.9509 \\
\hline 2 & 2 & 72 & 7 & 65.06 & 1440.83 & 49.23 & 1.8133 \\
\hline 2 & 2 & 72 & 8 & 49.23 & 1490.05 & 37.16 & 1.6922 \\
\hline 2 & 2 & 72 & 9 & 37.16 & 1527.21 & 28.44 & 1.5701 \\
\hline 2 & 2 & 72 & 10 & 28.44 & 1555.65 & & 1.4540 \\
\hline 2 & 3.5 & 70 & 1 & 704.85 & 704.85 & 545.71 & 2.8481 \\
\hline 2 & 3.5 & 70 & 2 & 545.71 & 1250.56 & 393.49 & 2.7370 \\
\hline 2 & 3.5 & 70 & 3 & 393.49 & 1644.05 & 281.73 & 2.5949 \\
\hline 2 & 3.5 & 70 & 4 & 281.73 & 1925.78 & 204.05 & 2.4498 \\
\hline
\end{tabular}


Table B2. Ammonia Test Results

(continued)

\begin{tabular}{|c|c|}
\hline Study & $\begin{array}{c}\text { Test } \\
\text { Sequence }\end{array}$ \\
\hline 1 & 27 \\
\hline 1 & 27 \\
\hline 1 & 27 \\
\hline 1 & 27 \\
\hline 1 & 27 \\
\hline 1 & 27 \\
\hline 1 & 28 \\
\hline 1 & 28 \\
\hline 1 & 28 \\
\hline 1 & 28 \\
\hline 1 & 28 \\
\hline 1 & 28 \\
\hline 1 & 28 \\
\hline 1 & 28 \\
\hline 1 & 28 \\
\hline 1 & 28 \\
\hline 1 & 29 \\
\hline 1 & 29 \\
\hline 1 & 29 \\
\hline 1 & 29 \\
\hline 1 & 29 \\
\hline 1 & 29 \\
\hline 1 & 29 \\
\hline 1 & 29 \\
\hline 1 & 29 \\
\hline 1 & 29 \\
\hline 1 & 30 \\
\hline 1 & 30 \\
\hline 1 & 30 \\
\hline 1 & 30 \\
\hline 1 & 30 \\
\hline 1 & 30 \\
\hline 1 & 30 \\
\hline 1 & 30 \\
\hline 1 & 30 \\
\hline 1 & 30 \\
\hline 1 & 31 \\
\hline 1 & 31 \\
\hline 1 & 31 \\
\hline 1 & 31 \\
\hline 1 & 31 \\
\hline 1 & 31 \\
\hline 1 & 31 \\
\hline 1 & 31 \\
\hline 1 & 31 \\
\hline 1 & 31 \\
\hline 1 & 32 \\
\hline 1 & 32 \\
\hline 1 & 32 \\
\hline 1 & 32 \\
\hline 1 & 32 \\
\hline 1 & 32 \\
\hline 1 & 32 \\
\hline 1 & 32 \\
\hline 1 & 32 \\
\hline 1 & 32 \\
\hline 1 & 33 \\
\hline 1 & 33 \\
\hline 1 & 33 \\
\hline 1 & 33 \\
\hline 1 & 33 \\
\hline 1 & 33 \\
\hline 1 & 33 \\
\hline 1 & 33 \\
\hline 1 & 33 \\
\hline 1 & 33 \\
\hline
\end{tabular}

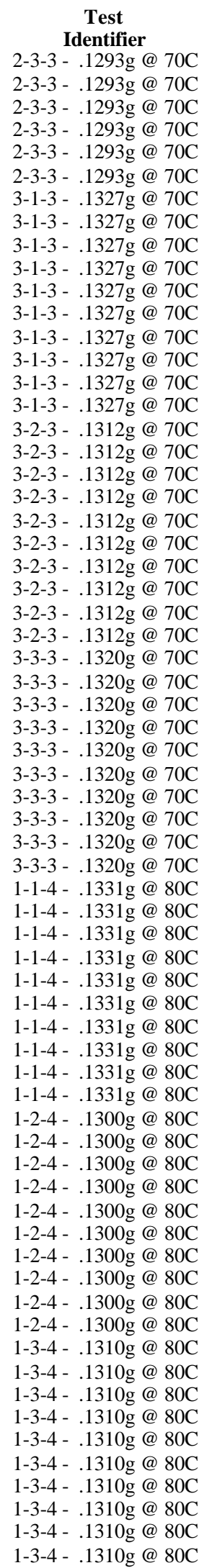

\begin{tabular}{|c|c|c|c|c|c|c|c|}
\hline $\begin{array}{c}\text { Salt } \\
\text { Solution }\end{array}$ & $\begin{array}{c}\mathrm{NH}_{3} \text { Conc } \\
\text { (molal) }\end{array}$ & $\begin{array}{c}\text { Temperature } \\
\left({ }^{\circ} \mathbf{C}\right)\end{array}$ & $\begin{array}{l}\text { Sampling } \\
\text { Sequence }\end{array}$ & $\begin{array}{c}\text { A } \\
\text { (peak area) }\end{array}$ & $\mathbf{y}$ & $\mathbf{x}$ & $\log (\mathbf{A})$ \\
\hline 2 & 3.5 & 70 & 5 & 204.05 & 2129.83 & 144.00 & 2.3097 \\
\hline 2 & 3.5 & 70 & 6 & 144.00 & 2273.83 & 102.25 & 2.1584 \\
\hline 2 & 3.5 & 70 & 7 & 102.25 & 2376.08 & 74.19 & 2.0097 \\
\hline 2 & 3.5 & 70 & 8 & 74.19 & 2450.28 & 56.05 & 1.8704 \\
\hline 2 & 3.5 & 70 & 9 & 56.05 & 2506.33 & 42.14 & 1.7486 \\
\hline 2 & 3.5 & 70 & 10 & 42.14 & 2548.47 & & 1.6247 \\
\hline 3 & 0.5 & 70 & 1 & 14.98 & 14.98 & 10.06 & 1.1756 \\
\hline 3 & 0.5 & 70 & 2 & 10.06 & 25.04 & 8.51 & 1.0024 \\
\hline 3 & 0.5 & 70 & 3 & 8.51 & 33.55 & 7.22 & 0.9300 \\
\hline 3 & 0.5 & 70 & 4 & 7.22 & 40.77 & 7.41 & 0.8587 \\
\hline 3 & 0.5 & 70 & 5 & 7.41 & 48.18 & 8.11 & 0.8696 \\
\hline 3 & 0.5 & 70 & 6 & 8.11 & 56.29 & 7.94 & 0.9092 \\
\hline 3 & 0.5 & 70 & 7 & 7.94 & 64.23 & 6.48 & 0.8997 \\
\hline 3 & 0.5 & 70 & 8 & 6.48 & 70.71 & 8.94 & 0.8117 \\
\hline 3 & 0.5 & 70 & 9 & 8.94 & 79.66 & 5.34 & 0.9515 \\
\hline 3 & 0.5 & 70 & 10 & 5.34 & 85.00 & & 0.7276 \\
\hline 3 & 2 & 70 & 1 & 26.05 & 26.05 & 26.09 & 1.4159 \\
\hline 3 & 2 & 70 & 2 & 26.09 & 52.14 & 21.38 & 1.4164 \\
\hline 3 & 2 & 70 & 3 & 21.38 & 73.52 & 17.29 & 1.3300 \\
\hline 3 & 2 & 70 & 4 & 17.29 & 90.82 & 11.89 & 1.2378 \\
\hline 3 & 2 & 70 & 5 & 11.89 & 102.70 & 9.56 & 1.0751 \\
\hline 3 & 2 & 70 & 6 & 9.56 & 112.26 & 9.08 & 0.9803 \\
\hline 3 & 2 & 70 & 7 & 9.08 & 121.33 & 9.11 & 0.9578 \\
\hline 3 & 2 & 70 & 8 & 9.11 & 130.44 & 8.67 & 0.9594 \\
\hline 3 & 2 & 70 & 9 & 8.67 & 139.11 & 7.75 & 0.9382 \\
\hline 3 & 2 & 70 & 10 & 7.75 & 146.86 & & 0.8890 \\
\hline 3 & 3.5 & 70 & 1 & 37.65 & 37.65 & 37.34 & 1.5757 \\
\hline 3 & 3.5 & 70 & 2 & 37.34 & 74.99 & 29.44 & 1.5722 \\
\hline 3 & 3.5 & 70 & 3 & 29.44 & 104.43 & 22.00 & 1.4690 \\
\hline 3 & 3.5 & 70 & 4 & 22.00 & 126.43 & 18.13 & 1.3424 \\
\hline 3 & 3.5 & 70 & 5 & 18.13 & 144.56 & 13.80 & 1.2584 \\
\hline 3 & 3.5 & 70 & 6 & 13.80 & 158.36 & 11.40 & 1.1398 \\
\hline 3 & 3.5 & 70 & 7 & 11.40 & 169.76 & 10.68 & 1.0569 \\
\hline 3 & 3.5 & 70 & 8 & 10.68 & 180.44 & 7.80 & 1.0285 \\
\hline 3 & 3.5 & 70 & 9 & 7.80 & 188.23 & 7.66 & 0.8920 \\
\hline 3 & 3.5 & 70 & 10 & 7.66 & 195.89 & & 0.8842 \\
\hline 1 & 0.5 & 80 & 1 & 188.40 & 188.40 & 172.45 & 2.2751 \\
\hline 1 & 0.5 & 80 & 2 & 172.45 & 360.85 & 136.62 & 2.2367 \\
\hline 1 & 0.5 & 80 & 3 & 136.62 & 497.47 & 107.56 & 2.1355 \\
\hline 1 & 0.5 & 80 & 4 & 107.56 & 605.04 & 86.08 & 2.0317 \\
\hline 1 & 0.5 & 80 & 5 & 86.08 & 691.12 & 67.40 & 1.9349 \\
\hline 1 & 0.5 & 80 & 6 & 67.40 & 758.52 & 54.44 & 1.8287 \\
\hline 1 & 0.5 & 80 & 7 & 54.44 & 812.96 & 45.18 & 1.7359 \\
\hline 1 & 0.5 & 80 & 8 & 45.18 & 858.14 & 36.96 & 1.6550 \\
\hline 1 & 0.5 & 80 & 9 & 36.96 & 895.10 & 31.67 & 1.5677 \\
\hline 1 & 0.5 & 80 & 10 & 31.67 & 926.77 & & 1.5007 \\
\hline 1 & 2 & 80 & 1 & 1011.43 & 1011.43 & 838.21 & 3.0049 \\
\hline 1 & 2 & 80 & 2 & 838.21 & 1849.64 & 654.57 & 2.9234 \\
\hline 1 & 2 & 80 & 3 & 654.57 & 2504.22 & 505.63 & 2.8160 \\
\hline 1 & 2 & 80 & 4 & 505.63 & 3009.84 & 390.61 & 2.7038 \\
\hline 1 & 2 & 80 & 5 & 390.61 & 3400.45 & 303.03 & 2.5917 \\
\hline 1 & 2 & 80 & 6 & 303.03 & 3703.49 & 237.21 & 2.4815 \\
\hline 1 & 2 & 80 & 7 & 237.21 & 3940.69 & 185.16 & 2.3751 \\
\hline 1 & 2 & 80 & 8 & 185.16 & 4125.85 & 143.07 & 2.2675 \\
\hline 1 & 2 & 80 & 9 & 143.07 & 4268.92 & 110.43 & 2.1556 \\
\hline 1 & 2 & 80 & 10 & 110.43 & 4379.35 & & 2.0431 \\
\hline 1 & 3.5 & 80 & 1 & 1967.45 & 1967.45 & 1599.65 & 3.2939 \\
\hline 1 & 3.5 & 80 & 2 & 1599.65 & 3567.10 & 1234.94 & 3.2040 \\
\hline 1 & 3.5 & 80 & 3 & 1234.94 & 4802.05 & 940.98 & 3.0916 \\
\hline 1 & 3.5 & 80 & 4 & 940.98 & 5743.02 & 718.07 & 2.9736 \\
\hline 1 & 3.5 & 80 & 5 & 718.07 & 6461.09 & 550.26 & 2.8562 \\
\hline 1 & 3.5 & 80 & 6 & 550.26 & 7011.35 & 422.23 & 2.7406 \\
\hline 1 & 3.5 & 80 & 7 & 422.23 & 7433.58 & 319.99 & 2.6255 \\
\hline 1 & 3.5 & 80 & 8 & 319.99 & 7753.56 & 246.21 & 2.5051 \\
\hline 1 & 3.5 & 80 & 9 & 246.21 & 7999.77 & 188.57 & 2.3913 \\
\hline 1 & 3.5 & 80 & 10 & 188.57 & 8188.34 & & 2.2755 \\
\hline
\end{tabular}


Table B2. Ammonia Test Results

(continued)

Test

Study Sequence

$1 \quad 34 \quad 2-1-4-.1305 \mathrm{~g} @ 80 \mathrm{C}$

2-1-4 -.1305g@80C

2-1-4 -.1305g@80C

2-1-4 -.1305g@80C

2-1-4 - .1305g@ 80C

2-1-4 - .1305g@80C

2-1-4 - .1305g@ 80C

2-1-4 - .1305g@80C

2-1-4 - .1305g@ 80C

2-1-4 - .1305g@80C

2-2-4a - .1293g@80C

2-2-4a - .1293g @ 80C

2-2-4a - .1293g@ 80C

$2-2-4 \mathrm{a}-.1293 \mathrm{~g} @ 80 \mathrm{C}$

2-2-4a - .1293g @ 80C

2-2-4a - .1293g@80C

2-2-4a - .1293g @ 80C

2-2-4a - .1293g@80C

2-2-4B - .1292g@80C

2-2-4B - .1292g@80C

2-2-4B - .1292g@80C

2-2-4B - .1292g @ 80C

2-2-4B - .1292g @ 80C

2-2-4B - .1292g@80C

2-2-4B - .1292g @ 80C

2-2-4B - .1292g @ 80C

2-2-4B - .1292g @ 80C

2-2-4B - .1292g @ 80C

2-3-4 - .1286g@80C

2-3-4 - .1286g@80C

2-3-4 - .1286g @ 80C

2-3-4 - .1286g @ 80C

2-3-4 - .1286g @ 80C

2-3-4 - .1286g @ 80C

2-3-4 - .1286g @ 80C

2-3-4 - .1286g@80C

2-3-4 - .1286g@80C

2-3-4 - .1286g@80C

3-1-4 - .1326g@80C

3-1-4 - .1326g@80C

3-1-4 - .1326g @ 80C

3-1-4 - .1326g @ 80C

$3-1-4$ - .1326g @ 80C

3-1-4 - .1326g @ 80C

3-1-4 - .1326g @ 80C

3-1-4 - .1326g @ 80C

$3-1-4$ - .1326g @ 80C

3-1-4 - .1326g @ 80C

3-2-4 - .1315g@80C

3-2-4 - .1315g@80C

3-2-4 - .1315g @ 80C

3-2-4 - .1315g @ 80C

3-2-4 - .1315g @ 80C

3-2-4 - .1315g @ 80C

3-2-4 - .1315g@80C

3-2-4 - .1315g@80C

3-2-4 - .1315g @ 80C

3-2-4 - .1315g @ 80C

3-3-4 - .1312g @ 80C

3-3-4 - .1312g @ 80C

3-3-4 - .1312g @ 80C

3-3-4 - .1312g @ 80C

3-3-4 - .1312g @ 80C

3-3-4 - .1312g @ 80C

3-3-4 - .1312g @ 80C

3-3-4 - .1312g@80C

\begin{tabular}{|c|c|c|c|c|c|c|c|}
\hline $\begin{array}{c}\text { Salt } \\
\text { Solution }\end{array}$ & $\underset{(\text { molal })}{\mathrm{NH}_{3} \text { Conc }}$ & $\begin{array}{c}\text { Temperature } \\
\left({ }^{\circ} \mathrm{C}\right)\end{array}$ & $\begin{array}{l}\text { Sampling } \\
\text { Sequence }\end{array}$ & $\underset{\text { (peak area) }}{\mathbf{A}}$ & $\mathbf{y}$ & $\mathbf{x}$ & $\log (A)$ \\
\hline 2 & 0.5 & & 1 & 117.01 & 117.01 & 93.74 & 2.0682 \\
\hline 2 & 0.5 & 80 & 2 & 93.74 & 210.75 & 71.79 & 1.9719 \\
\hline 2 & 0.5 & 80 & 3 & 71.79 & 282.54 & 56.62 & 1.8561 \\
\hline 2 & 0.5 & 80 & 4 & 56.62 & 339.16 & 44.02 & 1.7530 \\
\hline 2 & 0.5 & 80 & 5 & 44.02 & 383.19 & 36.68 & 1.6437 \\
\hline 2 & 0.5 & 80 & 6 & 36.68 & 419.87 & 31.35 & 1.5645 \\
\hline 2 & 0.5 & 80 & 7 & 31.35 & 451.22 & 24.42 & 1.4963 \\
\hline 2 & 0.5 & 80 & 8 & 24.42 & 475.65 & 20.40 & 1.3878 \\
\hline 2 & 0.5 & 80 & 9 & 20.40 & 496.05 & 17.08 & 1.3097 \\
\hline 2 & 0.5 & 80 & 10 & 17.08 & 513.13 & & 1.2326 \\
\hline 2 & 2 & 81 & 1 & 386.40 & 386.40 & 325.81 & 2.5870 \\
\hline 2 & 2 & 81 & 2 & 325.81 & 712.22 & 255.58 & 2.5130 \\
\hline 2 & 2 & 81 & 3 & 255.58 & 967.79 & 194.85 & 2.4075 \\
\hline 2 & 2 & 81 & 4 & 194.85 & 1162.64 & 150.95 & 2.2897 \\
\hline 2 & 2 & 81 & 5 & 150.95 & 1313.59 & 117.14 & 2.1788 \\
\hline 2 & 2 & 81 & 6 & 117.14 & 1430.73 & 86.55 & 2.0687 \\
\hline 2 & 2 & 81 & 7 & 86.55 & 1517.27 & 40.84 & 1.9372 \\
\hline 2 & 2 & 81 & 8 & 40.84 & 1558.12 & 450.83 & 1.6111 \\
\hline 2 & 2 & 80 & 1 & 450.83 & 450.83 & 381.67 & 2.6540 \\
\hline 2 & 2 & 80 & 2 & 381.67 & 832.49 & 296.05 & 2.5817 \\
\hline 2 & 2 & 80 & 3 & 296.05 & 1128.54 & 232.11 & 2.4714 \\
\hline 2 & 2 & 80 & 4 & 232.11 & 1360.65 & 179.13 & 2.3657 \\
\hline 2 & 2 & 80 & 5 & 179.13 & 1539.78 & 139.88 & 2.2532 \\
\hline 2 & 2 & 80 & 6 & 139.88 & 1679.66 & 106.42 & 2.1457 \\
\hline 2 & 2 & 80 & 7 & 106.42 & 1786.07 & 81.55 & 2.0270 \\
\hline 2 & 2 & 80 & 8 & 81.55 & 1867.62 & 64.36 & 1.9114 \\
\hline 2 & 2 & 80 & 9 & 64.36 & 1931.99 & 53.49 & 1.8086 \\
\hline 2 & 2 & 80 & 10 & 53.49 & 1985.48 & & 1.7283 \\
\hline 2 & 3.5 & 80 & 1 & 723.71 & 723.71 & 601.57 & 2.8596 \\
\hline 2 & 3.5 & 80 & 2 & 601.57 & 1325.28 & 465.87 & 2.7793 \\
\hline 2 & 3.5 & 80 & 3 & 465.87 & 1791.15 & 355.60 & 2.6683 \\
\hline 2 & 3.5 & 80 & 4 & 355.60 & 2146.75 & 275.23 & 2.5510 \\
\hline 2 & 3.5 & 80 & 5 & 275.23 & 2421.97 & 212.67 & 2.4397 \\
\hline 2 & 3.5 & 80 & 6 & 212.67 & 2634.65 & 165.66 & 2.3277 \\
\hline 2 & 3.5 & 80 & 7 & 165.66 & 2800.30 & 124.26 & 2.2192 \\
\hline 2 & 3.5 & 80 & 8 & 124.26 & 2924.56 & 99.69 & 2.0943 \\
\hline 2 & 3.5 & 80 & 9 & 99.69 & 3024.25 & 73.65 & 1.9987 \\
\hline 2 & 3.5 & 80 & 10 & 73.65 & 3097.90 & & 1.8672 \\
\hline 3 & 0.5 & 80 & 1 & 23.35 & 23.35 & 15.56 & 1.3683 \\
\hline 3 & 0.5 & 80 & 2 & 15.56 & 38.91 & 13.82 & 1.1919 \\
\hline 3 & 0.5 & 80 & 3 & 13.82 & 52.73 & 8.62 & 1.1405 \\
\hline 3 & 0.5 & 80 & 4 & 8.62 & 61.34 & 10.45 & 0.9353 \\
\hline 3 & 0.5 & 80 & 5 & 10.45 & 71.79 & 8.75 & 1.0189 \\
\hline 3 & 0.5 & 80 & 6 & 8.75 & 80.54 & 8.63 & 0.9419 \\
\hline 3 & 0.5 & 80 & 7 & 8.63 & 89.16 & 8.16 & 0.9358 \\
\hline 3 & 0.5 & 80 & 8 & 8.16 & 97.32 & 8.17 & 0.9116 \\
\hline 3 & 0.5 & 80 & 9 & 8.17 & 105.49 & 7.46 & 0.9123 \\
\hline 3 & 0.5 & 80 & 10 & 7.46 & 112.95 & & 0.8724 \\
\hline 3 & 2 & 80 & 1 & 22.92 & 22.92 & 28.49 & 1.3602 \\
\hline 3 & 2 & 80 & 2 & 28.49 & 51.41 & 26.97 & 1.4547 \\
\hline 3 & 2 & 80 & 3 & 26.97 & 78.38 & 22.46 & 1.4309 \\
\hline 3 & 2 & 80 & 4 & 22.46 & 100.84 & 18.64 & 1.3513 \\
\hline 3 & 2 & 80 & 5 & 18.64 & 119.48 & 17.90 & 1.2705 \\
\hline 3 & 2 & 80 & 6 & 17.90 & 137.38 & 16.18 & 1.2530 \\
\hline 3 & 2 & 80 & 7 & 16.18 & 153.56 & 12.40 & 1.2089 \\
\hline 3 & 2 & 80 & 8 & 12.40 & 165.96 & 11.23 & 1.0934 \\
\hline 3 & 2 & 80 & 9 & 11.23 & 177.18 & 11.96 & 1.0502 \\
\hline 3 & 2 & 80 & 10 & 11.96 & 189.15 & & 1.0779 \\
\hline 3 & 3.5 & 80 & 1 & 39.27 & 39.27 & 40.79 & 1.5940 \\
\hline 3 & 3.5 & 80 & 2 & 40.79 & 80.06 & 35.89 & 1.6106 \\
\hline 3 & 3.5 & 80 & 3 & 35.89 & 115.95 & 30.96 & 1.5550 \\
\hline 3 & 3.5 & 80 & 4 & 30.96 & 146.91 & 25.42 & 1.4908 \\
\hline 3 & 3.5 & 80 & 5 & 25.42 & 172.32 & 23.70 & 1.4051 \\
\hline 3 & 3.5 & 80 & 6 & 23.70 & 196.03 & 19.19 & 1.3748 \\
\hline 3 & 3.5 & 80 & 7 & 19.19 & 215.21 & 16.62 & 1.2830 \\
\hline 3 & 3.5 & 80 & 8 & 16.62 & 231.83 & 14.94 & 1.2207 \\
\hline
\end{tabular}


Table B2. Ammonia Test Results

(continued)

Test

Study Sequence

$1 \quad 40$

3-3-4 - .1312g@80C

1-1-5 - .1331g@90C

1-1-5 -.1331g@90C

$1-1-5-.1331 \mathrm{~g} @ 90 \mathrm{C}$

1-1-5 - .1331g@90C

1-1-5 - .1331g@90C

1-1-5 - .1331g@90C

1-1-5 - .1331g@90C

1-1-5 - .1331g@90C

1-1-5 -.1331g@90C

1-1-5 - .1331g@90C

1-2-5 - .1313g@90C

$1-2-5-.1313 \mathrm{~g} @ 90 \mathrm{C}$

1-2-5 - .1313g@90C

$1-2-5-.1313 \mathrm{~g} @ 90 \mathrm{C}$

1-2-5 - .1313g@90C

$1-2-5-.1313 \mathrm{~g} @ 90 \mathrm{C}$

1-2-5 - .1313g@90C

1-2-5 - .1313g@90C

1-2-5 - .1313g@90C

1-2-5 - .1313g @ 90C

1-3-5 - .1303g@90C

1-3-5 - .1303g@90C

1-3-5 - .1303g@90C

1-3-5 - .1303g@90C

1-3-5 - .1303g @ 90C

1-3-5 - .1303g@90C

1-3-5 - .1303g@90C

1-3-5 - .1303g@90C

1-3-5 - .1303g @ 90C

1-3-5 - .1303g@90C

2-1-5 - .1303g@90C

$2-1-5$ - .1303g @ 90C

2-1-5 - .1303g @ 90C

2-1-5 - .1303g@90C

2-1-5 - .1303g @ 90C

2-1-5 - .1303g@ @0C

2-1-5 - .1303g @ 90C

2-1-5 - .1303g@90C

2-1-5 - .1303g@90C

2-1-5 - .1303g@90C

2-2-5A - .1292g @ 90C

2-2-5A - .1292g @ 90C

2-2-5A - .1292g @ 90C

2-2-5A - .1292g @ 90C

2-2-5A - .1292g @ 90C

2-2-5A - .1292g @ 90C

2-2-5A - .1292g @ 90C

$2-2-5 \mathrm{~A}-.1292 \mathrm{~g} @ 90 \mathrm{C}$

2-2-5A - .1292g @ 90C

$2-2-5 \mathrm{~A}-.1292 \mathrm{~g} @ 90 \mathrm{C}$

2-2-5B - .1291g @ 90C

2-2-5B - .1291g@ 90C

2-2-5B - .1291g @ 90C

2-2-5B - .1291g @ 90C

2-2-5B - .1291g @ 90C

2-2-5B - .1291g@ 90C

2-2-5B - .1291g@90C

2-2-5B - .1291g@ 90C

2-2-5B - .1291g @ 90C

2-2-5B - .1291g @ 90C

2-3-5 - .1292g@90C

2-3-5 -.1292g@90C

2-3-5 - .1292g @ 90C

2-3-5 - .1292g@90C

\begin{tabular}{|c|c|c|c|c|c|c|c|}
\hline $\begin{array}{c}\text { Salt } \\
\text { Solution }\end{array}$ & $\begin{array}{c}\mathrm{NH}_{3} \text { Conc } \\
\text { (molal) }\end{array}$ & $\begin{array}{c}\text { Temperature } \\
\left({ }^{\circ} \mathbf{C}\right)\end{array}$ & $\begin{array}{l}\text { Sampling } \\
\text { Sequence }\end{array}$ & $\underset{\text { (peak area) }}{\mathbf{A}}$ & $\mathbf{y}_{77}$ & $x$ & $\log (\mathrm{A})$ \\
\hline 3 & 3.5 & 80 & 9 & 14.94 & 246.77 & 13.22 & 1.1742 \\
\hline 3 & 3.5 & 80 & 10 & 13.22 & 259.99 & & 1.1211 \\
\hline 1 & 0.5 & 90 & 1 & 226.69 & 226.69 & 187.36 & 2.3554 \\
\hline 1 & 0.5 & 90 & 2 & 187.36 & 414.05 & 139.38 & 2.2727 \\
\hline 1 & 0.5 & 90 & 3 & 139.38 & 553.43 & 103.77 & 2.1442 \\
\hline 1 & 0.5 & 90 & 4 & 103.77 & 657.20 & 75.83 & 2.0161 \\
\hline 1 & 0.5 & 90 & 5 & 75.83 & 733.02 & 54.71 & 1.8798 \\
\hline 1 & 0.5 & 90 & 6 & 54.71 & 787.73 & 47.17 & 1.7381 \\
\hline 1 & 0.5 & 90 & 7 & 47.17 & 834.90 & 39.85 & 1.6737 \\
\hline 1 & 0.5 & 90 & 8 & 39.85 & 874.76 & 31.55 & 1.6005 \\
\hline 1 & 0.5 & 90 & 9 & 31.55 & 906.30 & 25.80 & 1.4989 \\
\hline 1 & 0.5 & 90 & 10 & 25.80 & 932.11 & & 1.4117 \\
\hline 1 & 2 & 90 & 1 & 1302.00 & 1302.00 & 1007.00 & 3.1146 \\
\hline 1 & 2 & 90 & 2 & 1007.00 & 2309.00 & 738.00 & 3.0030 \\
\hline 1 & 2 & 90 & 3 & 738.00 & 3047.00 & 535.00 & 2.8681 \\
\hline 1 & 2 & 90 & 4 & 535.00 & 3582.00 & 386.00 & 2.7284 \\
\hline 1 & 2 & 90 & 5 & 386.00 & 3968.00 & 279.00 & 2.5866 \\
\hline 1 & 2 & 90 & 6 & 279.00 & 4247.00 & 206.66 & 2.4456 \\
\hline 1 & 2 & 90 & 7 & 206.66 & 4453.66 & 149.68 & 2.3153 \\
\hline 1 & 2 & 90 & 8 & 149.68 & 4603.34 & 108.45 & 2.1752 \\
\hline 1 & 2 & 90 & 9 & 108.45 & 4711.79 & 81.69 & 2.0352 \\
\hline 1 & 2 & 90 & 10 & 81.69 & 4793.48 & & 1.9122 \\
\hline 1 & 3.5 & 90 & 1 & 2175.00 & 2175.00 & 1657.00 & 3.3375 \\
\hline 1 & 3.5 & 90 & 2 & 1657.00 & 3832.00 & 1193.00 & 3.2193 \\
\hline 1 & 3.5 & 90 & 3 & 1193.00 & 5025.00 & 856.00 & 3.0766 \\
\hline 1 & 3.5 & 90 & 4 & 856.00 & 5881.00 & 617.00 & 2.9325 \\
\hline 1 & 3.5 & 90 & 5 & 617.00 & 6498.00 & 444.00 & 2.7903 \\
\hline 1 & 3.5 & 90 & 6 & 444.00 & 6942.00 & 320.00 & 2.6474 \\
\hline 1 & 3.5 & 90 & 7 & 320.00 & 7262.00 & 230.68 & 2.5052 \\
\hline 1 & 3.5 & 90 & 8 & 230.68 & 7492.68 & 168.84 & 2.3630 \\
\hline 1 & 3.5 & 90 & 9 & 168.84 & 7661.52 & 123.28 & 2.2275 \\
\hline 1 & 3.5 & 90 & 10 & 123.28 & 7784.80 & . & 2.0909 \\
\hline 2 & 0.5 & 90 & 1 & 118.37 & 118.37 & 88.61 & 2.0732 \\
\hline 2 & 0.5 & 90 & 2 & 88.61 & 206.99 & 69.52 & 1.9475 \\
\hline 2 & 0.5 & 90 & 3 & 69.52 & 276.51 & 53.73 & 1.8421 \\
\hline 2 & 0.5 & 90 & 4 & 53.73 & 330.24 & 44.84 & 1.7302 \\
\hline 2 & 0.5 & 90 & 5 & 44.84 & 375.08 & 35.49 & 1.6517 \\
\hline 2 & 0.5 & 90 & 6 & 35.49 & 410.57 & 26.59 & 1.5501 \\
\hline 2 & 0.5 & 90 & 7 & 26.59 & 437.15 & 25.85 & 1.4246 \\
\hline 2 & 0.5 & 90 & 8 & 25.85 & 463.00 & 21.77 & 1.4125 \\
\hline 2 & 0.5 & 90 & 9 & 21.77 & 484.77 & 16.88 & 1.3378 \\
\hline 2 & 0.5 & 90 & 10 & 16.88 & 501.65 & & 1.2275 \\
\hline 2 & 2 & 90 & 1 & 564.00 & 564.00 & 452.00 & 2.7513 \\
\hline 2 & 2 & 90 & 2 & 452.00 & 1016.00 & 329.06 & 2.6551 \\
\hline 2 & 2 & 90 & 3 & 329.06 & 1345.06 & 237.77 & 2.5173 \\
\hline 2 & 2 & 90 & 4 & 237.77 & 1582.84 & 170.58 & 2.3762 \\
\hline 2 & 2 & 90 & 5 & 170.58 & 1753.42 & 123.34 & 2.2319 \\
\hline 2 & 2 & 90 & 6 & 123.34 & 1876.76 & 90.67 & 2.0911 \\
\hline 2 & 2 & 90 & 7 & 90.67 & 1967.42 & 69.40 & 1.9574 \\
\hline 2 & 2 & 90 & 8 & 69.40 & 2036.82 & 55.38 & 1.8413 \\
\hline 2 & 2 & 90 & 9 & 55.38 & 2092.20 & 44.79 & 1.7434 \\
\hline 2 & 2 & 90 & 10 & 44.79 & 2136.99 & & 1.6512 \\
\hline 2 & 2 & 91 & 1 & 568.00 & 568.00 & 441.00 & 2.7543 \\
\hline 2 & 2 & 91 & 2 & 441.00 & 1009.00 & 323.00 & 2.6444 \\
\hline 2 & 2 & 91 & 3 & 323.00 & 1332.00 & 233.15 & 2.5092 \\
\hline 2 & 2 & 91 & 4 & 233.15 & 1565.15 & 168.11 & 2.3676 \\
\hline 2 & 2 & 91 & 5 & 168.11 & 1733.26 & 122.48 & 2.2256 \\
\hline 2 & 2 & 91 & 6 & 122.48 & 1855.74 & 85.11 & 2.0881 \\
\hline 2 & 2 & 91 & 7 & 85.11 & 1940.85 & 67.41 & 1.9300 \\
\hline 2 & 2 & 91 & 8 & 67.41 & 2008.26 & 55.07 & 1.8287 \\
\hline 2 & 2 & 91 & 9 & 55.07 & 2063.33 & 43.98 & 1.7409 \\
\hline 2 & 2 & 91 & 10 & 43.98 & 2107.31 & & 1.6432 \\
\hline 2 & 3.5 & 90 & 1 & 955.00 & 955.00 & 743.00 & 2.9800 \\
\hline 2 & 3.5 & 90 & 2 & 743.00 & 1698.00 & 540.00 & 2.8710 \\
\hline 2 & 3.5 & 90 & 3 & 540.00 & 2238.00 & 387.00 & 2.7324 \\
\hline 2 & 3.5 & 90 & 4 & 387.00 & 2625.00 & 276.00 & 2.5877 \\
\hline
\end{tabular}


Table B2. Ammonia Test Results

(continued)

Test

Study Sequence

1 S 47

$2-3-5-.1292 \mathrm{~g} @ 90 \mathrm{C}$

2-3-5 - .1292g@90C

$2-3-5$ - .1292g@90C

2-3-5 - .1292g @ 90C

$2-3-5-.1292 \mathrm{~g} @ 90 \mathrm{C}$

2-3-5 - .1292g@90C

3-1-5 - .1318g@90C

$3-1-5$ - .1318g@90C

$3-1-5-.1318 \mathrm{~g} @ 90 \mathrm{C}$

$3-1-5-.1318 \mathrm{~g} @ 90 \mathrm{C}$

$3-1-5-.1318 \mathrm{~g} @ 90 \mathrm{C}$

3-1-5 - .1318g@90C

3-1-5 - .1318g@90C

3-1-5 - .1318g@90C

$3-1-5$ - .1318g@90C

$3-1-5-.1318 \mathrm{~g} @ 90 \mathrm{C}$

3-2-5 - .1306g@90C

$3-2-5$ - .1306g @ 90C

3-2-5 - .1306g@90C

$3-2-5$ - .1306g@90C

3-2-5 - .1306g@90C

$3-2-5$ - .1306g @ 90C

3-2-5 - .1306g@90C

$3-2-5$ - .1306g @ 90C

3-2-5 - .1306g@90C

$3-2-5$ - .1306g@90C

3-3-5 - .1320g @ 90C

3-3-5 - .1320g @ 90C

3-3-5 - .1320g @ 90C

3-3-5 - .1320g@90C

3-3-5 - .1320g@90C

3-3-5 - .1320g @ 90C

3-3-5 - .1320g@90C

3-3-5 - .1320g @ 90C

$3-3-5-.1320 \mathrm{~g} @ 90 \mathrm{C}$

3-3-5 - .1320g@90C

3-1-1-1, 0.1313g@ @0C

3-1-1-2, 0.1313g @ 50C

3-1-1-3, 0.1313g @ 50C

3-1-1-4, 0.1313g @ 50C

3-1-1-5, 0.1313g @ 50C

3-1-1-6, 0.1313g @ 50C

3-1-1-7, 0.1313g @ 50C

3-1-1-8, 0.1313g @ 50C

3-1-1-9, 0.1313g @ 50C

3-1-1-10,0.1313g @ 50C

3-2-1-1, 0.1308g@ 50C

3-2-1-2, 0.1308g @ 50C

3-2-1-3, 0.1308g@ @0C

3-2-1-4, 0.1308g@ 50C

3-2-1-5, 0.1308g @ 50C

3-2-1-6, 0.1308g @ 50C

3-2-1-7, 0.1308g @ 50C

3-2-1-8, 0.1308g @ 50C

3-2-1-9, 0.1308g @ 50C

3-2-1-10, 0.1308g @ 50C

3-3-1-1, 0.1308g@ @0C

3-3-1-2, 0.1308g@50C

3-3-1-3, 0.1308g @ 50C

3-3-1-4, 0.1308g @ 50C

3-3-1-5, 0.1308g@50C

3-3-1-6, 0.1308g @ 50C

3-3-1-7, 0.1308g@ @0C

3-3-1-8, 0.1308g@ 50C

3-3-1-9, 0.1308g @ 50C

3-3-1-10, 0.1308g @ 50C

\begin{tabular}{|c|c|c|c|c|c|c|c|}
\hline $\begin{array}{c}\text { Salt } \\
\text { Solution } \\
2\end{array}$ & $\begin{array}{c}\mathrm{NH}_{3} \text { Conc } \\
\text { (molal) } \\
3.5\end{array}$ & $\begin{array}{c}\text { Temperature } \\
\left({ }^{\mathbf{O}} \mathbf{C}\right) \\
90\end{array}$ & $\begin{array}{l}\text { Sampling } \\
\text { Sequence } \\
5\end{array}$ & $\begin{array}{c}\mathbf{A} \\
\text { (peak area) } \\
276.00\end{array}$ & $\begin{array}{c}\mathbf{y} \\
2901.00\end{array}$ & $\begin{array}{c}\mathbf{x} \\
198.07\end{array}$ & $\begin{array}{l}\log (\mathbf{A}) \\
2.4409\end{array}$ \\
\hline 2 & 3.5 & 90 & 6 & 198.07 & 3099.07 & 139.48 & 2.2968 \\
\hline 2 & 3.5 & 90 & 7 & 139.48 & 3238.55 & 100.66 & 2.1445 \\
\hline 2 & 3.5 & 90 & 8 & 100.66 & 3339.21 & 74.70 & 2.0029 \\
\hline 2 & 3.5 & 90 & 9 & 74.70 & 3413.91 & 59.42 & 1.8733 \\
\hline 2 & 3.5 & 90 & 10 & 59.42 & 3473.33 & & 1.7739 \\
\hline 3 & 0.5 & 90 & 1 & 26.58 & 26.58 & 23.02 & 1.4246 \\
\hline 3 & 0.5 & 90 & 2 & 23.02 & 49.61 & 18.76 & 1.3621 \\
\hline 3 & 0.5 & 90 & 3 & 18.76 & 68.37 & 15.17 & 1.2733 \\
\hline 3 & 0.5 & 90 & 4 & 15.17 & 83.54 & 14.56 & 1.1810 \\
\hline 3 & 0.5 & 90 & 5 & 14.56 & 98.10 & 13.49 & 1.1631 \\
\hline 3 & 0.5 & 90 & 6 & 13.49 & 111.59 & 15.56 & 1.1301 \\
\hline 3 & 0.5 & 90 & 7 & 15.56 & 127.15 & 12.15 & 1.1919 \\
\hline 3 & 0.5 & 90 & 8 & 12.15 & 139.30 & 16.90 & 1.0845 \\
\hline 3 & 0.5 & 90 & 9 & 16.90 & 156.20 & 15.35 & 1.2279 \\
\hline 3 & 0.5 & 90 & 10 & 15.35 & 171.55 & & 1.1861 \\
\hline 3 & 2 & 90 & 1 & 36.56 & 36.56 & 39.35 & 1.5629 \\
\hline 3 & 2 & 90 & 2 & 39.35 & 75.90 & 33.57 & 1.5949 \\
\hline 3 & 2 & 90 & 3 & 33.57 & 109.48 & 29.37 & 1.5260 \\
\hline 3 & 2 & 90 & 4 & 29.37 & 138.85 & 24.62 & 1.4679 \\
\hline 3 & 2 & 90 & 5 & 24.62 & 163.47 & 20.09 & 1.3913 \\
\hline 3 & 2 & 90 & 6 & 20.09 & 183.56 & 17.46 & 1.3030 \\
\hline 3 & 2 & 90 & 7 & 17.46 & 201.03 & 15.06 & 1.2421 \\
\hline 3 & 2 & 90 & 8 & 15.06 & 216.09 & 15.92 & 1.1779 \\
\hline 3 & 2 & 90 & 9 & 15.92 & 232.01 & 15.90 & 1.2019 \\
\hline 3 & 2 & 90 & 10 & 15.90 & 247.91 & & 1.2013 \\
\hline 3 & 3.5 & 90 & 1 & 59.14 & 59.14 & 57.27 & 1.7718 \\
\hline 3 & 3.5 & 90 & 2 & 57.27 & 116.41 & 46.87 & 1.7579 \\
\hline 3 & 3.5 & 90 & 3 & 46.87 & 163.28 & 39.27 & 1.6709 \\
\hline 3 & 3.5 & 90 & 4 & 39.27 & 202.55 & 30.65 & 1.5941 \\
\hline 3 & 3.5 & 90 & 5 & 30.65 & 233.19 & 26.99 & 1.4864 \\
\hline 3 & 3.5 & 90 & 6 & 26.99 & 260.19 & 21.40 & 1.4312 \\
\hline 3 & 3.5 & 90 & 7 & 21.40 & 281.58 & 19.25 & 1.3303 \\
\hline 3 & 3.5 & 90 & 8 & 19.25 & 300.83 & 17.09 & 1.2845 \\
\hline 3 & 3.5 & 90 & 9 & 17.09 & 317.92 & 14.06 & 1.2328 \\
\hline 3 & 3.5 & 90 & 10 & 14.06 & 331.98 & & 1.1480 \\
\hline 3 & 0.5 & 50 & 1 & 19.52 & 19.52 & 19.12 & 1.2905 \\
\hline 3 & 0.5 & 50 & 2 & 19.12 & 38.64 & 15.99 & 1.2814 \\
\hline 3 & 0.5 & 50 & 3 & 15.99 & 54.63 & 14.29 & 1.2038 \\
\hline 3 & 0.5 & 50 & 4 & 14.29 & 68.92 & 12.66 & 1.1551 \\
\hline 3 & 0.5 & 50 & 5 & 12.66 & 81.58 & 11.86 & 1.1024 \\
\hline 3 & 0.5 & 50 & 6 & 11.86 & 93.43 & 10.47 & 1.0740 \\
\hline 3 & 0.5 & 50 & 7 & 10.47 & 103.90 & 8.89 & 1.0198 \\
\hline 3 & 0.5 & 50 & 8 & 8.89 & 112.79 & 9.41 & 0.9489 \\
\hline 3 & 0.5 & 50 & 9 & 9.41 & 122.21 & 9.90 & 0.9738 \\
\hline 3 & 0.5 & 50 & 10 & 9.90 & 132.10 & . & 0.9955 \\
\hline 3 & 2 & 50 & 1 & 17.86 & 17.86 & 16.09 & 1.2518 \\
\hline 3 & 2 & 50 & 2 & 16.09 & 33.95 & 15.31 & 1.2067 \\
\hline 3 & 2 & 50 & 3 & 15.31 & 49.26 & 13.32 & 1.1849 \\
\hline 3 & 2 & 50 & 4 & 13.32 & 62.58 & 12.17 & 1.1244 \\
\hline 3 & 2 & 50 & 5 & 12.17 & 74.74 & 12.18 & 1.0851 \\
\hline 3 & 2 & 50 & 6 & 12.18 & 86.92 & 11.71 & 1.0856 \\
\hline 3 & 2 & 50 & 7 & 11.71 & 98.63 & 11.90 & 1.0685 \\
\hline 3 & 2 & 50 & 8 & 11.90 & 110.52 & 10.89 & 1.0754 \\
\hline 3 & 2 & 50 & 9 & 10.89 & 121.41 & 9.56 & 1.0370 \\
\hline 3 & 2 & 50 & 10 & 9.56 & 130.97 & & 0.9805 \\
\hline 3 & 3.5 & 50 & 1 & 9.83 & 9.83 & 8.94 & 0.9927 \\
\hline 3 & 3.5 & 50 & 2 & 8.94 & 18.77 & 7.14 & 0.9513 \\
\hline 3 & 3.5 & 50 & 3 & 7.14 & 25.91 & 7.52 & 0.8538 \\
\hline 3 & 3.5 & 50 & 4 & 7.52 & 33.43 & 6.98 & 0.8762 \\
\hline 3 & 3.5 & 50 & 5 & 6.98 & 40.41 & 5.87 & 0.8439 \\
\hline 3 & 3.5 & 50 & 6 & 5.87 & 46.28 & 5.09 & 0.7683 \\
\hline 3 & 3.5 & 50 & 7 & 5.09 & 51.37 & 6.40 & 0.7064 \\
\hline 3 & 3.5 & 50 & 8 & 6.40 & 57.77 & 6.70 & 0.8062 \\
\hline 3 & 3.5 & 50 & 9 & 6.70 & 64.47 & 5.18 & 0.8263 \\
\hline 3 & 3.5 & 50 & 10 & 5.18 & 69.65 & . & 0.7145 \\
\hline
\end{tabular}


Table B2. Ammonia Test Results

(continued)

Test
Identifier

3-1-2-1, 0.1321g @ 60C

3-1-2-2, 0.1321g@60C

3-1-2-3, 0.1321g @ 60C

3-1-2-4, 0.1321g@60C

3-1-2-5, 0.1321g @ 60C

3-1-2-6, 0.1321g@60C

3-1-2-7, 0.1321g @ 60C

3-1-2-8, 0.1321g@60C

3-1-2-9, 0.1321g @ 60C

3-1-2-10,0.1321g@60C

3-2-2-1, 0.1318g@60C

3-2-2-2,0.1318g @60C

3-2-2-3, 0.1318g @ 60C

3-2-2-4, 0.1318g@60C

3-2-2-5, 0.1318g@60C

3-2-2-6, 0.1318g@60C

3-2-2-7, 0.1318g@60C

3-2-2-8, 0.1318g@60C

3-2-2-9, 0.1318g@60C

3-2-2-10, 0.1318g@60C

3-3-2-1, 0.1309g @ 60C

3-3-2-2, 0.1309g @ 60C

3-3-2-3, 0.1309g @60C

3-3-2-4, 0.1309g @ 60C

3-3-2-5, 0.1309g @ 60C

3-3-2-6, 0.1309g @ 60C

3-3-2-7, 0.1309g@60C

3-3-2-8, 0.1309g @ 60C

3-3-2-9, 0.1309g @ 60C

3-3-2-10,0.1309g @ 60C

3-1-3-1, 0.1312g@70C

3-1-3-2, 0.1312g@70C

3-1-3-3, 0.1312g @ 70C

3-1-3-4, 0.1312g@70C

3-1-3-5, 0.1312g @ 70C

3-1-3-6, 0.1312g @ 70C

3-1-3-7, 0.1312g@70C

3-1-3-8, 0.1312g @ 70C

3-1-3-9, 0.1312g@70C

3-1-3-10,0.1312g@70C

3-2-3-1, 0.1311g@70C

3-2-3-2, 0.1311g@70C

3-2-3-3, 0.1311g@70C

3-2-3-4, 0.1311g@70C

3-2-3-5, 0.1311g@70C

3-2-3-6, 0.1311g @ 70C

3-2-3-7, 0.1311g @ 70C

3-2-3-8, 0.1311g @ 70C

3-2-3-9, 0.1311g@70C

3-2-3-10,0.1311g@70C

2-2-4B-1, 0.1290g @ 80C

2-2-4B-2, 0.1290g @ 80C

2-2-4B-3, 0.1290g @ 80C

2-2-4B-4, 0.1290g @ 80C

2-2-4B-5, 0.1290g@80C

2-2-4B-6, 0.1290g @ 80C

2-2-4B-7, 0.1290g @ 80C

2-2-4B-8, 0.1290g @ 80C

2-2-4B-9, 0.1290g @ 80C

2-2-4B-10, 0.1290g @ 80C

3-1-4-1, 0.1326g @ 80C

3-1-4-2, 0.1326g @ 80C

3-1-4-3,0.1326g @ 80C

3-1-4-4, 0.1326g @80C

3-1-4-5, 0.1326g@80C

3-1-4-6, 0.1326g@80C

\begin{tabular}{|c|c|c|c|c|c|c|c|}
\hline $\begin{array}{c}\text { Salt } \\
\text { Solution }\end{array}$ & $\underset{(\text { molal })}{\mathrm{NH}_{3} \text { Conc }}$ & $\begin{array}{c}\text { Temperature } \\
\left({ }^{\circ} \mathbf{C}\right)\end{array}$ & $\begin{array}{l}\text { Sampling } \\
\text { Sequence }\end{array}$ & $\begin{array}{c}\text { A } \\
\text { (peak area) }\end{array}$ & $\mathbf{y}$ & $\mathbf{x}$ & \\
\hline 3 & 0.5 & 60 & 1 & 30.22 & 30.22 & 30.24 & 1.4803 \\
\hline 3 & 0.5 & 60 & 2 & 30.24 & 60.46 & 29.17 & 1.4806 \\
\hline 3 & 0.5 & 60 & 3 & 29.17 & 89.63 & 26.98 & 1.4649 \\
\hline 3 & 0.5 & 60 & 4 & 26.98 & 116.61 & 25.03 & 1.4310 \\
\hline 3 & 0.5 & 60 & 5 & 25.03 & 141.63 & 23.76 & 1.3984 \\
\hline 3 & 0.5 & 60 & 6 & 23.76 & 165.40 & 19.10 & 1.3759 \\
\hline 3 & 0.5 & 60 & 7 & 19.10 & 184.49 & 18.82 & 1.2809 \\
\hline 3 & 0.5 & 60 & 8 & 18.82 & 203.31 & 17.68 & 1.2746 \\
\hline 3 & 0.5 & 60 & 9 & 17.68 & 221.00 & 16.63 & 1.2476 \\
\hline 3 & 0.5 & 60 & 10 & 16.63 & 237.63 & & 1.2209 \\
\hline 3 & 2 & 60 & 1 & 20.98 & 20.98 & 19.14 & 1.3217 \\
\hline 3 & 2 & 60 & 2 & 19.14 & 40.12 & 20.82 & 1.2820 \\
\hline 3 & 2 & 60 & 3 & 20.82 & 60.94 & 18.83 & 1.3185 \\
\hline 3 & 2 & 60 & 4 & 18.83 & 79.77 & 16.34 & 1.2748 \\
\hline 3 & 2 & 60 & 5 & 16.34 & 96.11 & 16.08 & 1.2134 \\
\hline 3 & 2 & 60 & 6 & 16.08 & 112.19 & 11.71 & 1.2064 \\
\hline 3 & 2 & 60 & 7 & 11.71 & 123.90 & 13.18 & 1.0684 \\
\hline 3 & 2 & 60 & 8 & 13.18 & 137.08 & 12.96 & 1.1199 \\
\hline 3 & 2 & 60 & 9 & 12.96 & 150.04 & 12.57 & 1.1125 \\
\hline 3 & 2 & 60 & 10 & 12.57 & 162.61 & & 1.0992 \\
\hline 3 & 3.5 & 60 & 1 & 8.75 & 8.75 & 11.41 & 0.9419 \\
\hline 3 & 3.5 & 60 & 2 & 11.41 & 20.16 & 10.75 & 1.0574 \\
\hline 3 & 3.5 & 60 & 3 & 10.75 & 30.91 & 10.13 & 1.0315 \\
\hline 3 & 3.5 & 60 & 4 & 10.13 & 41.05 & 7.71 & 1.0057 \\
\hline 3 & 3.5 & 60 & 5 & 7.71 & 48.75 & 8.19 & 0.8870 \\
\hline 3 & 3.5 & 60 & 6 & 8.19 & 56.94 & 8.02 & 0.9131 \\
\hline 3 & 3.5 & 60 & 7 & 8.02 & 64.96 & 6.80 & 0.9040 \\
\hline 3 & 3.5 & 60 & 8 & 6.80 & 71.75 & 7.00 & 0.8323 \\
\hline 3 & 3.5 & 60 & 9 & 7.00 & 78.75 & 8.38 & 0.8450 \\
\hline 3 & 3.5 & 60 & 10 & 8.38 & 87.13 & & 0.9230 \\
\hline 3 & 0.5 & 70 & 1 & 63.22 & 63.22 & 68.33 & 1.8008 \\
\hline 3 & 0.5 & 70 & 2 & 68.33 & 131.55 & 61.50 & 1.8346 \\
\hline 3 & 0.5 & 70 & 3 & 61.50 & 193.04 & 56.69 & 1.7888 \\
\hline 3 & 0.5 & 70 & 4 & 56.69 & 249.74 & 51.86 & 1.7535 \\
\hline 3 & 0.5 & 70 & 5 & 51.86 & 301.59 & 45.98 & 1.7148 \\
\hline 3 & 0.5 & 70 & 6 & 45.98 & 347.57 & 39.21 & 1.6626 \\
\hline 3 & 0.5 & 70 & 7 & 39.21 & 386.78 & 36.38 & 1.5933 \\
\hline 3 & 0.5 & 70 & 8 & 36.38 & 423.16 & 33.21 & 1.5609 \\
\hline 3 & 0.5 & 70 & 9 & 33.21 & 456.36 & 26.62 & 1.5212 \\
\hline 3 & 0.5 & 70 & 10 & 26.62 & 482.98 & & 1.4252 \\
\hline 3 & 2 & 70 & 1 & 38.88 & 38.88 & 41.42 & 1.5897 \\
\hline 3 & 2 & 70 & 2 & 41.42 & 80.30 & 37.97 & 1.6173 \\
\hline 3 & 2 & 70 & 3 & 37.97 & 118.27 & 35.25 & 1.5794 \\
\hline 3 & 2 & 70 & 4 & 35.25 & 153.53 & 31.03 & 1.5472 \\
\hline 3 & 2 & 70 & 5 & 31.03 & 184.56 & 28.00 & 1.4918 \\
\hline 3 & 2 & 70 & 6 & 28.00 & 212.56 & 26.14 & 1.4471 \\
\hline 3 & 2 & 70 & 7 & 26.14 & 238.69 & 23.42 & 1.4172 \\
\hline 3 & 2 & 70 & 8 & 23.42 & 262.11 & 20.91 & 1.3696 \\
\hline 3 & 2 & 70 & 9 & 20.91 & 283.03 & 17.58 & 1.3204 \\
\hline 3 & 2 & 70 & 10 & 17.58 & 300.60 & & 1.2449 \\
\hline 2 & 2 & 80 & 1 & 234.49 & 234.49 & 212.22 & 2.3701 \\
\hline 2 & 2 & 80 & 2 & 212.22 & 446.71 & 181.32 & 2.3268 \\
\hline 2 & 2 & 80 & 3 & 181.32 & 628.03 & 152.76 & 2.2584 \\
\hline 2 & 2 & 80 & 4 & 152.76 & 780.79 & 131.17 & 2.1840 \\
\hline 2 & 2 & 80 & 5 & 131.17 & 911.95 & 109.02 & 2.1178 \\
\hline 2 & 2 & 80 & 6 & 109.02 & 1020.97 & 90.95 & 2.0375 \\
\hline 2 & 2 & 80 & 7 & 90.95 & 1111.92 & 75.31 & 1.9588 \\
\hline 2 & 2 & 80 & 8 & 75.31 & 1187.23 & 63.11 & 1.8769 \\
\hline 2 & 2 & 80 & 9 & 63.11 & 1250.34 & 51.29 & 1.8001 \\
\hline 2 & 2 & 80 & 10 & 51.29 & 1301.63 & & 1.7100 \\
\hline 3 & 0.5 & 80 & 1 & 119.40 & 119.40 & 110.72 & 2.0770 \\
\hline 3 & 0.5 & 80 & 2 & 110.72 & 230.12 & 95.00 & 2.0442 \\
\hline 3 & 0.5 & 80 & 3 & 95.00 & 325.12 & 78.38 & 1.9777 \\
\hline 3 & 0.5 & 80 & 4 & 78.38 & 403.50 & 67.17 & 1.8942 \\
\hline 3 & 0.5 & 80 & 5 & 67.17 & 470.67 & 57.52 & 1.8272 \\
\hline 3 & 0.5 & 80 & 6 & 57.52 & 528.19 & 48.95 & 1.7598 \\
\hline
\end{tabular}


Table B2. Ammonia Test Results

\author{
(continued)
}

Test Test

Study Sequence

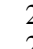

2

2

2

2

\begin{abstract}
Identifier
\end{abstract}
3-1-4-7, 0.1326g @ 80C 3-1-4-8, 0.1326g @ 80C 3-1-4-9, 0.1326g @ 80C 3-1-4-10,0.1326g @ 80C 3-2-4-1, 0.1316g@80C 3-2-4-2, 0.1316g @ 80C 3-2-4-3, 0.1316g@80C 3-2-4-4, 0.1316g @ 80C 3-2-4-5, 0.1316g @ 80C 3-2-4-6, 0.1316g @ 80C 3-2-4-7, 0.1316g@80C 3-2-4-8, 0.1316g@80C 3-2-4-9, 0.1316g @ 80C 3-2-4-10, 0.1316g @ 80C 3-1-5-1, 0.1314g@90C 3-1-5-2, 0.1314g @ 90C 3-1-5-3, 0.1314g@90C 3-1-5-4, 0.1314g @ 90C 3-1-5-5, 0.1314g@90C 3-1-5-6, 0.1314g @ 90C 3-1-5-7, 0.1314g@90C 3-1-5-8, 0.1314g@90C 3-1-5-9, 0.1314g@90C 3-1-5-10, 0.1314g@ 90C 3-2-5-1, 0.1309g@90C 3-2-5-2, 0.1309g @ 90C 3-2-5-3, 0.1309g @ 90C 3-2-5-4, 0.1309g @ 90C 3-2-5-5, 0.1309g @ 90C 3-2-5-6, 0.1309g@ 90C 3-2-5-7, 0.1309g @ 90C 3-2-5-8, 0.1309g @ 90C 3-2-5-9, 0.1309g @ 90C 3-2-5-10, 0.1309g@ @0C 2-2-4B-1, 0.1290g @ 80C 2-2-4B-2, 0.1290g @ 80C 2-2-4B-3, 0.1290g @ 80C 2-2-4B-4, 0.1290g @ 80C 2-2-4B-5, 0.1290g @ 80C 2-2-4B-6, 0.1290g @ 80C 2-2-4B-7, 0.1290g @ 80C 2-2-4B-8, 0.1290g @ 80C 2-2-4B-9, 0.1290g @ 80C 2-2-4B-10, 0.1290g @ 80C 3-1-4-1, 0.1326g @ 80C 3-1-4-2,0.1326g @ 80C 3-1-4-3, 0.1326g @ 80C 3-1-4-4, 0.1326g @ 80C 3-1-4-5, 0.1326g@80C 3-1-4-6, 0.1326g @ 80C 3-1-4-7, 0.1326g @ 80C 3-1-4-8, 0.1326g @ 80C 3-1-4-9, 0.1326g @ 80C 3-1-4-10, 0.1326g @ 80C 3-2-4-1,0.1316g @ 80C 3-2-4-2, 0.1316g@80C 3-2-4-3, 0.1316g @ 80C 3-2-4-4, 0.1316g @ 80C 3-2-4-5, 0.1316g@80C 3-2-4-6, 0.1316g @ 80C 3-2-4-7, 0.1316g@80C 3-2-4-8, 0.1316g @ 80C 3-2-4-9, 0.1316g @ 80C 3-2-4-10,0.1316g @ 80C 3-1-5-1, 0.1314g @ 90C 3-1-5-2, 0.1314g @ 90C 3-1-5-3, 0.1314g @ 90C 3-1-5-4, 0.1314g@ 90C 3-1-5-5, 0.1314g @ 90C 3-1-5-6, 0.1314g @ 90C 3-1-5-7, 0.1314g@90C
Salt $\mathrm{NH}_{3}$ Conc Temperature Sampling

$\begin{array}{lcccc}\text { Solution } & \text { (molal) } & \left({ }^{\mathbf{0}} \mathrm{C}\right) & \text { Sequence } & \text { (peak area) }\end{array}$

S

$\begin{array}{lllll}3 & 0.5 & 80 & 7 & 48.95 \\ 3 & 0.5 & 80 & 8 & 43.57 \\ 3 & 0.5 & 80 & 9 & 33.75 \\ 3 & 0.5 & 80 & 10 & 30.08\end{array}$

$\begin{array}{lll}3 & 2 & 80 \\ 3 & 2 & 80\end{array}$

$\begin{array}{lll}3 & 2 & 80 \\ 3 & 2 & 80\end{array}$

$\begin{array}{lll}3 & 2 & 80\end{array}$

$\begin{array}{lll}3 & 2 & 80\end{array}$

$\begin{array}{lc}80 & 10 \\ 80 & 1 \\ 80 & 2\end{array}$

33.33
32.21

35.87

25.53

19.87

17.66

16.33

14.18

12.68

10.27

59.16

52.42

43.94

34.16

29.97

23.14

19.82

15.41

13.78

10.41
51.29

67.67

54.95

40.13

34.58

26.13

19.95

17.84

14.98

13.84

218.28

212.89

192.31

172.89

152.49

135.14

118.04

105.78

91.64

82.30

82.01

67.98

60.67

50.31

42.79

34.27

28.76

22.85

20.99

17.29
119.40

110.72

95.00

78.38

67.17

57.52

48.95

43.57

33.75

30.08

33.33

32.21

35.87

25.53

19.87

17.66

16.33

\begin{tabular}{lcc}
\multicolumn{1}{c}{$\mathbf{y}$} & $\mathbf{x}$ & $\log (\mathbf{A})$ \\
577.14 & 43.57 & 1.6898 \\
620.71 & 33.75 & 1.6392 \\
654.46 & 30.08 & 1.5283 \\
684.55 &. & 1.4783 \\
33.33 & 32.21 & 1.5228 \\
65.53 & 35.87 & 1.5079 \\
101.40 & 25.53 & 1.5547 \\
126.93 & 19.87 & 1.4071 \\
146.80 & 17.66 & 1.2981 \\
164.46 & 16.33 & 1.2471 \\
180.79 & 14.18 & 1.2130 \\
194.97 & 12.68 & 1.1516 \\
207.65 & 10.27 & 1.1030 \\
217.91 & & 1.0114
\end{tabular}

$\begin{array}{lll}217.91 & . & 1.0114 \\ 59.16 & 52.42 & 1.7720\end{array}$

$\begin{array}{lll}59.16 & 52.42 & 1.7720 \\ 111.57 & 43.94 & 1.7195\end{array}$

$\begin{array}{lll}155.51 & 34.16 & 1.6429\end{array}$

$\begin{array}{lll}189.68 & 29.97 & 1.5336\end{array}$

$\begin{array}{lll}219.65 & 23.14 & 1.4767\end{array}$

$\begin{array}{lll}242.79 & 19.82 & 1.3644\end{array}$

$\begin{array}{lll}262.60 & 15.41 & 1.2970\end{array}$

$\begin{array}{lll}278.01 & 13.78 & 1.1878\end{array}$

$291.79 \quad 10.41 \quad 1.1392$

$\begin{array}{ccc}302.20 & . & 1.0173 \\ 51.29 & 67.67 & 1.7100\end{array}$

$\begin{array}{lll}118.96 & 54.95 & 1.8304\end{array}$

$\begin{array}{lll}173.91 & 40.13 \quad 1.7400\end{array}$

$\begin{array}{lll}214.04 & 34.58 & 1.6035\end{array}$

$\begin{array}{lll}248.62 & 26.13 & 1.5388\end{array}$

$\begin{array}{lll}274.75 & 19.95 & 1.4171\end{array}$

$\begin{array}{lll}294.70 & 17.84 & 1.2999\end{array}$

$\begin{array}{lll}312.54 & 14.98 & 1.2515\end{array}$

$\begin{array}{lll}327.53 & 13.84 & 1.1756\end{array}$

$\begin{array}{lll}341.37 & & 1.1413\end{array}$

$\begin{array}{lll}218.28 & 212.89 & 2.3390 \\ 431.17 & 192.31 & 2.3281\end{array}$

$\begin{array}{lll}623.48 & 172.89 & 2.2840\end{array}$

$\begin{array}{lll}796.36 & 152.49 & 2.2378\end{array}$

$\begin{array}{lll}948.85 & 135.14 & 2.1832\end{array}$

$\begin{array}{lll}1083.99 & 118.04 & 2.1308\end{array}$

$\begin{array}{lll}1202.03 & 105.78 & 2.0720\end{array}$

$\begin{array}{lll}1307.81 & 91.64 & 2.0244\end{array}$

$\begin{array}{lll}1399.44 & 82.30 & 1.9621\end{array}$

$\begin{array}{lll}1481.75 & \text {. } & 1.9154\end{array}$

$\begin{array}{lll}82.01 & 67.98 & 1.9139\end{array}$

$\begin{array}{lll}149.99 & 60.67 & 1.8324\end{array}$

$\begin{array}{lll}210.66 & 50.31 & 1.7830\end{array}$

$\begin{array}{lll}260.96 & 42.79 & 1.7016\end{array}$

$\begin{array}{lll}303.75 & 34.27 & 1.6313 \\ 338.02 & 28.76 & 1.5349\end{array}$

$\begin{array}{lll}366.78 & 22.85 & 1.4588\end{array}$

$\begin{array}{lll}389.62 & 20.99 & 1.3588\end{array}$

$\begin{array}{lll}410.61 & 17.29 & 1.3219\end{array}$

$\begin{array}{lcl}427.89 & . & 1.2377 \\ 119.40 & 110.72 & 2.0770\end{array}$

$\begin{array}{lll}230.12 & 95.00 & 2.0442\end{array}$

$\begin{array}{lll}325.12 & 78.38 & 1.9777\end{array}$

$\begin{array}{lll}403.50 & 67.17 & 1.8942\end{array}$

$\begin{array}{lll}470.67 & 57.52 & 1.8272\end{array}$ 
Table B2. Ammonia Test Results

(continued)

\begin{tabular}{|c|c|c|c|c|c|c|c|c|c|c|}
\hline Study & $\begin{array}{c}\text { Test } \\
\text { Sequence }\end{array}$ & $\begin{array}{c}\text { Test } \\
\text { Identifier }\end{array}$ & $\begin{array}{c}\text { Salt } \\
\text { Solution }\end{array}$ & $\begin{array}{c}\mathrm{NH}_{3} \text { Conc } \\
\text { (molal) }\end{array}$ & $\begin{array}{c}\text { Temperature } \\
\left({ }^{\circ} \mathrm{C}\right)\end{array}$ & $\begin{array}{l}\text { Sampling } \\
\text { Sequence }\end{array}$ & $\begin{array}{c}\mathbf{A} \\
\text { (peak area) }\end{array}$ & $\mathbf{y}$ & $\mathbf{x}$ & $\log (\mathbf{A})$ \\
\hline 3 & 4 & 3-1-5-8,0.1314g@90C & 3 & 0.5 & 90 & 8 & 14.18 & 194.97 & 12.68 & 1.1516 \\
\hline 3 & 4 & 3-1-5-9,0.1314g @90C & 3 & 0.5 & 90 & 9 & 12.68 & 207.65 & 10.27 & 1.1030 \\
\hline 3 & 4 & 3-1-5-10,0.1314g@90C & 3 & 0.5 & 90 & 10 & 10.27 & 217.91 & & 1.0114 \\
\hline 3 & 5 & 3-2-5-1, 0.1309g @ 90C & 3 & 2 & 90 & 1 & 111.65 & 111.65 & 99.37 & 2.0479 \\
\hline 3 & 5 & 3-2-5-2, 0.1309g@90C & 3 & 2 & 90 & 2 & 99.37 & 211.02 & 87.34 & 1.9973 \\
\hline 3 & 5 & 3-2-5-3, 0.1309g @ 90C & 3 & 2 & 90 & 3 & 87.34 & 298.37 & 74.08 & 1.9412 \\
\hline 3 & 5 & 3-2-5-4, 0.1309g @ 90C & 3 & 2 & 90 & 4 & 74.08 & 372.45 & 63.66 & 1.8697 \\
\hline 3 & 5 & 3-2-5-5, 0.1309g@90C & 3 & 2 & 90 & 5 & 63.66 & 436.11 & 56.27 & 1.8039 \\
\hline 3 & 5 & 3-2-5-6, 0.1309g @ 90C & 3 & 2 & 90 & 6 & 56.27 & 492.38 & 46.52 & 1.7502 \\
\hline 3 & 5 & 3-2-5-7, 0.1309g @ 90C & 3 & 2 & 90 & 7 & 46.52 & 538.89 & 41.42 & 1.6676 \\
\hline 3 & 5 & 3-2-5-8, 0.1309g @ 90C & 3 & 2 & 90 & 8 & 41.42 & 580.31 & 34.61 & 1.6172 \\
\hline 3 & 5 & 3-2-5-9, 0.1309g @90C & 3 & 2 & 90 & 9 & 34.61 & 614.92 & 30.43 & 1.5392 \\
\hline 3 & 5 & 3-2-5-10, 0.1309g@ @90C & 3 & 2 & 90 & 10 & 30.43 & 645.35 & & 1.4832 \\
\hline 3 & 6 & 2-2-4B-1, 0.1290g@80C & 2 & 2 & 80 & 1 & 234.49 & 234.49 & 212.22 & 2.3701 \\
\hline 3 & 6 & 2-2-4B-2, 0.1290g@80C & 2 & 2 & 80 & 2 & 212.22 & 446.71 & 181.32 & 2.3268 \\
\hline 3 & 6 & 2-2-4B-3, 0.1290g @ 80C & 2 & 2 & 80 & 3 & 181.32 & 628.03 & 152.76 & 2.2584 \\
\hline 3 & 6 & 2-2-4B-4, 0.1290g@80C & 2 & 2 & 80 & 4 & 152.76 & 780.79 & 131.17 & 2.1840 \\
\hline 3 & 6 & 2-2-4B-5, 0.1290g@80C & 2 & 2 & 80 & 5 & 131.17 & 911.95 & 109.02 & 2.1178 \\
\hline 3 & 6 & 2-2-4B-6, 0.1290g @ 80C & 2 & 2 & 80 & 6 & 109.02 & 1020.97 & 63.23 & 2.0375 \\
\hline 3 & 6 & 2-2-4B-7, 0.1290g@80C & 2 & 2 & 80 & 7 & 63.23 & 1084.20 & 75.31 & 1.8009 \\
\hline 3 & 6 & 2-2-4B-8, 0.1290g@80C & 2 & 2 & 80 & 8 & 75.31 & 1159.51 & 63.11 & 1.8769 \\
\hline 3 & 6 & 2-2-4B-9, 0.1290g@80C & 2 & 2 & 80 & 9 & 63.11 & 1222.63 & 51.29 & 1.8001 \\
\hline 3 & 6 & 2-2-4B-10,0.1290g@80C & 2 & 2 & 80 & 10 & 51.29 & 1273.91 & & 1.7100 \\
\hline 3 & 7 & 3-2-5-1 RERUN \#2 0.1321 & 3 & 2 & 90 & 1 & 51.13 & 419.72 & 45.34 & 1.7087 \\
\hline 3 & 7 & 3-2-5-1 RERUN \#2 0.1321 & 3 & 2 & 90 & 2 & 45.34 & 465.06 & 36.71 & 1.6565 \\
\hline 3 & 7 & 3-2-5-1 RERUN \#2 0.1321 & 3 & 2 & 90 & 3 & 36.71 & 501.77 & 29.92 & 1.5648 \\
\hline 3 & 7 & 3-2-5-1 RERUN \#2 0.1321 & 3 & 2 & 90 & 4 & 29.92 & 531.69 & 26.65 & 1.4760 \\
\hline 3 & 7 & 3-2-5-1 RERUN \#2 0.1321 & 3 & 2 & 90 & 5 & 26.65 & 558.34 & 20.42 & 1.4256 \\
\hline 3 & 7 & 3-2-5-1 RERUN \#2 0.1321 & 3 & 2 & 90 & 6 & 20.42 & 578.75 & 17.86 & 1.3100 \\
\hline 3 & 7 & 3-2-5-1 RERUN \#2 0.1321 & 3 & 2 & 90 & 7 & 17.86 & 596.61 & 14.61 & 1.2519 \\
\hline 3 & 7 & 3-2-5-1 RERUN \#2 0.1321 & 3 & 2 & 90 & 8 & 14.61 & 611.22 & 12.50 & 1.1645 \\
\hline 3 & 7 & 3-2-5-1 RERUN \#2 0.1321 & 3 & 2 & 90 & 9 & 12.50 & 623.72 & 12.30 & 1.0969 \\
\hline 3 & 7 & 3-2-5-1 RERUN \#2 0.1321 & 3 & 2 & 90 & 10 & 12.30 & 636.02 & & 1.0899 \\
\hline 3 & 8 & 1-1-5-1 RERUN \#10.1347 & 1 & 0.5 & 90 & 1 & 31.66 & 31.66 & 31.77 & 1.5005 \\
\hline 3 & 8 & 1-1-5-1 RERUN \#10.1347 & 1 & 0.5 & 90 & 2 & 31.77 & 63.43 & 23.80 & 1.5020 \\
\hline 3 & 8 & 1-1-5-1 RERUN \#10.1347 & 1 & 0.5 & 90 & 3 & 23.80 & 87.23 & 20.62 & 1.3765 \\
\hline 3 & 8 & 1-1-5-1 RERUN \#10.1347 & 1 & 0.5 & 90 & 4 & 20.62 & 107.85 & 16.66 & 1.3142 \\
\hline 3 & 8 & 1-1-5-1 RERUN \#10.1347 & 1 & 0.5 & 90 & 5 & 16.66 & 124.51 & 13.39 & 1.2218 \\
\hline 3 & 8 & 1-1-5-1 RERUN \#10.1347 & 1 & 0.5 & 90 & 6 & 13.39 & 137.91 & 12.21 & 1.1269 \\
\hline 3 & 8 & 1-1-5-1 RERUN \#10.1347 & 1 & 0.5 & 90 & 7 & 12.21 & 150.11 & 8.78 & 1.0865 \\
\hline 3 & 8 & 1-1-5-1 RERUN \#10.1347 & 1 & 0.5 & 90 & 8 & 8.78 & 158.89 & 8.41 & 0.9434 \\
\hline 3 & 8 & 1-1-5-1 RERUN \#10.1347 & 1 & 0.5 & 90 & 9 & 8.41 & 167.29 & 8.42 & 0.9245 \\
\hline 3 & 8 & 1-1-5-1 RERUN \#10.1347 & 1 & 0.5 & 90 & 10 & 8.42 & 175.71 & & 0.9253 \\
\hline 3 & 9 & 2-1-5-1 RERUN \#10.1311 & 2 & 0.5 & 90 & 1 & 30.39 & 30.39 & 25.80 & 1.4828 \\
\hline 3 & 9 & 2-1-5-1 RERUN \#10.1311 & 2 & 0.5 & 90 & 2 & 25.80 & 56.20 & 22.25 & 1.4117 \\
\hline 3 & 9 & 2-1-5-1 RERUN \#10.1311 & 2 & 0.5 & 90 & 3 & 22.25 & 78.44 & 19.24 & 1.3473 \\
\hline 3 & 9 & 2-1-5-1 RERUN \#10.1311 & 2 & 0.5 & 90 & 4 & 19.24 & 97.69 & 16.23 & 1.2843 \\
\hline 3 & 9 & 2-1-5-1 RERUN \#10.1311 & 2 & 0.5 & 90 & 5 & 16.23 & 113.91 & 14.00 & 1.2102 \\
\hline 3 & 9 & 2-1-5-1 RERUN \#10.1311 & 2 & 0.5 & 90 & 6 & 14.00 & 127.92 & 11.28 & 1.1463 \\
\hline 3 & 9 & 2-1-5-1 RERUN \#10.1311 & 2 & 0.5 & 90 & 7 & 11.28 & 139.20 & 9.28 & 1.0524 \\
\hline 3 & 9 & 2-1-5-1 RERUN \#10.1311 & 2 & 0.5 & 90 & 8 & 9.28 & 148.48 & 8.79 & 0.9673 \\
\hline 3 & 9 & 2-1-5-1 RERUN \#10.1311 & 2 & 0.5 & 90 & 9 & 8.79 & 157.26 & 8.31 & 0.9438 \\
\hline 3 & 9 & 2-1-5-1 RERUN \#10.1311 & 2 & 0.5 & 90 & 10 & 8.31 & 165.57 & & 0.9197 \\
\hline 4 & 1 & $1-1-1,70 \mathrm{C}, 0.1429$ & 1 & 0.5 & 70 & 1 & 159.54 & 159.54 & 155.20 & 2.2029 \\
\hline 4 & 1 & $1-1-2,70 \mathrm{C}, 0.1429$ & 1 & 0.5 & 70 & 2 & 155.20 & 314.74 & 134.55 & 2.1909 \\
\hline 4 & 1 & $1-1-3,70 \mathrm{C}, 0.1429$ & 1 & 0.5 & 70 & 3 & 134.55 & 449.29 & 116.61 & 2.1289 \\
\hline 4 & 1 & $1-1-4,70 \mathrm{C}, 0.1429$ & 1 & 0.5 & 70 & 4 & 116.61 & 565.90 & 100.57 & 2.0667 \\
\hline 4 & 1 & $1-1-5,70 \mathrm{C}, 0.1429$ & 1 & 0.5 & 70 & 5 & 100.57 & 666.47 & 86.76 & 2.0025 \\
\hline 4 & 1 & $1-1-6,70 \mathrm{C}, 0.1429$ & 1 & 0.5 & 70 & 6 & 86.76 & 753.23 & 73.10 & 1.9383 \\
\hline 4 & 1 & $1-1-7,70 \mathrm{C}, 0.1429$ & 1 & 0.5 & 70 & 7 & 73.10 & 826.33 & 62.00 & 1.8639 \\
\hline 4 & 1 & $1-1-8,70 \mathrm{C}, 0.1429$ & 1 & 0.5 & 70 & 8 & 62.00 & 888.32 & 56.49 & 1.7924 \\
\hline 4 & 1 & $1-1-9,70 \mathrm{C}, 0.1429$ & 1 & 0.5 & 70 & 9 & 56.49 & 944.81 & 45.24 & 1.7520 \\
\hline 4 & 1 & $1-1-10,70 \mathrm{C}, 0.1429$ & 1 & 0.5 & 70 & 10 & 45.24 & 990.05 & . & 1.6556 \\
\hline 4 & 2 & $1-2-1,70 \mathrm{C}, 0.1401$ & 1 & 2 & 70 & 1 & 771.74 & 771.74 & 680.94 & 2.8875 \\
\hline 4 & 2 & $1-2-2,70 \mathrm{C}, 0.1401$ & 1 & 2 & 70 & 2 & 680.94 & 1452.68 & 579.36 & 2.8331 \\
\hline 4 & 2 & $1-2-3,70 \mathrm{C}, 0.1401$ & 1 & 2 & 70 & 3 & 579.36 & 2032.04 & 482.13 & 2.7629 \\
\hline 4 & 2 & $1-2-4,70 \mathrm{C}, 0.1401$ & 1 & 2 & 70 & 4 & 482.13 & 2514.17 & 406.47 & 2.6832 \\
\hline 4 & 2 & $1-2-5,70 \mathrm{C}, 0.1401$ & 1 & 2 & 70 & 5 & 406.47 & 2920.64 & 339.22 & 2.6090 \\
\hline 4 & 2 & $1-2-6,70 \mathrm{C}, 0.1401$ & 1 & 2 & 70 & 6 & 339.22 & 3259.86 & 294.27 & 2.5305 \\
\hline 4 & 2 & $1-2-7,70 \mathrm{C}, 0.1401$ & 1 & 2 & 70 & 7 & 294.27 & 3554.13 & 247.67 & 2.4688 \\
\hline 4 & 2 & $1-2-8,70 \mathrm{C}, 0.1401$ & 1 & 2 & 70 & 8 & 247.67 & 3801.80 & 206.65 & 2.3939 \\
\hline
\end{tabular}


Table B2. Ammonia Test Results

Test

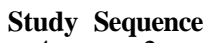

$4 \quad$ Identifier

$\begin{array}{lll}4 & 2 & 1-2-9,70 \mathrm{C}, 0.1401 \\ 4 & 2 & 1-2-10,70 \mathrm{C}, 0.1401\end{array}$

$43 \quad 1-3-1,70 \mathrm{C}, 0.1391$

$4 \quad 3 \quad 1-3-2,70 \mathrm{C}, 0.1391$

$\begin{array}{lll}4 & 3 & 1-3-3,70 \mathrm{C}, 0.1391 \\ 4 & 3 & 1-3-4,70 \mathrm{C}, 0.1391\end{array}$

$4 \quad 3 \quad 1-3-5,70 \mathrm{C}, 0.1391$

$4 \quad 3 \quad 1-3-6,70 \mathrm{C}, 0.1391$

$4 \quad 3 \quad 1-3-7,70 \mathrm{C}, 0.1391$

$4 \quad 3 \quad 1-3-8,70 \mathrm{C}, 0.1391$

$4 \quad 3 \quad 1-3-9,70 \mathrm{C}, 0.1391$

$\begin{array}{lll}4 & 3 & 1-3-10,70 \mathrm{C}, 0.1391 \\ 4 & 4 & 2-1-1,70 \mathrm{C}, 0.1395\end{array}$

$\begin{array}{lll}4 & 4 & 2-1-1,70 \mathrm{C}, 0.1395 \\ 4 & 4 & 2-1-2,70 \mathrm{C}, 0.1395\end{array}$

$\begin{array}{lll}4 & 4 & 2-1-2,70 \mathrm{C}, 0.1395 \\ 4 & 4 & 2-1-3,70 \mathrm{C}, 0.1395\end{array}$

$4 \quad 4 \quad 2-1-4,70 \mathrm{C}, 0.1395$

$4 \quad 4 \quad 2-1-5,70 \mathrm{C}, 0.1395$

$4 \quad 4 \quad 2-1-6,70 \mathrm{C}, 0.1395$

$4 \quad 4 \quad 2-1-7,70 \mathrm{C}, 0.1395$

$4 \quad 4 \quad 2-1-8,70 \mathrm{C}, 0.1395$

$4 \quad 4 \quad 2-1-9,70 \mathrm{C}, 0.1395$

$4 \quad 4 \quad 2-1-10,70 \mathrm{C}, 0.1395$

$4 \quad 5 \quad 2-2-170 \mathrm{C}, 0.1380$

$4 \quad 5 \quad 2-2-270 \mathrm{C}, 0.1380$

$4 \quad 5 \quad 2-2-370 \mathrm{C}, 0.1380$

$4 \quad 5 \quad 2-2-470 \mathrm{C}, 0.1380$

$4 \quad 5 \quad 2-2-570 \mathrm{C}, 0.1380$

$4 \quad 5 \quad 2-2-670 \mathrm{C}, 0.1380$

$4 \quad 5 \quad 2-2-770 \mathrm{C}, 0.1380$

$4 \quad 5 \quad 2-2-870 \mathrm{C}, 0.1380$

$4 \quad 5 \quad 2-2-970 \mathrm{C}, 0.1380$

$\begin{array}{lll}4 & 5 & 2-2-1070 \mathrm{C}, 0.1380 \\ 4 & 6 & 2-3-170 \mathrm{C}, 0.1380\end{array}$

$\begin{array}{lll}4 & 6 & 2-3-170 \mathrm{C}, 0.1380 \\ 4 & 6 & 2-3-270 \mathrm{C}, 0.1380\end{array}$

$4 \quad 6 \quad 2-3-370 \mathrm{C}, 0.1380$

$4 \quad 6 \quad 2-3-470 \mathrm{C}, 0.1380$

$4 \quad 6 \quad 2-3-570 \mathrm{C}, 0.1380$

$4 \quad 6 \quad 2-3-670 \mathrm{C}, 0.1380$

$4 \quad 6 \quad 2-3-770 \mathrm{C}, 0.1380$

$4 \quad 6 \quad 2-3-870 \mathrm{C}, 0.1380$

$4 \quad 6 \quad 2-3-970 \mathrm{C}, 0.1380$

$4 \quad 6 \quad 2-3-1070 \mathrm{C}, 0.1380$

$4 \quad 7 \quad 3-1-1,70 \mathrm{C}, 0.1408$

$4 \quad 7$ 3-1-2, 70C, 0.1408

$4 \quad 7 \quad 3-1-3,70 \mathrm{C}, 0.1408$

$473-1-4,70 \mathrm{C}, 0.1408$

$4 \quad 7 \quad 3-1-5,70 \mathrm{C}, 0.1408$

$4 \quad 7 \quad 3-1-6,70 \mathrm{C}, 0.1408$

$4 \quad 7 \quad 3-1-7,70 \mathrm{C}, 0.1408$

$4 \quad 73-1-8,70 \mathrm{C}, 0.1408$

$4 \quad 7$ 3-1-9, $70 \mathrm{C}, 0.1408$

$473-1-10,70 \mathrm{C}, 0.1408$

$4 \quad 8$ 3-2-1, 70C, 0.1419

$4 \quad 8 \quad 3-2-2,70 \mathrm{C}, 0.1419$

$4 \quad 8 \quad 3-2-3,70 \mathrm{C}, 0.1419$

$4 \quad 8 \quad 3-2-4,70 \mathrm{C}, 0.1419$

$4 \quad 8 \quad 3-2-5,70 \mathrm{C}, 0.1419$

$4 \quad 8 \quad 3-2-6,70 \mathrm{C}, 0.1419$

$4 \quad 8 \quad 3-2-7,70 \mathrm{C}, 0.1419$

$4 \quad 8 \quad 3-2-8,70 \mathrm{C}, 0.1419$

$4 \quad 8 \quad 3-2-9,70 \mathrm{C}, 0.1419$

3-2-10, 70C, 0.1419

3-3-1, 70C, 0.1405

3-3-2, 70C, 0.1405

$3-3-3,70 \mathrm{C}, 0.1405$

3-3-4, 70C, 0.1405

$3-3-5,70 \mathrm{C}, 0.1405$

3-3-6, 70C, 0.1405

$3-3-7,70 \mathrm{C}, 0.1405$

3-3-8, 70C, 0.1405

\author{
(continued)
}

Salt $\mathrm{NH}_{3}$ Conc Temperature Sampling Solution (molal) $\quad\left({ }^{\circ} \mathrm{C}\right) \quad$ Sequence (peak

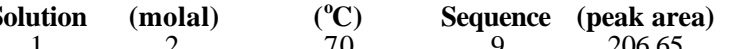

$\begin{array}{cccc}1 & 2 & 70 & 9 \\ 1 & 2 & 70 & 10 \\ 1 & 3.5 & 70 & 1 \\ 1 & 3.5 & 70 & 2\end{array}$

$\begin{array}{llll}1 & 3.5 & 70 & 2 \\ 1 & 3.5 & 70 & 3 \\ 1 & 3.5 & 70 & 4\end{array}$

$\begin{array}{llll}1 & 3.5 & 70 & 4 \\ 1 & 3.5 & 70 & 5 \\ 1 & 3.5 & 70 & 6\end{array}$

$\begin{array}{llll}1 & 3.5 & 70 & 6 \\ 1 & 3.5 & 70 & 7 \\ 1 & 3.5 & 70 & 8 \\ 1 & 3.5 & 70 & 9\end{array}$

$\begin{array}{cccc}1 & 3.5 & 70 & 9 \\ 1 & 3.5 & 70 & 10 \\ 2 & 0.5 & 70 & 1\end{array}$

$\begin{array}{llll}2 & 0.5 & 70 & 1 \\ 2 & 0.5 & 70 & 2 \\ 2 & 0.5 & 70 & 3\end{array}$

$\begin{array}{llll}2 & 0.5 & 70 & 3 \\ 2 & 0.5 & 70 & 4 \\ 2 & 0.5 & 70 & 5\end{array}$

$\begin{array}{llll}2 & 0.5 & 70 & 5 \\ 2 & 0.5 & 70 & 6 \\ 2 & 0.5 & 70 & 7\end{array}$

$\begin{array}{llll}2 & 0.5 & 70 & 6 \\ 2 & 0.5 & 70 & 8 \\ 2 & 0.5 & 70 & 9\end{array}$

$2 \quad 0.5$


Table B2. Ammonia Test Results

(continued)

\begin{tabular}{|c|c|c|}
\hline Study & $\begin{array}{c}\text { Test } \\
\text { Sequence }\end{array}$ & $\begin{array}{c}\text { Test } \\
\text { Identifier }\end{array}$ \\
\hline 4 & 9 & $3-3-9,70 \mathrm{C}, 0.1405$ \\
\hline 4 & 9 & $3-3-10,70 \mathrm{C}, 0.1405$ \\
\hline 4 & 10 & $3-1-1,60 \mathrm{C}, 0.1420$ \\
\hline 4 & 10 & $3-1-2,60 \mathrm{C}, 0.1420$ \\
\hline 4 & 10 & $3-1-3,60 \mathrm{C}, 0.1420$ \\
\hline 4 & 10 & $3-1-4,60 \mathrm{C}, 0.1420$ \\
\hline 4 & 10 & $3-1-5,60 C, 0.1420$ \\
\hline 4 & 10 & $3-1-6,60 \mathrm{C}, 0.1420$ \\
\hline 4 & 10 & $3-1-7,60 \mathrm{C}, 0.1420$ \\
\hline 4 & 10 & $3-1-8,60 \mathrm{C}, 0.1420$ \\
\hline 4 & 10 & $3-1-9,60 \mathrm{C}, 0.1420$ \\
\hline 4 & 10 & $3-1-10,60 \mathrm{C}, 0.1420$ \\
\hline 4 & 11 & $3-2-1,60 \mathrm{C}, 0.1410$ \\
\hline 4 & 11 & $3-2-2,60 \mathrm{C}, 0.1410$ \\
\hline 4 & 11 & $3-2-3,60 \mathrm{C}, 0.1410$ \\
\hline 4 & 11 & $3-2-4,60 C, 0.1410$ \\
\hline 4 & 11 & $3-2-5,60 C, 0.1410$ \\
\hline 4 & 11 & $3-2-6,60 \mathrm{C}, 0.1410$ \\
\hline 4 & 11 & $3-2-7,60 \mathrm{C}, 0.1410$ \\
\hline 4 & 11 & $3-2-8,60 \mathrm{C}, 0.1410$ \\
\hline 4 & 11 & $3-2-9,60 \mathrm{C}, 0.1410$ \\
\hline 4 & 11 & $3-2-10,60 \mathrm{C}, 0.1410$ \\
\hline 4 & 12 & $3-3-1,60 \mathrm{C}, 0.1407$ \\
\hline 4 & 12 & $3-3-2,60 \mathrm{C}, 0.1407$ \\
\hline 4 & 12 & $3-3-3,60 C, 0.1407$ \\
\hline 4 & 12 & $3-3-4,60 \mathrm{C}, 0.1407$ \\
\hline 4 & 12 & $3-3-5,60 C, 0.1407$ \\
\hline 4 & 12 & $3-3-6,60 \mathrm{C}, 0.1407$ \\
\hline 4 & 12 & $3-3-7,60 \mathrm{C}, 0.1407$ \\
\hline 4 & 12 & $3-3-8,60 \mathrm{C}, 0.1407$ \\
\hline 4 & 12 & $3-3-9,60 \mathrm{C}, 0.1407$ \\
\hline 4 & 12 & $3-3-10,60 \mathrm{C}, 0.1407$ \\
\hline 4 & 13 & $3-1-1,50 \mathrm{C}, 0.1423$ \\
\hline 4 & 13 & $3-1-2,50 \mathrm{C}, 0.1423$ \\
\hline 4 & 13 & $3-1-3,50 \mathrm{C}, 0.1423$ \\
\hline 4 & 13 & $3-1-4,50 \mathrm{C}, 0.1423$ \\
\hline 4 & 13 & $3-1-5,50 \mathrm{C}, 0.1423$ \\
\hline 4 & 13 & $3-1-6,50 \mathrm{C}, 0.1423$ \\
\hline 4 & 13 & $3-1-7,50 \mathrm{C}, 0.1423$ \\
\hline 4 & 13 & $3-1-8,50 \mathrm{C}, 0.1423$ \\
\hline 4 & 13 & $3-1-9,50 \mathrm{C}, 0.1423$ \\
\hline 4 & 13 & $3-1-10,50 \mathrm{C}, 0.1423$ \\
\hline 5 & 1 & $3-2-1,50 C, 0.1418$ \\
\hline 5 & 1 & $3-2-2,50 \mathrm{C}, 0.1418$ \\
\hline 5 & 1 & $3-2-3,50 C, 0.1418$ \\
\hline 5 & 1 & $3-2-4,50 \mathrm{C}, 0.1418$ \\
\hline 5 & 1 & $3-2-5,50 \mathrm{C}, 0.1418$ \\
\hline 5 & 1 & $3-2-6,50 \mathrm{C}, 0.1418$ \\
\hline 5 & 1 & $3-2-7,50 \mathrm{C}, 0.1418$ \\
\hline 5 & 1 & $3-2-8,50 \mathrm{C}, 0.1418$ \\
\hline 5 & 1 & $3-2-9,50 \mathrm{C}, 0.1418$ \\
\hline 5 & 1 & $3-2-10,50 C, 0.1418$ \\
\hline 5 & 2 & $3-3-1,50 C, 0.1402$ \\
\hline 5 & 2 & $3-3-2,50 C, 0.1402$ \\
\hline 5 & 2 & $3-3-3,50 \mathrm{C}, 0.1402$ \\
\hline 5 & 2 & $3-3-4,50 \mathrm{C}, 0.1402$ \\
\hline 5 & 2 & $3-3-5,50 \mathrm{C}, 0.1402$ \\
\hline 5 & 2 & $3-3-6,50 \mathrm{C}, 0.1402$ \\
\hline 5 & 2 & $3-3-7,50 \mathrm{C}, 0.1402$ \\
\hline 5 & 2 & $3-3-8,50 \mathrm{C}, 0.1402$ \\
\hline 5 & 2 & $3-3-9,50 \mathrm{C}, 0.1402$ \\
\hline 5 & 2 & $3-3-10,50 \mathrm{C}, 0.1402$ \\
\hline 5 & 3 & $1-1-6-1,100 \mathrm{C}, 0.1427$ \\
\hline 5 & 3 & $1-1-6-2,100 \mathrm{C}, 0.1427$ \\
\hline 5 & 3 & $1-1-6-3,100 \mathrm{C}, 0.1427$ \\
\hline 5 & 3 & $1-1-6-4,100 \mathrm{C}, 0.1427$ \\
\hline 5 & 3 & $1-1-6-5,100 \mathrm{C}, 0.1427$ \\
\hline 5 & 3 & $1-1-6-6,100 \mathrm{C}, 0.1427$ \\
\hline
\end{tabular}

\begin{tabular}{|c|c|c|c|c|c|c|c|}
\hline $\begin{array}{c}\text { Salt } \\
\text { Solution }\end{array}$ & $\begin{array}{c}\mathrm{NH}_{3} \text { Conc } \\
(\text { molal })\end{array}$ & $\begin{array}{c}\text { Temperature } \\
\left({ }^{\circ} \mathbf{C}\right)\end{array}$ & $\begin{array}{l}\text { Sampling } \\
\text { Sequence }\end{array}$ & $\underset{\text { (peak area) }}{\mathbf{A}}$ & $\mathbf{y}$ & $\mathbf{x}$ & $\log (A)$ \\
\hline 3 & 3.5 & 70 & 9 & 14.23 & 263.21 & 12.00 & 1.1531 \\
\hline 3 & 3.5 & 70 & 10 & 12.00 & 275.21 & & 1.0793 \\
\hline 3 & 0.5 & 60 & 1 & 9.57 & 9.57 & 8.14 & 0.9810 \\
\hline 3 & 0.5 & 60 & 2 & 8.14 & 17.71 & 8.98 & 0.9106 \\
\hline 3 & 0.5 & 60 & 3 & 8.98 & 26.69 & 10.29 & 0.9531 \\
\hline 3 & 0.5 & 60 & 4 & 10.29 & 36.98 & 7.19 & 1.0125 \\
\hline 3 & 0.5 & 60 & 5 & 7.19 & 44.17 & 7.67 & 0.8567 \\
\hline 3 & 0.5 & 60 & 6 & 7.67 & 51.84 & 7.52 & 0.8848 \\
\hline 3 & 0.5 & 60 & 7 & 7.52 & 59.36 & 5.75 & 0.8763 \\
\hline 3 & 0.5 & 60 & 8 & 5.75 & 65.12 & 5.61 & 0.7600 \\
\hline 3 & 0.5 & 60 & 9 & 5.61 & 70.72 & 3.83 & 0.7487 \\
\hline 3 & 0.5 & 60 & 10 & 3.83 & 74.55 & & 0.5834 \\
\hline 3 & 2 & 60 & 1 & 22.64 & 22.64 & 29.47 & 1.3549 \\
\hline 3 & 2 & 60 & 2 & 29.47 & 52.11 & 22.09 & 1.4693 \\
\hline 3 & 2 & 60 & 3 & 22.09 & 74.19 & 19.17 & 1.3441 \\
\hline 3 & 2 & 60 & 4 & 19.17 & 93.36 & 18.78 & 1.2826 \\
\hline 3 & 2 & 60 & 5 & 18.78 & 112.14 & 17.97 & 1.2738 \\
\hline 3 & 2 & 60 & 6 & 17.97 & 130.12 & 11.90 & 1.2546 \\
\hline 3 & 2 & 60 & 7 & 11.90 & 142.02 & 12.54 & 1.0756 \\
\hline 3 & 2 & 60 & 8 & 12.54 & 154.56 & 11.44 & 1.0984 \\
\hline 3 & 2 & 60 & 9 & 11.44 & 166.00 & 10.13 & 1.0584 \\
\hline 3 & 2 & 60 & 10 & 10.13 & 176.13 & & 1.0054 \\
\hline 3 & 3.5 & 60 & 1 & 41.02 & 41.02 & 39.64 & 1.6130 \\
\hline 3 & 3.5 & 60 & 2 & 39.64 & 80.66 & 36.35 & 1.5981 \\
\hline 3 & 3.5 & 60 & 3 & 36.35 & 117.01 & 34.61 & 1.5605 \\
\hline 3 & 3.5 & 60 & 4 & 34.61 & 151.62 & 29.05 & 1.5392 \\
\hline 3 & 3.5 & 60 & 5 & 29.05 & 180.67 & 23.47 & 1.4632 \\
\hline 3 & 3.5 & 60 & 6 & 23.47 & 204.15 & 22.77 & 1.3706 \\
\hline 3 & 3.5 & 60 & 7 & 22.77 & 226.92 & 20.70 & 1.3573 \\
\hline 3 & 3.5 & 60 & 8 & 20.70 & 247.62 & 17.37 & 1.3160 \\
\hline 3 & 3.5 & 60 & 9 & 17.37 & 264.99 & 14.64 & 1.2398 \\
\hline 3 & 3.5 & 60 & 10 & 14.64 & 279.63 & & 1.1655 \\
\hline 3 & 0.5 & 50 & 1 & 10.99 & 10.99 & 6.44 & 1.0410 \\
\hline 3 & 0.5 & 50 & 2 & 6.44 & 17.44 & 11.04 & 0.8092 \\
\hline 3 & 0.5 & 50 & 3 & 11.04 & 28.48 & 7.69 & 1.0431 \\
\hline 3 & 0.5 & 50 & 4 & 7.69 & 36.17 & 5.85 & 0.8860 \\
\hline 3 & 0.5 & 50 & 5 & 5.85 & 42.02 & 8.37 & 0.7670 \\
\hline 3 & 0.5 & 50 & 6 & 8.37 & 50.39 & 4.41 & 0.9228 \\
\hline 3 & 0.5 & 50 & 7 & 4.41 & 54.80 & 3.99 & 0.6445 \\
\hline 3 & 0.5 & 50 & 8 & 3.99 & 58.79 & 4.51 & 0.6006 \\
\hline 3 & 0.5 & 50 & 9 & 4.51 & 63.30 & 4.12 & 0.6543 \\
\hline 3 & 0.5 & 50 & 10 & 4.12 & 67.42 & . & 0.6148 \\
\hline 3 & 2 & 50 & 1 & 16.35 & 16.35 & 19.64 & 1.2136 \\
\hline 3 & 2 & 50 & 2 & 19.64 & 36.00 & 19.78 & 1.2932 \\
\hline 3 & 2 & 50 & 3 & 19.78 & 55.77 & 14.53 & 1.2961 \\
\hline 3 & 2 & 50 & 4 & 14.53 & 70.30 & 15.54 & 1.1622 \\
\hline 3 & 2 & 50 & 5 & 15.54 & 85.84 & 12.90 & 1.1915 \\
\hline 3 & 2 & 50 & 6 & 12.90 & 98.75 & 13.36 & 1.1107 \\
\hline 3 & 2 & 50 & 7 & 13.36 & 112.11 & 11.18 & 1.1259 \\
\hline 3 & 2 & 50 & 8 & 11.18 & 123.29 & 9.14 & 1.0485 \\
\hline 3 & 2 & 50 & 9 & 9.14 & 132.43 & 6.89 & 0.9610 \\
\hline 3 & 2 & 50 & 10 & 6.89 & 139.33 & & 0.8385 \\
\hline 3 & 3.5 & 50 & 1 & 30.56 & 30.56 & 27.43 & 1.4852 \\
\hline 3 & 3.5 & 50 & 2 & 27.43 & 57.99 & 25.20 & 1.4382 \\
\hline 3 & 3.5 & 50 & 3 & 25.20 & 83.20 & 21.95 & 1.4015 \\
\hline 3 & 3.5 & 50 & 4 & 21.95 & 105.15 & 23.34 & 1.3415 \\
\hline 3 & 3.5 & 50 & 5 & 23.34 & 128.49 & 18.92 & 1.3680 \\
\hline 3 & 3.5 & 50 & 6 & 18.92 & 147.40 & 16.72 & 1.2768 \\
\hline 3 & 3.5 & 50 & 7 & 16.72 & 164.12 & 15.62 & 1.2233 \\
\hline 3 & 3.5 & 50 & 8 & 15.62 & 179.74 & 16.04 & 1.1937 \\
\hline 3 & 3.5 & 50 & 9 & 16.04 & 195.78 & 12.51 & 1.2051 \\
\hline 3 & 3.5 & 50 & 10 & 12.51 & 208.29 & & 1.0972 \\
\hline 1 & 0.5 & 100 & 1 & 167.44 & 167.44 & 145.79 & 2.2238 \\
\hline 1 & 0.5 & 100 & 2 & 145.79 & 313.23 & 114.91 & 2.1637 \\
\hline 1 & 0.5 & 100 & 3 & 114.91 & 428.14 & 89.63 & 2.0604 \\
\hline 1 & 0.5 & 100 & 4 & 89.63 & 517.77 & 65.81 & 1.9524 \\
\hline 1 & 0.5 & 100 & 5 & 65.81 & 583.58 & 51.07 & 1.8183 \\
\hline 1 & 0.5 & 100 & 6 & 51.07 & 634.65 & 38.15 & 1.7081 \\
\hline
\end{tabular}


Table B2. Ammonia Test Results

(continued)

\begin{tabular}{|c|c|}
\hline Study & $\begin{array}{c}\text { Test } \\
\text { Sequence }\end{array}$ \\
\hline 5 & \\
\hline 5 & 3 \\
\hline 5 & 3 \\
\hline 5 & 3 \\
\hline 5 & 4 \\
\hline 5 & 4 \\
\hline 5 & 4 \\
\hline 5 & 4 \\
\hline 5 & 4 \\
\hline 5 & 4 \\
\hline 5 & 4 \\
\hline 5 & 4 \\
\hline 5 & 4 \\
\hline 5 & 4 \\
\hline 5 & 5 \\
\hline 5 & 5 \\
\hline 5 & 5 \\
\hline 5 & 5 \\
\hline 5 & 5 \\
\hline 5 & 5 \\
\hline 5 & 5 \\
\hline 5 & 5 \\
\hline 5 & 5 \\
\hline 5 & 5 \\
\hline 5 & 6 \\
\hline 5 & 6 \\
\hline 5 & 6 \\
\hline 5 & 6 \\
\hline 5 & 6 \\
\hline 5 & 6 \\
\hline 5 & 6 \\
\hline 5 & 6 \\
\hline 5 & 6 \\
\hline 5 & 6 \\
\hline 5 & 7 \\
\hline 5 & 7 \\
\hline 5 & 7 \\
\hline 5 & 7 \\
\hline 5 & 7 \\
\hline 5 & 7 \\
\hline 5 & 7 \\
\hline 5 & 7 \\
\hline 5 & 7 \\
\hline 5 & 7 \\
\hline 5 & 8 \\
\hline 5 & 8 \\
\hline 5 & 8 \\
\hline 5 & 8 \\
\hline 5 & 8 \\
\hline 5 & 8 \\
\hline 5 & 8 \\
\hline 5 & 8 \\
\hline 5 & 8 \\
\hline 5 & 8 \\
\hline 5 & 9 \\
\hline 5 & 9 \\
\hline 5 & 9 \\
\hline 5 & 9 \\
\hline 5 & 9 \\
\hline 5 & 9 \\
\hline 5 & 9 \\
\hline 5 & 9 \\
\hline 5 & 9 \\
\hline 5 & 9 \\
\hline 5 & 10 \\
\hline 5 & 10 \\
\hline
\end{tabular}

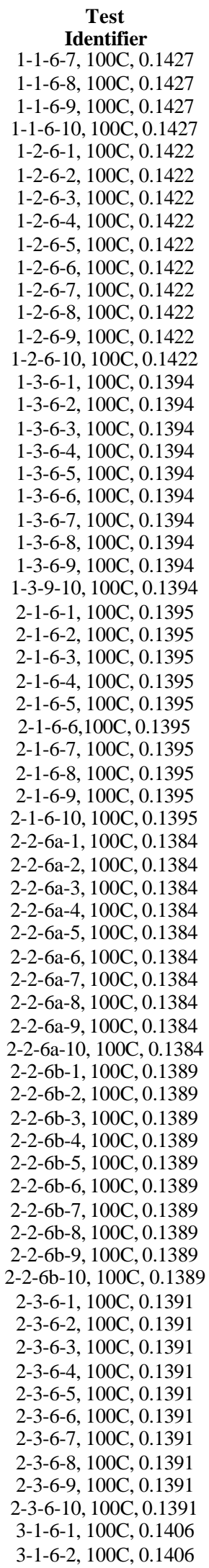

\begin{tabular}{|c|c|c|c|c|c|c|c|}
\hline $\begin{array}{c}\text { Salt } \\
\text { Solution }\end{array}$ & $\begin{array}{c}\mathrm{NH}_{3} \text { Conc } \\
(\text { molal })\end{array}$ & $\begin{array}{c}\text { Temperature } \\
\left({ }^{\circ} \mathbf{C}\right)\end{array}$ & $\begin{array}{l}\text { Sampling } \\
\text { Sequence }\end{array}$ & $\underset{\text { (peak area) }}{\mathbf{A}}$ & $\mathbf{y}$ & $\mathbf{x}$ & $\log (\mathrm{A})$ \\
\hline 1 & 0.5 & 100 & 7 & 38.15 & 672.79 & 26.31 & 1.5815 \\
\hline 1 & 0.5 & 100 & 8 & 26.31 & 699.10 & 20.17 & 1.4201 \\
\hline 1 & 0.5 & 100 & 9 & 20.17 & 719.27 & 16.35 & 1.3046 \\
\hline 1 & 0.5 & 100 & 10 & 16.35 & 735.62 & & 1.2135 \\
\hline 1 & 2 & 100 & 1 & 838.55 & 838.55 & 686.00 & 2.9235 \\
\hline 1 & 2 & 100 & 2 & 686.00 & 1524.55 & 517.00 & 2.8363 \\
\hline 1 & 2 & 100 & 3 & 517.00 & 2041.55 & 395.00 & 2.7135 \\
\hline 1 & 2 & 100 & 4 & 395.00 & 2436.55 & 295.00 & 2.5966 \\
\hline 1 & 2 & 100 & 5 & 295.00 & 2731.55 & 220.00 & 2.4698 \\
\hline 1 & 2 & 100 & 6 & 220.00 & 2951.55 & 165.00 & 2.3424 \\
\hline 1 & 2 & 100 & 7 & 165.00 & 3116.55 & 104.66 & 2.2175 \\
\hline 1 & 2 & 100 & 8 & 104.66 & 3221.21 & 90.55 & 2.0198 \\
\hline 1 & 2 & 100 & 9 & 90.55 & 3311.76 & 70.44 & 1.9569 \\
\hline 1 & 2 & 100 & 10 & 70.44 & 3382.20 & & 1.8478 \\
\hline 1 & 3.5 & 100 & 1 & 1338.00 & 1338.00 & 1049.00 & 3.1265 \\
\hline 1 & 3.5 & 100 & 2 & 1049.00 & 2387.00 & 797.00 & 3.0208 \\
\hline 1 & 3.5 & 100 & 3 & 797.00 & 3184.00 & 599.00 & 2.9015 \\
\hline 1 & 3.5 & 100 & 4 & 599.00 & 3783.00 & 447.00 & 2.7774 \\
\hline 1 & 3.5 & 100 & 5 & 447.00 & 4230.00 & 329.00 & 2.6503 \\
\hline 1 & 3.5 & 100 & 6 & 329.00 & 4559.00 & 242.00 & 2.5172 \\
\hline 1 & 3.5 & 100 & 7 & 242.00 & 4801.00 & 177.00 & 2.3838 \\
\hline 1 & 3.5 & 100 & 8 & 177.00 & 4978.00 & 131.00 & 2.2480 \\
\hline 1 & 3.5 & 100 & 9 & 131.00 & 5109.00 & 99.81 & 2.1173 \\
\hline 1 & 3.5 & 100 & 10 & 99.81 & 5208.81 & & 1.9992 \\
\hline 2 & 0.5 & 100 & 1 & 139.35 & 139.35 & 119.06 & 2.1441 \\
\hline 2 & 0.5 & 100 & 2 & 119.06 & 258.41 & 87.23 & 2.0758 \\
\hline 2 & 0.5 & 100 & 3 & 87.23 & 345.63 & 67.32 & 1.9406 \\
\hline 2 & 0.5 & 100 & 4 & 67.32 & 412.95 & 49.94 & 1.8282 \\
\hline 2 & 0.5 & 100 & 5 & 49.94 & 462.89 & 36.23 & 1.6984 \\
\hline 2 & 0.5 & 100 & 6 & 36.23 & 499.12 & 26.87 & 1.5590 \\
\hline 2 & 0.5 & 100 & 7 & 26.87 & 525.98 & 19.29 & 1.4292 \\
\hline 2 & 0.5 & 100 & 8 & 19.29 & 545.27 & 14.35 & 1.2852 \\
\hline 2 & 0.5 & 100 & 9 & 14.35 & 559.62 & 9.00 & 1.1568 \\
\hline 2 & 0.5 & 100 & 10 & 9.00 & 568.62 & & 0.9544 \\
\hline 2 & 2 & 100 & 1 & 361.70 & 361.70 & 296.00 & 2.5583 \\
\hline 2 & 2 & 100 & 2 & 296.00 & 657.70 & 227.00 & 2.4713 \\
\hline 2 & 2 & 100 & 3 & 227.00 & 884.70 & 173.16 & 2.3560 \\
\hline 2 & 2 & 100 & 4 & 173.16 & 1057.85 & 130.58 & 2.2384 \\
\hline 2 & 2 & 100 & 5 & 130.58 & 1188.43 & 95.00 & 2.1159 \\
\hline 2 & 2 & 100 & 6 & 95.00 & 1283.44 & 70.79 & 1.9777 \\
\hline 2 & 2 & 100 & 7 & 70.79 & 1354.22 & 52.73 & 1.8499 \\
\hline 2 & 2 & 100 & 8 & 52.73 & 1406.95 & 37.41 & 1.7220 \\
\hline 2 & 2 & 100 & 9 & 37.41 & 1444.36 & 27.68 & 1.5729 \\
\hline 2 & 2 & 100 & 10 & 27.68 & 1472.04 & & 1.4422 \\
\hline 2 & 2 & 100 & 1 & 393.04 & 1865.08 & 325.00 & 2.5944 \\
\hline 2 & 2 & 100 & 2 & 325.00 & 2190.08 & 159.66 & 2.5119 \\
\hline 2 & 2 & 100 & 3 & 159.66 & 2349.74 & 186.49 & 2.2032 \\
\hline 2 & 2 & 100 & 4 & 186.49 & 2536.23 & 138.63 & 2.2707 \\
\hline 2 & 2 & 100 & 5 & 138.63 & 2674.86 & 104.24 & 2.1419 \\
\hline 2 & 2 & 100 & 6 & 104.24 & 2779.09 & 75.82 & 2.0180 \\
\hline 2 & 2 & 100 & 7 & 75.82 & 2854.91 & 55.13 & 1.8798 \\
\hline 2 & 2 & 100 & 8 & 55.13 & 2910.04 & 39.69 & 1.7414 \\
\hline 2 & 2 & 100 & 9 & 39.69 & 2949.73 & 28.66 & 1.5987 \\
\hline 2 & 2 & 100 & 10 & 28.66 & 2978.39 & & 1.4572 \\
\hline 2 & 3.5 & 100 & 1 & 635.00 & 635.00 & 523.00 & 2.8028 \\
\hline 2 & 3.5 & 100 & 2 & 523.00 & 1158.00 & 394.90 & 2.7185 \\
\hline 2 & 3.5 & 100 & 3 & 394.90 & 1552.90 & 294.11 & 2.5965 \\
\hline 2 & 3.5 & 100 & 4 & 294.11 & 1847.01 & 216.10 & 2.4685 \\
\hline 2 & 3.5 & 100 & 5 & 216.10 & 2063.10 & 156.64 & 2.3347 \\
\hline 2 & 3.5 & 100 & 6 & 156.64 & 2219.74 & 104.74 & 2.1949 \\
\hline 2 & 3.5 & 100 & 7 & 104.74 & 2324.48 & 80.35 & 2.0201 \\
\hline 2 & 3.5 & 100 & 8 & 80.35 & 2404.83 & 57.20 & 1.9050 \\
\hline 2 & 3.5 & 100 & 9 & 57.20 & 2462.04 & 41.28 & 1.7574 \\
\hline 2 & 3.5 & 100 & 10 & 41.28 & 2503.32 & & 1.6157 \\
\hline 3 & 0.5 & 100 & 1 & 21.05 & 21.05 & 17.50 & 1.3232 \\
\hline 3 & 0.5 & 100 & 2 & 17.50 & 38.55 & 11.76 & 1.2430 \\
\hline
\end{tabular}


Table B2. Ammonia Test Results

(continued)

\begin{tabular}{|c|c|}
\hline Study & $\begin{array}{c}\text { Test } \\
\text { Sequence }\end{array}$ \\
\hline 5 & 10 \\
\hline 5 & 10 \\
\hline 5 & 10 \\
\hline 5 & 10 \\
\hline 5 & 10 \\
\hline 5 & 10 \\
\hline 5 & 10 \\
\hline 5 & 10 \\
\hline 5 & 11 \\
\hline 5 & 11 \\
\hline 5 & 11 \\
\hline 5 & 11 \\
\hline 5 & 11 \\
\hline 5 & 11 \\
\hline 5 & 11 \\
\hline 5 & 11 \\
\hline 5 & 11 \\
\hline 5 & 11 \\
\hline 5 & 12 \\
\hline 5 & 12 \\
\hline 5 & 12 \\
\hline 5 & 12 \\
\hline 5 & 12 \\
\hline 5 & 12 \\
\hline 5 & 12 \\
\hline 5 & 12 \\
\hline 5 & 12 \\
\hline 5 & 12 \\
\hline 5 & 13 \\
\hline 5 & 13 \\
\hline 5 & 13 \\
\hline 5 & 13 \\
\hline 5 & 13 \\
\hline 5 & 13 \\
\hline 5 & 13 \\
\hline 5 & 13 \\
\hline 5 & 13 \\
\hline 5 & 13 \\
\hline 5 & 14 \\
\hline 5 & 14 \\
\hline 5 & 14 \\
\hline 5 & 14 \\
\hline 5 & 14 \\
\hline 5 & 14 \\
\hline 5 & 14 \\
\hline 5 & 14 \\
\hline 5 & 14 \\
\hline 5 & 14 \\
\hline 5 & 15 \\
\hline 5 & 15 \\
\hline 5 & 15 \\
\hline 5 & 15 \\
\hline 5 & 15 \\
\hline 5 & 15 \\
\hline 5 & 15 \\
\hline 5 & 15 \\
\hline 5 & 15 \\
\hline 5 & 15 \\
\hline 5 & 16 \\
\hline 5 & 16 \\
\hline 5 & 16 \\
\hline 5 & 16 \\
\hline 5 & 16 \\
\hline 5 & 16 \\
\hline 5 & 16 \\
\hline 5 & 16 \\
\hline
\end{tabular}

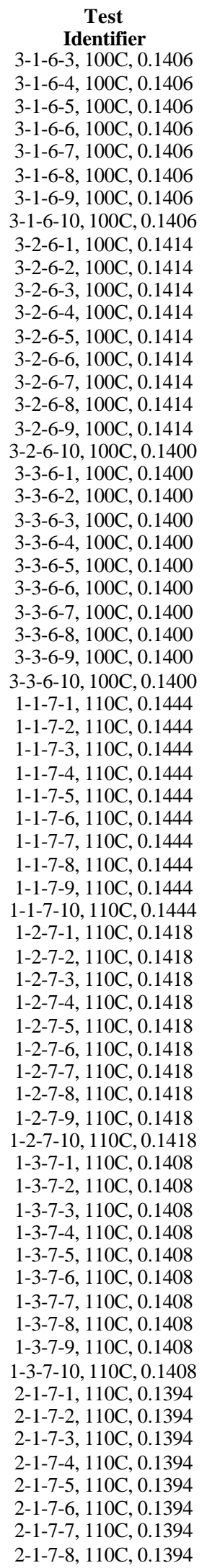

\begin{tabular}{|c|c|}
\hline Salt & $\mathbf{N H}_{3}$ Conc \\
\hline Solution & (molal) \\
\hline 3 & 0.5 \\
\hline 3 & 0.5 \\
\hline 3 & 0.5 \\
\hline 3 & 0.5 \\
\hline 3 & 0.5 \\
\hline 3 & 0.5 \\
\hline 3 & 0.5 \\
\hline 3 & 0.5 \\
\hline 3 & 2 \\
\hline 3 & 2 \\
\hline 3 & 2 \\
\hline 3 & 2 \\
\hline 3 & 2 \\
\hline 3 & 2 \\
\hline 3 & 2 \\
\hline 3 & 2 \\
\hline 3 & 2 \\
\hline 3 & 2 \\
\hline 3 & 3.5 \\
\hline 3 & 3.5 \\
\hline 3 & 3.5 \\
\hline 3 & 3.5 \\
\hline 3 & 3.5 \\
\hline 3 & 3.5 \\
\hline 3 & 3.5 \\
\hline 3 & 3.5 \\
\hline 3 & 3.5 \\
\hline 3 & 3.5 \\
\hline 1 & 0.5 \\
\hline 1 & 0.5 \\
\hline 1 & 0.5 \\
\hline 1 & 0.5 \\
\hline 1 & 0.5 \\
\hline 1 & 0.5 \\
\hline 1 & 0.5 \\
\hline 1 & 0.5 \\
\hline 1 & 0.5 \\
\hline 1 & 0.5 \\
\hline 1 & 2 \\
\hline 1 & 2 \\
\hline 1 & 2 \\
\hline 1 & 2 \\
\hline 1 & 2 \\
\hline 1 & 2 \\
\hline 1 & 2 \\
\hline 1 & 2 \\
\hline 1 & 2 \\
\hline 1 & 2 \\
\hline 1 & 3.5 \\
\hline 1 & 3.5 \\
\hline 1 & 3.5 \\
\hline 1 & 3.5 \\
\hline 1 & 3.5 \\
\hline 1 & 3.5 \\
\hline 1 & 3.5 \\
\hline 1 & 3.5 \\
\hline 1 & 3.5 \\
\hline 1 & 3.5 \\
\hline 2 & 0.5 \\
\hline 2 & 0.5 \\
\hline 2 & 0.5 \\
\hline 2 & 0.5 \\
\hline 2 & 0.5 \\
\hline 2 & 0.5 \\
\hline 2 & 0.5 \\
\hline 2 & 0.5 \\
\hline
\end{tabular}

emperature Sampling

A

$\begin{array}{ccc}\left({ }^{\mathbf{C}} \mathbf{C}\right) & \text { Sequence } & \text { (peak area) } \\ 100 & 3 & 11.76 \\ 100 & 4 & 0.00 \\ 100 & 5 & 0.00 \\ 100 & 6 & 0.00 \\ 100 & 7 & 0.00 \\ 100 & 8 & 3.30 \\ 100 & 9 & 0.00 \\ 100 & 10 & 0.00 \\ 100 & 1 & 25.04 \\ 100 & 2 & 25.33\end{array}$

100
100

100
100

100

100

100
100

100

100

100
100

100

100

100

110

110

110

110
110

110

110

110

110

110

110

110

110

110

110
110

110

110

110
110

110

110

110

110

110
110

110

110

110
110

110

110

110
110

110

$\underset{50.31}{\mathbf{y}}$

50.31

50.31

50.31
50.31

53.61

53.61

53.61

25.04
50.37

50.37

18.59

12.29

41.88

0.00

68.95
81.24

123.12

0.00
0.00

123.12

123.12

123.12

0.00

0.00

123.12

44.14
41.86

29.74

22.71

15.17

11.50

7.57

0.00

0.00

0.00

223.11
162.61

123.26

91.91

76.28

34.47

23.07

56.35

29.78

34.83

1045.00

766.00

535.07

358.00

170.74

102.45

105.10

75.88

65.81

35.57

1601.00

771.00

524.00

346.00

235.00

123.12

44.14
86.00

115.74

138.45

153.62

165.12

172.69

172.69

172.69

172.69
223.11

385.72

508.98

600.90

677.17

711.64

734.71

791.06

820.84

855.67

1045.00

1811.00

2346.07

2704.07

2874.80

2977.25

3082.35

3158.23

3224.04

1601.00

2755.00

3526.00

4050.00

4396.00

4631.00

160.13

4791.13

4898.64

73.39

4972.03

51.47

5023.50

122.84

94.05

216.89

281.96

62.15

344.11

396.67

47.12

443.78

4.19

447.97

$\begin{array}{cc}\mathbf{x} & \log (\mathbf{A}) \\ 0.00 & 1.0706\end{array}$

$0.00 \quad 1.0706$

0.00

0.00

0.00

0.5184

$25.33 \quad 1.3985$

$18.59 \quad 1.4036$

$12.29 \quad 1.2692$

$41.88 \quad 1.0895$

$0.00 \quad 1.6220$

0.00

0.00

0.00

$\begin{array}{ll}41.86 & 1.6449\end{array}$

$29.74 \quad 1.6218$

$22.71 \quad 1.4734$

$15.17 \quad 1.3562$

$11.50 \quad 1.1809$

$\begin{array}{ll}7.57 & 1.0607\end{array}$

$0.00 \quad 0.8792$

0.00
0.00

$162.61 \quad 2.3485$

$123.26 \quad 2.2112$

$\begin{array}{ll}91.91 & 2.0908\end{array}$

$\begin{array}{ll}76.28 & 1.9634\end{array}$

$34.47 \quad 1.8824$

$23.07 \quad 1.5374$

$56.35 \quad 1.3631$

$29.78 \quad 1.7509$

$34.83 \quad 1.4739$

$\begin{array}{ll}766.00 & 3.0191\end{array}$

$\begin{array}{ll}535.07 & 2.8842\end{array}$

$\begin{array}{ll}358.00 & 2.7284\end{array}$

$\begin{array}{ll}170.74 & 2.5539\end{array}$

$102.45 \quad 2.2323$

$105.10 \quad 2.0105$

$\begin{array}{ll}75.88 & 2.0216\end{array}$

$65.81 \quad 1.8801$

$35.57 \quad 1.8183$

$1154.00-1.5511$

$771.00 \quad 3.0622$

$524.00 \quad 2.8871$

$\begin{array}{ll}346.00 & 2.7193\end{array}$

$\begin{array}{ll}235.00 & 2.5391\end{array}$

$\begin{array}{ll}160.13 & 2.3711\end{array}$

$107.51 \quad 2.2045$

$\begin{array}{ll}73.39 & 2.0314 \\ 51.47 & 1.8656\end{array}$

$51.47 \quad 1.8656$

$94.05 \quad 2.0893$

$65.07 \quad 1.9734$

$62.15 \quad 1.8134$

$52.55 \quad 1.7935$

$47.12 \quad 1.7206$

$\begin{array}{rr}4.19 & 1.6732 \\ 30.18 & 0.6226\end{array}$

$\begin{array}{llll}30.18 & 478.15 & 34.69 & 1.4797\end{array}$ 
Table B2. Ammonia Test Results

(continued)

\begin{tabular}{|c|c|c|}
\hline Study & $\begin{array}{c}\text { Test } \\
\text { Sequence }\end{array}$ & $\begin{array}{c}\text { Test } \\
\text { Identifier }\end{array}$ \\
\hline 5 & 16 & $2-1-7-9,110 \mathrm{C}, 0.1394$ \\
\hline 5 & 16 & $2-1-7-10,110 \mathrm{C}, 0.1394$ \\
\hline 6 & 1 & $1-3-7-1,110 \mathrm{C}, 0.1408$ \\
\hline 6 & 1 & $1-3-7-2,110 \mathrm{C}, 0.1408$ \\
\hline 6 & 1 & $1-3-7-3,110 \mathrm{C}, 0.1408$ \\
\hline 6 & 1 & $1-3-7-4,110 \mathrm{C}, 0.1408$ \\
\hline 6 & 1 & $1-3-7-5,110 \mathrm{C}, 0.1408$ \\
\hline 6 & 1 & $1-3-7-6,110 \mathrm{C}, 0.1408$ \\
\hline 6 & 1 & $1-3-7-7,110 \mathrm{C}, 0.1408$ \\
\hline 6 & 1 & $1-3-7-8,110 \mathrm{C}, 0.1408$ \\
\hline 6 & 1 & $1-3-7-9,110 \mathrm{C}, 0.1408$ \\
\hline 6 & 1 & $1-3-7-10,110 \mathrm{C}, 0.1408$ \\
\hline 6 & 2 & $2-1-7-1,110 \mathrm{C}, 0.1394$ \\
\hline 6 & 2 & $2-1-7-2,110 \mathrm{C}, 0.1394$ \\
\hline 6 & 2 & $2-1-7-3,110 \mathrm{C}, 0.1394$ \\
\hline 6 & 2 & $2-1-7-4,110 \mathrm{C}, 0.1394$ \\
\hline 6 & 2 & $2-1-7-5,110 \mathrm{C}, 0.1394$ \\
\hline 6 & 2 & $2-1-7-6,110 \mathrm{C}, 0.1394$ \\
\hline 6 & 2 & $2-1-7-7,110 \mathrm{C}, 0.1394$ \\
\hline 6 & 2 & $2-1-7-8,110 \mathrm{C}, 0.1394$ \\
\hline 6 & 2 & $2-1-7-9,110 \mathrm{C}, 0.1394$ \\
\hline 6 & 2 & $2-1-7-10,110 \mathrm{C}, 0.1394$ \\
\hline 6 & 3 & $2-2-7 a-1,110 C, 0.1378$ \\
\hline 6 & 3 & $2-2-7 a-2,110 C, 0.1378$ \\
\hline 6 & 3 & $2-2-7 a-3,110 C, 0.1378$ \\
\hline 6 & 3 & $2-2-7 \mathrm{a}-4,110 \mathrm{C}, 0.1378$ \\
\hline 6 & 3 & $2-2-7 a-5,110 C, 0.1378$ \\
\hline 6 & 3 & $2-2-7 a-6,110 C, 0.1378$ \\
\hline 6 & 3 & $2-2-7 a-7,110 C, 0.1378$ \\
\hline 6 & 3 & $2-2-7 a-8,110 C, 0.1378$ \\
\hline 6 & 3 & 2-2-7a-9, 110C, 0.1378 \\
\hline 6 & 3 & $2-2-7 a-10,110 C, 0.1378$ \\
\hline 6 & 4 & $2-2-7 b-1,110 C, 0.1383$ \\
\hline 6 & 4 & $2-2-7 b-2,110 \mathrm{C}, 0.1383$ \\
\hline 6 & 4 & $2-2-7 b-3,110 \mathrm{C}, 0.1383$ \\
\hline 6 & 4 & $2-2-7 b-4,110 \mathrm{C}, 0.1383$ \\
\hline 6 & 4 & $2-2-7 b-5,110 C, 0.1383$ \\
\hline 6 & 4 & $2-2-7 b-6,110 \mathrm{C}, 0.1383$ \\
\hline 6 & 4 & $2-2-7 b-7,110 C, 0.1383$ \\
\hline 6 & 4 & $2-2-7 b-8,110 C, 0.1383$ \\
\hline 6 & 4 & $2-2-7 b-9,110 \mathrm{C}, 0.1383$ \\
\hline 6 & 4 & $2-2-7 b-10,110 C, 0.1383$ \\
\hline 6 & 5 & $2-3-7-1,110 \mathrm{C}, 0.1375$ \\
\hline 6 & 5 & $2-3-7-2,110 \mathrm{C}, 0.1375$ \\
\hline 6 & 5 & $2-3-7-3,110 \mathrm{C}, 0.1375$ \\
\hline 6 & 5 & $2-3-7-4,110 \mathrm{C}, 0.1375$ \\
\hline 6 & 5 & $2-3-7-5,110 \mathrm{C}, 0.1375$ \\
\hline 6 & 5 & $2-3-7-6,110 \mathrm{C}, 0.1375$ \\
\hline 6 & 5 & $2-3-7-7,110 \mathrm{C}, 0.1375$ \\
\hline 6 & 5 & $2-3-7-8,110 \mathrm{C}, 0.1375$ \\
\hline 6 & 5 & $2-3-7-9,110 \mathrm{C}, 0.1375$ \\
\hline 6 & 5 & $2-3-7-10,110 \mathrm{C}, 0.1375$ \\
\hline 7 & 1 & $3-1-1-1,0.1390 \mathrm{~g}, 50 \mathrm{C}$ \\
\hline 7 & 1 & $3-1-1-2,0.1390 \mathrm{~g}, 50 \mathrm{C}$ \\
\hline 7 & 1 & $3-1-1-3,0.1390 \mathrm{~g}, 50 \mathrm{C}$ \\
\hline 7 & 1 & $3-1-1-4,0.1390 \mathrm{~g}, 50 \mathrm{C}$ \\
\hline 7 & 1 & $3-1-1-5,0.1390 \mathrm{~g}, 50 \mathrm{C}$ \\
\hline 7 & 1 & $3-1-1-6,0.1390 \mathrm{~g}, 50 \mathrm{C}$ \\
\hline 7 & 1 & $3-1-1-7,0.1390 \mathrm{~g}, 50 \mathrm{C}$ \\
\hline 7 & 1 & $3-1-1-8,0.1390 \mathrm{~g}, 50 \mathrm{C}$ \\
\hline 7 & 1 & $3-1-1-9,0.1390 \mathrm{~g}, 50 \mathrm{C}$ \\
\hline 7 & 1 & $3-1-1-10,0.1390 \mathrm{~g}, 50 \mathrm{C}$ \\
\hline 7 & 2 & $3-2-1-1,0.1380 \mathrm{~g}, 50 \mathrm{C}$ \\
\hline 7 & 2 & $3-2-1-2,0.1380 \mathrm{~g}, 50 \mathrm{C}$ \\
\hline 7 & 2 & $3-2-1-3,0.1380 \mathrm{~g}, 50 \mathrm{C}$ \\
\hline 7 & 2 & $3-2-1-4,0.1380 \mathrm{~g}, 50 \mathrm{C}$ \\
\hline
\end{tabular}

\begin{tabular}{|c|c|c|c|c|c|c|c|}
\hline $\begin{array}{c}\text { Salt } \\
\text { Solution }\end{array}$ & $\begin{array}{c}\mathrm{NH}_{3} \text { Conc } \\
(\text { molal })\end{array}$ & $\begin{array}{c}\text { Temperature } \\
\left({ }^{\circ} \mathbf{C}\right)\end{array}$ & $\begin{array}{l}\text { Sampling } \\
\text { Sequence }\end{array}$ & $\underset{\text { (peak area) }}{\mathbf{A}}$ & $\mathbf{y}$ & $\mathbf{x}$ & $\log (\mathrm{A})$ \\
\hline 2 & 0.5 & 110 & & 34.69 & 512.84 & 36.05 & 1.5402 \\
\hline 2 & 0.5 & 110 & 10 & 36.05 & 548.89 & & 1.5569 \\
\hline 1 & 3.5 & 110 & 1 & 1600.92 & 1600.92 & 1153.99 & 3.2044 \\
\hline 1 & 3.5 & 110 & 2 & 1153.99 & 2754.90 & 770.94 & 3.0622 \\
\hline 1 & 3.5 & 110 & 3 & 770.94 & 3525.84 & 523.65 & 2.8870 \\
\hline 1 & 3.5 & 110 & 4 & 523.65 & 4049.49 & 346.42 & 2.7190 \\
\hline 1 & 3.5 & 110 & 5 & 346.42 & 4395.91 & 235.34 & 2.5396 \\
\hline 1 & 3.5 & 110 & 6 & 235.34 & 4631.25 & 160.13 & 2.3717 \\
\hline 1 & 3.5 & 110 & 7 & 160.13 & 4791.39 & 107.51 & 2.2045 \\
\hline 1 & 3.5 & 110 & 8 & 107.51 & 4898.89 & 73.39 & 2.0314 \\
\hline 1 & 3.5 & 110 & 9 & 73.39 & 4972.28 & 51.47 & 1.8656 \\
\hline 1 & 3.5 & 110 & 10 & 51.47 & 5023.75 & & 1.7115 \\
\hline 2 & 0.5 & 110 & 1 & 122.84 & 122.84 & 94.05 & 2.0893 \\
\hline 2 & 0.5 & 110 & 2 & 94.05 & 216.89 & 65.07 & 1.9734 \\
\hline 2 & 0.5 & 110 & 3 & 65.07 & 281.96 & 62.15 & 1.8134 \\
\hline 2 & 0.5 & 110 & 4 & 62.15 & 344.11 & 52.55 & 1.7935 \\
\hline 2 & 0.5 & 110 & 5 & 52.55 & 396.67 & 47.12 & 1.7206 \\
\hline 2 & 0.5 & 110 & 6 & 47.12 & 443.78 & 20.00 & 1.6732 \\
\hline 2 & 0.5 & 110 & 7 & 20.00 & 463.78 & 30.18 & 1.3010 \\
\hline 2 & 0.5 & 110 & 8 & 30.18 & 493.96 & 34.69 & 1.4797 \\
\hline 2 & 0.5 & 110 & 9 & 34.69 & 528.65 & 36.05 & 1.5402 \\
\hline 2 & 0.5 & 110 & 10 & 36.05 & 564.70 & & 1.5569 \\
\hline 2 & 2 & 110 & 1 & 383.92 & 383.92 & 288.37 & 2.5842 \\
\hline 2 & 2 & 110 & 2 & 288.37 & 672.29 & 204.81 & 2.4599 \\
\hline 2 & 2 & 110 & 3 & 204.81 & 877.10 & 138.09 & 2.3114 \\
\hline 2 & 2 & 110 & 4 & 138.09 & 1015.19 & 98.26 & 2.1402 \\
\hline 2 & 2 & 110 & 5 & 98.26 & 1113.45 & 111.86 & 1.9924 \\
\hline 2 & 2 & 110 & 6 & 111.86 & 1225.31 & 37.00 & 2.0487 \\
\hline 2 & 2 & 110 & 7 & 37.00 & 1262.31 & 43.00 & 1.5682 \\
\hline 2 & 2 & 110 & 8 & 43.00 & 1305.31 & 30.00 & 1.6335 \\
\hline 2 & 2 & 110 & 9 & 30.00 & 1335.31 & 18.00 & 1.4771 \\
\hline 2 & 2 & 110 & 10 & 18.00 & 1353.31 & & 1.2553 \\
\hline 2 & 2 & 110 & 1 & 342.96 & 1696.26 & 258.88 & 2.5352 \\
\hline 2 & 2 & 110 & 2 & 258.88 & 1955.14 & 128.99 & 2.4131 \\
\hline 2 & 2 & 110 & 3 & 128.99 & 2084.12 & 107.27 & 2.1105 \\
\hline 2 & 2 & 110 & 4 & 107.27 & 2191.40 & 75.07 & 2.0305 \\
\hline 2 & 2 & 110 & 5 & 75.07 & 2266.47 & 53.82 & 1.8755 \\
\hline 2 & 2 & 110 & 6 & 53.82 & 2320.29 & 54.46 & 1.7310 \\
\hline 2 & 2 & 110 & 7 & 54.46 & 2374.75 & 40.01 & 1.7361 \\
\hline 2 & 2 & 110 & 8 & 40.01 & 2414.76 & 36.02 & 1.6021 \\
\hline 2 & 2 & 110 & 9 & 36.02 & 2450.78 & 19.51 & 1.5566 \\
\hline 2 & 2 & 110 & 10 & 19.51 & 2470.29 & 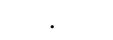 & 1.2902 \\
\hline 2 & 3.5 & 110 & 1 & 538.78 & 538.78 & 395.44 & 2.7314 \\
\hline 2 & 3.5 & 110 & 2 & 395.44 & 934.22 & 221.70 & 2.5971 \\
\hline 2 & 3.5 & 110 & 3 & 221.70 & 1155.92 & 147.40 & 2.3458 \\
\hline 2 & 3.5 & 110 & 4 & 147.40 & 1303.32 & 105.59 & 2.1685 \\
\hline 2 & 3.5 & 110 & 5 & 105.59 & 1408.91 & 80.78 & 2.0236 \\
\hline 2 & 3.5 & 110 & 6 & 80.78 & 1489.69 & 72.77 & 1.9073 \\
\hline 2 & 3.5 & 110 & 7 & 72.77 & 1562.46 & 32.87 & 1.8620 \\
\hline 2 & 3.5 & 110 & 8 & 32.87 & 1595.33 & 45.23 & 1.5167 \\
\hline 2 & 3.5 & 110 & 9 & 45.23 & 1640.55 & 0.00 & 1.6554 \\
\hline 2 & 3.5 & 110 & 10 & 0.00 & 1640.55 & & \\
\hline 3 & 0.5 & 50 & 1 & 243.72 & 243.72 & 211.69 & 2.3869 \\
\hline 3 & 0.5 & 50 & 2 & 211.69 & 455.41 & 160.77 & 2.3257 \\
\hline 3 & 0.5 & 50 & 3 & 160.77 & 616.19 & 121.69 & 2.2062 \\
\hline 3 & 0.5 & 50 & 4 & 121.69 & 737.88 & 91.34 & 2.0853 \\
\hline 3 & 0.5 & 50 & 5 & 91.34 & 829.21 & 45.43 & 1.9606 \\
\hline 3 & 0.5 & 50 & 6 & 45.43 & 874.64 & 49.09 & 1.6573 \\
\hline 3 & 0.5 & 50 & 7 & 49.09 & 923.73 & 40.03 & 1.6910 \\
\hline 3 & 0.5 & 50 & 8 & 40.03 & 963.76 & 24.29 & 1.6024 \\
\hline 3 & 0.5 & 50 & 9 & 24.29 & 988.05 & 0.00 & 1.3854 \\
\hline 3 & 0.5 & 50 & 10 & 0.00 & 988.05 & & \\
\hline 3 & 2 & 50 & 1 & 1038.38 & 1038.38 & 816.57 & 3.0164 \\
\hline 3 & 2 & 50 & 2 & 816.57 & 1854.95 & 624.01 & 2.9120 \\
\hline 3 & 2 & 50 & 3 & 624.01 & 2478.96 & 517.28 & 2.7952 \\
\hline 3 & 2 & 50 & 4 & 517.28 & 2996.23 & 401.45 & 2.7137 \\
\hline
\end{tabular}


Table B2. Ammonia Test Results

(continued)

Test Study Sequence $7 \quad 2$

$3-2-1-6,0.1380 \mathrm{~g}, 50 \mathrm{C}$

3-2-1-7, 0.1380g, 50C

$3-2-1-8,0.1380 \mathrm{~g}, 50 \mathrm{C}$

3-2-1-9, 0.1380g, 50C

$3-2-1-10,0.1380 \mathrm{~g}, 50 \mathrm{C}$

3-3-1-1, 0.1372g, 50C

3-3-1-2, 0.1372g, 50C

3-3-1-3, 0.1372g, 50C

3-3-1-4, 0.1372g, 50C

3-3-1-5, 0.1372g, 50C

3-3-1-6, 0.1372g, 50C

3-3-1-7, 0.1372g, 50C

$3-3-1-8,0.1372 \mathrm{~g}, 50 \mathrm{C}$

$3-3-1-9,0.1372 \mathrm{~g}, 50 \mathrm{C}$

3-3-1-10, 0.1372g, 50C

3-1-2-1, 0.1396g, 60C

3-1-2-2, 0.1396g, 60C

$3-1-2-3,0.1396 \mathrm{~g}, 60 \mathrm{C}$

3-1-2-4, 0.1396g, 60C

$3-1-2-5,0.1396 \mathrm{~g}, 60 \mathrm{C}$

3-1-2-6, 0.1396g, 60C

$3-1-2-7,0.1396 \mathrm{~g}, 60 \mathrm{C}$

$3-1-2-8,0.1396 \mathrm{~g}, 60 \mathrm{C}$

3-1-2-9, 0.1396g, 60C

3-1-2-10, 0.1396g, 60C

3-2-2-1, 0.1368g, 60C

3-2-2-2, 0.1368g, 60C

3-2-2-3, 0.1368g, 60C

$3-2-2-4,0.1368 \mathrm{~g}, 60 \mathrm{C}$

3-2-2-5, 0.1368g, 60C

3-2-2-6, 0.1368g, 60C

3-2-2-7, 0.1368g, 60C

$3-2-2-8,0.1368 \mathrm{~g}, 60 \mathrm{C}$

3-2-2-9, 0.1368g, 60C

3-2-2-10, 0.1368g, 60C

3-1-2-1, 60C, 0.1383

$3-1-2-2,60 \mathrm{C}, 0.1383$

3-1-2-3, 60C, 0.1383

$3-1-2-4,60 \mathrm{C}, 0.1383$

3-1-2-5, 60C, 0.1383

$3-1-2-6,60 \mathrm{C}, 0.1383$

3-1-2-7, 60C, 0.1383

$3-1-2-8,60 \mathrm{C}, 0.1383$

3-1-2-9, 60C, 0.1383

$3-1-2-10,60 \mathrm{C}, 0.1383$

3-2-2-1, 60C, 0.1373

$3-2-2-2,60 \mathrm{C}, 0.1373$

$3-2-2-3,60 \mathrm{C}, 0.1373$

3-2-2-4, 60C, 0.1373

3-2-2-5, 60C, 0.1373

3-2-2-6, 60C, 0.1373

$3-2-2-7,60 \mathrm{C}, 0.1373$

$3-2-2-8,60 \mathrm{C}, 0.1373$

$3-2-2-9,60 \mathrm{C}, 0.1373$

3-2-2-10, 60C, 0.1373

3-3-2-1, 60C, 0.1370

3-3-2-2, 60C, 0.1370

3-3-2-3, 60C, 0.1370

3-3-2-4, 60C, 0.1370

$3-3-2-5,60 \mathrm{C}, 0.1370$

3-3-2-6, 60C, 0.1370

$3-3-2-7,60 \mathrm{C}, 0.1370$

3-3-2-8, 60C, 0.1370

3-3-2-9, 60C, 0.1370

3-3-2-10, 60C, 0.1370

\begin{tabular}{|c|c|c|c|c|c|c|c|}
\hline $\begin{array}{c}\text { Salt } \\
\text { Solution }\end{array}$ & $\begin{array}{c}\mathrm{NH}_{3} \text { Conc } \\
(\mathrm{molal})\end{array}$ & $\begin{array}{c}\text { Temperature } \\
\left({ }^{\circ} \mathbf{C}\right)\end{array}$ & $\begin{array}{l}\text { Sampling } \\
\text { Sequence }\end{array}$ & $\underset{\text { (peak area) }}{\mathbf{A}}$ & $\mathbf{y}$ & $\mathbf{x}$ & $\log (A)$ \\
\hline 3 & 2 & 50 & 5 & 401.45 & 3397.68 & 318.36 & 2.6036 \\
\hline 3 & 2 & 50 & 6 & 318.36 & 3716.04 & 254.33 & 2.5029 \\
\hline 3 & 2 & 50 & 7 & 254.33 & 3970.37 & 206.19 & 2.4054 \\
\hline 3 & 2 & 50 & 8 & 206.19 & 4176.56 & 165.69 & 2.3143 \\
\hline 3 & 2 & 50 & 9 & 165.69 & 4342.24 & 135.05 & 2.2193 \\
\hline 3 & 2 & 50 & 10 & 135.05 & 4477.29 & & 2.1305 \\
\hline 3 & 3.5 & 50 & 1 & 1698.12 & 1698.12 & 1330.85 & 3.2300 \\
\hline 3 & 3.5 & 50 & 2 & 1330.85 & 3028.97 & 1021.98 & 3.1241 \\
\hline 3 & 3.5 & 50 & 3 & 1021.98 & 4050.95 & 841.95 & 3.0094 \\
\hline 3 & 3.5 & 50 & 4 & 841.95 & 4892.90 & 645.87 & 2.9253 \\
\hline 3 & 3.5 & 50 & 5 & 645.87 & 5538.77 & 506.75 & 2.8101 \\
\hline 3 & 3.5 & 50 & 6 & 506.75 & 6045.52 & 401.90 & 2.7048 \\
\hline 3 & 3.5 & 50 & 7 & 401.90 & 6447.41 & 326.98 & 2.6041 \\
\hline 3 & 3.5 & 50 & 8 & 326.98 & 6774.39 & 274.88 & 2.5145 \\
\hline 3 & 3.5 & 50 & 9 & 274.88 & 7049.27 & 241.16 & 2.4391 \\
\hline 3 & 3.5 & 50 & 10 & 241.16 & 7290.42 & & 2.3823 \\
\hline 3 & 0.5 & 60 & 1 & 308.88 & 308.88 & 238.51 & 2.4898 \\
\hline 3 & 0.5 & 60 & 2 & 238.51 & 547.39 & 175.67 & 2.3775 \\
\hline 3 & 0.5 & 60 & 3 & 175.67 & 723.05 & 132.38 & 2.2447 \\
\hline 3 & 0.5 & 60 & 4 & 132.38 & 855.43 & 38.73 & 2.1218 \\
\hline 3 & 0.5 & 60 & 5 & 38.73 & 894.16 & 65.89 & 1.5881 \\
\hline 3 & 0.5 & 60 & 6 & 65.89 & 960.05 & 41.57 & 1.8188 \\
\hline 3 & 0.5 & 60 & 7 & 41.57 & 1001.62 & 29.98 & 1.6188 \\
\hline 3 & 0.5 & 60 & 8 & 29.98 & 1031.60 & 2.50 & 1.4768 \\
\hline 3 & 0.5 & 60 & 9 & 2.50 & 1034.10 & 0.00 & 0.3976 \\
\hline 3 & 0.5 & 60 & 10 & 0.00 & 1034.10 & & \\
\hline 3 & 2 & 60 & 1 & 644.18 & 644.18 & 432.99 & 2.8090 \\
\hline 3 & 2 & 60 & 2 & 432.99 & 1077.17 & 283.46 & 2.6365 \\
\hline 3 & 2 & 60 & 3 & 283.46 & 1360.62 & 181.25 & 2.4525 \\
\hline 3 & 2 & 60 & 4 & 181.25 & 1541.87 & 116.31 & 2.2583 \\
\hline 3 & 2 & 60 & 5 & 116.31 & 1658.18 & 72.96 & 2.0656 \\
\hline 3 & 2 & 60 & 6 & 72.96 & 1731.14 & 46.64 & 1.8631 \\
\hline 3 & 2 & 60 & 7 & 46.64 & 1777.77 & 30.30 & 1.6687 \\
\hline 3 & 2 & 60 & 8 & 30.30 & 1808.07 & 0.00 & 1.4814 \\
\hline 3 & 2 & 60 & 9 & 0.00 & 1808.07 & 0.00 & 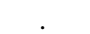 \\
\hline 3 & 2 & 60 & 10 & 0.00 & 1808.07 & & \\
\hline 3 & 0.5 & 60 & 1 & 71.33 & 71.33 & 56.33 & 1.8533 \\
\hline 3 & 0.5 & 60 & 2 & 56.33 & 127.66 & 33.73 & 1.7507 \\
\hline 3 & 0.5 & 60 & 3 & 33.73 & 161.39 & 32.59 & 1.5280 \\
\hline 3 & 0.5 & 60 & 4 & 32.59 & 193.98 & 24.73 & 1.5131 \\
\hline 3 & 0.5 & 60 & 5 & 24.73 & 218.71 & 18.85 & 1.3932 \\
\hline 3 & 0.5 & 60 & 6 & 18.85 & 237.57 & 16.13 & 1.2754 \\
\hline 3 & 0.5 & 60 & 7 & 16.13 & 253.69 & 14.48 & 1.2075 \\
\hline 3 & 0.5 & 60 & 8 & 14.48 & 268.17 & 11.36 & 1.1608 \\
\hline 3 & 0.5 & 60 & 9 & 11.36 & 279.53 & 9.29 & 1.0555 \\
\hline 3 & 0.5 & 60 & 10 & 9.29 & 288.82 & 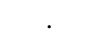 & 0.9679 \\
\hline 3 & 2 & 60 & 1 & 322.88 & 322.88 & 247.77 & 2.5090 \\
\hline 3 & 2 & 60 & 2 & 247.77 & 570.65 & 181.75 & 2.3940 \\
\hline 3 & 2 & 60 & 3 & 181.75 & 752.40 & 134.50 & 2.2595 \\
\hline 3 & 2 & 60 & 4 & 134.50 & 886.90 & 99.82 & 2.1287 \\
\hline 3 & 2 & 60 & 5 & 99.82 & 986.72 & 76.20 & 1.9992 \\
\hline 3 & 2 & 60 & 6 & 76.20 & 1062.92 & 48.16 & 1.8819 \\
\hline 3 & 2 & 60 & 7 & 48.16 & 1111.08 & 40.16 & 1.6827 \\
\hline 3 & 2 & 60 & 8 & 40.16 & 1151.23 & 30.22 & 1.6038 \\
\hline 3 & 2 & 60 & 9 & 30.22 & 1181.45 & 26.51 & 1.4803 \\
\hline 3 & 2 & 60 & 10 & 26.51 & 1207.96 & & 1.4235 \\
\hline 3 & 3.5 & 60 & 1 & 570.03 & 570.03 & 440.60 & 2.7559 \\
\hline 3 & 3.5 & 60 & 2 & 440.60 & 1010.64 & 327.88 & 2.6440 \\
\hline 3 & 3.5 & 60 & 3 & 327.88 & 1338.52 & 244.22 & 2.5157 \\
\hline 3 & 3.5 & 60 & 4 & 244.22 & 1582.73 & 183.92 & 2.3878 \\
\hline 3 & 3.5 & 60 & 5 & 183.92 & 1766.65 & 140.44 & 2.2646 \\
\hline 3 & 3.5 & 60 & 6 & 140.44 & 1907.09 & 109.45 & 2.1475 \\
\hline 3 & 3.5 & 60 & 7 & 109.45 & 2016.54 & 87.75 & 2.0392 \\
\hline 3 & 3.5 & 60 & 8 & 87.75 & 2104.29 & 63.69 & 1.9432 \\
\hline 3 & 3.5 & 60 & 9 & 63.69 & 2167.98 & 50.23 & 1.8041 \\
\hline 3 & 3.5 & 60 & 10 & 50.23 & 2218.21 & . & 1.7010 \\
\hline
\end{tabular}


Table B2. Ammonia Test Results (continued)

Test

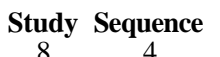

8 - 4

3-1-3-2, 70C, 0.1392

3-1-3-3, 70C, 0.1392

3-1-3-4, 70C, 0.1392

3-1-3-5, 70C, 0.1392

3-1-3-6, 70C, 0.1392

3-1-3-7, 70C, 0.1392

$3-1-3-8,70 \mathrm{C}, 0.1392$

3-1-3-9, 70C, 0.1392

$3-1-3-10,70 \mathrm{C}, 0.1392$

3-2-3-1, 70C, 0.1366

$3-2-3-2,70 \mathrm{C}, 0.1366$

3-2-3-3, 70C, 0.1366

$3-2-3-4,70 \mathrm{C}, 0.1366$

3-2-3-5, 70C, 0.1366

$3-2-3-6,70 \mathrm{C}, 0.1366$

$3-2-3-7,70 \mathrm{C}, 0.1366$

3-2-3-8, 70C, 0.1366

3-2-3-9, 70C, 0.1366

3-2-3-10, 70C, 0.1366

3-3-3-1, 70C, 0.1366

3-3-3-2, 70C, 0.1366

3-3-3-3, 70C, 0.1366

3-3-3-4, 70C, 0.1366

3-3-3-5, 70C, 0.1366

3-3-3-6, 70C, 0.1366

3-3-3-7, 70C, 0.1366

3-3-3-8, 70C, 0.1366

3-3-3-9, 70C, 0.1366

3-3-3-10, 70C, 0.1366

3-1-4-1, 80C, 0.1392

3-1-4-2, 80C, 0.1392

3-1-4-3, 80C, 0.1392

3-1-4-4, 80C, 0.1392

$3-1-4-5,80 \mathrm{C}, 0.1392$

3-1-4-6, 80C, 0.1392

3-1-4-7, 80C, 0.1392

3-1-4-8, 80C, 0.1392

3-1-4-9, 80C, 0.1392

3-1-4-10, 80C, 0.1392

3-2-4-1, 80C, 0.1379

3-2-4-2, 80C, 0.1379

3-2-4-3, 80C, 0.1379

3-2-4-4, 80C, 0.1379

3-2-4-5, 80C, 0.1379

3-2-4-6, 80C, 0.1379

3-2-4-7, 80C, 0.1379

$3-2-4-8,80 \mathrm{C}, 0.1379$

3-2-4-9, 80C, 0.1379

$3-2-4-10,80 \mathrm{C}, 0.1379$

3-3-4-1, 80C, 0.1359

3-3-4-2, 80C, 0.1359

3-3-4-3, 80C, 0.1359

3-3-4-4, 80C, 0.1359

3-3-4-5, 80C, 0.1359

3-3-4-6, 80C, 0.1359

3-3-4-7, 80C, 0.1359

3-3-4-8, 80C, 0.1359

3-3-4-9, 80C, 0.1359

3-3-4-10, 80C, 0.1359

\begin{tabular}{|c|c|c|c|c|c|c|c|}
\hline $\begin{array}{c}\text { Salt } \\
\text { Solution }\end{array}$ & $\begin{array}{c}\mathrm{NH}_{3} \text { Conc } \\
\text { (molal) }\end{array}$ & $\begin{array}{c}\text { Temperature } \\
\left({ }^{\circ} \mathrm{C}\right)\end{array}$ & $\begin{array}{l}\text { Sampling } \\
\text { Sequence }\end{array}$ & $\underset{\text { (peak area) }}{\mathbf{A}}$ & & $\mathbf{x}$ & $\log (\mathrm{A})$ \\
\hline 3 & 0.5 & 70 & 1 & 91.58 & 91.58 & 72.74 & 1.9618 \\
\hline 3 & 0.5 & 70 & 2 & 72.74 & 164.32 & 52.52 & 1.8618 \\
\hline 3 & 0.5 & 70 & 3 & 52.52 & 216.84 & 38.64 & 1.7203 \\
\hline 3 & 0.5 & 70 & 4 & 38.64 & 255.48 & 31.43 & 1.5870 \\
\hline 3 & 0.5 & 70 & 5 & 31.43 & 286.91 & 21.37 & 1.4974 \\
\hline 3 & 0.5 & 70 & 6 & 21.37 & 308.28 & 17.68 & 1.3298 \\
\hline 3 & 0.5 & 70 & 7 & 17.68 & 325.96 & 14.23 & 1.2475 \\
\hline 3 & 0.5 & 70 & 8 & 14.23 & 340.19 & 14.86 & 1.1531 \\
\hline 3 & 0.5 & 70 & 9 & 14.86 & 355.05 & 10.94 & 1.1720 \\
\hline 3 & 0.5 & 70 & 10 & 10.94 & 365.99 & & 1.0390 \\
\hline 3 & 2 & 70 & 1 & 434.27 & 434.27 & 336.38 & 2.6378 \\
\hline 3 & 2 & 70 & 2 & 336.38 & 770.64 & 246.44 & 2.5268 \\
\hline 3 & 2 & 70 & 3 & 246.44 & 1017.09 & 179.17 & 2.3917 \\
\hline 3 & 2 & 70 & 4 & 179.17 & 1196.25 & 131.30 & 2.2533 \\
\hline 3 & 2 & 70 & 5 & 131.30 & 1327.56 & 97.62 & 2.1183 \\
\hline 3 & 2 & 70 & 6 & 97.62 & 1425.18 & 80.37 & 1.9896 \\
\hline 3 & 2 & 70 & 7 & 80.37 & 1505.55 & 46.06 & 1.9051 \\
\hline 3 & 2 & 70 & 8 & 46.06 & 1551.61 & 27.71 & 1.6633 \\
\hline 3 & 2 & 70 & 9 & 27.71 & 1579.32 & 35.77 & 1.4426 \\
\hline 3 & 2 & 70 & 10 & 35.77 & 1615.08 & & 1.5535 \\
\hline 3 & 3.5 & 70 & 1 & 796.55 & 796.55 & 611.35 & 2.9012 \\
\hline 3 & 3.5 & 70 & 2 & 611.35 & 1407.90 & 442.73 & 2.7863 \\
\hline 3 & 3.5 & 70 & 3 & 442.73 & 1850.62 & 318.24 & 2.6461 \\
\hline 3 & 3.5 & 70 & 4 & 318.24 & 2168.87 & 231.31 & 2.5028 \\
\hline 3 & 3.5 & 70 & 5 & 231.31 & 2400.17 & 171.51 & 2.3642 \\
\hline 3 & 3.5 & 70 & 6 & 171.51 & 2571.68 & 130.50 & 2.2343 \\
\hline 3 & 3.5 & 70 & 7 & 130.50 & 2702.18 & 99.60 & 2.1156 \\
\hline 3 & 3.5 & 70 & 8 & 99.60 & 2801.78 & 79.03 & 1.9983 \\
\hline 3 & 3.5 & 70 & 9 & 79.03 & 2880.81 & 49.01 & 1.8978 \\
\hline 3 & 3.5 & 70 & 10 & 49.01 & 2929.82 & . & 1.6903 \\
\hline 3 & 0.5 & 80 & 1 & 131.58 & 131.58 & 97.18 & 2.1192 \\
\hline 3 & 0.5 & 80 & 2 & 97.18 & 228.76 & 63.39 & 1.9876 \\
\hline 3 & 0.5 & 80 & 3 & 63.39 & 292.15 & 35.12 & 1.8020 \\
\hline 3 & 0.5 & 80 & 4 & 35.12 & 327.27 & 34.47 & 1.5455 \\
\hline 3 & 0.5 & 80 & 5 & 34.47 & 361.74 & 19.39 & 1.5375 \\
\hline 3 & 0.5 & 80 & 6 & 19.39 & 381.13 & 18.91 & 1.2876 \\
\hline 3 & 0.5 & 80 & 7 & 18.91 & 400.04 & 3.23 & 1.2768 \\
\hline 3 & 0.5 & 80 & 8 & 3.23 & 403.27 & 2.29 & 0.5085 \\
\hline 3 & 0.5 & 80 & 9 & 2.29 & 405.56 & 0.00 & 0.3598 \\
\hline 3 & 0.5 & 80 & 10 & 0.00 & 405.56 & & \\
\hline 3 & 2 & 80 & 1 & 539.49 & 539.49 & 417.14 & 2.7320 \\
\hline 3 & 2 & 80 & 2 & 417.14 & 956.63 & 286.70 & 2.6203 \\
\hline 3 & 2 & 80 & 3 & 286.70 & 1243.33 & 206.98 & 2.4574 \\
\hline 3 & 2 & 80 & 4 & 206.98 & 1450.31 & 145.37 & 2.3159 \\
\hline 3 & 2 & 80 & 5 & 145.37 & 1595.68 & 105.03 & 2.1625 \\
\hline 3 & 2 & 80 & 6 & 105.03 & 1700.70 & 74.27 & 2.0213 \\
\hline 3 & 2 & 80 & 7 & 74.27 & 1774.97 & 57.86 & 1.8708 \\
\hline 3 & 2 & 80 & 8 & 57.86 & 1832.83 & 44.64 & 1.7624 \\
\hline 3 & 2 & 80 & 9 & 44.64 & 1877.47 & 37.48 & 1.6497 \\
\hline 3 & 2 & 80 & 10 & 37.48 & 1914.95 & & 1.5738 \\
\hline 3 & 3.5 & 80 & 1 & 948.40 & 948.40 & 712.66 & 2.9770 \\
\hline 3 & 3.5 & 80 & 2 & 712.66 & 1661.06 & 493.13 & 2.8529 \\
\hline 3 & 3.5 & 80 & 3 & 493.13 & 2154.19 & 342.70 & 2.6930 \\
\hline 3 & 3.5 & 80 & 4 & 342.70 & 2496.89 & 237.75 & 2.5349 \\
\hline 3 & 3.5 & 80 & 5 & 237.75 & 2734.64 & 171.83 & 2.3761 \\
\hline 3 & 3.5 & 80 & 6 & 171.83 & 2906.47 & 124.94 & 2.2351 \\
\hline 3 & 3.5 & 80 & 7 & 124.94 & 3031.41 & 88.58 & 2.0967 \\
\hline 3 & 3.5 & 80 & 8 & 88.58 & 3119.99 & 60.36 & 1.9473 \\
\hline 3 & 3.5 & 80 & 9 & 60.36 & 3180.34 & 34.30 & 1.7807 \\
\hline 3 & 3.5 & 80 & 10 & 34.30 & 3214.64 & 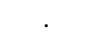 & 1.5353 \\
\hline
\end{tabular}


Table B2. Ammonia Test Results

$\begin{array}{ccc}\text { Study } & \begin{array}{c}\text { Test } \\ \text { Sequence }\end{array} & \begin{array}{c}\text { Test } \\ \text { Identifier }\end{array} \\ 8 & 10 & 3-1-5-1,90 \mathrm{C}, 0.1402 \\ 8 & 10 & 3-1-5-2,90 \mathrm{C}, 0.1402 \\ 8 & 10 & 3-1-5-3,90 \mathrm{C}, 0.1402 \\ 8 & 10 & 3-1-5-4,90 \mathrm{C}, 0.1402 \\ 8 & 10 & 3-1-5-5,90 \mathrm{C}, 0.1402 \\ 8 & 10 & 3-1-5-6,90 \mathrm{C}, 0.1402 \\ 8 & 10 & 3-1-5-7,90 \mathrm{C}, 0.1402 \\ 8 & 10 & 3-1-5-8,90 \mathrm{C}, 0.1402 \\ 8 & 10 & 3-1-5-9,90 \mathrm{C}, 0.1402 \\ 8 & 10 & 3-1-5-10,90 \mathrm{C}, 0.1402 \\ 8 & 11 & 3-2-5-1,90 \mathrm{C}, 0.1377 \\ 8 & 11 & 3-2-5-2,90 \mathrm{C}, 0.1377 \\ 8 & 11 & 3-2-5-3,90 \mathrm{C}, 0.1377 \\ 8 & 11 & 3-2-5-4,90 \mathrm{C}, 0.1377 \\ 8 & 11 & 3-2-5-5,90 \mathrm{C}, 0.1377 \\ 8 & 11 & 3-2-5-6,90 \mathrm{C}, 0.1377 \\ 8 & 11 & 3-2-5-7,90 \mathrm{C}, 0.1377 \\ 8 & 11 & 3-2-5-8,90 \mathrm{C}, 0.1377 \\ 8 & 11 & 3-2-5-9,90 \mathrm{C}, 0.1377 \\ 8 & 11 & 3-2-5-10,90 \mathrm{C}, 0.1377 \\ 8 & 12 & 3-3-5-1,90 \mathrm{C}, 0.1370 \\ 8 & 12 & 3-3-5-2,90 \mathrm{C}, 0.1370 \\ 8 & 12 & 3-3-5-3,90 \mathrm{C}, 0.1370 \\ 8 & 12 & 3-3-5-4,90 \mathrm{C}, 0.1370 \\ 8 & 12 & 3-3-5-5,90 \mathrm{C}, 0.1370 \\ 8 & 12 & 3-3-5-6,90 \mathrm{C}, 0.1370 \\ 8 & 12 & 3-3-5-7,90 \mathrm{C}, 0.1370 \\ 8 & 12 & 3-3-5-8,90 \mathrm{C}, 0.1370 \\ 8 & 12 & 3-3-5-9,90 \mathrm{C}, 0.1370 \\ 8 & 12 & 3-3-5-10,90 \mathrm{C}, 0.1370\end{array}$

$\begin{array}{ccc}\begin{array}{c}\text { Salt } \\ \text { Solution }\end{array} & \begin{array}{c}\mathbf{N H}_{\mathbf{3}} \text { Conc } \\ (\mathbf{m o l a l})\end{array} & \begin{array}{c}\text { Temperature } \\ \left({ }^{\mathbf{}} \mathbf{C}\right)\end{array} \\ 3 & 0.5 & 90 \\ 3 & 0.5 & 90 \\ 3 & 0.5 & 90 \\ 3 & 0.5 & 90 \\ 3 & 0.5 & 90 \\ 3 & 0.5 & 90 \\ 3 & 0.5 & 90 \\ 3 & 0.5 & 90 \\ 3 & 0.5 & 90 \\ 3 & 0.5 & 90 \\ 3 & 2 & 90 \\ 3 & 2 & 90 \\ 3 & 2 & 90 \\ 3 & 2 & 90 \\ 3 & 2 & 90 \\ 3 & 2 & 90 \\ 3 & 2 & 90 \\ 3 & 2 & 90 \\ 3 & 2 & 90 \\ 3 & 2 & 90 \\ 3 & 3.5 & 90 \\ 3 & 3.5 & 90 \\ 3 & 3.5 & 90 \\ 3 & 3.5 & 90 \\ 3 & 3.5 & 90 \\ 3 & 3.5 & 90 \\ 3 & 3.5 & 90 \\ 3 & 3.5 & 90 \\ 3 & 3.5 & 90 \\ 3 & 3.5 & 90 \\ & & \end{array}$

Sampling
Sequence
1
2
3
4
5
6
7
8
9
10
1
2
3
4
5
6
7
8
9
10
1
2
3
4
5
6
7
8
9
10

A

$\begin{array}{llll}\text { (peak area) } & \mathbf{y} & \mathbf{x} & \log (\mathrm{A})\end{array}$ $\begin{array}{llll}160.23 & 160.23 & 117.12 & 2.2047\end{array}$ $\begin{array}{llll}117.12 & 277.35 & 81.41 & 2.0686\end{array}$ $\begin{array}{llll}81.41 & 358.76 & 40.42 & 1.9107\end{array}$ $\begin{array}{llll}40.42 & 399.18 & 20.68 & 1.6066\end{array}$ $\begin{array}{llll}20.68 & 419.86 & 0.00 & 1.3155\end{array}$ $\begin{array}{llll}0.00 & 419.86 & 0.00\end{array}$ 0.00 0.00 2.70 0.00 672.46 508.45 361.39

247.30

169.94

118.30

78.18

47.77

28.54

0.00 1093.40

856.04

611.02

428.65

297.94

207.28

148.50

103.73

72.82

419.86

419.86

$\begin{array}{lll}422.56 & 0.00 \quad 0.4320\end{array}$

422.56

$\begin{array}{lll}672.46 & 508.45 & 2.8277\end{array}$

$\begin{array}{lll}1180.91 & 361.39 & 2.7062\end{array}$

$\begin{array}{lll}1542.29 & 247.30 & 2.5580\end{array}$

$\begin{array}{lll}1789.59 & 169.94 & 2.3932\end{array}$

$\begin{array}{llll}1959.53 & 118.30 & 2.2303\end{array}$

$\begin{array}{lll}2077.83 & 78.18 & 2.0730\end{array}$

$\begin{array}{lll}2156.01 & 47.77 & 1.8931\end{array}$

$\begin{array}{lll}2203.78 & 28.54 & 1.6792\end{array}$

$\begin{array}{lll}2232.32 & 0.00 & 1.4554\end{array}$

2232.32

$\begin{array}{lll}1093.40 & 856.04 & 3.0388\end{array}$

$\begin{array}{lll}1949.44 & 611.02 & 2.9325\end{array}$

$\begin{array}{llll}2560.46 & 428.65 & 2.7861\end{array}$

$\begin{array}{lll}2989.11 & 297.94 & 2.6321\end{array}$

$\begin{array}{lll}3287.04 & 207.28 & 2.4741\end{array}$

$\begin{array}{lll}3494.32 & 148.50 & 2.3166\end{array}$

$\begin{array}{lll}3642.82 & 103.73 & 2.1717\end{array}$

$\begin{array}{lll}3746.55 & 72.82 & 2.0159\end{array}$

$\begin{array}{lll}3819.37 & 45.10 & 1.8623\end{array}$

45.10

$3864.48 \quad$. $\quad 1.6542$ 
Table B3. Test Results for Special Salt Solutions

\begin{tabular}{|c|c|c|c|c|c|c|c|c|c|}
\hline Study & $\begin{array}{c}\text { Test } \\
\text { Sequence }\end{array}$ & $\begin{array}{c}\text { Test } \\
\text { Identifier }\end{array}$ & $\begin{array}{c}\text { Salt } \\
\text { Solution }\end{array}$ & $\begin{array}{c}\text { Temperature } \\
\left({ }^{\circ} \mathrm{C}\right)\end{array}$ & $\begin{array}{l}\text { Sampling } \\
\text { Sequence }\end{array}$ & $\underset{\text { (peak area) }}{\mathbf{A}}$ & $\mathbf{y}$ & $\mathbf{x}$ & $\log (\mathbf{A})$ \\
\hline Special Salt Solutions & 1 & $50 \mathrm{C} \mathrm{NaAlO} 2 \# 1$ 1-1 & NaAlO2\#1 & 50 & 1 & 597.10 & 597.10 & 702.69 & 2.7760 \\
\hline Special Salt Solutions & 1 & 50C NaAlO2 \#1 1-2 & NaAlO2\#1 & 50 & 2 & 702.69 & 1299.79 & 655.46 & 2.8468 \\
\hline Special Salt Solutions & 1 & $50 \mathrm{C} \mathrm{NaAlO} 2 \# 1$ 1-3 & NaAlO2\#1 & 50 & 3 & 655.46 & 1955.25 & 595.13 & 2.8165 \\
\hline Special Salt Solutions & 1 & $50 \mathrm{C} \mathrm{NaAlO} 2 \# 1$ 1-4 & NaAlO2\#1 & 50 & 4 & 595.13 & 2550.38 & 547.73 & 2.7746 \\
\hline Special Salt Solutions & 1 & 50C NaAlO2 \#1 1-5 & NaAlO2\#1 & 50 & 5 & 547.73 & 3098.11 & 503.67 & 2.7386 \\
\hline Special Salt Solutions & 1 & $50 \mathrm{C}$ NaAlO2 \#1 1-6 & NaAlO2\#1 & 50 & 6 & 503.67 & 3601.78 & 467.03 & 2.7021 \\
\hline Special Salt Solutions & 1 & 50C NaAlO2 \#1 1-7 & NaAlO2 \#1 & 50 & 7 & 467.03 & 4068.81 & 428.36 & 2.6693 \\
\hline Special Salt Solutions & 1 & 50C NaAlO2 \#1 1-8 & $\mathrm{NaAlO} 2 \# 1$ & 50 & 8 & 428.36 & 4497.17 & 394.11 & 2.6318 \\
\hline Special Salt Solutions & 1 & 50C NaAlO2 \#1 1-9 & NaAlO2\#1 & 50 & 9 & 394.11 & 4891.28 & 363.15 & 2.5956 \\
\hline Special Salt Solutions & 1 & $50 \mathrm{C}$ NaAlO2 \#1 $1-10$ & NaAlO2\#1 & 50 & 10 & 363.15 & 5254.43 & & 2.5601 \\
\hline Special Salt Solutions & 2 & 50C NaAlO2 \#1 2-1 & $\mathrm{NaAlO} 2 \# 1$ & 50 & 1 & 715.34 & 715.34 & 660.70 & 2.8545 \\
\hline Special Salt Solutions & 2 & $50 \mathrm{C} \mathrm{NaAlO} 2 \# 1$ 2-2 & NaAlO2\#1 & 50 & 2 & 660.70 & 1376.05 & 605.41 & 2.8200 \\
\hline Special Salt Solutions & 2 & $50 \mathrm{C}$ NaAlO2 \#1 2-3 & NaAlO2\#1 & 50 & 3 & 605.41 & 1981.46 & 562.18 & 2.7821 \\
\hline Special Salt Solutions & 2 & 50C NaAlO2 \#1 2-4 & NaAlO2\#1 & 50 & 4 & 562.18 & 2543.64 & 521.32 & 2.7499 \\
\hline Special Salt Solutions & 2 & $50 \mathrm{C} \mathrm{NaAlO} 2 \# 1$ 2-5 & $\mathrm{NaAlO2 \# 1}$ & 50 & 5 & 521.32 & 3064.95 & 480.55 & 2.7171 \\
\hline Special Salt Solutions & 2 & 50C NaAlO2 \#1 2-6 & $\mathrm{NaAlO} 2 \# 1$ & 50 & 6 & 480.55 & 3545.50 & 441.15 & 2.6817 \\
\hline Special Salt Solutions & 2 & 50C NaAlO2 \#1 2-7 & NaAlO2\#1 & 50 & 7 & 441.15 & 3986.65 & 405.70 & 2.6446 \\
\hline Special Salt Solutions & 2 & 50C NaAlO2 \#1 2-8 & $\mathrm{NaAlO} 2 \# 1$ & 50 & 8 & 405.70 & 4392.35 & 374.75 & 2.6082 \\
\hline Special Salt Solutions & 2 & 50C NaAlO2 \#1 2-9 & NaAlO2\#1 & 50 & 9 & 374.75 & 4767.09 & 343.96 & 2.5737 \\
\hline Special Salt Solutions & 2 & $50 \mathrm{C}$ NaAlO2 \#1 2-10 & NaAlO2\#1 & 50 & 10 & 343.96 & 5111.06 & & 2.5365 \\
\hline Special Salt Solutions & 3 & $50 \mathrm{C} \mathrm{NaAlO} 2$ \#2 1-1 & $\mathrm{NaAlO} 2 \# 2$ & 50 & 1 & 582.11 & 582.11 & 561.05 & 2.7650 \\
\hline Special Salt Solutions & 3 & $50 \mathrm{C}$ NaAlO2 \#2 1-2 & $\mathrm{NaAlO} 2 \# 2$ & 50 & 2 & 561.05 & 1143.16 & 515.98 & 2.7490 \\
\hline Special Salt Solutions & 3 & $50 \mathrm{C} \mathrm{NaAlO} 2$ \#2 1 1-3 & $\mathrm{NaAlO} 2 \# 2$ & 50 & 3 & 515.98 & 1659.13 & 478.99 & 2.7126 \\
\hline Special Salt Solutions & 3 & 50C NaAlO2 \#2 1-4 & $\mathrm{NaAlO} 2 \# 2$ & 50 & 4 & 478.99 & 2138.13 & 443.84 & 2.6803 \\
\hline Special Salt Solutions & 3 & $50 \mathrm{C}$ NaAlO2 \#2 1-5 & $\mathrm{NaAlO} 2 \# 2$ & 50 & 5 & 443.84 & 2581.97 & 414.88 & 2.6472 \\
\hline Special Salt Solutions & 3 & 50C NaAlO2 \#2 1-6 & $\mathrm{NaAlO} 2 \# 2$ & 50 & 6 & 414.88 & 2996.85 & 386.87 & 2.6179 \\
\hline Special Salt Solutions & 3 & $50 \mathrm{C} \mathrm{NaAlO} 2 \# 2$ 1-7 & $\mathrm{NaAlO} 2 \# 2$ & 50 & 7 & 386.87 & 3383.71 & 357.94 & 2.5876 \\
\hline Special Salt Solutions & 3 & 50C NaAlO2 \#2 1-8 & $\mathrm{NaAlO} 2 \# 2$ & 50 & 8 & 357.94 & 3741.66 & 331.87 & 2.5538 \\
\hline Special Salt Solutions & 3 & 50C NaAlO2 \#2 1-9 & $\mathrm{NaAlO} 2 \# 2$ & 50 & 9 & 331.87 & 4073.53 & 312.78 & 2.5210 \\
\hline Special Salt Solutions & 3 & $50 \mathrm{C}$ NaAlO2 \#2 1-10 & $\mathrm{NaAlO} 2 \# 2$ & 50 & 10 & 312.78 & 4386.31 & & 2.4952 \\
\hline Special Salt Solutions & 4 & $50 \mathrm{C}$ NaAlO2 \#2 2-1 & $\mathrm{NaAlO} 2 \# 2$ & 50 & 1 & 607.34 & 607.34 & 537.05 & 2.7834 \\
\hline Special Salt Solutions & 4 & $50 \mathrm{C} \mathrm{NaAlO} 2 \# 2$ 2-1 & $\mathrm{NaAlO} 2 \# 2$ & 50 & 2 & 537.05 & 1144.39 & 496.73 & 2.7300 \\
\hline Special Salt Solutions & 4 & $50 \mathrm{C}$ NaAlO2 \#2 2-3 & $\mathrm{NaAlO} 2 \# 2$ & 50 & 3 & 496.73 & 1641.12 & 462.58 & 2.6961 \\
\hline Special Salt Solutions & 4 & $50 \mathrm{C} \mathrm{NaAlO} 2 \# 22-4$ & $\mathrm{NaAlO} 2 \# 2$ & 50 & 4 & 462.58 & 2103.70 & 428.97 & 2.6652 \\
\hline Special Salt Solutions & 4 & $50 \mathrm{C} \mathrm{NaAlO} 2 \# 2$ 2-5 & $\mathrm{NaAlO} 2 \# 2$ & 50 & 5 & 428.97 & 2532.67 & 398.67 & 2.6324 \\
\hline Special Salt Solutions & 4 & $50 \mathrm{C}$ NaAlO2 \#2 2-6 & $\mathrm{NaAlO} 2 \# 2$ & 50 & 6 & 398.67 & 2931.34 & 372.25 & 2.6006 \\
\hline Special Salt Solutions & 4 & 50C NaAlO2 \#2 2-7 & $\mathrm{NaAlO} 2 \# 2$ & 50 & 7 & 372.25 & 3303.59 & 345.27 & 2.5708 \\
\hline Special Salt Solutions & 4 & $50 \mathrm{C} \mathrm{NaAlO} 2 \# 22-8$ & $\mathrm{NaAlO} 2 \# 2$ & 50 & 8 & 345.27 & 3648.86 & 317.43 & 2.5382 \\
\hline Special Salt Solutions & 4 & $50 \mathrm{C} \mathrm{NaAlO} 2$ \#2 2-9 & $\mathrm{NaAlO} 2 \# 2$ & 50 & 9 & 317.43 & 3966.29 & 295.02 & 2.5016 \\
\hline Special Salt Solutions & 4 & $50 \mathrm{C}$ NaAlO2 \#2 2-10 & $\mathrm{NaAlO} 2 \# 2$ & 50 & 10 & 295.02 & 4261.31 & & 2.4699 \\
\hline Special Salt Solutions & 5 & 50C NaAlO2 \#2 3-1 & $\mathrm{NaAlO} 2 \# 2$ & 50 & 1 & 613.04 & 613.04 & 547.35 & 2.7875 \\
\hline Special Salt Solutions & 5 & $50 \mathrm{C} \mathrm{NaAlO} 2 \# 2$ 3-2 & $\mathrm{NaAlO} 2 \# 2$ & 50 & 2 & 547.35 & 1160.39 & 499.87 & 2.7383 \\
\hline Special Salt Solutions & 5 & $50 \mathrm{C} \mathrm{NaAlO} 2 \# 23-3$ & $\mathrm{NaAlO} 2 \# 2$ & 50 & 3 & 499.87 & 1660.26 & 464.21 & 2.6989 \\
\hline Special Salt Solutions & 5 & 50C NaAlO2 \#2 3-4 & $\mathrm{NaAlO} 2 \# 2$ & 50 & 4 & 464.21 & 2124.47 & 430.42 & 2.6667 \\
\hline Special Salt Solutions & 5 & $50 \mathrm{C} \mathrm{NaAlO} 2 \# 2$ 3-5 & $\mathrm{NaAlO} 2 \# 2$ & 50 & 5 & 430.42 & 2554.90 & 398.04 & 2.6339 \\
\hline Special Salt Solutions & 5 & $50 \mathrm{C}$ NaAlO2 \#2 3-6 & $\mathrm{NaAlO} 2 \# 2$ & 50 & 6 & 398.04 & 2952.94 & 368.33 & 2.5999 \\
\hline Special Salt Solutions & 5 & $50 \mathrm{C} \mathrm{NaAlO} 2 \# 23-7$ & $\mathrm{NaAlO} 2 \# 2$ & 50 & 7 & 368.33 & 3321.27 & 345.74 & 2.5662 \\
\hline Special Salt Solutions & 5 & $50 \mathrm{C} \mathrm{NaAlO} 2 \# 2$ 3-8 & $\mathrm{NaAlO} 2 \# 2$ & 50 & 8 & 345.74 & 3667.01 & 317.74 & 2.5387 \\
\hline Special Salt Solutions & 5 & $50 \mathrm{C}$ NaAlO2 \#2 3-9 & $\mathrm{NaAlO} 2 \# 2$ & 50 & 9 & 317.74 & 3984.75 & 288.96 & 2.5021 \\
\hline Special Salt Solutions & 5 & $50 \mathrm{C} \mathrm{NaAlO} 2 \# 2$ 3-10 & $\mathrm{NaAlO} 2 \# 2$ & 50 & 10 & 288.96 & 4273.71 & & 2.4608 \\
\hline Special Salt Solutions & 6 & 50C NaNO3 1-1 & NaNO3 & 50 & 1 & 1086.82 & 1086.82 & 1001.37 & 3.0362 \\
\hline Special Salt Solutions & 6 & $50 \mathrm{C}$ NaNO3 1-2 & NaNO3 & 50 & 2 & 1001.37 & 2088.19 & 923.46 & 3.0006 \\
\hline Special Salt Solutions & 6 & $50 \mathrm{C}$ NaNO3 1-3 & NaNO3 & 50 & 3 & 923.46 & 3011.65 & 859.83 & 2.9654 \\
\hline Special Salt Solutions & 6 & $50 \mathrm{C}$ NaNO3 1-4 & NaNO3 & 50 & 4 & 859.83 & 3871.48 & 795.22 & 2.9344 \\
\hline Special Salt Solutions & 6 & 50C NaNO3 1-5 & NaNO3 & 50 & 5 & 795.22 & 4666.70 & 745.91 & 2.9005 \\
\hline Special Salt Solutions & 6 & $50 \mathrm{C}$ NaNO3 1-6 & NaNO3 & 50 & 6 & 745.91 & 5412.61 & 693.72 & 2.8727 \\
\hline Special Salt Solutions & 6 & $50 \mathrm{C}$ NaNO3 1-7 & NaNO3 & 50 & 7 & 693.72 & 6106.33 & 647.92 & 2.8412 \\
\hline Special Salt Solutions & 6 & 50C NaNO3 1-8 & NaNO3 & 50 & 8 & 647.92 & 6754.25 & 605.75 & 2.8115 \\
\hline Special Salt Solutions & 6 & $50 \mathrm{C}$ NaNO3 1-9 & NaNO3 & 50 & 9 & 605.75 & 7359.99 & 561.05 & 2.7823 \\
\hline Special Salt Solutions & 6 & $50 \mathrm{C} \mathrm{NaNO} 31-10$ & $\mathrm{NaNO} 3$ & 50 & 10 & 561.05 & 7921.04 & & 2.7490 \\
\hline Special Salt Solutions & 7 & $50 \mathrm{C}$ NaNO3 2-1 & NaNO3 & 50 & 1 & 1113.23 & 1113.23 & 982.85 & 3.0466 \\
\hline Special Salt Solutions & 7 & $50 \mathrm{C}$ NaNO3 2-2 & NaNO3 & 50 & 2 & 982.85 & 2096.08 & 888.76 & 2.9925 \\
\hline Special Salt Solutions & 7 & $50 \mathrm{C}$ NaNO3 2-3 & NaNO3 & 50 & 3 & 888.76 & 2984.84 & 830.42 & 2.9488 \\
\hline Special Salt Solutions & 7 & $50 \mathrm{C}$ NaNO3 2-4 & NaNO3 & 50 & 4 & 830.42 & 3815.26 & 768.81 & 2.9193 \\
\hline Special Salt Solutions & 7 & $50 \mathrm{C}$ NaNO3 2-5 & NaNO3 & 50 & 5 & 768.81 & 4584.07 & 722.34 & 2.8858 \\
\hline Special Salt Solutions & 7 & $50 \mathrm{C}$ NaNO3 2-6 & NaNO3 & 50 & 6 & 722.34 & 5306.40 & 669.29 & 2.8587 \\
\hline
\end{tabular}


Table B3. Test Results for Special Salt Solutions

(continued)

\begin{tabular}{|c|c|c|c|c|c|c|c|c|c|}
\hline $\begin{array}{c}\text { Study } \\
\text { Special Salt Solutions }\end{array}$ & $\begin{array}{c}\text { Test } \\
\text { Sequence }\end{array}$ & $\begin{array}{c}\text { Test } \\
\text { Identifier } \\
\text { 50C NaNO3 2-7 }\end{array}$ & $\begin{array}{c}\text { Salt } \\
\text { Solution } \\
\text { NaNO3 }\end{array}$ & $\begin{array}{c}\text { Temperature } \\
\left({ }^{\circ} \mathbf{C}\right) \\
50\end{array}$ & $\begin{array}{l}\text { Sampling } \\
\text { Sequence } \\
7\end{array}$ & $\begin{array}{c}\text { A } \\
\text { (peak area) } \\
669.29\end{array}$ & $\begin{array}{c}\mathbf{y} \\
5975.69\end{array}$ & $\begin{array}{c}\mathbf{x} \\
624.58\end{array}$ & $\begin{array}{l}\log (\mathbf{A}) \\
2.8256\end{array}$ \\
\hline $\begin{array}{l}\text { Special Salt Solutions } \\
\text { Special Salt Solutions }\end{array}$ & 7 & $\begin{array}{l}50 \mathrm{C} \text { NaNO3 } 2-7 \\
50 \mathrm{C} \text { NaNO3 2-8 }\end{array}$ & $\begin{array}{l}\mathrm{NaNO3} \\
\mathrm{NaNO} 3\end{array}$ & $\begin{array}{l}50 \\
50\end{array}$ & 8 & $\begin{array}{l}069.29 \\
624.58\end{array}$ & $\begin{array}{l}5975.69 \\
6600.27\end{array}$ & $\begin{array}{l}024.38 \\
578.30\end{array}$ & $\begin{array}{l}2.8256 \\
2.7956\end{array}$ \\
\hline Special Salt Solutions & 7 & $50 \mathrm{C}$ NaNO3 2-9 & $\mathrm{NaNO} 3$ & 50 & 9 & 578.30 & 7178.57 & 533.54 & 2.7622 \\
\hline Special Salt Solutions & 7 & $50 \mathrm{C}$ NaNO3 2-10 & $\mathrm{NaNO} 3$ & 50 & 10 & 533.54 & 7712.10 & & 2.7272 \\
\hline Special Salt Solutions & 8 & $50 \mathrm{C}$ NaNO3 3-1 & NaNO3 & 50 & 1 & 1133.66 & 1133.66 & 991.35 & 3.0545 \\
\hline Special Salt Solutions & 8 & $50 \mathrm{C}$ NaNO3 3-2 & NaNO3 & 50 & 2 & 991.35 & 2125.00 & 889.68 & 2.9962 \\
\hline Special Salt Solutions & 8 & 50C NaNO3 3-3 & $\mathrm{NaNO} 3$ & 50 & 3 & 889.68 & 3014.68 & 834.55 & 2.9492 \\
\hline Special Salt Solutions & 8 & $50 \mathrm{C}$ NaNO3 3-4 & NaNO3 & 50 & 4 & 834.55 & 3849.23 & 773.81 & 2.9215 \\
\hline Special Salt Solutions & 8 & 50C NaNO3 3-5 & NaNO3 & 50 & 5 & 773.81 & 4623.04 & 724.01 & 2.8886 \\
\hline Special Salt Solutions & 8 & $50 \mathrm{C}$ NaNO3 3-6 & NaNO3 & 50 & 6 & 724.01 & 5347.05 & 670.17 & 2.8597 \\
\hline Special Salt Solutions & 8 & $50 \mathrm{C}$ NaNO3 3-7 & $\mathrm{NaNO} 3$ & 50 & 7 & 670.17 & 6017.22 & 623.95 & 2.8262 \\
\hline Special Salt Solutions & 8 & $50 \mathrm{C}$ NaNO3 3-8 & NaNO3 & 50 & 8 & 623.95 & 6641.17 & 578.08 & 2.7951 \\
\hline Special Salt Solutions & 8 & $50 \mathrm{C}$ NaNO3 3-9 & $\mathrm{NaNO} 3$ & 50 & 9 & 578.08 & 7219.25 & 539.08 & 2.7620 \\
\hline Special Salt Solutions & 8 & 50C NaNO3 3-10 & $\mathrm{NaNO} 3$ & 50 & 10 & 539.08 & 7758.33 & & 2.7317 \\
\hline Special Salt Solutions & 9 & $50 \mathrm{C}$ NaNO2 $1-1$ & $\mathrm{NaNO} 2$ & 50 & 1 & 501.99 & 501.99 & 468.58 & 2.7007 \\
\hline Special Salt Solutions & 9 & $50 \mathrm{C}$ NaNO2 $1-2$ & $\mathrm{NaNO} 2$ & 50 & 2 & 468.58 & 970.57 & 411.45 & 2.6708 \\
\hline Special Salt Solutions & 9 & $50 \mathrm{C}$ NaNO2 $1-3$ & $\mathrm{NaNO} 2$ & 50 & 3 & 411.45 & 1382.02 & 385.32 & 2.6143 \\
\hline Special Salt Solutions & 9 & $50 \mathrm{C}$ NaNO2 $1-4$ & $\mathrm{NaNO} 2$ & 50 & 4 & 385.32 & 1767.34 & 358.31 & 2.5858 \\
\hline Special Salt Solutions & 9 & 50C NaNO2 1-5 & $\mathrm{NaNO} 2$ & 50 & 5 & 358.31 & 2125.64 & 330.89 & 2.5543 \\
\hline Special Salt Solutions & 9 & $50 \mathrm{C}$ NaNO2 1-6 & $\mathrm{NaNO} 2$ & 50 & 6 & 330.89 & 2456.53 & 311.20 & 2.5197 \\
\hline Special Salt Solutions & 9 & $50 \mathrm{C}$ NaNO2 $1-7$ & $\mathrm{NaNO} 2$ & 50 & 7 & 311.20 & 2767.74 & 290.29 & 2.4930 \\
\hline Special Salt Solutions & 9 & $50 \mathrm{C}$ NaNO2 $1-8$ & $\mathrm{NaNO} 2$ & 50 & 8 & 290.29 & 3058.02 & 268.43 & 2.4628 \\
\hline Special Salt Solutions & 9 & $50 \mathrm{C}$ NaNO2 $1-9$ & $\mathrm{NaNO} 2$ & 50 & 9 & 268.43 & 3326.45 & 253.61 & 2.4288 \\
\hline Special Salt Solutions & 9 & $50 \mathrm{C}$ NaNO2 $1-10$ & $\mathrm{NaNO} 2$ & 50 & 10 & 253.61 & 3580.06 & & 2.4042 \\
\hline Special Salt Solutions & 10 & $50 \mathrm{C}$ NaNO2 2-1 & $\mathrm{NaNO} 2$ & 50 & 1 & 500.09 & 500.09 & 440.64 & 2.6990 \\
\hline Special Salt Solutions & 10 & $50 \mathrm{C}$ NaNO2 2-2 & $\mathrm{NaNO} 2$ & 50 & 2 & 440.64 & 940.73 & 387.85 & 2.6441 \\
\hline Special Salt Solutions & 10 & $50 \mathrm{C}$ NaNO2 $2-3$ & $\mathrm{NaNO} 2$ & 50 & 3 & 387.85 & 1328.58 & 359.67 & 2.5887 \\
\hline Special Salt Solutions & 10 & $50 \mathrm{C}$ NaNO2 2-4 & $\mathrm{NaNO} 2$ & 50 & 4 & 359.67 & 1688.25 & 340.72 & 2.5559 \\
\hline Special Salt Solutions & 10 & $50 \mathrm{C}$ NaNO2 $2-5$ & $\mathrm{NaNO} 2$ & 50 & 5 & 340.72 & 2028.96 & 314.25 & 2.5324 \\
\hline Special Salt Solutions & 10 & $50 \mathrm{C}$ NaNO2 2-6 & $\mathrm{NaNO} 2$ & 50 & 6 & 314.25 & 2343.22 & 293.92 & 2.4973 \\
\hline Special Salt Solutions & 10 & $50 \mathrm{C}$ NaNO2 2-7 & $\mathrm{NaNO} 2$ & 50 & 7 & 293.92 & 2637.13 & 274.15 & 2.4682 \\
\hline Special Salt Solutions & 10 & $50 \mathrm{C}$ NaNO2 2-8 & $\mathrm{NaNO} 2$ & 50 & 8 & 274.15 & 2911.29 & 251.63 & 2.4380 \\
\hline Special Salt Solutions & 10 & $50 \mathrm{C}$ NaNO2 2-9 & $\mathrm{NaNO} 2$ & 50 & 9 & 251.63 & 3162.91 & 233.69 & 2.4008 \\
\hline Special Salt Solutions & 10 & $50 \mathrm{C}$ NaNO2 2-10 & $\mathrm{NaNO} 2$ & 50 & 10 & 233.69 & 3396.60 & & 2.3686 \\
\hline Special Salt Solutions & 11 & $50 \mathrm{C}$ NaNO2 $3-1$ & $\mathrm{NaNO} 2$ & 50 & 1 & 489.47 & 489.47 & 426.61 & 2.6897 \\
\hline Special Salt Solutions & 11 & $50 \mathrm{C}$ NaNO2 $3-2$ & $\mathrm{NaNO} 2$ & 50 & 2 & $\begin{array}{l}409.41 \\
426.61\end{array}$ & $\begin{array}{l}409.41 \\
916.08\end{array}$ & 376.54 & 2.6300 \\
\hline Special Salt Solutions & 11 & $50 \mathrm{C}$ NaNO2 $3-3$ & $\mathrm{NaNO} 2$ & 50 & 3 & 376.54 & 1292.62 & 350.26 & 2.5758 \\
\hline Special Salt Solutions & 11 & $50 \mathrm{C}$ NaNO2 $3-4$ & $\mathrm{NaNO} 2$ & 50 & 4 & 350.26 & 1642.88 & 330.44 & 2.5444 \\
\hline Special Salt Solutions & 11 & $50 \mathrm{C}$ NaNO2 $3-5$ & $\mathrm{NaNO} 2$ & 50 & 5 & 330.44 & 1973.32 & 303.52 & 2.5191 \\
\hline Special Salt Solutions & 11 & $50 \mathrm{C}$ NaNO2 $3-6$ & $\mathrm{NaNO} 2$ & 50 & 6 & 303.52 & 2276.84 & 284.58 & 2.4822 \\
\hline Special Salt Solutions & 11 & $50 \mathrm{C}$ NaNO2 $3-7$ & $\mathrm{NaNO} 2$ & 50 & 7 & 284.58 & 2561.42 & 264.54 & 2.4542 \\
\hline Special Salt Solutions & 11 & $50 \mathrm{C}$ NaNO2 3-8 & $\mathrm{NaNO} 2$ & 50 & 8 & 264.54 & 2825.96 & 249.36 & 2.4225 \\
\hline Special Salt Solutions & 11 & $50 \mathrm{C}$ NaNO2 $3-9$ & $\mathrm{NaNO} 2$ & 50 & 9 & 249.36 & 3075.32 & 226.18 & 2.3968 \\
\hline Special Salt Solutions & 11 & $50 \mathrm{C}$ NaNO2 3-10 & $\mathrm{NaNO} 2$ & 50 & 10 & 226.18 & 3301.50 & & 2.3545 \\
\hline Special Salt Solutions & 12 & $50 \mathrm{C} \mathrm{Na} 2 \mathrm{CO} 31-1$ & $\mathrm{Na} 2 \mathrm{CO} 3$ & 50 & 1 & 1188.21 & 1188.21 & 1080.51 & 3.0749 \\
\hline Special Salt Solutions & 12 & $50 \mathrm{C} \mathrm{Na} 2 \mathrm{CO} 31-2$ & $\mathrm{Na} 2 \mathrm{CO} 3$ & 50 & 2 & 1080.51 & 2268.73 & 932.46 & 3.0336 \\
\hline Special Salt Solutions & 12 & $50 \mathrm{C} \mathrm{Na} 2 \mathrm{CO} 31-3$ & $\mathrm{Na} 2 \mathrm{CO} 3$ & 50 & 3 & 932.46 & 3201.19 & 859.07 & 2.9696 \\
\hline $\begin{array}{l}\text { Special Salt Solutions } \\
\text { Salo }\end{array}$ & 12 & $50 \mathrm{C} \mathrm{Na} 2 \mathrm{CO} 31-4$ & $\mathrm{Na} 2 \mathrm{CO} 3$ & 50 & 4 & 859.07 & 4060.26 & 780.79 & 2.9340 \\
\hline Special Salt Solutions & 12 & $50 \mathrm{C} \mathrm{Na} 2 \mathrm{CO} 31-5$ & $\mathrm{Na} 2 \mathrm{CO} 3$ & 50 & 5 & 780.79 & 4841.05 & 711.11 & 2.8925 \\
\hline Special Salt Solutions & 12 & $50 \mathrm{C}$ Na2CO3 1-6 & $\mathrm{Na} 2 \mathrm{CO} 3$ & 50 & 6 & 711.11 & 5552.16 & 650.55 & 2.8519 \\
\hline Special Salt Solutions & 12 & $50 \mathrm{C} \mathrm{Na} 2 \mathrm{CO} 31-7$ & $\mathrm{Na} 2 \mathrm{CO} 3$ & 50 & 7 & 650.55 & 6202.71 & 591.31 & 2.8133 \\
\hline Special Salt Solutions & 12 & $50 \mathrm{C}$ Na2CO3 $1-8$ & $\mathrm{Na} 2 \mathrm{CO} 3$ & 50 & 8 & 591.31 & 6794.01 & 534.91 & 2.7718 \\
\hline Special Salt Solutions & 12 & $50 \mathrm{C} \mathrm{Na} 2 \mathrm{CO} 31-9$ & $\mathrm{Na} 2 \mathrm{CO} 3$ & 50 & 9 & 534.91 & 7328.92 & 484.30 & 2.7283 \\
\hline Special Salt Solutions & 12 & $50 \mathrm{C} \mathrm{Na} 2 \mathrm{CO} 31-10$ & $\mathrm{Na} 2 \mathrm{CO} 3$ & 50 & 10 & 484.30 & 7813.22 & & 2.6851 \\
\hline Special Salt Solutions & 13 & $50 \mathrm{C}$ Na2 $2 \mathrm{CO} 32-1$ & $\mathrm{Na} 2 \mathrm{CO} 3$ & 50 & 1 & 1223.04 & 1223.04 & 1085.94 & 3.0874 \\
\hline Special Salt Solutions & 13 & $50 \mathrm{C} \mathrm{Na} 2 \mathrm{CO} 32-2$ & $\mathrm{Na} 2 \mathrm{CO} 3$ & 50 & 2 & 1085.94 & 2308.98 & 937.66 & 3.0358 \\
\hline Special Salt Solutions & 13 & $50 \mathrm{C} \mathrm{Na} 2 \mathrm{CO} 32-3$ & $\mathrm{Na} 2 \mathrm{CO} 3$ & 50 & 3 & 937.66 & 3246.64 & 852.58 & 2.9720 \\
\hline Special Salt Solutions & 13 & $50 \mathrm{C} \mathrm{Na} 2 \mathrm{CO} 32-4$ & $\mathrm{Na} 2 \mathrm{CO} 3$ & 50 & 4 & 852.58 & $\begin{array}{l}32409.04 \\
4099\end{array}$ & $\begin{array}{l}02.00 \\
779.63\end{array}$ & 2.9307 \\
\hline Special Salt Solutions & 13 & $50 \mathrm{C}$ Na2CO3 2-5 & $\mathrm{Na} 2 \mathrm{CO} 3$ & 50 & 5 & 779.63 & 4878.85 & 707.66 & 2.8919 \\
\hline Special Salt Solutions & 13 & $50 \mathrm{C} \mathrm{Na} 2 \mathrm{CO} 32-6$ & $\mathrm{Na} 2 \mathrm{CO} 3$ & 50 & 6 & 707.66 & 5586.50 & 645.78 & 2.8498 \\
\hline Special Salt Solutions & 13 & $50 \mathrm{C} \mathrm{Na} 2 \mathrm{CO} 32-7$ & $\mathrm{Na} 2 \mathrm{CO} 3$ & 50 & 7 & 645.78 & 6232.28 & 584.00 & 2.8101 \\
\hline Special Salt Solutions & 13 & $50 \mathrm{C} \mathrm{Na} 2 \mathrm{CO} 32-8$ & $\mathrm{Na} 2 \mathrm{CO} 3$ & 50 & 8 & 584.00 & 6816.28 & 530.10 & 2.7664 \\
\hline Special Salt Solutions & 13 & $50 \mathrm{C} \mathrm{Na} 2 \mathrm{CO} 32-9$ & $\mathrm{Na} 2 \mathrm{CO} 3$ & 50 & 9 & 530.10 & 7346.38 & 476.29 & 2.7244 \\
\hline Special Salt Solutions & 13 & $50 \mathrm{C} \mathrm{Na} 2 \mathrm{CO} 32-10$ & $\mathrm{Na} 2 \mathrm{CO} 3$ & 50 & 10 & 476.29 & 7822.67 & & 2.6779 \\
\hline Special Salt Solutions & 14 & $50 \mathrm{C} \mathrm{Na} 2 \mathrm{CO} 33-1$ & $\mathrm{Na} 2 \mathrm{CO} 3$ & 50 & 1 & 1316.76 & 1316.76 & 1133.07 & 3.1195 \\
\hline Special Salt Solutions & 14 & $50 \mathrm{C} \mathrm{Na} 2 \mathrm{CO} 33-2$ & $\mathrm{Na} 2 \mathrm{CO} 3$ & 50 & 2 & 1133.07 & 2449.83 & 977.24 & 3.0543 \\
\hline
\end{tabular}


Table B3. Test Results for Special Salt Solutions

(continued)

\begin{tabular}{|c|c|c|c|c|c|c|c|c|c|}
\hline Study & $\begin{array}{c}\text { Test } \\
\text { Sequence }\end{array}$ & $\begin{array}{c}\text { Test } \\
\text { Identifier }\end{array}$ & $\begin{array}{c}\text { Salt } \\
\text { Solution }\end{array}$ & $\begin{array}{c}\text { Temperature } \\
\left({ }^{\circ} \mathrm{C}\right)\end{array}$ & $\begin{array}{l}\text { Sampling } \\
\text { Sequence }\end{array}$ & $\begin{array}{c}\mathrm{A} \\
\text { (peak area) }\end{array}$ & & & $\log (\mathrm{A})$ \\
\hline Special Salt Solutions & 14 & $50 \mathrm{C} \mathrm{Na} 2 \mathrm{CO} 33-3$ & $\mathrm{Na} 2 \mathrm{CO} 3$ & 50 & 3 & 977.24 & 3427.07 & $\begin{array}{c}x \\
861.89\end{array}$ & 2.9900 \\
\hline Special Salt Solutions & 14 & $50 \mathrm{C} \mathrm{Na} 2 \mathrm{CO} 33-4$ & $\mathrm{Na} 2 \mathrm{CO} 3$ & 50 & 4 & 861.89 & 4288.96 & 793.64 & 2.9355 \\
\hline Special Salt Solutions & 14 & $50 \mathrm{C} \mathrm{Na} 2 \mathrm{CO} 33-5$ & $\mathrm{Na} 2 \mathrm{CO} 3$ & 50 & 5 & 793.64 & 5082.60 & 713.87 & 2.8996 \\
\hline Special Salt Solutions & 14 & $50 \mathrm{C} \mathrm{Na} 2 \mathrm{CO} 33-6$ & $\mathrm{Na} 2 \mathrm{CO} 3$ & 50 & 6 & 713.87 & 5796.46 & 654.52 & 2.8536 \\
\hline Special Salt Solutions & 14 & $50-\mathrm{C} \mathrm{Na} 2 \mathrm{CO} 33-7$ & $\mathrm{Na} 2 \mathrm{CO} 3$ & 50 & 7 & 654.52 & 6450.98 & 592.76 & 2.8159 \\
\hline Special Salt Solutions & 14 & $50 \mathrm{C} \mathrm{Na} 2 \mathrm{CO} 33-8$ & $\mathrm{Na} 2 \mathrm{CO} 3$ & 50 & 8 & 592.76 & 7043.74 & 535.57 & 2.7729 \\
\hline Special Salt Solutions & 14 & $50 \mathrm{C} \mathrm{Na} 2 \mathrm{CO} 33-9$ & $\mathrm{Na} 2 \mathrm{CO} 3$ & 50 & 9 & 535.57 & 7579.31 & 485.94 & 2.7288 \\
\hline Special Salt Solutions & 14 & $50 \mathrm{C} \mathrm{Na} 2 \mathrm{CO} 33-10$ & $\mathrm{Na} 2 \mathrm{CO} 3$ & 50 & 10 & 485.94 & 8065.25 & & 2.6866 \\
\hline Special Salt Solutions & 15 & $50 \mathrm{C} \mathrm{Na} 2 \mathrm{SO} 4$ 1-1 & $\mathrm{Na} 2 \mathrm{SO} 4$ & 50 & 1 & 669.30 & 669.30 & 596.14 & 2.8256 \\
\hline Special Salt Solutions & 15 & $50 \mathrm{C} \mathrm{Na} 2 \mathrm{SO} 41-2$ & $\mathrm{Na} 2 \mathrm{SO} 4$ & 50 & 2 & 596.14 & 1265.43 & 520.45 & 2.7753 \\
\hline Special Salt Solutions & 15 & $50 \mathrm{C}$ Na2SO4 1-3 & $\mathrm{Na} 2 \mathrm{SO} 4$ & 50 & 3 & 520.45 & 1785.88 & 467.77 & 2.7164 \\
\hline Special Salt Solutions & 15 & $50 \mathrm{C} \mathrm{Na} 2 \mathrm{SO} 41-4$ & $\mathrm{Na} 2 \mathrm{SO} 4$ & 50 & 4 & 467.77 & 2253.65 & 426.55 & 2.6700 \\
\hline Special Salt Solutions & 15 & $50 \mathrm{C} \mathrm{Na} 2 \mathrm{SO} 41-5$ & $\mathrm{Na} 2 \mathrm{SO} 4$ & 50 & 5 & 426.55 & 2680.20 & 388.96 & 2.6300 \\
\hline Special Salt Solutions & 15 & $50 \mathrm{C} \mathrm{Na} 2 \mathrm{SO} 41-6$ & $\mathrm{Na} 2 \mathrm{SO} 4$ & 50 & 6 & 388.96 & 3069.16 & 353.12 & 2.5899 \\
\hline Special Salt Solutions & 15 & $50 \mathrm{C}$ Na2SO4 1-7 & $\mathrm{Na} 2 \mathrm{SO} 4$ & 50 & 7 & 353.12 & 3422.28 & 328.05 & 2.5479 \\
\hline Special Salt Solutions & 15 & $50 \mathrm{C} \mathrm{Na2SO} 41-8$ & $\mathrm{Na} 2 \mathrm{SO} 4$ & 50 & 8 & 328.05 & 3750.33 & 297.16 & 2.5159 \\
\hline Special Salt Solutions & 15 & $50 \mathrm{C} \mathrm{Na} 2 \mathrm{SO} 41-9$ & $\mathrm{Na} 2 \mathrm{SO} 4$ & 50 & 9 & 297.16 & 4047.49 & 272.59 & 2.4730 \\
\hline Special Salt Solutions & 15 & $50 \mathrm{C}$ Na2SO4 $1-10$ & $\mathrm{Na} 2 \mathrm{SO} 4$ & 50 & 10 & 272.59 & 4320.08 & & 2.4355 \\
\hline Special Salt Solutions & 16 & $50 \mathrm{C} \mathrm{Na} 2 \mathrm{SO} 42-1$ & $\mathrm{Na} 2 \mathrm{SO} 4$ & 50 & 1 & 655.74 & 655.74 & 575.56 & 2.8167 \\
\hline Special Salt Solutions & 16 & $50 \mathrm{C}$ Na2SO4 2-2 & $\mathrm{Na} 2 \mathrm{SO} 4$ & 50 & 2 & 575.56 & 1231.30 & 499.79 & 2.7601 \\
\hline Special Salt Solutions & 16 & $50 \mathrm{C}$ Na2SO4 2-3 & $\mathrm{Na} 2 \mathrm{SO} 4$ & 50 & 3 & 499.79 & 1731.10 & 450.25 & 2.6988 \\
\hline Special Salt Solutions & 16 & $50 \mathrm{C}$ Na2SO4 2-4 & $\mathrm{Na} 2 \mathrm{SO} 4$ & 50 & 4 & 450.25 & 2181.35 & 410.38 & 2.6535 \\
\hline Special Salt Solutions & 16 & $50 \mathrm{C}$ Na2SO4 2-5 & $\mathrm{Na} 2 \mathrm{SO} 4$ & 50 & 5 & 410.38 & 2591.73 & 376.78 & 2.6132 \\
\hline Special Salt Solutions & 16 & $50 \mathrm{C} \mathrm{Na} 2 \mathrm{SO} 42-6$ & $\mathrm{Na} 2 \mathrm{SO} 4$ & 50 & 6 & 376.78 & 2968.51 & 346.89 & 2.5761 \\
\hline Special Salt Solutions & 16 & $50 \mathrm{C} \mathrm{Na} 2 \mathrm{SO} 42-7$ & $\mathrm{Na} 2 \mathrm{SO} 4$ & 50 & 7 & 346.89 & 3315.39 & 312.13 & 2.5402 \\
\hline Special Salt Solutions & 16 & $50 \mathrm{C}$ Na2SO4 2-8 & $\mathrm{Na} 2 \mathrm{SO} 4$ & 50 & 8 & 312.13 & 3627.53 & 284.74 & 2.4943 \\
\hline Special Salt Solutions & 16 & $50 \mathrm{C}$ Na2SO4 2-9 & $\mathrm{Na} 2 \mathrm{SO} 4$ & 50 & 9 & 284.74 & 3912.26 & 260.80 & 2.4544 \\
\hline Special Salt Solutions & 16 & $50 \mathrm{C} \mathrm{Na} 2 \mathrm{SO} 42-10$ & $\mathrm{Na} 2 \mathrm{SO} 4$ & 50 & 10 & 260.80 & 4173.07 & & 2.4163 \\
\hline Special Salt Solutions & 17 & $50 \mathrm{C} \mathrm{Na} 2 \mathrm{SO} 43-1$ & $\mathrm{Na} 2 \mathrm{SO} 4$ & 50 & 1 & 677.23 & 677.23 & 590.44 & 2.8307 \\
\hline Special Salt Solutions & 17 & $50 \mathrm{C} \mathrm{Na} 2 \mathrm{SO} 43-2$ & $\mathrm{Na} 2 \mathrm{SO} 4$ & 50 & 2 & 590.44 & 1267.67 & 504.01 & 2.7712 \\
\hline Special Salt Solutions & 17 & $50 \mathrm{C} \mathrm{Na} 2 \mathrm{SO} 43-3$ & $\mathrm{Na} 2 \mathrm{SO} 4$ & 50 & 3 & 504.01 & 1771.67 & 444.07 & 2.7024 \\
\hline Special Salt Solutions & 17 & $50 \mathrm{C} \mathrm{Na} 2 \mathrm{SO} 43-4$ & $\mathrm{Na} 2 \mathrm{SO} 4$ & 50 & 4 & 444.07 & 2215.74 & 410.59 & 2.6474 \\
\hline Special Salt Solutions & 17 & $50 \mathrm{C}$ Na2SO4 3-5 & $\mathrm{Na} 2 \mathrm{SO} 4$ & 50 & 5 & 410.59 & 2626.33 & 371.19 & 2.6134 \\
\hline Special Salt Solutions & 17 & $50 \mathrm{C} \mathrm{Na} 2 \mathrm{SO} 43-6$ & $\mathrm{Na} 2 \mathrm{SO} 4$ & 50 & 6 & 371.19 & 2997.52 & 340.71 & 2.5696 \\
\hline Special Salt Solutions & 17 & $50 \mathrm{C}$ Na2SO4 3-7 & $\mathrm{Na} 2 \mathrm{SO} 4$ & 50 & 7 & 340.71 & 3338.23 & 313.90 & 2.5324 \\
\hline Special Salt Solutions & 17 & $50 \mathrm{C} \mathrm{Na} 2 \mathrm{SO} 43-8$ & $\mathrm{Na} 2 \mathrm{SO} 4$ & 50 & 8 & 313.90 & 3652.13 & 284.55 & 2.4968 \\
\hline Special Salt Solutions & 17 & $50 \mathrm{C} \mathrm{Na} 2 \mathrm{SO} 43-9$ & $\mathrm{Na} 2 \mathrm{SO} 4$ & 50 & 9 & 284.55 & 3936.67 & 258.34 & 2.4542 \\
\hline Special Salt Solutions & 17 & $50 \mathrm{C} \mathrm{Na} 2 \mathrm{SO} 43-10$ & $\mathrm{Na} 2 \mathrm{SO} 4$ & 50 & 10 & 258.34 & 4195.01 & & 2.4122 \\
\hline Special Salt Solutions & 18 & $60 \mathrm{C} \mathrm{Na} 2 \mathrm{CO} 31-1$ & $\mathrm{Na} 2 \mathrm{CO} 3$ & 60 & 1 & 692.25 & 692.25 & 694.32 & 2.8403 \\
\hline Special Salt Solutions & 18 & $60 \mathrm{C} \mathrm{Na} 2 \mathrm{CO} 31-1$ & $\mathrm{Na} 2 \mathrm{CO} 3$ & 60 & 2 & 694.32 & 1386.56 & 620.26 & 2.8416 \\
\hline Special Salt Solutions & 18 & $60 \mathrm{C} \mathrm{Na} 2 \mathrm{CO} 31-3$ & $\mathrm{Na} 2 \mathrm{CO} 3$ & 60 & 3 & 620.26 & 2006.82 & 545.45 & 2.7926 \\
\hline Special Salt Solutions & 18 & $60 \mathrm{C}$ Na2CO3 1-4 & $\mathrm{Na} 2 \mathrm{CO} 3$ & 60 & 4 & 545.45 & 2552.27 & 482.08 & 2.7368 \\
\hline Special Salt Solutions & 18 & $60 \mathrm{C}$ Na2CO3 $1-5$ & $\mathrm{Na} 2 \mathrm{CO} 3$ & 60 & 5 & 482.08 & 3034.35 & 425.12 & 2.6831 \\
\hline Special Salt Solutions & 18 & $60 \mathrm{C} \mathrm{Na} 2 \mathrm{CO} 31-6$ & $\mathrm{Na} 2 \mathrm{CO} 3$ & 60 & 6 & 425.12 & 3459.46 & 373.28 & 2.6285 \\
\hline Special Salt Solutions & 18 & $60 \mathrm{C} \mathrm{Na} 2 \mathrm{CO} 31-7$ & $\mathrm{Na} 2 \mathrm{CO} 3$ & 60 & 7 & 373.28 & 3832.74 & 327.41 & 2.5720 \\
\hline Special Salt Solutions & 18 & $60 \mathrm{C} \mathrm{Na} 2 \mathrm{CO} 31-8$ & $\mathrm{Na} 2 \mathrm{CO} 3$ & 60 & 8 & 327.41 & 4160.15 & 287.12 & 2.5151 \\
\hline Special Salt Solutions & 18 & $60 \mathrm{C} \mathrm{Na} 2 \mathrm{CO} 31-9$ & $\mathrm{Na} 2 \mathrm{CO} 3$ & 60 & 9 & 287.12 & 4447.27 & 252.94 & 2.4581 \\
\hline Special Salt Solutions & 18 & $60 \mathrm{C} \mathrm{Na} 2 \mathrm{CO} 31-10$ & $\mathrm{Na} 2 \mathrm{CO} 3$ & 60 & 10 & 252.94 & 4700.21 & & 2.4030 \\
\hline Special Salt Solutions & 19 & $60 \mathrm{C} \mathrm{Na} 2 \mathrm{CO} 32-1$ & $\mathrm{Na} 2 \mathrm{CO} 3$ & 60 & 1 & 721.20 & 721.20 & 680.47 & 2.8581 \\
\hline Special Salt Solutions & 19 & $60 \mathrm{C} \mathrm{Na} 2 \mathrm{CO} 32-2$ & $\mathrm{Na} 2 \mathrm{CO} 3$ & 60 & 2 & 680.47 & 1401.67 & 606.65 & 2.8328 \\
\hline Special Salt Solutions & 19 & $60 \mathrm{C} \mathrm{Na} 2 \mathrm{CO} 32-3$ & $\mathrm{Na} 2 \mathrm{CO} 3$ & 60 & 3 & 606.65 & 2008.32 & 535.69 & 2.7829 \\
\hline Special Salt Solutions & 19 & $60 \mathrm{C}$ Na2CO3 2-4 & $\mathrm{Na} 2 \mathrm{CO} 3$ & 60 & 4 & 535.69 & 2544.02 & 472.82 & 2.7289 \\
\hline Special Salt Solutions & 19 & $60 \mathrm{C} \mathrm{Na} 2 \mathrm{CO} 32-5$ & $\mathrm{Na} 2 \mathrm{CO} 3$ & 60 & 5 & 472.82 & 3016.84 & 415.71 & 2.6747 \\
\hline Special Salt Solutions & 19 & $60 \mathrm{C}$ Na2CO3 2-6 & $\mathrm{Na} 2 \mathrm{CO} 3$ & 60 & 6 & 415.71 & 3432.55 & 366.68 & 2.6188 \\
\hline Special Salt Solutions & 19 & $60 \mathrm{C} \mathrm{Na} 2 \mathrm{CO} 32-7$ & $\mathrm{Na} 2 \mathrm{CO} 3$ & 60 & 7 & 366.68 & 3799.23 & 323.48 & 2.5643 \\
\hline Special Salt Solutions & 19 & $60 \mathrm{C} \mathrm{Na} 2$ & $\mathrm{Na} 2 \mathrm{CO} 3$ & 60 & 8 & 323.48 & 4122.71 & 286.69 & 2.5098 \\
\hline Special Salt Solutions & 19 & $60 \mathrm{C} \mathrm{Na} 2 \mathrm{CO} 32-9$ & $\mathrm{Na} 2 \mathrm{CO} 3$ & 60 & 9 & 286.69 & 4409.39 & 249.95 & 2.4574 \\
\hline Special Salt Solutions & 19 & $60 \mathrm{C} \mathrm{Na} 2 \mathrm{CO} 32-10$ & $\mathrm{Na} 2 \mathrm{CO} 3$ & 60 & 10 & 249.95 & 4659.34 & & 2.3978 \\
\hline Special Salt Solutions & 20 & $60 \mathrm{C} \mathrm{Na} 2 \mathrm{CO} 33-1$ & $\mathrm{Na} 2 \mathrm{CO} 3$ & 60 & 1 & 680.45 & 680.45 & 640.17 & 2.8328 \\
\hline Special Salt Solutions & 20 & $60 \mathrm{C} \mathrm{Na} 2 \mathrm{CO} 33-2$ & $\mathrm{Na} 2 \mathrm{CO} 3$ & 60 & 2 & 640.17 & 1320.62 & 570.35 & 2.8063 \\
\hline Special Salt Solutions & 20 & $60 \mathrm{C} \mathrm{Na} 2 \mathrm{CO} 33-3$ & $\mathrm{Na} 2 \mathrm{CO} 3$ & 60 & 3 & 570.35 & 1890.97 & 513.00 & 2.7561 \\
\hline Special Salt Solutions & 20 & $60 \mathrm{C} \mathrm{Na} 2$ & N & 60 & 4 & 513.00 & 2403.96 & 451.84 & 2.7101 \\
\hline Special Salt Solutions & 20 & $60 \mathrm{C} \mathrm{Na} 2 \mathrm{CO} 33-5$ & $\mathrm{Na} 2 \mathrm{CO} 3$ & 60 & 5 & 451.84 & 2855.80 & 395.31 & 2.6550 \\
\hline Special Salt Solutions & 20 & $60 \mathrm{C} \mathrm{Na} 2 \mathrm{CO} 33-6$ & $\mathrm{Na} 2 \mathrm{CO} 3$ & 60 & 6 & 395.31 & 3251.11 & 355.98 & 2.5969 \\
\hline Special Salt Solutions & 20 & $60 \mathrm{C} \mathrm{Na} 2 \mathrm{CO} 33-7$ & $\mathrm{Na} 2 \mathrm{CO} 3$ & 60 & 7 & 355.98 & 3607.09 & 313.11 & 2.5514 \\
\hline
\end{tabular}


Table B3. Test Results for Special Salt Solutions

(continued)

\begin{tabular}{|c|c|c|c|c|c|c|c|c|c|}
\hline Study & $\begin{array}{c}\text { Test } \\
\text { Sequence }\end{array}$ & $\begin{array}{c}\text { Test } \\
\text { Identifier }\end{array}$ & $\begin{array}{c}\text { Salt } \\
\text { Solution }\end{array}$ & $\begin{array}{c}\text { Temperature } \\
\left({ }^{\circ} \mathrm{C}\right)\end{array}$ & $\begin{array}{l}\text { Sampling } \\
\text { Sequence }\end{array}$ & $\begin{array}{c}\text { A } \\
\text { (peak area) }\end{array}$ & $\mathrm{y}$ & $\mathrm{x}$ & $\log (\mathrm{A})$ \\
\hline Special Salt Solutions & 20 & $60 \mathrm{C} \mathrm{Na} 2 \mathrm{CO} 3$ 3-8 & $\mathrm{Na} 2 \mathrm{CO} 3$ & 60 & 8 & 313.11 & 3920.21 & 272.61 & 2.4957 \\
\hline Special Salt Solutions & 20 & $60 \mathrm{C} \mathrm{Na} 2 \mathrm{CO} 3$ 3-9 & $\mathrm{Na} 2 \mathrm{CO} 3$ & 60 & 9 & 272.61 & 4192.82 & 244.46 & 2.4355 \\
\hline Special Salt Solutions & 20 & $60 \mathrm{C} \mathrm{Na} 2 \mathrm{CO} 33-10$ & $\mathrm{Na} 2 \mathrm{CO} 3$ & 60 & 10 & 244.46 & 4437.27 & & 2.3882 \\
\hline Special Salt Solutions & 21 & 60C Na2SO4 1-1 & $\mathrm{Na} 2 \mathrm{SO} 4$ & 60 & 1 & 368.63 & 368.63 & 335.44 & 2.5666 \\
\hline Special Salt Solutions & 21 & $60 \mathrm{C} \mathrm{Na} 2 \mathrm{SO} 41-2$ & $\mathrm{Na} 2 \mathrm{SO} 4$ & 60 & 2 & 335.44 & 704.07 & 294.93 & 2.5256 \\
\hline Special Salt Solutions & 21 & $60 \mathrm{C} \mathrm{Na} 2 \mathrm{SO} 41-3$ & $\mathrm{Na} 2 \mathrm{SO} 4$ & 60 & 3 & 294.93 & 999.00 & 256.24 & 2.4697 \\
\hline Special Salt Solutions & 21 & $60 \mathrm{C} \mathrm{Na} 2 \mathrm{SO} 41-4$ & $\mathrm{Na} 2 \mathrm{SO} 4$ & 60 & 4 & 256.24 & 1255.24 & 226.65 & 2.4086 \\
\hline Special Salt Solutions & 21 & $60 \mathrm{C} \mathrm{Na} 2 \mathrm{SO} 41-5$ & $\mathrm{Na} 2 \mathrm{SO} 4$ & 60 & 5 & 226.65 & 1481.88 & 198.20 & 2.3554 \\
\hline Special Salt Solutions & 21 & $60 \mathrm{C} \mathrm{Na} 2 \mathrm{SO} 4$ 1-6 & $\mathrm{Na} 2 \mathrm{SO} 4$ & 60 & 6 & 198.20 & 1680.08 & 175.32 & 2.2971 \\
\hline Special Salt Solutions & 21 & 60C Na2SO4 1-7 & $\mathrm{Na} 2 \mathrm{SO} 4$ & 60 & 7 & 175.32 & 1855.40 & 154.44 & 2.2438 \\
\hline Special Salt Solutions & 21 & 60C Na2SO4 1-8 & $\mathrm{Na} 2 \mathrm{SO} 4$ & 60 & 8 & 154.44 & 2009.84 & 138.87 & 2.1888 \\
\hline Special Salt Solutions & 21 & $60 \mathrm{C} \mathrm{Na} 2 \mathrm{SO} 4$ 1-9 & $\mathrm{Na} 2 \mathrm{SO} 4$ & 60 & 9 & 138.87 & 2148.72 & 122.65 & 2.1426 \\
\hline Special Salt Solutions & 21 & 60C Na2SO4 1-10 & $\mathrm{Na} 2 \mathrm{SO} 4$ & 60 & 10 & 122.65 & 2271.36 & & 2.0887 \\
\hline Special Salt Solutions & 22 & 60C Na2SO4 2-1 & $\mathrm{Na} 2 \mathrm{SO} 4$ & 60 & 1 & 324.83 & 324.83 & 294.97 & 2.5117 \\
\hline Special Salt Solutions & 22 & $60 \mathrm{C} \mathrm{Na} 2 \mathrm{SO} 42-2$ & $\mathrm{Na} 2 \mathrm{SO} 4$ & 60 & 2 & 294.97 & 619.80 & 267.15 & 2.4698 \\
\hline Special Salt Solutions & 22 & $60 \mathrm{C} \mathrm{Na} 2 \mathrm{SO} 42-3$ & $\mathrm{Na} 2 \mathrm{SO} 4$ & 60 & 3 & 267.15 & 886.95 & 242.86 & 2.4268 \\
\hline Special Salt Solutions & 22 & 60C Na2SO4 2-4 & $\mathrm{Na} 2 \mathrm{SO} 4$ & 60 & 4 & 242.86 & 1129.81 & 216.98 & 2.3854 \\
\hline Special Salt Solutions & 22 & $60 \mathrm{C} \mathrm{Na} 2 \mathrm{SO} 42-5$ & $\mathrm{Na} 2 \mathrm{SO} 4$ & 60 & 5 & 216.98 & 1346.79 & 191.94 & 2.3364 \\
\hline Special Salt Solutions & 22 & $60 \mathrm{C} \mathrm{Na} 2 \mathrm{SO} 42-6$ & $\mathrm{Na} 2 \mathrm{SO} 4$ & 60 & 6 & 191.94 & 1538.73 & 169.26 & 2.2832 \\
\hline Special Salt Solutions & 22 & 60C Na2SO4 2-7 & $\mathrm{Na} 2 \mathrm{SO} 4$ & 60 & 7 & 169.26 & 1707.99 & 150.66 & 2.2286 \\
\hline Special Salt Solutions & 22 & $60 \mathrm{C} \mathrm{Na} 2 \mathrm{SO} 42-8$ & $\mathrm{Na} 2 \mathrm{SO} 4$ & 60 & 8 & 150.66 & 1858.65 & 135.13 & 2.1780 \\
\hline Special Salt Solutions & 22 & $60 \mathrm{C} \mathrm{Na} 2 \mathrm{SO} 42-9$ & $\mathrm{Na} 2 \mathrm{SO} 4$ & 60 & 9 & 135.13 & 1993.78 & 119.62 & 2.1307 \\
\hline Special Salt Solutions & 22 & 60C Na2SO4 2-10 & $\mathrm{Na} 2 \mathrm{SO} 4$ & 60 & 10 & 119.62 & 2113.40 & & 2.0778 \\
\hline Special Salt Solutions & 23 & 60CNa2SO4 3-1 & $\mathrm{Na} 2 \mathrm{SO} 4$ & 60 & 1 & 324.14 & 324.14 & 294.86 & 2.5107 \\
\hline Special Salt Solutions & 23 & $60 \mathrm{C} \mathrm{Na} 2 \mathrm{SO} 43-2$ & $\mathrm{Na} 2 \mathrm{SO} 4$ & 60 & 2 & 294.86 & 619.00 & 273.60 & 2.4696 \\
\hline Special Salt Solutions & 23 & $60 \mathrm{C} \mathrm{Na} 2 \mathrm{SO} 43-2$ & $\mathrm{Na} 2 \mathrm{SO} 4$ & 60 & 3 & 273.60 & 892.60 & 250.92 & 2.4371 \\
\hline Special Salt Solutions & 23 & $60 \mathrm{C} \mathrm{Na} 2 \mathrm{SO} 43-4$ & $\mathrm{Na} 2 \mathrm{SO} 4$ & 60 & 4 & 250.92 & 1143.51 & 226.82 & 2.3995 \\
\hline Special Salt Solutions & 23 & $60 \mathrm{C} \mathrm{Na} 2 \mathrm{SO} 43-5$ & $\mathrm{Na} 2 \mathrm{SO} 4$ & 60 & 5 & 226.82 & 1370.34 & 206.25 & 2.3557 \\
\hline Special Salt Solutions & 23 & 60C Na2SO4 3-6 & $\mathrm{Na} 2 \mathrm{SO} 4$ & 60 & 6 & 206.25 & 1576.58 & 187.67 & 2.3144 \\
\hline Special Salt Solutions & 23 & 60C Na2SO4 3-7 & $\mathrm{Na} 2 \mathrm{SO} 4$ & 60 & 7 & 187.67 & 1764.25 & 168.71 & 2.2734 \\
\hline Special Salt Solutions & 23 & $60 \mathrm{C} \mathrm{Na} 2 \mathrm{SO} 43-8$ & $\mathrm{Na} 2 \mathrm{SO} 4$ & 60 & 8 & 168.71 & 1932.96 & 150.65 & 2.2272 \\
\hline Special Salt Solutions & 23 & $60 \mathrm{C} \mathrm{Na} 2 \mathrm{SO} 43-9$ & $\mathrm{Na} 2 \mathrm{SO} 4$ & 60 & 9 & 150.65 & 2083.61 & 136.37 & 2.1780 \\
\hline Special Salt Solutions & 23 & 60C Na2SO4 3-10 & $\mathrm{Na} 2 \mathrm{SO} 4$ & 60 & 10 & 136.37 & 2219.98 & & 2.1347 \\
\hline Special Salt Solutions & 24 & 60C NaNO2 1-1 & NaNO2 & 60 & 1 & 281.17 & 281.17 & 258.99 & 2.4490 \\
\hline Special Salt Solutions & 24 & 60C NaNO2 1-2 & $\mathrm{NaNO} 2$ & 60 & 2 & 258.99 & 540.16 & 239.69 & 2.4133 \\
\hline Special Salt Solutions & 24 & $60 \mathrm{C}$ NaNO2 1-3 & $\mathrm{NaNO} 2$ & 60 & 3 & 239.69 & 779.85 & 225.51 & 2.3796 \\
\hline Special Salt Solutions & 24 & 60C NaNO2 1-4 & $\mathrm{NaNO} 2$ & 60 & 4 & 225.51 & 1005.36 & 208.81 & 2.3532 \\
\hline Special Salt Solutions & 24 & $60 \mathrm{C}$ NaNO2 1-5 & $\mathrm{NaNO} 2$ & 60 & 5 & 208.81 & 1214.17 & 191.12 & 2.3198 \\
\hline Special Salt Solutions & 24 & 60C NaNO2 1-6 & $\mathrm{NaNO} 2$ & 60 & 6 & 191.12 & 1405.30 & 177.06 & 2.2813 \\
\hline Special Salt Solutions & 24 & 60C NaNO2 1-7 & $\mathrm{NaNO} 2$ & 60 & 7 & 177.06 & 1582.36 & 164.21 & 2.2481 \\
\hline Special Salt Solutions & 24 & 60C NaNO2 1-8 & NaNO2 & 60 & 8 & 164.21 & 1746.57 & 149.74 & 2.2154 \\
\hline Special Salt Solutions & 24 & $60 \mathrm{C}$ NaNO2 1-9 & $\mathrm{NaNO} 2$ & 60 & 9 & 149.74 & 1896.31 & 134.52 & 2.1753 \\
\hline Special Salt Solutions & 24 & 60C NaNO2 1-10 & $\mathrm{NaNO} 2$ & 60 & 10 & 134.52 & 2030.83 & & 2.1288 \\
\hline Special Salt Solutions & 25 & 60C NaNO2 2-1 & $\mathrm{NaNO} 2$ & 60 & 1 & 302.02 & 302.02 & 276.98 & 2.4800 \\
\hline Special Salt Solutions & 25 & $60 \mathrm{C}$ NaNO2 2-2 & $\mathrm{NaNO} 2$ & 60 & 2 & 276.98 & 579.00 & 259.34 & 2.4425 \\
\hline Special Salt Solutions & 25 & $60 \mathrm{C}$ NaNO2 2-3 & $\mathrm{NaNO} 2$ & 60 & 3 & 259.34 & 838.34 & 241.04 & 2.4139 \\
\hline Special Salt Solutions & 25 & $60 \mathrm{C}$ NaNO2 2-4 & NaNO2 & 60 & 4 & 241.04 & 1079.38 & 223.33 & 2.3821 \\
\hline Special Salt Solutions & 25 & 60C NaNO2 2-5 & $\mathrm{NaNO} 2$ & 60 & 5 & 223.33 & 1302.71 & 206.89 & 2.3489 \\
\hline Special Salt Solutions & 25 & 60C NaNO2 2-6 & $\mathrm{NaNO} 2$ & 60 & 6 & 206.89 & 1509.60 & 187.05 & 2.3157 \\
\hline Special Salt Solutions & 25 & 60C NaNO2 2-7 & $\mathrm{NaNO} 2$ & 60 & 7 & 187.05 & 1696.64 & 174.24 & 2.2719 \\
\hline Special Salt Solutions & 25 & $60 \mathrm{C}$ NaNO2 2-8 & $\mathrm{NaNO} 2$ & 60 & 8 & 174.24 & 1870.89 & 158.62 & 2.2412 \\
\hline Special Salt Solutions & 25 & $60 \mathrm{C}$ NaNO2 2-9 & NaNO2 & 60 & 9 & 158.62 & 2029.50 & 141.98 & 2.2004 \\
\hline Special Salt Solutions & 25 & 60C NaNO2 2-10 & $\mathrm{NaNO} 2$ & 60 & 10 & 141.98 & 2171.48 & & 2.1522 \\
\hline Special Salt Solutions & 26 & $60 \mathrm{C}$ NaNO2 3-1 & NaNO2 & 60 & 1 & 334.60 & 334.60 & 297.62 & 2.5245 \\
\hline Special Salt Solutions & 26 & $60 \mathrm{C}$ NaNO2 3-2 & $\mathrm{NaNO} 2$ & 60 & 2 & 297.62 & 632.22 & 271.49 & 2.4737 \\
\hline Special Salt Solutions & 26 & 60C NaNO2 3-3 & $\mathrm{NaNO} 2$ & 60 & 3 & 271.49 & 903.71 & 255.18 & 2.4338 \\
\hline Special Salt Solutions & 26 & $60 \mathrm{C}$ NaNO2 3-4 & $\mathrm{NaNO} 2$ & 60 & 4 & 255.18 & 1158.89 & 236.36 & 2.4068 \\
\hline Special Salt Solutions & 26 & 60C NaNO2 3-5 & $\mathrm{NaNO} 2$ & 60 & 5 & 236.36 & 1395.25 & 217.00 & 2.3736 \\
\hline Special Salt Solutions & 26 & 60C NaNO2 3-6 & NaNO2 & 60 & 6 & 217.00 & 1612.25 & 201.41 & 2.3365 \\
\hline Special Salt Solutions & 26 & $60 \mathrm{C}$ NaNO2 3-7 & NaNO2 & 60 & 7 & 201.41 & 1813.66 & 182.88 & 2.3041 \\
\hline Special Salt Solutions & 26 & 60C NaNO2 3-8 & NaNO2 & 60 & 8 & 182.88 & 1996.54 & 167.26 & 2.2622 \\
\hline Special Salt Solutions & 26 & 60C NaNO2 3-9 & $\mathrm{NaNO} 2$ & 60 & 9 & 167.26 & 2163.80 & 152.36 & 2.2234 \\
\hline Special Salt Solutions & 26 & $60 \mathrm{C}$ NaNO2 3-10 & $\mathrm{NaNO} 2$ & 60 & 10 & 152.36 & 2316.16 & . & 2.1829 \\
\hline Special Salt Solutions & 27 & 60C NaNO3 1-1 & NaNO3 & 60 & 1 & 814.32 & 814.32 & 726.76 & 2.9108 \\
\hline Special Salt Solutions & 27 & $60 \mathrm{C}$ NaNO3 $1-2$ & NaNO3 & 60 & 2 & 726.76 & 1541.08 & 644.51 & 2.8614 \\
\hline Special Salt Solutions & 27 & $60 \mathrm{C}$ NaNO3 $1-3$ & NaNO3 & 60 & 3 & 644.51 & 2185.59 & 614.21 & 2.8092 \\
\hline
\end{tabular}


Table B3. Test Results for Special Salt Solutions

(continued)

\begin{tabular}{|c|c|c|c|c|c|c|c|c|c|}
\hline Special Salt Solutions & 27 & $\begin{array}{l}\text { Identifier } \\
\text { 60C NaNO3 1-4 }\end{array}$ & $\begin{array}{l}\text { Solution } \\
\text { NaNO3 }\end{array}$ & $\begin{array}{c}(\mathrm{c}) \\
60\end{array}$ & 4 & $\begin{array}{c}\text { (peak area) } \\
614.21\end{array}$ & $\begin{array}{c}\mathrm{y} \\
2799.80\end{array}$ & $\begin{array}{c}\mathrm{x} \\
570.13\end{array}$ & 2.7883 \\
\hline Special Salt Solutions & 27 & $60 \mathrm{C}$ NaNO3 $1-5$ & $\mathrm{NaNO} 3$ & 60 & 5 & 570.13 & 3369.93 & 519.44 & 2.7560 \\
\hline Special Salt Solutions & 27 & 60C NaNO3 $1-6$ & NaNO3 & 60 & 6 & 519.44 & 3889.38 & 474.52 & 2.7155 \\
\hline Special Salt Solutions & 27 & $60 \mathrm{C}$ NaNO3 $1-7$ & NaNO3 & 60 & 7 & 474.52 & 4363.90 & 429.16 & 2.6763 \\
\hline Special Salt Solutions & 27 & 60C NaNO3 1-8 & $\mathrm{NaNO} 3$ & 60 & 8 & 429.16 & 4793.05 & 391.91 & 2.6326 \\
\hline Special Salt Solutions & 27 & 60C NaNO3 1-9 & NaNO3 & 60 & 9 & 391.91 & 5184.97 & 354.89 & 2.5932 \\
\hline Special Salt Solutions & 27 & 60C NaNO3 1-10 & $\mathrm{NaNO} 3$ & 60 & 10 & 354.89 & 5539.86 & & 2.5501 \\
\hline Special Salt Solutions & 28 & 60C NaNO3 2-1 & $\mathrm{NaNO} 3$ & 60 & 1 & 812.07 & 812.07 & 728.44 & 2.9096 \\
\hline Special Salt Solutions & 28 & 60C NaNO3 2-2 & $\mathrm{NaNO} 3$ & 60 & 2 & 728.44 & 1540.50 & 644.22 & 2.8624 \\
\hline Special Salt Solutions & 28 & 60C NaNO3 2-3 & $\mathrm{NaNO} 3$ & 60 & 3 & 644.22 & 2184.72 & 599.84 & 2.8090 \\
\hline Special Salt Solutions & 28 & $60 \mathrm{C}$ NaNO3 2-4 & NaNO3 & 60 & 4 & 599.84 & 2784.56 & 557.89 & 2.7780 \\
\hline Special Salt Solutions & 28 & $60 \mathrm{C}$ NaNO3 2-5 & $\mathrm{NaNO} 3$ & 60 & 5 & 557.89 & 3342.44 & 518.12 & 2.7465 \\
\hline Special Salt Solutions & 28 & 60C NaNO3 2-6 & NaNO3 & 60 & 6 & 518.12 & 3860.56 & 476.72 & 2.7144 \\
\hline Special Salt Solutions & 28 & $60 \mathrm{C}$ NaNO3 2-7 & $\mathrm{NaNO} 3$ & 60 & 7 & 476.72 & 4337.29 & 432.98 & 2.6783 \\
\hline Special Salt Solutions & 28 & 60C NaNO3 2-8 & $\mathrm{NaNO} 3$ & 60 & 8 & 432.98 & 4770.27 & 389.91 & 2.6365 \\
\hline Special Salt Solutions & 28 & 60C NaNO3 2-9 & $\mathrm{NaNO} 3$ & 60 & 9 & 389.91 & 5160.18 & 356.63 & 2.5910 \\
\hline Special Salt Solutions & 28 & 60C NaNO3 2-10 & NaNO3 & 60 & 10 & 356.63 & 5516.81 & & 2.5522 \\
\hline Special Salt Solutions & 29 & 60C NaNO3 3-1 & $\mathrm{NaNO} 3$ & 60 & 1 & 847.88 & 847.88 & 761.18 & 2.9283 \\
\hline Special Salt Solutions & 29 & 60C NaNO3 3-2 & $\mathrm{NaNO} 3$ & 60 & 2 & 761.18 & 1609.06 & 659.21 & 2.8815 \\
\hline Special Salt Solutions & 29 & 60C NaNO3 3-3 & NaNO3 & 60 & 3 & 659.21 & 2268.27 & 619.91 & 2.8190 \\
\hline Special Salt Solutions & 29 & 60C NaNO3 3-4 & $\mathrm{NaNO} 3$ & 60 & 4 & 619.91 & 2888.18 & 580.21 & 2.7923 \\
\hline Special Salt Solutions & 29 & 60C NaNO3 3-5 & $\mathrm{NaNO} 3$ & 60 & 5 & 580.21 & 3468.39 & 533.20 & 2.7636 \\
\hline Special Salt Solutions & 29 & 60C NaNO3 3-6 & NaNO3 & 60 & 6 & 533.20 & 4001.60 & 485.00 & 2.7269 \\
\hline Special Salt Solutions & 29 & 60C NaNO3 3-7 & $\mathrm{NaNO} 3$ & 60 & 7 & 485.00 & 4486.59 & 441.93 & 2.6857 \\
\hline Special Salt Solutions & 29 & 60C NaNO3 3-8 & NaNO3 & 60 & 8 & 441.93 & 4928.52 & 402.04 & 2.6454 \\
\hline Special Salt Solutions & 29 & 60C NaNO3 3-9 & NaNO3 & 60 & 9 & 402.04 & 5330.56 & 363.62 & 2.6043 \\
\hline Special Salt Solutions & 29 & 60C NaNO3 3-10 & $\mathrm{NaNO} 3$ & 60 & 10 & 363.62 & 5694.18 & & 2.5606 \\
\hline Special Salt Solutions & 30 & $70 \mathrm{C} \mathrm{Na} 2 \mathrm{CO} 31-1$ & $\mathrm{Na} 2 \mathrm{CO} 3$ & 70 & 1 & 949.65 & 949.65 & 822.03 & 2.9776 \\
\hline Special Salt Solutions & 30 & $70 \mathrm{C} \mathrm{Na} 2 \mathrm{CO} 31-2$ & $\mathrm{Na} 2 \mathrm{CO} 3$ & 70 & 2 & 822.03 & 1771.68 & 698.83 & 2.9149 \\
\hline Special Salt Solutions & 30 & $70 \mathrm{C} \mathrm{Na} 2 \mathrm{CO} 31-3$ & $\mathrm{Na} 2 \mathrm{CO} 3$ & 70 & 3 & 698.83 & 2470.51 & 640.83 & 2.8444 \\
\hline Special Salt Solutions & 30 & $70 \mathrm{C} \mathrm{Na} 2 \mathrm{CO} 31-4$ & $\mathrm{Na} 2 \mathrm{CO} 3$ & 70 & 4 & 640.83 & 3111.34 & 555.39 & 2.8067 \\
\hline Special Salt Solutions & 30 & $70 \mathrm{C} \mathrm{Na} 2 \mathrm{CO} 31-5$ & $\mathrm{Na} 2 \mathrm{CO} 3$ & 70 & 5 & 555.39 & 3666.72 & 476.14 & 2.7446 \\
\hline Special Salt Solutions & 30 & $70 \mathrm{C}$ Na2CO3 1-6 & $\mathrm{Na} 2 \mathrm{CO} 3$ & 70 & 6 & 476.14 & 4142.87 & 410.30 & 2.6777 \\
\hline Special Salt Solutions & 30 & $70 \mathrm{C} \mathrm{Na} 2 \mathrm{CO} 31-7$ & $\mathrm{Na} 2 \mathrm{CO} 3$ & 70 & 7 & 410.30 & 4553.16 & 348.07 & 2.6131 \\
\hline Special Salt Solutions & 30 & $70 \mathrm{C} \mathrm{Na} 2 \mathrm{CO} 31-8$ & $\mathrm{Na} 2 \mathrm{CO} 3$ & 70 & 8 & 348.07 & 4901.24 & 296.97 & 2.5417 \\
\hline Special Salt Solutions & 30 & $70 \mathrm{C} \mathrm{Na} 2 \mathrm{CO} 31-9$ & $\mathrm{Na} 2 \mathrm{CO} 3$ & 70 & 9 & 296.97 & 5198.21 & 252.84 & 2.4727 \\
\hline Special Salt Solutions & 30 & $70 \mathrm{C} \mathrm{Na} 2 \mathrm{CO} 31-10$ & $\mathrm{Na} 2 \mathrm{CO} 3$ & 70 & 10 & 252.84 & 5451.05 & & 2.4028 \\
\hline Special Salt Solutions & 31 & $70 \mathrm{C} \mathrm{Na} 2 \mathrm{CO} 32-1$ & $\mathrm{Na} 2 \mathrm{CO} 3$ & 70 & 1 & 1041.55 & 1041.55 & 835.50 & 3.0177 \\
\hline Special Salt Solutions & 31 & $70 \mathrm{C} \mathrm{Na} 2 \mathrm{CO} 32-2$ & $\mathrm{Na} 2 \mathrm{CO} 3$ & 70 & 2 & 835.50 & 1877.04 & 673.99 & 2.9219 \\
\hline Special Salt Solutions & 31 & $70 \mathrm{C} \mathrm{Na} 2 \mathrm{CO} 32-3$ & $\mathrm{Na} 2 \mathrm{CO} 3$ & 70 & 3 & 673.99 & 2551.04 & 593.62 & 2.8287 \\
\hline Special Salt Solutions & 31 & $70 \mathrm{C} \mathrm{Na} 2 \mathrm{CO} 32-4$ & $\mathrm{Na} 2 \mathrm{CO} 3$ & 70 & 4 & 593.62 & 3144.66 & 545.11 & 2.7735 \\
\hline Special Salt Solutions & 31 & $70 \mathrm{C} \mathrm{Na} 2 \mathrm{CO} 32-5$ & $\mathrm{Na} 2 \mathrm{CO} 3$ & 70 & 5 & 545.11 & 3689.77 & 467.76 & 2.7365 \\
\hline Special Salt Solutions & 31 & $70 \mathrm{C} \mathrm{Na} 2 \mathrm{CO} 32-6$ & $\mathrm{Na} 2 \mathrm{CO} 3$ & 70 & 6 & 467.76 & 4157.52 & 395.40 & 2.6700 \\
\hline Special Salt Solutions & 31 & $70 \mathrm{C} \mathrm{Na} 2 \mathrm{CO} 32-7$ & $\mathrm{Na} 2 \mathrm{CO} 3$ & 70 & 7 & 395.40 & 4552.92 & 333.71 & 2.5970 \\
\hline Special Salt Solutions & 31 & $70 \mathrm{C} \mathrm{Na} 2 \mathrm{CO} 32-8$ & $\mathrm{Na} 2 \mathrm{CO} 3$ & 70 & 8 & 333.71 & 4886.64 & 280.80 & 2.5234 \\
\hline Special Salt Solutions & 31 & $70 \mathrm{C}$ Na2 $2 \mathrm{CO} 32-9$ & $\mathrm{Na} 2 \mathrm{CO} 3$ & 70 & 9 & 280.80 & 5167.43 & 237.87 & 2.4484 \\
\hline Special Salt Solutions & 31 & $70 \mathrm{C} \mathrm{Na} 2 \mathrm{CO} 32-10$ & $\mathrm{Na} 2 \mathrm{CO} 3$ & 70 & 10 & 237.87 & 5405.31 & & 2.3763 \\
\hline Special Salt Solutions & 32 & $70 \mathrm{C} \mathrm{Na} 2 \mathrm{CO} 33-1$ & $\mathrm{Na} 2 \mathrm{CO} 3$ & 70 & 1 & 919.61 & 919.61 & 736.59 & 2.9636 \\
\hline Special Salt Solutions & 32 & $70 \mathrm{C} \mathrm{Na} 2 \mathrm{CO} 33-2$ & $\mathrm{Na} 2 \mathrm{CO} 3$ & 70 & 2 & 736.59 & 1656.20 & 624.80 & 2.8672 \\
\hline Special Salt Solutions & 32 & $70 \mathrm{C} \mathrm{Na} 2 \mathrm{CO} 33-3$ & $\mathrm{Na} 2 \mathrm{CO} 3$ & 70 & 3 & 624.80 & 2281.00 & 544.55 & 2.7957 \\
\hline Special Salt Solutions & 32 & $70 \mathrm{C} \mathrm{Na} 2 \mathrm{CO} 33-4$ & $\mathrm{Na} 2 \mathrm{CO} 3$ & 70 & 4 & 544.55 & 2825.56 & 478.60 & 2.7360 \\
\hline Special Salt Solutions & 32 & $70 \mathrm{C}$ Na2CO3 3-5 & $\mathrm{Na} 2 \mathrm{CO} 3$ & 70 & 5 & 478.60 & 3304.15 & 405.26 & 2.6800 \\
\hline Special Salt Solutions & 32 & $70 \mathrm{C} \mathrm{Na} 2 \mathrm{CO} 33-6$ & $\mathrm{Na} 2 \mathrm{CO} 3$ & 70 & 6 & 405.26 & 3709.41 & 339.16 & 2.6077 \\
\hline Special Salt Solutions & 32 & $70 \mathrm{C} \mathrm{Na} 2 \mathrm{CO} 33-7$ & $\mathrm{Na} 2 \mathrm{CO} 3$ & 70 & 7 & 339.16 & 4048.57 & 287.46 & 2.5304 \\
\hline Special Salt Solutions & 32 & $70 \mathrm{C} \mathrm{Na} 2 \mathrm{CO} 33-8$ & $\mathrm{Na} 2 \mathrm{CO} 3$ & 70 & 8 & 287.46 & 4336.03 & 240.14 & 2.4586 \\
\hline Special Salt Solutions & 32 & $70 \mathrm{C} \mathrm{Na} 2 \mathrm{CO} 33-9$ & $\mathrm{Na} 2 \mathrm{CO} 3$ & 70 & 9 & 240.14 & 4576.17 & 197.48 & 2.3805 \\
\hline Special Salt Solutions & 32 & $70 \mathrm{C} \mathrm{Na} 2 \mathrm{CO} 33-10$ & $\mathrm{Na} 2 \mathrm{CO} 3$ & 70 & 10 & 197.48 & 4773.65 & & 2.2955 \\
\hline Special Salt Solutions & 33 & 70C Na2SO4 1-1 & $\mathrm{Na} 2 \mathrm{SO} 4$ & 70 & 1 & 398.94 & 398.94 & 334.75 & 2.6009 \\
\hline Special Salt Solutions & 33 & $70 \mathrm{C}$ Na2SO4 1-2 & $\mathrm{Na} 2 \mathrm{SO} 4$ & 70 & 2 & 334.75 & 733.68 & 286.90 & 2.5247 \\
\hline Special Salt Solutions & 33 & $70 \mathrm{C} \mathrm{Na} 2 \mathrm{SO} 4$ 1-3 & $\mathrm{Na} 2 \mathrm{SO} 4$ & 70 & 3 & 286.90 & 1020.58 & 242.32 & 2.4577 \\
\hline Special Salt Solutions & 33 & 70C Na2SO4 1-4 & $\mathrm{Na} 2 \mathrm{SO} 4$ & 70 & 4 & 242.32 & 1262.90 & 209.42 & 2.3844 \\
\hline Special Salt Solutions & 33 & $70 \mathrm{C} \mathrm{Na} 2 \mathrm{SO} 41-5$ & $\mathrm{Na} 2 \mathrm{SO} 4$ & 70 & 5 & 209.42 & 1472.32 & 180.03 & 2.3210 \\
\hline Special Salt Solutions & 33 & $70 \mathrm{C} \mathrm{Na} 2 \mathrm{SO} 41-6$ & $\mathrm{Na} 2 \mathrm{SO} 4$ & 70 & 6 & 180.03 & 1652.35 & 160.80 & 2.2554 \\
\hline Special Salt Solutions & 33 & $70 \mathrm{C} \mathrm{Na} 2 \mathrm{SO} 41-7$ & $\mathrm{Na} 2 \mathrm{SO} 4$ & 70 & 7 & 160.80 & 1813.16 & 136.15 & 2.2063 \\
\hline Special Salt Solutions & 33 & $70 \mathrm{C} \mathrm{Na} 2 \mathrm{SO} 41-8$ & $\mathrm{Na} 2 \mathrm{SO} 4$ & 70 & 8 & 136.15 & 1949.31 & 118.97 & 2.1340 \\
\hline Special Salt Solutions & 33 & $70 \mathrm{C} \mathrm{Na2SO} 41-9$ & $\mathrm{Na} 2 \mathrm{SO} 4$ & 70 & 9 & 118.97 & 2068.28 & 103.59 & 2.0754 \\
\hline
\end{tabular}


Table B3. Test Results for Special Salt Solutions

(continued)

\begin{tabular}{|c|c|c|c|c|c|c|c|c|c|}
\hline Study & $\begin{array}{c}\text { Test } \\
\text { Sequence }\end{array}$ & Test & Salt & Temperature & Sampling & A & & $\mathrm{x}$ & \\
\hline Special Salt Solutions & 33 & $\begin{array}{c}\text { Identifier } \\
\text { 70C Na2SO4 1-10 }\end{array}$ & $\begin{array}{l}\text { Solution } \\
\text { Na2SO4 }\end{array}$ & $\begin{array}{c}\left({ }^{\circ} \mathrm{C}\right) \\
70\end{array}$ & $\begin{array}{l}\text { Sequence } \\
10\end{array}$ & $\begin{array}{c}\text { (peak area) } \\
103.59\end{array}$ & $\begin{array}{c}\mathrm{y} \\
2171.87\end{array}$ & & 2.0153 \\
\hline Special Salt Solutions & 34 & $70 \mathrm{C}$ Na2SO4 2-1 & $\mathrm{Na} 2 \mathrm{SO} 4$ & 70 & 1 & 391.24 & 391.24 & 314.48 & 2.5924 \\
\hline Special Salt Solutions & 34 & $70 \mathrm{C} \mathrm{Na} 2 \mathrm{SO} 42-2$ & $\mathrm{Na} 2 \mathrm{SO} 4$ & 70 & 2 & 314.48 & 705.72 & 267.78 & 2.4976 \\
\hline Special Salt Solutions & 34 & $70 \mathrm{C}$ Na2SO $42-3$ & $\mathrm{Na} 2 \mathrm{SO} 4$ & 70 & 3 & 267.78 & 973.50 & 226.03 & 2.4278 \\
\hline Special Salt Solutions & 34 & $70 \mathrm{C}$ Na2SO4 2-4 & $\mathrm{Na} 2 \mathrm{SO} 4$ & 70 & 4 & 226.03 & 1199.52 & 212.10 & 2.3542 \\
\hline Special Salt Solutions & 34 & $70 \mathrm{C}$ Na2SO4 2-5 & $\mathrm{Na} 2 \mathrm{SO} 4$ & 70 & 5 & 212.10 & 1411.62 & 187.15 & 2.3265 \\
\hline Special Salt Solutions & 34 & $70 \mathrm{C}$ Na2SO4 2-6 & $\mathrm{Na} 2 \mathrm{SO} 4$ & 70 & 6 & 187.15 & 1598.77 & 162.91 & 2.2722 \\
\hline Special Salt Solutions & 34 & $70 \mathrm{C}$ Na2SO4 2-7 & $\mathrm{Na} 2 \mathrm{SO} 4$ & 70 & 7 & 162.91 & 1761.68 & 137.53 & 2.2119 \\
\hline Special Salt Solutions & 34 & $70 \mathrm{C}$ Na2SO4 2-8 & $\mathrm{Na} 2 \mathrm{SO} 4$ & 70 & 8 & 137.53 & 1899.20 & 118.11 & 2.1384 \\
\hline Special Salt Solutions & 34 & 70C Na2SO4 2-9 & $\mathrm{Na} 2 \mathrm{SO} 4$ & 70 & 9 & 118.11 & 2017.31 & 99.68 & 2.0723 \\
\hline Special Salt Solutions & 34 & $70 \mathrm{C} \mathrm{Na} 2 \mathrm{SO} 42-10$ & $\mathrm{Na} 2 \mathrm{SO} 4$ & 70 & 10 & 99.68 & 2117.00 & & 1.9986 \\
\hline Special Salt Solutions & 35 & $70 \mathrm{C} \mathrm{Na} 2 \mathrm{SO} 43-1$ & $\mathrm{Na} 2 \mathrm{SO} 4$ & 70 & 1 & 375.74 & 375.74 & 308.92 & 2.5749 \\
\hline Special Salt Solutions & 35 & $70 \mathrm{C} \mathrm{Na} 2 \mathrm{SO} 43-2$ & $\mathrm{Na} 2 \mathrm{SO} 4$ & 70 & 2 & 308.92 & 684.65 & 272.02 & 2.4898 \\
\hline Special Salt Solutions & 35 & $70 \mathrm{C} \mathrm{Na} 2 \mathrm{SO} 43-3$ & $\mathrm{Na} 2 \mathrm{SO} 4$ & 70 & 3 & 272.02 & 956.67 & 231.94 & 2.4346 \\
\hline Special Salt Solutions & 35 & $70 \mathrm{C}$ Na2SO4 3-4 & $\mathrm{Na} 2 \mathrm{SO} 4$ & 70 & 4 & 231.94 & 1188.61 & 205.06 & 2.3654 \\
\hline Special Salt Solutions & 35 & $70 \mathrm{C}$ Na2SO4 3-5 & $\mathrm{Na} 2 \mathrm{SO} 4$ & 70 & 5 & 205.06 & 1393.67 & 183.42 & 2.3119 \\
\hline Special Salt Solutions & 35 & 70C Na2SO4 3-6 & $\mathrm{Na} 2 \mathrm{SO} 4$ & 70 & 6 & 183.42 & 1577.09 & 160.53 & 2.2635 \\
\hline Special Salt Solutions & 35 & 70C Na2SO4 3-7 & $\mathrm{Na} 2 \mathrm{SO} 4$ & 70 & 7 & 160.53 & 1737.63 & 137.63 & 2.2056 \\
\hline Special Salt Solutions & 35 & 70C Na2SO4 3-8 & $\mathrm{Na} 2 \mathrm{SO} 4$ & 70 & 8 & 137.63 & 1875.26 & 117.26 & 2.1387 \\
\hline Special Salt Solutions & 35 & $70 \mathrm{C}$ Na2SO4 3-9 & $\mathrm{Na} 2 \mathrm{SO} 4$ & 70 & 9 & 117.26 & 1992.52 & 102.32 & 2.0692 \\
\hline Special Salt Solutions & 35 & $70 \mathrm{C} \mathrm{Na} 2 \mathrm{SO} 43-10$ & $\mathrm{Na} 2 \mathrm{SO} 4$ & 70 & 10 & 102.32 & 2094.84 & & 2.0100 \\
\hline Special Salt Solutions & 36 & 70C NaNO2 1-1 & $\mathrm{NaNO} 2$ & 70 & 1 & 295.69 & 295.69 & 267.45 & 2.4708 \\
\hline Special Salt Solutions & 36 & $70 \mathrm{C}$ NaNO2 $1-2$ & $\mathrm{NaNO} 2$ & 70 & 2 & 267.45 & 563.14 & 225.07 & 2.4272 \\
\hline Special Salt Solutions & 36 & 70C NaNO2 1-3 & $\mathrm{NaNO} 2$ & 70 & 3 & 225.07 & 788.20 & 200.41 & 2.3523 \\
\hline Special Salt Solutions & 36 & $70 \mathrm{C}$ NaNO2 $1-4$ & $\mathrm{NaNO} 2$ & 70 & 4 & 200.41 & 988.61 & 191.91 & 2.3019 \\
\hline Special Salt Solutions & 36 & 70C NaNO2 1-5 & $\mathrm{NaNO} 2$ & 70 & 5 & 191.91 & 1180.52 & 165.92 & 2.2831 \\
\hline Special Salt Solutions & 36 & 70C NaNO2 1-6 & $\mathrm{NaNO} 2$ & 70 & 6 & 165.92 & 1346.44 & 153.24 & 2.2199 \\
\hline Special Salt Solutions & 36 & 70C NaNO2 1-7 & $\mathrm{NaNO} 2$ & 70 & 7 & 153.24 & 1499.68 & 134.77 & 2.1854 \\
\hline Special Salt Solutions & 36 & 70C NaNO2 1-8 & $\mathrm{NaNO} 2$ & 70 & 8 & 134.77 & 1634.44 & 115.37 & 2.1296 \\
\hline Special Salt Solutions & 36 & 70C NaNO2 1-9 & $\mathrm{NaNO} 2$ & 70 & 9 & 115.37 & 1749.81 & 100.67 & 2.0621 \\
\hline Special Salt Solutions & 36 & $70 \mathrm{C}$ NaNO2 $1-10$ & $\mathrm{NaNO} 2$ & 70 & 10 & 100.67 & 1850.48 & & 2.0029 \\
\hline Special Salt Solutions & 37 & $70 \mathrm{C}$ NaNO2 2-1 & $\mathrm{NaNO} 2$ & 70 & 1 & 311.01 & 311.01 & 269.23 & 2.4928 \\
\hline Special Salt Solutions & 37 & $70 \mathrm{C}$ NaNO2 2-2 & $\mathrm{NaNO} 2$ & 70 & 2 & 269.23 & 580.24 & 228.05 & 2.4301 \\
\hline Special Salt Solutions & 37 & 70C NaNO2 2-3 & $\mathrm{NaNO} 2$ & 70 & 3 & 228.05 & 808.29 & 211.63 & 2.3580 \\
\hline Special Salt Solutions & 37 & $70 \mathrm{C}$ NaNO2 2-4 & $\mathrm{NaNO} 2$ & 70 & 4 & 211.63 & 1019.93 & 196.74 & 2.3256 \\
\hline Special Salt Solutions & 37 & 70C NaNO2 2-5 & $\mathrm{NaNO} 2$ & 70 & 5 & 196.74 & 1216.67 & 176.95 & 2.2939 \\
\hline Special Salt Solutions & 37 & $70 \mathrm{C}$ NaNO2 2-6 & $\mathrm{NaNO} 2$ & 70 & 6 & 176.95 & 1393.61 & 157.48 & 2.2478 \\
\hline Special Salt Solutions & 37 & 70C NaNO2 2-7 & $\mathrm{NaNO} 2$ & 70 & 7 & 157.48 & 1551.09 & 139.31 & 2.1972 \\
\hline Special Salt Solutions & 37 & 70C NaNO2 2-8 & $\mathrm{NaNO} 2$ & 70 & 8 & 139.31 & 1690.40 & 122.97 & 2.1440 \\
\hline Special Salt Solutions & 37 & 70C NaNO2 2-9 & $\mathrm{NaNO} 2$ & 70 & 9 & 122.97 & 1813.37 & 106.71 & 2.0898 \\
\hline Special Salt Solutions & 37 & 70C NaNO2 2-10 & $\mathrm{NaNO} 2$ & 70 & 10 & 106.71 & 1920.08 & & 2.0282 \\
\hline Special Salt Solutions & 38 & $70 \mathrm{C}$ NaNO2 3-1 & $\mathrm{NaNO} 2$ & 70 & 1 & 321.41 & 321.41 & 264.52 & 2.5071 \\
\hline Special Salt Solutions & 38 & $70 \mathrm{C}$ NaNO2 3-2 & $\mathrm{NaNO} 2$ & 70 & 2 & 264.52 & 585.93 & 234.14 & 2.4225 \\
\hline Special Salt Solutions & 38 & $70 \mathrm{C}$ NaNO2 3-3 & $\mathrm{NaNO} 2$ & 70 & 3 & 234.14 & 820.08 & 203.18 & 2.3695 \\
\hline Special Salt Solutions & 38 & 70C NaNO2 3-4 & $\mathrm{NaNO} 2$ & 70 & 4 & 203.18 & 1023.25 & 189.82 & 2.3079 \\
\hline Special Salt Solutions & 38 & $70 \mathrm{C}$ NaNO2 3-5 & $\mathrm{NaNO} 2$ & 70 & 5 & 189.82 & 1213.08 & 172.50 & 2.2783 \\
\hline Special Salt Solutions & 38 & $70 \mathrm{C}$ NaNO2 3-6 & $\mathrm{NaNO} 2$ & 70 & 6 & 172.50 & 1385.58 & 155.88 & 2.2368 \\
\hline Special Salt Solutions & 38 & 70C NaNO2 3-7 & $\mathrm{NaNO} 2$ & 70 & 7 & 155.88 & 1541.45 & 135.23 & 2.1928 \\
\hline Special Salt Solutions & 38 & 70C NaNO2 3-8 & $\mathrm{NaNO} 2$ & 70 & 8 & 135.23 & 1676.68 & 120.67 & 2.1311 \\
\hline Special Salt Solutions & 38 & $70 \mathrm{C}$ NaNO2 3-9 & $\mathrm{NaNO} 2$ & 70 & 9 & 120.67 & 1797.35 & 106.59 & 2.0816 \\
\hline Special Salt Solutions & 38 & 70C NaNO2 3-10 & $\mathrm{NaNO} 2$ & 70 & 10 & 106.59 & 1903.94 & & 2.0277 \\
\hline Special Salt Solutions & 39 & 70C NaNO3 1-1 & $\mathrm{NaNO} 3$ & 70 & 1 & 724.77 & 724.77 & 652.57 & 2.8602 \\
\hline Special Salt Solutions & 39 & 70C NaNO3 1-2 & $\mathrm{NaNO} 3$ & 70 & 2 & 652.57 & 1377.34 & 561.29 & 2.8146 \\
\hline Special Salt Solutions & 39 & $70 \mathrm{C}$ NaNO3 $1-3$ & NaNO3 & 70 & 3 & 561.29 & 1938.63 & 496.36 & 2.7492 \\
\hline Special Salt Solutions & 39 & $70 \mathrm{C}$ NaNO3 1-4 & $\mathrm{NaNO} 3$ & 70 & 4 & 496.36 & 2434.99 & 468.41 & 2.6958 \\
\hline Special Salt Solutions & 39 & 70C NaNO3 1-5 & $\mathrm{NaNO} 3$ & 70 & 5 & 468.41 & 2903.40 & 409.11 & 2.6706 \\
\hline Special Salt Solutions & 39 & $70 \mathrm{C}$ NaNO3 $1-6$ & NaNO3 & 70 & 6 & 409.11 & 3312.51 & 361.36 & 2.6118 \\
\hline Special Salt Solutions & 39 & $70 \mathrm{C}$ NaNO3 1-7 & $\mathrm{NaNO} 3$ & 70 & 7 & 361.36 & 3673.87 & 318.63 & 2.5579 \\
\hline Special Salt Solutions & 39 & $70 \mathrm{C}$ NaNO3 $1-8$ & NaNO3 & 70 & 8 & 318.63 & 3992.50 & 283.79 & 2.5033 \\
\hline Special Salt Solutions & 39 & 70C NaNO3 1-9 & $\mathrm{NaNO} 3$ & 70 & 9 & 283.79 & 4276.29 & 249.02 & 2.4530 \\
\hline Special Salt Solutions & 39 & 70C NaNO3 1-10 & NaNO3 & 70 & 10 & 249.02 & 4525.31 & & 2.3962 \\
\hline Special Salt Solutions & 40 & $70 \mathrm{C}$ NaNO3 2-1 & $\mathrm{NaNO} 3$ & 70 & 1 & 748.85 & 748.85 & 659.92 & 2.8744 \\
\hline Special Salt Solutions & 40 & 70C NaNO3 2-2 & NaNO3 & 70 & 2 & 659.92 & 1408.77 & 587.70 & 2.8195 \\
\hline Special Salt Solutions & 40 & $70 \mathrm{C}$ NaNO3 $2-3$ & $\mathrm{NaNO} 3$ & 70 & 3 & 587.70 & 1996.47 & 546.87 & 2.7692 \\
\hline Special Salt Solutions & 40 & $70 \mathrm{C}$ NaNO3 2-4 & $\mathrm{NaNO} 3$ & 70 & 4 & 546.87 & 2543.34 & 492.56 & 2.7379 \\
\hline Special Salt Solutions & 40 & $70 \mathrm{C}$ NaNO3 2-5 & $\mathrm{NaNO} 3$ & 70 & 5 & 492.56 & 3035.91 & 433.89 & 2.6925 \\
\hline
\end{tabular}


Table B3. Test Results for Special Salt Solutions

(continued)

\begin{tabular}{|c|c|c|c|c|c|c|c|c|c|}
\hline & Test & Test & Salt & Temperature & Sampling & A & & & \\
\hline Special Salt Solutions & 40 & $\begin{array}{c}\text { Identifier } \\
\text { 70C NaNO3 2-6 }\end{array}$ & $\begin{array}{l}\text { Solution } \\
\text { NaNO33 }\end{array}$ & $\begin{array}{l}\left({ }^{\circ} \mathrm{C}\right) \\
70\end{array}$ & $\begin{array}{l}\text { Sequence } \\
6\end{array}$ & $\begin{array}{c}\text { (peak area) } \\
433.89\end{array}$ & $\begin{array}{c}\mathrm{y} \\
3469.79\end{array}$ & $\begin{array}{c}\mathrm{x} \\
382.64\end{array}$ & 2.6374 \\
\hline Special Salt Solutions & 40 & 70C NaNO3 2-7 & $\mathrm{NaNO} 3$ & 70 & 7 & 382.64 & 3852.44 & 340.27 & 2.5828 \\
\hline Special Salt Solutions & 40 & $70 \mathrm{C}$ NaNO3 $2-8$ & NaNO3 & 70 & 8 & 340.27 & 4192.71 & 298.83 & 2.5318 \\
\hline Special Salt Solutions & 40 & $70 \mathrm{C}$ NaNO3 2-9 & NaNO3 & 70 & 9 & 298.83 & 4491.54 & 261.68 & 2.4754 \\
\hline Special Salt Solutions & 40 & 70C NaNO3 2-10 & NaNO3 & 70 & 10 & 261.68 & 4753.22 & & 2.4178 \\
\hline Special Salt Solutions & 41 & 70C NaNO3 3-1 & NaNO3 & 70 & 1 & 807.71 & 807.71 & 727.80 & 2.9073 \\
\hline Special Salt Solutions & 41 & 70C NaNO3 3-2 & $\mathrm{NaNO} 3$ & 70 & 2 & 727.80 & 1535.52 & 626.50 & 2.8620 \\
\hline Special Salt Solutions & 41 & 70C NaNO3 3-3 & $\mathrm{NaNO} 3$ & 70 & 3 & 626.50 & 2162.02 & 542.85 & 2.7969 \\
\hline Special Salt Solutions & 41 & $70 \mathrm{C}$ NaNO3 3-4 & $\mathrm{NaNO} 3$ & 70 & 4 & 542.85 & 2704.87 & 520.32 & 2.7347 \\
\hline Special Salt Solutions & 41 & 70C NaNO3 3-5 & $\mathrm{NaNO} 3$ & 70 & 5 & 520.32 & 3225.19 & 463.17 & 2.7163 \\
\hline Special Salt Solutions & 41 & 70C NaNO3 3-6 & NaNO3 & 70 & 6 & 463.17 & 3688.36 & 406.31 & 2.6657 \\
\hline Special Salt Solutions & 41 & 70C NaNO3 3-7 & $\mathrm{NaNO} 3$ & 70 & 7 & 406.31 & 4094.67 & 355.25 & 2.6089 \\
\hline Special Salt Solutions & 41 & 70C NaNO3 3-8 & NaNO3 & 70 & 8 & 355.25 & 4449.92 & 312.64 & 2.5505 \\
\hline Special Salt Solutions & 41 & 70C NaNO3 3-9 & $\mathrm{NaNO} 3$ & 70 & 9 & 312.64 & 4762.55 & 273.59 & 2.4950 \\
\hline Special Salt Solutions & 41 & 70C NaNO3 3-10 & NaNO3 & 70 & 10 & 273.59 & 5036.15 & & 2.4371 \\
\hline Special Salt Solutions & 42 & $80 \mathrm{C} \mathrm{Na} 2 \mathrm{CO} 31-1$ & $\mathrm{Na} 2 \mathrm{CO} 3$ & 80 & 1 & 898.44 & 898.44 & 721.93 & 2.9535 \\
\hline Special Salt Solutions & 42 & $80 \mathrm{C} \mathrm{Na} 2 \mathrm{CO} 31-2$ & $\mathrm{Na} 2 \mathrm{CO} 3$ & 80 & 2 & 721.93 & 1620.37 & 598.44 & 2.8585 \\
\hline Special Salt Solutions & 42 & $80 \mathrm{C} \mathrm{Na} 2 \mathrm{CO} 31-3$ & $\mathrm{Na} 2 \mathrm{CO} 3$ & 80 & 3 & 598.44 & 2218.81 & 503.05 & 2.7770 \\
\hline Special Salt Solutions & 42 & $80 \mathrm{C} \mathrm{Na} 2 \mathrm{CO} 31-4$ & $\mathrm{Na} 2 \mathrm{CO} 3$ & 80 & 4 & 503.05 & 2721.86 & 428.03 & 2.7016 \\
\hline Special Salt Solutions & 42 & $80 \mathrm{C} \mathrm{Na} 2 \mathrm{CO} 31-5$ & $\mathrm{Na} 2 \mathrm{CO} 3$ & 80 & 5 & 428.03 & 3149.88 & 355.19 & 2.6315 \\
\hline Special Salt Solutions & 42 & $80 \mathrm{C} \mathrm{Na} 2 \mathrm{CO} 31-6$ & $\mathrm{Na} 2 \mathrm{CO} 3$ & 80 & 6 & 355.19 & 3505.07 & 289.48 & 2.5505 \\
\hline Special Salt Solutions & 42 & $80 \mathrm{C} \mathrm{Na} 2 \mathrm{CO} 31-7$ & $\mathrm{Na} 2 \mathrm{CO} 3$ & 80 & 7 & 289.48 & 3794.55 & 239.86 & 2.4616 \\
\hline Special Salt Solutions & 42 & $80 \mathrm{C} \mathrm{Na} 2 \mathrm{CO} 31-8$ & $\mathrm{Na} 2 \mathrm{CO} 3$ & 80 & 8 & 239.86 & 4034.41 & 190.28 & 2.3800 \\
\hline Special Salt Solutions & 42 & $80 \mathrm{C} \mathrm{Na} 2 \mathrm{CO} 31-9$ & $\mathrm{Na} 2 \mathrm{CO} 3$ & 80 & 9 & 190.28 & 4224.69 & 155.51 & 2.2794 \\
\hline Special Salt Solutions & 42 & $80 \mathrm{C} \mathrm{Na} 2 \mathrm{CO} 31-10$ & $\mathrm{Na} 2 \mathrm{CO} 3$ & 80 & 10 & 155.51 & 4380.20 & & 2.1918 \\
\hline Special Salt Solutions & 43 & $80 \mathrm{C} \mathrm{Na} 2 \mathrm{CO} 32-1$ & $\mathrm{Na} 2 \mathrm{CO} 3$ & 80 & 1 & 956.27 & 956.27 & 721.86 & 2.9806 \\
\hline Special Salt Solutions & 43 & 80C Na2CO3 2-2 & $\mathrm{Na} 2 \mathrm{CO} 3$ & 80 & 2 & 721.86 & 1678.13 & 597.49 & 2.8585 \\
\hline Special Salt Solutions & 43 & $80 \mathrm{C} \mathrm{Na} 2 \mathrm{CO} 32-3$ & $\mathrm{Na} 2 \mathrm{CO} 3$ & 80 & 3 & 597.49 & 2275.62 & 514.86 & 2.7763 \\
\hline Special Salt Solutions & 43 & $80 \mathrm{C} \mathrm{Na} 2 \mathrm{CO} 32-4$ & $\mathrm{Na} 2 \mathrm{CO} 3$ & 80 & 4 & 514.86 & 2790.48 & 439.64 & 2.7117 \\
\hline Special Salt Solutions & 43 & $80 \mathrm{C} \mathrm{Na} 2 \mathrm{CO} 32-5$ & $\mathrm{Na} 2 \mathrm{CO} 3$ & 80 & 5 & 439.64 & 3230.12 & 364.64 & 2.6431 \\
\hline Special Salt Solutions & 43 & $80 \mathrm{C}$ Na2CO3 2-6 & $\mathrm{Na} 2 \mathrm{CO} 3$ & 80 & 6 & 364.64 & 3594.76 & 291.41 & 2.5619 \\
\hline Special Salt Solutions & 43 & $80 \mathrm{C} \mathrm{Na} 2 \mathrm{CO} 32-7$ & $\mathrm{Na} 2 \mathrm{CO} 3$ & 80 & 7 & 291.41 & 3886.17 & 235.53 & 2.4645 \\
\hline Special Salt Solutions & 43 & $80 \mathrm{C} \mathrm{Na} 2 \mathrm{CO} 32-8$ & $\mathrm{Na} 2 \mathrm{CO} 3$ & 80 & 8 & 235.53 & 4121.69 & 190.19 & 2.3720 \\
\hline Special Salt Solutions & 43 & $80 \mathrm{C} \mathrm{Na} 2 \mathrm{CO} 32-9$ & $\mathrm{Na} 2 \mathrm{CO} 3$ & 80 & 9 & 190.19 & 4311.88 & 154.76 & 2.2792 \\
\hline Special Salt Solutions & 43 & $80 \mathrm{C} \mathrm{Na} 2 \mathrm{CO} 32-10$ & $\mathrm{Na} 2 \mathrm{CO} 3$ & 80 & 10 & 154.76 & 4466.64 & & 2.1897 \\
\hline Special Salt Solutions & 44 & $80 \mathrm{C} \mathrm{Na} 2 \mathrm{CO} 33-1$ & $\mathrm{Na} 2 \mathrm{CO} 3$ & 80 & 1 & 897.69 & 897.69 & 719.02 & 2.9531 \\
\hline Special Salt Solutions & 44 & $80 \mathrm{C} \mathrm{Na} 2 \mathrm{CO} 33-2$ & $\mathrm{Na} 2 \mathrm{CO} 3$ & 80 & 2 & 719.02 & 1616.71 & 604.45 & 2.8567 \\
\hline Special Salt Solutions & 44 & $80 \mathrm{C} \mathrm{Na} 2 \mathrm{CO} 33-3$ & $\mathrm{Na} 2 \mathrm{CO} 3$ & 80 & 3 & 604.45 & 2221.15 & 504.69 & 2.7814 \\
\hline Special Salt Solutions & 44 & $80 \mathrm{C} \mathrm{Na} 2 \mathrm{CO} 33-4$ & $\mathrm{Na} 2 \mathrm{CO} 3$ & 80 & 4 & 504.69 & 2725.85 & 408.17 & 2.7030 \\
\hline Special Salt Solutions & 44 & $80 \mathrm{C} \mathrm{Na} 2 \mathrm{CO} 33-5$ & $\mathrm{Na} 2 \mathrm{CO} 3$ & 80 & 5 & 408.17 & 3134.02 & 333.40 & 2.6108 \\
\hline Special Salt Solutions & 44 & $80 \mathrm{C} \mathrm{Na} 2 \mathrm{CO} 33-6$ & $\mathrm{Na} 2 \mathrm{CO} 3$ & 80 & 6 & 333.40 & 3467.42 & 273.14 & 2.5230 \\
\hline Special Salt Solutions & 44 & $80 \mathrm{C} \mathrm{Na} 2 \mathrm{CO} 33-7$ & $\mathrm{Na} 2 \mathrm{CO} 3$ & 80 & 7 & 273.14 & 3740.55 & 223.57 & 2.4364 \\
\hline Special Salt Solutions & 44 & $80 \mathrm{C} \mathrm{Na} 2 \mathrm{CO} 33-8$ & $\mathrm{Na} 2 \mathrm{CO} 3$ & 80 & 8 & 223.57 & 3964.13 & 180.58 & 2.3494 \\
\hline Special Salt Solutions & 44 & $80 \mathrm{C}$ Na2CO3 3-9 & $\mathrm{Na} 2 \mathrm{CO} 3$ & 80 & 9 & 180.58 & 4144.71 & 145.22 & 2.2567 \\
\hline Special Salt Solutions & 44 & $80 \mathrm{C} \mathrm{Na} 2 \mathrm{CO} 33-10$ & $\mathrm{Na} 2 \mathrm{CO} 3$ & 80 & 10 & 145.22 & 4289.93 & & 2.1620 \\
\hline Special Salt Solutions & 45 & 80C Na2SO4 1-1 & $\mathrm{Na} 2 \mathrm{SO} 4$ & 80 & 1 & 454.18 & 454.18 & 356.97 & 2.6572 \\
\hline Special Salt Solutions & 45 & $80 \mathrm{C}$ Na2SO4 1-2 & $\mathrm{Na} 2 \mathrm{SO} 4$ & 80 & 2 & 356.97 & 811.14 & 298.14 & 2.5526 \\
\hline Special Salt Solutions & 45 & $80 \mathrm{C} \mathrm{Na} 2 \mathrm{SO} 4$ 1-3 & $\mathrm{Na} 2 \mathrm{SO} 4$ & 80 & 3 & 298.14 & 1109.28 & 252.59 & 2.4744 \\
\hline Special Salt Solutions & 45 & $80 \mathrm{C} \mathrm{Na} 2 \mathrm{SO} 41-4$ & $\mathrm{Na} 2 \mathrm{SO} 4$ & 80 & 4 & 252.59 & 1361.87 & 215.58 & 2.4024 \\
\hline Special Salt Solutions & 45 & $80 \mathrm{C}$ Na2SO4 1-5 & $\mathrm{Na} 2 \mathrm{SO} 4$ & 80 & 5 & 215.58 & 1577.44 & 182.06 & 2.3336 \\
\hline Special Salt Solutions & 45 & 80C Na2SO4 1-6 & $\mathrm{Na} 2 \mathrm{SO} 4$ & 80 & 6 & 182.06 & 1759.50 & 150.95 & 2.2602 \\
\hline Special Salt Solutions & 45 & $80 \mathrm{C}$ Na2SO4 1-7 & $\mathrm{Na} 2 \mathrm{SO} 4$ & 80 & 7 & 150.95 & 1910.45 & 124.84 & 2.1788 \\
\hline Special Salt Solutions & 45 & 80C Na2SO4 1-8 & $\mathrm{Na} 2 \mathrm{SO} 4$ & 80 & 8 & 124.84 & 2035.29 & 98.90 & 2.0964 \\
\hline Special Salt Solutions & 45 & $80 \mathrm{C}$ Na2SO4 1-9 & $\mathrm{Na} 2 \mathrm{SO} 4$ & 80 & 9 & 98.90 & 2134.19 & 78.28 & 1.9952 \\
\hline Special Salt Solutions & 45 & $80 \mathrm{C} \mathrm{Na} 2 \mathrm{SO} 41-10$ & $\mathrm{Na} 2 \mathrm{SO} 4$ & 80 & 10 & 78.28 & 2212.47 & & 1.8936 \\
\hline Special Salt Solutions & 46 & $80 \mathrm{C} \mathrm{Na} 2 \mathrm{SO} 42-1$ & $\mathrm{Na} 2 \mathrm{SO} 4$ & 80 & 1 & 506.04 & 506.04 & 399.31 & 2.7042 \\
\hline Special Salt Solutions & 46 & $80 \mathrm{C}$ Na2SO4 2-2 & $\mathrm{Na} 2 \mathrm{SO} 4$ & 80 & 2 & 399.31 & 905.35 & 320.82 & 2.6013 \\
\hline Special Salt Solutions & 46 & $80 \mathrm{C} \mathrm{Na} 2 \mathrm{SO} 42-3$ & $\mathrm{Na} 2 \mathrm{SO} 4$ & 80 & 3 & 320.82 & 1226.17 & 274.50 & 2.5063 \\
\hline Special Salt Solutions & 46 & $80 \mathrm{C}$ Na2SO4 2-4 & $\mathrm{Na} 2 \mathrm{SO} 4$ & 80 & 4 & 274.50 & 1500.68 & 241.15 & 2.4385 \\
\hline Special Salt Solutions & 46 & $80 \mathrm{C} \mathrm{Na} 2 \mathrm{SO} 42-5$ & $\mathrm{Na} 2 \mathrm{SO} 4$ & 80 & 5 & 241.15 & 1741.83 & 201.89 & 2.3823 \\
\hline Special Salt Solutions & 46 & $80 \mathrm{C} \mathrm{Na} 2 \mathrm{SO} 42-6$ & $\mathrm{Na} 2 \mathrm{SO} 4$ & 80 & 6 & 201.89 & 1943.71 & 166.03 & 2.3051 \\
\hline Special Salt Solutions & 46 & $80 \mathrm{C} \mathrm{Na} 2 \mathrm{SO} 42-7$ & $\mathrm{Na} 2 \mathrm{SO} 4$ & 80 & 7 & 166.03 & 2109.74 & 136.46 & 2.2202 \\
\hline Special Salt Solutions & 46 & $80 \mathrm{C} \mathrm{Na} 2 \mathrm{SO} 42-8$ & $\mathrm{Na} 2 \mathrm{SO} 4$ & 80 & 8 & 136.46 & 2246.20 & 108.66 & 2.1350 \\
\hline Special Salt Solutions & 46 & $80 \mathrm{C} \mathrm{Na} 2 \mathrm{SO} 42-9$ & $\mathrm{Na} 2 \mathrm{SO} 4$ & 80 & 9 & 108.66 & 2354.86 & 88.14 & 2.0361 \\
\hline Special Salt Solutions & 46 & $80 \mathrm{C} \mathrm{Na} 2 \mathrm{SO} 4$ 2-10 & $\mathrm{Na} 2 \mathrm{SO} 4$ & 80 & 10 & 88.14 & 2443.00 & & 1.9452 \\
\hline Special Salt Solutions & 47 & 80C Na2SO4 3-1 & $\mathrm{Na} 2 \mathrm{SO} 4$ & 80 & 1 & 464.60 & 464.60 & 346.92 & 2.6671 \\
\hline
\end{tabular}


Table B3. Test Results for Special Salt Solutions

(continued)

\begin{tabular}{|c|c|c|c|c|c|c|c|c|c|}
\hline Study & $\begin{array}{c}\text { Test } \\
\text { Sequence }\end{array}$ & $\begin{array}{c}\text { Test } \\
\text { Identifier }\end{array}$ & $\begin{array}{c}\text { Salt } \\
\text { Solution }\end{array}$ & $\begin{array}{c}\text { Temperature } \\
\left({ }^{\circ} \mathrm{C}\right)\end{array}$ & $\begin{array}{l}\text { Sampling } \\
\text { Sequence }\end{array}$ & $\begin{array}{c}\text { A } \\
\text { (peak area) }\end{array}$ & $\mathrm{y}$ & $\mathrm{x}$ & $\log (\mathrm{A})$ \\
\hline Special Salt Solutions & 47 & 80C Na2SO4 3-2 & $\mathrm{Na} 2 \mathrm{SO} 4$ & 80 & 2 & 346.92 & 811.52 & 289.30 & 2.5402 \\
\hline Special Salt Solutions & 47 & 80C Na2SO4 3-3 & $\mathrm{Na} 2 \mathrm{SO} 4$ & 80 & 3 & 289.30 & 1100.82 & 243.28 & 2.4614 \\
\hline Special Salt Solutions & 47 & 80C Na2SO4 3-4 & $\mathrm{Na} 2 \mathrm{SO} 4$ & 80 & 4 & 243.28 & 1344.10 & 210.49 & 2.3861 \\
\hline Special Salt Solutions & 47 & 80C Na2SO4 3-5 & $\mathrm{Na} 2 \mathrm{SO} 4$ & 80 & 5 & 210.49 & 1554.60 & 176.48 & 2.3232 \\
\hline Special Salt Solutions & 47 & 80C Na2SO4 3-6 & $\mathrm{Na} 2 \mathrm{SO} 4$ & 80 & 6 & 176.48 & 1731.08 & 145.88 & 2.2467 \\
\hline Special Salt Solutions & 47 & 80C Na2SO4 3-7 & $\mathrm{Na} 2 \mathrm{SO} 4$ & 80 & 7 & 145.88 & 1876.96 & 115.61 & 2.1640 \\
\hline Special Salt Solutions & 47 & 80C Na2SO4 3-8 & $\mathrm{Na} 2 \mathrm{SO} 4$ & 80 & 8 & 115.61 & 1992.57 & 90.89 & 2.0630 \\
\hline Special Salt Solutions & 47 & $80 \mathrm{C} \mathrm{Na} 2 \mathrm{SO} 43-9$ & $\mathrm{Na} 2 \mathrm{SO} 4$ & 80 & 9 & 90.89 & 2083.46 & 72.81 & 1.9585 \\
\hline Special Salt Solutions & 47 & $80 \mathrm{C} \mathrm{Na} 2 \mathrm{SO} 4$ 3-10 & $\mathrm{Na} 2 \mathrm{SO} 4$ & 80 & 10 & 72.81 & 2156.27 & & 1.8622 \\
\hline Special Salt Solutions & 48 & 80C NaNO2 1-1 & $\mathrm{NaNO} 2$ & 80 & 1 & 346.00 & 346.00 & 292.84 & 2.5391 \\
\hline Special Salt Solutions & 48 & 80C NaNO2 1-2 & $\mathrm{NaNO} 2$ & 80 & 2 & 292.84 & 638.84 & 248.64 & 2.4666 \\
\hline Special Salt Solutions & 48 & 80C NaNO2 1-3 & $\mathrm{NaNO} 2$ & 80 & 3 & 248.64 & 887.48 & 216.75 & 2.3956 \\
\hline Special Salt Solutions & 48 & 80C NaNO2 1-4 & $\mathrm{NaNO} 2$ & 80 & 4 & 216.75 & 1104.23 & 186.98 & 2.3360 \\
\hline Special Salt Solutions & 48 & 80C NaNO2 1-5 & $\mathrm{NaNO} 2$ & 80 & 5 & 186.98 & 1291.21 & 158.91 & 2.2718 \\
\hline Special Salt Solutions & 48 & 80C NaNO2 1-6 & $\mathrm{NaNO} 2$ & 80 & 6 & 158.91 & 1450.12 & 133.02 & 2.2012 \\
\hline Special Salt Solutions & 48 & 80C NaNO2 1-7 & $\mathrm{NaNO} 2$ & 80 & 7 & 133.02 & 1583.15 & 111.30 & 2.1239 \\
\hline Special Salt Solutions & 48 & 80C NaNO2 1-8 & $\mathrm{NaNO} 2$ & 80 & 8 & 111.30 & 1694.44 & 90.48 & 2.0465 \\
\hline Special Salt Solutions & 48 & $80 \mathrm{C}$ NaNO2 1-9 & $\mathrm{NaNO} 2$ & 80 & 9 & 90.48 & 1784.92 & 74.07 & 1.9565 \\
\hline Special Salt Solutions & 48 & 80C NaNO2 1-10 & $\mathrm{NaNO} 2$ & 80 & 10 & 74.07 & 1858.99 & & 1.8696 \\
\hline Special Salt Solutions & 49 & 80C NaNO2 2-1 & $\mathrm{NaNO} 2$ & 80 & 1 & 308.65 & 308.65 & 233.89 & 2.4895 \\
\hline Special Salt Solutions & 49 & $80 \mathrm{C}$ NaNO2 2-2 & $\mathrm{NaNO} 2$ & 80 & 2 & 233.89 & 542.54 & 225.04 & 2.3690 \\
\hline Special Salt Solutions & 49 & $80 \mathrm{C}$ NaNO2 2-3 & $\mathrm{NaNO} 2$ & 80 & 3 & 225.04 & 767.58 & 202.70 & 2.3523 \\
\hline Special Salt Solutions & 49 & 80C NaNO2 2-4 & $\mathrm{NaNO} 2$ & 80 & 4 & 202.70 & 970.28 & 169.17 & 2.3069 \\
\hline Special Salt Solutions & 49 & $80 \mathrm{C}$ NaNO2 2-5 & $\mathrm{NaNO} 2$ & 80 & 5 & 169.17 & 1139.45 & 145.24 & 2.2283 \\
\hline Special Salt Solutions & 49 & $80 \mathrm{C}$ NaNO2 2-6 & $\mathrm{NaNO} 2$ & 80 & 6 & 145.24 & 1284.69 & 119.09 & 2.1621 \\
\hline Special Salt Solutions & 49 & 80C NaNO2 2-7 & $\mathrm{NaNO} 2$ & 80 & 7 & 119.09 & 1403.79 & 99.39 & 2.0759 \\
\hline Special Salt Solutions & 49 & $80 \mathrm{C}$ NaNO2 2-8 & $\mathrm{NaNO} 2$ & 80 & 8 & 99.39 & 1503.17 & 82.29 & 1.9973 \\
\hline Special Salt Solutions & 49 & 80C NaNO2 2-9 & $\mathrm{NaNO} 2$ & 80 & 9 & 82.29 & 1585.46 & 66.78 & 1.9153 \\
\hline Special Salt Solutions & 49 & 80C NaNO2 2-10 & $\mathrm{NaNO} 2$ & 80 & 10 & 66.78 & 1652.24 & & 1.8246 \\
\hline Special Salt Solutions & 50 & 80C NaNO2 3-1 & $\mathrm{NaNO} 2$ & 80 & 1 & 320.80 & 320.80 & 257.69 & 2.5062 \\
\hline Special Salt Solutions & 50 & 80C NaNO2 3-2 & $\mathrm{NaNO} 2$ & 80 & 2 & 257.69 & 578.48 & 222.14 & 2.4111 \\
\hline Special Salt Solutions & 50 & 80C NaNO2 3-3 & $\mathrm{NaNO} 2$ & 80 & 3 & 222.14 & 800.63 & 205.45 & 2.3466 \\
\hline Special Salt Solutions & 50 & 80C NaNO2 3-4 & $\mathrm{NaNO} 2$ & 80 & 4 & 205.45 & 1006.07 & 172.31 & 2.3127 \\
\hline Special Salt Solutions & 50 & 80C NaNO2 3-5 & $\mathrm{NaNO} 2$ & 80 & 5 & 172.31 & 1178.38 & 146.03 & 2.2363 \\
\hline Special Salt Solutions & 50 & 80C NaNO2 3-6 & $\mathrm{NaNO} 2$ & 80 & 6 & 146.03 & 1324.41 & 125.76 & 2.1644 \\
\hline Special Salt Solutions & 50 & 80C NaNO2 3-7 & $\mathrm{NaNO} 2$ & 80 & 7 & 125.76 & 1450.17 & 102.96 & 2.0995 \\
\hline Special Salt Solutions & 50 & 80C NaNO2 3-8 & $\mathrm{NaNO} 2$ & 80 & 8 & 102.96 & 1553.13 & 85.88 & 2.0126 \\
\hline Special Salt Solutions & 50 & 80C NaNO2 3-9 & $\mathrm{NaNO} 2$ & 80 & 9 & 85.88 & 1639.01 & 70.21 & 1.9339 \\
\hline Special Salt Solutions & 50 & 80C NaNO2 3-10 & $\mathrm{NaNO} 2$ & 80 & 10 & 70.21 & 1709.22 & & 1.8464 \\
\hline Special Salt Solutions & 51 & 80C NaNO3 1-1 & NaNO3 & 80 & 1 & 710.68 & 710.68 & 655.92 & 2.8517 \\
\hline Special Salt Solutions & 51 & 80C NaNO3 1-2 & $\mathrm{NaNO} 3$ & 80 & 2 & 655.92 & 1366.60 & 601.34 & 2.8169 \\
\hline Special Salt Solutions & 51 & $80 \mathrm{C}$ NaNO3 1-3 & NaNO3 & 80 & 3 & 601.34 & 1967.95 & 529.65 & 2.7791 \\
\hline Special Salt Solutions & 51 & 80C NaNO3 1-4 & NaNO3 & 80 & 4 & 529.65 & 2497.60 & 447.16 & 2.7240 \\
\hline Special Salt Solutions & 51 & $80 \mathrm{C}$ NaNO3 1-5 & NaNO3 & 80 & 5 & 447.16 & 2944.76 & 370.74 & 2.6505 \\
\hline Special Salt Solutions & 51 & $80 \mathrm{C}$ NaNO3 1-6 & NaNO3 & 80 & 6 & 370.74 & 3315.50 & 310.05 & 2.5691 \\
\hline Special Salt Solutions & 51 & 80C NaNO3 1-7 & NaNO3 & 80 & 7 & 310.05 & 3625.55 & 256.45 & 2.4914 \\
\hline Special Salt Solutions & 51 & $80 \mathrm{C}$ NaNO3 1-8 & $\mathrm{NaNO} 3$ & 80 & 8 & 256.45 & 3882.00 & 217.97 & 2.4090 \\
\hline Special Salt Solutions & 51 & 80C NaNO3 1-9 & NaNO3 & 80 & 9 & 217.97 & 4099.97 & 180.25 & 2.3384 \\
\hline Special Salt Solutions & 51 & 80C NaNO3 1-10 & NaNO3 & 80 & 10 & 180.25 & 4280.22 & & 2.2559 \\
\hline Special Salt Solutions & 52 & 80C NaNO3 2-1 & NaNO3 & 80 & 1 & 696.45 & 696.45 & 601.81 & 2.8429 \\
\hline Special Salt Solutions & 52 & 80C NaNO3 2-2 & NaNO3 & 80 & 2 & 601.81 & 1298.26 & 517.46 & 2.7795 \\
\hline Special Salt Solutions & 52 & $80 \mathrm{C}$ NaNO3 2-3 & $\mathrm{NaNO} 3$ & 80 & 3 & 517.46 & 1815.72 & 449.75 & 2.7139 \\
\hline Special Salt Solutions & 52 & 80C NaNO3 2-4 & NaNO3 & 80 & 4 & 449.75 & 2265.46 & 382.68 & 2.6530 \\
\hline Special Salt Solutions & 52 & $80 \mathrm{C}$ NaNO3 2-5 & $\mathrm{NaNO} 3$ & 80 & 5 & 382.68 & 2648.14 & 321.75 & 2.5828 \\
\hline Special Salt Solutions & 52 & 80C NaNO3 2-6 & NaNO3 & 80 & 6 & 321.75 & 2969.89 & 271.39 & 2.5075 \\
\hline Special Salt Solutions & 52 & 80C NaNO3 2-7 & NaNO3 & 80 & 7 & 271.39 & 3241.28 & 224.77 & 2.4336 \\
\hline Special Salt Solutions & 52 & $80 \mathrm{C}$ NaNO3 2-8 & NaNO3 & 80 & 8 & 224.77 & 3466.05 & 182.84 & 2.3517 \\
\hline Special Salt Solutions & 52 & 80C NaNO3 2-9 & NaNO3 & 80 & 9 & 182.84 & 3648.89 & 151.27 & 2.2621 \\
\hline Special Salt Solutions & 52 & 80C NaNO3 2-10 & $\mathrm{NaNO} 3$ & 80 & 10 & 151.27 & 3800.15 & . & 2.1797 \\
\hline Special Salt Solutions & 53 & $90 \mathrm{C} 1.0 \mathrm{M} \# 1$ & $\mathrm{NaOH} 1 \mathrm{M}$ & 90 & 1 & 236084.37 & 236084.40 & 215171.40 & 5.3731 \\
\hline Special Salt Solutions & 53 & $90 \mathrm{C} 1.0 \mathrm{M} \mathrm{\# 2}$ & $\mathrm{NaOH} 1 \mathrm{M}$ & 90 & 2 & 215171.37 & 451255.70 & 180961.30 & 5.3328 \\
\hline Special Salt Solutions & 53 & $90 \mathrm{C} 1.0 \mathrm{M} \mathrm{\# 3}$ & $\mathrm{NaOH} 1 \mathrm{M}$ & 90 & 3 & 180961.33 & 632217.10 & 151123.10 & 5.2576 \\
\hline Special Salt Solutions & 53 & $90 \mathrm{C} 1.0 \mathrm{M} \# 4$ & $\mathrm{NaOH} 1 \mathrm{M}$ & 90 & 4 & 151123.06 & 783340.10 & 120544.70 & 5.1793 \\
\hline Special Salt Solutions & 53 & $90 \mathrm{C} 1.0 \mathrm{M} \mathrm{\# 5}$ & $\mathrm{NaOH} 1 \mathrm{M}$ & 90 & 5 & 120544.68 & 903884.80 & 93596.50 & 5.0811 \\
\hline Special Salt Solutions & 53 & $90 \mathrm{C} 1.0 \mathrm{M} \mathrm{\# 6}$ & $\mathrm{NaOH} 1 \mathrm{M}$ & 90 & 6 & 93596.50 & 997481.30 & 74706.07 & 4.9713 \\
\hline Special Salt Solutions & 53 & $90 \mathrm{C} 1.0 \mathrm{M} \# 7$ & $\mathrm{NaOH} 1 \mathrm{M}$ & 90 & 7 & 74706.07 & 1072187.00 & 59468.16 & 4.8734 \\
\hline
\end{tabular}


Table B3. Test Results for Special Salt Solutions

(continued)

\begin{tabular}{|c|c|c|c|c|c|c|c|c|c|}
\hline & Test & Test & Salt & Temperature & Sampling & A & & & \\
\hline Study & Sequence & Identifier & Solution & $\left({ }^{\circ} \mathrm{C}\right)$ & Sequence & (peak area) & $4121 \mathrm{y} 50$ & $\mathrm{x}$ & $\log (\mathrm{A})$ \\
\hline Special Salt Solutions & 53 & $90 \mathrm{C} 1.0 \mathrm{M} \mathrm{\# 8}$ & $\mathrm{NaOH} 1 \mathrm{M}$ & 90 & 8 & 59468.16 & 1131656.00 & 46039.00 & 4.7743 \\
\hline Special Salt Solutions & 53 & $90 \mathrm{C} 1.0 \mathrm{M} \# 9$ & $\mathrm{NaOH} 1 \mathrm{M}$ & 90 & 9 & 46039.00 & 1177695.00 & 37236.29 & 4.6631 \\
\hline Special Salt Solutions & 53 & $90 \mathrm{C} 1.0 \mathrm{M} \# 10$ & $\mathrm{NaOH} 1 \mathrm{M}$ & 90 & 10 & 37236.29 & 1214931.00 & & 4.5710 \\
\hline Special Salt Solutions & 54 & $80 \mathrm{C} 1.0 \mathrm{M} \# 1$ & $\mathrm{NaOH} 1 \mathrm{M}$ & 80 & 1 & 232484.77 & 232484.80 & 217313.10 & 5.3664 \\
\hline Special Salt Solutions & 54 & $80 \mathrm{C} 1.0 \mathrm{M} \mathrm{\# 2}$ & $\mathrm{NaOH} 1 \mathrm{M}$ & 80 & 2 & 217313.06 & 449797.80 & 190089.30 & 5.3371 \\
\hline Special Salt Solutions & 54 & $80 \mathrm{C} 1.0 \mathrm{M} \mathrm{\# 3}$ & $\mathrm{NaOH} 1 \mathrm{M}$ & 80 & 3 & 190089.32 & 639887.10 & 158860.60 & 5.2790 \\
\hline Special Salt Solutions & 54 & $80 \mathrm{C} 1.0 \mathrm{M} \# 4$ & $\mathrm{NaOH} 1 \mathrm{M}$ & 80 & 4 & 158860.55 & 798747.70 & 126264.90 & 5.2010 \\
\hline Special Salt Solutions & 54 & $80 \mathrm{C} 1.0 \mathrm{M} \# 5$ & $\mathrm{NaOH} 1 \mathrm{M}$ & 80 & 5 & 126264.92 & 925012.60 & 104900.70 & 5.1013 \\
\hline Special Salt Solutions & 54 & $80 \mathrm{C} 1.0 \mathrm{M} \# 6$ & $\mathrm{NaOH} 1 \mathrm{M}$ & 80 & 6 & 104900.69 & 1029913.00 & 87558.87 & 5.0208 \\
\hline Special Salt Solutions & 54 & $80 \mathrm{C} 1.0 \mathrm{M} \# 7$ & $\mathrm{NaOH} 1 \mathrm{M}$ & 80 & 7 & 87558.87 & 1117472.00 & 71590.96 & 4.9423 \\
\hline Special Salt Solutions & 54 & $80 \mathrm{C} 1.0 \mathrm{M} \# 8$ & $\mathrm{NaOH} 1 \mathrm{M}$ & 80 & 8 & 71590.96 & 1189063.00 & 58985.81 & 4.8549 \\
\hline Special Salt Solutions & 54 & $80 \mathrm{C} 1.0 \mathrm{M} \# 9$ & $\mathrm{NaOH} 1 \mathrm{M}$ & 80 & 9 & 58985.81 & 1248049.00 & 48295.98 & 4.7707 \\
\hline Special Salt Solutions & 54 & $80 \mathrm{C} 1.0 \mathrm{M} \# 10$ & $\mathrm{NaOH} 1 \mathrm{M}$ & 80 & 10 & 48295.98 & 1296345.00 & & 4.6839 \\
\hline Special Salt Solutions & 55 & $80 \mathrm{C} 1.0 \mathrm{M} \# 1 \mathrm{D}$ & $\mathrm{NaOH} 1 \mathrm{M}$ & 80 & 1 & 238057.24 & 238057.20 & 216294.90 & 5.3767 \\
\hline Special Salt Solutions & 55 & $80 \mathrm{C} 1.0 \mathrm{M} \mathrm{\# 2 \textrm {D }}$ & $\mathrm{NaOH} 1 \mathrm{M}$ & 80 & 2 & 216294.90 & 454352.10 & 181365.50 & 5.3350 \\
\hline Special Salt Solutions & 55 & $80 \mathrm{C} 1.0 \mathrm{M} \# 3 \mathrm{D}$ & $\mathrm{NaOH} 1 \mathrm{M}$ & 80 & 3 & 181365.51 & 635717.60 & 153117.40 & 5.2586 \\
\hline Special Salt Solutions & 55 & $80 \mathrm{C} 1.0 \mathrm{M} \# 4 \mathrm{D}$ & $\mathrm{NaOH} 1 \mathrm{M}$ & 80 & 4 & 153117.40 & 788835.10 & 126044.10 & 5.1850 \\
\hline Special Salt Solutions & 55 & $80 \mathrm{C} 1.0 \mathrm{M} \mathrm{\# 5 \textrm {D }}$ & $\mathrm{NaOH} 1 \mathrm{M}$ & 80 & 5 & 126044.06 & 914879.10 & 104861.60 & 5.1005 \\
\hline Special Salt Solutions & 55 & $80 \mathrm{C} 1.0 \mathrm{M} \# 6 \mathrm{D}$ & $\mathrm{NaOH} 1 \mathrm{M}$ & 80 & 6 & 104861.57 & 1019741.00 & 87318.30 & 5.0206 \\
\hline Special Salt Solutions & 55 & $80 \mathrm{C} 1.0 \mathrm{M} \# 7 \mathrm{D}$ & $\mathrm{NaOH} 1 \mathrm{M}$ & 80 & 7 & 87318.30 & 1107059.00 & 73333.14 & 4.9411 \\
\hline Special Salt Solutions & 55 & $80 \mathrm{C} 1.0 \mathrm{M} \# 8 \mathrm{D}$ & $\mathrm{NaOH} 1 \mathrm{M}$ & 80 & 8 & 73333.14 & 1180392.00 & 59094.25 & 4.8653 \\
\hline Special Salt Solutions & 55 & $80 \mathrm{C} 1.0 \mathrm{M} \# 9 \mathrm{D}$ & $\mathrm{NaOH} 1 \mathrm{M}$ & 80 & 9 & 59094.25 & 1239486.00 & 50047.21 & 4.7715 \\
\hline Special Salt Solutions & 55 & $80 \mathrm{C} 1.0 \mathrm{M} \# 10 \mathrm{D}$ & $\mathrm{NaOH} 1 \mathrm{M}$ & 80 & 10 & 50047.21 & 1289534.00 & & 4.6994 \\
\hline Special Salt Solutions & 56 & $70 \mathrm{C} 1.0 \mathrm{M} \# 1$ & $\mathrm{NaOH} 1 \mathrm{M}$ & 70 & 1 & 221686.84 & 221686.80 & 187540.10 & 5.3457 \\
\hline Special Salt Solutions & 56 & $70 \mathrm{C} 1.0 \mathrm{M} \mathrm{\# 2}$ & $\mathrm{NaOH} 1 \mathrm{M}$ & 70 & 2 & 187540.15 & 409227.00 & 168686.50 & 5.2731 \\
\hline Special Salt Solutions & 56 & $70 \mathrm{C} 1.0 \mathrm{M} \# 3$ & $\mathrm{NaOH} 1 \mathrm{M}$ & 70 & 3 & 168686.46 & 577913.40 & 143938.10 & 5.2271 \\
\hline Special Salt Solutions & 56 & $70 \mathrm{C} 1.0 \mathrm{M} \# 4$ & $\mathrm{NaOH} 1 \mathrm{M}$ & 70 & 4 & 143938.13 & 721851.60 & 124630.40 & 5.1582 \\
\hline Special Salt Solutions & 56 & $70 \mathrm{C} 1.0 \mathrm{M} \# 5$ & $\mathrm{NaOH} 1 \mathrm{M}$ & 70 & 5 & 124630.41 & 846482.00 & 100485.00 & 5.0956 \\
\hline Special Salt Solutions & 56 & $70 \mathrm{C} 1.0 \mathrm{M} \# 6$ & $\mathrm{NaOH} 1 \mathrm{M}$ & 70 & 6 & 100484.96 & 946966.90 & 85451.75 & 5.0021 \\
\hline Special Salt Solutions & 56 & $70 \mathrm{C} 1.0 \mathrm{M} \mathrm{\# 7}$ & $\mathrm{NaOH} 1 \mathrm{M}$ & 70 & 7 & 85451.75 & 1032419.00 & 72950.24 & 4.9317 \\
\hline Special Salt Solutions & 56 & $70 \mathrm{C} 1.0 \mathrm{M} \# 8$ & $\mathrm{NaOH} 1 \mathrm{M}$ & 70 & 8 & 72950.25 & 1105369.00 & 60974.27 & 4.8630 \\
\hline Special Salt Solutions & 56 & $70 \mathrm{C} 1.0 \mathrm{M} \# 9$ & $\mathrm{NaOH} 1 \mathrm{M}$ & 70 & 9 & 60974.27 & 1166343.00 & 51217.11 & 4.7851 \\
\hline Special Salt Solutions & 56 & $70 \mathrm{C} 1.0 \mathrm{M} \# 10$ & $\mathrm{NaOH} 1 \mathrm{M}$ & 70 & 10 & 51217.11 & 1217560.00 & & 4.7094 \\
\hline Special Salt Solutions & 57 & $70 \mathrm{C} 1.0 \mathrm{M} \# 1 \mathrm{D}$ & $\mathrm{NaOH} 1 \mathrm{M}$ & 70 & 1 & 211766.32 & 211766.30 & 183953.80 & 5.3259 \\
\hline Special Salt Solutions & 57 & $70 \mathrm{C} 1.0 \mathrm{M} \mathrm{\# 2 \textrm {D }}$ & $\mathrm{NaOH} 1 \mathrm{M}$ & 70 & 2 & 183953.75 & 395720.10 & 158172.80 & 5.2647 \\
\hline Special Salt Solutions & 57 & $70 \mathrm{C} 1.0 \mathrm{M} \# 3 \mathrm{D}$ & $\mathrm{NaOH} 1 \mathrm{M}$ & 70 & 3 & 158172.81 & 553892.90 & 135260.10 & 5.1991 \\
\hline Special Salt Solutions & 57 & $70 \mathrm{C} 1.0 \mathrm{M} \mathrm{\# 4 \textrm {D }}$ & $\mathrm{NaOH} 1 \mathrm{M}$ & 70 & 4 & 135260.13 & 689153.00 & 115093.50 & 5.1312 \\
\hline Special Salt Solutions & 57 & $70 \mathrm{C} 1.0 \mathrm{M} \# 5 \mathrm{D}$ & $\mathrm{NaOH} 1 \mathrm{M}$ & 70 & 5 & 115093.46 & 804246.50 & 98205.96 & 5.0611 \\
\hline Special Salt Solutions & 57 & $70 \mathrm{C} 1.0 \mathrm{M} \# 6 \mathrm{D}$ & $\mathrm{NaOH} 1 \mathrm{M}$ & 70 & 6 & 98205.96 & 902452.40 & 83345.50 & 4.9921 \\
\hline Special Salt Solutions & 57 & $70 \mathrm{C} 1.0 \mathrm{M} \# 7 \mathrm{D}$ & $\mathrm{NaOH} 1 \mathrm{M}$ & 70 & 7 & 83345.50 & 985797.90 & 70905.86 & 4.9209 \\
\hline Special Salt Solutions & 57 & $70 \mathrm{C} 1.0 \mathrm{M} \# 8 \mathrm{D}$ & $\mathrm{NaOH} 1 \mathrm{M}$ & 70 & 8 & 70905.86 & 1056704.00 & 58732.14 & 4.8507 \\
\hline Special Salt Solutions & 57 & $70 \mathrm{C} 1.0 \mathrm{M} \# 9 \mathrm{D}$ & $\mathrm{NaOH} 1 \mathrm{M}$ & 70 & 9 & 58732.14 & 1115436.00 & 50614.42 & 4.7689 \\
\hline Special Salt Solutions & 57 & $70 \mathrm{C} 1.0 \mathrm{M} \# 10 \mathrm{D}$ & $\mathrm{NaOH} 1 \mathrm{M}$ & 70 & 10 & 50614.42 & 1166050.00 & & 4.7043 \\
\hline Special Salt Solutions & 58 & $60 \mathrm{C} 1.0 \mathrm{M} \# 1$ & $\mathrm{NaOH} 1 \mathrm{M}$ & 60 & 1 & 181339.26 & 181339.30 & 163197.30 & 5.2585 \\
\hline Special Salt Solutions & 58 & $60 \mathrm{C} 1.0 \mathrm{M} \mathrm{\# 2}$ & $\mathrm{NaOH} 1 \mathrm{M}$ & 60 & 2 & 163197.27 & 344536.50 & 143753.60 & 5.2127 \\
\hline Special Salt Solutions & 58 & $60 \mathrm{C} 1.0 \mathrm{M} \# 3$ & $\mathrm{NaOH} 1 \mathrm{M}$ & 60 & 3 & 143753.59 & 488290.10 & 117160.80 & 5.1576 \\
\hline Special Salt Solutions & 58 & $60 \mathrm{C} 1.0 \mathrm{M} \# 4$ & $\mathrm{NaOH} 1 \mathrm{M}$ & 60 & 4 & 117160.80 & 605450.90 & 101905.70 & 5.0688 \\
\hline Special Salt Solutions & 58 & $60 \mathrm{C} 1.0 \mathrm{M} \# 5$ & $\mathrm{NaOH} 1 \mathrm{M}$ & 60 & 5 & 101905.73 & 707356.60 & 89175.60 & 5.0082 \\
\hline Special Salt Solutions & 58 & $60 \mathrm{C} 1.0 \mathrm{M} \# 6$ & $\mathrm{NaOH} 1 \mathrm{M}$ & 60 & 6 & 89175.60 & 796532.20 & 76788.73 & 4.9502 \\
\hline Special Salt Solutions & 58 & $60 \mathrm{C} 1.0 \mathrm{M} \mathrm{\# 7}$ & $\mathrm{NaOH} 1 \mathrm{M}$ & 60 & 7 & 76788.73 & 873321.00 & 65157.26 & 4.8853 \\
\hline Special Salt Solutions & 58 & $60 \mathrm{C} 1.0 \mathrm{M} \# 8$ & $\mathrm{NaOH} 1 \mathrm{M}$ & 60 & 8 & 65157.26 & 938478.20 & 55766.06 & 4.8140 \\
\hline Special Salt Solutions & 58 & $60 \mathrm{C} 1.0 \mathrm{M} \# 9$ & $\mathrm{NaOH} 1 \mathrm{M}$ & 60 & 9 & 55766.06 & 994244.30 & 49648.32 & 4.7464 \\
\hline Special Salt Solutions & 58 & $60 \mathrm{C} 1.0 \mathrm{M} \# 10$ & $\mathrm{NaOH} 1 \mathrm{M}$ & 60 & 10 & 49648.32 & 1043893.00 & & 4.6959 \\
\hline Special Salt Solutions & 59 & $60 \mathrm{C} 1.0 \mathrm{M} \# 1 \mathrm{D}$ & $\mathrm{NaOH} 1 \mathrm{M}$ & 60 & 1 & 186737.91 & 186737.90 & 163028.50 & 5.2712 \\
\hline Special Salt Solutions & 59 & $60 \mathrm{C} 1.0 \mathrm{M} \# 2 \mathrm{D}$ & $\mathrm{NaOH} 1 \mathrm{M}$ & 60 & 2 & 163028.50 & 349766.40 & 133682.00 & 5.2123 \\
\hline Special Salt Solutions & 59 & $60 \mathrm{C} 1.0 \mathrm{M} \# 3 \mathrm{D}$ & $\mathrm{NaOH} 1 \mathrm{M}$ & 60 & 3 & 133682.04 & 483448.50 & 115157.20 & 5.1261 \\
\hline Special Salt Solutions & 59 & $60 \mathrm{C} 1.0 \mathrm{M} \# 4 \mathrm{D}$ & $\mathrm{NaOH} 1 \mathrm{M}$ & 60 & 4 & 115157.19 & 598605.60 & 99671.53 & 5.0613 \\
\hline Special Salt Solutions & 59 & $60 \mathrm{C} 1.0 \mathrm{M} \# 5 \mathrm{D}$ & $\mathrm{NaOH} 1 \mathrm{M}$ & 60 & 5 & 99671.53 & 698277.20 & 87287.32 & 4.9986 \\
\hline Special Salt Solutions & 59 & $60 \mathrm{C} 1.0 \mathrm{M} \# 6 \mathrm{D}$ & $\mathrm{NaOH} 1 \mathrm{M}$ & 60 & 6 & 87287.33 & 785564.50 & 74930.16 & 4.9410 \\
\hline Special Salt Solutions & 59 & $60 \mathrm{C} 1.0 \mathrm{M} \# 7 \mathrm{D}$ & $\mathrm{NaOH} 1 \mathrm{M}$ & 60 & 7 & 74930.16 & 860494.70 & 62395.15 & 4.8747 \\
\hline & 59 & $60 \mathrm{C}$ & 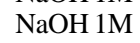 & 60 & 8 & 620515 & 922889.80 & 54025.08 & 4.7952 \\
\hline Special Salt Solutions & 59 & $60 \mathrm{C} 1.0 \mathrm{M} \# 9 \mathrm{D}$ & $\mathrm{NaOH} 1 \mathrm{M}$ & 60 & 9 & 54025.08 & 976914.90 & 47249.78 & 4.7326 \\
\hline Special Salt Solutions & 59 & $60 \mathrm{C} 1.0 \mathrm{M} \# 10 \mathrm{D}$ & $\mathrm{NaOH} 1 \mathrm{M}$ & 60 & 10 & 47249.78 & 1024165.00 & & 4.6744 \\
\hline Special Salt Solutions & 60 & $50 \mathrm{C} 1$. & $\mathrm{NaOH} 1 \mathrm{M}$ & 50 & 1 & 135911.43 & 135911.40 & 117221.10 & 5.1333 \\
\hline Special Salt Solutions & 60 & $50 \mathrm{C} 1.0 \mathrm{M} \# 2$ & $\mathrm{NaOH} 1$ & 50 & 2 & 117221.14 & 253132.60 & 95013.75 & 5.0690 \\
\hline Special Salt Solutions & 60 & $50 \mathrm{C} 1.0 \mathrm{M} \# 3$ & $\mathrm{NaOH} 1 \mathrm{M}$ & 50 & 3 & 95013.75 & 348146.30 & 81506.85 & 4.9778 \\
\hline
\end{tabular}


Table B3. Test Results for Special Salt Solutions (continued)

\begin{tabular}{|c|c|c|}
\hline & Test & Test \\
\hline Study & Sequence & Identifier \\
\hline Special Salt Solutions & 60 & $50 \mathrm{C} 1.0 \mathrm{M} \mathrm{\# 4}$ \\
\hline Special Salt Solutions & 60 & $50 \mathrm{C} 1.0 \mathrm{M} \mathrm{\# 5}$ \\
\hline Special Salt Solutions & 0 & $50 \mathrm{C} 1.0 \mathrm{M} \# 6$ \\
\hline Special Salt Solutions & 60 & $50 \mathrm{C} 1.0 \mathrm{M} \# 7$ \\
\hline Special Salt Solutions & 60 & $50 \mathrm{C} 1.0 \mathrm{M} \# 8$ \\
\hline Special Salt Solutions & 60 & $50 \mathrm{C} 1.0 \mathrm{M} \# 9$ \\
\hline Special Salt Solutions & 60 & $50 \mathrm{C} 1.0 \mathrm{M} \# 10$ \\
\hline Special Salt Solutions & 61 & $50 \mathrm{C} 1.0 \mathrm{M} \# 1 \mathrm{D}$ \\
\hline Special Salt Solutions & 61 & $50 \mathrm{C} 1.0 \mathrm{M} \mathrm{\# 2D}$ \\
\hline Special Salt Solutions & 61 & $50 \mathrm{C} 1.0 \mathrm{M} \# 3 \mathrm{D}$ \\
\hline Special Salt Solutions & 61 & $50 \mathrm{C} 1.0 \mathrm{M} \mathrm{\# 4D}$ \\
\hline Special Salt Solutions & 61 & $50 \mathrm{C} 1.0 \mathrm{M} \# 5 \mathrm{D}$ \\
\hline Special Salt Solutions & 61 & $50 \mathrm{C} 1.0 \mathrm{M} \# 6 \mathrm{D}$ \\
\hline Special Salt Solutions & 61 & $50 \mathrm{C} 1.0 \mathrm{M} \# 7 \mathrm{D}$ \\
\hline Special Salt Solutions & 61 & $50 \mathrm{C} 1.0 \mathrm{M} \# 8 \mathrm{D}$ \\
\hline Special Salt Solutions & 61 & $50 \mathrm{C} 1.0 \mathrm{M} \# 9 \mathrm{D}$ \\
\hline Special Salt Solutions & 61 & $50 \mathrm{C} 1.0 \mathrm{M} \# 10 \mathrm{D}$ \\
\hline
\end{tabular}

Salt
Solution
$\mathrm{NaOH} 1 \mathrm{M}$
$\mathrm{NaOH} 1 \mathrm{M}$
$\mathrm{NaOH} 1 \mathrm{M}$
$\mathrm{NaOH} 1 \mathrm{M}$
$\mathrm{NaOH} 1 \mathrm{M}$
$\mathrm{NaOH} 1 \mathrm{M}$
$\mathrm{NaOH} 1 \mathrm{M}$
$\mathrm{NaOH} 1 \mathrm{M}$
$\mathrm{NaOH} 1 \mathrm{M}$
$\mathrm{NaOH} 1 \mathrm{M}$
$\mathrm{NaOH} 1 \mathrm{M}$
$\mathrm{NaOH} 1 \mathrm{M}$
$\mathrm{NaOH} 1 \mathrm{M}$
$\mathrm{NaOH} 1 \mathrm{M}$
$\mathrm{NaOH} 1 \mathrm{M}$
$\mathrm{NaOH} 1 \mathrm{M}$
$\mathrm{NaOH} 1 \mathrm{M}$

Temperature

$\left({ }^{\circ} \mathrm{C}\right)$
50
50
50
50
50
50
50
50
50
50
50
50
50
50
50
50
50

Sampling
Sequence
4
5
6
7
8
9
10
1
2
3
4
5
6
7
8
9
10

A

(peak area) 81506.85

70918.37

61352.26

52986.39

45089.95

39958.47

35659.79

124211.48

111530.68

89832.60

75653.99

65910.53

56403.63

47594.06

39078.42

32710.78

27811.665

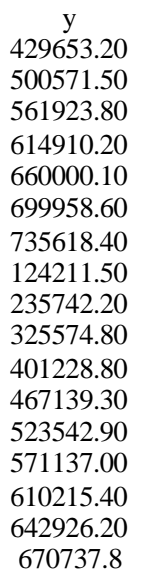

$\begin{array}{cc}\mathrm{x} & \log (\mathrm{A}) \\ 70918.37 & 4.9112 \\ 61352.26 & 4.8508 \\ 52986.39 & 4.7878 \\ 45089.95 & 4.7242 \\ 39958.47 & 4.6541 \\ 35659.78 & 4.6016 \\ . & 4.5522 \\ 111530.70 & 5.0942 \\ 89832.60 & 5.0474 \\ 75653.99 & 4.9534 \\ 65910.53 & 4.8788 \\ 56403.63 & 4.8190 \\ 47594.06 & 4.7513 \\ 39078.42 & 4.6776 \\ 32710.78 & 4.5919 \\ 27811.67 & 4.5147 \\ . & 4.4442\end{array}$ 


\section{Exhibit B1: Common Logarithm of Peak Area Versus Sample Sequence for Methanol Studies: Study 1; Tests 1-8}

Methanol Study 1 (Test Sequence=1)

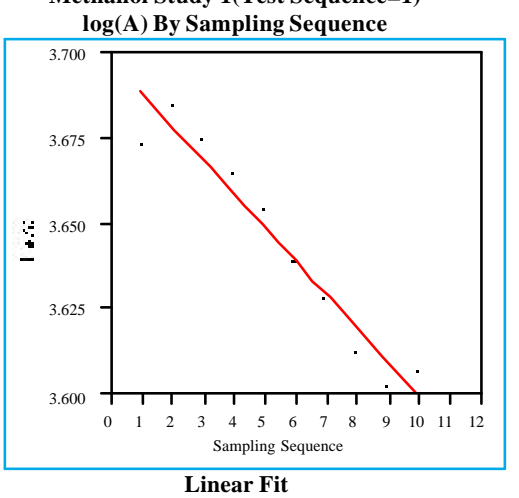

$\log (\mathrm{A})=3.69794-0.0098$ Sampling Sequence Summary of Fit

RSquare

RSquare Adj

Root Mean Square Error

Mean of Response

0.938377

0.930674

0.00806

3.64406

Observations (or Sum Wgts)

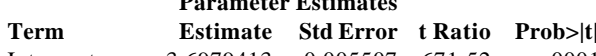
$\begin{array}{llllll}\text { Intercept } & 3.6979413 & 0.005507 & 671.52 & <.000\end{array}$ Sampling Seq. $\begin{array}{lllll}3.0 .009796 & 0.000888 & -11.04 & <.0001\end{array}$

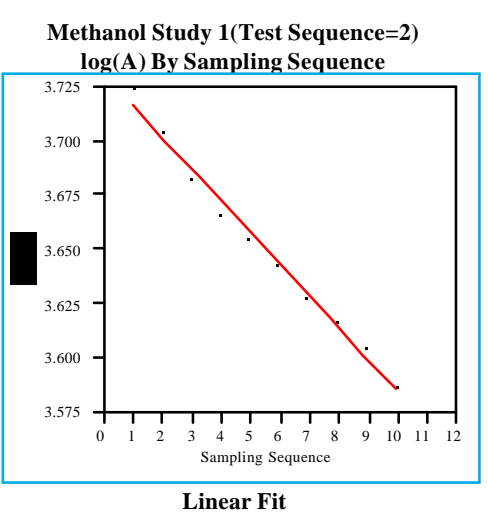

$\log (\mathrm{A})=3.73112-0.01458$ Sampling Sequence

Summary of Fit

RSquare

RSquare Adj

Root Mean Square Er

0.990419

Mean of Response
Observations (or Sum Wgts)

0.98922

3.650943

Parameter Estimates

Estimate Std Error t Ratio Prob $>|\mathbf{t}|$

$\begin{array}{lrrrr}\text { Intercept } & 3.7311173 & 0.003145 & 1186.3 & <.0001 \\ \text { Sampling eq. } & -0.014577 & 0.000507 & -28.76 & <.0001\end{array}$
Methanol Study 1 (Test Sequence=3)

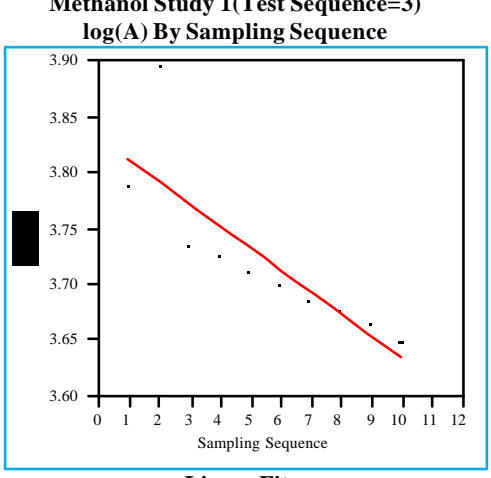

Linear Fit

$\log (A)=3.83173-0.01985$ Sampling Sequence \begin{tabular}{lr}
\multicolumn{1}{c}{ Summary of Fit } \\
RSquare & 0.692786 \\
RSquare Adj & 0.654384 \\
Root Mean Square Error & 0.042459 \\
Mean of Response & 3.722527 \\
Observations (or Sum Wgts) & 10
\end{tabular}

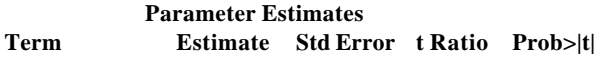
$\begin{array}{lrrrr}\text { Term } & \text { Estimate } & \text { Std Error } & \text { t Ratio } & \text { Prob }>\mid \text { t } \mid \\ \text { Intercept } & 3.8317279 & 0.029005 & 132.11 & <.0001 \\ \text { Sampling eq. } & -0.019855 & 0.004675 & -4.25 & 0.0028\end{array}$

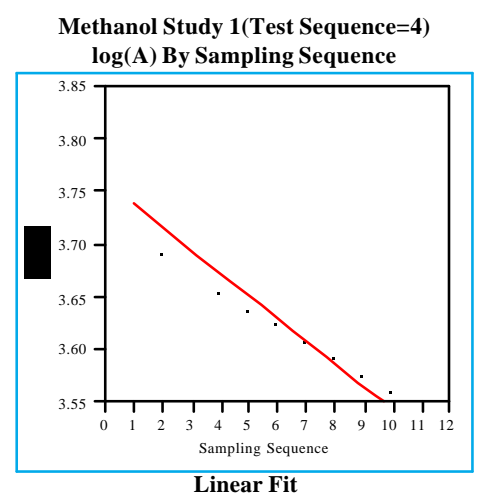

$\log (\mathrm{A})=3.76008-0.02151$ Sampling Sequence

RSquare Summary of Fit

0.852719

RSquare Adj

0.834309

0.02871
3.641762
1

Observations (or Sum Wgts)

10

Parameter Estimates

Estimate Std Error t Ratio Prob>|

$\begin{array}{llllll} & \text { Intercept } & 3.7600791 & 0.019613 & 191.71 & <.000\end{array}$

$\begin{array}{lrrrr}\text { Sampling Seq. } & -0.021512 & 0.003161 & -6.81 & 0.0001\end{array}$
Methanol Study 1 (Test Sequence=5)

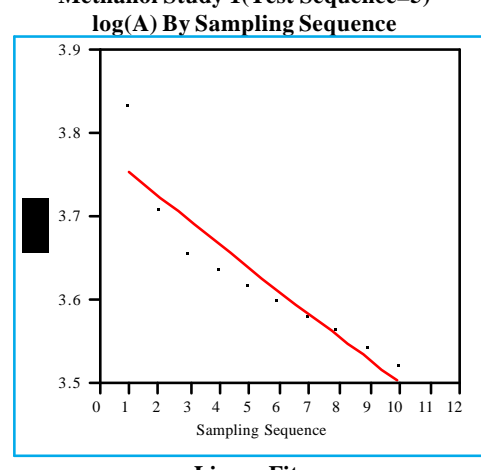

Linear Fit

$\log (\mathrm{A})=3.7795-0.02793$ Sampling Sequence

Summary of Fit

RSquare

0.854701

\begin{tabular}{ll}
0.856538 \\
\hline
\end{tabular}

Root Mean Square Error

0.036978

Observations (or Sum Wgts)

$\begin{array}{lrrrr}\text { Term } & \text { Parameter Estimates } \\ \text { Estimate } & \text { Std Error } & \text { t Ratio } & \text { Prob }>|t|\end{array}$

$\begin{array}{lllll}\text { Sampling Seq. } & -0.027928 & 0.004071 & -6.86 & 0.0001\end{array}$

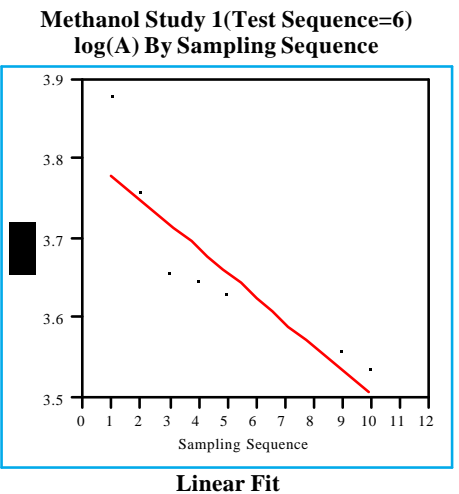

$\log (\mathrm{A})=3.81261-0.03063$ Sampling Sequence

Square Summary of Fit

Methanol Study 1(Test Sequence=7) $\log (A)$ By Sampling Sequence

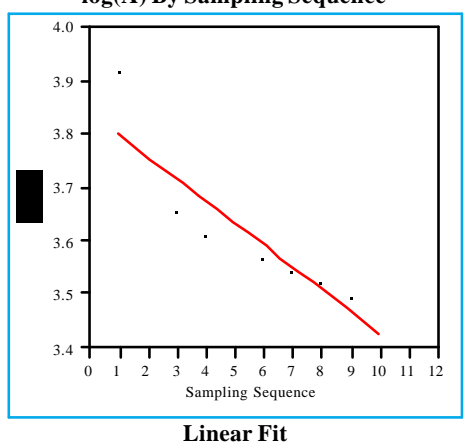

$\log (\mathrm{A})=3.83817-0.04143$ Sampling Sequence Summary of Fit

RSquare

RSquare Adj
Root Mean Square Error

Mean of Response

0.832979

0.812102

0.059572

Observations (or Sum Wgts)

Term Estimate Std Error t Ratio Prob $>|\mathbf{t}|$

$\begin{array}{llllll}\text { Intercept } & 3.8381714 & 0.040695 & 94.31 & <.0001\end{array}$ $\begin{array}{lllll}\text { Sampling Seq. } & -0.041428 & 0.006559 & -6.32 & 0.0002\end{array}$

Methanol Study 1 (Test Sequence=8) $\log (\mathrm{A})$ By Sampling Sequence

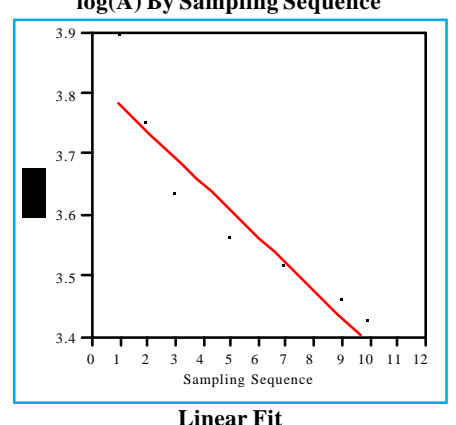
$\log (\mathrm{A})=3.82693-0.04375$ Sampling Sequence
Summary of Fit RSquare 857962 RSquare Adj $\quad 0.812862$ $\begin{array}{ll} & 0.789469 \\ \text { Root Mean Square Error } & 0.047189\end{array}$ Mean of Response 3.64416 Observations (or Sum Wgts)

Term Estimate Std Error t Ratio Prob $>|\mathbf{t}|$ $\begin{array}{lrrrrr} & \text { Estimate } & \text { Std Error } & \text { t Ratio } & \text { Prob }>\mid \boldsymbol{M} \\ \text { Intercept } & 3.8126058 & 0.032236 & 118.27 & <.0001\end{array}$ $\begin{array}{lllll}\text { Sampling Seq. } & -0.030626 & 0.005195 & -5.89 & 0.0004\end{array}$ 


\section{Exhibit B2: Common Logarithm of Peak Area Versus Sample Sequence for Methanol Studies: Study 1; Tests 9-16}

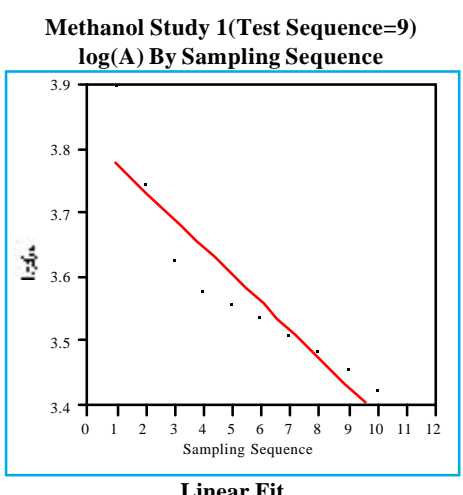

$\log (\mathrm{A})=3.82126-0.04364$ Sampling Sequence

Summary of Fit $\quad 0.845147$

$\begin{array}{lr}\text { RSquare } & 0.845147 \\ \text { RSquare Adj } & 0.825791 \\ \text { Root Mean Square Error } & 0.059984 \\ \text { Mean of Response } & 3.58125\end{array}$

3.58125

Observations (or Sum Wgts)

10

$\begin{array}{lrrrrr}\text { Term } & \text { Estimate } & \text { Std Error } & \text { t Ratio } & \text { Prob }>|\mathbf{t}| \\ \text { Intercept } & 3.821258 & 0.040977 & 93.25 & <.0001\end{array}$ Sampling Seq. $-0.043638 \quad 0.006604 \quad-6.61 \quad 0.0002$

Methanol Study 1 (Test Sequence=10)
$\log (\mathbf{A})$ By Sampling Sequence

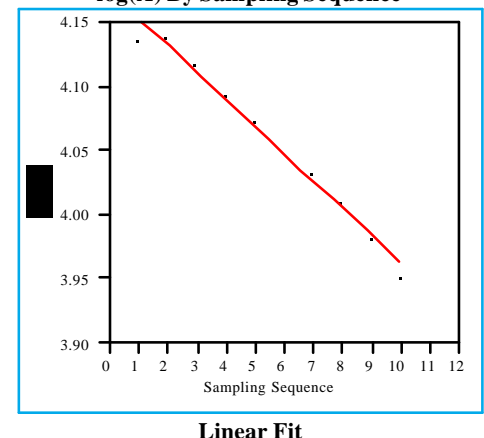

$\log (\mathrm{A})=4.17472-0.02131$ Sampling Sequence RSquare Summary of Fit

RSquare Adj

Root Mean Square Error

Mean of Response

0.983506

Observations (or Sum Wgts)

0.981445

4.057497

Parameter Estimates

10

$\begin{array}{llllll}\text { Intercept } \quad 4.1747201 & 0.006055 & 689.48 & <.000\end{array}$ $\begin{array}{lllll}\text { Sampling Seq. } & -0.021313 & 0.000976 & -21.84 & <.000\end{array}$
Methanol Study 1 (Test Sequence=11)

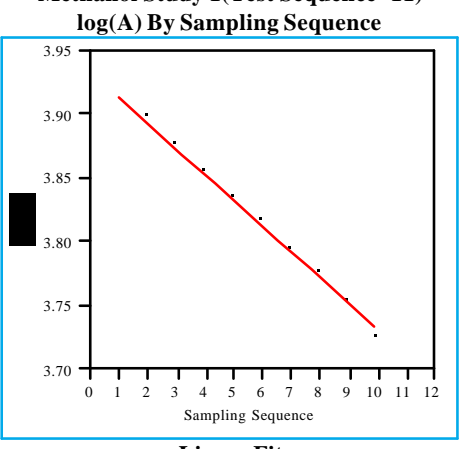

\section{Linear Fit}

$\log (\mathrm{A})=3.93296-0.01977$ Sampling Sequence

\begin{tabular}{lr}
\multicolumn{1}{c}{ Summary of Fit } \\
RSquare & 0.985969 \\
RSquare Adj & 0.984215 \\
Root Mean Square Error & 0.007575 \\
Mean of Response & 3.824214 \\
& 10
\end{tabular}

Mean of Response

Observations (or Sum Wgts)

0.984215

10

\begin{tabular}{lrrrr} 
Term & Parameter Estimates & & \\
Estimate & Std Error & t Ratio & Prob $>|t|$ \\
\hline & 3.9329628 & 0.005174 & 760.08 & $<0001$
\end{tabular}

$\begin{array}{lrrrrr} & 3.9329628 & 0.005174 & 760.08 & <.0001 \\ \text { Sampling Seq } & -0.019773 & 0.000834 & -23.71 & <.000\end{array}$

Methanol Study 1 (Test Sequence=12)

$\log (\mathrm{A})$ By Sampling Sequence

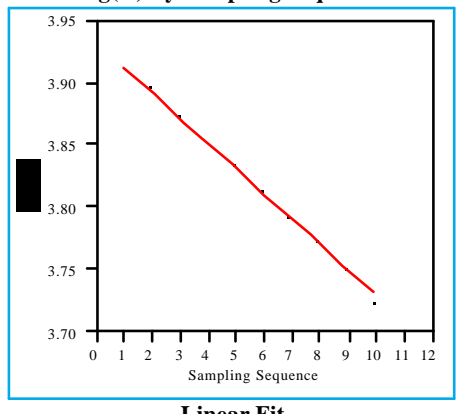

$\log (\mathrm{A})=3.93138-0.02017 \mathrm{~S}$

Sequence

$\begin{array}{ll}\text { RSquare } & \text { Summary of Fit } \\ \text { RSquare Adj } & 0.993254 \\ \text { Root Mean Square Error } & 0.992411 \\ \text { Mean of Response } & 0.005339 \\ \text { Observations (or Sum Wgts) } & 3.820417\end{array}$

Mean of Response

Observations (or Sum Wgts)

0.992411

10

$\begin{array}{lrrrrr}\text { Term } & \text { Parameter Estimates } & & \\ \text { Intercept } & 3.9313771 & \text { Std Error } & \text { t Ratio } & \text { Prob }>|t| \\ & 0.003647 & 1077.9 & <.0001\end{array}$

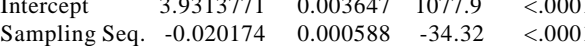

Methanol Study 1 (Test Sequence=13)

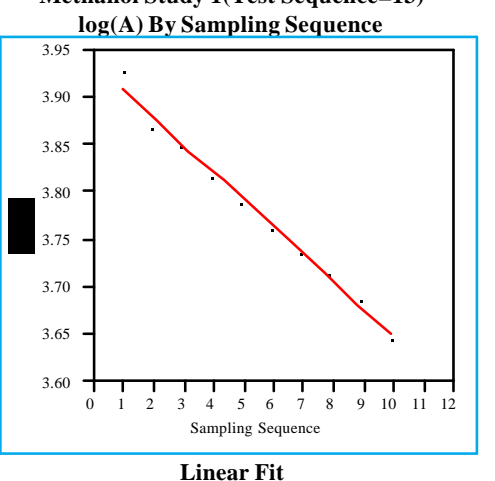

$\log (A)=3.93594-0.02872$ Sampling Sequence Summary of Fit

RSquare

RSquare Adj

Root Mean Square Erro

Observations (or Sum Wgts)
Oatam

0.989356

0.988026

3.777968

Term Estimate Std Error t Ratio Prob $>\mid \mathbf{t}$ $\begin{array}{lrrrrr}\text { Intercept } & 3.9359428 & 0.006536 & 602.24 & <.000\end{array}$ $\begin{array}{lllll}\text { Sampling Seq. } & -0.028723 & 0.001053 & -27.27 & <.0001\end{array}$
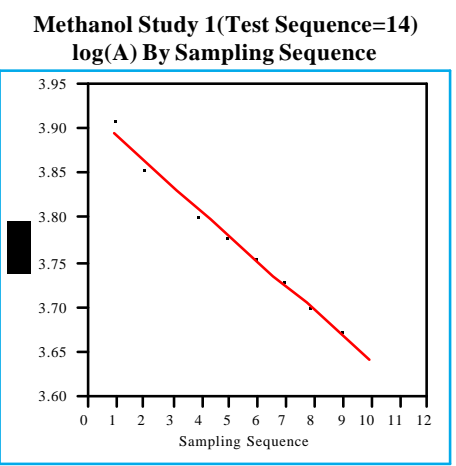

Linear Fit
$\log (\mathrm{A})=3.92042-0.02812$ Sampling Sequenc Summary of Fit

RSquare

\section{RSquare Adj}

Root Mean Square Error

Mean of Response

0.992207

3.765759

10

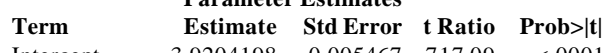

$\begin{array}{llllll}\text { Intercept } & 3.9204198 & 0.005467 & 717.09 & <.0001\end{array}$

$\begin{array}{lrlll}\text { Sampling Seq. } & -0.02812 & 0.000881 & -31.91 & <.0001\end{array}$
Methanol Study 1 (Test Sequence=15)

$\log (\mathrm{A})$ By Sampling Sequence

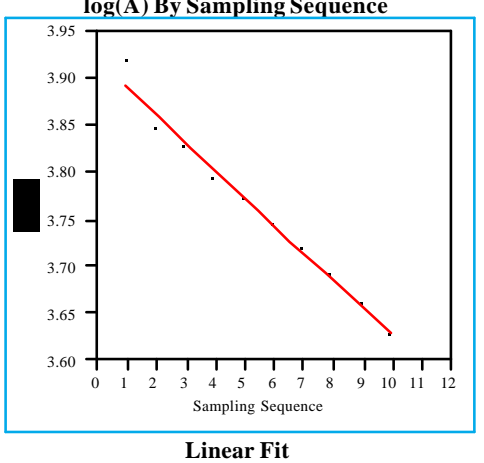

$\log (\mathrm{A})=3.92091-0.02929$ Sampling Sequence RSquare

RSquare Adj Summary of Fit

Root Mean Square Error

0.984129

Mean of Response

0.011945

Observations (or Sum Wgts)

$$
10
$$

Term Estimate Std Error t Ratio Prob $>|t|$ $\begin{array}{lrrrr}\text { Intercept } & 3.9209128 & 0.00816 & 480.51 & <.000\end{array}$ $\begin{array}{lllll}\text { Sampling Seq. } & -0.02929 & 0.001315 & -22.27 & <.0001\end{array}$

Methanol Study 1 (Test Sequence=16)

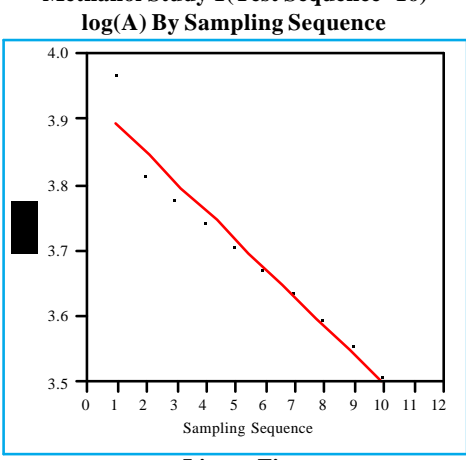

$\log (A)=3.9387-0.04392$ Sampling Sequence

\section{Summary of Fit}

RSquare

Root Mean Squre Eror

Mean of Response

Observations (or Sum Wgts)

0.949633

0.032482

Term Estimate Std Error t Ratio Prob $>|\mathbf{t}|$

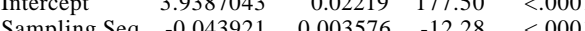


Exhibit B3: Common Logarithm of Peak Area Versus Sample Sequence for Methanol Studies: Study 1; Tests 17-24

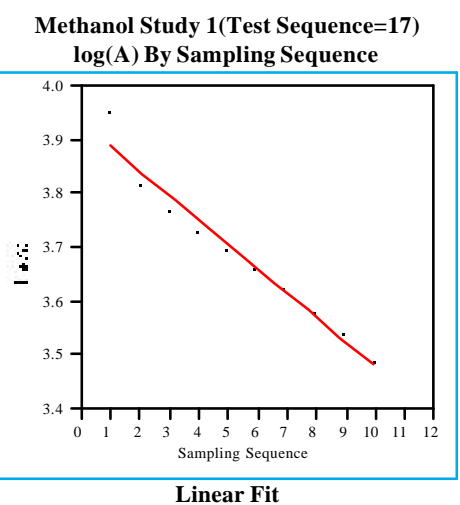

$\log (\mathrm{A})=3.92941-0.04474$ Sampling Sequence

\begin{tabular}{ll}
$\log (\mathrm{A})=3.929 \mathrm{Summary}$ of Fit & \\
RSquare & 0.959363 \\
RSquare Adj & 0.954283 \\
Root Mean Square Error & 0.029571 \\
Mean of Response & 3.683332 \\
\hline
\end{tabular}

Root Mean Square Error

Mean of Response
Observations (or Sum Wgts) \begin{tabular}{lrrrrr}
\multicolumn{7}{c}{ Parameter Estimates } & & \\
Term & Estimate & Std Error & t Ratio & Prob $>|\mathbf{t}|$ \\
Intercept & 3.9294094 & 0.020201 & 194.52 & $<.0001$ \\
Sampling Seq & -0.044741 & 0.003256 & -13.74 & $<.0001$
\end{tabular}

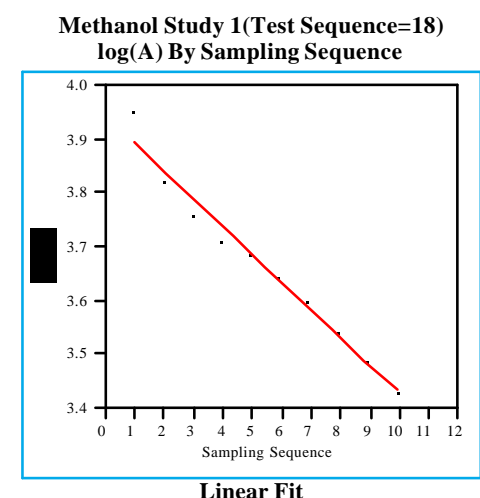

$\log (\mathrm{A})=3.9441-0.05157$ Sampling Sequence Summary of Fit

\section{RSquare}

RSquare Adj

Root Mean Square Error

Mean of Response

Observations (or Sum Wgts)

Parameter Estimates

Term Estimate Std Error t Ratio Prob $>\mid \mathbf{t}$ $\begin{array}{lrrrrr}\text { Intercept } & 3.9441036 & 0.019418 & 203.12 & <.0001 \\ \text { Sampling Seq. } & -0.051568 & 0.003129 & -16.48 & <.0001\end{array}$
Methanol Study 1(Test Sequence=19)

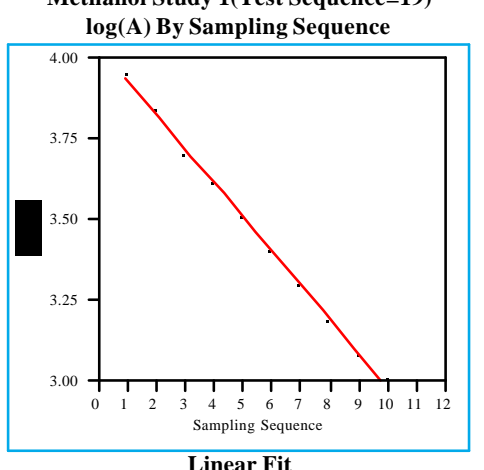

Linear Fit
$\log (\mathrm{A})=4.03773-0.10586$ Sampling Sequence

$\begin{array}{ll}\text { RSquare } \quad \text { Summary of Fit } & \\ \text { RSquare Adj } & 0.998014 \\ \text { Root Mean Square Error } & 0.997766 \\ \text { Mean of Response } & 0.015165 \\ \text { Oren } & 3.455524\end{array}$

Root Mean Square Error $\quad 0.015165$

Observations (or Sum Wgts)

10

\begin{tabular}{lrrrr} 
& \multicolumn{2}{c}{ Parameter Estimates } & & \\
Term & Estimate & Std Error & t Ratio & Prob $>|\mathbf{t}|$ \\
Intercept & 4.0377325 & 0.01036 & 389.76 & $<.0001$ \\
Sampling Seq & -0.105856 & 0.00167 & -63.40 & $<.0001$
\end{tabular}

Methanol Study 1(Test Sequence=20)

\begin{tabular}{l}
$\log (\mathrm{A})$ By Sampling Sequence \\
\hline
\end{tabular}

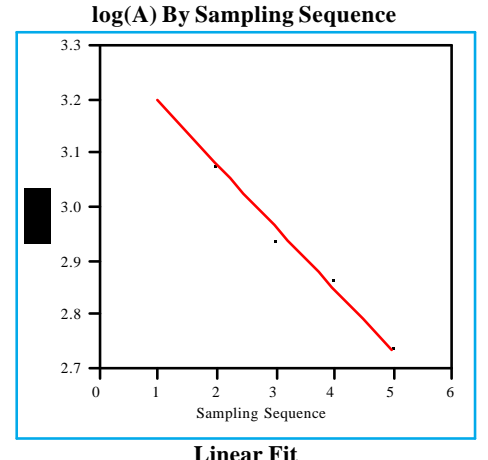

$\log (\mathrm{A})=3.31261-0.11613$ Sampling Sequence

RSquare Summary of Fit

RSquare Adj

Root Mean Square Error

Mean of Response

0.990514

0.987352
0.020749

Observations (or Sum Wgts)

2.964209

Term Pstimate Std Error t Ratio Prob $>|t|$

$\begin{array}{llllll}\text { Intercept } & 3.3126087 & 0.021762 & 152.22 & <.0001\end{array}$ $\begin{array}{lllll}\text { Sampling Seq. } & -0.116133 & 0.006561 & -17.70 & 0.0004\end{array}$
Methanol Study 1 (Test Sequence=21)

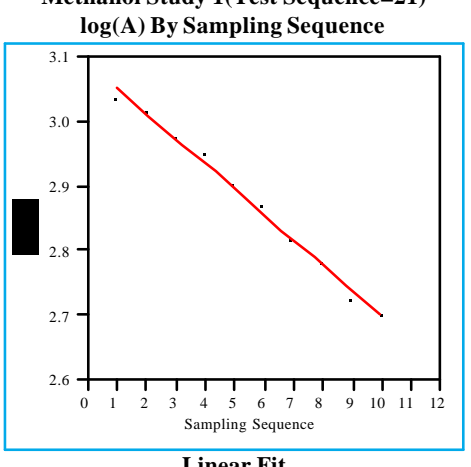

Linear Fit
$\log (\mathrm{A})=3.09002-0.03905$ Sampling Sequence

$\begin{array}{cc}\log (\mathrm{A})=3.09002-0.03905 \text { Sampling Sequence } \\ \text { RSquare } & \text { Summary of Fit }\end{array}$

$\begin{array}{lr}\text { RSquare } & 0.99247 \\ \text { RSquare Adj } & 0.99153\end{array}$

RSquare Adj $\quad 0.9915$

$\begin{array}{lr}\text { Root Mean Square Error } & 0.010921 \\ \text { Mean of Response } & 2.87526 \\ \text { Observations (or Sum Wgts) } & 10\end{array}$ \begin{tabular}{lrrrr}
\multicolumn{1}{c}{ Parameter Estimates } & & \\
Term & Estimate & Std Error & t Ratio & Prob $>|\mathbf{t}|$ \\
Intercept & 3.0900166 & 0.007461 & 414.17 & $<.0001$ \\
Sampling Seq. & -0.039047 & 0.001202 & -32.47 & $<.0001$
\end{tabular}

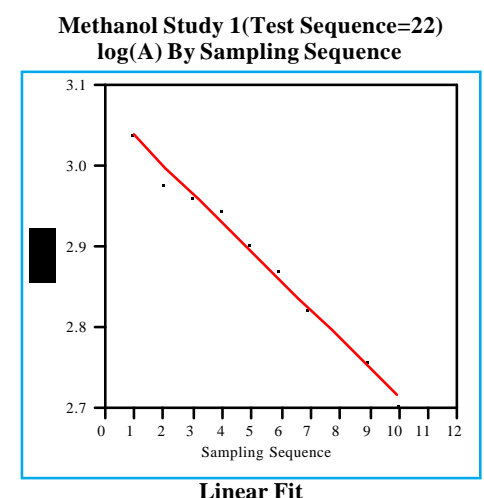

$\log (A)=3.07075-0.03545$ Sampling Sequence Summary of Fit

RSquare

RSquare Adj

Root Mean Square Error

Mean of Response

0.988385
0.986933
0.01234

Observations (or

0.01234

(or Sum Wgts)

Term Estimate Std Error t Ratio Prob $>|t|$ $\begin{array}{lrrrrr}\text { Intercept } & 3.0707485 & 0.00843 & 364.26 & <.0001\end{array}$ $\begin{array}{lllll}\text { Sampling Seq. } & -0.035448 & 0.001359 & -26.09 & <.0001\end{array}$
Methanol Study 1(Test Sequence=23) $\log (\mathrm{A})$ By Sampling Sequence

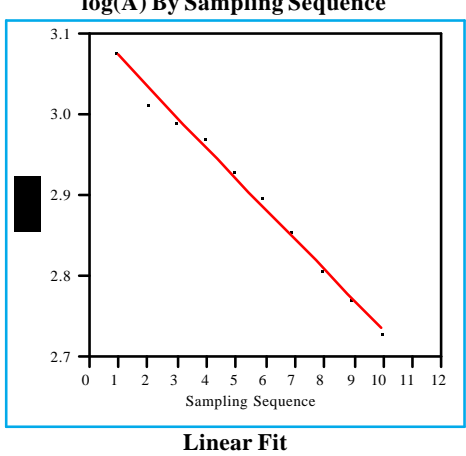

Linear Fit
$\log (\mathrm{A})=3.1079-0.03729$ Sampling Sequence

$$
\begin{array}{lr}
\multicolumn{2}{c}{\text { Summary of Fit }} \\
\text { RSquare } & 0.990629 \\
\text { RSquare Adj } & 0.989457 \\
\text { Root Mean Square Error } & 0.011646 \\
\text { Mean of Response } & 2.902817 \\
\text { Observations (or Sum Wgts) } & 10
\end{array}
$$

10

\begin{tabular}{lrrrr}
\multicolumn{7}{c}{ Parameter Estimates } & & \\
Term & Estimate & Std Error & t Ratio & Prob $>|t|$ \\
Intercept & 3.1078961 & 0.007956 & 390.64 & $<.0001$ \\
Sampling Seq & -0.037287 & 0.001282 & -29.08 & $<.0001$
\end{tabular}

Methanol Study 1 (Test Sequence=24)

Methanol Stady 1 (Test Sequence $=24)$

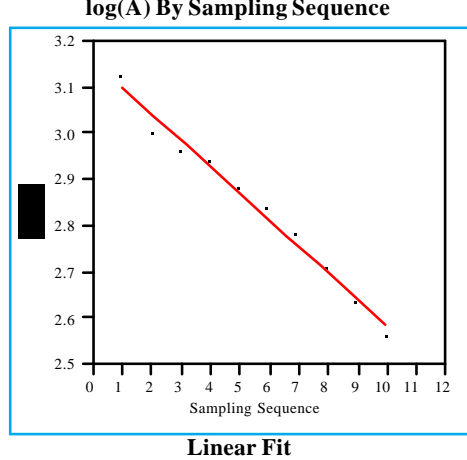

$\log (\mathrm{A})=3.15567-0.05727$ Sampling Sequence

RSquare

RSquare Adj

Summary of Fit

Root Mean Square Error

Mean of Response

0.980747
0.97834

Observations (or Sum Wgts)

0.025768
2.840686

Parameter Estimates

10

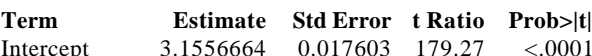
$\begin{array}{lrrrr} & 3.1556664 & 0.017603 & 179.27 & <.0001 \\ \text { Sampling Seq. } & -0.057269 & 0.002837 & -20.19 & <.0001\end{array}$ 
Exhibit B4: Common Logarithm of Peak Area Versus Sample Sequence for Methanol Studies: Study 1; Tests 25-32

Methanol Study 1 (Test Sequence=25)

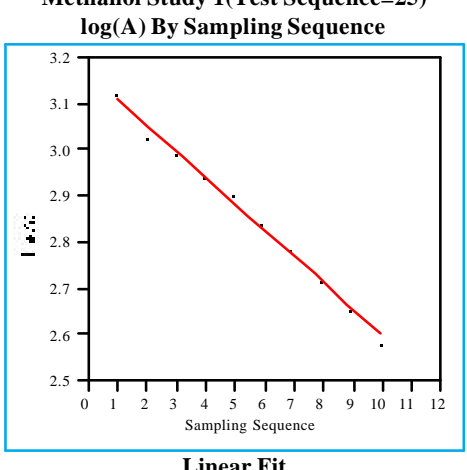

$\log (\mathrm{A})=3.16343-0.05659$ Sampling Sequence Summary of Fit

RSquare RSquare Adj

Root Mean Square Error

Mean of Response

0.992093

Observations (or Sum Wgts)

0.016223

2.852214

$\begin{array}{lrrrr}\text { Term } & \text { Estimate } & \text { Std Error } & \text { t Ratio } & \text { Prob }>|t|\end{array}$ $\begin{array}{lrrrr}\text { Sampling Seq. } & -0.056585 & 0.001786 & -31.68 & <.0001\end{array}$

Methanol Study $\mathbf{1}$ (Test Sequence $=26$ ) $\log (\mathrm{A})$ By Sampling Sequence

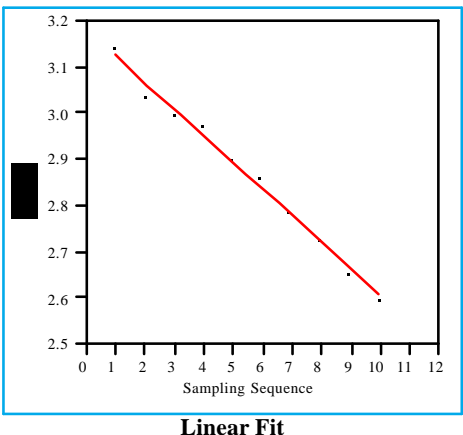

$\log (A)=3.18281-0.05782$ Sampling Sequence RSquare Summary of Fit

RSquare Adj

Root Mean Square Error

Mean of Response

0.989769

Observations (or Sum Wgts)

0.08876

2.864824

Parameter Estimates

Term Estimate Std Error t Ratio Prob $>|\mathbf{t}|$

$\begin{array}{llllll}\text { Intercept } & 3.182808 & 0.012895 & 246.83 & <.000\end{array}$ $\begin{array}{lllll}\text { Sampling Seq. } & -0.057815 & 0.002078 & -27.82 & <.000\end{array}$
Methanol Study 1(Test Sequence=27)

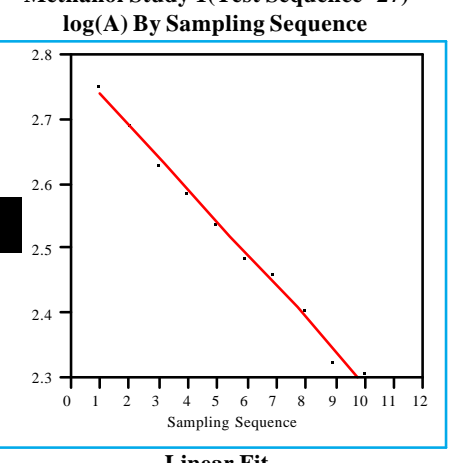

$\log (\mathrm{A})=2.78807-0.04935$ Sampling Sequence Summary of Fit

RSquare

RSquare Adj

Error

0.993722
0.992938
0.01259

Root Mean Square Error $\quad 0.012595$

Mean of Response

2.516664

Observations (or Sum Wgts)$$
\begin{array}{r}
664 \\
10
\end{array}
$$

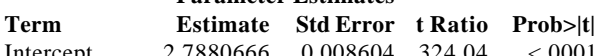
$\begin{array}{lrrrr} & 2.7880666 & 0.008604 & 324.04 & <.000 \\ \text { Sampling Seq. } & -0.049346 & 0.001387 & -35.59 & <.0001\end{array}$

Methanol Study 1 (Test Sequence=28) $\log (\mathrm{A})$ By Sampling Sequence

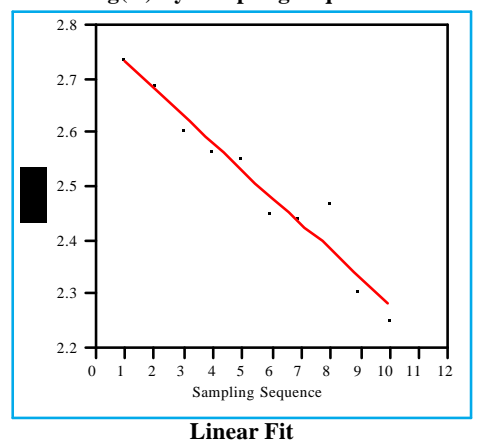

$\log (\mathrm{A})=2.78068-0.05005$ Sampling Sequence RS Summary of Fit

RSquare Adj

Root Mean Square Erro

Mean of Response

0.947796
0.94127

0.037718

Observations (or Sum Wgts)

2.505427

Parameter Estimates

Term Estimate Std Error t Ratio Prob $>|t|$

$\begin{array}{llllll}\text { Intercept } & 2.7806788 & 0.025766 & 107.92 & <.000\end{array}$

$\begin{array}{lllll}\text { Sampling Seq. } & -0.050046 & 0.004153 & -12.05 & <.0001\end{array}$
Methanol Study 1 (Test Sequence=29)

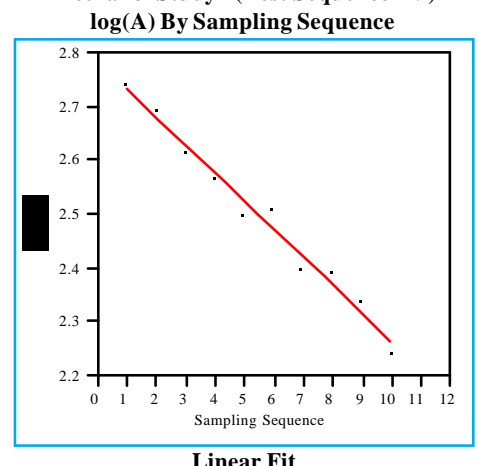

$\log (A)=2.78396-0.05211$ Sampling Sequence Summary of Fit

RSquare

RSquare Adj

Root Mean Square Error

Mean of Response

0.979238

Observations (or Sum Wgts)

0.024365

$\begin{array}{lrrrr}\text { Term } & \text { Estimate } & \text { Std Error } & \text { t Ratio } & \text { Prob }>|t|\end{array}$ $\begin{array}{lrrrrr} & 2.7839577 & 0.016644 & 167.26 & <.0001 \\ \text { Sampling Seq. } & -0.052107 & 0.002682 & -19.42 & <.0001\end{array}$

Methanol Study 1(Test Sequence=30) $\log (\mathrm{A})$ By Sampling Sequence

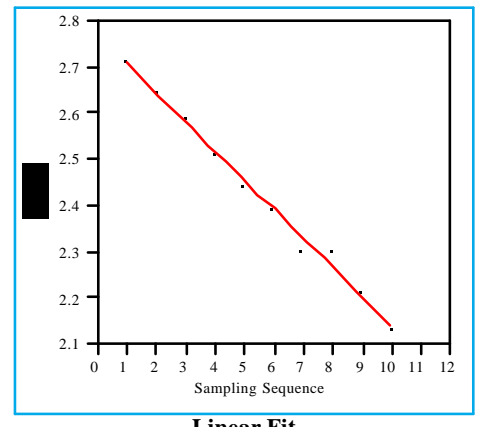

$\log (A)=2.76989-0.06307$ Sampling Sequence RSquare Summary of F

\section{RSquare Adj}

Root Mean Square Erro

Mean of Response

0.992847

0.01719

Observations (or Sum Wgts)

2.42303

Term Estimate Std Error t Ratio Prob $>|\mathbf{t}|$

$\begin{array}{lrrrrr}\text { Intercept } & 2.7698881 & 0.011743 & 235.88 & <.0001\end{array}$

$\begin{array}{lllll}\text { Sampling Seq. } & -0.063065 & 0.001893 & -33.32 & <.0001\end{array}$
Methanol Study 1(Test Sequence=31) $\log (A)$ By Sampling Sequence

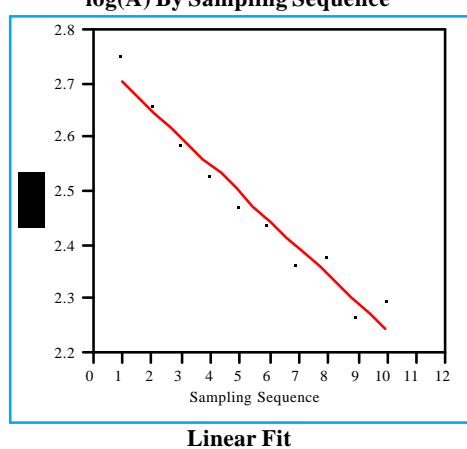

$\log (\mathrm{A})=2.75138-0.05089$ Sampling Sequence RSquare

RSquare Adj

Summary of Fit

0.955307

Root Mean Square Error

0.949721

Mean of Response

2.47147

Observations (or Sum Wgts)
Parameter Estimates

$\begin{array}{lrrrr}\text { Term } & \text { Estimate } & \text { Std Error } & \text { t Ratio } & \text { Prob }>|t|\end{array}$ $\begin{array}{lllll}\text { Sampling Seq. } & -0.050894 & 0.003892 & -13.08 & <.0001\end{array}$

Methanol Study 1(Test Sequence=32) $\log (\mathrm{A})$ By Sampling Sequence

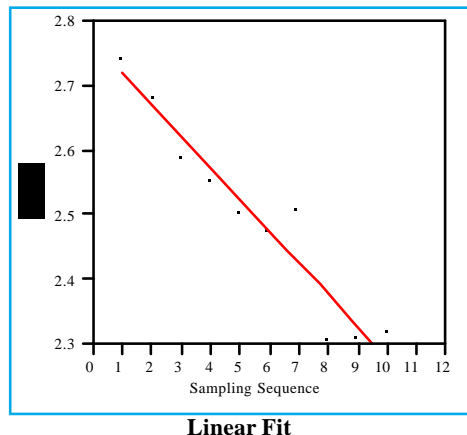

$\log (A)=2.76661-0.04865$ Sampling Sequence RSquare Summary of Fit

RSquare Adj

Root Mean Square Erro

Mean of Response

0.923839

Observations (or Sum Wgts)

0.044857

2.499037

Parameter Estimate

Term Estimate Std Error t Ratio Prob $>|\mathbf{t}|$ $\begin{array}{lrrrr}\text { Intercept } & 2.7666118 & 0.030643 & 90.28 & <.0001 \\ \text { Sampling Seq. } & -0.04865 & 0.004939 & -9.85 & <.0001\end{array}$ 
Exhibit B5: Common Logarithm of Peak Area Versus Sample Sequence for Methanol Studies: Study 1; Tests 33-40

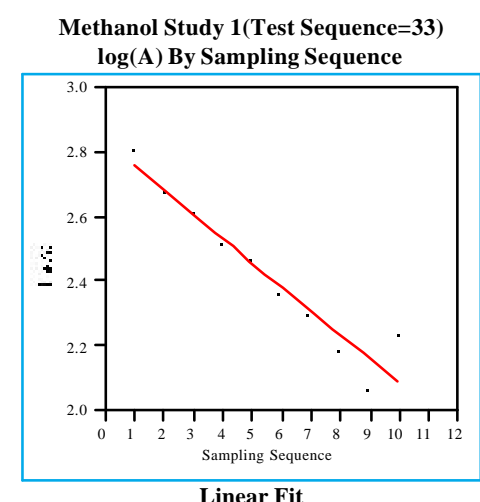

Linear Fit
$\log (\mathrm{A})=2.82942-0.07447$ Sampling Sequence

$\begin{array}{lr}\log (\mathrm{A})= & 2.82942-0.07447 \text { Sampling Sequence } \\ \text { RSquare } & \text { Summary of Fit }\end{array}$

RSquare Adj

Root Mean Square Error

0.923662
0.91412
0.068753

Mean of Response

2.419825

Observations (or Sum Wgts)

10

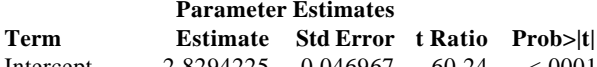
$\begin{array}{lrrrr}\text { Intercept } & 2.8294225 & 0.046967 & 60.24 & <.0001 \\ \text { Sampling Seq. } & -0.074472 & 0.007569 & -9.84 & <.0001\end{array}$

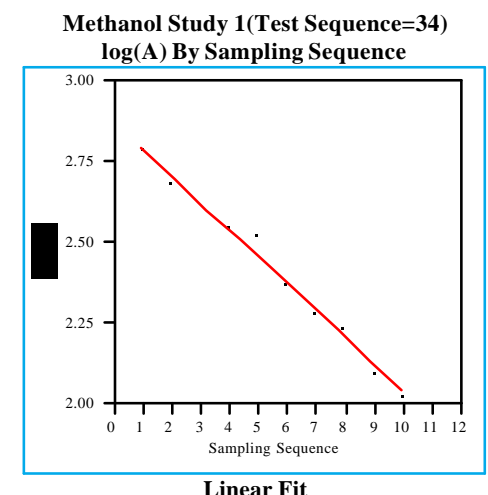

$\log (\mathrm{A})=2.87107-0.08344$ Sampling Sequence

RSquare Summary of Fit

RSquare Adj

0.988142

Root Mean Square Error

0.98665

Mean of Response

0.029354
2.412146

Observations (or Sum Wgts)

10

Parameter Estimates

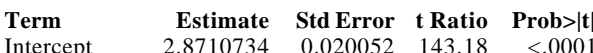
$\begin{array}{lrrrr} & 2.8710734 & 0.020052 & 143.18 & <.0001 \\ \text { Sampling Seq. } & -0.083441 & 0.003232 & -25.82 & <.0001\end{array}$
Methanol Study 1 (Test Sequence=35) $\log (\mathrm{A})$ By Sampling Sequence

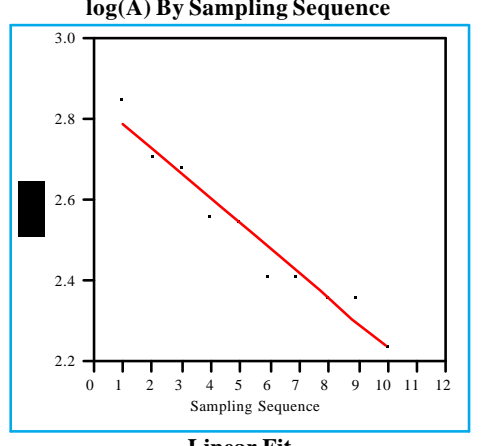

Linear Fit
$\log (\mathrm{A})=2.84946-0.06143$ Sampling Sequence

$\log (\mathrm{A})=2.84946-0.06143$ Sampling Sequence
RSquare

0.95613
0.950646

$\begin{array}{ll}\text { RSquare Adj } & 0.950646 \\ \text { Root Mean Square Error } & 0.042255 \\ \end{array}$

2.511603

Observations (or Sum Wgts)

$$
10
$$

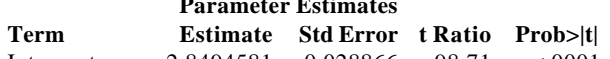
$\begin{array}{llllll}\text { Intercept } & 2.8494581 & 0.028866 & 98.71 & <.0001\end{array}$

Sampling Seq. $-0.061428 \quad 0.004652 \quad-13.20$

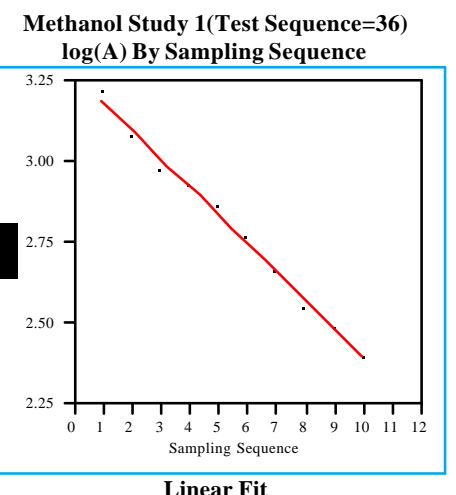

$\log (\mathrm{A})=3.27226-0.08805$ Sampling Sequence

RSquare Summary of Fit

$\begin{array}{ll} & 0.993652 \\ \text { RSquare Adj } & 0.992858\end{array}$

Root Mean Square Error

Mean of Response

0.0226
2.787996

Observations (or Sum Wgts)

10

Term $\quad$ Parameter Estimates

$\begin{array}{lrrrr}\text { Term } & \text { Estimate } & \text { Std Error } & \text { t Ratio } & \text { Prob }>|t| \\ \text { Intercept } & 3.2722566 & 0.015438 & 211.95 & <.0001\end{array}$ $\begin{array}{lllll}\text { Sampling Seq. } & -0.088047 & 0.002488 & -35.39 & <.0001\end{array}$
Methanol Study 1 (Test Sequence=37)

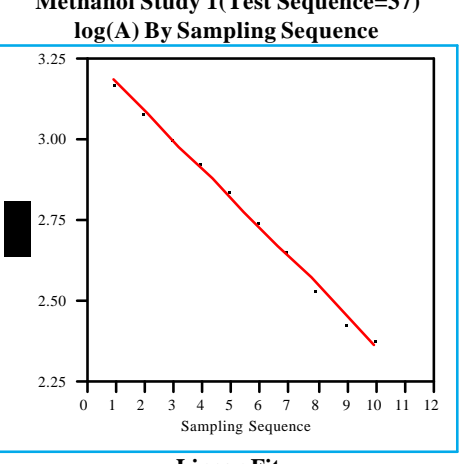

Linear Fit
$\log (\mathrm{A})=3.26898-0.09061$ Sampling Sequence

\begin{tabular}{ll}
$\log (\mathrm{A})=$ & \multicolumn{2}{c}{ Summary of Fit } \\
RSquare & 0.99509
\end{tabular}

$\begin{array}{lr}\text { RSquare } & 0.99509 \\ \text { RSquare Adj } & 0.994476\end{array}$

Root Mean Square Error $\quad 0.02043$

Observations (or Sum Wgts)

.

Term $\quad \begin{aligned} & \text { Parameter Estimates } \\ & \text { Estimate }\end{aligned}$ Std Error t Ratio Prob $>|t|$

$\begin{array}{lrrrrr}\text { Term } & \text { Estimate } & \text { Std Error } & \text { t Ratio } & \text { Prob }>|\mathbf{t}| \\ \text { Intercept } & 3.2689774 & 0.013963 & 234.12 & <.0001\end{array}$ $\begin{array}{lrrrr}\text { Sampling Seq. } & -0.090607 & 0.00225 & -40.26 & <.0001\end{array}$

Methanol Study 1 (Test Sequence=38) $\log (\mathrm{A})$ By Sampling Sequence

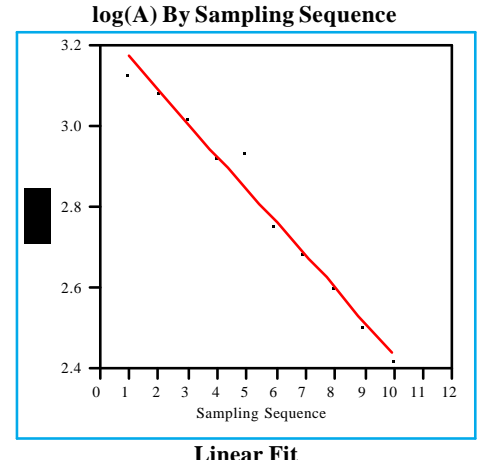

$\log (\mathrm{A})=3.25109-0.08139$ Sampling Sequence Summary of Fit

RSquare

are Error

Root Mean Square Erro

Mean of Response

0.980447
0.978003
0.03691

Observations (or

2.8034

Term Parameter Estimates atio Prob $>$ t

$\begin{array}{lrrrr}\text { Estimate } & \text { Std Error } & \text { t Ratio } & \text { Prob }>|t| \\ \text { Intercept } & 3.2510861 & 0.025214 & 128.94 & <.0001\end{array}$ $\begin{array}{lllll}\text { Sampling Seq. } & -0.08139 & 0.004064 & -20.03 & <.0001\end{array}$
Methanol Study 1(Test Sequence=39) $\log ($ A) By Sampling Sequence

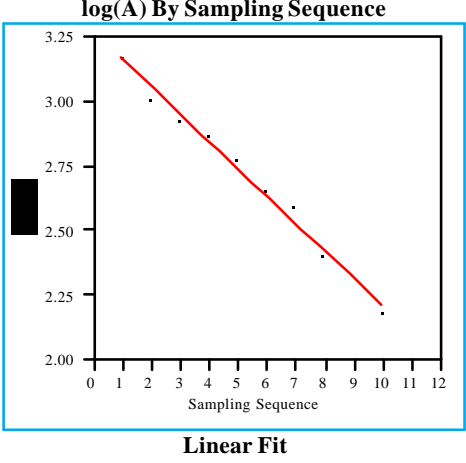

Linear Fit
$\log (A)=3.2688-0.10633$ Sampling Sequence

$$
\text { Summary of Fit }
$$

RSquare

RSquare Adj

0.986471
0.98478

Root Mean Square Erro

0.98478

Observations (or Sum Wgts)

2.684011

Observations (or Sum Wgts)

$\begin{array}{lllll}\text { Term } & \text { Parameter Estimates } & & \\ \text { Estimate } & \text { Std Error } & \text { t Ratio } & \text { Prob }>|t|\end{array}$

$\begin{array}{lllll}0.027316 & 119.67 & <.000\end{array}$

Methanol Study 1(Test Sequence=40)

Metha( Study $1($ Test Sequence $=40)$

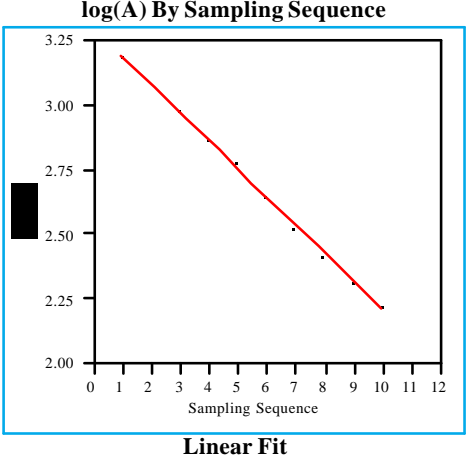

$\log (\mathrm{A})=3.29592-0.10903$ Sampling Sequence

RSquare Summary of Fit

RSquare Adj

Root Mean Square Error

Mean of Response
Observations (or Sum Wgts)

0.998887

0.998748

2.696277

Parameter Estimates

Term Estimate Std Error t Ratio Prob $>|\mathbf{t}|$

$\begin{array}{lrrrr}\text { Intercept } & 3.2959217 & 0.007983 & 412.86 & <.0001 \\ \text { Sampling Seq. } & -0.109026 & 0.001287 & -84.74 & <.0001\end{array}$ 
Exhibit B6: Common Logarithm of Peak Area Versus Sample Sequence for Methanol Studies: Study 1; Tests 41-44; Study 2; Tests 1-4

Methanol Study 1 (Test Sequence=41)

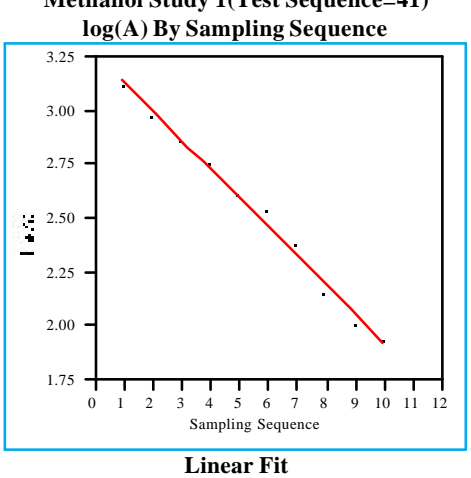

$\log (A)=3.26724-0.13491$ Sampling Sequence

RSquare

Summary of Fit

$\begin{array}{lr}\text { RSquare Adj } & 0.989358 \\ \text { Root Mean Square Error } & 0.042338 \\ \text { Mean of Response } & 2.525232 \\ \text { Observations (or Sum Wgts) } & 10\end{array}$

Parameter Estimates

Term Estimate Std Error t Ratio Prob $>|t|$ $\begin{array}{lrrrrr} & 3.2672383 & 0.028922 & 112.97 & <.0001 \\ \text { Sampling Seq. } & -0.13491 & 0.004661 & -28.94 & <.0001\end{array}$

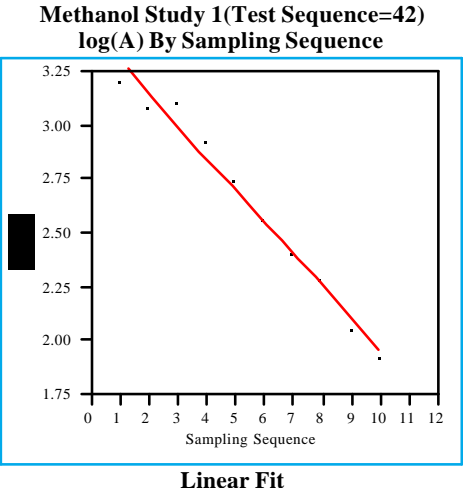

$\log (\mathrm{A})=3.44113-0.14894$ Sampling Sequence

RSquare

Summary of Fit

RSquare Adj

Root Mean Square Error

Mean of Respons

0.980144

Observations (or Sum Wgts)

0.068075

2.621977

$\begin{array}{lrrrr} & \text { Parameter Estimates } & & \\ \text { Term } & \text { Estimate } & \text { Std Error } & \text { t Ratio } & \text { Prob }>|\mathbf{t}| \\ \text { Intercept } & 3.4411344 & 0.046504 & 74.00 & <.0001\end{array}$ $\begin{array}{lrrrr}\text { Intercept } & 3.4411344 & 0.046504 & 74.00 & <.0001 \\ \text { Sampling Seq. } & -0.148938 & 0.007495 & -19.87 & <.0001\end{array}$
Methanol Study 1(Test Sequence=43)

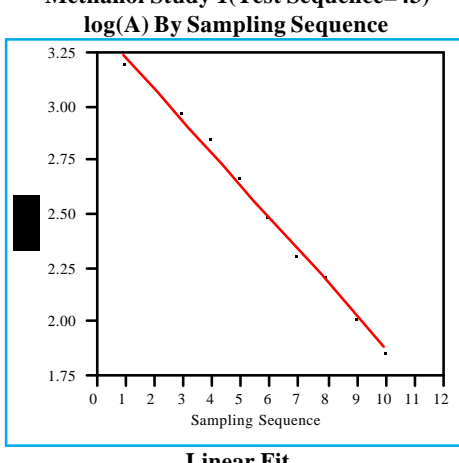

$\log (\mathrm{A})=3.38362-0.15039$ Sampling Sequence Summary of Fit

RSquare

0.992643

RSquare Adj 0.991723

Root Mean Square Error $\quad 0.041579$

$\begin{array}{lr}\text { Mean of Response } & 2.55646 \\ \text { Observations (or Sum Wgts) } & 10\end{array}$

$$
\begin{array}{r}
55646 \\
10
\end{array}
$$

Term Estimate $\quad$ Std Error t Ratio Prob $>|t|$ $\begin{array}{lllll}\text { Intercept } & 3.3836175 & 0.028404 & 119.13 & <.0001 \\ \text { Sampling Seq } & -0.150392 & 0.004578 & -32.85 & <.0001\end{array}$

\section{Methanol Study 1 (Test Sequence=44)} $\log (\mathrm{A})$ By Sampling Sequence

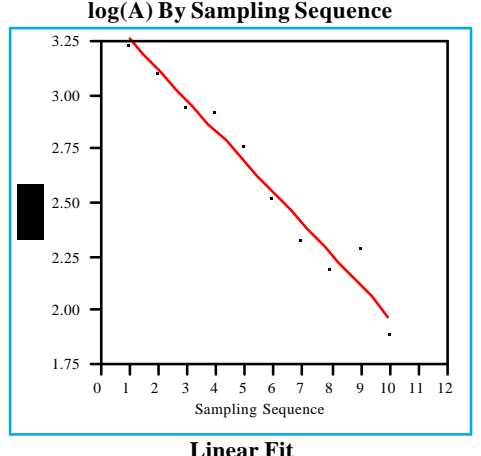

$\log (A)=3.40643-0.14359$ Sampling Sequence BSquate Summary of Fit

Root Mean Square Error

Mean of Response

0.964379

0.088624 2.616659

Parameter Estimates

Intercept Estimate Std Error t Ratio Prob $>|\mathbf{t}|$ \begin{tabular}{llllll} 
Intercept & 3.4064287 & 0.060542 & 56.27 & $<.0001$ \\
\hline Sampling & & &
\end{tabular} $\begin{array}{lllll}\text { Sampling Seq. } & -0.143594 & 0.009757 & -14.72 & <.0001\end{array}$
Methanol Study $2($ Test Sequence $=1)$

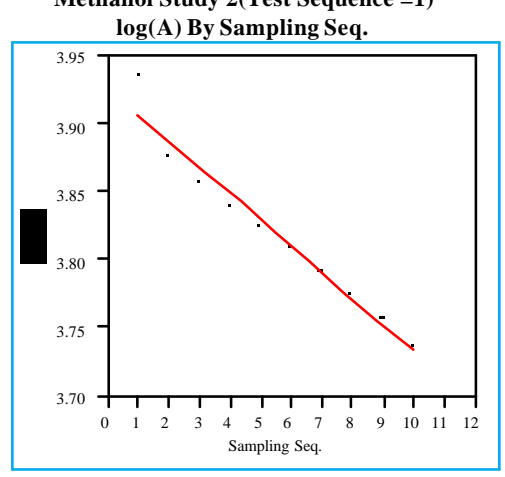

Linear $\mathbf{F}$

$\log (A)=3.92686-0.01931$ Sampling Seq Summary of Fit

\section{RSquare}

Root Mean Square Error

Mean of Response

0.96033

0.955371

0.01260

Observations (or Sum Wgts)

3.820682
10

Parameter Estimates

Term Estimate Std Error t Ratio Prob $>\mid \mathbf{t}$ $\begin{array}{lllllll}\text { Intercept } & 3.9268637 & 0.008608 & 456.20 & <.0001\end{array}$ $\begin{array}{llllll}\text { Sampling Seq. } & -0.019306 & 0.001387 & -13.92 & <.0001\end{array}$

Methanol Study 2 (Test Sequence $=2$ )

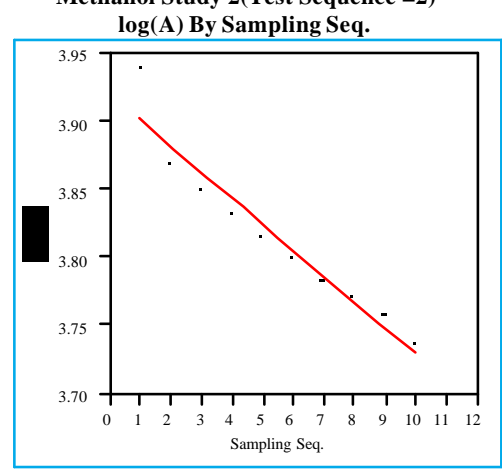

Linear Fit
$\log (A)=3.92072-0.0191$ Sampling Sec

Square

Summary of Fit

0.926779

RSquare Adj

Root Mean Square Erro

Mean of Response

0.01723

Observations (or Sum Wgts)

8.815675

$\begin{array}{lrrrr}\text { Term } & \text { Estimate } & \text { Std Error } & \text { t Ratio } & \text { Prob }>|\mathbf{t}| \\ \text { Intercept } & 3.9207153 & 0.011776 & 332.93 & <.0001\end{array}$ Sampling Seq. $\quad-0.019098 \quad 0.001898 \quad-10.06 \quad<.0001$
Methanol Study $2($ Test Sequence $=3$ )

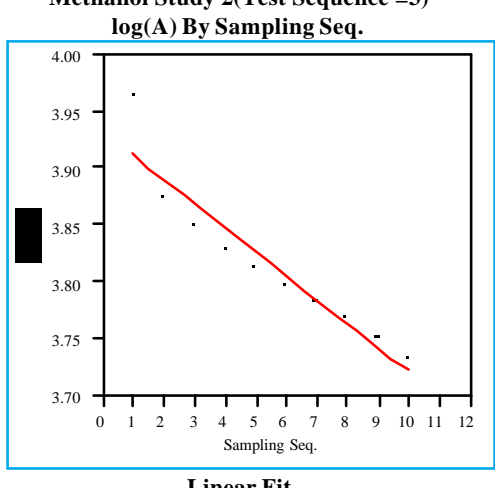

$\log (\mathrm{A})=3.9334-0.02125$ Sampling Sec Summary of Fit

RSquare

RSquare Adj

Root Mean Square Error

Mean of Response

0.898774

0.88612

0.022906

.816504

Observations (or Sum Wgts)

Term Estimate Std Error t Ratio Prob $>|\mathbf{t}|$

$\begin{array}{llllll}\text { Intercept } & 3.9334028 & 0.015648 & 251.37 & <.0001\end{array}$ $\begin{array}{lrrrr}\text { Sampling Seq. } & -0.021254 & 0.002522 & -8.43 & <.0001\end{array}$

Methanol Study 2 (Test Sequence $=4$ )

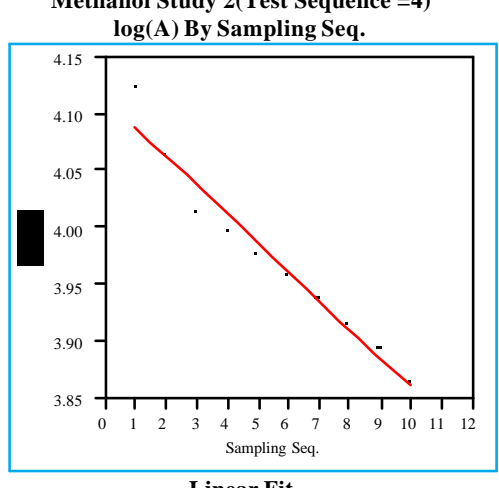
Linear Fit
$\log (\mathrm{A})=4.11471-0.02549$ Sampling Seq.
Summary of Fit

RSquare

Root Mean Square Error

0.954854

Mean of Response

0.016738

Observations (or Sum Wgts)

$\begin{array}{llll}\text { Term Estimate Std Error t Ratio Prob }>|t| & \end{array}$ $\begin{array}{llllll} & 4.1147122 & 0.011434 & 359.87 & <.0001\end{array}$ 
Exhibit B7: Common Logarithm of Peak Area Versus Sample Sequence for Methanol Studies: Study 2; Tests 5-12

Methanol Study 2 (Test Sequence =5)

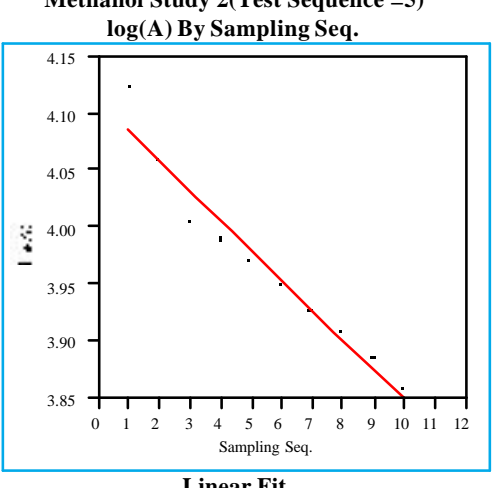

$\log (\mathrm{A})=4.11169-0.02624$ Sampling Seq. RSquare Summary of Fit

RSquare Adj

Root Mean Square Error

0.952451

Mean of Response

0.018826

3.967385

Observations (or Sum Wgts) 10

\begin{tabular}{lrrrr} 
Term & Parameter Estimates & & \\
Intercept & 4.1116929 & 0.012861 & 319.71 & $<$ \\
\hline
\end{tabular} $\begin{array}{lrrrr}\text { Intercept } & 4.1116929 & 0.012861 & 319.71 & <.0001 \\ \text { Sampling Seq. } & -0.026238 & 0.002073 & -12.66 & <.0001\end{array}$

Methanol Study 2 (Test Sequence =6)

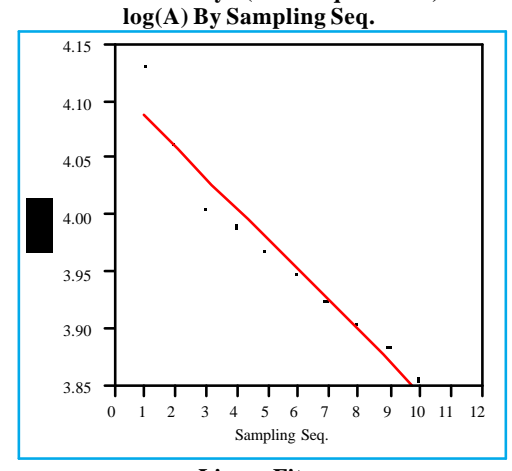

Linear Fit
$\log (\mathrm{A})=4.11656-0.02712$ Sampling Seq.

$\begin{array}{ll}\text { RSquare } \quad \text { Summary of Fit } & 0.946344 \\ \text { RSquare Adj } & 0.939637 \\ \text { Root Mean Square Error } & 0.020739 \\ \text { Mean of Response } & 3.967384\end{array}$

Mean of Response

Observations (or Sum Wgts)

0.939637

3.967384

Parameter Estimates

Term Estimate Std Error t Ratio Prob $>|\mathbf{t}|$ $\begin{array}{lrrrrr}\text { Intercept } & 4.1165576 & 0.014168 & 290.56 & <.0001 \\ \text { Sampling Seq. } & -0.027123 & 0.002283 & -11.88 & <.0001\end{array}$
Methanol Study 2(Test Sequence $=7$ )

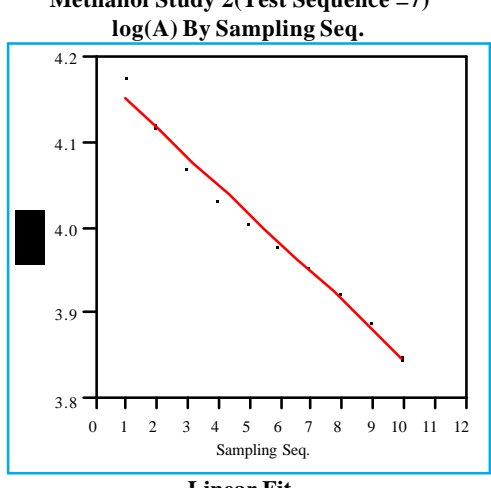

Linear Fit

$\log (\mathrm{A})=4.18538-0.03392$ Sampling Seq.

\begin{tabular}{lr} 
RSquare $\quad$ Summary of Fit & \\
RSquare Adj & 0.985143 \\
Root Mean Square Error & 0.983286 \\
Mean of Response & 0.013377 \\
Observations (or Sum Wots) & 3.998818 \\
\hline
\end{tabular}

Mean of Response

Observations (or Sum Wgts)

983286

998818

$\begin{array}{lllll}\text { Term } & \text { Parameter Estimates } & & \\ \text { Estimate } & \text { Std Error } & \text { t Ratio } & \text { Prob }>|t|\end{array}$ $\begin{array}{lrrrrr}\text { Intercept } & 4.1853795 & 0.009138 & 458.00 & <.0001 \\ \text { Sampling Seq. } & -0.03392 & 0.001473 & -23.03 & <.0001\end{array}$

Methanol Study $2($ Test Sequence $=8$ )

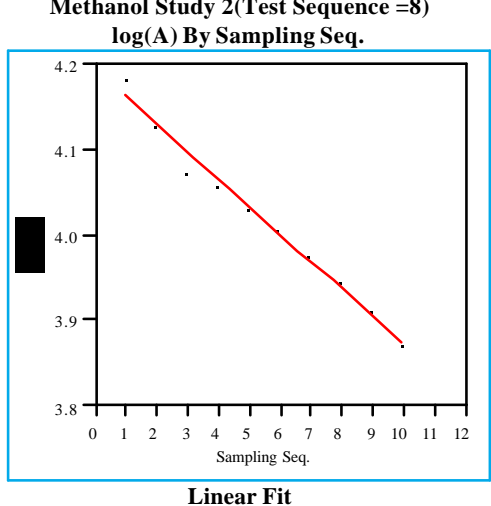

$\log (\mathrm{A})=4.19222-0.03193$ Sampling Seq RSquare

RSquare Adj ummary of Fit

Mean of Respare Erro

Mean of Response
Observations (or Sum Wgts)

0.98555

0.012417

4.016579

Parameter Estimates

Term Estimate Std Error t Ratio Prob $>|t|$

$\begin{array}{lrrrr}\text { Intercept } & 4.1922178 & 0.008483 & 494.21 & <.0001 \\ \text { Sampling Seq. } & -0.031934 & 0.001367 & -23.36 & <.0001\end{array}$
Methanol Study 2(Test Sequence =9)

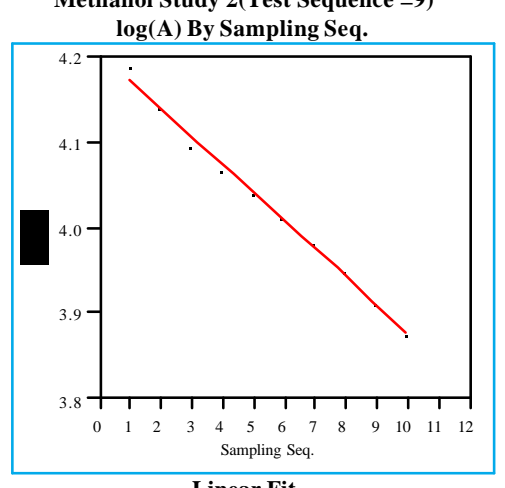

Linear Fit

$\log (A)=4.20469-0.0328$ Sampling Seq \begin{tabular}{ll}
\multicolumn{1}{c}{ Summary of Fit } & \\
RSquare & 0.993216 \\
RSquare Adj & 0.992368 \\
Root Mean Square Error & 0.008705 \\
Mean of Response & 4.024305 \\
\hline
\end{tabular}

Observations (or Sum Wgts)

Parameter Estimates

$\begin{array}{lllll}\text { Term } & \text { Estimate } & \text { Std Error } & \text { t Ratio } & \text { Prob }>|t|\end{array}$ $\begin{array}{lrrrrr} & \text { Estercept } & 4.204695 & 0.005947 & 707.08 & <.0001 \\ \text { Sampling Seq. } & -0.032798 & 0.000958 & -34.22 & <.0001\end{array}$

Methanol Study 2 (Test Sequence $=10$ )

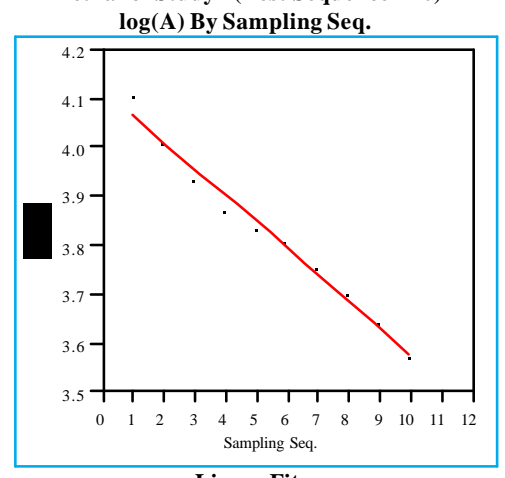

$\log (A)=4.11912-0.05422$ Sampling Seq RSquare Summary of Fit

RSquare Ad

Root Mean Square Erro

Mean of Response

0.985674

bservations (or Sum Wgts)

0.020991

Parameter Estimates

Term Estimate Std Error t Ratio Prob $>|t|$ $\begin{array}{lrrrrr}\text { Intercept } & 4.119116 & 0.014339 & 287.26 & <.0001 \\ \text { Sampling } & -0.054219 & 0.002311 & -23.46 & <.0001\end{array}$ $\begin{array}{lllll} & -1.054219 & 0.002311 & -23.46 & <.0001\end{array}$
Methanol Study $2($ Test Sequence $=11)$

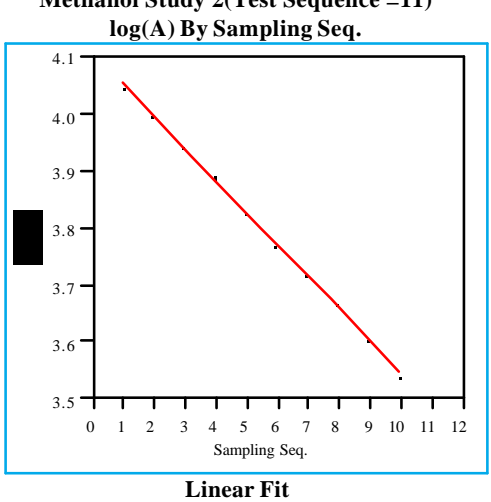

$\log (\mathrm{A})=4.10569-0.05599 \mathrm{~S}$

99 Sampling Seq.

$\begin{array}{lr}\text { RSquare } & 0.999001 \\ \text { RSquare Adj } & 0.998876 \\ \text { Root Mean Square Error } & 0.005685 \\ \text { Mean of Response } & 3.797765 \\ \text { Observations (or Sum Wots) } & 10\end{array}$

Mean of Response

Observations (or Sum Wgts)

$\begin{array}{lrrrr}\text { Term } & \text { Estimate } & \text { Std Error } & \text { t Ratio } & \text { Prob }>|t|\end{array}$ $\begin{array}{lrrrr}\text { Intercept } & 4.1056865 & 0.003883 & 1057.3 & <.0001 \\ \text { Sampling Seq. } & -0.055986 & 0.000626 & -89.45 & <.0001\end{array}$

Methanol Study 2(Test Sequence $=12$ )

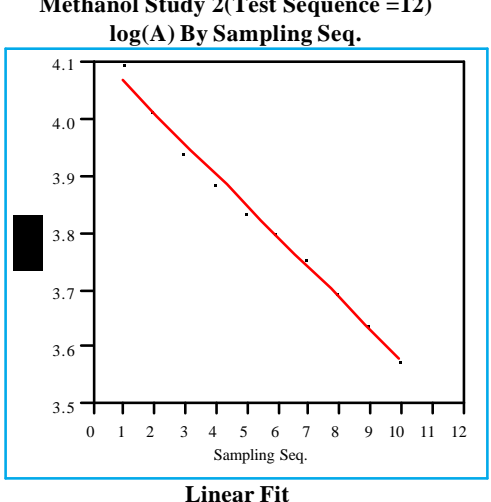

$\log (A)=4.12056-0.05433$ Sampling Seq.

RSquare

RSquare Adj

Summary of Fit

Root Mean Square Error

Mean of Response

0.991756 0.990726 3.821732

\section{Parameter Estimates}

$\begin{array}{lrrrr}\text { Term } & \text { Estimate } & \text { Std Error } & \text { t Ratio } & \text { Prob }>|\mathbf{t}|\end{array}$ $\begin{array}{lrrrrr}\text { Intercept } & 4.1205614 & 0.010867 & 379.18 & <.0001 \\ \text { Sampling Seq. } & -0.054333 & 0.001751 & -31.02 & <.0001\end{array}$ 
Exhibit B8: Common Logarithm of Peak Area Versus Sample Sequence for Methanol Studies: Study 2; Tests 13-15; Study 3; Tests 1-5

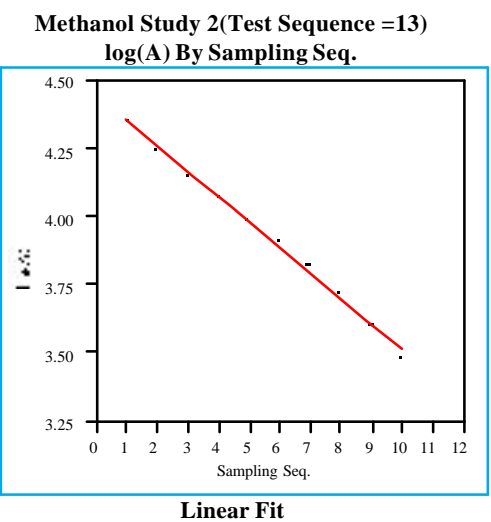

$\log (A)=4.45195-0.09343$ Sampling Seq.

RSquare$$
\text { Summary of Fit }
$$

$\begin{array}{ll}\text { RSquare } & 0.99536 \\ \text { RSquare Adj } & 0.092783\end{array}$

Root Mean Square Error

0.99536

Mean of Response

0.020483

Observations (or Sum Wgts)

3.938074

$\begin{array}{lllll} & \text { Parameter Estimates } & & \\ \text { Term } & \text { Estimate } & \text { Std Error } & \text { t Ratio } & \text { Prob }>|t|\end{array}$ $\begin{array}{llllll}\text { Intercept } & 4.4519504 & 0.013992 & 318.17 & <.0001 \\ \text { Sampling Seq. } & -0.093432 & 0.002255 & -41.43 & <.0001\end{array}$

Methanol Study 2 (Test Sequence $=14$ )

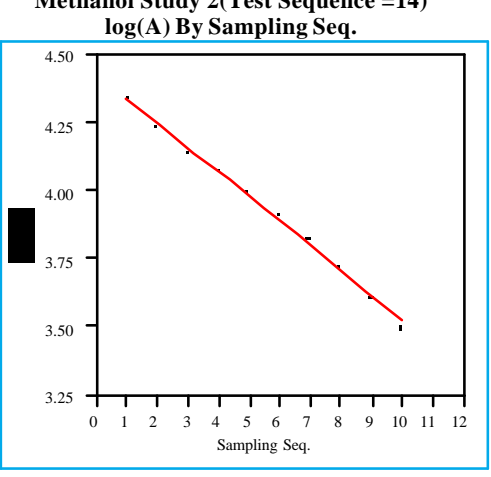

Linear Fit

$\log (\mathrm{A})=4.43886-0.09097$ Sampling Seq.

$\begin{array}{ll}\text { RSquare } & \text { Summary of Fit } \\ \text { RSquare Adj } & 0.995696 \\ \text { Root Mean Square Error } & 0.995158 \\ \text { Mean of Response } & 0.019207 \\ \text { O } & 3.938509\end{array}$

Root Mean Square Enor

0.995158

Observations (or Sum Wgts)

$\begin{array}{lrrrrr} & \text { Parameter Estimates } & & \\ \text { Term } & \text { Estimate } & \text { Std Error } & \text { t Ratio } & \text { Prob }>|\mathbf{t}| \\ \text { Intercept } & 4.4388609 & 0.013121 & 338.31 & <.0001\end{array}$ $\begin{array}{lrrrr}\text { Intercept } & 4.4388609 & 0.013121 & 338.31 & <.0001 \\ \text { Sampling Seq. } & -0.090973 & 0.002115 & -43.02 & <.0001\end{array}$
Methanol Study 2 (Test Sequence $=15$ )

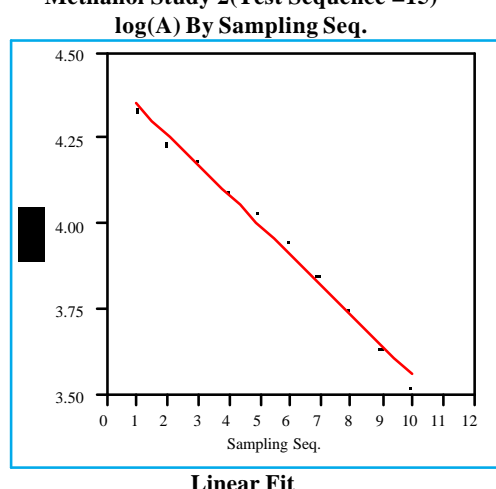

$\log (\mathrm{A})=4.44126-0.08823$ Sampling Seq$$
\text { Summary of Fit }
$$

RSquare

Summary of Fit

0.990328

RSquare Adj

Mean of Response

0.989119

Observations (or Sum Wgts)

3.956008

\begin{tabular}{lrrrr}
\multicolumn{5}{c}{} \\
Term & Parameter Estimates & & \\
Intercept & 4.4412639 & 0.019128 & 232.19 & $<.0001$ \\
Sampling Seq. & -0.088228 & 0.003083 & -28.62 & $<.0001$
\end{tabular}

Methanol Study 3(Test Sequence=1)

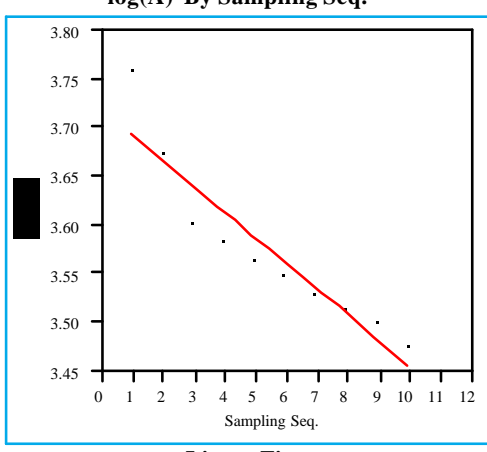

$\log (\mathrm{A})=3.72082-0$.

$\log (\mathrm{A})=3.72082-0.02661$ Sampling Seq

RSquare Summary of Fit

RSquare Adj
Root Mean Square Error

Mean of Response

Observations (or Sum Wgts)

0.876043
0.860548

Parameter Estimates

Term Estimate Std Error t Ratio Prob $>\mid \mathbf{t}$

$\begin{array}{lrrrrr} & 3.720822 & 0.021957 & 169.46 & <.0001 \\ & \text { Intercept } & -0.001539 & -7.52 & <.001\end{array}$

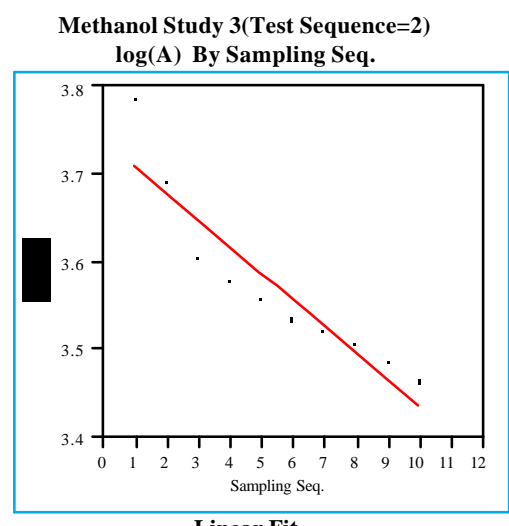

Linear Fit
$\log (\mathrm{A})=3.73923-0.0305$ Sampling Seq \begin{tabular}{lr}
\multicolumn{2}{c}{ Summary of Fit } \\
RSquare & 0.865387 \\
RSquare Adj & 0.84856 \\
Root Mean Square Error & 0.03863 \\
Mean of Response & 3.571475 \\
Observations (or Sum Wgts) & 10
\end{tabular}

\begin{tabular}{lrrrr} 
& \multicolumn{2}{c}{ Parameter Estimates } & & \\
Term & Estimate & Std Error & t Ratio & Prob $>|\mathbf{t}|$ \\
Intercept & 3.7392255 & 0.026389 & 141.70 & $<.0001$
\end{tabular} $\begin{array}{lrrrr}\text { Intercept } & 3.7392255 & 0.026389 & 141.70 & <.0001 \\ \text { Sampling Seq. } & -0.0305 & 0.004253 & -7.17 & <.0001\end{array}$

Methanol Study 3(Test Sequence=3)

Methanol Study 3(Test Sequence
$\log ($ A) By Sampling Seq.

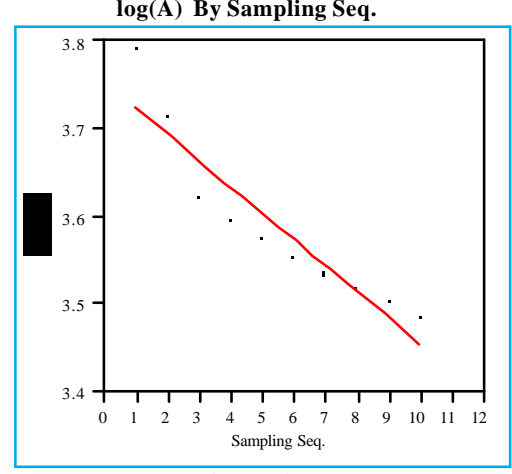

Linear Fit

$\log (\mathrm{A})=3.75459-0.03014$ Sampling Seq$$
\text { Square }
$$$$
\text { Summary of Fit }
$$

0.873818

RSquare
RSquare Adj

Root Mean Square Error

0.85804

Root Mean Square

Observations (or Sum Wgts)

Parameter Estimates

Term Estimate Std Error t Ratio Prob $>1$

$\begin{array}{llllll}\text { Intercept } & 3.754587 & 0.025124 & 149.44 & <.0001\end{array}$

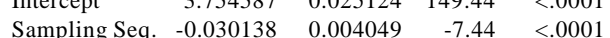

Methanol Study 3(Test Sequence $=4$ ) $\log (\mathrm{A})$ By Sampling Seq.

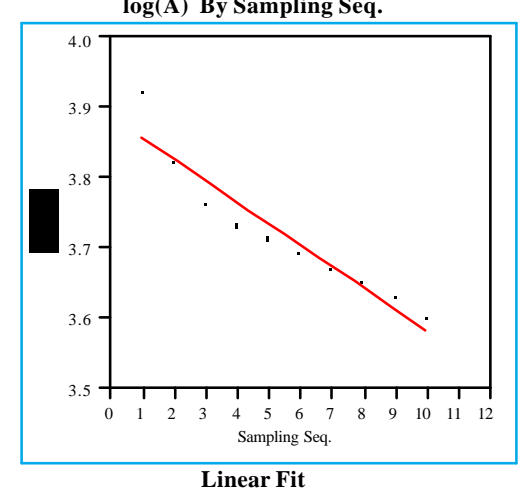

$\log (\mathrm{A})=3.88666-0.03053$ Sampling Seq RSquare

RSquare Adj Summary of Fit

0.906758 0.895103

Root Mean Square Error

Mean of Response

3.718724

Observations (or Sum Wgts)

$\begin{array}{llll} & \text { Parameter Estimates } & & \\ \text { Tertimate } & \text { Std Error } & \text { Ratio } & \text { Prob }>|t|\end{array}$ $\begin{array}{lrrrrr}\text { Intercept } & 3.8866556 & 0.021479 & 180.95 & <.000\end{array}$ $\begin{array}{lllll}\text { Sampling Seq. } & -0.030533 & 0.003462 & -8.82 & <.000\end{array}$

Methanol Study 3(Test Sequence=5)

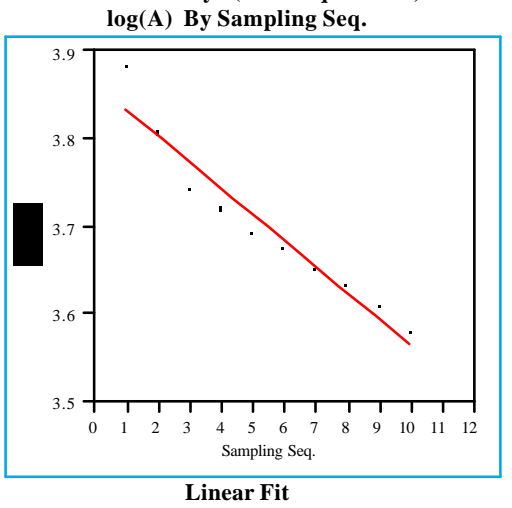

$\log (A)=\begin{gathered}3.86226-0.02971 \text { Sampling Seq. } \\ \text { Summary of Fit }\end{gathered}$

RSquare

0.93672

Root Mean Square Error

Mean of Response

0.92881

Observations (or Sum Wgts)

Parameter Estimates

Term Estimate Std Error t Ratio Prob $>|\mathbf{t}|$

Sampling Seq $-0.029713 \quad 0.00273-10.88<.0001$ 


\section{Exhibit B9: Common Logarithm of Peak Area Versus Sample Sequence for Methanol Studies: Study 3; Tests 6-13}

Methanol Study 3(Test Sequence=6)

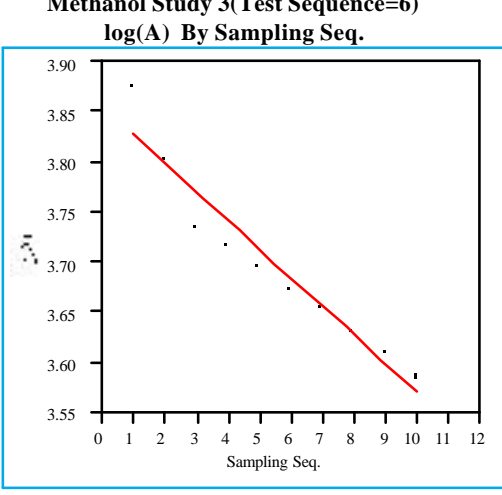

Linear Fit

Linear Fit 5 - 0.02848 Sampling Seq.

RSquare
RSquare Ad $j$

Summary of Fit

Root Mean Square Error

Mean of Response

0.92905

0.02527

3.69896

Observations (or Sum Wgts)

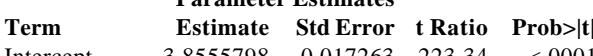

$\begin{array}{lllll}3.855798 & 0.017263 & 223.34 & <.0001\end{array}$

Methanol Study 3(Test Sequence=7)

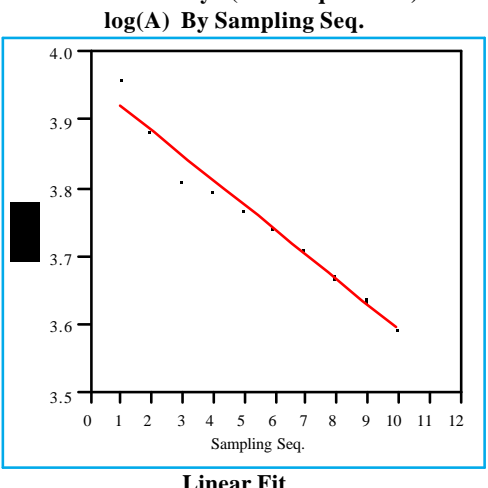

Linear Fit
$\log (A)=3.95651-0.03641$ Sampling Seq

RSquare
RSquare Adj ummary of Fit

Root Mean Square Er

Mean of Response
Observations (or Sum Wgt

0.968901

0.965014

3.75625

Parameter Estimates

10

$\begin{array}{lrrrrr}\text { Intercept } & 3.9565092 & 0.01431 & 276.49 & <.000\end{array}$ $\begin{array}{lllll}\text { Sampling Seq. } & -0.03641 & 0.002306 & -15.79 & <.0001\end{array}$
Methanol Study 3(Test Sequence=8) $\log (\mathrm{A})$ By Sampling Seq.

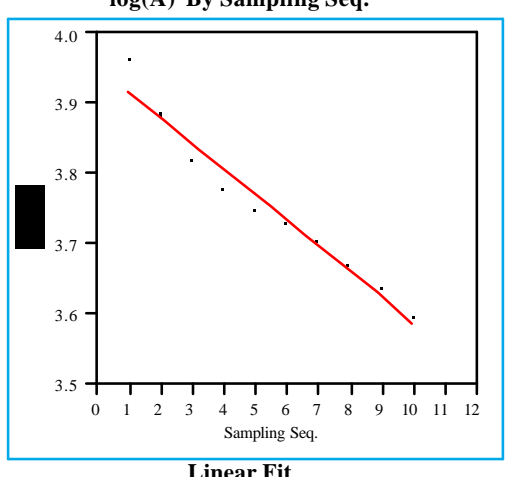

$\log (\mathrm{A})=3.95384-0.03$

S5384-0.03671 Sampling Seq

$\begin{array}{lll}\text { RSquare } & \text { Summary of Fit } & 0.959998 \\ \text { RSquare Adj } & & 0.954998\end{array}$

RSquare Adj $\quad 0.954998$

Root Mean Square Error $\quad 0.024066$

Observations (or Sum Wgts)

Parameter Estimates

Term Estimate Std Error t Ratio Prob $>|t|$ $\begin{array}{lrrrr}\text { Intercept } & 3.9538445 & 0.01644 & 240.50 & <.0001 \\ \text { Sampling Seq } & -0.036712 & 0.00265 & -13.86 & <.0001\end{array}$

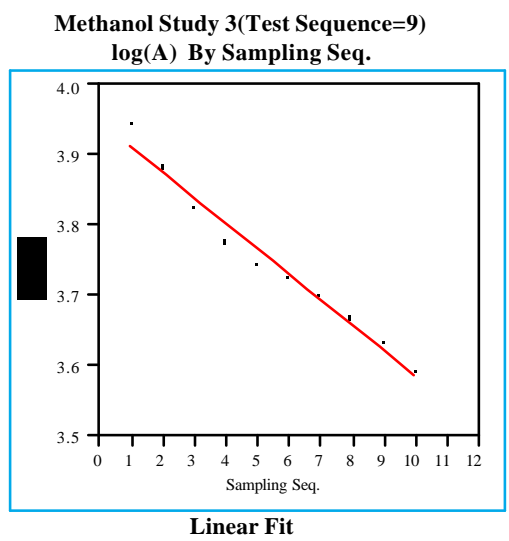

$\log (A)=3.94767-0.03625$ Sampling Seq

$\begin{array}{ll} & \\ \text { RSquare } & 0.973665 \\ \text { RSquare Adj } & 0.970373 \\ \text { Root Mean Square Error } & 0.019144 \\ \text { Mean of Response } & 3.748308\end{array}$

Mean of Response

3.748308

Observations (or Sum Wgts)

$\begin{array}{lllll}\text { Term } & \text { Estimate } & \text { Std Error } & \text { t Ratio } & \text { Prob }>|t|\end{array}$

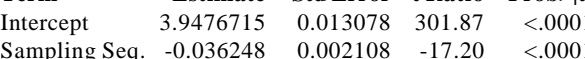

Methanol Study 3(Test Sequence $=10$ ) $\log (\mathrm{A})$ By Sampling Seq.

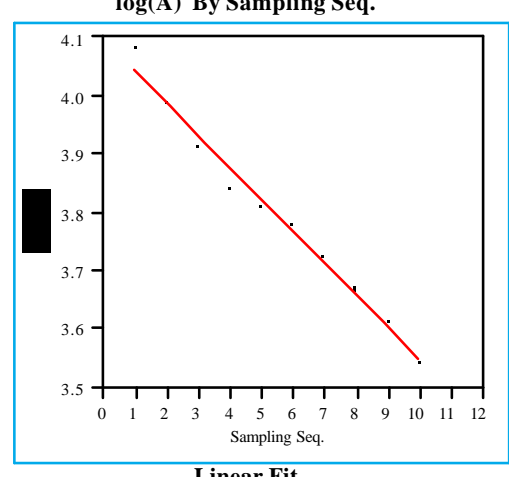

Linear Fit

$\log (A)=4.09847-0.05495$ Sampling Seq.

$\begin{array}{lr}\text { RSquare Summary of Fit } & \\ \text { RSquare Adj } & 0.983454 \\ \text { Root Mean Square Error } & 0.981385 \\ \text { Mean of Response } & 0.022889 \\ \text { Observations } & 3.796242\end{array}$

Mean of Response

.98138

Observations (or Sum Wgts) \begin{tabular}{lrrrr}
\multicolumn{5}{c}{ Parameter Estimates } \\
Term & Estimate & Std Error & t Ratio & Prob $>|\mathbf{t}|$ \\
Intercept & 4.0984682 & 0.015636 & 262.12 & $<.0001$ \\
Sampling Seq & -0.05495 & 0.00252 & -21.81 & $<.0001$
\end{tabular}

Methanol Study 3(Test Sequence=11)

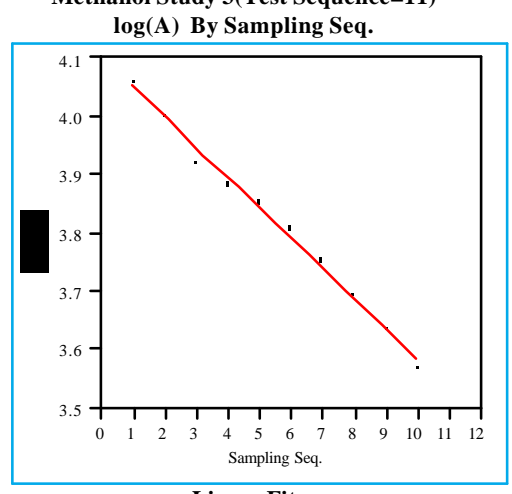

$\log (\mathrm{A})=4.1035-0.0519$ Sampling Seq

Square

$$
\text { Summary of Fit }
$$

0.992326

RSquare Adj

0.991367

3.818026

Observations (or Sum Wgts)

Term Estimate Std Error t Ratio Prob $>\mid \mathbf{t}$ $\begin{array}{lllllll}\text { Intercept } & 4.1035016 & 0.010013 & 409.82 & <.0001\end{array}$ Sampling Seq. $\begin{array}{lllll}-0.051905 & 0.001614 & -32.16 & <.0001\end{array}$
Methanol Study 3(Test Sequence=12) $\log (\mathrm{A})$ By Sampling Seq.

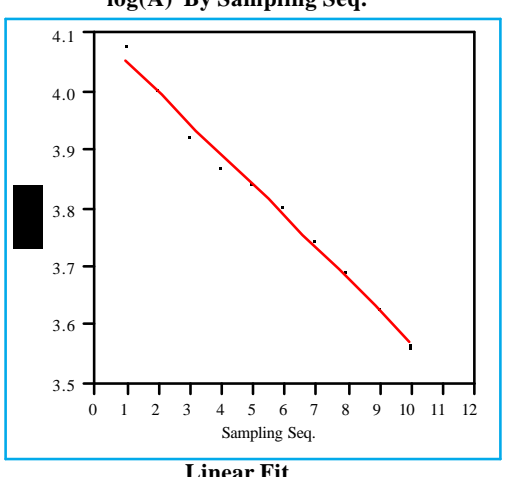

$\log (\mathrm{A})=4.10832-0 . \mathrm{d}$

361 Sampling Seq.

\begin{tabular}{ll}
\multicolumn{1}{c}{ Summary of Fit } \\
RSquare & 0.989522 \\
RSquare Adj & 0.988212 \\
Root Mean Square Error & 0.017715 \\
Mean of Response & 3.813462 \\
Obs
\end{tabular}

Mean of Response
Observations (or Sum Wgts)

Parameter Estimates

Term Estimate Std Error t Ratio Prob $>|t|$

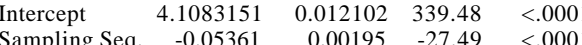

Methanol Study 3(Test Sequence=13)

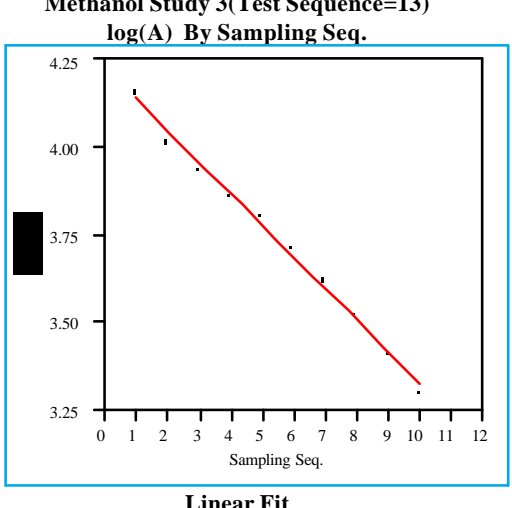

$\log (\mathrm{A})=4.23099-0.09037$ Sampling Seq.
Summary of Fit

$\begin{array}{ll}\text { RSquare } & 0.992454 \\ \text { RSquare Adj } & 0.991511\end{array}$

Root Mean Square Error $\quad 0.025305$

Mean of Response

0.025305
3.733947

Observations (or Sum Wgts)

Parameter Estimates

Term Estimate Std Error t Ratio Prob $>\mid \mathbf{t}$ $\begin{array}{llllll}\text { Intercept } & 4.2309897 & 0.017287 & 244.76 & <.0001\end{array}$ $\begin{array}{lllll}\text { Sampling Seq. } & -0.090371 & 0.002786 & -32.44 & <.0001\end{array}$ 
Exhibit B10: Common Logarithm of Peak Area Versus Sample Sequence for Methanol Studies: Study 3; Tests 14-15; Study 4; Tests 1-6

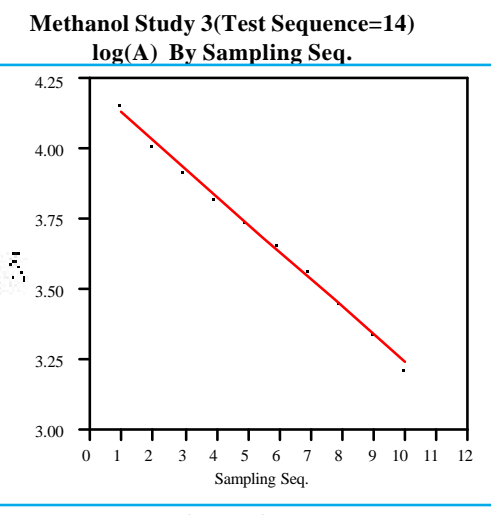

Linear Fit
$\log (\mathrm{A})=4.23156-0.09918$ Sampling Sec

$\begin{array}{lr}\text { RSquare } & \text { Summary of Fit } \\ \text { RSquare Adj } & 0.99573 \\ \text { Root Mean Square Error } & 0.995196 \\ \text { Mean of Response } & 0.020858 \\ \text { Orens } & 3.686047\end{array}$

$\begin{array}{ll}\text { Root Mean Square Error } & 0.020858 \\ \text { Mean of Response } & 3.686047\end{array}$

Observations (or Sum Wgts)

10 \begin{tabular}{lrrrr}
\multicolumn{1}{c}{ Parameter Estimates } & & \\
Term & Estimate & Std Error & t Ratio & Prob $>|\mathbf{t}|$ \\
Intercept & 4.2315566 & 0.014249 & 296.97 & $<.0001$ \\
Sampling & -0.099184 & 0.002296 & -43.19 & $<.0001$
\end{tabular} Sampling Seq $\quad-0.099184 \quad 0.002296 \quad-43.19<000$

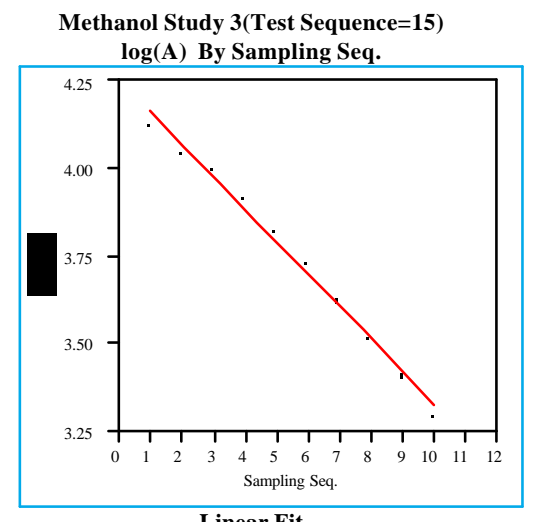

Linear Fit
$\log (\mathrm{A})=4.25397-0.09255$ Sampling Seq.

$\begin{aligned} \log (\mathrm{A})= & 4.25397-0.09255 \text { Sampling Seq. } \\ & \text { Summary of Fit }\end{aligned}$

$\begin{array}{ll}\text { RSquare } & 0.989512 \\ \text { RSquare Adj } & 0.988201 \\ \text { Root Mean Square Error } & 0.030598 \\ \text { Mean of Response } & 3.744943\end{array}$

Root Mean of Square Eritor

Observations (or Sum Wgts)

10

$\begin{array}{lllll} & \text { Parameter Estimates } & & \\ \text { Term } & \text { Estimate } & \text { Std Error } & \text { t Ratio } & \text { Prob }>|t|\end{array}$ $\begin{array}{llllll}\text { Intercept } & 4.2539684 & 0.020903 & 203.51 & <.000 \\ \text { Sampling Seq } & -0.09255 & 0.003369 & -27.47 & <.000\end{array}$
Methanol Study 4(Test Sequence=1)

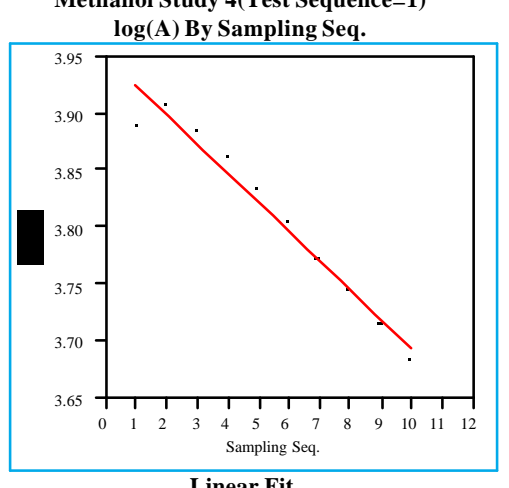

$\log (\mathrm{A})=3.95102-0.02569$ Sampling Seq

$$
\text { Summary of Fit }
$$$$
\text { RSquare }
$$

RSquare Adj

Root Mean Square Error

Mean of Response

0.963494 0.958931

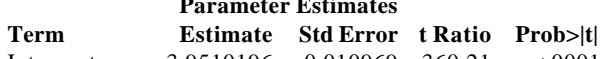
$\begin{array}{lrrrr}\text { Intercept } & 3.9510196 & 0.010969 & 360.21 & <.0001 \\ \text { Samp } & & 0.001768 & -14.53 & <.0001\end{array}$

Sampling Seq. $-0.025687 \quad 0.001768 \quad-14.53$

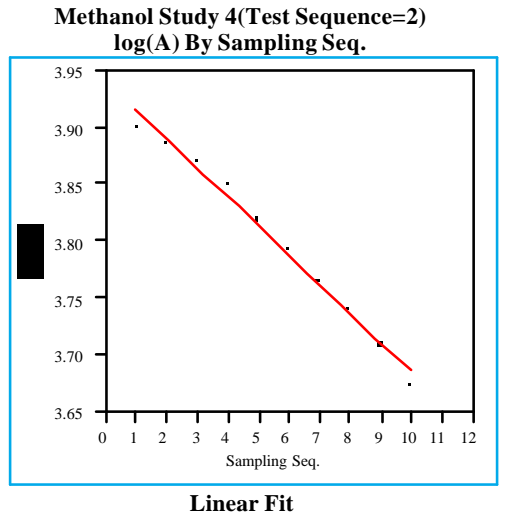

$\log (\mathrm{A})=3.9424-0.02566$

$\log (\mathrm{A})=3.9424-0.02566$ Sampling Seq.

\begin{tabular}{lrrrr}
\multicolumn{2}{c}{ Summary of Fit } & \\
RSquare & & 0.989625 \\
RSquare Adj & & 0.988328 \\
Root Mean Square Error & 0.008435 \\
Mean of Response & 3.801299 \\
Observations (or Sum Wgts) & \multicolumn{2}{c}{10} \\
\multicolumn{4}{c}{ Parameter Estimates } \\
Term & Estimate & Std Error & $\mathbf{t}$ Ratio & Prob $>|\mathbf{t}|$ \\
ntercept & 3.9424015 & 0.005762 & 684.15 & $<.0001$ \\
Sampling Seq. & -0.025655 & 0.000929 & -27.62 & $<.0001$
\end{tabular}

Methanol Study 4 (Test Sequence $=3$ )

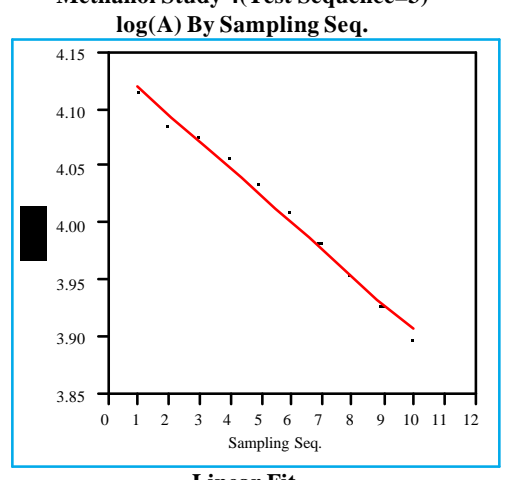

Linear Fit
$\log (\mathrm{A})=4.1448-0.02388$ Sampling Sec

RSquare

Summary of Fit

0.989214

RSquare Adj

Root Mean Square Error

Mean of Response

0.987866

Observations (or Sum Wgts)

4.013458

$\begin{array}{lrrrr} & \text { Estimate } & \text { Std Error } & \text { t Ratio } & \text { Prob }>|\mathbf{t}| \\ \text { Intercept } & 4.1448046 & 0.00547 & 757.68 & <.0001\end{array}$

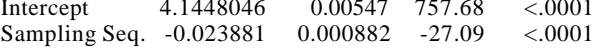

Methanol Study 4 (Test Sequence $=4$ )

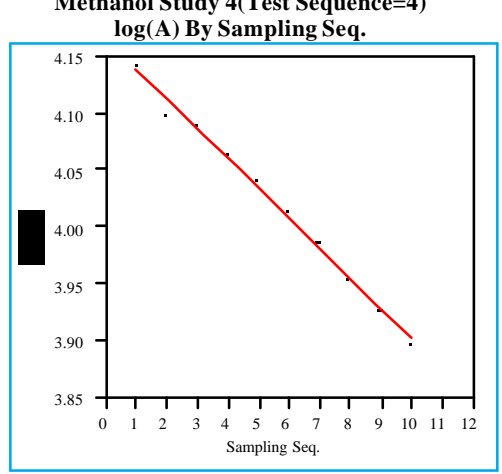

Linear Fit

$\log (\mathrm{A})=4.16677-0.02639$ Sampling Seq

\begin{tabular}{lrrrr}
\multicolumn{4}{c}{ Summary of Fit } & \\
RSquare & & 0.992796 \\
RSquare Adj & & 0.991896 \\
Root Mean Square Error & 0.007219 \\
Mean of Response & 4.02162 \\
Observations (or Sum Wgts) & \multicolumn{2}{c}{10} \\
\multicolumn{5}{c}{ Parameter Estimates } \\
Term & Estimate & Std Error & t Ratio & Prob $>|\mathbf{t}|$ \\
Intercept & 4.1667675 & 0.004932 & 844.92 & $<.0001$ \\
Sampling Seq. & -0.02639 & 0.000795 & -33.20 & $<.0001$
\end{tabular}

Methanol Study 4(Test Sequence=5) $\log (A)$ By Sampling Seq.

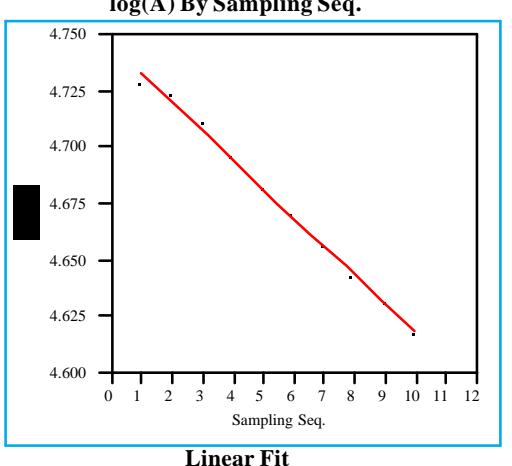

$\log (A)=4.74586-0.0128$ Sampling Seq

RSquare

RSquare Adj

Summary of Fit

Root Mean Square Error

Mean of Response

0.995702 0.002701 4.67544

Observations (or Sum Wgts)

Term Estimate Std Error t Ratio Prob $>\mid \mathbf{t}$ $\begin{array}{lrrrrr}\text { Intercept } & 4.7458561 & 0.001845 & 2572 & <.0001\end{array}$ $\begin{array}{lllll}\text { Sampling Seq. } & -0.012803 & 0.000297 & -43.05 & <.0001\end{array}$

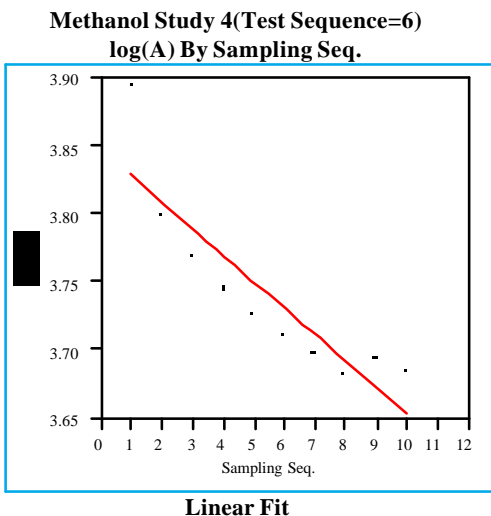

$\log (A)=3.84865-0.01954$ Sampling Seq.

$\begin{array}{lll} & \text { Summary of Fit } & \\ \text { RSquare } & & 0.786283\end{array}$

$\begin{array}{ll}\text { RSquare } & 0.786283 \\ \text { RSquare Adj } & 0.759569\end{array}$

Root Mean Square Error $\quad 0.03272$

Mean of Response

3.741165

Observations (or Sum Wgts)

Term Estimate Std Error t Ratio Prob $>|\mathbf{t}|$

$\begin{array}{lrrrr}\text { Intercept } & 3.8486528 & 0.022352 & 172.19 & <.0001 \\ \text { Sampling Seq } & -0.019543 & 0.003602 & -5.43 & 0.0006\end{array}$ 
Exhibit B11: Common Logarithm of Peak Area Versus Sample Sequence for Methanol Studies: Study 4; Tests 7-14

Methanol Study 4 (Test Sequence=7)

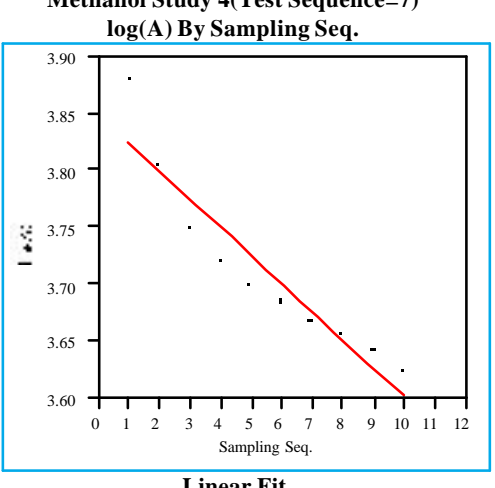

$\log (\mathrm{A})=3.85012-0.02496$ Sampling Seq.

RSquare

Summary of Fit

RSquare Adj

Root Mean Square Error

0.889881

Mean of Response

0.876116

3.712855

Observations (or Sum Wgts)

$\begin{array}{lllll} & \text { Parameter Estimates } & & \\ \text { Term } & \text { Estimate } & \text { Std Error } & \text { t Ratio } & \text { Prob }>|t|\end{array}$ $\begin{array}{lrrrrr}\text { Intercept } & 3.8501187 & 0.019259 & 199.91 & <.0001 \\ \text { Sampling Seq } & -0.024957 & 0.003104 & -8.04 & <.0001\end{array}$

Methanol Study 4 (Test Sequence=8)

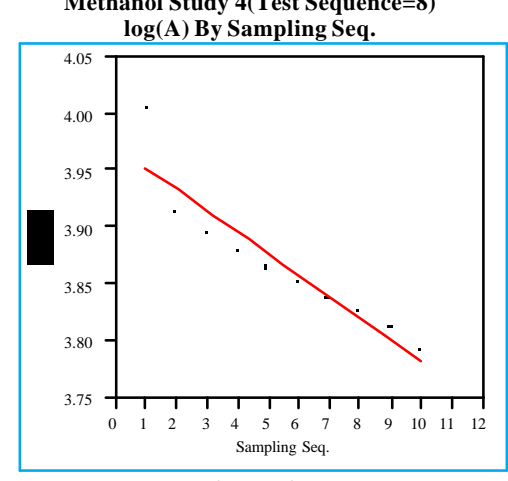

$\log (\mathrm{A})=3.97192-0.01892$

$\log (A)=3.97192-0.01892$ Sampling Seq.

RSquare

Summary of Fit $\quad 0.867241$

RSquare Adj $\quad 0.850646$

Root Mean Square Error $\quad 0.023766$

Observations (or $\mathrm{S}$

3.867883
10

\section{Parameter Estimates}

$\begin{array}{lrrr}\text { Term } & \text { Estimate } & \text { Std Error t Ratio Prob }>|t|\end{array}$ $\begin{array}{lrrrr}\text { Intercept } & 3.9719165 & 0.016235 & 244.65 & <.0001 \\ \text { Sampling Seq. } & -0.018915 & 0.002617 & -7.23 & <.0001\end{array}$
Methanol Study 4(Test Sequence=9)

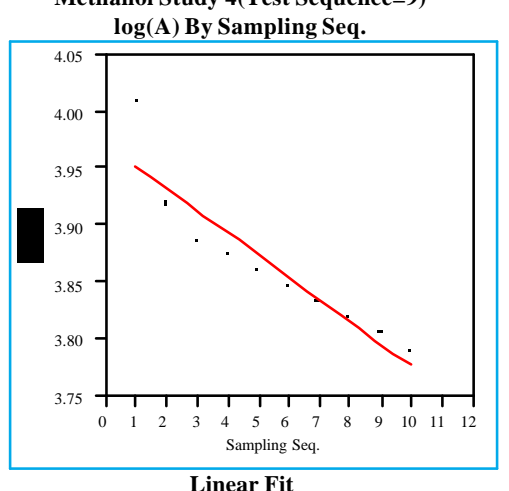

$\log (\mathrm{A})=3.97341-0.01977$ Sampling Seq

$$
\begin{aligned}
& \log (\mathrm{A})=3.97341-0.01977 \mathrm{~S} \\
& \text { RSquare }
\end{aligned}
$$

RSquare Adj

Root Mean Square Error

Mean of Response

Parameter Estimates

Term Estimate Std Error t Ratio Prob $>|t|$

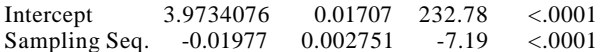

Methanol Study 4(Test Sequence=10)

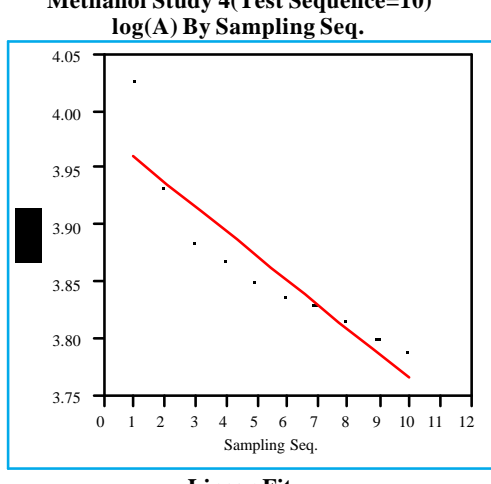

Linear Fit
$\log (\mathrm{A})=3.98194-0.02161$ Sampling Seq.

\begin{tabular}{lrrrr} 
& Summary of Fit & \\
RSquare & & 0.830224 \\
RSquare Adj & & 0.809002 \\
Root Mean Square Error & 0.031378 \\
Mean of Response & 3.863098 \\
Observations (or Sum Wgts) & \multicolumn{2}{c}{10} \\
\multicolumn{5}{c}{ Parameter Estimates } \\
Term & Estimate & Std Error & $\mathbf{t}$ Ratio & Prob $>|\mathbf{t}|$ \\
ntercept & 3.9819404 & 0.021435 & 185.76 & $<.0001$ \\
Sampling Seq. & -0.021608 & 0.003455 & -6.25 & 0.0002
\end{tabular}

Methanol Study 4 (Test Sequence=11)

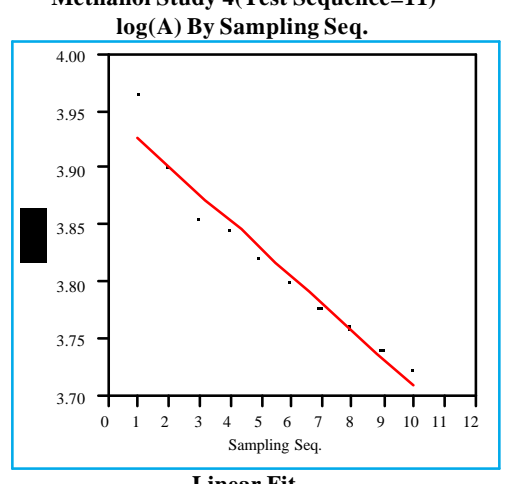

Linear Fit
$\log (\mathrm{A})=3.95226-0.0244$ Sampling Seq Summary of Fit

RSquare

RSquare Adj

Root Mean Square Erro

Mean of Response

0.952249

Observations (or Sum Wgts)

0.94628

3.818083

$\begin{array}{lrrrr}\text { Term } & \text { Estimate } & \text { Std Error } & \text { t Ratio } & \text { Prob }>|\mathbf{t}|\end{array}$ $\begin{array}{lrrrrr} & & 3.9522585 & 0.011984 & 329.79 & <.0001 \\ \text { Sampling Seq. } & -0.024396 & 0.001931 & -12.63 & <.0001\end{array}$

Methanol Study 4(Test Sequence=12)

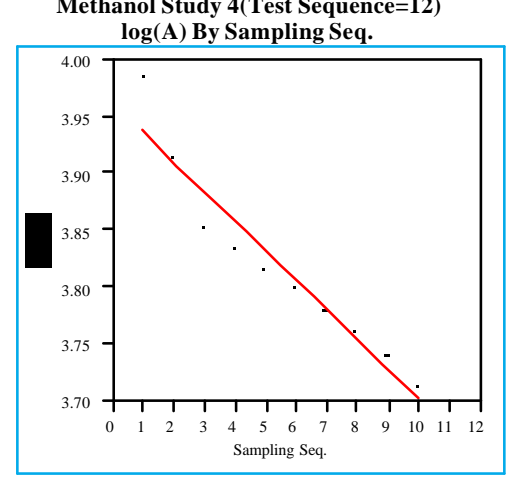

Linear Fit

$\log (\mathrm{A})=3.96364-0.02619$ Sampling Seq

\begin{tabular}{llrrr}
\multicolumn{4}{c}{ Summary of Fit } \\
RSquare & & 0.926323 \\
RSquare Adj & & 0.917114 \\
Root Mean Square Error & 0.023722 \\
Mean of Response & 3.819577 \\
Observations (or Sum Wgts) & \multicolumn{2}{c}{10} \\
\multicolumn{5}{r}{ Parameter Estimates } \\
Term & Estimate & Std Error & t Ratio & Prob $>|\mathbf{t}|$ \\
Intercept & 3.9636367 & 0.016205 & 244.59 & $<.0001$ \\
Sampling Seq. & -0.026193 & 0.002612 & -10.03 & $<.0001$
\end{tabular}

Methanol Study 4(Test Sequence=13)

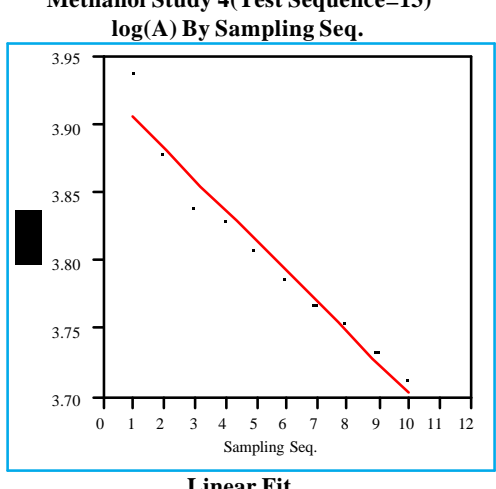

$\log (\mathrm{A})=3.92805-0.02244$ Sampling Seq.

RSquare

RSquare Adj

Summary of Fit

0.958289 0.953075 0.015036

Mean of Response

Observations (or Sum Wgts)
Parameter Estimate

$\begin{array}{lrrrr}\text { Term } & \text { Estimate } & \text { Std Error } & \text { t Ratio } & \text { Prob }>|t|\end{array}$ $\begin{array}{lrrrr}\text { Sampling Seq. } & -0.022443 & 0.001655 & -13.56 & <.0001\end{array}$

Methanol Study 4(Test Sequence=14)

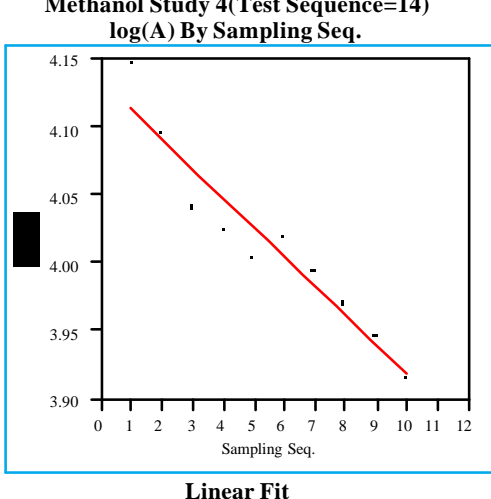

$\log (A)=4.13484-0.02163$ Sampling Seq.

\begin{tabular}{ll}
\multicolumn{2}{c}{ Summary of Fit } \\
RSquare & 0.916626 \\
RSquare Adj & 0.906204 \\
Root Mean Square Error & 0.020949 \\
Mean of Response & 4.015872
\end{tabular}

Observations (or Sum Wgts)$$
\begin{array}{r}
-15872 \\
10
\end{array}
$$

Term

Parameter Estimates

$\begin{array}{llllll}\text { Intercept } & 3.9636367 & 0.016205 & 244.59 & <.000\end{array}$

Estimate Std Error t Ratio Prob $>|\mathbf{t}|$

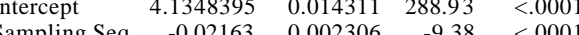


Exhibit B12: Common Logarithm of Peak Area Versus Sample Sequence for Methanol Studies: Study 4; Tests 15-16

Methanol Study 4(Test Sequence=15)

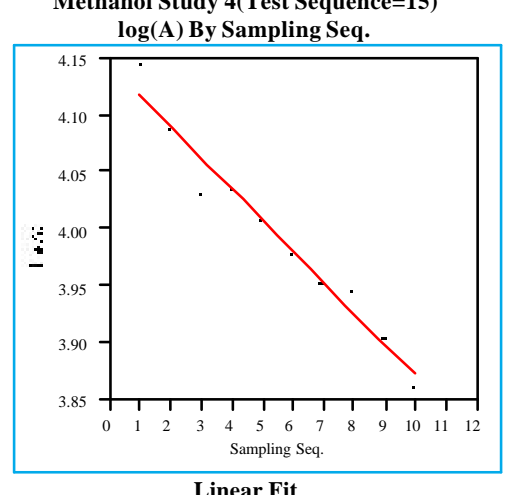

$\log (\mathrm{A})=4.14587-0.0275$ Sampling Seq.

RSquare

RSquare Adj

Root Mean Square Errot

Mean of Response

Observations (or Sum Wgts)

0.963802 0.959277 0.017114 3.99463

Parameter Estimates

$\begin{array}{lrrrrr}\text { Term } & \text { Estimate } & \text { Std Error } & \text { t Ratio } & \text { Prob }>|t| \\ \text { Intercept } & 4.1458747 & 0.011691 & 354.62 & <.0001\end{array}$ $\begin{array}{lllll}\text { Intercept } & 4.1458747 & 0.011691 & 354.62 & <.0001 \\ \text { Sampling Seq. } & -0.027499 & 0.001884 & -14.59 & <.0001\end{array}$
Methanol Study 4(Test Sequence=16)

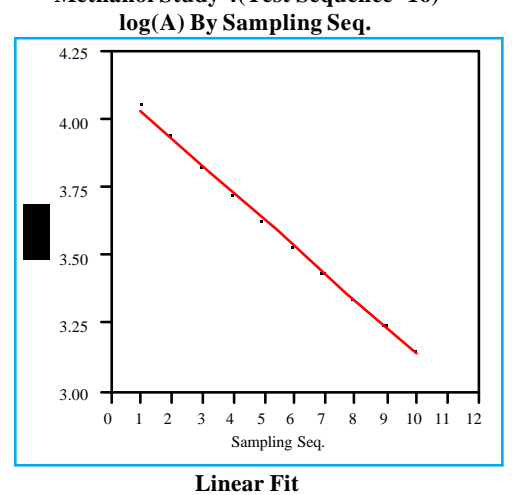

$\log (A)=4.13558-0.09963$ Sampling Seq

Summary of Fit

$\begin{array}{lr}0.99903 \\ \text { RSquare Adj } & 0.998909\end{array}$

Root Mean Square Error $\quad 0.00997$

$\begin{array}{lr}\text { Mean of Response } & 3.587595 \\ \text { Observations (or Sum Wgts) } & 10\end{array}$$$
10
$$

$\begin{array}{lllll} & \text { Estimate } & \text { Std Error } & \text { t Ratio } & \text { Prob }>|\mathbf{t}|\end{array}$

$\begin{array}{lrrrrr}\text { Sampling Seq. } & -0.099633 & 0.006811 & 607.18 & <.0001 \\ \text { S } & 0.001098 & -90.76 & <.0001\end{array}$ 


\section{Exhibit B13: $y$ versus $x$ for Methanol Studies: Study 1; Tests 1-8}

Methanol Study 1 x vs $y$ (Test Sequence=1) y By x

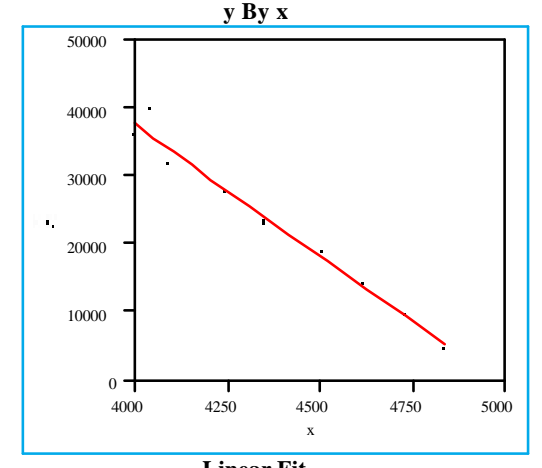

Linear Fit $y=193001-38.7892$

RSquare Summary of Fit

RSquare Adj

Root Mean Square Error

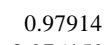

Observations (or Sum Wgts)

0.97914
0.976159

1872.82

22989.12

$\begin{array}{lrrrr}\text { Term } & \text { Estimate } & \text { Std Error } & \text { t Ratio } & \text { Prob }>|\mathbf{t}|\end{array}$

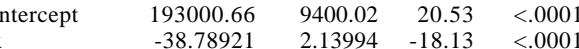

Methanol Study $1 \times$ vs $y$ (Test Sequence=2)

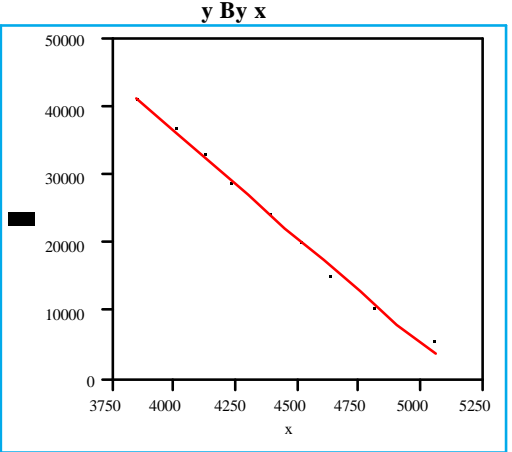

Linear Fit

$y=161723-31.2688 x$

RSquare

Summary of Fit

J

Root Mean Square Erro

Mean of Response

0.993016

Observations (or Sum Wgts)

0.992018

1093.427
23879.2

Parameter Estimates

Parameter Estimates

$\begin{array}{lrrrr}\text { Term } & \text { Estimate } & \text { Std Error } & \text { t Ratio } & \text { Prob }>|t| \\ \text { Intercept } & 161723.21 & 4384.638 & 36.88 & <.0001 \\ \mathrm{x} & -31.26878 & 0.991177 & -31.55 & <.0001\end{array}$
Methanol Study $1 \times$ vs $y($ Test Sequence $=3)$

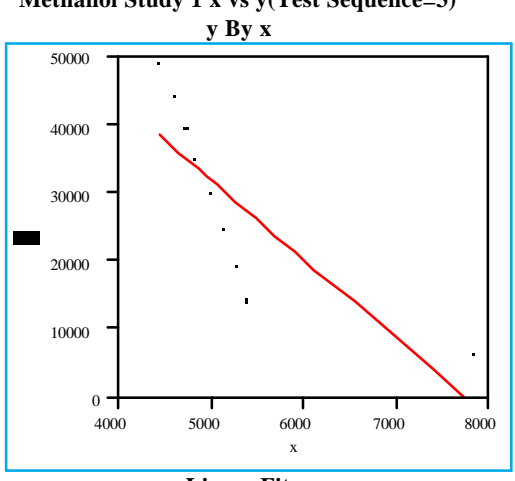

Linear Fit

$$
y=89907.9-11.5478
$$

Summary of Fit

$\begin{array}{lr}\text { RSquare } & 0.670764 \\ \text { RSquare Adj } & 0.62373 \\ \text { Root Mean Square Error } & 8810.997 \\ \text { Mean of Response } & 29111.52 \\ \text { Observations (or Sum Wots) } & 9\end{array}$

Mean of Respon

Observations (or Sum Wgts)
Parameter Estimates

Term Estimate Std Error t Ratio Prob $>|t|$ $\begin{array}{lrrrr}\text { Intercept } & 89907.931 & 16364.67 & 5.49 & 0.0009 \\ & -11.54777 & 3.057863 & -3.78 & 0.0069\end{array}$

Methanol Study 1 x vs $y($ Test Sequence $=4)$

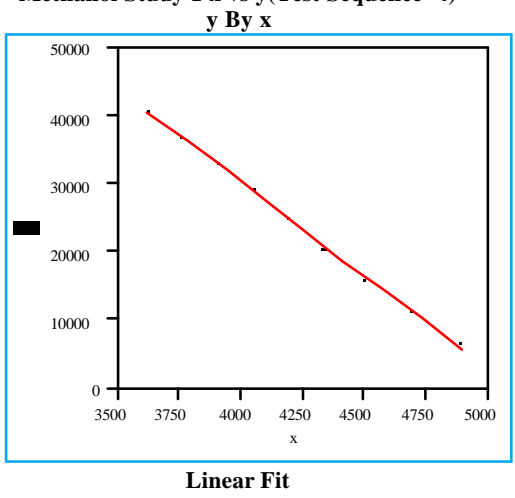
Linear Fit $y=140351-27.4751$

\begin{tabular}{lr} 
RSquare & Summary of Fit \\
RSquare Adj & 0.999137 \\
Root Mean Square Error & 0.999014 \\
Mean of Response & 369.2988 \\
Obserations (or Sum Wgts) & 24305.25 \\
\hline
\end{tabular}

Observations (or Sum Wgts)

Parameter Estimates

Term Estimate Std Error t Ratio Prob $>|t|$

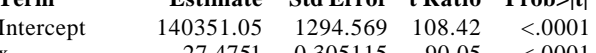

Methanol Study 1 x vs y(Test Sequence=5)

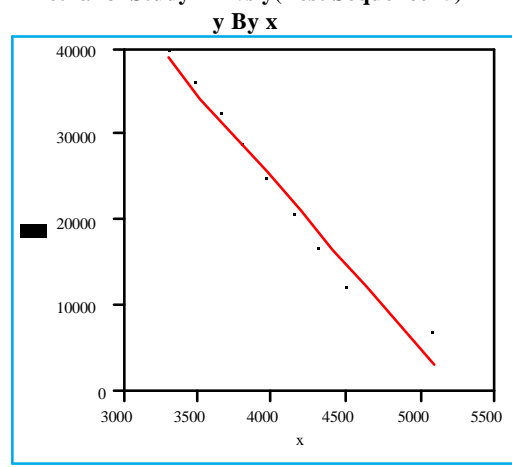

Linear Fit

$y=104706-19.9023 x$

RSquare

RSquare Adj

Mean of Response

0.967371

0.962709

Observations (or Sum Wgts)

24295

Parameter Estimates

$\begin{array}{lllll} & \text { Estimate } & \text { Std Error } & \text { t Ratio } & \text { Prob }>|t|\end{array}$

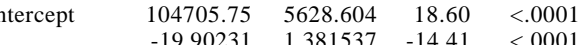

Methanol Study 1 x vs y(Test Sequence=6)

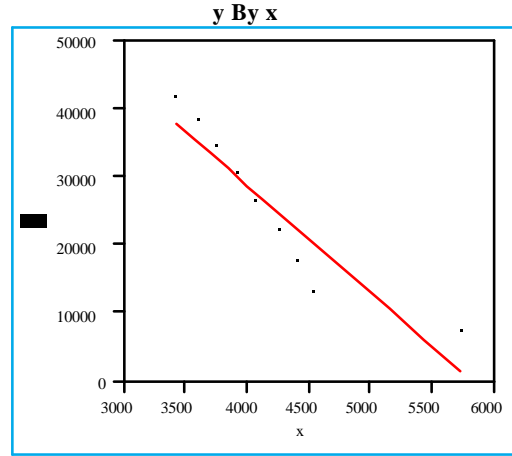

\section{Linear Fit}

$y=92055.3-15.7763$

$\begin{array}{lr}\text { RSquare } \quad \text { Summary of Fit } & 0.86301 \\ \text { RSquare Adj } & 0.84344 \\ \text { Root Mean Square Error } & 4597.338 \\ \text { Mean of Response } & 25835.54 \\ \text { Ore } & 9\end{array}$

Mean of Response

Parameter Estimates

Term Estimate Std Error t Ratio Prob $>|t|$

$\begin{array}{lrrrr}\text { Intercept } & 92055.348 & 10088.9 & 9.12 & <.0001 \\ \mathrm{x} & -15.77626 & 2.375698 & -6.64 & 0.0003\end{array}$
Methanol Study 1 x vs $y($ Test Sequence $=7)$ $\mathbf{y}$ By $x$

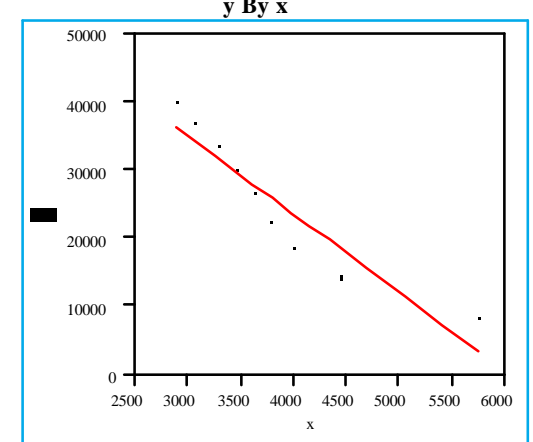

Linear Fit

$y=69770.4-11.4862 x$

RSquare

RSquare Adj

Root Mean Square Error

Mean of Response

0.886091

0.869819

3847.616

Observations (or Sum Wgts)

$\begin{array}{lllll}\text { Term } & \text { Parameter Estimates } & & \\ \text { Estimate } & \text { Std Error } & \text { t Ratio } & \text { Prob }>|t|\end{array}$

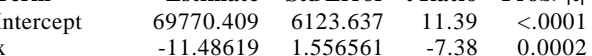

Methanol Study 1 x vs $y($ Test Sequence $=8)$

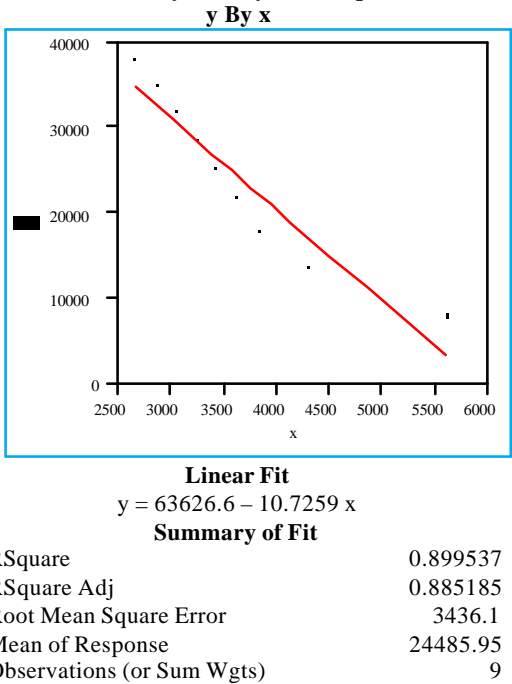

Observations (or Sum Wgts)

Parameter Estimates

Term Estimate Std Error t Ratio Prob $>\mid \mathbf{t}$

$\begin{array}{lrrrr}\text { Intercept } & 63626.619 & 5074.889 & 12.54 & <.000 \\ \mathrm{x} & -10.7259 & 1.354813 & -7.92 & <.0001\end{array}$ 
Exhibit B14: y versus x for Methanol Studies: Study 1; Tests 8-16

Methanol Study 1 x vs $y$ (Test Sequence=9)

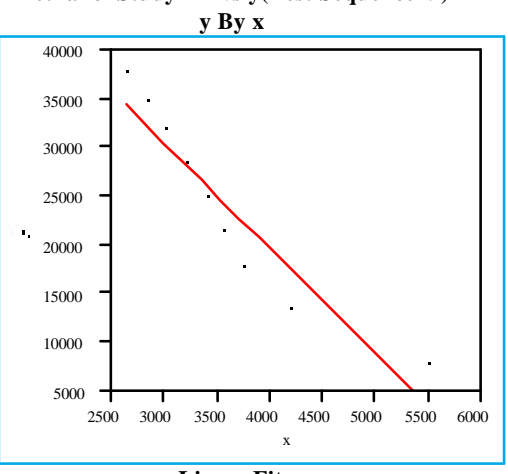

Linear Fit

$$
y=63229.8-10.8366
$$

RSquare
RSquare Adj

Summary of Fit

Root Mean Square Error

Mean of Response

0.891088

0.875529

3527.149

24237.27

Observations (or Sum Wgts)

$\begin{array}{lrrrr} & \text { Parameter Estimates } & & \\ \text { Term } & \text { Estimate } & \text { Std Error } & \text { t Ratio } & \text { Prob }>|t|\end{array}$ $\begin{array}{lrrrr} & 63229.836 & 5284.845 & 11.96 & <.000 \\ \mathrm{x} \text { tercept } & -10.83662 & 1.431931 & -7.57 & 0.000\end{array}$

Methanol Study $1 \times$ vs $y($ Test Sequence $=10)$

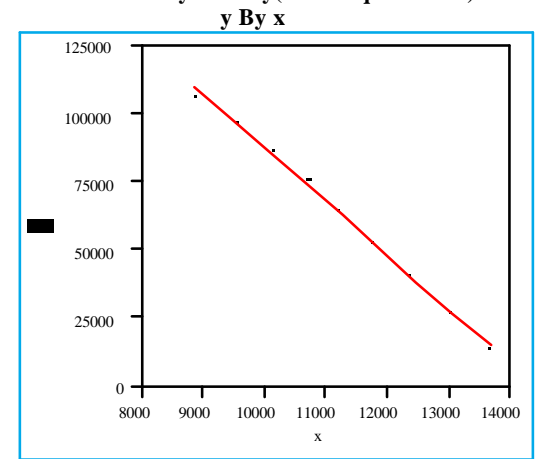

\section{Linear Fit}

$\mathrm{y}=285306-19.7078$

RSquare

Summary of Fit

Rquare Adj

Root Mean Square Er

Observations (or Sum Wgts)

Observations (or Sum Wgts)
Parameter Estimates

$\begin{array}{lrrrr}\text { Term } & \text { Estimate } & \text { Std Error } & \text { t Ratio } & \text { Prob }>|t|\end{array}$

$\begin{array}{lrrrr}\text { Intercept } & 285306.44 & 4853.502 & 58.78 & <.0001 \\ \mathrm{x} & -19.70782 & 0.425956 & -46.27 & <.0001\end{array}$

0.996741 0.996275

1938.521

62747.27
Methanol Study 1 x vs $y($ Test Sequence=11)

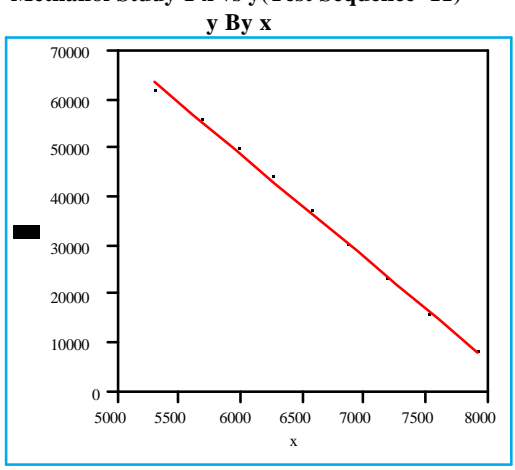

Linear Fit

$$
\mathrm{y}=176287-21.1954
$$

RSquare

$\begin{array}{ll}\text { RSquare } & 0.99795 \\ \text { RSquare Adj } & 0.997557\end{array}$

Root Mean Square Error $\quad 0.997657$

Mean of Response

895.9203

Observations (or Sum Wgts)

Term Parameter Estimates

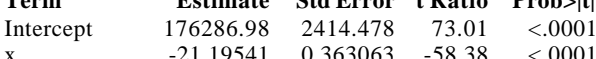

$\begin{array}{lll}-21.19541 & 0.363063 & -58.38 \\ & & <.\end{array}$

Methanol Study 1 x vs $\mathbf{y}(\mathrm{T}$

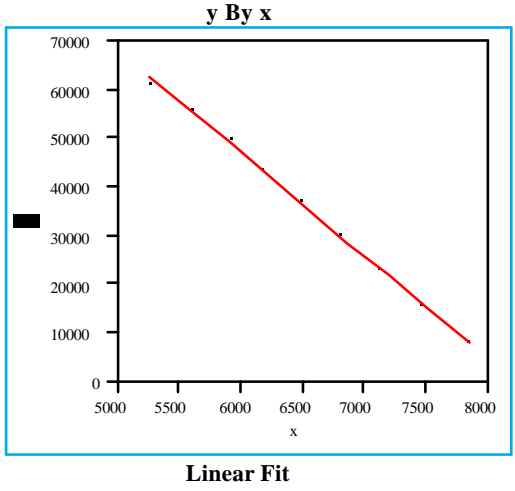
Linear Fit $=174508-21.1828$
Summary of Fit

RSquare

Root Mean Square Error

Root Mean Square Erro

Observations (or Sum Wgts)

673.0875

Parameter Estimates
Pats

Term Estimate Std Error t Ratio Prob $>\mid \mathbf{t}$
Methanol Study 1 x vs $y($ Test Sequence= $=13)$

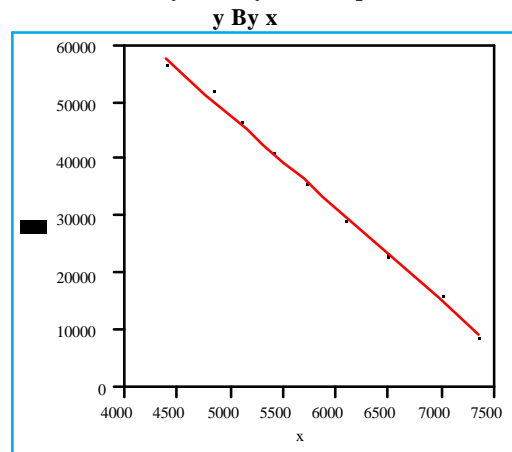

Linear Fit

$$
y=131096-16.5578 x
$$

RSquare

RSquare Adj
Root Mean Square Error

Mean of Response

0.997479

0.997119

884.9458

Observations (or Sum Wgts)

Parameter Estimates

$\begin{array}{lrrrr} & \text { Estimate } & \text { Std Error } & \text { t Ratio } & \text { Prob }>|t|\end{array}$ $\begin{array}{lrrrr} & 131096.47 & 1863.525 & 70.35 & <.0001 \\ \mathrm{x} & -16.55779 & 0.314614 & -52.63 & <.0001\end{array}$

Methanol Study 1 x vs y(Test Sequence=14)

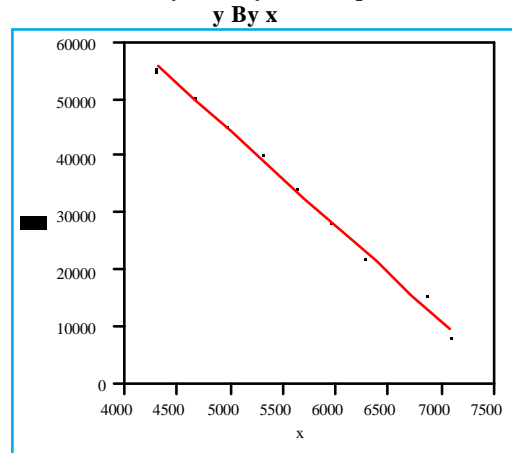

\section{Linear Fit} $y=128652-16.7621$

\begin{tabular}{lr} 
& \\
RSquare & Summary of Fit \\
RSquare Adj & 0.99599 \\
Root Mean Square Error & 0.995418 \\
Mean of Response & 1087.96 \\
Obe & 33188.98 \\
\hline
\end{tabular}

$\begin{array}{lrrrrr} & \text { Intercept } & 174508.43 & 1812.705 & 96.27 & <.0001 \\ \mathrm{x} & -21.18284 & 0.275435 & -76.91 & <.0001\end{array}$
Observations (or Sum Wgts)

Parameter Estimates

$\begin{array}{lrrrr}\text { Term } & \text { Estimate } & \text { Std Error } & \text { t Ratio } & \text { Prob }>|t|\end{array}$

$\begin{array}{lrrrr}\text { Intercept } & 128651.64 & 2317.884 & 55.50 & <.0001 \\ \mathrm{X} & -16.7621 & 0.40198 & -41.70 & <.0001\end{array}$
Methanol Study 1 x vs $y$ (Test Sequence $=15$

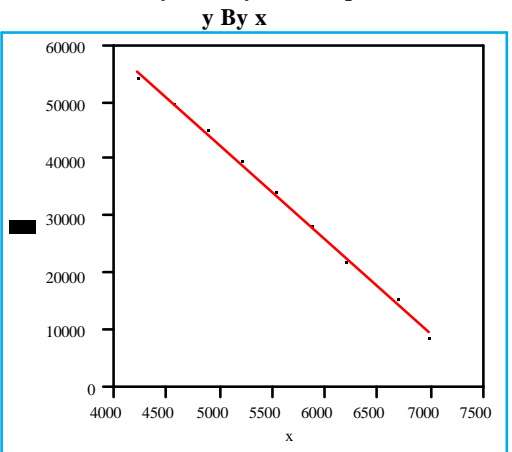

Linear Fit

$$
y=126096-16.642 x
$$

RSquare

Summary of Fit

RSquare Adj

Root Mean Square Error

Mean of Response

0.997386

807.8685

Observations (or Sum Wgts)

$\begin{array}{lrrrr} & \text { Parameter Estimates } & & \\ \text { Term } & \text { Estimate } & \text { Std Error } & \text { t Ratio } & \text { Prob }>|t| \\ \text { Intercept } & 126096.17 & 1706.919 & 73.87 & <.0001\end{array}$ $\begin{array}{lrrrr} & 126096.17 & 1706.919 & 73.87 & <.0001 \\ \mathrm{x} & -16.64203 & 0.301191 & -55.25 & <.0001\end{array}$

Methanol Study 1 x vs $y($ Test Sequence=16)

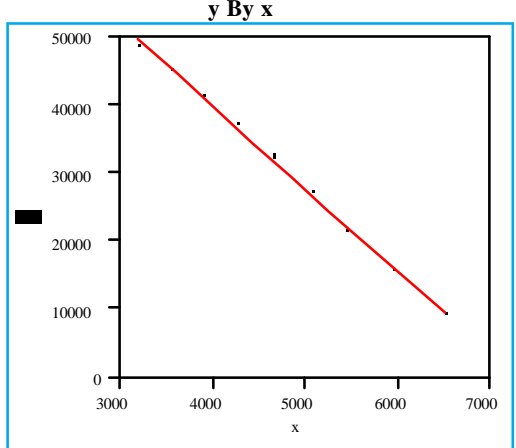

\section{Linear Fit}

$y=88879.4-12.1395$

RSquare

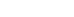

0.998728

RSquare Adj $\quad 0.998546$

Root Mean Square Error

517.8053

Observations (or Sum Wgts)

Parameter Estimates

Term Estimate Std Error t Ratio Prob $>\mid \mathbf{t}$

$\begin{array}{llllll}\text { Intercept } & 88879.429 & 798.7581 & 111.27 & <.0001 \\ \mathrm{x} & -12.13947 & 0.163752 & -74.13 & <.0001\end{array}$ 
Exhibit B15: y versus x for Methanol Studies: Study 1; Tests 17-24

Methanol Study 1 x vs $y($ Test Sequence $=17)$

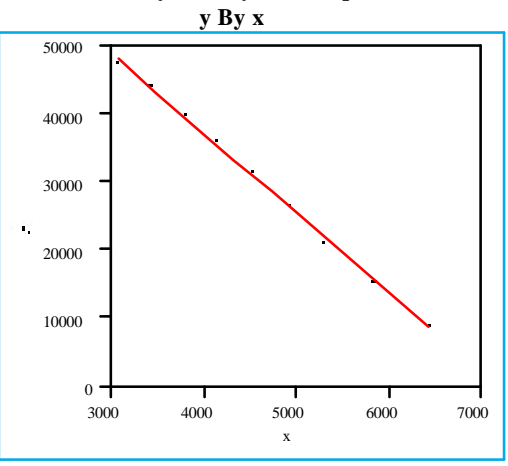

Linear Fit

$y=84175.9-11.6707 x$

RSquare

Summary of Fit

RSquare Adj

Root Mean Square Error

0.998369

0.998136

569.561

Observations (or Sum Wgts)

$\begin{array}{lrrrr}\text { Term } & \text { Parameter Estimates } & & \\ \text { Estimate } & \text { Std Error } & \text { t Ratio } & \text { Prob> }|\mathbf{t}|\end{array}$ $\begin{array}{lrrrr} & 84175.907 & 846.3358 & 99.46 & <.000 \\ & -11.67073 & 0.17828 & -65.46 & <.000\end{array}$

Methanol Study $1 \times$ vs $y($ Test Sequence $=18)$

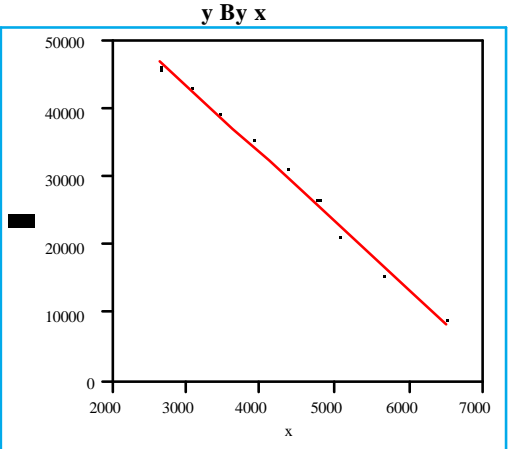

\section{Linear Fit}

$y=73576.7-9.9542 x$

RSquare

uare Adj

Root Mean Square Erro

0.993575

Mean of Response

0.992657

Observations (or Sum Wgts)

1086.117
29676.73

Term
Intercep

Parameter Estimates

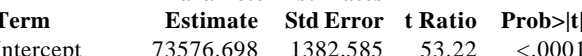

$\begin{array}{llrrr} & & & \\ \mathrm{x} & -9.9546 .698 & 1382.585 & 53.22 & <.0001 \\ & & 0.302558 & -32.90 & <.0001\end{array}$
Methanol Study 1 x vs $y($ Test Sequence $=19)$

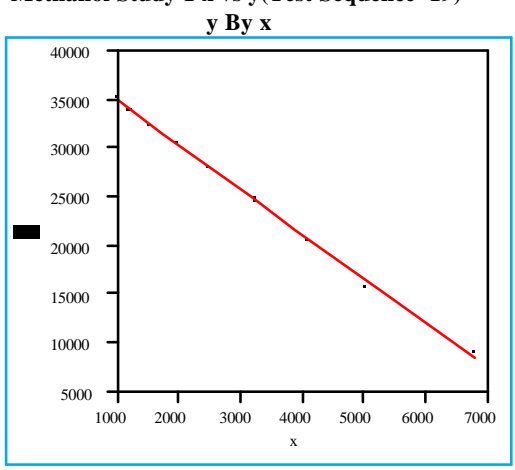

Linear Fit

$$
y=39533.4-4.58921
$$

RSquare

Summary of Fi

$\begin{array}{ll}\text { RSquare Adj } & 0.998016 \\ \text { Root Mean Square Error } & 0.997733 \\ \text { Mean of Response } & 426.1953 \\ \text { Observations (or Sum Wgts) } & 25617.78\end{array}$

Parameter Estimates

$\begin{array}{lllll}\text { Term } & \text { Estimate } & \text { Std Error } & \text { t Ratio } & \text { Prob }>|t|\end{array}$

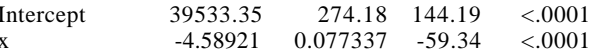

Methanol Study $1 \times$ vs $y($ Test Sequence $=20)$

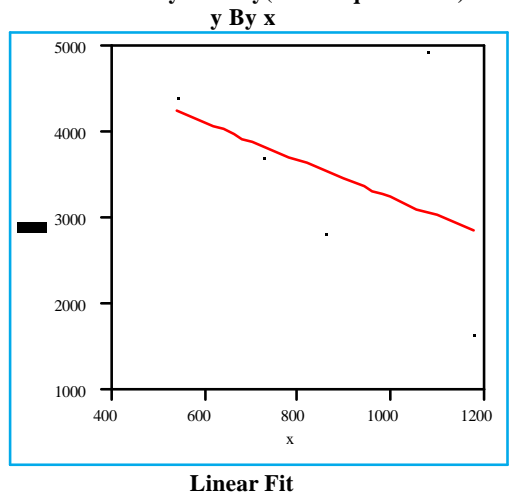

$y=5429.28-2.19469$

RSquare

RSquare Adj

Met Mean Square Error

Mean of Response

0.186992

servations (or Sum Wgts)

1370.313

Parameter Estimates

$\begin{array}{lrrrr}\text { Term } & \text { Estimate } & \text { Std Error } & \text { t Ratio } & \text { Prob }>|t|\end{array}$

$\begin{array}{lrrrr}\text { Intercept } & 5429.2798 & 2407.544 & 2.26 & 0.1094 \\ \mathrm{x} & -2.194687 & 2.642088 & -0.83 & 0.4671\end{array}$
Methanol Study 1 x vs y(Test Sequence=21)

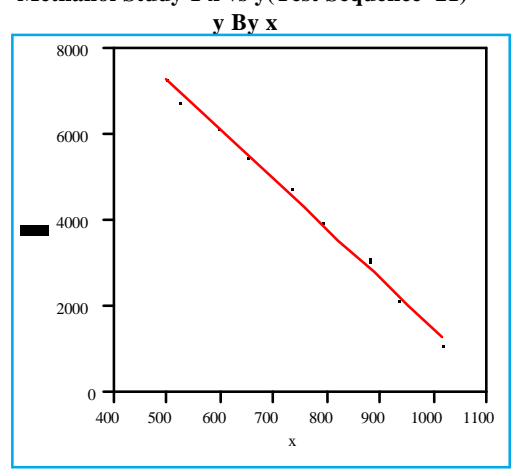

\section{Linear Fit}

$\mathrm{y}=12952.2-11.4062 \mathrm{x}$

RSquare

Summary of Fit

0.994486

RSquare Adj

Root Mean Square Error

Observations (or Sum Wgts)

0.99369

168.392

Parameter Estimates

Term $\quad \begin{array}{llll}\text { Estimate } & \text { Std Error } & \text { t Ratio } & \text { Prob }>|t|\end{array}$ $\begin{array}{lrrrrr} & \text { Entercept } & 12952.178 & 244.4483 & 52.99 & <.0001\end{array}$

Methanol Study 1 x vs $\mathbf{y}($ Test Sequence $=22)$

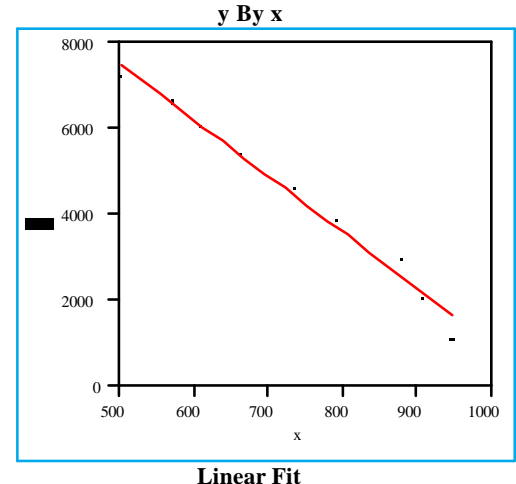

$$
y=14063.6-13.0882 x
$$

RSquare Summary of Fit

RSquare Adj

Root Mean Square Error

Mean of Response
Observations (or Sum Wgts)

Parameter Estimates

$\begin{array}{lrrrr}\text { Term } & \text { Estimate } & \text { Std Error } & \text { t Ratio } & \text { Prob }>|\mathbf{t}|\end{array}$

$\begin{array}{lrrrr}\text { Intercept } & 14063.558 & 510.71 & 27.54 & <.0001 \\ \mathrm{X} & -13.08823 & 0.679525 & -19.26 & <.0001\end{array}$

0.98148

0.978835

306.6104
Methanol Study 1 x vs $y$ (Test Sequence $=23$

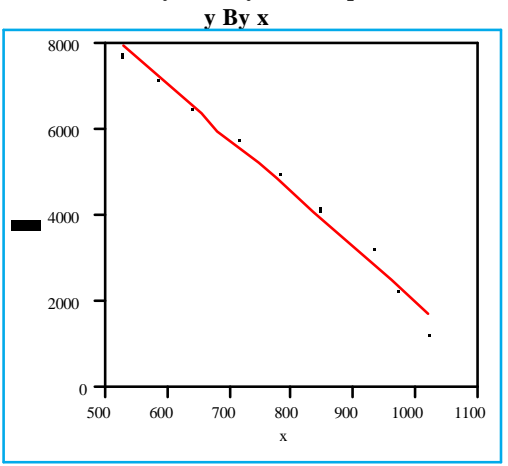

Linear Fit

$=14609.4-12.5826$

RSquare

are Error

0.985158

0.983038

Root Mean Square

Observations (or Sum Wgts)

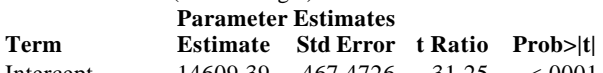

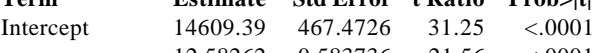

Methanol Study 1 x vs $\mathbf{y}($ Test Sequence $=24)$

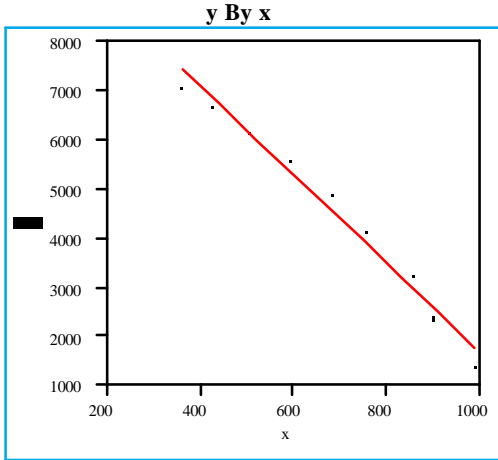

$$
\begin{array}{lr}
\hline \multicolumn{3}{c}{\begin{array}{c}
\text { Linear Fit } \\
\text { y }=10619-8.88918 x \\
\text { Summary of Fit }
\end{array}} \\
\text { RSquare } \quad 0.978487 \\
\text { RSquare Adj } & 0.975414 \\
\text { Root Mean Square Error } & 311.6478 \\
\text { Mean of Response } & 4588.45 \\
\text { Observations (or Sum Wgts) } & 9
\end{array}
$$

Parameter Estimates

Tstimate Std Error t Ratio Prob $>|\mathbf{t}|$

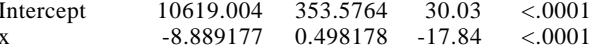


Exhibit B16: y versus x for Methanol Studies: Study 1; Tests 25-32

Methanol Study 1 x vs $y($ Test Sequence=25)

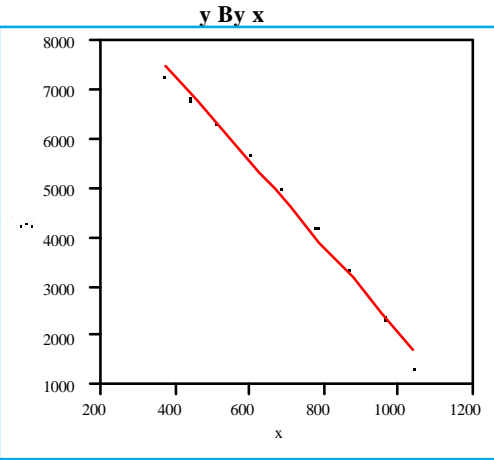

Linear Fit

$=10771.7-8.67823$

RSquare

RSquare Ad

Summary of Fit

RSquare Adj
Root Mean Sq

Mean of Response Erior

Observations (or Sum Wgts)

\begin{tabular}{lrrrrr}
\multicolumn{7}{c}{ Parameter Estimates } & & \\
Term & Estimate & Std Error & t Ratio & Prob $>|\mathbf{t}|$ \\
Intercept & 10771.695 & 246.6248 & 43.68 & $<.0001$ \\
& -8.678234 & 0.335395 & -25.87 & $<.000$
\end{tabular}

Methanol Study 1 x vs $\mathbf{y}($ Test Sequence $=26)$

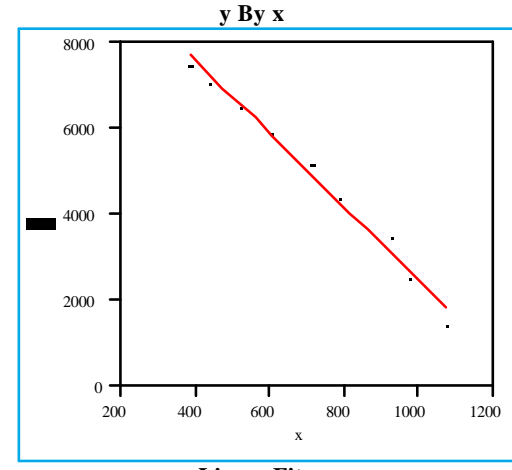

$$
y=\begin{aligned}
& 11009.2-8.51875 x \\
& \text { Summary of Fit }
\end{aligned}
$$

RSquare

RSquare Adj

Root Mean Square Erro

Mean of Response

0.984625

Response

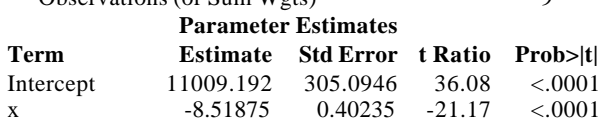

Methanol Study 1 x vs $y($ Test Sequence $=27)$

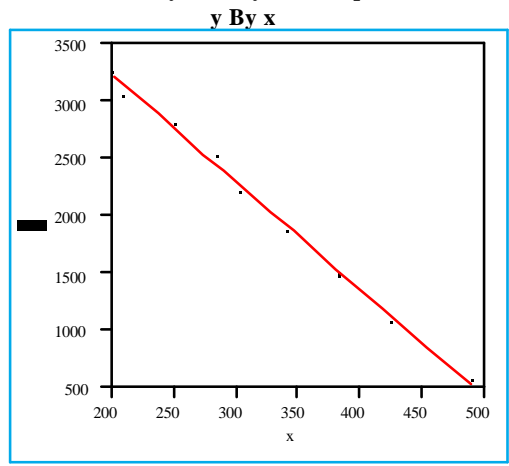

Linear Fit

$y=5119.95-9.39544$

\begin{tabular}{ll}
\multicolumn{2}{c}{$y=5119.95-9.39544 x$} \\
RSquare $\quad$ Summary of Fit \\
RSquare Adj & 0.995236 \\
Root Mean Square Error & 0.994555 \\
Mean of Response & 68.17416 \\
Oren & 2089.215
\end{tabular}

ions (or 5

Observations (or Sum Wgts)

$\begin{array}{lrrrr}\text { Term } & \text { Estimate } & \text { Std Error } & \text { t Ratio } & \text { Prob }>|\mathbf{t}| \\ \text { Intercept } & 5119.9456 & 82.44895 & 62.10 & <.000\end{array}$

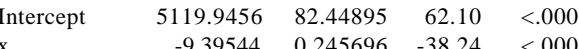

Methanol Study 1 x vs $y($ Test Sequence $=28$ )

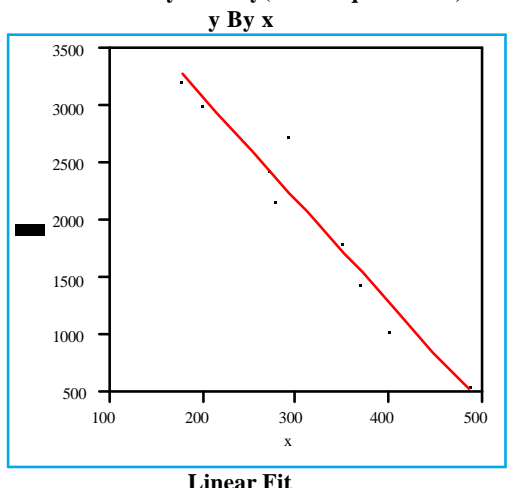$$
y=4871.91-8.96445
$$$$
\begin{aligned}
& =4871.91-8.96445 x \\
& \text { Summary of Fit }
\end{aligned}
$$

BSquare

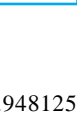

RSquare Adj

0.948125

Root Mean Square Erro

Mean of Response
Observations (or Sum Wgts)

221.3331

Parameter Estimates

Estimate Std Error t Ratio Prob $>\mid \mathbf{t}$

$\begin{array}{lrrrr}\text { Intercept } & 4871.9086 & 261.077 & 18.66 & <.0001 \\ \mathrm{X} & -8.964449 & 0.792541 & -11.31 & <.0001\end{array}$
Methanol Study 1 x vs y (Test Sequence=29)

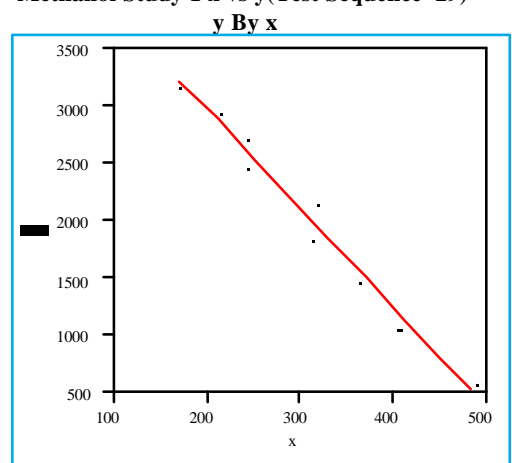

\section{Linear Fit}

$\mathrm{y}=4723.46-8.69785 \mathrm{x}$

Summary of Fit

0.976991

RSquare

RSquare Adj

Meot Mean of Response

Observations (or Sum Wgts)

144.2074

Parameter Estimates

Term Estimate Std Error t Ratio Prob $>|t|$ $\begin{array}{llllll}\text { Intercept } & 4723.4566 & 163.5786 & 28.88 & <.0001\end{array}$

Methanol Study 1 x vs $\mathbf{y}($ Test Sequence $=30$ )

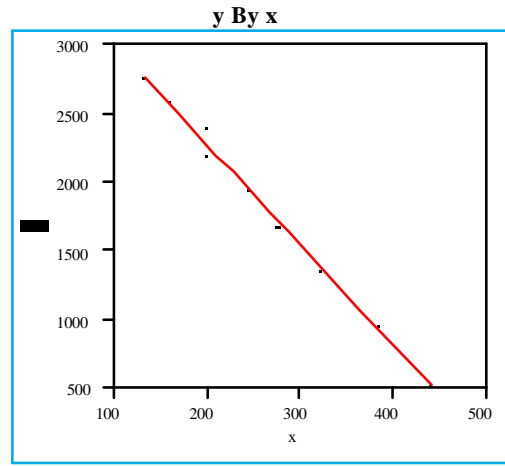

$$
y=3745.78-7.31382 x
$$

RSquare

RSquare Adj

Root Mean Square Erro

Mean of Response
Observations (or Sum Wgts)

0.994448 0.993655 60.6696

$\begin{array}{lrrrr} & \text { Parameter Estimates } & & \\ \text { Term } & \text { Estimate } & \text { Std Error } & \text { t Ratio } & \text { Prob }>|\mathbf{t}| \\ \text { Intercept } & 3745.7817 & 58.09037 & 64.48 & <.0001\end{array}$ $\begin{array}{lrrrrr}\text { Intercept } & 3745.7817 & 58.09037 & 64.48 & <.0001 \\ \mathrm{x} & -7.313823 & 0.206544 & -35.41 & <.0001\end{array}$
Methanol Study 1 x vs $\mathbf{y}$ (Test Sequence $=31$ )

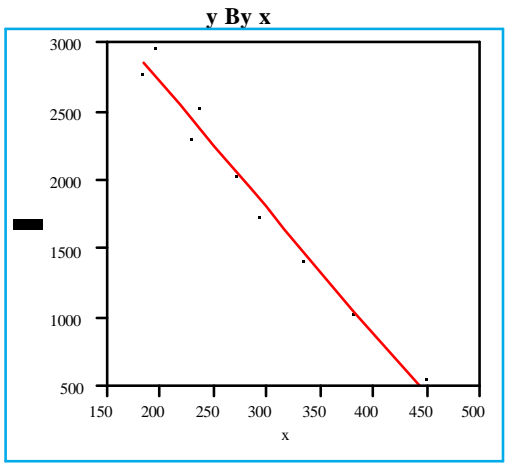

Linear Fit

$y=4503.99-8.99484 x$

RSquare

RSquare Adj

Error

Root Mean Square

Observations (or Sum Wgts)

Parameter Estimates

9

$\begin{array}{lrrrr} & \text { Estimate } & \text { Std Error } & \text { t Ratio } & \text { Prob }>|t|\end{array}$

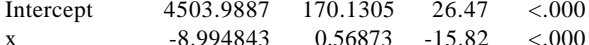

Methanol Study $1 \times$ vs $y$ (Test Sequence $=32$ )

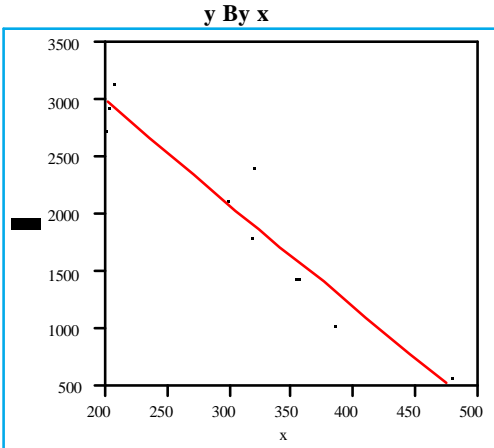

$$
\begin{array}{ll} 
& \multicolumn{1}{c}{\text { Linear Fit }} \\
& y=4783.03-8.97847 x \\
& \text { Summary of Fit } \\
\text { RSquare } & \\
\text { RSquare Adj } & \\
\text { Root Mean Square Error } \\
\text { Mean of Response } \\
\text { Observations (or Sum Wgts) }
\end{array}
$$

2008.081

$\begin{array}{lllll} & \text { Estimate } & \text { Std Error t Ratio Prob }>|\mathbf{t}|\end{array}$ $\begin{array}{lrrrr} & & \text { Es } & \\ \text { lntercept } & 4783.0344 & 316.1333 & 15.13 & <.0001 \\ \mathrm{X} & -8.978465 & 0.982972 & -9.13 & <.0001\end{array}$ 
Exhibit B17: y versus x for Methanol Studies: Study 1; Tests 33-40

Methanol Study 1 x vs $y($ Test Sequence $=33)$

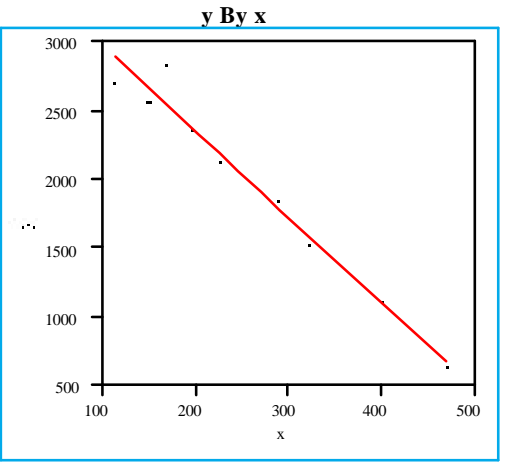

Linear Fit

$y=3580.93-6.16336 x$

RSquare

RSquare Adj

RSquare Adj
Root Mean Square Error

Mean of Response

0.970743

0.966564

137.8448

Observations (or Sum Wgts)

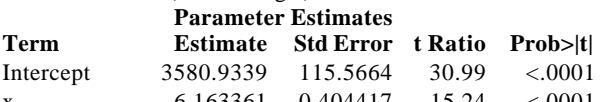

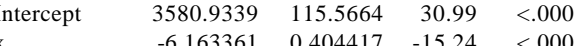

Methanol Study 1 x vs $y($ Test Sequence $=34)$

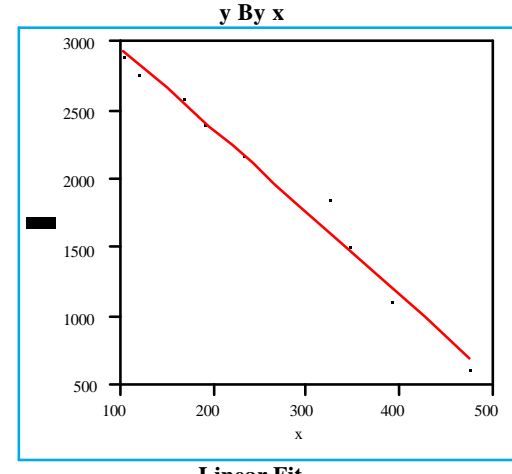$$
y=3567.72-6.0094 x
$$

RSquare Summary of Fit

RSquare Adj

Root Mean Square Error

Mean of Response

0.982659

Parameter Estimates

Term Estimate Std Error t Ratio Prob $>|\mathbf{t}|$ $\begin{array}{lrrrrr} & & 3567.716 & 87.73793 & 40.66 & <.0001 \\ \mathrm{X} & -6.009396 & 0.301732 & -19.92 & <.0001\end{array}$
Methanol Study 1 x vs $y($ Test Sequence $=35$ )

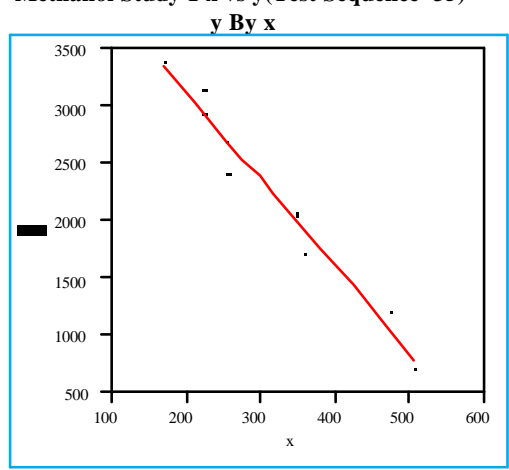
$y=4632.31-7.54973$

\begin{tabular}{lr} 
& \\
RSquare & Summary of Fit \\
RSquare Adj & 0.965008 \\
Root Mean Square Error & 0.96001 \\
Mean of Response & 181.1204 \\
Obere & 2241.973 \\
\hline
\end{tabular}

Observations (or Sum Wgts)

$\begin{array}{lrrrr}\text { Term } & \text { Parameter Estimates } & & \\ \text { Estimate } & \text { Std Error } & \text { t Ratio } & \text { Prob }>\mid \mathbf{t}\end{array}$

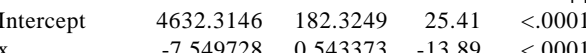

Methanol Study $1 \mathrm{x}$ vs $\mathrm{y}$ (Test Sequence=36)

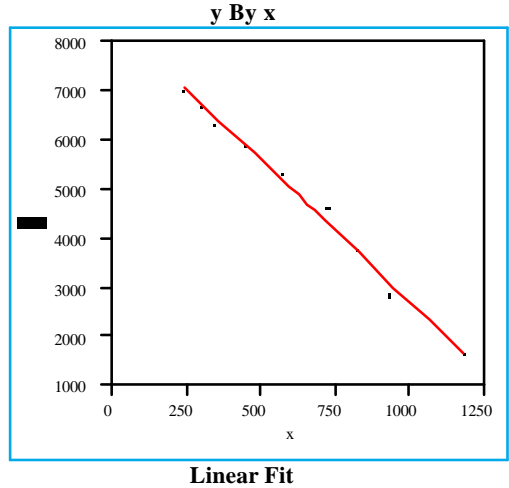$$
\mathrm{y}=8468.6-5.72915
$$

RS Summary of Fit

RSquare

Root Mean Square Error

Mean of Response

Observations (or Sum Wgts)

$$
\text { Parameter Estimates }
$$

$\begin{array}{lrrrr}\text { Term } & \text { Estimate } & \text { Std Error } & \text { t Ratio } & \text { Prob }>|t| \\ \text { Intercept } & 8468.6038 & 131.415 & 64.44 & <.0001\end{array}$

$\begin{array}{lrrrr}\text { Intercept } & 8468.6038 & 131.4151 & 64.44 & <.0001 \\ \mathrm{x} & -5.729154 & 0.189106 & -30.30 & <.0001\end{array}$
Methanol Study 1 x vs y(Test Sequence $=37$ )

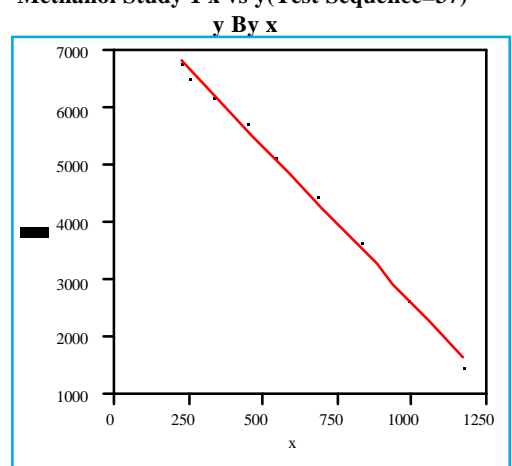

Linear Fit
$y=8067.69-5.43936 x$

Summary of Fit

RSquare

0.995779

RSquare Adj

Meot Mean of Response

Observations (or Sum Wgts)

0.995176

127.19
4718.008

Parameter Estimates

$\begin{array}{llll}\text { Term Estimate } & \text { Std Error } & \text { t Ratio Prob }>|t|\end{array}$ $\begin{array}{lllll}\text { Intercept } \quad 8067.6892 & 92.69647 & 87.03 & <.0001\end{array}$

Methanol Study 1 x vs $y($ Test Sequence $=38$ )

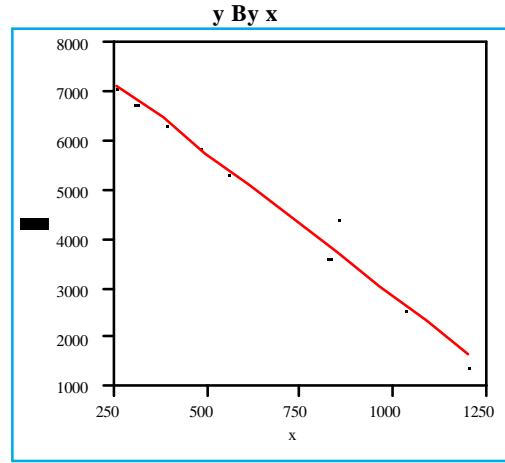

$x$

$$
y=8632.03-5.79524 x
$$

RSquare

RSquare Adj

Root Mean Square Erro

Mean of Response
Observations (or Sum Wgts)

Parameter Estimates

Term Estimate Std Error t Ratio Prob $>|\mathbf{t}|$ $\begin{array}{lrrrr}\text { Intercept } & 8632.0297 & 249.524 & 34.59 & <.0001 \\ \mathrm{x} & -5.795241 & 0.33959 & -17.07 & <.0001\end{array}$
Methanol Study 1 x vs $\mathbf{y}$ (Test Sequence $=39$ )

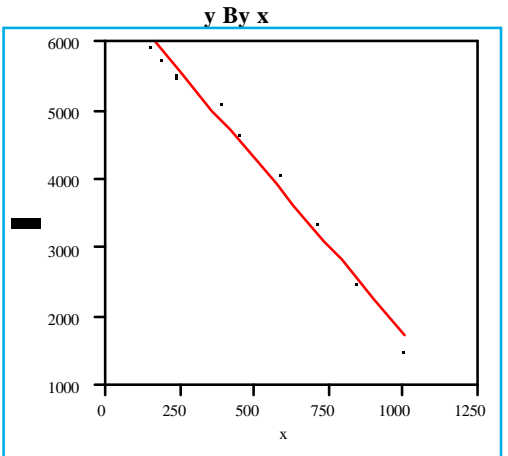

Linear Fit

$y=6859.42-5.08625 x$

RSquare

re Error

0.986762

Root Mean Square Err

0.984871

Mean of Response

Observations (or Sum Wgts)

Term $\begin{array}{llll}\text { Parameter Estimates } & & \\ \text { Estimate } & \text { Std Error } & \text { Ratio } & \text { Prob }>|t|\end{array}$

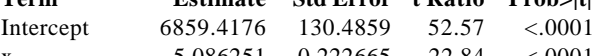

Methanol Study $1 \times$ vs $y($ Test Sequence $=40)$

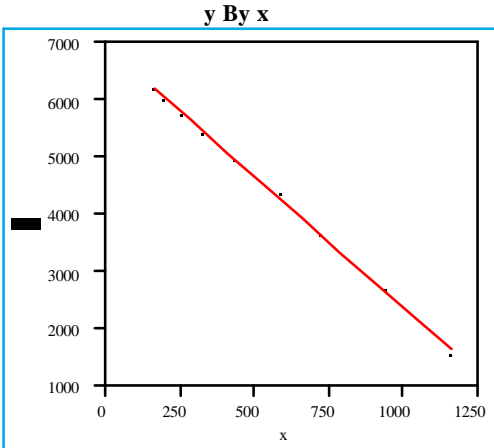

$$
\begin{aligned}
& \text { Linear Fit } \\
& y=6946.16-4.54679 x \\
& \text { Summary of Fit }
\end{aligned}
$$

$\begin{array}{llll} & \text { Parameter Estimates } & & \\ \text { Term } & \text { Estimate Std Error } & \text { t Ratio } & \text { Prob }>|t|\end{array}$ $\begin{array}{llllll}\text { Intercept } & 6946.158 & 39.33057 & 176.61 & <.0001\end{array}$ $\begin{array}{lllll}\mathrm{x} & -4.546789 & 0.062362 & -72.91 & <.000\end{array}$ 


\section{Exhibit B18: y versus x for Methanol Studies: Study 1; Tests 41-44; Study 2; Tests 1-4}

Methanol Study 1 x vs $y($ Test Sequence $=41)$

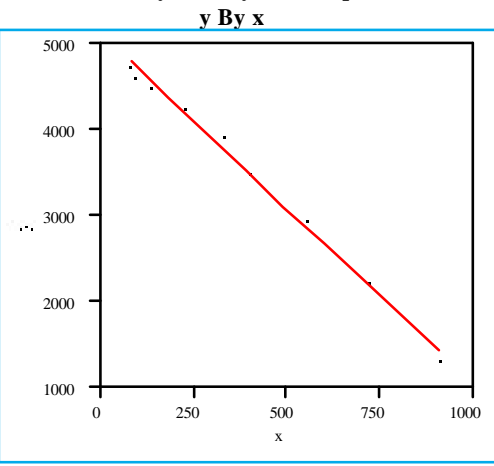

Linear Fit
$\mathrm{y}=5108.58-4.01972 \mathrm{x}$

RSquare
RSquare Ad $j$

Summary of Fit

0.993599

Root Mean Square Error

0.993599

Mean of Response

3544.741

Observations (or Sum Wgts) $\begin{array}{lrrrr} & \text { Parameter Estimates } & & \\ \text { Term } & \begin{array}{r}\text { Estimate } \\ \text { Intd Error }\end{array} & \text { t Ratio } & \text { Prob }>|\mathbf{t}| \\ \text { Intercept } & 5108.5753 & 58.20588 & 87.77 & <.0001 \\ \text { x } & -4.019721 & 0.121942 & -32.96 & <.0001\end{array}$

Methanol Study 1 x vs $y($ Test Sequence $=42)$

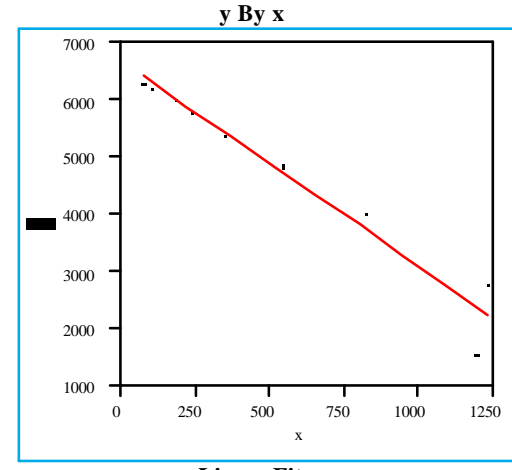

$$
y=6663.07-3.5681
$$

RSquare Summary of Fit

RSquare Adj

Root Mean Square Erro

Mean of Response

Observations (or Sum Wgts)

Estimate Std Error t Ratio Prob $>\mid \mathbf{t}$

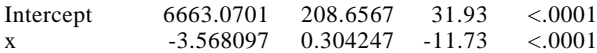

Methanol Study 1 x vs $y($ Test Sequence $=43$ )

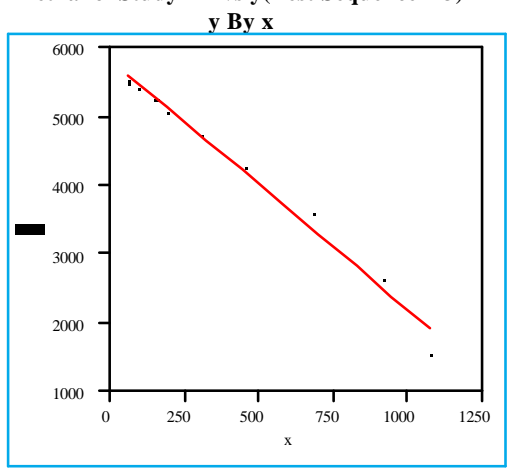

Linear Fit

$=5826.63-3.62815$

$\begin{array}{ll}\text { RSquare } & 0.983371 \\ \text { RSquare Adj } & 0.980996 \\ \text { Root Mean Square Error } & 189.2631 \\ \text { Mean of Response } & 4204.506\end{array}$

Mean of Response

Observations (or Sum Wgts)

$\begin{array}{lrrrrr}\text { Term } & \text { Parameter Estimates } & & \\ \text { Intercept } & \text { Estimate } & \text { Std Error } & \text { t Ratio } & \text { Prob }>|t| \\ x & 5826.6295 & 101.6681 & 57.31 & <.0001\end{array}$ $\begin{array}{lrrrr} & 5826.6295 & 101.6681 & 57.31 & <.000 \\ \mathrm{x} & -3.628153 & 0.178322 & -20.35 & <.000\end{array}$

Methanol Study 1 x vs $y($ Test Sequence $=44)$

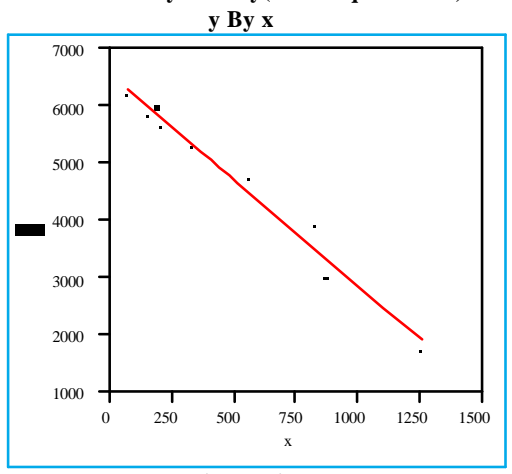

\section{Linear $\mathrm{F}$}

$$
y=6532.57-3.67306 x
$$

RSquare Summary of Fit

RSquare Adj

Root Mean Square Erro

Mean of Response

Observations (or Sum Wgts) Parameter Estimates

Term Estimate Std Error t Ratio Prob $>|\mathbf{t}|$

$\begin{array}{lrrrr}\text { Intercept } & 6532.5719 & 135.1305 & 48.34 & <.0001 \\ \mathrm{x} & -3.67306 & 0.213761 & -17.18 & <.0001\end{array}$
Methanol Study $2 x$ vs $y($ Test Sequence $=1)$

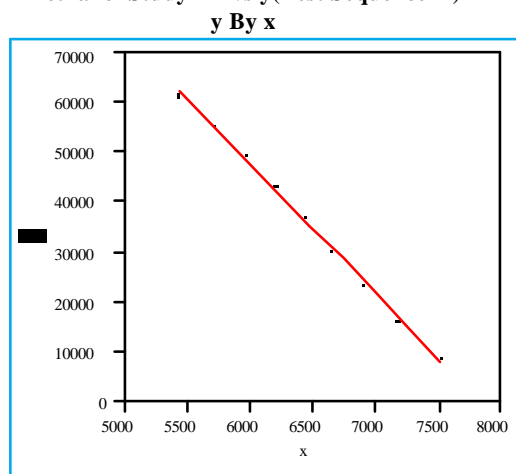

Linear Fit

$y=205070-26.1605 x$

RSquare

RSquare Adj

Error

Mean of Respons

Observations (or Sum Wgts)

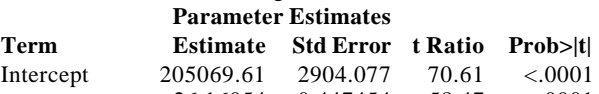

$\begin{array}{llllll}\text { Intercept } & 205069.61 & 2904.077 & 70.61 & <.000 \\ \mathrm{x} & & 26.16054 & 0.447454 & -58.47 & <.0001\end{array}$

Methanol Study 2 x vs y(Test Sequence $=2)$

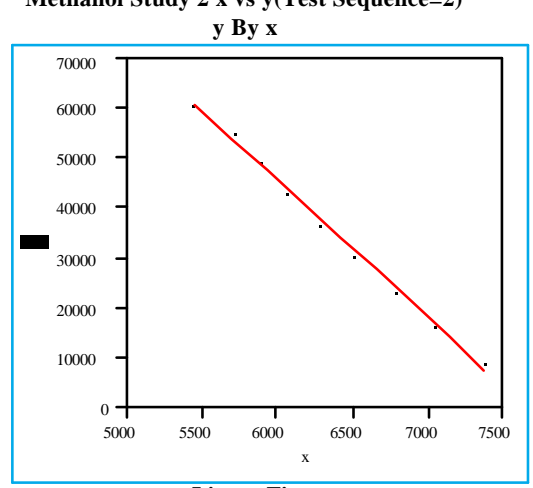

Linear Fit

$$
y=211648-27.6427 x
$$

Summary of Fit

RSquare

0.996526

RSquare Adj

Root Mean Square Erro

0.996029

Mean of Response

1115.702

Observations (or Sum Wgts)

$\begin{array}{lrrrr}\text { Term } & \text { Estimate } & \text { Std Error } & \text { t Ratio } & \text { Prob }>|\mathbf{t}| \\ \text { Intercept } & 211647.76 & 3943.559 & 53.67 & <.0001\end{array}$ $\begin{array}{lrrrr}\text { Intercept } & 211647.76 & 3943.559 & 53.67 & <.0001 \\ \mathrm{x} & -27.64273 & 0.6169 & -44.81 & <.0001\end{array}$
Methanol Study 2 x vs $\mathbf{y}$ (Test Sequence $=3$ ) y By x

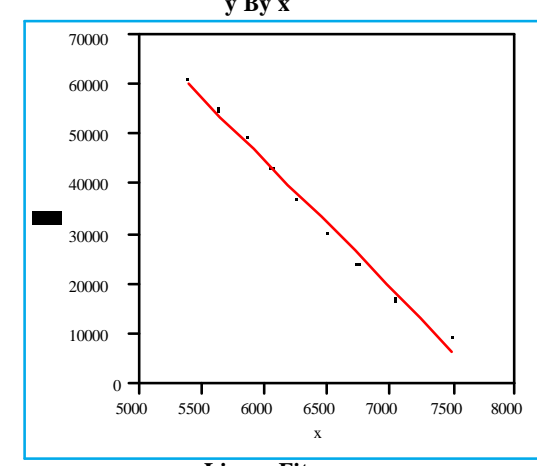

\section{$\mathrm{y}=199115-25.6753$}

Summary of Fit

RSquare

RSquare Adj

Root Mean Square Error

Mean of Response

Observations (or Sum Wgts)

0.992474

0.991399

36232.25

Parameter Estimates

Term Estimate Std Error t Ratio Prob $>\mid \mathbf{t}$ $\begin{array}{lrrrr}\text { Intercept } & 199114.89 & 5388.57 & 36.95 & <.000 \\ \mathrm{x} & -25.67527 & 0.845036 & -30.38 & <.0001\end{array}$

Methanol Study 2 x vs $y$ (Test Sequence $=4$ )

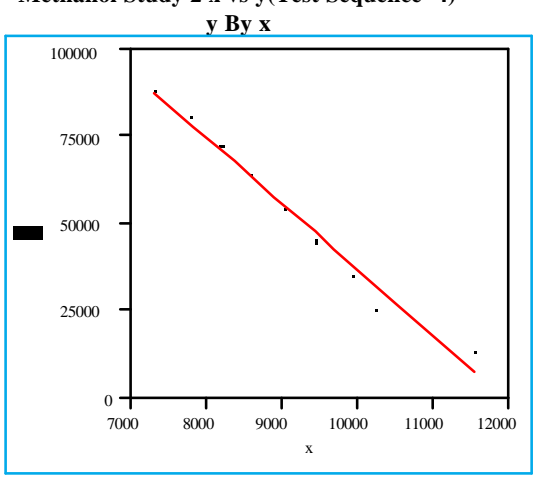

Linear Fit

$$
\mathrm{y}=227669-19.0502
$$

RSquare

Summary of Fit

0.980838

RSquare Adj

Root Mean Square Error

0.978

Mean of Response

Observations (or Sum Wgts)

$$
\text { (5) }
$$

Term Parameter Estimates

\begin{tabular}{rrrrr} 
Estimate & Std Error & t Ratio & Prob $>|t| t$ \\
\hline 227669.28 & 9307.224 & 24.46 & $<.0001$
\end{tabular} $\begin{array}{rrrrr}\mathrm{x} & -19.05022 & 1.00641 & -18.93 & <.0001\end{array}$ 
Exhibit B19: y versus x for Methanol Studies: Study 2; Tests 5-12
Methanol Study $2 x$ vs $y$ (Test Sequence=5)

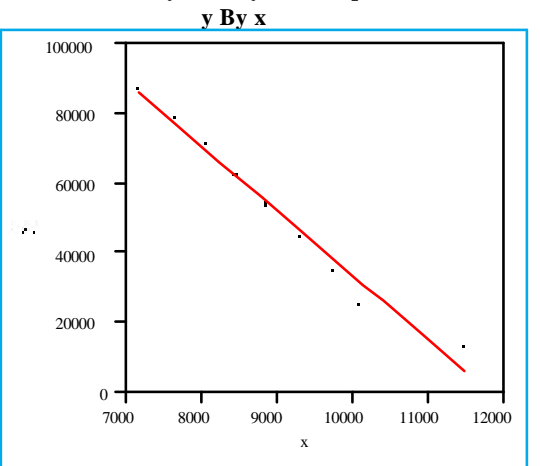

\section{Linear Fit
$y=218820-18.4794 x$ \\ ummary of Fit}

RSquare

RSquare Adj

Root Mean Square Erro

0.974546

0.9709

Mean of Response

4290.872
52492.13

Observations (or Sum Wgts)

\begin{tabular}{lrrrr} 
& \multicolumn{1}{c}{ Parameter Estimates } & & \\
Term & Estimate & Std Error & t Ratio & Prob $>|\mathbf{t}|$ \\
Intercept & 218819.58 & 10260.07 & 21.33 & $<.0001$ \\
$\mathrm{X}$ & -18.47943 & 1.128791 & -16.37 & $<.0001$
\end{tabular}

Methanol Study 2 x vs y $($ Test Sequence $=6)$

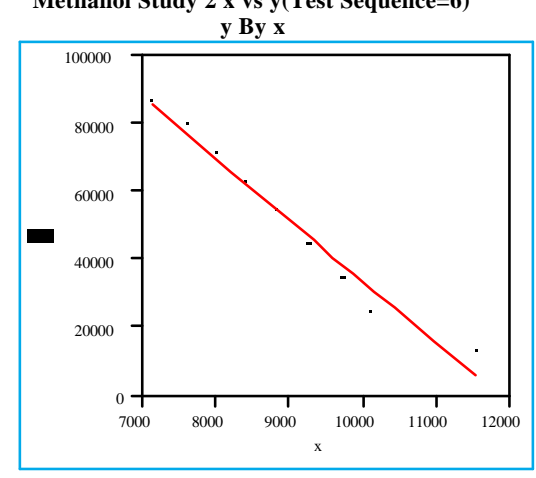

$$
\begin{gathered}
\text { Linear Fit } \\
y=214922-18.0484 x \\
\text { Summary of Fit }
\end{gathered}
$$

RSquare

RSquare Adj

Root Mean Square Erro

Mean of Response

0.974055

Observations (or Sum Wgts)

0.970348

4324.27

52761.48

$\begin{array}{llll}\text { Term Estimate } & \text { Std Error t Ratio } & \text { Prob }>|t|\end{array}$

\begin{tabular}{lllll} 
Intercept & 214921.8 & 10106.35 & 21.27 & $<.000$ \\
\hline & -18.04845 & 1.113337 & -16.21 & $<.0001$
\end{tabular}

$\begin{array}{lllll}\mathrm{x} & -18.04845 & 1.113337 & -16.21 & <.000\end{array}$
Parameter Estimates
Methanol Study $2 x$ vs $y$ (Test Sequence=7)

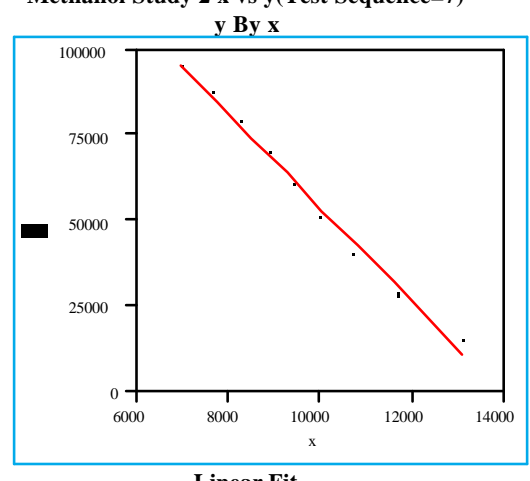

Linear Fit

$$
y=192844-13.8382
$$

RSquare

RSquare Adj

Summary of Fit

Root Mean Square Error

Mean of Response

0.990783

0.989466

58562.43

Observations (or Sum Wgts)

$\begin{array}{lrrrr}\text { Term } & \text { Estimate } & \text { Std Error } & \text { t Ratio } & \text { Prob }>|\mathbf{t}| \\ \text { Intercept } & 192844.39 & 4984.023 & 38.69 & <.0001\end{array}$

$\begin{array}{llllll}\mathrm{x} & -13.83815 & 0.504473 & -27.43 & <.0001\end{array}$

Methanol Study $2 x$ vs $\mathbf{y}($ Test Sequence $=8$ )

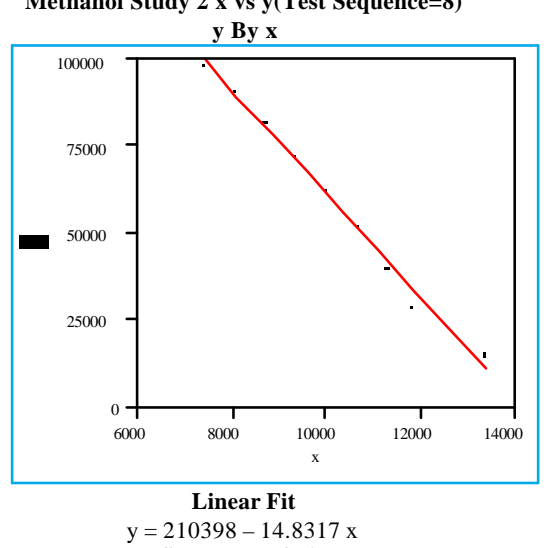

RSquare

RSquare Adj

Mean of Response

0.989809

0.988354

3085.596
60331.2

Observations (or Sum Wgts)

Parameter Estimates $\begin{array}{llll}-14.83174 & 0.568812 & -26.07 & <.0001\end{array}$

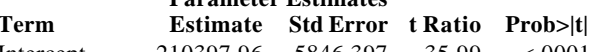

$\begin{array}{llllll}\text { Intercept } & 210397.96 & 5846.397 & 35.99 & <.0001\end{array}$
Methanol Study $2 x$ vs $y($ Test Sequence $=9$ )

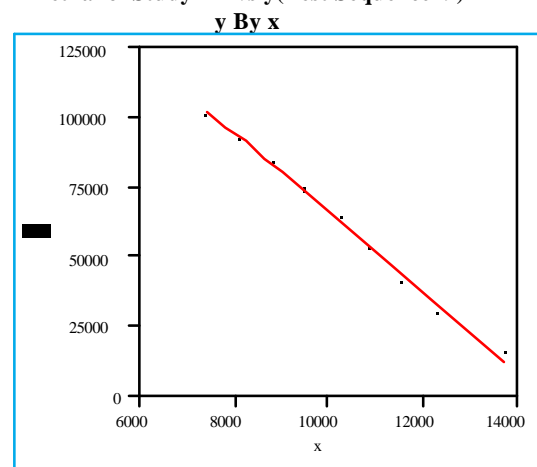

\section{Linear Fit}

$y=208243-14.1948 x$

$\begin{array}{lr}\text { RSquare } \quad \text { Summary of Fit } & \\ \text { RSquare Adj } & 0.994036 \\ \text { Root Mean Square Error } & 0.993184 \\ \text { Mean of Response } & 2414.13 \\ \text { Ober } & 61617.84\end{array}$

Mean of Response

(or Sum Wgts) $\begin{array}{lrrrr} & \text { Parameter Estimates } & & \\ \text { Term } & \text { Estimate } & \text { Std Error } & \text { t Ratio } & \text { Prob }>|\mathbf{t}| \\ \text { Intercept } & 208243.04 & 4367.582 & 47.68 & <.0001\end{array}$

Methanol Study 2 x vs y(Test Sequence $=10$ )

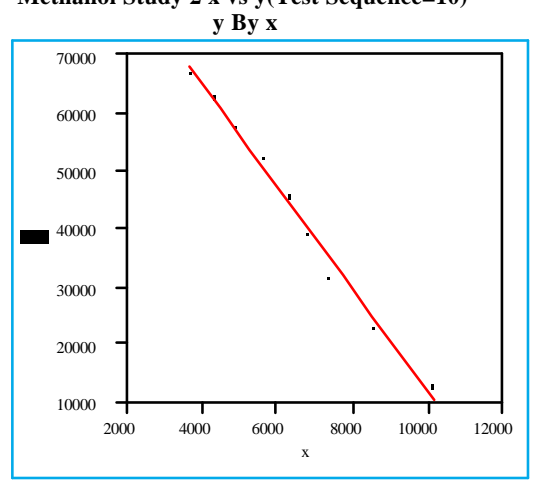

Linear Fi

$y=100777-8.88866 x$

Summary of Fit

0.989742

RSquare

Root Mean Square Erro

Mean of Respons

0.988277

Observations (or Sum Wgts)

43409.03

Parameter Estimates

$\begin{array}{lrrrr}\text { Estimate } & \text { Std Error } & \text { t Ratio } & \text { Prob }>|t|\end{array}$ $\begin{array}{lrrrrr}\mathrm{x} & -8.88866 & 0.342019 & -25.99 & <.0001\end{array}$
Methanol Study $2 x$ vs $y$ (Test Sequence=11)

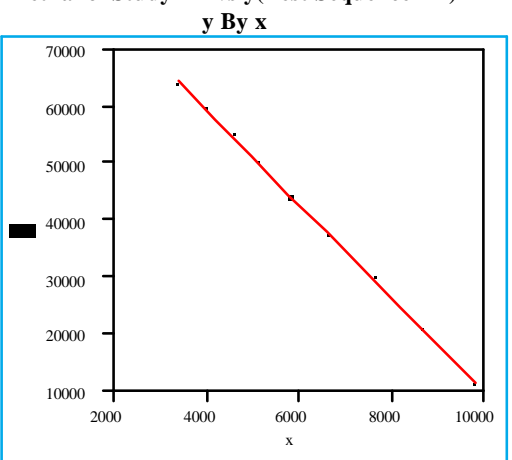

Linear Fit
$y=92607.4-8.24197 x$

Summary of Fit

RSquare

RSquare Adj

Root Mean Square Error

Mean of Response

0.999506

0.999436

427.5053

Observations (or Sum Wgts)

$\begin{array}{lrrrr}\text { Term } & \text { Parameter Estimates } & & \\ & \text { Estimate } & \text { Std Error } & \text { t Ratio } & \text { Prob }>|\mathbf{t}|\end{array}$ $\begin{array}{llllll} & & \text { E2607.415 } & 454.3138 & 203.84 & <.0001 \\ \mathrm{x} & -8.241971 & 0.069226 & -119.1 & <.0001\end{array}$

Methanol Study $2 \times$ vs $y($ Test Sequence $=12)$

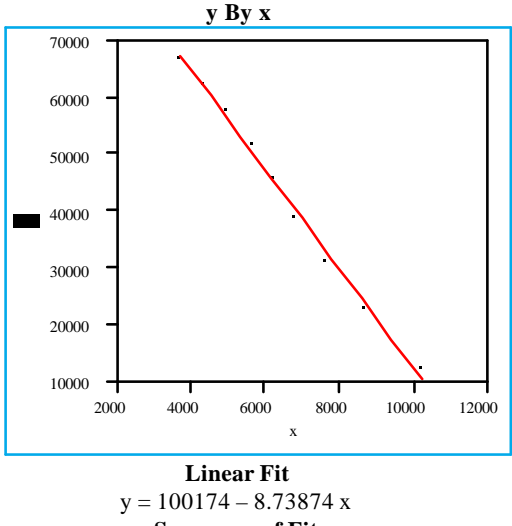

RSquare

RSquare Adj

Reot Mean Square Error

Observations (or Sum Wgts)

0.993999

0.993142

1542.153

Parameter Estimates

Term Estimate Std Error t Ratio Prob $>\mid \mathbf{t}$

$x$ 


\section{Exhibit B20: y versus x for Methanol Studies: Study 2; Tests 13-15; Study 3; Test 1-5}

Methanol Study $2 x$ vs $y($ Test Sequence=13)

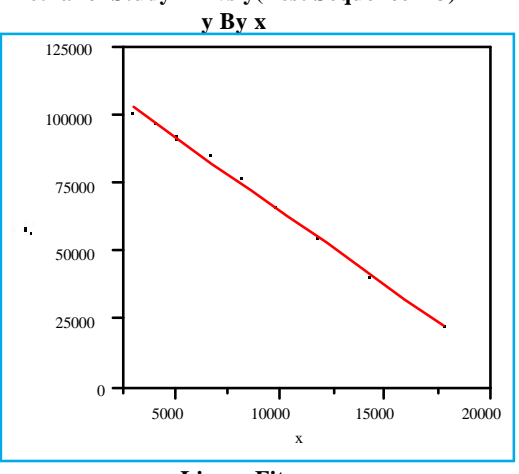

Linear Fit

$y=119264-5.38691 x$

RSquare

RSquare Adj

Root Mean Square Error

Mean of Response

0.99746

0.997097

70690.72

Observations (or Sum Wgts)

$\begin{array}{lrrrrr}\text { Term } & \text { Parameter Estimates } & & \\ \text { Intercept } & 119263.84 & 1043.753 & 114.26 & <.0001\end{array}$ $\begin{array}{lllll}\mathrm{X} & -5.386912 & 0.102749 & -52.43 & <.000\end{array}$

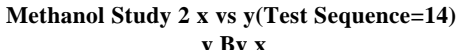

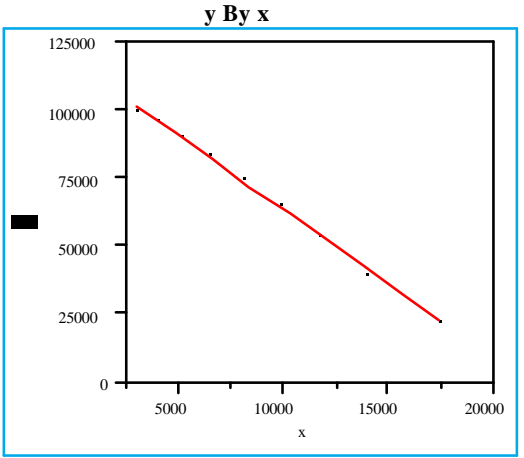

$$
\begin{gathered}
\text { Linear Fit } \\
y=119403-5.53406 x
\end{gathered}
$$

RSquare

Summary of Fit

RSquare Adj

ean Square Erro

0.996959

Mean of Response

0.996524

Observations (or Sum Wgts)

(Param Wgts)

Term Estimate Std Error t Ratio Prob $>|\mathbf{t}|$

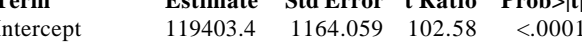

$\begin{array}{lllll}\mathrm{x} & -5.534062 & 0.115528 & -47.90 & <.0001\end{array}$
Methanol Study $2 \mathrm{x}$ vs $\mathrm{y}($ Test Sequence $=15)$

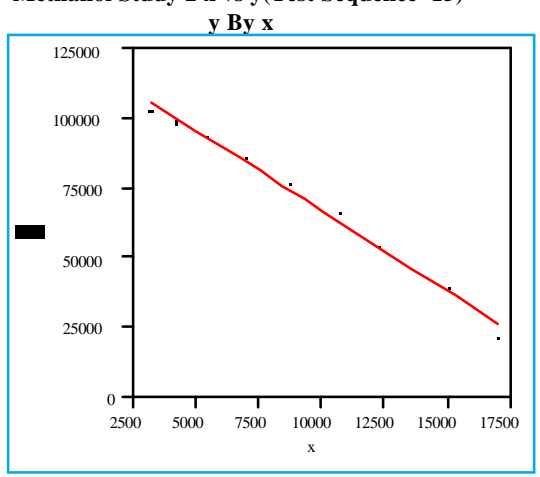

\section{Linear Fit}

$y=124875-5.76699 x$

RSquare

RSquare Adj mmary of Fi

Root Mean Square Erro

Observations (or Sum Wgts)

\begin{tabular}{lrrrr} 
& \multicolumn{1}{c}{ Parameter Estimates } & & \\
Term & $\begin{array}{r}\text { Estimate } \\
\text { Std Error }\end{array}$ & t Ratio & Prob $>|\mathbf{t}|$ \\
Intercept & 124875.24 & 2235.303 & 55.87 & $<.0001$ \\
$\mathrm{x}$ & -5.766986 & 0.214246 & -26.92 & $<.0001$
\end{tabular}

Methanol Study $3 x$ vs $y($ Test Sequence $=1)$

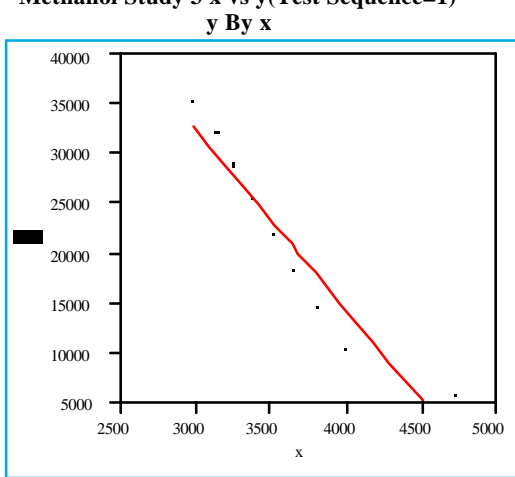

Linear Fit

$$
\mathrm{y}=87214.6-18.2158 \mathrm{x}
$$

Summary of Fit

$\begin{array}{lr}\text { RSquare } & 0.918349 \\ \text { RSquare Adj } & 0.906684 \\ \text { Root Mean Square Error } & 3058.97 \\ \text { Mean of Response } & 21392.24 \\ \text { Observations (or Sum Wots) } & 9\end{array}$

Observations (or Sum

Parameter Estimates

$\begin{array}{lrrrr}\text { Term } & \text { Estimate } & \text { Std Error } & \text { t Ratio } & \text { Prob }>|\mathbf{t}| \\ \text { Intercept } & 87214.559 & 7487.997 & 11.65 & <.0001\end{array}$

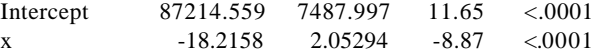

Methanol Study $3 x$ vs $y($ Test Sequence $=2)$

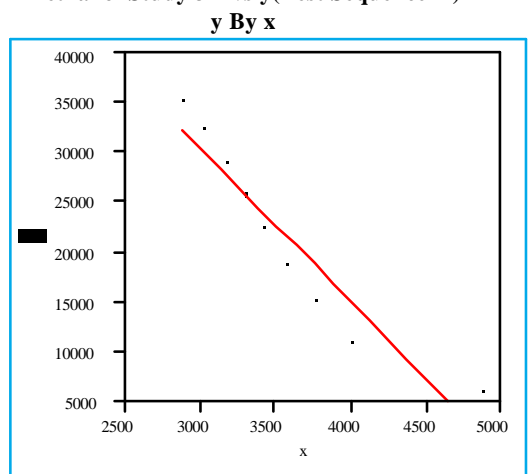

$$
\begin{aligned}
& \text { Linear Fit } \\
& y=77032.2-15.4758 x
\end{aligned}
$$

RSquare Summary of Fit

Root Mean Square Error

Mean of Response

Observations (or Sum Wgts)

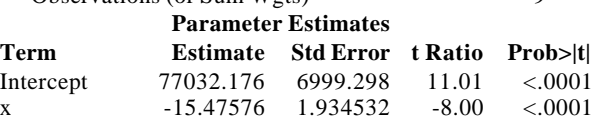

Methanol Study $3 x$ vs y(Test Sequence $=3)$

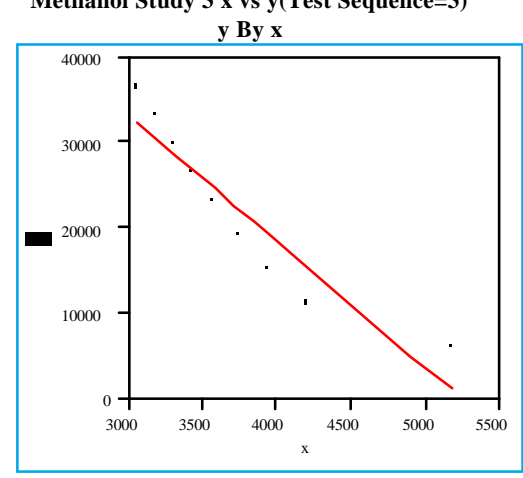

$$
\mathrm{y}=77310.3-14.669 \mathrm{x}
$$$$
\text { Summary of Fit }
$$

RSquare

0.883128

RSquare Adj

Root Mean Square Error

Mean of Response
Observations (or Sum Wgts)

3766.86

22565.6 $\begin{array}{lrrrrr}\text { Term } & \text { Estimate } & \text { Std Error } & \text { t Ratio } & \text { Prob }>|\mathbf{t}| \\ \text { Intercept } & 77310.284 & 7631.254 & 10.13 & <.0001\end{array}$

Intercept

$\begin{array}{rrrr}-14.66901 & 2.016951 & -7.27 & 0.0002\end{array}$
Methanol Study $3 x$ vs $y($ Test Sequence $=4)$

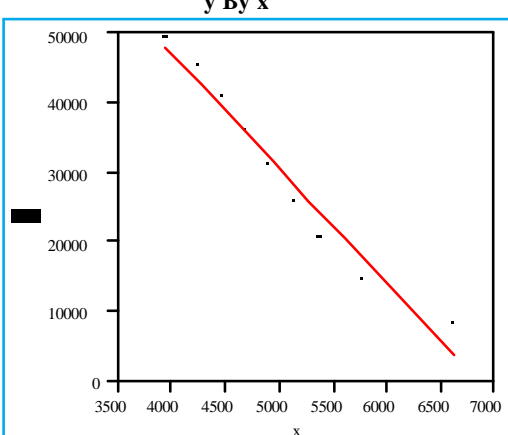

\section{Linear Fit}

$y=113921-16.6183 x$

RSquare Summary of Fit

Root Mean Square Error

Mean of Response

Observations (or Sum Wgts)

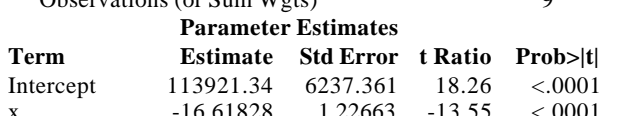

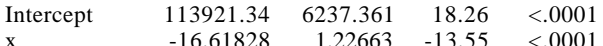

Methanol Study $3 x$ vs $y$ (Test Sequence $=5$ )

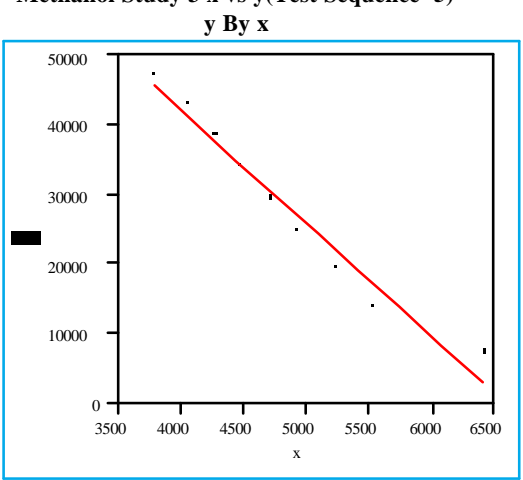

Linear Fit

$$
y=107407-16.2718 x
$$

Summary of Fit

RSquare

RSquare Adj

Root Mean Square Error

Mean of Response

0.964248

0.95914

28854.91

Parameter Estimates

$\begin{array}{lrrrr} & \text { Estimate } & \text { Std Error } & \text { t Ratio } & \text { Prob }>|\mathbf{t}| \\ \text { Tntercept } & 10740677 & 5788.624 & 18.55 & <.0001\end{array}$ $\begin{array}{lrrrr}\text { Intercept } & 107406.77 & 5788.624 & 18.55 & <.000 \\ \mathrm{x} & -16.27179 & 1.184254 & -13.74 & <.0001\end{array}$ 
Exhibit B21: $y$ versus x for Methanol Studies: Study 3; Test 6-13

Methanol Study $3 x$ vs $y($ Test Sequence $=6$ ) y By $\mathbf{x}$

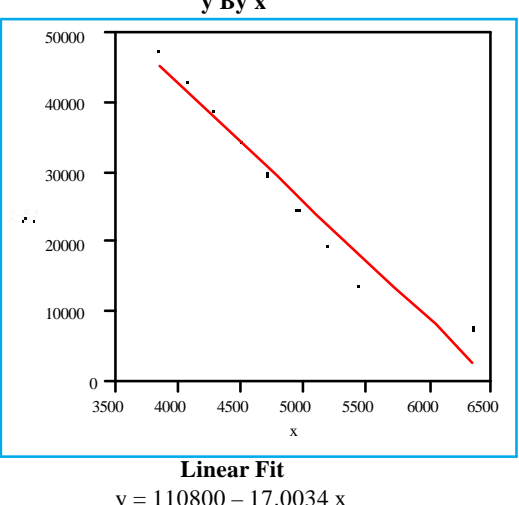
$y=110800-17.0034$

RSquare

RSquare Adj

Rean Square Error

0.95764

Mean of Response

2962.969
28684.81

Observations (or Sum Wgts)

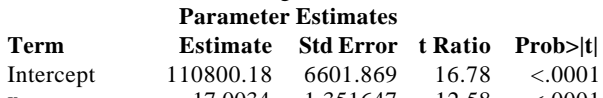

$\begin{array}{lrrrr}\text { Intercept } & 110800.18 & 6601.869 & 16.78 & <.000 \\ \mathrm{x} & -17.0034 & 1.351647 & -12.58 & <.0001\end{array}$

Methanol Study $3 x$ vs $y($ Test Sequence $=7)$ y By $x$

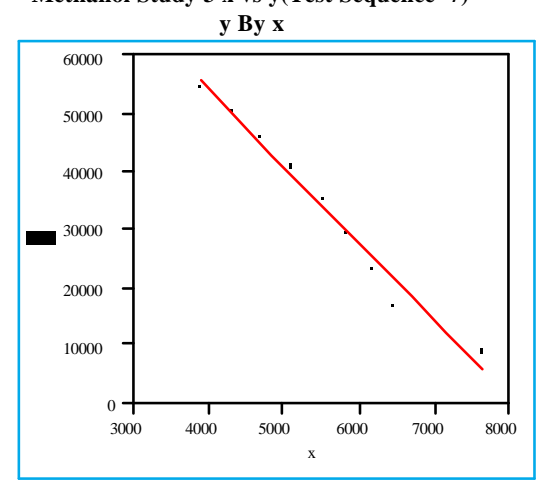

Linear Fit

$$
y=107317-13.2756 x
$$

RSquare

Summary of Fit

RSquare Adj

0.978254

Root Mean Square Error

0.975147

Mean of Response

33995.83

Observations (or Sum Wgts)

$\begin{array}{lrrrr}\text { Term } & \text { Estimate } & \text { Std Error } & \text { t Ratio } & \text { Prob }>|t|\end{array}$

$\begin{array}{lrrrr}\text { Intercept } & 107317.08 & 4212.714 & 25.47 & <.000 \\ \mathrm{x} & -13.27563 & 0.748123 & -17.75 & <.000\end{array}$
Methanol Study $3 x$ vs $y($ Test Sequence $=8$ )

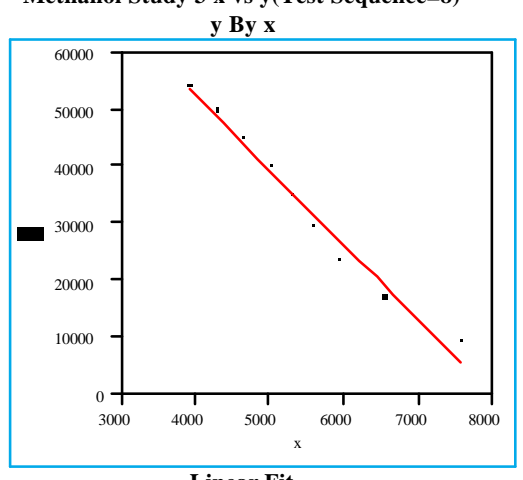

Linear Fit

$y=105267-13.104 x$

RSquare

Summary of Fit

RSquare Adj
Root Mean Square Error

Mean of Response

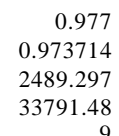

Observations (or Sum Wgts)

$\begin{array}{lllll}\text { Term } & \text { Parameter Estimates } & & \\ \text { Estimate } & \text { Std Error } & \text { t Ratio } & \text { Prob }>|t|\end{array}$

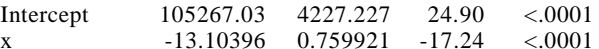

Methanol Study 3 x vs y $($ Test Sequence $=9)$

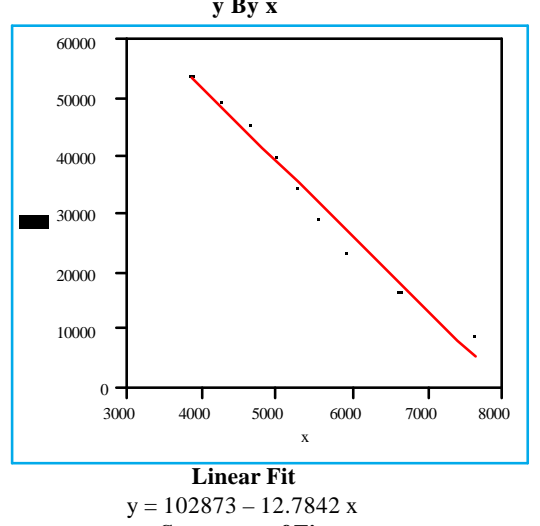

Summary of Fit

RSquare

RSquare Adj

Root Mean Square Error

Mean of Response

0.977539

0.97433

2451.736

Observations (or Sum Wgts)

33361.84
9

Estimate Std Error t Ratio Prob $>|t|$

$\begin{array}{lrrrr}\text { Intercept } & 102872.98 & 4065.488 & 25.30 & <.0001 \\ \mathrm{x} & -12.78424 & 0.732447 & -17.45 & <.0001\end{array}$
Methanol Study 3 x vs $y($ Test Sequence $=10$ )

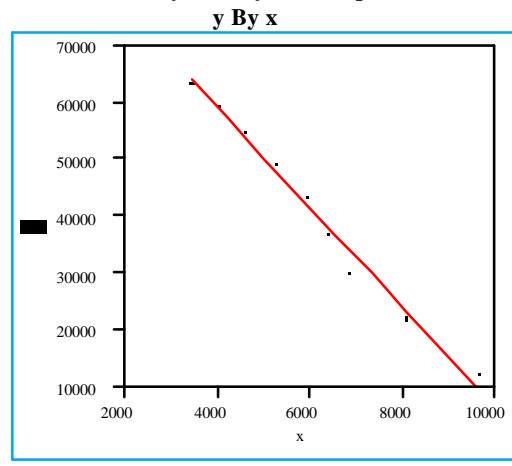

Linear Fit

$y=94548.7-8.75015 x$

RSquare

Summary of Fit

RSquare Ad

Root Mean Square Erro

Mean of Response

0.987195

0.985366

2115.314

Observations (or Sum Wgts)

$\begin{array}{lllll} & \text { Estimate } & \text { Std Error } & \text { t Ratio } & \text { Prob }>|\mathbf{t}|\end{array}$ $\begin{array}{lrrrrr} & & 94548.72 & 2401.324 & 39.37 & <.0001 \\ \mathrm{x} & -8.750152 & 0.376663 & -23.23 & <.0001\end{array}$

Methanol Study 3 x vs $y($ Test Sequence=11)

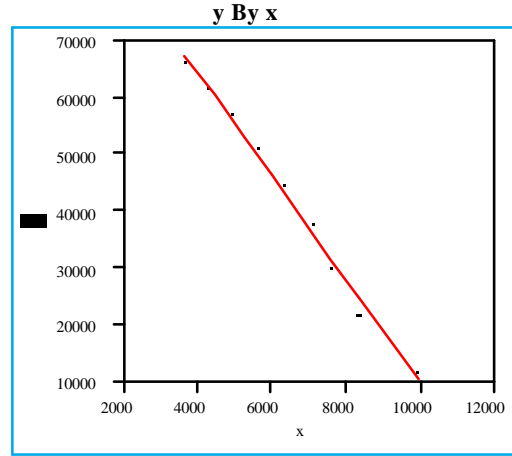

\section{Linear Fit}

$y=101412-9.14224 x$

$\begin{array}{lr}\text { RSquare } & \text { Summary of Fit } \\ \text { RSquare Adj } & 0.990414 \\ \text { Root Mean Square Error } & 0.989044 \\ \text { Mean of Response } & 1953.107 \\ \text { Obsere } & 42302.77\end{array}$

Mean of Response

Parameter Estimates

Term Estimate Std Error t Ratio Prob $>|\mathbf{t}|$

$\begin{array}{llllll}\text { Intercept } & 101412.15 & 2292.377 & 44.24 & <.0001 \\ \mathrm{x} & -9.142236 & 0.339955 & -26.89 & <.0001\end{array}$
Methanol Study 3 x vs $y($ Test Sequence=12)

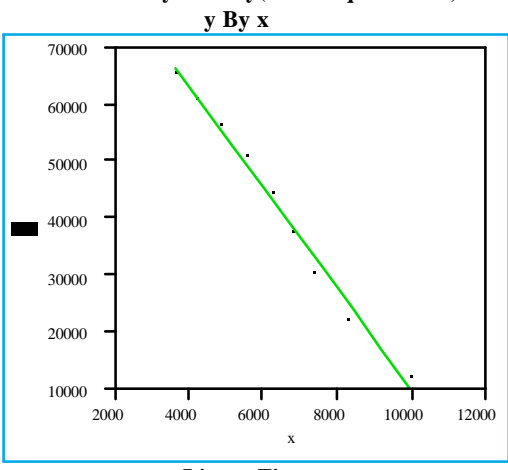

Linear Fit

$y=99281.7-8.93182 x$

Summary of Fit

0.989562

RSquare

RSquare Adj

Root Mean Square Error

0.988071

Mean of Response

2001.356

Observations (or Sum Wgts)

$\begin{array}{lrlll}\text { Term } & \text { Parameter Estimates } & & \\ \text { Estimate } & \text { Std Error } & \text { t Ratio } & \text { Prob }>|t|\end{array}$ $\begin{array}{lrrrrr} & 99281.724 & 2306.277 & 43.05 & <.0001 \\ \mathrm{x} & -8.931823 & 0.34672 & -25.76 & <.0001\end{array}$

Methanol Study $3 \times$ vs $y($ Test Sequence $=13)$

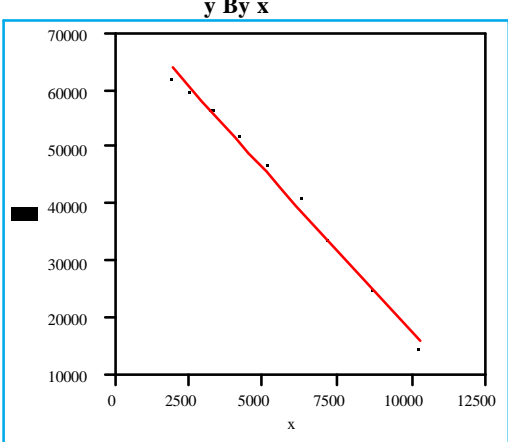

\section{Linear Fit}

$y=75456.2-5.76836 x$

RSquare

RSquare Adj

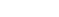

0.994078

Root Mean Square Error

0.993232

Mean of Response

1358.924

(or Sum Wgts)

Term Estimate Std Error t Ratio Prob $>|\mathbf{t}|$

$\begin{array}{lllll}\text { Intercept } & 75456.165 & 1037.123 & 72.76 & <.0001 \\ \mathrm{x} & -5.768356 & 0.168275 & -34.28 & <.0001\end{array}$ 


\section{Exhibit B22: y versus x for Methanol Studies: Study 3; Test 14-15; Study 4; Tests 1-6}

Methanol Study $3 x$ vs $y($ Test Sequence=14)

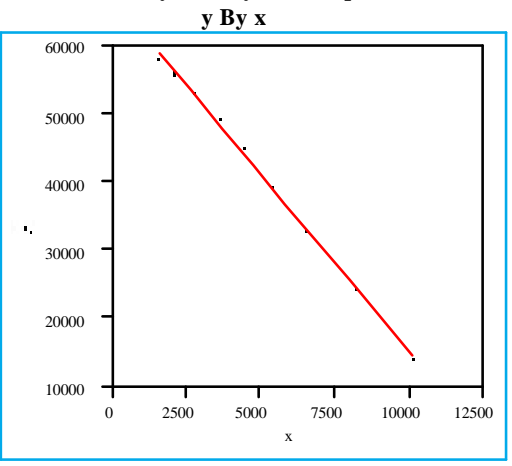

Linear Fit

$$
\mathrm{y}=67469.6-5.18471
$$

RSquare

Summary of Fit

RSquare Ad

Root Mean Square Error

0.998361

649.9023

Observations (or Sum Wgts)

41286.79

$\begin{array}{lrrrrr}\text { Term } & \text { Parameter Estimates } & & \\ \text { Estimate } & \text { Std Error } & \text { t Ratio } & \text { Prob }>|\mathbf{t}| \\ \text { Intercept } & 67469.624 & 455.7619 & 148.04 & <.0001\end{array}$ $\begin{array}{lrrrrr} & 67469.624 & 455.7619 & 148.04 & <.000 \\ \text { Intercept } & -5.184708 & 0.079403 & -65.30 & <.000\end{array}$

Methanol Study $3 \times$ vs $y($ Test Sequence $=15)$

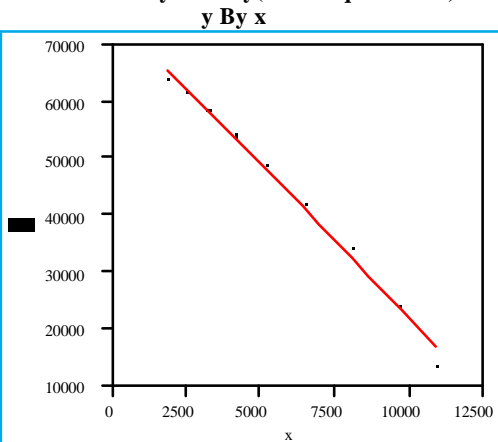

$y=76384.2-5.4165 x$

RSquare

0.988972

$\begin{array}{lr}\text { RSquare Adj } & 0.987397 \\ \text { Root Mean Square Error } & 1982.526\end{array}$

1982.526

Observations (or Sum Wgts)

Parameter Estimates

$\begin{array}{lrrrr}\text { Term } & \text { Estimate } & \text { Std Error } & \text { t Ratio } & \text { Prob }>|t| \\ \text { Intercept } & 76384.247 & 1433.279 & 53.29 & <.000\end{array}$

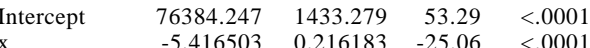

Methanol Study $4 x$ vs $y($ Test Sequence $=1)$

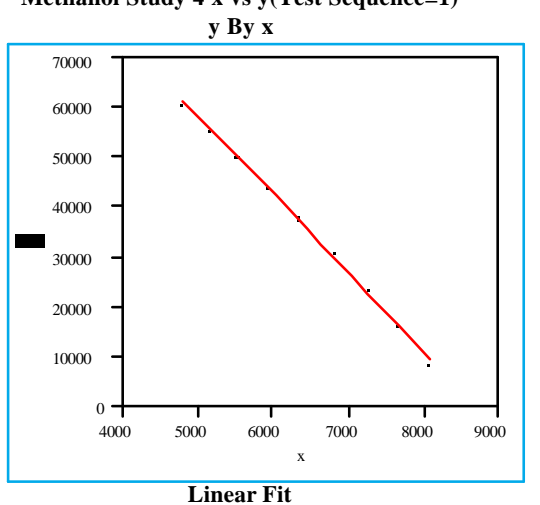

Linear Fit
$y=137739-15.832$ Summary of Fit

RSquare Adj

Root Mean Square Error

Mean of Response

Observations (or Sum Wgts)

Term Estimate Std Error t Ratio Prob $>|t|$ $\begin{array}{lllll}\text { Intercept } & 137739.16 & 2167.695 & 63.54 & <.000\end{array}$

Methanol Study $4 x$ vs $y($ Test Sequence $=2)$

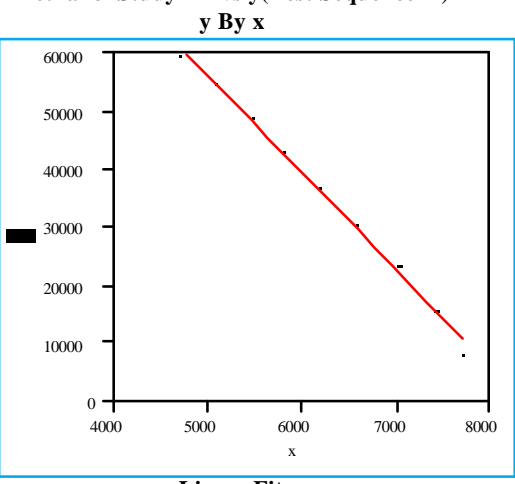

Linear Fit

$$
y=140620-16.8247 x
$$

RSquare

Summary of Fit

RSquare Adj

Root Mean Square Error

Mean of Response

Observations (or Sum Wgts)

0.994936

0.994936

1343.954

35506.83

Parameter Estimates
Parts

$\begin{array}{lrrrr}\text { Term } & \text { Estimate } & \text { Std Error } & \text { t Ratio } & \text { Prob }>|t|\end{array}$

$\begin{array}{lrrrr} & 140619.93 & 2869.636 & 49.00 & <.0001 \\ \mathrm{In} & -16.82471 & 0.453691 & -37.08 & <.0001\end{array}$
Methanol Study $4 x$ vs $y($ Test Sequence $=3)$

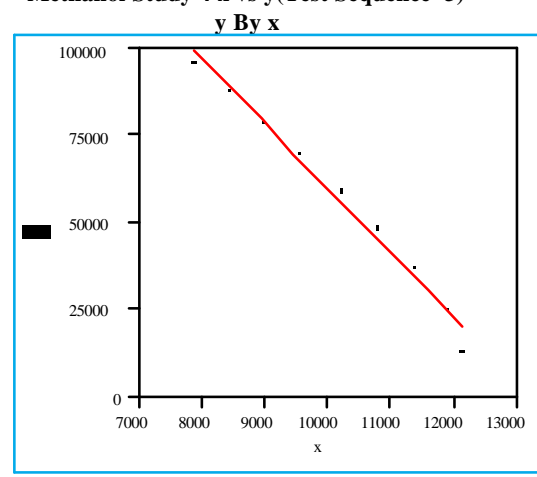

Linear Fit

$y=244994-18.4655 x$

$\begin{array}{lr} & \\ \text { RSquare } \quad \text { Summary of Fit } & 0.986221 \\ \text { RSquare Adj } & 0.984253 \\ \text { Root Mean Square Error } & 3604.68 \\ \text { Mean of Response } & 57389.17\end{array}$

Mean of Response

\begin{tabular}{lrrrrr}
\multicolumn{5}{c}{ Observations (or Sum Wgts) } & \multicolumn{1}{c}{9} \\
Term & Parameter Estimates & & \\
Intercept & Estimate & Std Error & t Ratio & Prob $>|t|$ \\
$x$ & 244993.76 & 8467.026 & 28.94 & $<.0001$
\end{tabular} $\begin{array}{lrrrrr}\text { Intercept } & 244993.76 & 8467.026 & 28.94 & <.000\end{array}$

Methanol Study $4 x$ vs $y($ Test Sequence $=4)$

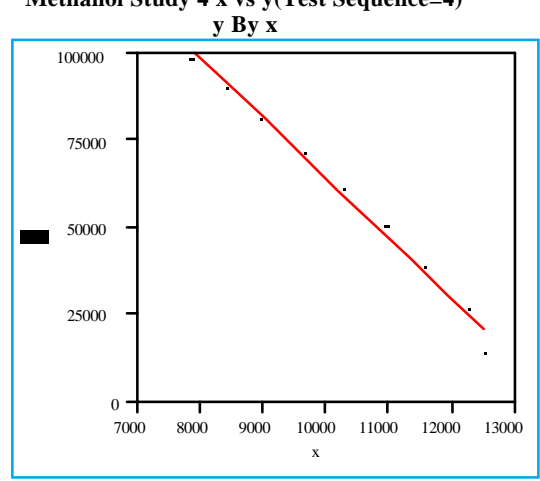

Linear Fit

$$
y=237458-17.2813 x
$$

RSquare Summary of Fit

RSquare Ad

Root Mean Square Error

Mean of Response

0.988304

Observations (or Sum Wgts)

0.986633

3375.286

$\begin{array}{lllll} & \text { Estimate } & \text { Std Error } & \text { t Ratio } & \text { Prob }>|t|\end{array}$ $\begin{array}{lrrrr} & 237457.84 & 7414.395 & 32.03 & <.0001 \\ \mathrm{x} & -17.28133 & 0.710569 & -24.32 & <.0001\end{array}$
Methanol Study $4 x$ vs $y($ Test Sequence $=5$ )

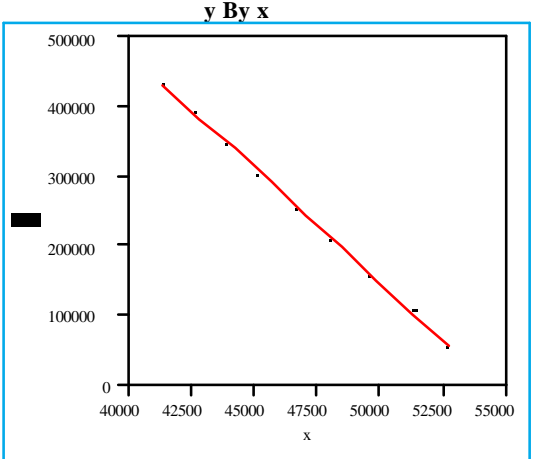

Linear Fit

$y=1802864-33.1103$

RSquare

RSquare Adj

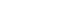

0.999348

Root Mean Square Error

0.999255

Mean of Response

3553.979

Observations (or Sum Wgts)

Parameter Estimates

Term Estimate Std Error t Ratio Prob> $>\mathbf{t} \mid$ $\mathrm{x} x-33.11031-0.319556-103.6<0001$

Methanol Study $4 x$ vs $y$ (Test Sequence $=6$ )

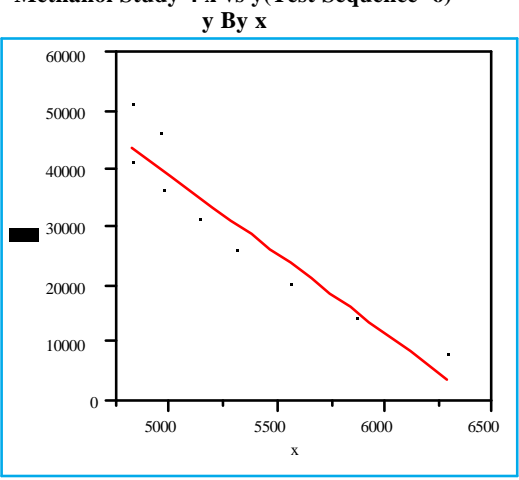

$$
\begin{gathered}
\text { Linear Fit } \\
\mathrm{y}=174718-27.1689 x
\end{gathered}
$$

RSquare

0.89752

RSquare Adj $\quad 0.88288$

Root Mean Square Er

4999.169

Observations (or Sum Wgts)

Parameter Estimates

$\begin{array}{lllll}\text { Term Estimate } & \text { Std Error t Ratio Prob }>|t|\end{array}$ $\begin{array}{lrrrr}\text { Intercept } & 174718.43 & 18518.3 & 9.43 & <.0001 \\ \mathrm{X} & -27.16886 & 3.469916 & -7.83 & 0.0001\end{array}$ 
Exhibit B23: y versus x for Methanol Studies: Study 4; Tests 7-14

Methanol Study $4 \mathrm{x}$ vs $\mathbf{y}($ Test Sequence $=7)$ y By $\mathbf{x}$

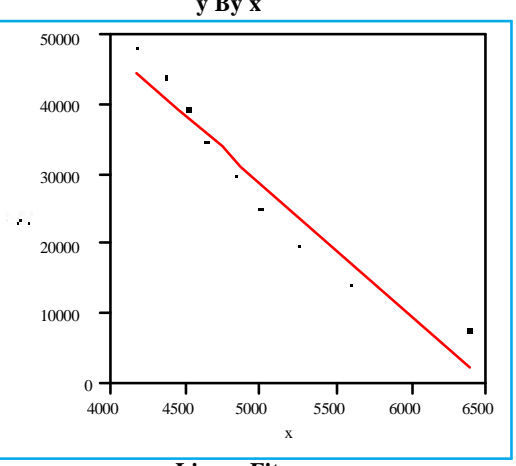

$$
\begin{gathered}
\text { Linear Fit } \\
y=125455-19.3215 x
\end{gathered}
$$

RSquare

ummary of Fit

Root Mean Square Error

0.931264

Mean of Response

Observations (or Sum Wgts)

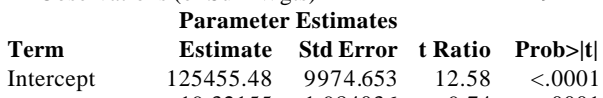

$\begin{array}{lllll}\text { Intercept } & 125455.48 & 9974.653 & 12.58 & <.0001 \\ \mathrm{x} & -19.32155 & 1.984036 & -9.74 & <.0001\end{array}$

Methanol Study 4 x vs y(Test Sequence $=8$ )

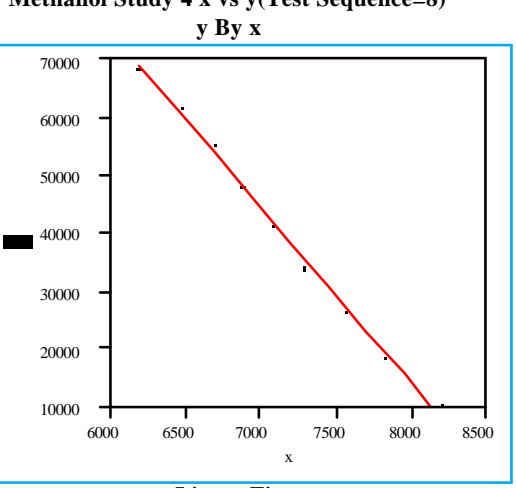

Linear Fit

$$
y=257280-30.3628 x
$$

RSquare

Summary of Fit

0.995261

Mean of Response

0.994584

1461.469

Observations (or Sum Wgts)

$\begin{array}{lrrrr} & \text { Estimate } & \text { Std Error } & \text { t Ratio } & \text { Prob }>\mid \mathbf{t} \text { | }\end{array}$ $\begin{array}{lrrrr}\text { Intercept } & 257280.16 & 5679.397 & 45.30 & <.000 \\ x & -30.36278 & 0.791905 & -38.34 & <.000\end{array}$
Methanol Study $4 \mathrm{x}$ vs $\mathrm{y}($ Test Sequence $=9$ )

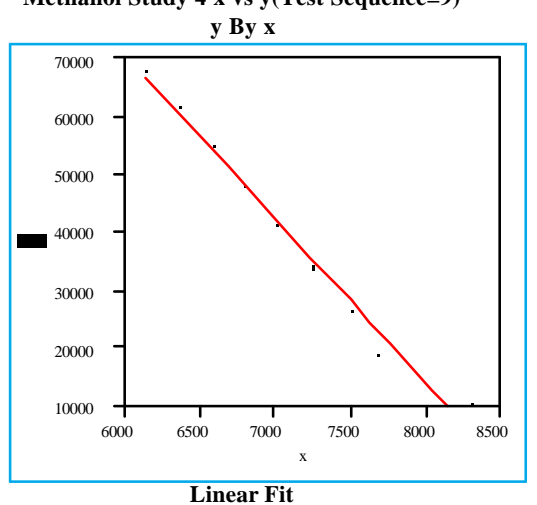

$$
\begin{gathered}
\text { Linear Fit } \\
y=241093-28.3509 x
\end{gathered}
$$

RSquare Summary of Fit

RSquare Adj

Root Mean Square Erro

Observations (or Sum Wgts)

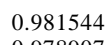

0.981544

2857.074

40215.34

Parameter Estimates

Term Estimate Std Error t Ratio Prob $>|t|$ $\begin{array}{lrrrr}\text { Intercept } & 241092.83 & 10454.66 & 23.06 & <.0001 \\ \mathrm{x} & -28.35091 & 1.469386 & -19.29 & <.0001\end{array}$

Methanol Study 4 x vs $y($ Test Sequence $=10)$ y By $x$

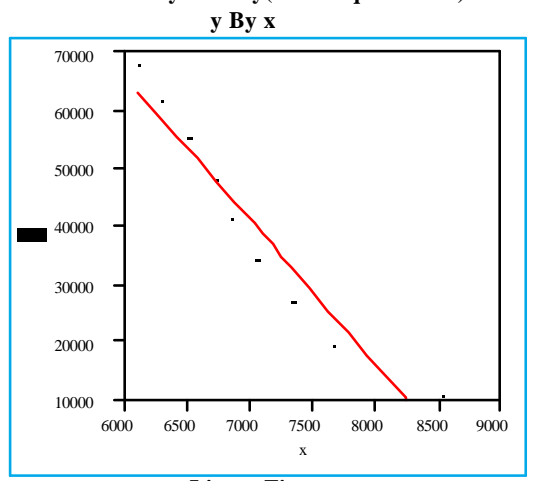

Linear $\mathbf{F}$

$$
y=215985-24.9589 x
$$

$\begin{array}{lr} & \\ \text { RSquare } & \text { Summary of Fit } \\ \text { RSquare Adj } & 0.936055 \\ \text { Root Mean Square Error } & 0.92692 \\ \text { Mean of Response } & 5253.986 \\ \text { Observations (or Sum Wgs) } & 40532.98\end{array}$

Parameter Estimates

Term Estimate Std Error t Ratio Prob $>|t|$

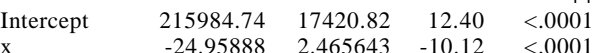

Methanol Study $4 \mathrm{x}$ vs $\mathrm{y}($ Test Sequence=11)

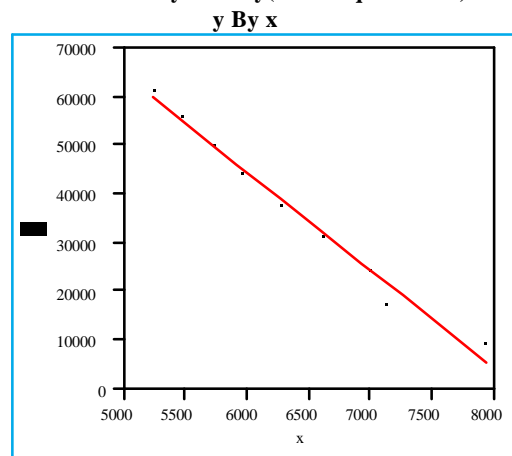

Linear Fit

$y=165852-20.1916 x$

RSquare

Root Mean Square Error

Mean of Response

0.983222

0.980825

2469.23

Observations (or Sum Wgts)

36873.15

Parameter Estimates

$\begin{array}{lrrrr} & \text { Estimate } & \text { Std Error } & \text { t Ratio } & \text { Prob }>|t|\end{array}$ $\begin{array}{rrrrr} & 165852.15 & 6421.174 & 25.83 & <.0001 \\ \mathrm{X} & -20.19159 & 0.996939 & -20.25 & <.0001\end{array}$

Methanol Study $4 x$ vs $y($ Test Sequence $=12)$

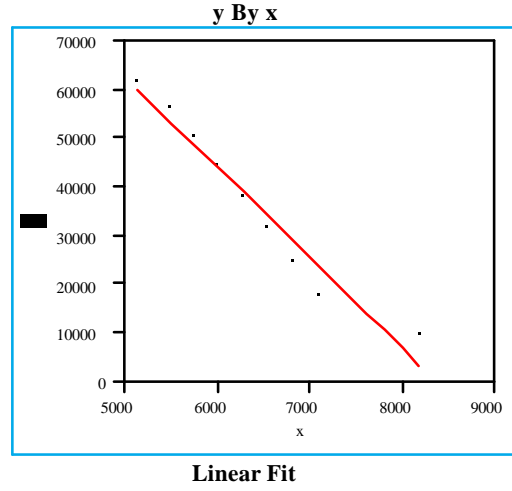

\section{Linear Fit} $y=156344-18.6262 x$

$\begin{array}{lr}\text { RSquare } \quad \text { Summary of Fit } & \\ \text { RSquare Adj } & 0.956815 \\ \text { Root Mean Square Error } & 0.950646 \\ \text { Mean of Response } & 3953.92 \\ \text { Ore } & 37404.42\end{array}$

Observations (or Sum Wgts)

\section{Parameter Estimates}

Term Estimate Std Error t Ratio Prob $>|t|$

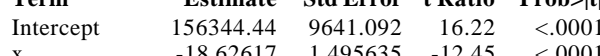

Methanol Study $4 \mathrm{x}$ vs $\mathrm{y}($ Test Sequence $=13$ )

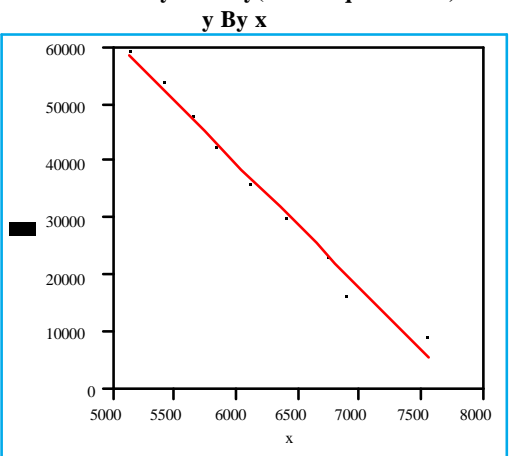

Linear Fit

$$
y=171678-21.9587
$$

RSquare

RSquare Adj

Summary of F

0.987465 0.985674

Root Mean Square Error 0.985674
2073.487 Mean of Response

35378.64

Parameter Estimates
Psts

$\begin{array}{lrrrr}\text { Term } & \text { Estimate } & \text { Std Error } & \text { t Ratio } & \text { Prob }>|t| \\ \text { Intercept } & 171678 & 5845.27 & 29.37 & <.0001\end{array}$ $\begin{array}{lrrrrr}\mathrm{x} & -21.95869 & 0.935104 & -23.48 & <.0001 \\ & & & & & \end{array}$

Methanol Study $4 x$ vs $y($ Test Sequence $=14)$

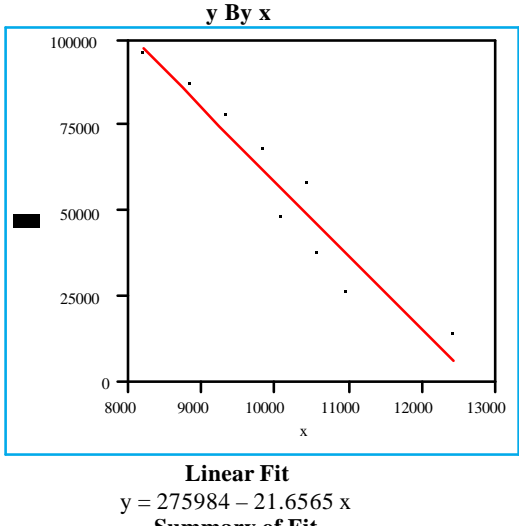

RSquare

RSquare Adj

0.920594

Root Mean Square Error

0.90925

Observations (or $\mathrm{S}$

8492.34

Observations (or Sum Wgts)

Parameter Estimates

$\begin{array}{lrrrr}\text { Term } & \text { Estimate } & \text { Std Error } & \text { t Ratio } & \text { Prob }>\mid \mathbf{t} \\ \text { Intercept } & 275983.57 & 24435.01 & 11.29 & <.000\end{array}$

$x \quad-21.65654 \quad 2.403989 \quad-9.01<.0001$ 
Exhibit B24: y versus $x$ for Methanol Studies: Study 4; Tests 15-16

Methanol Study $4 x$ vs $y($ Test Sequence $=15)$

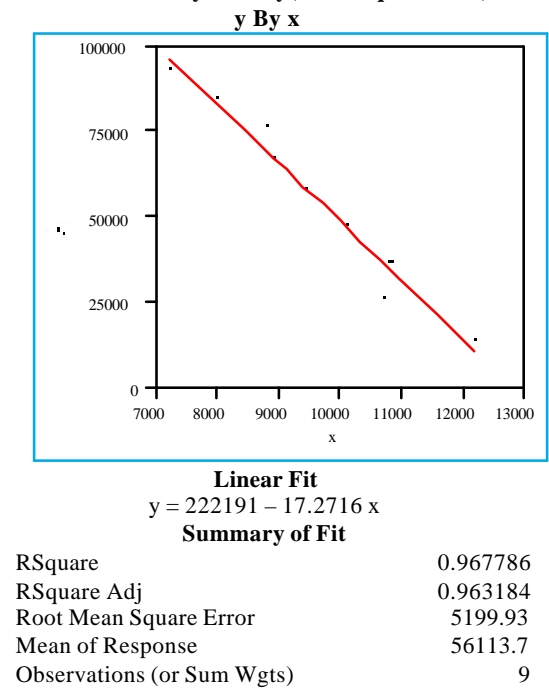

Observations (or Sum Wgts)

Parameter Estimates

Estimate Std Error t Ratio Prob $>|\mathbf{t}|$

$\begin{array}{lrrrr}\text { Intercept } & 222191 & 11582.8 & 19.18 & <.0001 \\ & -17.27157 & 1.191014 & -14.50 & <.0001\end{array}$
Methanol Study $4 \mathrm{x}$ vs $\mathrm{y}($ Test Sequence=16)

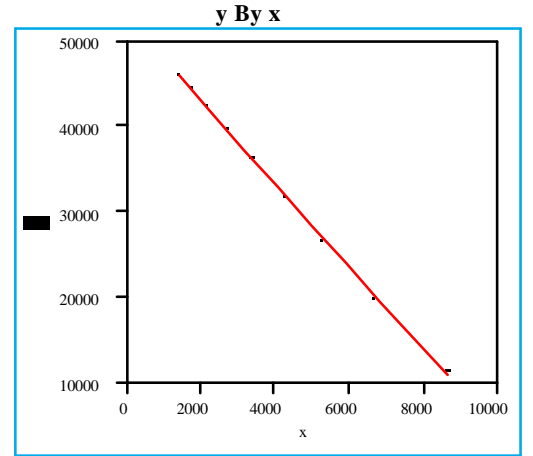

Linear Fit

$y=52939.5-4.83758 x$

RSquare

0.999138 RSquare Adj $\quad 0.999015$ Root Mean Square Error $\quad 374.4663$ Mean of Response $\quad 33320.07$ Observations (or Sum Wgts)

Parameter Estimates

Estimate Std Error t Ratio Prob $>\mid \mathbf{t}$ $\begin{array}{rrrr}52939.51 & 251.0349 & 210.89 & <.0001 \\ -4.837584 & 0.053704 & -90.08 & <.0001\end{array}$ 


\section{Exhibit B25: Common Logarithm of Peak Area Versus Sample Sequence for Ammonia Studies: Study 1; Tests 1-8}

Ammonia Data(Study=1 Test Sequence=1)

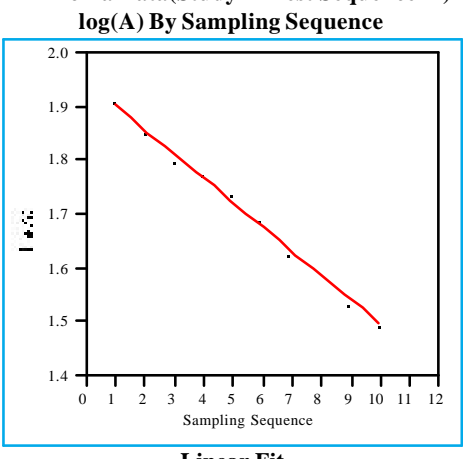

Linear Fit

$\log (\mathrm{A})=1.94481-0.0448$ Sampling Sequence RSquare

$$
\text { Summary of Fit }
$$

\begin{tabular}{lr} 
RSquare Adj & 0.992825 \\
Root Mean Square Error & 0.991928 \\
Mean of Response & 0.012229 \\
Obervations (or Sum Wgts) & 1.698429 \\
\hline
\end{tabular}

Mean of Response

Parameter Estimates

$$
10
$$

$\begin{array}{lrrrr}\text { Term } & \text { Estimate } & \text { Std Error } & \text { t Ratio } & \text { Prob }>|\mathbf{t}| \\ \text { Intercept } & 1.9448139 & 0.008354 & 232.80 & <.0001\end{array}$

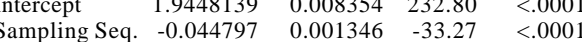

Ammonia Data(Study=1 Test Sequence=2)

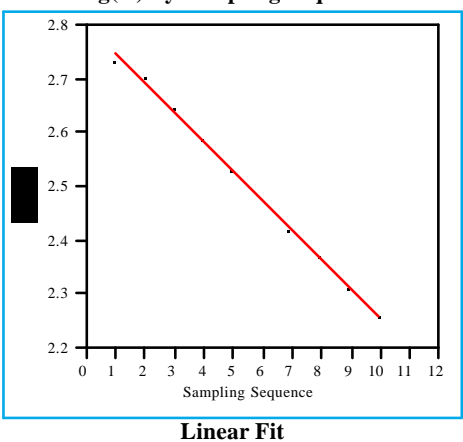

$\log (\mathrm{A})=2.80028-0.0544$ Sampling Sequence RSquare Summary of Fit

RSquare Adj

Root Mean Square Error

Mean of Response

\subsection{3}

Observations (or Sum Wgts)

0.006418

2.501059

Parameter Estimate

Term Estimate Std Error t Ratio Prob $>|\mathbf{t}|$ $\begin{array}{lrrrrr}\text { Intercept } & 2.8002835 & 0.004384 & 638.74 & <.000\end{array}$ $\begin{array}{lllll}\text { Sampling Seq. } & -0.054404 & 0.000707 & -77.00 & <.000\end{array}$
Ammonia Data(Study=1 Test Sequence=3)

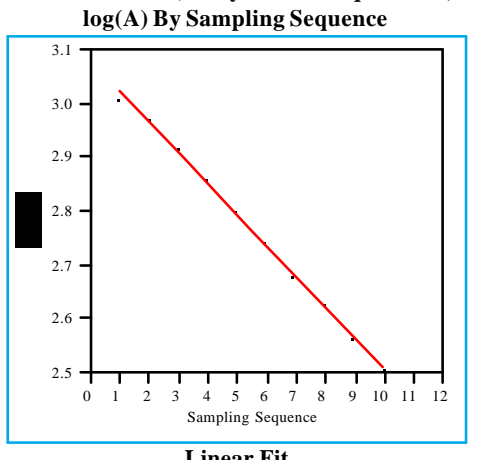

Linear Fit
$\log (\mathrm{A})=3.07801-0.05708$ Sampling Sequence

$$
\text { Summary of Fit }
$$$$
\text { RSquar }
$$

\section{RSquare Adj}

Root Mean Square Error

Mean of Response

$$
\begin{array}{r}
0.998569 \\
0.99839 \\
0.00694 \\
2.76407 \\
10
\end{array}
$$

Observations (or Sum Wgts)

$\begin{array}{lrrrr}\text { Term } & \text { Estimate } & \text { Std Error } & \text { t Ratio } & \text { Prob }>|\mathbf{t}|\end{array}$ $\begin{array}{lrrrrr} & 3.0780107 & 0.004741 & 649.26 & <.0001 \\ \text { Sampling Seq. } & -0.05708 & 0.000764 & -74.71 & <.0001\end{array}$

Ammonia Data(Study=1 Test Sequence=4) $\log (\mathrm{A})$ By Sampling Sequence

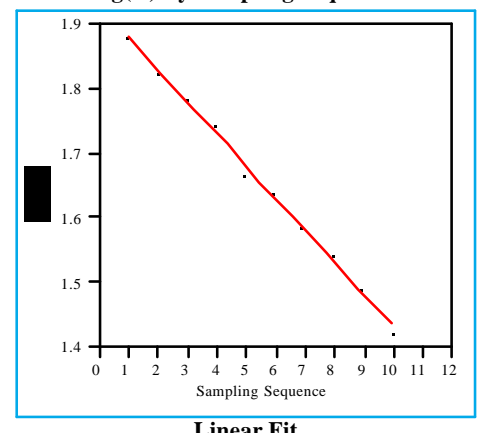

$\log (\mathrm{A})=1.9276-0.04956$ Sampling Sequence Summary of Fit

RSquare

Root Mean Square Erro

Mean of Response

0.996247
0.995778

0.995778
0.009768

1.655023

Observations (or Sum Wgts)

10

Estimate Std Error t Ratio Prob $>|\mathbf{t}|$

$\begin{array}{llllll}\text { Intercept } & 1.927597 & 0.006673 & 288.89 & <.0001\end{array}$

$\begin{array}{lllll}\text { Sampling Seq. } & -0.049559 & 0.001075 & -46.09 & <.0001\end{array}$
Ammonia Data(Study=1 Test Sequence=5)

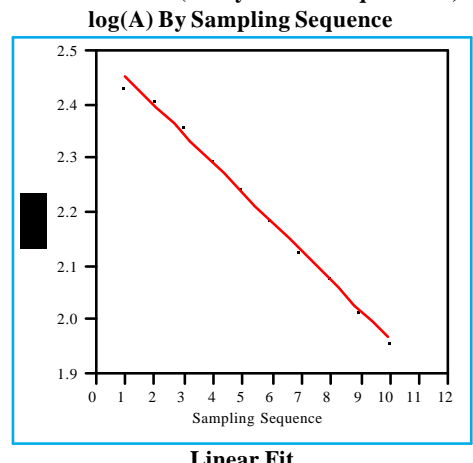

$\log (\mathrm{A})=2.50674-0.0542$ Sampling Sequence Summary of Fit

RSquare

0.996734 RSquare Adj

Root Mean Square Error

Mean of Response

0.996326

Observations (or Sum Wgts)

208638

Parameter Estimates

$\begin{array}{lrrrrr}\text { Term } & \text { Estimate } & \text { Std Error } & \text { t Ratio } & \text { Prob }>|\mathbf{t}| \\ \text { Intercept } & 2.5067443 & 0.006806 & 368.32 & <.0001\end{array}$ $\begin{array}{llllll}\text { Sampling Seq. } & -0.054201 & 0.001097 & -49.41 & <.0001\end{array}$

Ammonia Data(Study $=1$ Test Sequence $=6$ ) $\log ($ A) By Sampling Sequence

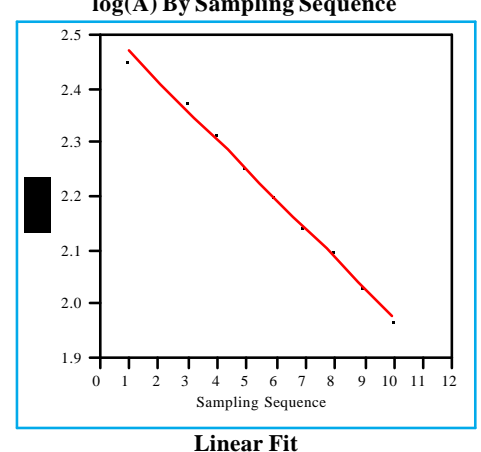

$\log (\mathrm{A})=2.52202-0.05453$ Sampling Sequence RSquare Summary of $F$

\section{RSquare Adj}

Root Mean Square Erro

Mean of Response
Observations (or Sum Wgts)

0.996719

0.010046

2.222132

Estimate Std Error t Ratio Prob $>|t|$ $\begin{array}{lrrrr}\text { Intercept } & 2.5220246 & 0.006863 & 367.48 & <.0001 \\ \text { Sampling Seq. } & -0.054526 & 0.001106 & -49.30 & <.0001\end{array}$
Ammonia Data(Study=1 Test Sequence=7)

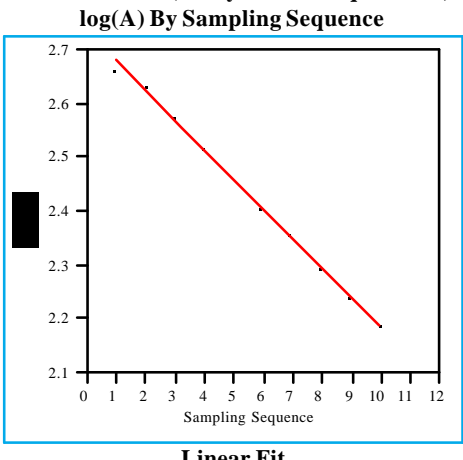

$\log (A)=2.73115-0.05468$ Sampling Sequence

RSquare

Summary of Fit

0.998395

RSquare Adj

Root Mean Square Error

0.998194

Mean of Response

0.00704

Observations (or Sum Wgts)

10

$\begin{array}{lllll}\text { Term } & \text { Parameter Estimates } & & \\ & \text { Std Error } & \text { t Ratio } & \text { Prob }>|t|\end{array}$ $\begin{array}{llllll} & 2.7311512 & 0.004809 & 567.88 & <.0001 \\ \text { Sampling Seq. } & -0.054676 & 0.000775 & -70.54 & <.0001\end{array}$

Ammonia Data(Study=1 Test Sequence=8) $\log (\mathrm{A})$ By Sampling Sequence

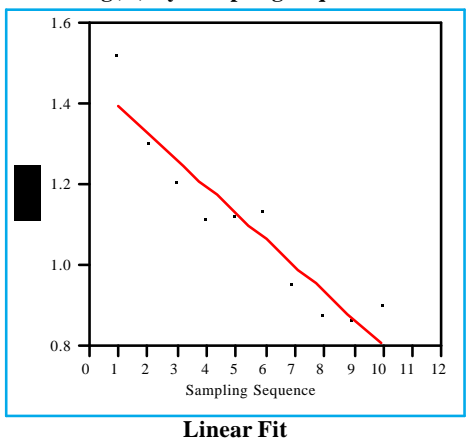

$\log (A)=1.45702-0.06527$ Sampling Sequence RSquare Summary of Fi

RSquare Adj

Root Mean Square Error

Mean of Response

0.885361

Observations (or Sum Wgts)

0.075421

Estimate Std Error t Ratio Prob $>|\mathbf{t}|$ $\begin{array}{lrrrr}\text { Intercept } & 1.4570199 & 0.051522 & 28.28 & <.0001 \\ \text { Sampling Seq. } & -0.065268 & 0.008304 & -7.86 & <.0001\end{array}$ 
Exhibit B26: Common Logarithm of Peak Area Versus Sample Sequence for Ammonia Studies: Study 1; Tests 9-16

Ammonia Data(Study=1 Test Sequence=9) $\log (\mathbf{A})$ By Sampling Sequence

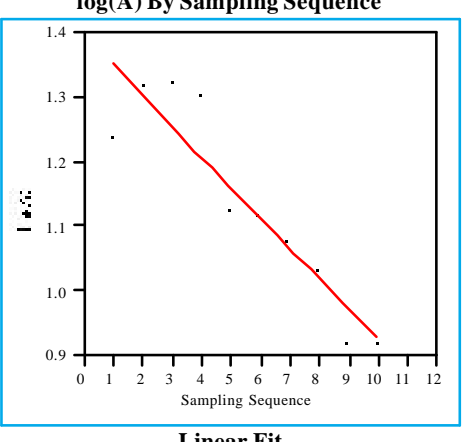

Linear Fit
$\log (\mathrm{A})=1.39522-0.04711$ Sampling Sequence

\begin{tabular}{lr}
$\log (\mathrm{A})=1.39522-0.04711$ Sampling Sequence \\
RSquare & Summary of Fit \\
RSquare Adj & 0.856632 \\
\hline & 0.838711
\end{tabular}

Root Mean Square Error

0.061894

Mean of Response

1.136101

Observations (or Sum Wgts)

10 $\begin{array}{lrrrr} & \text { Parameter Estimates } & & \\ \text { Term } & \text { Estimate } & \text { Std Error } & \text { t Ratio } & \text { Prob }>|\mathbf{t}| \\ \text { Intercept } & 1.3952216 & 0.042282 & 33.00 & <.0001 \\ \text { Sampling } & -0.047113 & 0.006814 & -6.91 & 0.0001\end{array}$ $\begin{array}{llllll}\text { Sampling Seq } & -0.047113 & 0.006814 & -6.91 & 0.0001\end{array}$

Ammonia Data(Study=1 Test Sequence $=10$ )

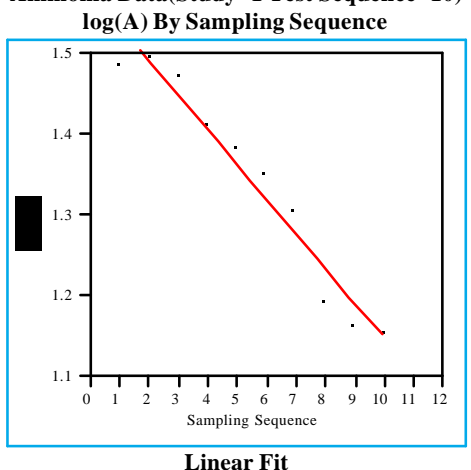

$\log (\mathrm{A})=1.57599-0.04275$ Sampling Sequence
Summary of Fit

RSquare

\begin{tabular}{lr} 
RSquare Adj & 0.944922 \\
Root Mean Square Error & 0.031151 \\
Mean of Response & 1.340848 \\
\hline & 10
\end{tabular}

Observations (or Sum Wgts)

10

$\begin{array}{lrrrr}\text { Term } & \text { Parameter Estimates } & & \\ \text { Intimate } & \text { Std Error } & \text { t Ratio } & \text { Prob }>|\mathbf{t}|\end{array}$ $\begin{array}{lrrrr} & 1.5759938 & 0.02128 & 74.06 & <.0001 \\ \text { Sampling Seq. } & -0.042754 & 0.00343 & -12.47 & <.0001\end{array}$
Ammonia Data (Study=1 Test Sequence=11) $\log ($ A) By Sampling Sequence

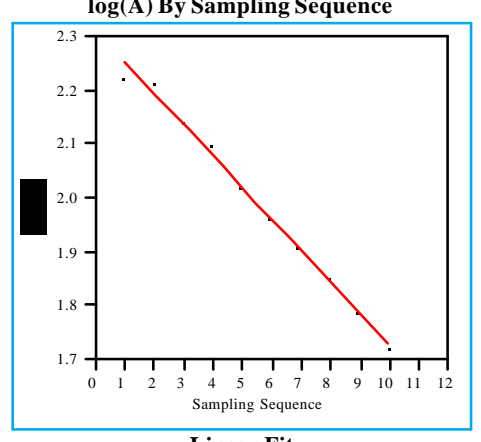

Linear Fit
$\log (\mathrm{A})=2.31062-0.05828$ Sampling Sequence

$\begin{array}{lr}\text { RSquare } \quad \text { Summary of Fit } & \\ \text { RSquare Adj } & 0.994514 \\ \text { Root Mean Square Error } & 0.993828 \\ \text { Mean of Response } & 0.0139 \\ & 1.990096\end{array}$

Observations (or Sum Wgts)
Parameter Estimates

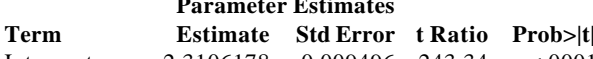

$\begin{array}{lrlllll}\text { Intercept } & 2.3106178 & 0.009496 & 243.34 & <.0001\end{array}$

Ammonia Data(Study=1 Test Sequence=12) \begin{tabular}{l}
$\log (A)$ By Sampling Sequence \\
\hline
\end{tabular}

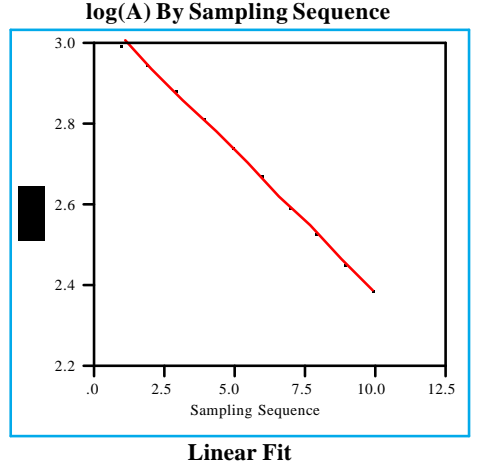

$\log (A)=\underset{3.08249-0.06966 \text { Sampling Sequence }}{\text { Summary of Fit }}$

RSquare

Summary of Fit

RSquare Adj

Root Mean Square Error

Mean of Response

0.998721
0.998562
0.008004
2.699367
10

Observations (or Sum Wgts)

10

Term $\quad$ Estimate Std Error t Ratio Prob $>|t|$

$\begin{array}{lrrrrr}\text { Intercept } & 3.0824869 & 0.005468 & 563.77 & <.0001 \\ \text { Sampling Seq } & -0.069658 & 0.000881 & -79.05 & <.0001\end{array}$
Ammonia Data(Study=1 Test Sequence=13)

$\log (\mathrm{A})$ By Sampling Sequence

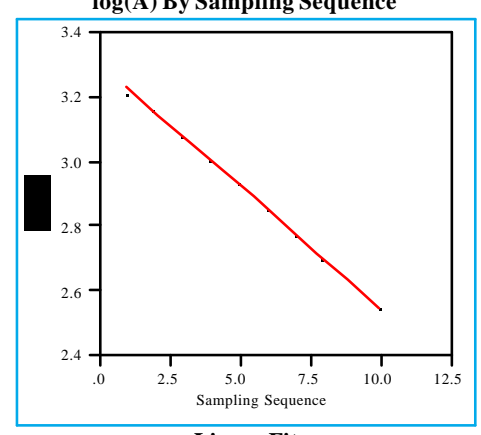

Linear Fit

$\log (A)=3.29914-0.07539$ Sampling Sequence
Summary of Fit

RSquare

\section{Root Mean Square Error}

Mean of Response

0.998784
0.998632

0.008448

Observations (or Sum Wgts)

2.884521

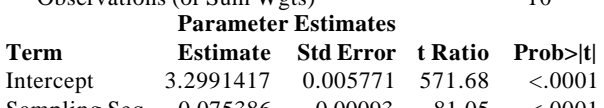

Ammonia Data(Study=1 Test Sequence=14)

Ammonia Data(Study $=1$ Test Sequence
$\log ($ A) By Sampling Sequence

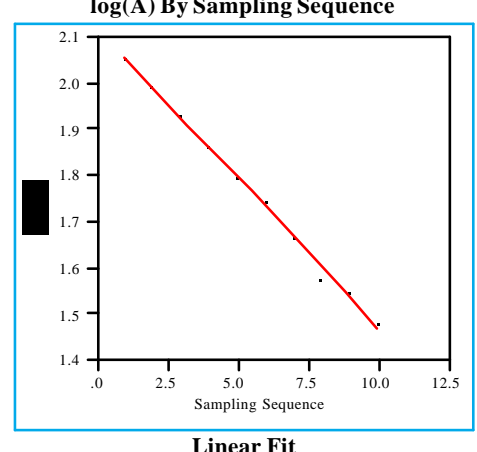

$\log (\mathrm{A})=2.11744-0.06475$ Sampling Sequence RSquare Summary of Fit

Root Mean Square Error

Mean of Response

0.996906

Observations (or

0.01158

Term Estimate Std Error t Ratio Prob $>|t|$ $\begin{array}{llllll}\text { Intercept } & 2.1174402 & 0.007913 & 267.58 & <.0001\end{array}$ $\begin{array}{lllll}\text { Sampling Seq. } & -0.064749 & 0.001275 & -50.77 & <.0001\end{array}$
Ammonia Data(Study=1 Test Sequence $=15$ $\log (A)$ By Sampling Sequence

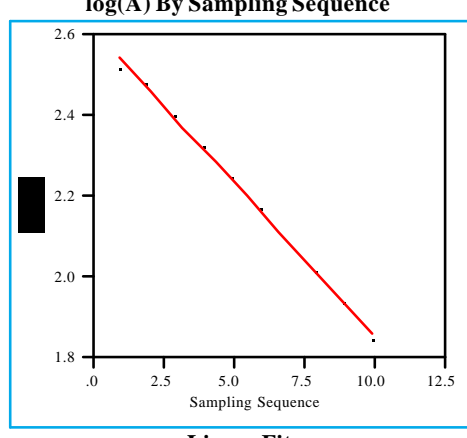

$\log (A)=2.61446-0.07606$ Sampling Sequence

$$
\begin{array}{r}
\log (\mathrm{A})=2.61446-0.07606 \text { Sam } \\
\text { Summary of Fit }
\end{array}
$$

RSquare

are Adj

Root Mean Square Error

0.997494

Mean of Response

0.997181

Observations (or Sum Wgts)

2.196148

Term Estimate Std Error t Ratio Prob $>|t|$

$\begin{array}{llllll}\text { Intercept } & 2.6144609 & 0.008363 & 312.63 & <.0001\end{array}$

Ammonia Data(Study=1 Test Sequence=16)

Ammonia Data(Study=1 Test Sequence

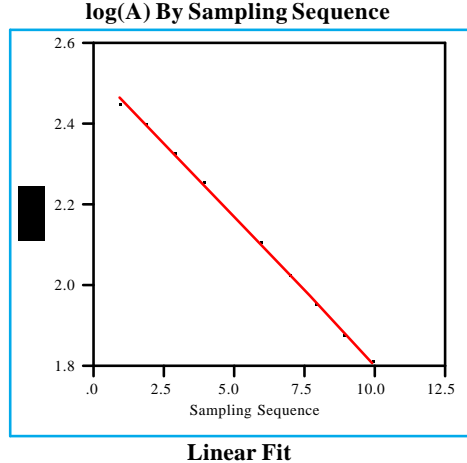

$\log (\mathrm{A})=2.53668-0.07291$ Sampling Sequence

RSquare

RSquare Adj

Summary of Fit

Root Mean Square Error

Mean of Response

0.999008

Observations (or Sum Wgts)

0.007378

$\begin{array}{llll}\text { Term } & \text { Parameter Estimates } & & \\ \text { Estimate } & \text { Std Error } & \mathbf{t} \text { Ratio } & \text { Prob }>|t|\end{array}$

$\begin{array}{lrrrrr}\text { Intercept } & 2.5366833 & 0.00504 & 503.31 & <.0001\end{array}$ $\begin{array}{lllll}\text { Sampling Seq. } & -0.072912 & 0.000812 & -89.76 & <.0001\end{array}$ 
Exhibit B27: Common Logarithm of Peak Area Versus Sample Sequence for Ammonia Studies: Study 1; Tests 17-24

Ammonia Data(Study=1 Test Sequence=17) $\log ($ A) By Sampling Sequence

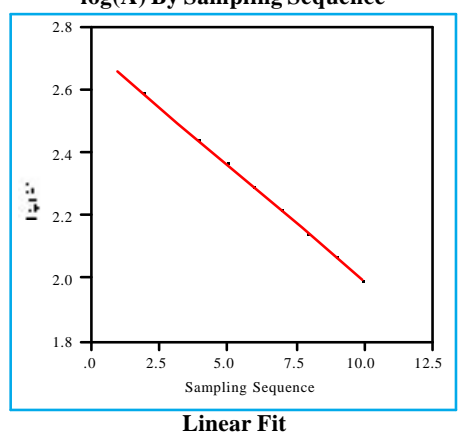

$\log (\mathrm{A})=2.72748-0.07317$ Sampling Sequence

RSquare
RSquare Adj

Summary of Fit

Root Mean Square Error

Mean of Response

0.998529
0.998345

0.00901

Observations (or Sum Wgts)

2.325037

$\begin{array}{lrrrr}\text { Term } & \text { Earameter Estimates } & & \\ \text { Intercept } & 2.7274756 & 0.006161 & 442.69 & <0001\end{array}$ $\begin{array}{lrrrr} & 2.7274756 & 0.006161 & 442.69 & <.000 \\ \text { Sampling Seq. } & -0.073171 & 0.000993 & -73.69 & <.000\end{array}$

Ammonia Data(Study=1 Test Sequence=18) $\log (A)$ By Sampling Sequence

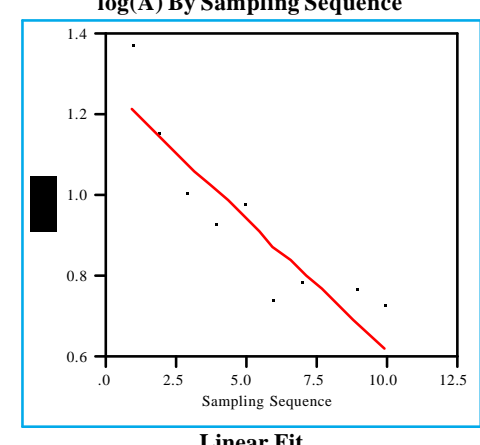

$\log (\mathrm{A})=1.2727-0.06596$ Sampling Sequence RSquare Summary of Fit

RSquare Adj

Root Mean Square Error

Mean of Response

0.806196

Observations (or Sum Wgts)

0.103858

0.909901

Parameter Estimates

Term Estimate Std Error t Ratio Prob $>|\mathbf{t}|$ $\begin{array}{lllll}\text { Intercept } & 1.2726962 & 0.070949 & 17.94 & <.000\end{array}$ $\begin{array}{lllll}\text { Sampling Seq. } & -0.065963 & 0.011434 & -5.77 & 0.0004\end{array}$
Ammonia Data(Study=1 Test Sequence=19) $\log ($ A) By Sampling Sequence

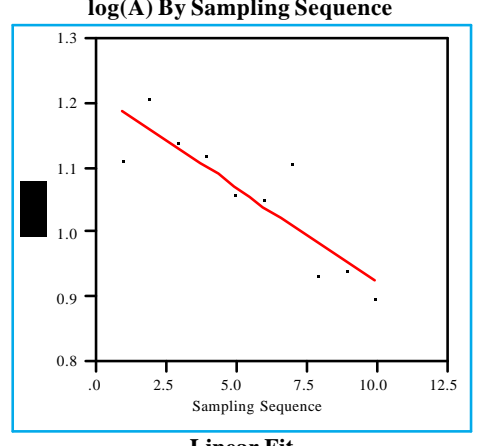

$\operatorname{Linear~Fit~}$

$\log (\mathrm{A})=1.21635-0.02952$ Sampling Sequence

\begin{tabular}{lr}
\multicolumn{2}{c}{ Summary of Fit } \\
RSquare & 0.76985 \\
RSquare Adj & 0.741082 \\
Root Mean Square Error & 0.051838 \\
Mean of Response & 1.053975
\end{tabular}

Mean of Response

Mean of Response

Parameter Estimates

Term Estimate Std Error t Ratio Prob $>|t|$

$\begin{array}{llllll}\text { Intercept } & 1.2163538 & 0.035412 & 34.35 & <.0001\end{array}$ $\begin{array}{lllll}\text { Sampling Seq. } & -0.029523 & 0.005707 & -5.17 & 0.0009\end{array}$

Ammonia Data(Study=1 Test Sequence=20

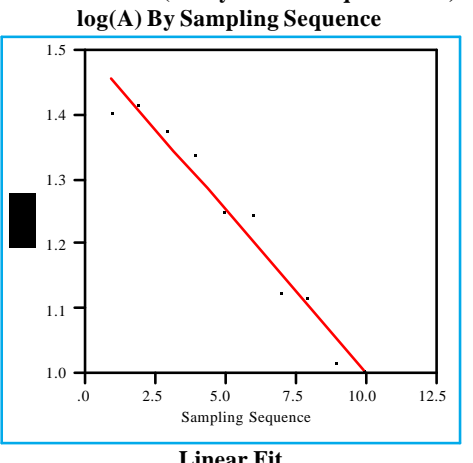

$\log (A)=1.50417-0.05025$ Sampling Sequence
Summary of Fit

RSquare

$\begin{array}{lr}\text { RSquare Adj } & 0.958971 \\ & 0.953842\end{array}$

Root Mean Square Error $\quad 0.03338$

Mean of Response $\quad 1.227774$

Observations (or Sum Wgts)

Term Estimate Std Error t Ratio Prob $>|t|$

$\begin{array}{lrrrrr}\text { Intercept } & 1.5041655 & 0.022803 & 65.96 & <.0001 \\ \text { Sampling Seq } & -0.050253 & 0.003675 & -13.67 & <.0001\end{array}$
Ammonia Data(Study=1 Test Sequence $=21$ )

$\log (A)$ By Sampling Sequence

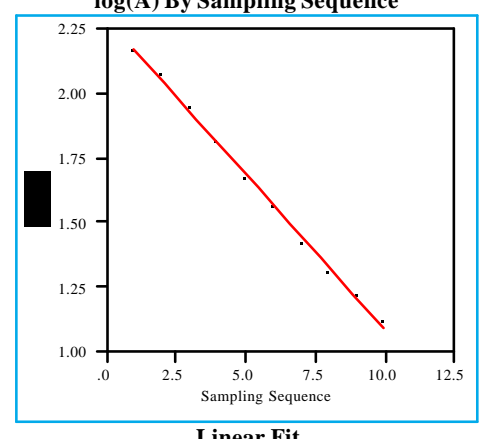

Linear Fit
$\log (\mathrm{A})=2.29128-0.12072$ Sampling Sequence

$\log (\mathrm{A})=2.29128-0.12072$ Sampling Sequence

0.997278

RSquare Adj $\quad 0.996938$

Mean of Response

1.627332

Observations (or Sum Wgts)

$\begin{array}{lllll} & \text { Parameter Estimates } & & \\ \text { Term } & \text { Estimate } & \text { Std Error } & \text { t Ratio } & \text { Prob }>|t|\end{array}$ $\begin{array}{llllll} & \text { Elercept } & 2.2912824 & 0.013835 & 165.61 & <.000 \\ & & \end{array}$

Ammonia Data(Study $=1$ Test Sequence $=22$ )

Ammonia Data(Study=1 Test Sequence
$\log ($ A) By Sampling Sequence

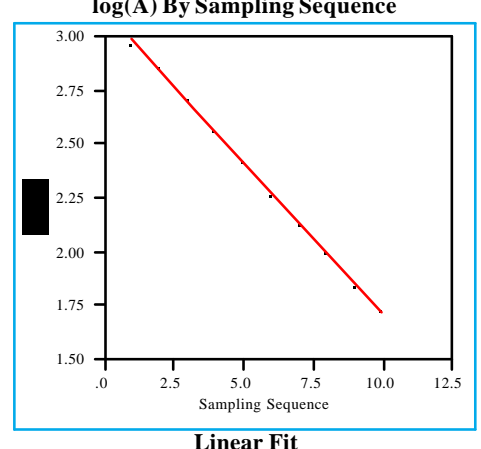

0.14135 Sampling Sequence

Square Summary of Fit

Root Mean Square Error

Mean of Response

0.999155
0.999049

Observations (or

0.013203

2.342014
10

Parameter Estimates

$\begin{array}{llll}\text { Estimate Std Error t Ratio Prob }>|t| & \end{array}$ $\begin{array}{lrrrr} & \text { E } & & \\ \text { Sampling Seq. } & -0.1141351 & 0.009019 & 345.87 & <.0001 \\ \text { Sa } & 0.001454 & -97.24 & <.0001\end{array}$
Ammonia Data(Study=1 Test Sequence=23

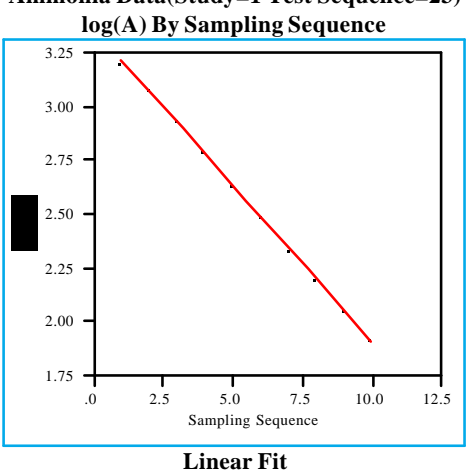

Linear Fit
$\log (A)=3.36109-0.14562$ Sampling Sequence

$$
\begin{array}{r}
\log (\mathrm{A})=3.36109-0.14562 \text { Samp } \\
\text { Summary of Fit }
\end{array}
$$

RSquare

0.999605

$\begin{array}{lr}\text { RSquare Adj } & 0.999556 \\ \text { Root Mean Square Error } & 0.00929\end{array}$

Mean of Response

2.560202

Observations (or Sum Wgts)

$\begin{array}{lrrrrr}\text { Term } & \text { Parameter Estimates } & & \\ \text { Estimate } & \text { Std Error } & \text { t Ratio } & \text { Prob }>|\mathbf{t}| \\ \text { Intercept } & 3.3610889 & 0.006346 & 529.61 & <.0001\end{array}$

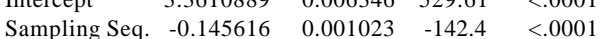

Ammonia Data(Study=1 Test Sequence=24) $\log (A)$ By Sampling Sequence

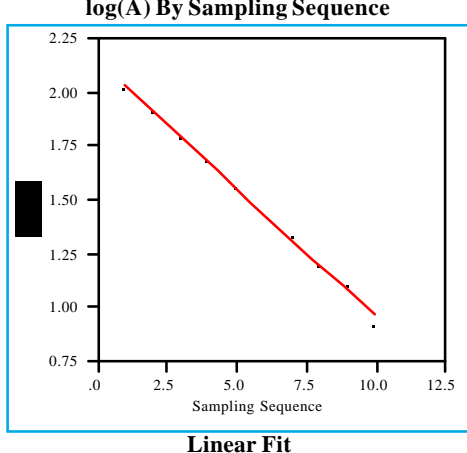

$\log (\mathrm{A})=2.14296-0.11866$ Sampling Sequence

RSquare Summary of Fit

RSquare Adj

Root Mean Square Error

Mean of Response
Observations (or Sum Wgts)

0.997711
0.997425

0.018253

1.490324

Term Estimate Std Error t Ratio Prob $>|\mathbf{t}|$

$\begin{array}{lrrrrr}\text { Intercept } & 2.1429629 & 0.012469 & 171.86 & <.0001\end{array}$

$\begin{array}{lrrrr}\text { Sampling Seq. } & -0.118662 & 0.00201 & -59.05 & <.0001\end{array}$ 
Exhibit B28: Common Logarithm of Peak Area Versus Sample Sequence for Ammonia Studies: Study 1; Tests 25-32

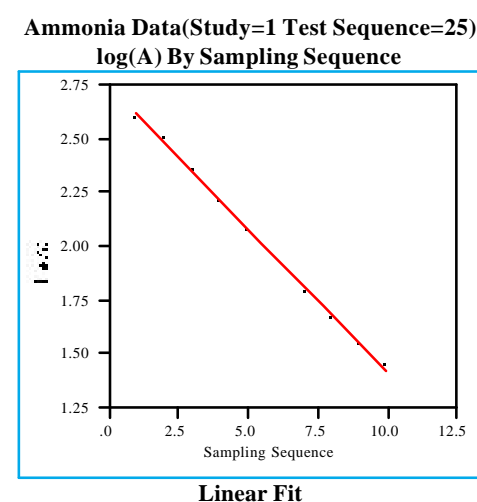

$\log (\mathrm{A})=2.74614-0.13302$ Sampling Sequence

\begin{tabular}{ll}
$\log (\mathrm{A})=2.74614-0.1397986$ \\
RSquare & Summary of Fit \\
RSquare Adj & 0.997734 \\
Root Mean Square Error & 0.019192 \\
Mean of Response & 2.014541 \\
\hline
\end{tabular}

Mean of Response

Observations (or Sum Wgts)

\begin{tabular}{lrrrr} 
Term & \multicolumn{2}{c}{ Parameter Estimates } & & \\
Estimate & Std Error & $\mathbf{t}$ Ratio & Prob $>|\mathbf{t}|$ \\
Intercept & 2.7461369 & 0.01311 & 209.46 & $<.0001$
\end{tabular} $\begin{array}{lllll}\text { Sampling Seq. } & -0.133017 & 0.002113 & -62.95 & <.000\end{array}$

Ammonia Data(Study=1 Test Sequence=26)

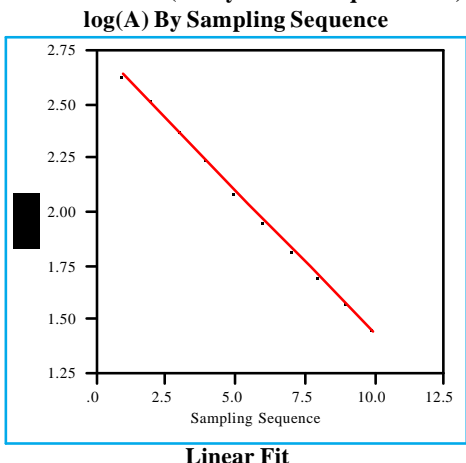

$\log (\mathrm{A})=2.76697-0.13351$ Sampling Sequence

RSquare

Summary of Fit

$\begin{array}{lr}\text { RSquare Adj } & 0.998646 \\ \text { Root Mean Square Error } & 0.998477 \\ \text { Mean of Response } & 0.015784 \\ \text { Observations (or Sum Wgts) } & 2.032675 \\ \text { Par } & 10\end{array}$

Observations (or Sum Wgts)

Term Estimate Std Error t Ratio Prob $>|\mathbf{t}|$ $\begin{array}{llllll}\text { Intercept } & 2.7669673 & 0.010783 & 256.61 & <.0001\end{array}$ $\begin{array}{lllll}\text { Sampling Seq. } & -0.133508 & 0.001738 & -76.83 & <.0001\end{array}$
Ammonia Data(Study=1 Test Sequence=27)

\begin{tabular}{c}
$\log (\mathbf{A})$ By Sampling Sequence \\
\hline
\end{tabular}

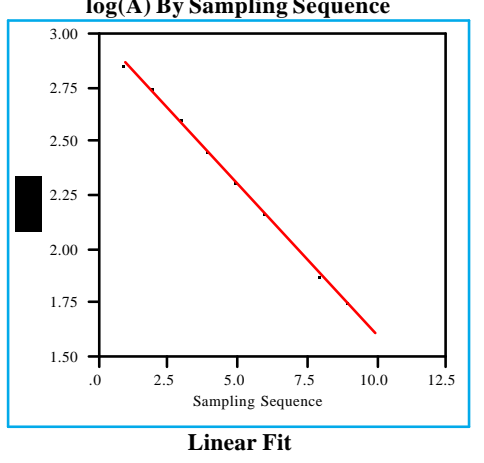

Linear Fit
$\log (\mathrm{A})=3.00258-0.13954$ S

$\log (\mathrm{A})=3.00258-0.13954$ Sampling Sequence

$\begin{array}{lll} & \text { Summary of Fit } & \\ \text { RSquare } & & 0.999098\end{array}$

$\begin{array}{ll}\text { RSquare Adj } & 0.998985 \\ \text { Root Mean Square Error } & 0.013466\end{array}$

Root Mean Square Error $\quad 0.013466$

Observations (or Sum Wgts)

$$
10
$$

$\begin{array}{llrrr} & \text { Parameter Estimates } & & \\ \text { Term } & \text { Estimate } & \text { Std Error } & \text { t Ratio } & \text { Prob }>|\mathbf{t}| \\ \text { Intercept } & 3.002584 & 0.009199 & 326.41 & <.0001 \\ \text { Sampling } & -0.139538 & 0.001483 & -94.12 & <.0001\end{array}$

Ammonia Data(Study=1 Test Sequence=28) $\log (A)$ By Sampling Sequence

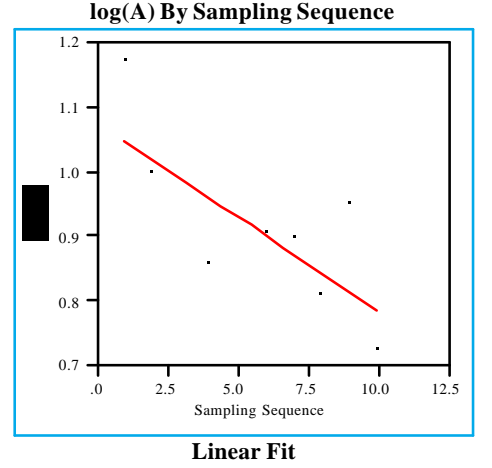

$\log (\mathrm{A})=1.07416-0.02919$ Sampling Sequence Summary of Fit

RSquare

0.547734

RSquare Adj

Root Mean Square Error

Mean of Response

0.491201
0.085183

Observations (or Sum Wgts)

0.085183

Parameter Estimates

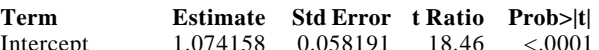

$\begin{array}{lrrrr} & 1.074158 & 0.058191 & 18.46 & <.0001 \\ \text { Sampling Seq. } & -0.029192 & 0.009378 & -3.11 & 0.0144\end{array}$
Ammonia Data(Study=1 Test Sequence=29)

$\log ($ A) By Sampling Sequence

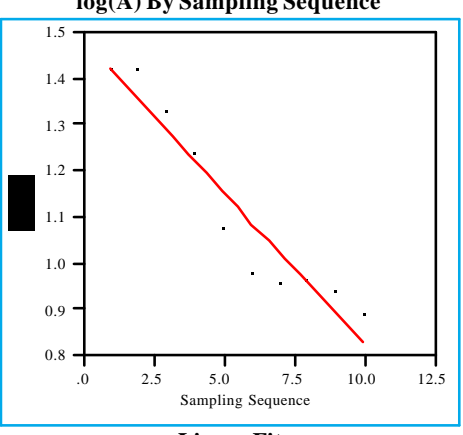

Linear Fit

$\log (\mathrm{A})=1.48259-0.06593$ Sampling Sequence

$$
\text { Summary of Fit }
$$

\section{RSquare
RSquare Adj}

(n)

Root Mean Square Error

Mean of Response

Observations (or Sum Wgts)

0.911284

0.066055
1.119999

Term $\begin{array}{lllll}\text { Intercept } & 1.4825874 & 0.045124 & 32.86 & <.0001\end{array}$ $\begin{array}{lllll}\text { Sampling Seq. } & -0.065925 & 0.007272 & -9.07 & <.0001\end{array}$

Ammonia Data(Study=1 Test Sequence=30)

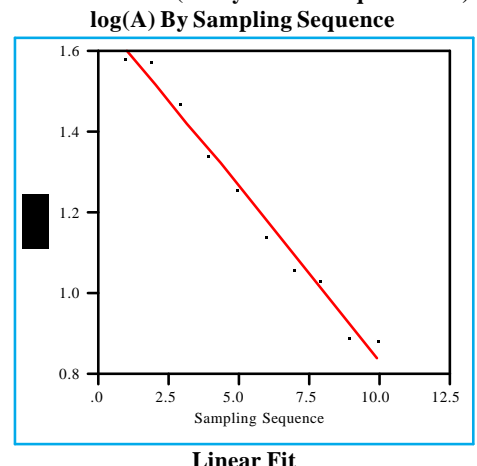

$\log (\mathrm{A})=1.694-0.08583$ Sampling Sequence

RSquare Summary of Fit

RSquare Adj

Root Mean Square Erro

Mean of Response

0.981872

Observations (or

0.03745

Term Estimate Std Error t Ratio Prob $>|\mathbf{t}|$ $\begin{array}{llllll}\text { Intercept } & 1.6939976 & 0.025586 & 66.21 & <.0001\end{array}$ $\begin{array}{lllll}\text { Sampling Seq. } & -0.085834 & 0.004124 & -20.82 & <.0001\end{array}$
Ammonia Data(Study=1 Test Sequence=31

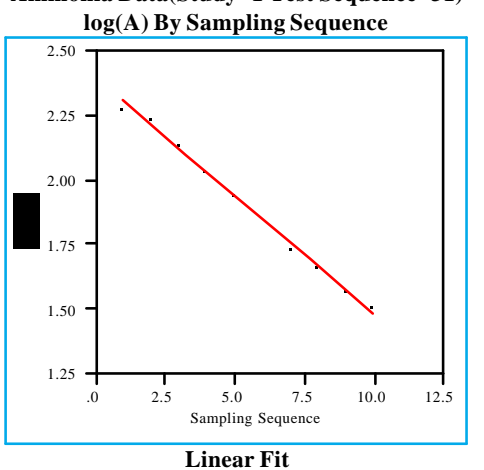

Linear Fit
$\log (\mathrm{A})=2.3918-0.09121$ Sampling Sequence

$\log (\mathrm{A})=2.3918-0.09121$ Sampling Sequence

RSquare

0.995972

$\begin{array}{ll}\text { RSquare Adj } & 0.995469 \\ \text { Rot Mean Square Error } & 0.018626\end{array}$

Root Mean Square Error

0.018626
1.890175

Observations (or Sum Wgts)

\begin{tabular}{lrrrrr} 
& \multicolumn{2}{c}{ Parameter Estimates } & & \\
Term & Estimate & Std Error & t Ratio & Prob $>|\mathbf{t}|$ \\
Intercept & 2.3918039 & 0.012724 & 187.98 & $<.0001$
\end{tabular}

$\begin{array}{lllll}\text { Sampling Seq. } & -0.091205 & 0.002051 & -44.48 & <.0001\end{array}$

Ammonia Data(Study=1 Test Sequence=32) $\log (A)$ By Sampling Sequence

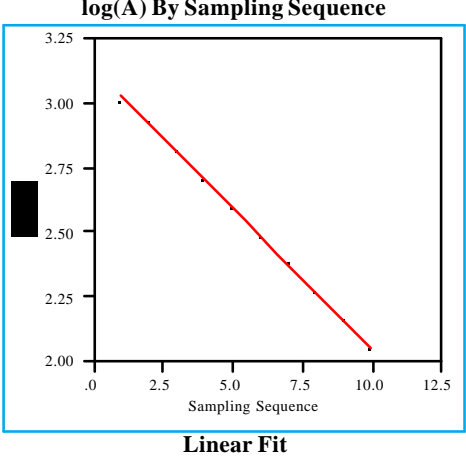

$\log (\mathrm{A})=3.13192-0.1083$ Sampling Sequence

RSquare Summary of Fit

Root Mean Square Error

Mean of Response

0.999417

Observations (or Sum Wgts)

0.008401

2.53626

Term Estimate Std Error t Ratio Prob $>|\mathbf{t}|$

$\begin{array}{llllll}\text { Intercept } & 3.1319218 & 0.005739 & 545.73 & <.0001\end{array}$

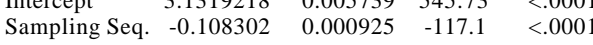


Exhibit B29: Common Logarithm of Peak Area Versus Sample Sequence for Ammonia Studies: Study 1; Tests 25-32

Ammonia Data(Study=1 Test Sequence $=33$ ) $\log (\mathbf{A})$ By Sampling Sequence

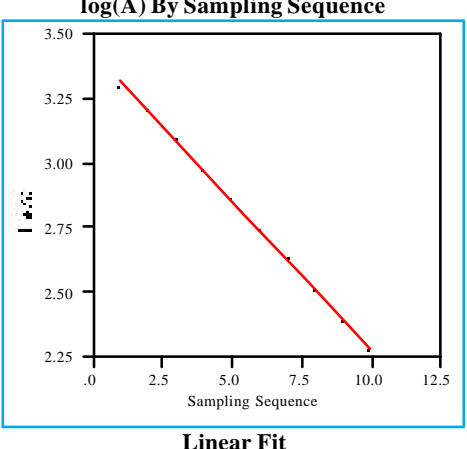

$\log (\mathrm{A})=3.42731-0.11483$ Sampling Sequence RSquare

RSquare Adj mmary of Fit

Mean Square Erro

Mean of Response
Observations (or Sum Wgts)

0.9995

0.00825

2.795733 $\begin{array}{lrrrrr} & \text { Parameter Estimates } & & \\ \text { Term } & \text { Estimate } & \text { Std Error } & \text { t Ratio } & \text { Prob }>|\mathbf{t}| \\ \text { Intercept } & 3.4273091 & 0.005636 & 608.09 & <.0001 \\ \text { Sant } & -114832 & 0.000908 & -126.4 & r .0001\end{array}$ $\begin{array}{lllll}\text { Sampling Seq. } & -0.114832 & 0.000908 & -126.4 & <.000\end{array}$

Ammonia Data(Study=1 Test Sequence=34)

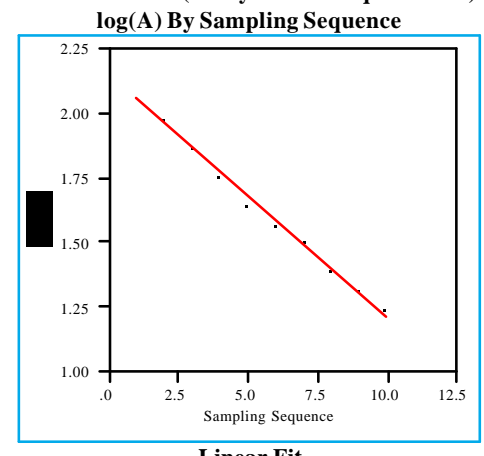

Linear Fit
$\log (\mathrm{A})=2.13994-0.09301$ Sampling Sequence
Summary of Fit

RSquare

RSquare Adj

0.995833

Root Mean Square Error

0.019322

Mean of Response

1.628373

Observations (or Sum Wgts)

$$
10
$$

$\begin{array}{lrrrrr}\text { Term } & \text { Estimate } & \text { Std Error } & \text { t Ratio } & \text { Prob }>|\mathbf{t}| \\ \text { Intercept } & 2.1399365 & 0.013199 & 162.13 & <.0001\end{array}$ $\begin{array}{lllll}\text { Sampling Seq. } & -0.093012 & 0.002127 & -43.72 & <.0001\end{array}$
Ammonia Data(Study=1 Test Sequence=35)

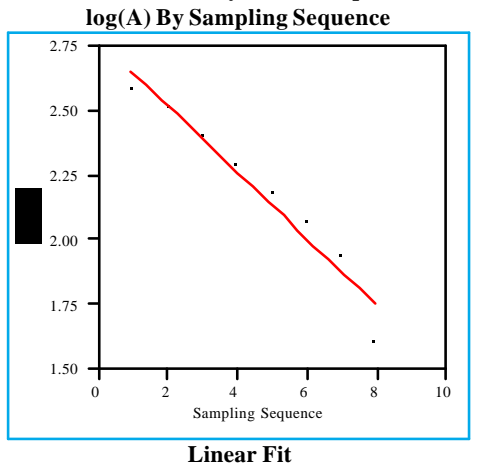
$\log (\mathrm{A})=2.77972-0.12902$ Sampling Sequence RSquare

Root Mean Square Error

Mean of Response

Observations (or Sum Wgts)

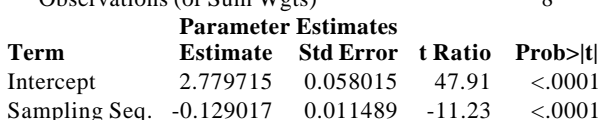

Ammonia Data(Study=1 Test Sequence=36) $\log (\mathbf{A})$ By Sampling Sequence

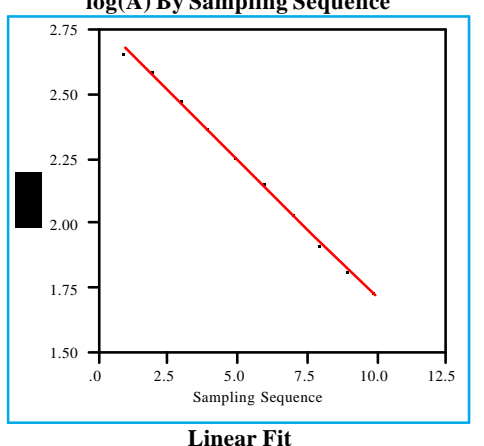

$\log (\mathrm{A})=2.78357-0.10707$ Sampling Sequence

RSquare

Summary of Fi 0.998364
0.99816
0.013916
2.194702
10

Root Mean Square Erro

Mean of Response

Observations (or Sum Wgts)

$$
10
$$

$\begin{array}{lrrrr}\text { Term } & \text { Estimate } & \text { Std Error } & \text { t Ratio } & \text { Prob }>|t| \\ \text { Intercept } & 2.7835663 & 0.009507 & 292.80 & <.0001\end{array}$ Sampling Seq. $-0.107066 \quad 0.001532 \quad-69.88<.000$
Ammonia Data(Study=1 Test Sequence $=37$ )

$\log (\mathbf{A})$ By Sampling Sequence

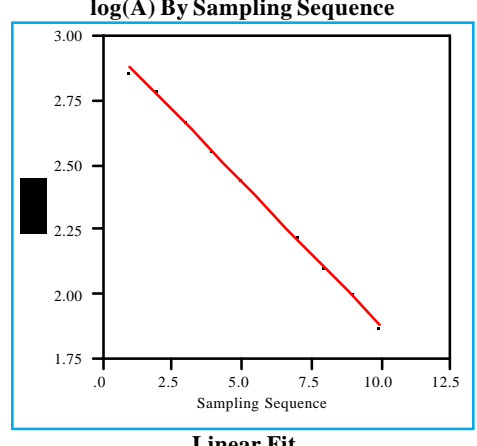

$\log (\mathrm{A})=2.99291-0.11135$ Sampling Sequence Summary of Fit

\subsection{3}

RSquare Ads

oot Mean Square Erro
Mean of Response

Mean of Response

0.998909

ervations (or Sum Wgts) 2.38048

\begin{tabular}{lrrrr}
\multicolumn{1}{c}{ Parameter Estimates } & & \\
Term & Estimate & Std Error & t Ratio & Prob $>|\mathbf{t}|$ \\
Intercept & 2.9929145 & 0.007612 & 393.20 & $<.0001$
\end{tabular} $\begin{array}{lllll}\text { Sampling Seq. } & -0.111351 & 0.001227 & -90.77 & <.0001\end{array}$

Ammonia Data(Study=1 Test Sequence $=38$ ) $\log ($ A) By Sampling Sequence

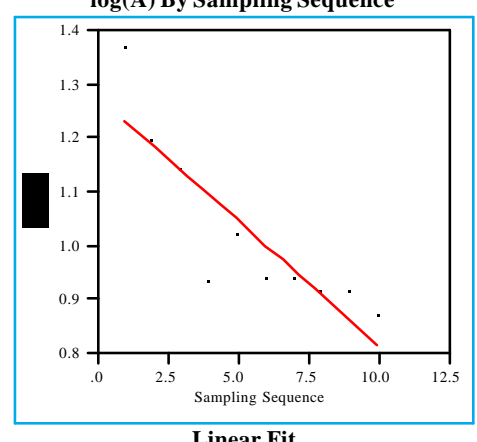

$\log (\mathrm{A})=1.27756-0.04$

0.0463 Sampling Sequence

RSquare

ummary of Fit

Mean of Response

0.766294

Observations (or

0.73708

(or Sum Wgts)

Term Parameter Estimates

$\begin{array}{rrrrr} & \text { Estimate } & \text { Std Error } & \text { t Ratio } & \text { Prob }>|\mathbf{t}| \\ \text { Intercept } & 1.2775613 & 0.056094 & 22.78 & <.0001\end{array}$

Sampling Seq. $-0.046301 \quad 0.00904 \quad-5.12 \quad 0.0009$
Ammonia Data(Study=1 Test Sequence=39) $\log (\mathrm{A})$ By Sampling Sequence

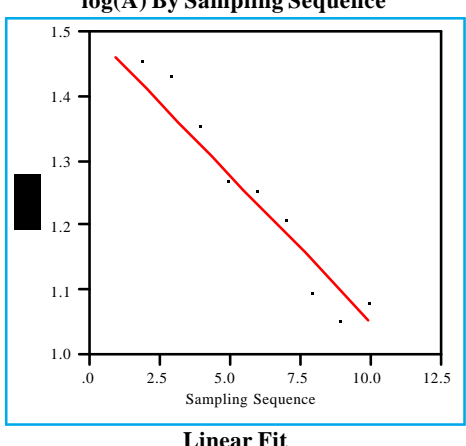

$\log (\mathrm{A})=1.50525-0.04548$ Sampling Sequence RSquare

RSquare Adj

Observations (or Sum Wgts)

0.052804

Term Estimate Std Error t Ratio Prob $>|\mathbf{t}|$ $\begin{array}{llllll}\text { Intercept } & 1.505246 & 0.036072 & 41.73 & <.0001\end{array}$ $\begin{array}{lllll}\text { Sampling Seq. } & -0.045484 & 0.005814 & -7.82 & <.0001\end{array}$

Ammonia Data(Study=1 Test Sequence $=40$ )

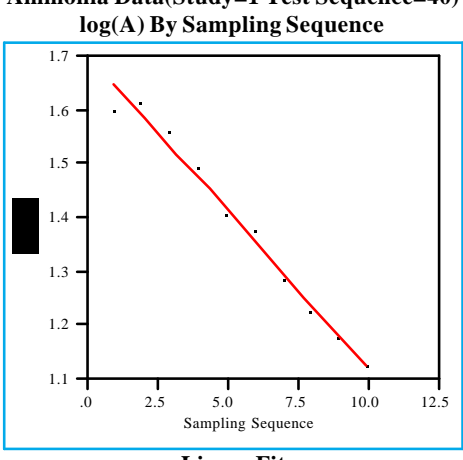

$\log (A)=1.70412-0.0584$ Sampling Sequence

Summary of Fit

Root Mean Square Error

0.98174
0.979458

Mean of Response

0.979458

Observations (or Sum Wgts)

Parameter Estimates

$\begin{array}{lrrrr}\text { Term } & \text { Estimate } & \text { Std Error } & \text { t Ratio } & \text { Prob }>|\mathbf{t}|\end{array}$

$\begin{array}{lllll}\text { Sampling Seq } & -0.058398 & 0.002816 & -20.74 & <.0001\end{array}$ 
Exhibit B30: Common Logarithm of Peak Area Versus Sample Sequence for Ammonia Studies: Study 1; Tests 41-48

Ammonia Data(Study=1 Test Sequence $=41)$

$\log (\mathbf{A})$ By Sampling Sequence

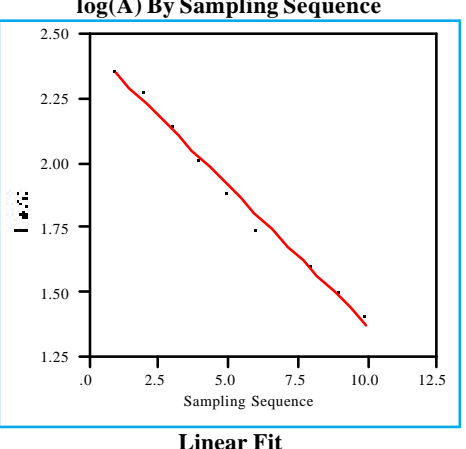

$\log (\mathrm{A})=2.45235-0.10786$ Sampling Sequence RSquare Summary of Fit

\subsection{5} $\begin{array}{lr}\text { RSquare Adj } & 0.988733 \\ \text { Root Mean Square Error } & 0.034839\end{array}$$$
\begin{array}{r}
0.034839 \\
1.8591 \\
10
\end{array}
$$

Mean of Response

$\begin{array}{lrrrrr} & \text { Parameter Estimates } & & \\ \text { Term } & \text { Estimate } & \text { Std Error } & \text { t Ratio } & \text { Prob }>|\mathbf{t}| \\ \text { Intercept } & 2.4523518 & 0.0238 & 103.04 & <.0001\end{array}$ $\begin{array}{lrrrr}\text { Intercept } & 2.4523518 & 0.0238 & 103.04 & <.0001 \\ \text { Sampling Seq. } & -0.107864 & 0.003836 & -28.12 & <.0001\end{array}$

Ammonia Data(Study=1 Test Sequence=42)

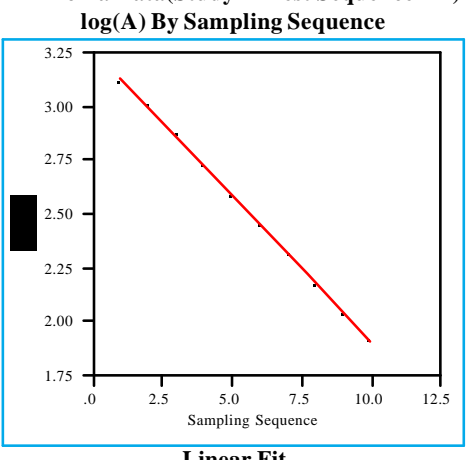

$\log (A)=3.26645-0.13601$ Sampling Sequence

RSquare

Summary of Fit

\begin{tabular}{lr} 
RSquare Adj & 0.999626 \\
Root Mean Square Error & 0.999579 \\
Mean of Response & 0.008454 \\
\hline
\end{tabular}

Mean of Response
Observations (or S

Parameter Estimates

$\begin{array}{lrrrr}\text { Term } & \text { Estimate } & \text { Std Error } & \text { t Ratio } & \text { Prob }>|t|\end{array}$

$\begin{array}{lrrrrr} & \text { Estercept } & 3.2664498 & 0.005775 & 565.62 & <.0001 \\ \text { Sampling Seq. } & -0.136008 & 0.000931 & -146.1 & <.0001\end{array}$
Ammonia Data(Study=1 Test Sequence=43)

$\log (\mathrm{A})$ By Sampling Sequence

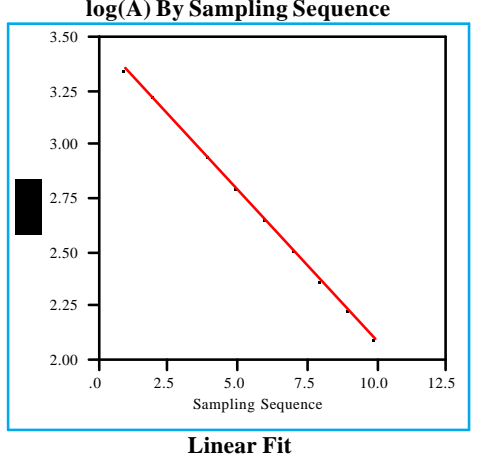

$\log (\mathrm{A})=3.49084-0.14033$ Sampling Sequence RSquare Summary of Fit

Root Mean Square Eror

0.999777

Root Mean Square Erro

Observations (or Sum Wgts)

0.006725

2.71901

Parameter Estimates

Tstimate Std Error t Ratio Prob>|t $\begin{array}{lrrrrr}\text { Intercept } & 3.4908432 & 0.004594 & 759.81 & <.0001\end{array}$ $\begin{array}{lllll}\text { Sampling Seq. } & -0.140333 & 0.00074 & -189.5 & <.0001\end{array}$

Ammonia Data(Study=1 Test Sequence=44)

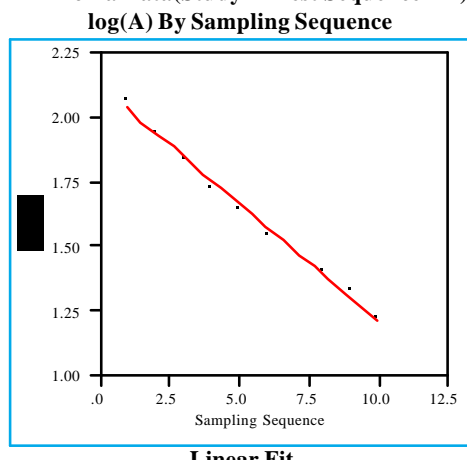

$\log (\mathrm{A})=2.12129-0.09119$ Sampling Sequence

RSquare

Summary of Fi

RSquare Adj

are Error

0.987103
0.985491

Root Mean Square Error

0.033475

Mean of Response

1.619717

Observations (or Sum Wgts)

Parameter Estimates

$\begin{array}{lrrrr}\text { Term } & \text { Estimate } & \text { Std Error } & \text { t Ratio } & \text { Prob }>|t|\end{array}$

$\begin{array}{lrrrr} & 2.1212855 & 0.022868 & 92.76 & <.0001 \\ \text { Sampling Seq. } & -0.091194 & 0.003685 & -24.74 & <.0001\end{array}$
Ammonia Data(Study=1 Test Sequence=45)

$\log (\mathbf{A})$ By Sampling Sequence

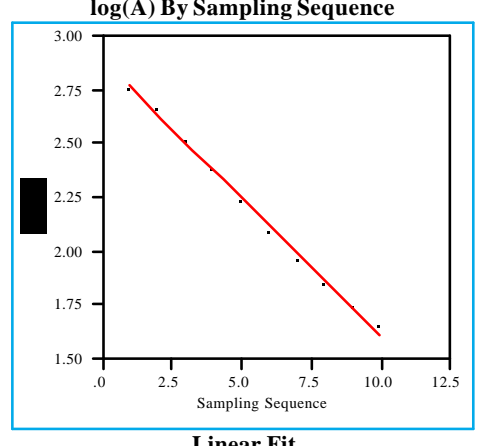
$\log (A)=2.88362-0.12764$ Sampling Sequence
Summary of Fit RSquare

\subsection{9} RSquare Adj Root Mean Square Erro Mean of Response 0.995465 Observations (or Sum Was) 2.18162

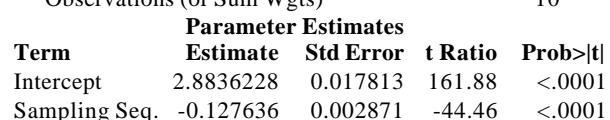

\section{Ammonia Data $($ Study $=1$ Test Sequence $=46$ )}

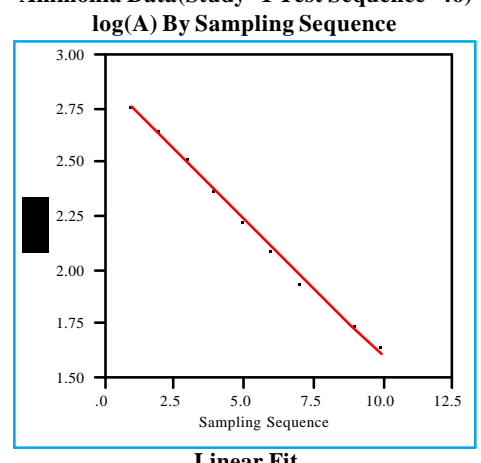

$\log (\mathrm{A})=2.87913-0.12835$ Sampling Sequence RSquare Summary of Fit

0.994884 RSquare Ad

Root Mean Square Erro

Mean of Response

0.994244

Observations (or Sum Wgts)

$\begin{array}{lrrrr} & \text { Estimate } & \text { Std Error } & \text { t Ratio } & \text { Prob }>|\mathbf{t}| \\ \text { Intercept } & 2.8791316 & 0.020192 & 142.59 & <.0001\end{array}$ $\begin{array}{lllll}\text { Intercept } & 2.8791316 & 0.020192 & 142.59 & <.0001 \\ \text { Sampling Seq. } & -0.128349 & 0.003254 & -39.44 & <.0001\end{array}$
Ammonia Data(Study=1 Test Sequence $=47$ )

$\log (\mathrm{A})$ By Sampling Sequence

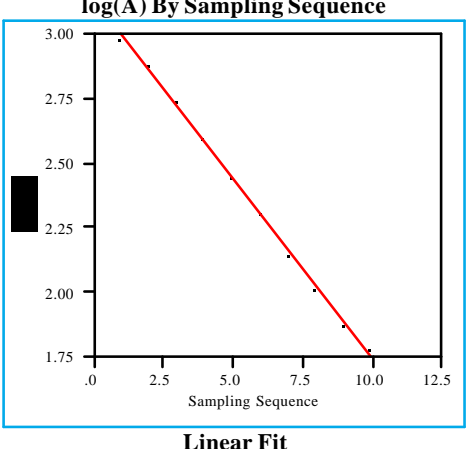

$\log (A)=3.13568-0.13915$ Sampling Sequence RSquare

RSquare Adj Summary of Fit

0.998492 Root Mean Square Error $\quad 0.998304$ Mean of Response

0.017364
2.37034

Observations (or Sum Wgts)

Term $\quad \begin{array}{lll}\text { Parameter Estimates } & & \\ \text { Estimate Std Error } & \text { Ratio } & \text { Prob }>|t|\end{array}$ $\begin{array}{lrrrrr} & \text { Entercept } & 3.1356761 & 0.011862 & 264.35 & <.0001\end{array}$ $\begin{array}{llllll}\text { Sampling Seq. } & -0.139152 & 0.001912 & -72.79 & <.0001\end{array}$

Ammonia Data(Study=1 Test Sequence $=48$ )

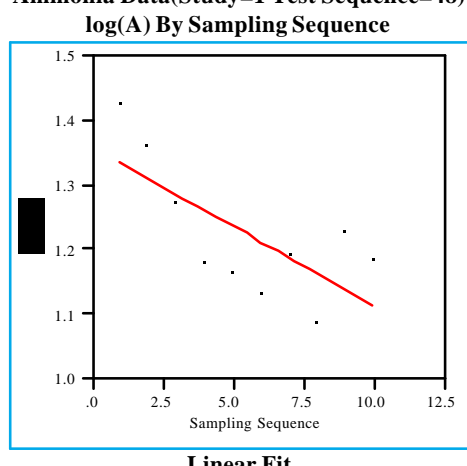

$\log (\mathrm{A})=1.35684-0.02443$ Sampling Sequence

$$
\text { Summary of Fit }
$$

RSquare Adj

Error

0.502232

Root Mean Sq

Mean of Response

0.440011

Observations (or Sum Wgts)

Parameter Estimates

Term Estimate Std Error t Ratio Prob $>\mid$ t $\begin{array}{lrrrrr} & 1.3568355 & 0.053354 & 25.43 & <.000 \\ & -0.02443 & 0.008599 & -2.84 & 0.0218\end{array}$ 
Exhibit B31: Common Logarithm of Peak Area Versus Sample Sequence for Ammonia Studies: Study 1; Tests 49-50; Study 2; Tests 1-6

Ammonia Data(Study=1 Test Sequence=49) $\log (\mathrm{A})$ By Sampling Sequence

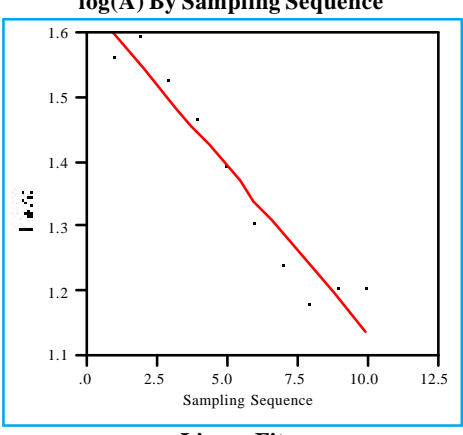

$\log (\mathrm{A})=1.65068-0.05159$ Sampling Sequence RSquare Summary of Fit RSquare Adj

Root Mean Square Erro

Mean of Response

0.928177

Observations (or Sum Wgts)

0.04608

1.366943

$\begin{array}{lrrrr} & \text { Parameter Estimates } & & \\ \text { Term } & \text { Estimate } & \text { Std Error } & \text { t Ratio } & \text { Prob }>|\mathbf{t}| \\ \text { Intercept } & 1.6506784 & 0.031481 & 52.43 & <.0001 \\ \text { Sampling Seq. } & -0.051588 & 0.005074 & -10.17 & <.0001\end{array}$

Ammonia Data(Study $=1$ Test Sequence $=50$ )

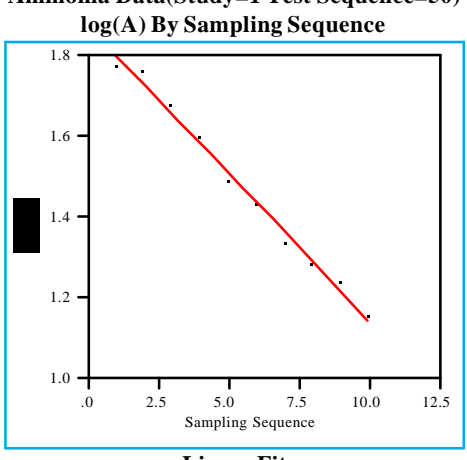

$\log (\mathrm{A})=1.87311-0.07315$ Sampling Sequence

$\begin{array}{ll}\log (\mathrm{A})= & 1.87311-0.07315 \text { Sampling Sequence } \\ \text { RSquare } & \text { Summary of Fit }\end{array}$

RSquare
RSquare Ad

$\begin{array}{ll}\text { RSquare Adj } & 0.989865 \\ \text { Root Mean Square Error } & 0.022397\end{array}$

Mean of Response

1.470789

Observations (or Sum Wgts)

10

\begin{tabular}{lrrrrr} 
& \multicolumn{2}{c}{ Parameter Estimates } & & \\
Term & Estimate & Std Error & t Ratio & Prob $>|\mathbf{t}|$ \\
Intercept & 1.8731063 & 0.0153 & 122.43 & $<.0001$
\end{tabular} $\begin{array}{lllll}\text { Sampling Seq. } & -0.073149 & 0.002466 & -29.67 & <.0001\end{array}$
Ammonia Data(Study=2 Test Sequence=1)

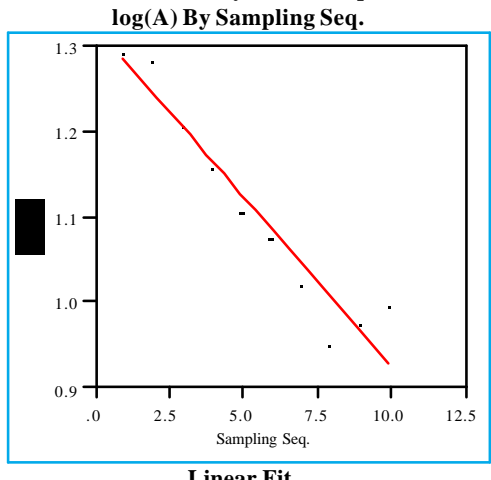

Linear Fit
$\log (\mathrm{A})=1.32175-0.0395$ Sampling Seq.

Summary of Fit

RSquare

0.921918

0.912157

Root Mean Square

0.036912
1.104523

Observations (or Sum Wgts)

10

Parameter Estimates

Term Estimate Std Error t Ratio Prob $>\mid t$ $\begin{array}{lllll}\text { Intercept } & 1.3217522 & 0.025216 & 52.42 & <.0001\end{array}$

- A nonia Data(Study=2 Test Sequence=2)

Ammonia Data(Study=2 Test Sequence

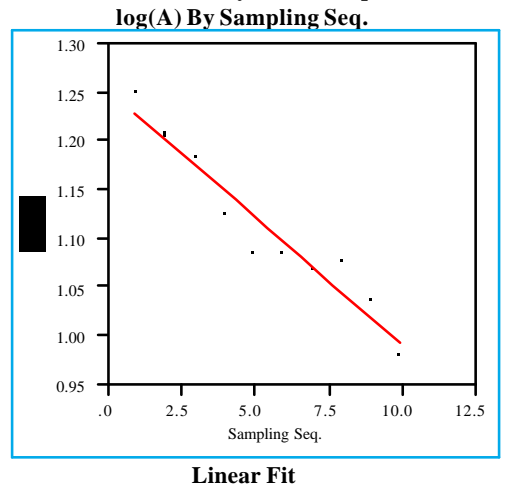

$\log (A)=1.25478-0.02633$ Sampling Seq

RSquare Summary of Fit

\begin{tabular}{lrrrr} 
Intercept & 0.9740206 & 0.036965 & 26.35 & $<.0001$ \\
\hline Sampling Seq. & -0.025467 & 0.005958 & -4.27 & 0.0027
\end{tabular}

RSquare Adj

0.929887

Root Mean Square Error

Mean of Response

0.023214

Observations (or Sum Wgts)

1.109992

Parameter Estimates

10

Term Estimate Std Error t Ratio Prob $>|t|$

$\begin{array}{lllll}\text { Intercept } & 1.2547827 & 0.015858 & 79.13 & <.000\end{array}$

$\begin{array}{lrrrr}\text { Sampling Seq. } & -0.026326 & 0.002556 & -10.30 & <.0001\end{array}$ $\begin{array}{lllll}\text { Sampling Seq } & -0.025467 & 0.036965 & 26.35 & <.0001 \\ & & & & \\ & & & \end{array}$

Ammonia Data(Study=2 Test Sequence $=4$ )

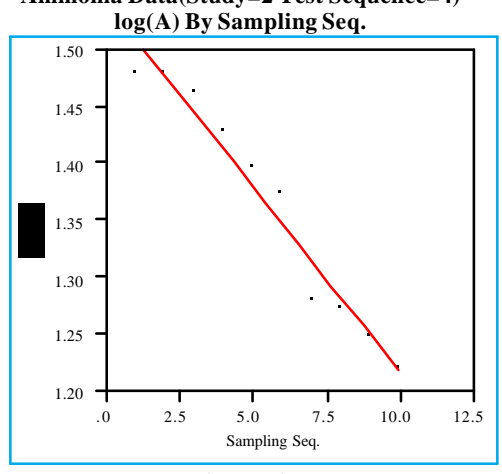

$\log (\mathrm{A})=1.54518-0.03267$ Sampling Seq RS Summary of Fit

Ammonia Data(Study=2 Test Sequence=3)

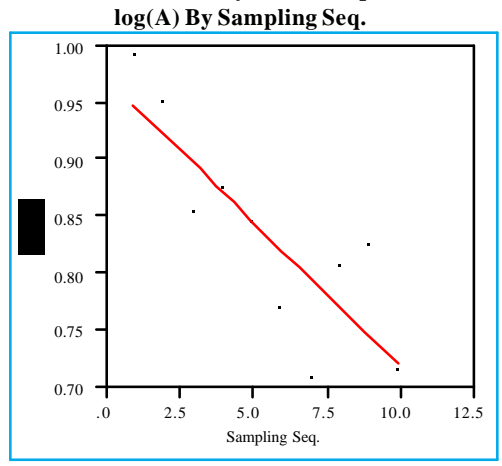

$\log (\mathrm{A})=0.97402-0.02547$ Sampling Se

RSquare Summary of Fit $\quad 0.695513$

RSquare Adj $\quad 0.657452$

of Response

0.833952

Square

0.956715

Root Mean Square Error

1.365515
10

Parameter Estimates
Pats

Term Estimate Std Error t Ratio Prob $>|\mathbf{t}|$ $\begin{array}{lrrrrr}\text { Intercept } & 1.5451773 & 0.015242 & 101.37 & <.0001 \\ \text { Sampling Seq. } & -0.032666 & 0.002457 & -13.30 & <.0001\end{array}$
Ammonia Data(Study=2 Test Sequence $=5$ )

$\log (A)$ By Sampling Seq.

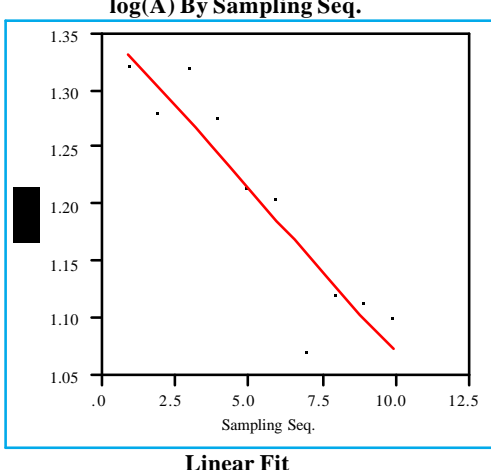

$\log (\mathrm{A})=1.36194-0.02914$ Sampling Seq.

$\begin{array}{lr}\text { RSquare Summary of Fit } & \\ \text { RSquare Adj } & 0.844818 \\ \text { Root Mean Square Error } & 0.82542 \\ \text { Mean of Response } & 0.040102 \\ \text { Obs } & 1.201683\end{array}$

Observations (or Sum Wgts)

Term $\quad \begin{array}{lll}\text { Parameter Estimates } \\ \text { Estimate }\end{array}$ $\begin{array}{lrrrr}\text { Intercept } & 1.3619371 & 0.027395 & 49.71 & <.0001 \\ & & & \end{array}$

Ammonia Data(Study=2 Test Sequence=6)

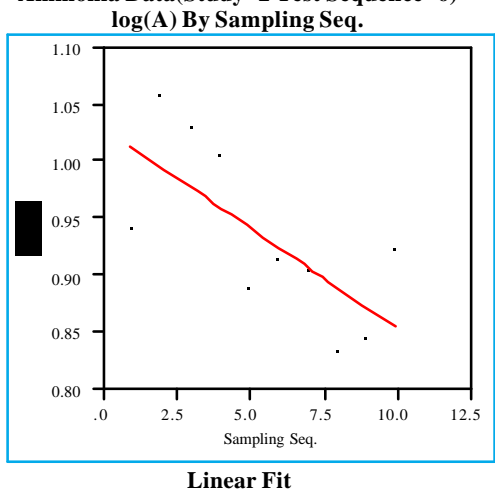

$\log (\mathrm{A})=1.0318-0.01777$ Sampling Seq RSquare Summary of Fit

0.501978

RSquare Adj $\quad 0.439725$

Root Mean Square Error

0.056831

Mean of Response

0.934083

Observations (or Sum Wgts)

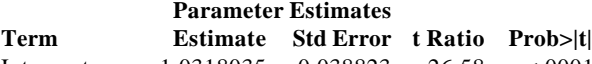

$\begin{array}{lrrrrr}\text { Intercept } & 1.0318035 & 0.038823 & 26.58 & <.0001\end{array}$ $\begin{array}{lllll}\text { Sampling Seq. } & -0.017767 & 0.006257 & -2.84 & 0.0218\end{array}$ 
Exhibit B32: Common Logarithm of Peak Area Versus Sample Sequence for Ammonia Studies: Study 2; Tests 7-13; Study 3; Test 1

Ammonia Data(Study=2 Test Sequence=7)

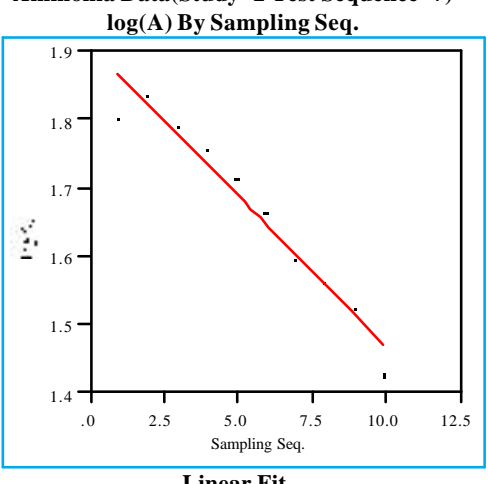

$\log (\mathrm{A})=1.90715-0.04392$ Sampling Seq.

\begin{tabular}{lr} 
RSquare $\quad$ Summary of Fit & \\
RSquare Adj & 0.952834 \\
Root Mean Square Error & 0.946939 \\
Mean of Response & 0.03138 \\
Observations (or Sum Wgts) & 1.665583 \\
\hline
\end{tabular}

Meot Mean of Response

Observations (or Sum Wgts)

$\begin{array}{lrrrr}\text { Term } & \text { Parameter Estimates } & & \\ \text { Intercept } & \text { Estimate } & \text { Std Error } & \text { t Ratio } & \text { Prob }>|\mathbf{t}|\end{array}$ $\begin{array}{lrrrr}\text { Intercept } & 1.9071467 & 0.021437 & 88.97 & <.000 \\ \text { Sampling Seq. } & -0.043921 & 0.003455 & -12.71 & <.000\end{array}$

Ammonia Data(Study=2 Test Sequence=8)

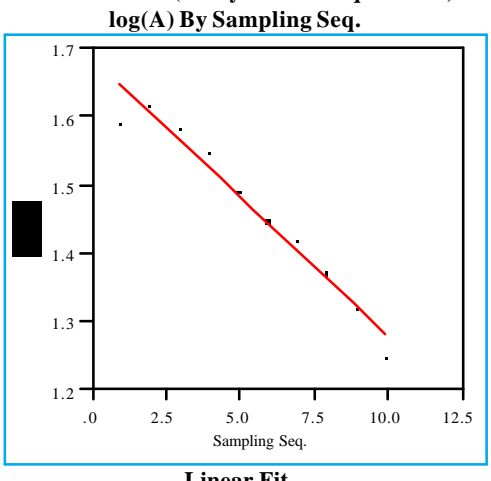

$\log (\mathrm{A})=1.68464-0.0404$ Sampling Seq \begin{tabular}{lr}
\multicolumn{2}{c}{ Summary of Fit } \\
RSquare & 0.9595 \\
RSquare Adj & 0.954437 \\
Root Mean Square Error & 0.026651 \\
Mean of Response & 1.462469 \\
Observations (or Sum Wgts) & 10
\end{tabular}

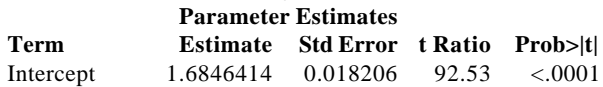
$\begin{array}{lrrrr}\text { Intercept } & 1.6846414 & 0.018206 & 92.53 & <.0001 \\ \text { Sampling Seq. } & -0.040395 & 0.002934 & -13.77 & <.0001\end{array}$
Ammonia Data(Study $=2$ Test Sequence $=9$ )

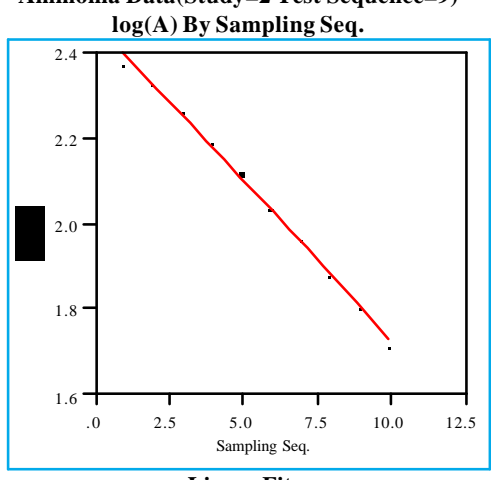

$\log (\mathrm{A})=2.47377-0.0745$

$\log (\mathrm{A})=2.47377-0.0745$ Sampling Seq.

$\begin{array}{ll}\text { RSquare } & 0.996122 \\ \text { RSquare Adj } & 0.995637 \\ \text { Root Mean Square Error } & 0.014927 \\ \text { Mean of Response } & 2.064043\end{array}$

Mean of Response

Observations (or Sum Wgts)

.995637

2.064043

$\begin{array}{lllll} & \text { Parameter Estimates } & & \\ \text { Term } & \text { Estimate } & \text { Std Error } & \text { t Ratio } & \text { Prob }>|\mathbf{t}|\end{array}$ $\begin{array}{lllll}\text { Intercept } & 2.4737682 & 0.010197 & 242.59 & <.0001 \\ \text { Sampling Seq. } & -0.074495 & 0.001643 & -45.33 & <.0001\end{array}$

Ammonia Data(Study $=2$ Test Sequence $=10$ )

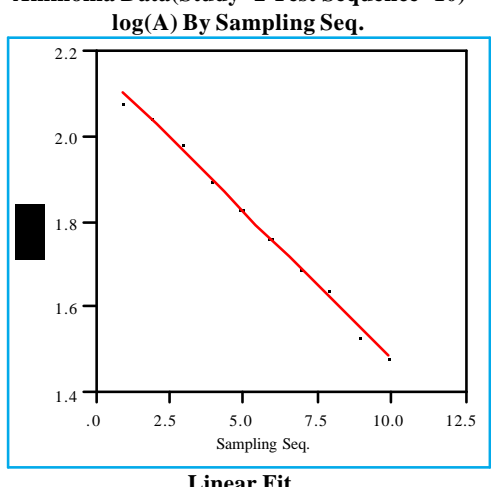

$\log (\mathrm{A})=2.17068-0.06893$ Sampling Seq RSquare

RSquare Adj Summary of Fi

Ammonia Data(Study=2 Test Sequence=11)

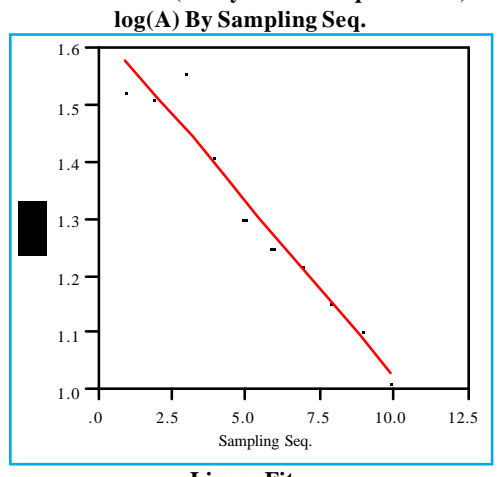

Linear Fit

$\log (\mathrm{A})=1.63785-0.06112$ Sampling Seq

$\begin{array}{ll} & \text { Summary of Fit } \\ \text { RSquare } & 0.952335 \\ \text { RSquare Adj } & 0.946377 \\ \text { Root Mean Square Error } & 0.043913 \\ \text { Mean of Response } & 1.301674 \\ \text { Obserats } & \end{array}$

Mean of Respons

0.94637

Observations (or Sum Wgts)

$\begin{array}{lrrrr}\text { Term } & \text { Parameter Estimates } & & \\ \text { Intercept } & \text { Estimate } & \text { Std Error } & \text { t Ratio } & \text { Prob }>|t| \\ & 1.6378546 & 0.029998 & 54.60 & <.0001\end{array}$ $\begin{array}{lrrrr}\text { Intercept } & 1.6378546 & 0.029998 & 54.60 & <.0001 \\ \text { Sampling Seq. } & -0.061124 & 0.004835 & -12.64 & <.0001\end{array}$

Ammonia Data(Study=2 Test Sequence=12)

$\log ($ A $)$ By Sampling Seq.

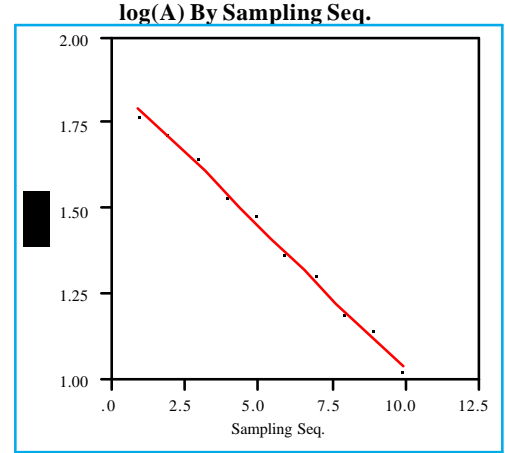

Linear Fit

$\log (A)=1.88007-0.08455$ Sampling Seq

RSivare

$$
\text { Summary of Fit }
$$

0.994752

Root Mean Square Erro

0.015153

Mean of Response

$$
\begin{array}{r}
0.015153 \\
1.791571 \\
10
\end{array}
$$

Parameter Estimates

Term Estimate Std Error t Ratio Prob $>\mid \mathbf{t}$

$\begin{array}{lrrrr}\text { Intercept } & 2.1706753 & 0.010351 & 209.70 & <.0001 \\ \text { Sampling Seq. } & -0.068928 & 0.001668 & -41.32 & <.0001\end{array}$
RSquare Adj

Parameter Estimates

Term Estimate Std Error t Ratio Prob $>|t|$ $\begin{array}{llllll}\text { Intercept } & 1.8800728 & 0.011939 & 157.47 & <.0001 \\ \text { Sampling } & -0.084553 & 0.001924 & -43.94 & <.0001\end{array}$

\section{Reor meare Eror \\ Observations (or Sum Wgts) \\ 0.017477}

Ammonia Data(Study=2 Test Sequence=13)

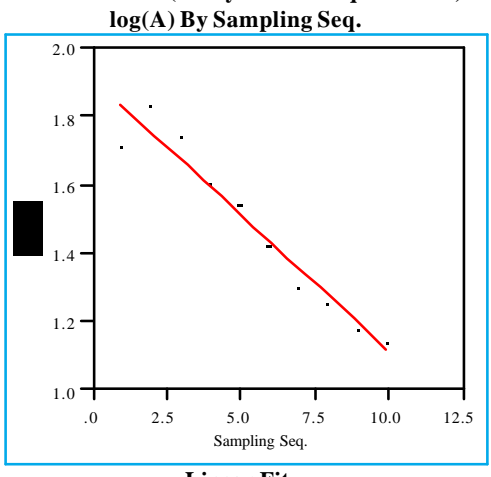

$\log (\mathrm{A})=1.91004-0.07986 \mathrm{~S}$

$004-0.07986$ Sampling Seq.

$\begin{array}{lr}\text { RSquare } & \text { Summary of Fit } \\ \text { RSquare Adj } & 0.944412 \\ \text { Root Mean Square Error } & 0.937463 \\ \text { Mean of Response } & 0.06222 \\ \text { Oberat } & 1.470809\end{array}$

Mean of Response

Observations (or Sum Wgts)

0.06222

Parameter Estimates

$\begin{array}{lrrrr}\text { Term } & \text { Estimate } & \text { Std Error } & \text { t Ratio } & \text { Prob }>|\mathbf{t}|\end{array}$ $\begin{array}{lrrrr}\text { Intercept } & 1.9100448 & 0.042504 & 44.94 & <.0001 \\ \text { Sampling Seq. } & -0.079861 & 0.00685 & -11.66 & <.0001\end{array}$

Ammonia Data(Study $=3$ Test Sequence=1)

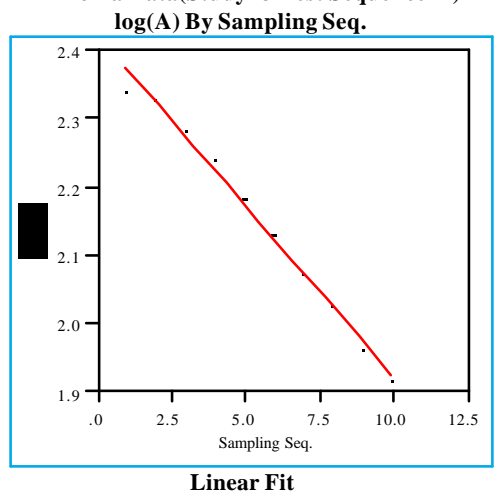

$\log (A)=2.42177-0.04983$ Sampling Seq. RSquare

RSquare Adj Summary of Fi

0.990992

Root Mean Square Error $\quad 0.989866$

Mean of Response

0.015257
2.147684

Observations (or Sum Wgts)

2.147684

$\begin{array}{lrrrr}\text { Term } & \text { Estimate } & \text { Std Error } & \text { t Ratio } & \text { Prob }>|t|\end{array}$ $\begin{array}{lrrrr}\text { Intercept } & 2.4217733 & 0.010423 & 232.35 & <.0001 \\ \text { Sampling Seq. } & -0.049834 & 0.00168 & -29.67 & <.0001\end{array}$ 
Exhibit B33: Common Logarithm of Peak Area Versus Sample Sequence for Ammonia Studies: Study 3; Tests 2-9

Ammonia Data(Study=3 Test Sequence=2)

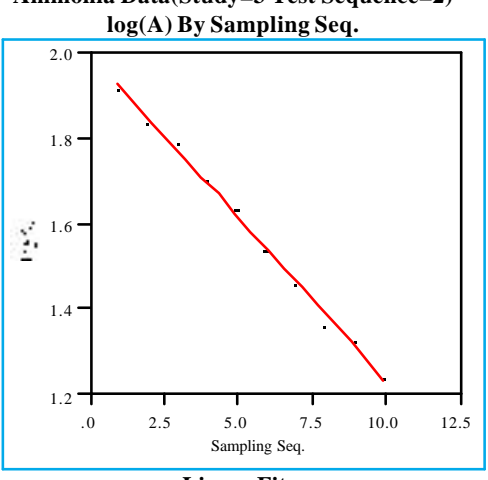

$\log ($ A $)=1.99757-0.07639$

$\log (A)=1.99757-0.07639$ Sampling Seq.

$\begin{array}{lr} & \text { Summary of Fit } \\ \text { RSquare } & 0.996456 \\ \text { RSquare Adj } & 0.996013 \\ \text { Root Mean Square Error } & 0.01463 \\ \text { Mean of Response } & 1.577422 \\ \text { Observations (or Sum Wots) } & 10\end{array}$

Reot Mean of Response

Observations (or Sum Wgts)

10

$\begin{array}{lrrrr}\text { Term } & \text { Estimate } & \text { Std Error } & \mathbf{t} \text { Ratio } & \text { Prob }>|\mathbf{t}|\end{array}$ Sampling Seq. $-0.076391 \quad 0.001611 \quad-47.43<<000$

Ammonia Data(Study=3 Test Sequence=3)

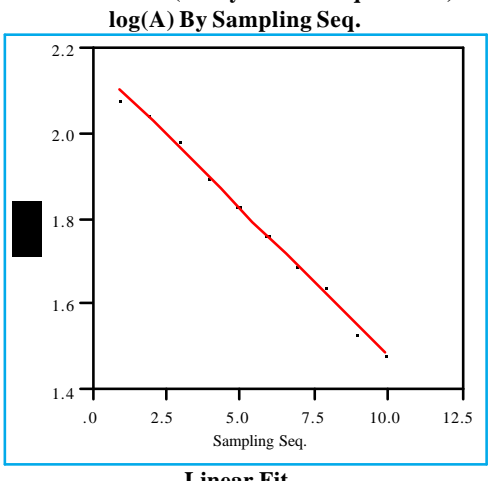

$\log (A)=2.17068-0.06893$ Sampling Seq RSquare Summary of Fit RSquare Adj

Root Mean Square Error 0.995335 Mean of Response 0.994752

Observations (or Sum Wgts)

$$
1.791571
$$

\begin{tabular}{lrrrrr}
\multicolumn{1}{c}{ Parameter Estimates } & & \\
Term & Estimate & Std Error & t Ratio & Prob $>|\mathbf{t}|$ \\
Intercept & 2.1706753 & 0.010351 & 209.70 & $<.0001$
\end{tabular} $\begin{array}{lrrrr}\text { Intercept } & 2.1706753 & 0.010351 & 209.70 & <.0001 \\ \text { Sampling Seq. } & -0.068928 & 0.001668 & -41.32 & <.0001\end{array}$
Ammonia Data $($ Study $=3$ Test Sequence $=4$ )

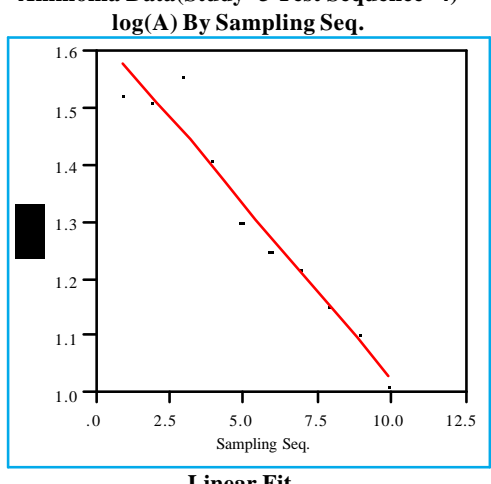

Linear Fit

$\log (\mathrm{A})=1.63785-0.06112$ Sampling Seq.

$\begin{array}{lr}\text { RSquare } \quad \text { Summary of Fit } & 0.952335 \\ \text { RSquare Adj } & 0.946377 \\ \text { Root Mean Square Error } & 0.043913 \\ \text { Mean of Response } & 1.301674 \\ \text { Obserations } & 10\end{array}$

Mean of Response

Observations (or Sum Wgts)

0.946377

301674

$\begin{array}{lllll}\text { Term } & \text { Parameter Estimates } & & \\ \text { Estimate } & \text { Std Error } & \text { t Ratio } & \text { Prob }>|t|\end{array}$ $\begin{array}{lrrrrr}\text { Intercept } & 1.6378546 & 0.029998 & 54.60 & <.0001 \\ \text { Sampling Seq. } & -0.061124 & 0.004835 & -12.64 & <.0001\end{array}$

Ammonia Data(Study=3 Test Sequence=5)

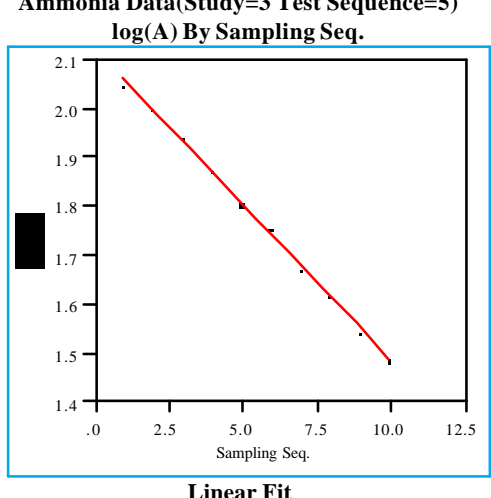

$\log (\mathrm{A})=2.12401-0.06405$ Sampling Seq RSquare

RSquare Adj Summary of Fit

Ammonia Data(Study=3 Test Sequence=6)

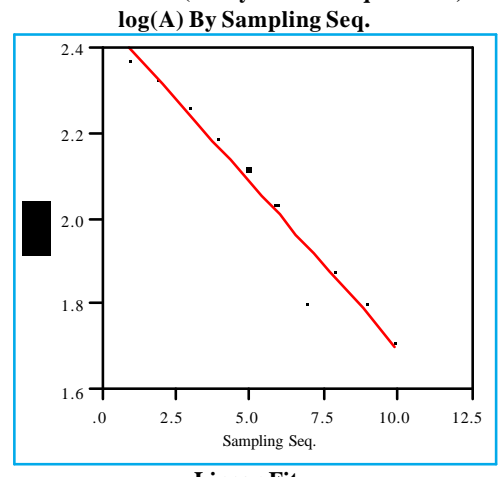

Linear Fit

$\log (\mathrm{A})=2.47377-0.07737$ Sampling Seq

RSquare

RSquare Adj

Summary of Fit

0.958303

Root Mean Square Error

0.9530

Observations (or Sum Wgts)

0.051824

$\begin{array}{lrrrr}\text { Term } & \text { Estimate } & \text { Std Error } & \text { t Ratio } & \text { Prob }>|\mathbf{t}|\end{array}$ $\begin{array}{lrrrr}\text { Sampling Seq. } & -0.077366 & 0.005706 & -13.56 & <.0001\end{array}$

Ammonia Data(Study=3 Test Sequence=7)

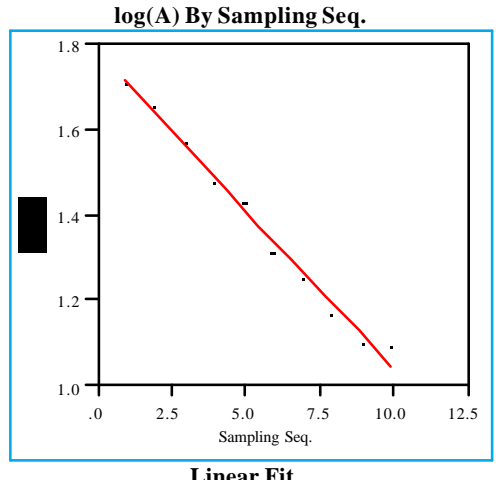

$\log (\mathrm{A})=1.78364-0.07439$ Sampling Seq RSquare Summary of $F$

0.998478

Mean of Response

0.00803

Observations (or Sum Wgts)

$$
1.771743
$$

Estimate Std Error t Ratio Prob $>|\mathbf{t}|$

$\begin{array}{lrrrr}\text { Intercept } & 2.1240144 & 0.005485 & 387.21 & <.0001 \\ \text { Sampling Seq. } & -0.064049 & 0.000884 & -72.45 & <.0001\end{array}$
RSquare

Root Mean Square Error

Mean of Response

Observations (or Sum Wgts)

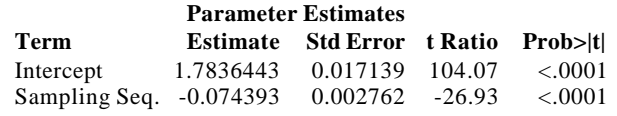

Ammonia Data(Study $=3$ Test Sequence $=8$ )

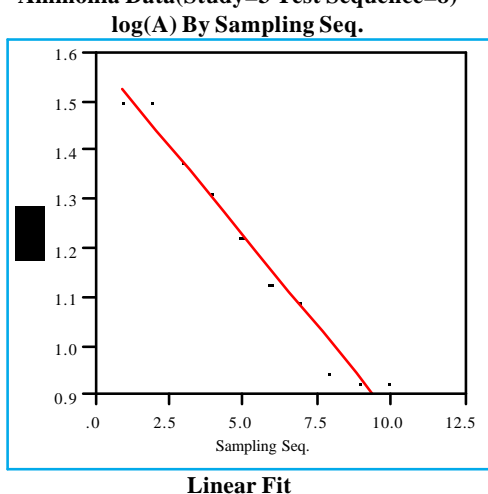

$\log (\mathrm{A})=1.59761-0.07371$ Sampling Seq.

\begin{tabular}{ll} 
RSquare $\quad$ Summary of Fit & \\
RSquare Adj & 0.972446 \\
Root Mean Square Error & 0.969002 \\
Mean of Response & 0.039847 \\
Observations (or Sum Wgts) & 1.192183 \\
\hline
\end{tabular}

0.969002

Observations (or Sum Wgts) 10

$\begin{array}{lrrrr}\text { Term } & \text { Parameter Estimates } & & \\ \text { Instimate } & \text { Std Error } & \text { t Ratio } & \text { Prob }>|t| \\ \text { Intercept } & 1.5976118 & 0.027221 & 58.69 & <.0001\end{array}$ $\begin{array}{lrrrr}\text { Intercept } & 1.5976118 & 0.027221 & 58.69 & <.0001 \\ \text { Sampling Seq. } & -0.073714 & 0.004387 & -16.80 & <.0001\end{array}$

Ammonia Data(Study $=3$ Test Sequence=9)

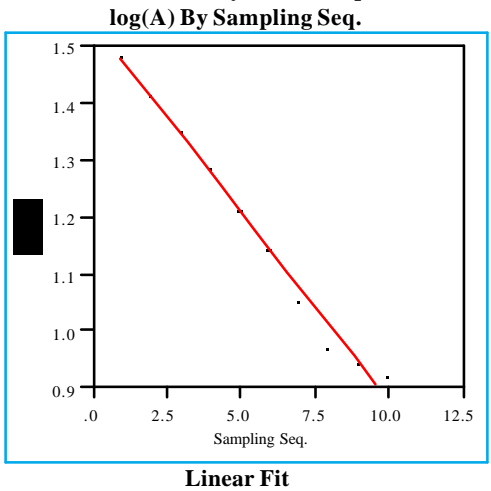

$\log (A)=1.54332-0.06668$ Sampling Seq. RSquare

RSquare Adj Summary of Fit

Root Mean Square Error

0.988202

Mean of Response

0.023398

Observations (or Sum Wgts)

1.176568

Parameter Estimates

$\begin{array}{lrrrr}\text { Term } & \text { Estimate } & \text { Std Error } & \text { t Ratio } & \text { Prob }>|\mathbf{t}| \\ \text { Intercept } & 1.5433235 & 0.015984 & 96.55 & \langle .000\end{array}$ $\begin{array}{lrrrr}\text { Intercept } & 1.5433235 & 0.015984 & 96.55 & <.0001 \\ \text { Sampling Seq. } & -0.066683 & 0.002576 & -25.89 & <.0001\end{array}$ 
Exhibit B34: Common Logarithm of Peak Area Versus Sample Sequence for Ammonia Studies: Study 4; Tests 1-8

Ammonia Data(Study=4 Test Sequence=1)

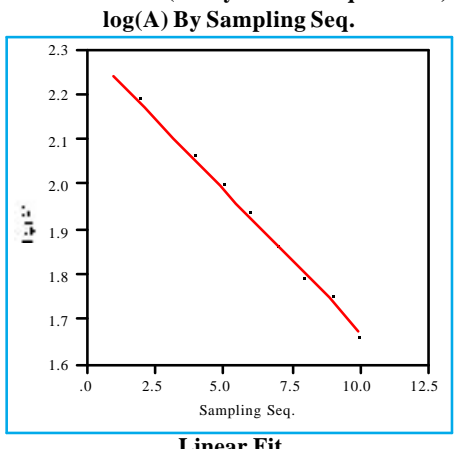

$\log (\mathrm{A})=2.30451-0.06275$ Sampling Seq. Summary of Fit

RSquare

RSquare Adj

Root Mean Square Error

Mean of Response

0.991019 0.989897 1.959395

Observations (or Sum Wgts)

Term Estimate Std Error t Ratio Prob $>|\mathbf{t}|$ $\begin{array}{lrrrrr}\text { Intercept } & 2.3045133 & 0.013104 & 175.86 & <.0001\end{array}$ $\begin{array}{lllll}\text { Sampling Seq. } & -0.062749 & 0.002112 & -29.71 & <.0001\end{array}$

Ammonia Data(Study=4 Test Sequence=2)
$\log ($ A $)$ By Sampling Seq.

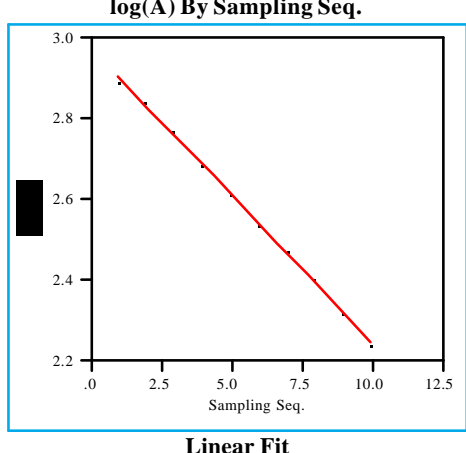

$\log (\mathrm{A})=2.97371-0.07302$ Sampling Seq.

RSquare Summary of Fit

\subsection{7}

RSquare Adj $\quad 0.998962$

Root Mean Square Error $\quad 0.007127$

Mean of Response

2.57208
10

Observations (or Sum Wgts)

10

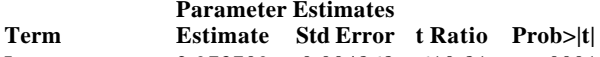
$\begin{array}{lrrrrr}\text { Intercept } & 2.973709 & 0.004868 & 610.81 & <.000\end{array}$ $\begin{array}{lllll}\text { Sampling Seq. } & -0.073023 & 0.000785 & -93.07 & <.0001\end{array}$
Ammonia Data(Study=4 Test Sequence=3) $\log ($ A) By Sampling Seq.

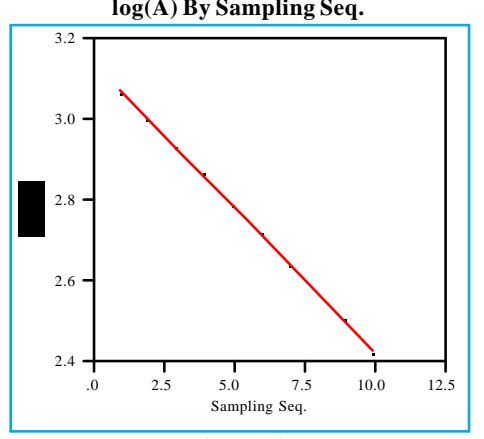

Linear Fit
$\log (\mathrm{A})=3.13887-0.07137$

$\log (\mathrm{A})=3.13887-0.07137$ Sampling Seq.

\begin{tabular}{|c|c|c|}
\hline \multirow{2}{*}{\multicolumn{3}{|c|}{$\begin{array}{ll}\text { RSquare } & \text { Summary of Fit }\end{array}$}} \\
\hline & & \\
\hline Root Mean Squ & & 0.005389 \\
\hline Uean of Rest & & \\
\hline
\end{tabular}

Root Mean Square Erro

Observations (or Sum Wgts)

Term $\quad \begin{array}{lll}\text { Parameter Estimates } \\ \text { Estimate }\end{array}$ Std Error $\mathbf{t}$ Ratio Prob $>|t|$

$\begin{array}{lrrrr}\text { Term } & \text { Estimate } & \text { Std Error } & \text { t Ratio } & \text { Prob }>|t| \\ \text { Intercept } & 3.1388731 & 0.003682 & 852.60 & <.0001\end{array}$

$\begin{array}{lllll}\text { Intercept } & 3.1388731 & 0.003682 & 852.60 & <.0001 \\ \text { Sampling Seq. } & -0.071368 & 0.000593 & -120.3 & <.0001\end{array}$

Ammonia Data(Study=4 Test Sequence=4)

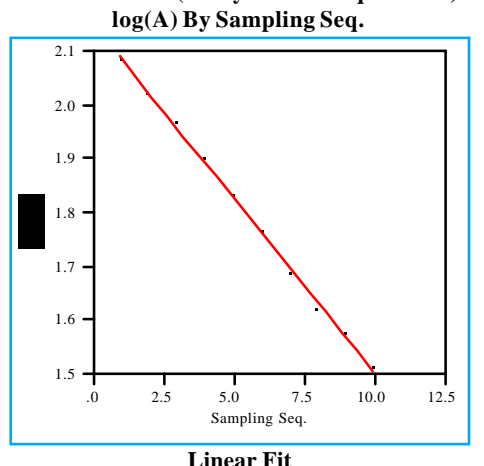

$\log (A)=2.15219-0.0648$ Sampling Seq.

RSquare Summary of Fit

$\begin{array}{ll}\text { RSquare } & 0.998153 \\ \text { RSquare Adj } & 0.997922\end{array}$

Root Mean Square Error $\quad 0.008953$

Mean of Response

1.795777
10

Observations (or Sum Wgts)

Parameter Estimates

Term Estimate Std Error t Ratio Prob $>|t|$

$\begin{array}{lrrrr}\text { Intercept } & 2.1521881 & 0.006116 & 351.90 & <.0001 \\ \text { Sampling Seq } & -0.064802 & 0.000986 & -65.74 & <.0001\end{array}$
Ammonia Data(Study=4 Test Sequence=5)

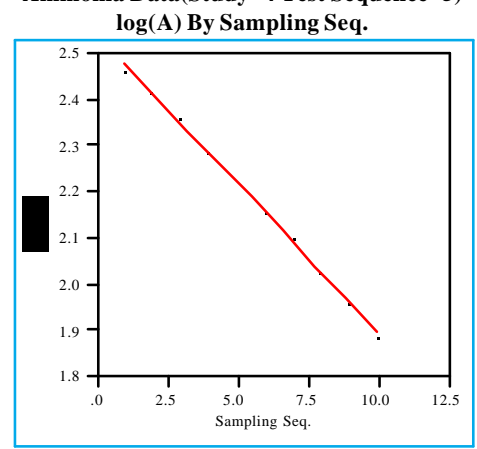

Linear Fit

$\log (\mathrm{A})=2.5391-0.06457$ Sampling Seq

\begin{tabular}{ll}
\multicolumn{2}{c}{ Summary of Fit } \\
RSquare & 0.998319 \\
RSquare Adj & 0.998109 \\
Root Mean Square Error & 0.008509 \\
Mean of Response & 2.183972
\end{tabular}

Mean of Response

0.998109

$\begin{array}{lllll} & \text { Parameter Estimates } & & \\ \text { Term } & \text { Estimate } & \text { Std Error } & \text { t Ratio } & \text { Prob }>|t|\end{array}$ $\begin{array}{lllllr}\text { Intercept } & 2.5391025 & 0.005813 & 436.83 & <.0001\end{array}$ $\begin{array}{lllll}\text { Sampling Seq. } & -0.064569 & 0.000937 & -68.93 & <.0001\end{array}$

Ammonia Data (Study=4 Test Sequence=6)

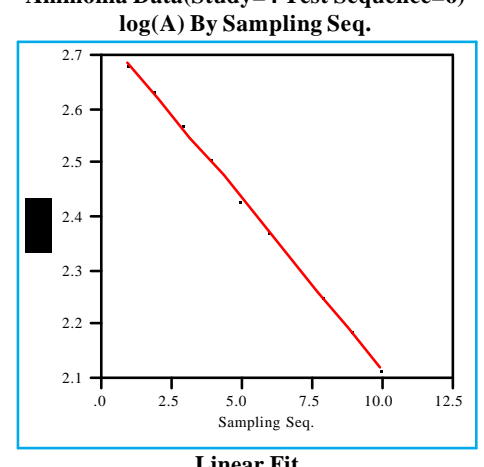

$\log (\mathrm{A})=2.74936-0.06317$ Sampling Seq

RSquare Summary of Fit

$\begin{array}{ll} & 0.999062 \\ \text { RSquare Adj } & 0.998945\end{array}$ Root Mean Square Error $\quad 0.006216$ Mean of Response 2.401926

Observations (or Sum Wgts)

Term Parameter Estimates t Ratio Prob>|t

$\begin{array}{lrrrrr} & \text { Estimate } & \text { Std Error } & \text { t Ratio } & \text { Prob }>\mid \mathbb{|} \\ \text { Intercept } & 2.7493615 & 0.004247 & 647.42 & <.0001\end{array}$ $\begin{array}{llllll}\text { Sampling Seq. } & -0.06317 & 0.000684 & -92.30 & <.0001\end{array}$
Ammonia Data(Study=4 Test Sequence=7)

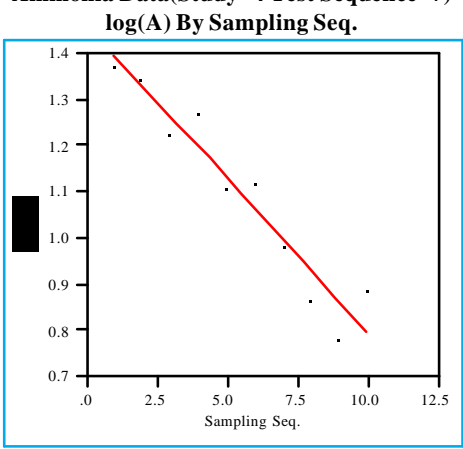

Linear Fit

$\log (A)=1.46253-0.067$ Sampling Seq

\begin{tabular}{lr}
\multicolumn{2}{c}{ Summary of Fit } \\
RSquare & 0.923557 \\
RSquare Adj & 0.914002 \\
Root Mean Square Error & 0.061897 \\
Mean of Response & 1.094054 \\
Observations (or Sum Wgts) & 10
\end{tabular}

Observation (or Sum Wgts)

$\begin{array}{lrrrr} & \text { Parameter Estimates } & & \\ \text { Term } & \text { Estimate } & \text { Std Error } & \text { t Ratio } & \text { Prob }>|\mathbf{t}| \\ & 1.4625336 & 0.042284 & 34.59 & <.0001\end{array}$ $\begin{array}{lrrrr}\text { Intercept } & 1.4625336 & 0.042284 & 34.59 & <.0001 \\ \text { Sampling } & -0.066996 & 0.006815 & -9.83 & <.0001\end{array}$

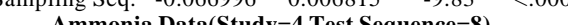
Ammonia Data(Study $=4$ Test Sequence $=8)$
$\log ($ A $)$ By Sampling Seq.

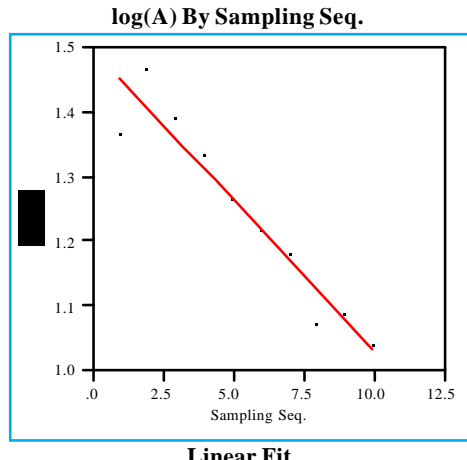

$\log (A)=1.49869-0.04687$ Sampling Seq.

RSquare

Summary of Fit

$\begin{array}{ll} & 0.920728 \\ \text { RSquare Adj } & 0.910819\end{array}$

Root Mean Square Error $\quad 0.044162$

Mean of Response

1.24092
10

Observations (or Sum Wgts)

Parameter Estimates

$\begin{array}{llll}\text { Term } & \text { Estimate } & \text { Std Error } & \text { t Ratio Prob }>\mid \mathbf{t} \text { | }\end{array}$

$\begin{array}{lrrrrr} & & \text { Ester6938 } & 0.030169 & 49.68 & <.0001 \\ \text { Sampling Seq. } & -0.046868 & 0.004862 & -9.64 & <.0001\end{array}$ 
Exhibit B35: Common Logarithm of Peak Area Versus Sample Sequence for Ammonia Studies: Study 4; Tests 9-13; Study 5; Tests 1-3

Ammonia Data(Study=4 Test Sequence=9) $\log ($ A) By Sampling Seq.

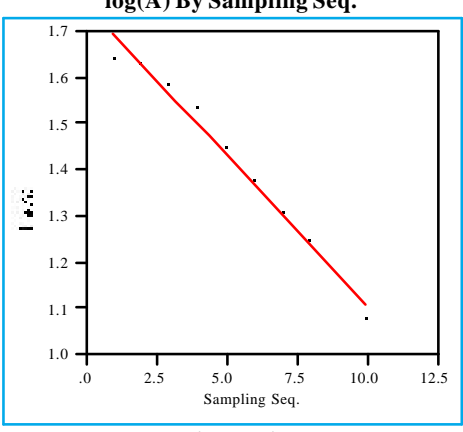

$\log (\mathrm{A})=1.76192-0.06577$

RSquare

Summary of Fit

RSquare Adj

Root Mean Square Erro

Mean of Response

0.98253
0.980346

0.980346

1.400175

Parameter Estimates

10

Term Estimate Std Error t Ratio Prob $>|t|$ $\begin{array}{lllllll} & 1.7619167 & 0.01924 & 91.58 & <.000\end{array}$ Sampling Seq. $\begin{array}{rllll}0.065771 & 0.003101 & -21.21 & <.0001\end{array}$

Ammonia Data(Study=4 Test Sequence $=10$ )

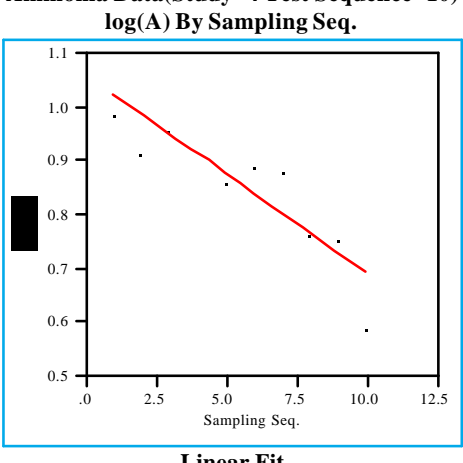

$\log (A)=1.05865-0.03672$ Sampling Seq. RSquare Summary of Fit

\section{RSquare Ad $j$}

are Error

0.747275

Root Mean Square Erro

0.715684

Mean of Response

0.856709

Observations (or Sum Wgts)$$
10
$$

$\begin{array}{lrrrr}\text { Term } & \text { Estimater } & \text { Std Error } & \text { t Ratio } & \text { Prob }>|\mathbf{t}| \\ \text { Intercept } & 1.0586454 & 0.04684 & 22.60 & <.0001\end{array}$ $\begin{array}{lrrrr} & 1.0586454 & 0.04684 & 22.60 & <.000 \\ \text { Sampling Seq. } & -0.036716 & 0.007549 & -4.86 & 0.0013\end{array}$
Ammonia Data(Study=4 Test Sequence=11)

$\log (\mathrm{A})$ By Sampling Seq.

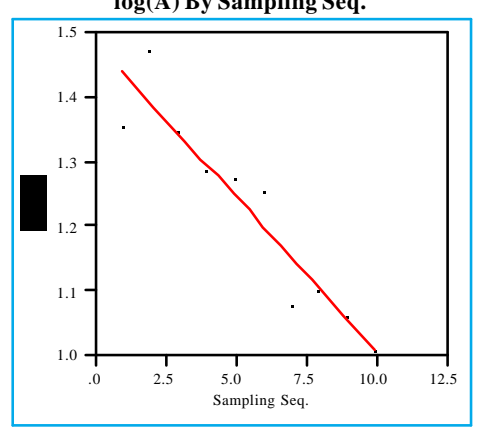

Linear Fit

$\log (\mathrm{A})=1.48472-0.04782$ Sampling Seq $\begin{array}{lr}\text { RSquare } & \text { Summary of Fit } \\ \text { RSquare Adj } & 0.89188 \\ \text { Root Mean Square Error } & 0.878365 \\ \text { Mean of Response } & 0.053468 \\ \text { Ore } & 1.221708\end{array}$ Observations (or Sum Wgts) 10 $\begin{array}{lrrrr} & \text { Parameter Estimates } & & \\ \text { Term } & \begin{array}{r}\text { Estimate } \\ \text { Std Error }\end{array} & \text { t Ratio } & \text { Prob }>|\mathbf{t}| \\ \text { Intercept } & 1.4847198 & 0.036526 & 40.65 & <.0001 \\ \text { Sampling } & -0.04782 & 0.005887 & -8.12 & <.0001\end{array}$

Ammonia Data(Study=4 Test Sequence=12)

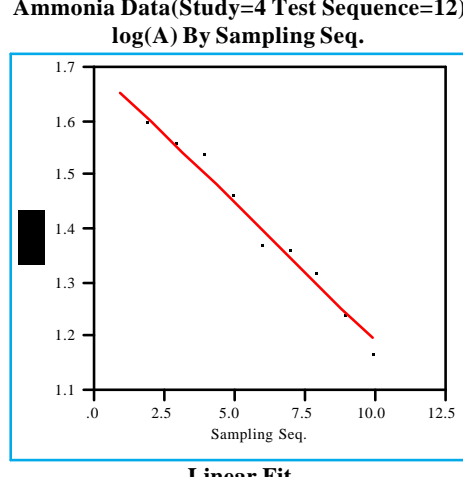

$\log (A)=1.70222-0.05089$ Sampling Seq

RSquare

RSquare Adj Summary of Fi

Root Mean Square Erro

Observations (or Sum Wgts)

Term $\quad \begin{aligned} & \text { Parameter Estimates } \\ & \text { Estimate }\end{aligned}$ Std Error $t$ Ratio Prob $>|\mathbf{t}|$

$\begin{array}{lrrrr}\text { Intercept } & 1.7022169 & 0.01795 & 94.83 & <.0001 \\ \text { Sampling Seq } & -0.05089 & 0.002893 & -17.59 & <.0001\end{array}$
Ammonia Data(Study $=4$ Test Sequence $=13$ )

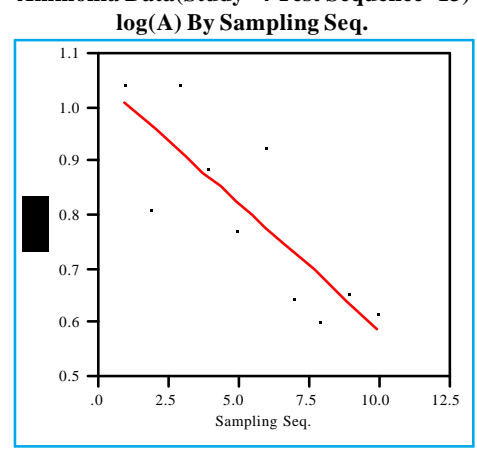

Linear Fit

$\log (\mathrm{A})=1.05505-0.04667$ Sampling Seq \begin{tabular}{lrrrr}
\multicolumn{3}{c}{ Summary of Fit } \\
RSquare & & 0.690551 \\
RSquare Adj & 0.65187 \\
Root Mean Square Error & 0.100337 \\
Mean of Response & 0.798333 \\
Observations (or Sum Wgts) & \multicolumn{1}{c}{10} \\
\multicolumn{5}{c}{ Parameter Estimates } \\
Term & Estimate & Std Error & $\mathbf{t}$ Ratio & Prob $>|\mathbf{t}|$ \\
Intercept & 1.0550453 & 0.068543 & 15.39 & $<.0001$ \\
Sampling Seq. & -0.046675 & 0.011047 & -4.23 & 0.0029
\end{tabular}

Ammonia Data(Study=5 Test Sequence=1)

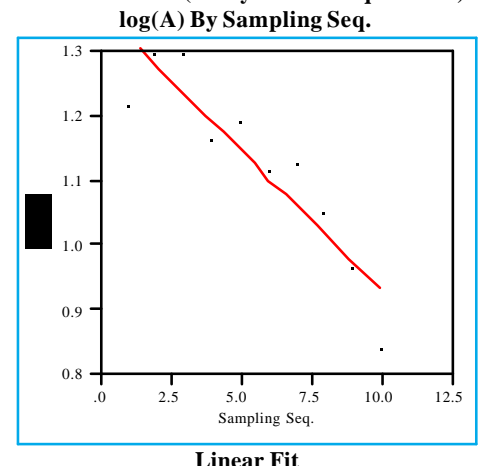

$\log (\mathrm{A})=1.36178-0.04321$ Sampling Seq RSquare Summary of Fit

RSquare Adj

Root Mean Square Erro

Mean of Response

0.826948

0.063479

Term $\quad \begin{array}{lll}\text { Parameter Estimates } & & \\ \text { Estimate Std Error t Ratio Prob }>|t|\end{array}$ $\begin{array}{llllll}\text { Intercept } & 1.3617847 & 0.043364 & 31.40 & <.0001\end{array}$ $\begin{array}{lllll}\text { Sampling Seq. } & -0.043211 & 0.006989 & -6.18 & 0.0003\end{array}$
Ammonia Data(Study $=5$ Test Sequence $=2$ ) $\log (A)$ By Sampling Seq.

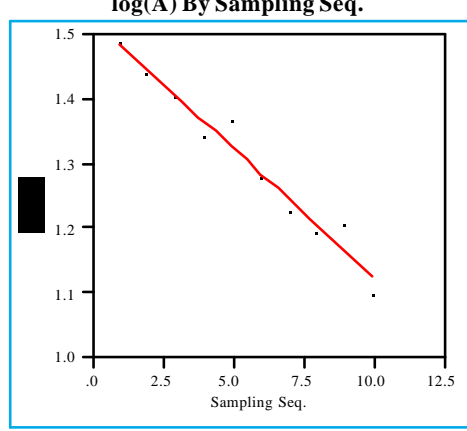

Linear Fit
$\log (\mathrm{A})=1.52333-0.04005$ Sampling Seq.

$$
\log (\mathrm{A})=\begin{gathered}
1.52333-0.04005 \text { Sam } \\
\text { Summary of Fit }
\end{gathered}
$$

$\begin{array}{lr}\text { RSquare } & \text { Summary of Fit } \\ \text { RSquare Adj } & 0.9603 \\ \text { Root Mean Square Error } & 0.955337 \\ \text { Mean of Response } & 1.30305 \\ \text { Obser } & \end{array}$

Observations (or Sum Wgts)

$\begin{array}{lrrrr} & \text { Parameter Estimates } & & \\ \text { Term } & \begin{array}{r}\text { Estimate } \\ \text { Std Error }\end{array} & \text { t Ratio } & \text { Prob }>|\mathbf{t}| \\ \text { Intercept } & 1.5233348 & 0.017865 & 85.27 & <.0001 \\ \text { Sampling Seq } & -0.040052 & 0.002879 & -13.91 & <.0001\end{array}$

Ammonia Data(Study $=5$ Test Sequence $=3$ )

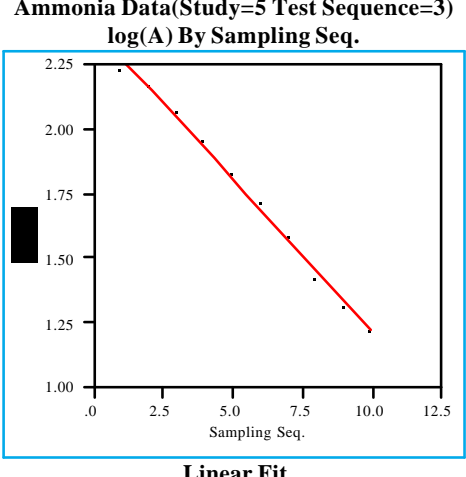

$\log (A)=2.39568-0.11837$ Sampling Seq

RSquare Summary of Fit

$\begin{array}{ll}\text { RSquare Adj } & 0.994323 \\ \text { Rquan } & 0.993613\end{array}$

Root Mean Square Error

Mean of Response

0.028722

Observations (or Sum Wgts)

Term Estimate Std Error t Ratio Prob $>\mid \mathbf{t}$

$\begin{array}{lrrrrr}\text { Term } & \text { Estimate } & \text { Std Error } & \text { t Ratio } & \text { Prob }>\mid \mathfrak{t} \\ \text { Intercept } & 2.3956841 & 0.019621 & 122.10 & <.0001\end{array}$ $\begin{array}{lllll}\text { Sampling Seq. } & -0.118369 & 0.003162 & -37.43 & <.0001\end{array}$ 
Exhibit B36: Common Logarithm of Peak Area Versus Sample Sequence for Ammonia Studies: Study 5; Tests 4-11

Ammonia Data(Study=5 Test Sequence=4)

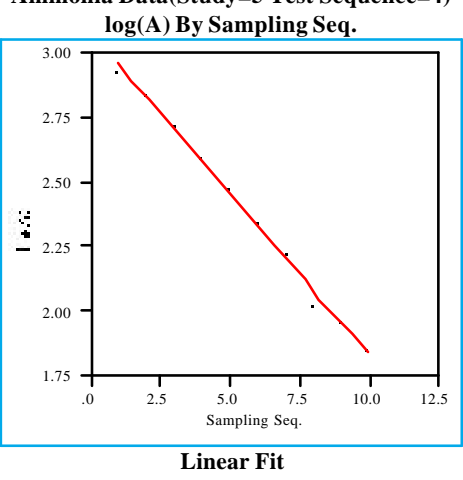

Linear Fit
$\log (\mathrm{A})=3.07811-0.12467$ Sampling Seq.

$$
\text { Summary of Fit }
$$

RSquare

Summary of Fit $\quad 0.99546$

$\begin{array}{ll}\text { RSquare Adj } & 0.994893 \\ \text { Root Mean Square Error } & 0.027037\end{array}$

Root Mean Square Error

Observations (or Sum Wgts)

2.392412

$\begin{array}{lrrrrr}\text { Term } & \text { Parameter Estimates } & & \\ \text { Estimate } & \text { Std Error } & \text { t Ratio } & \text { Prob }>|\mathbf{t}|\end{array}$ $\begin{array}{llllll} & \text { Estercept } & 3.0781117 & 0.01847 & 166.66 & <.000 \\ \end{array}$ $\begin{array}{lllll}\text { Sampling Seq. } & -0.124673 & 0.002977 & -41.88 & <.000\end{array}$

Ammonia Data(Study=5 Test Sequence=5)

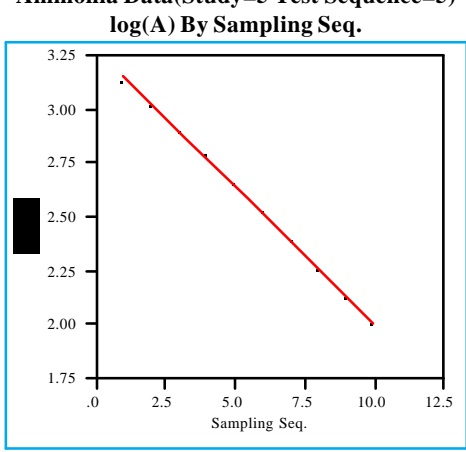

Linear Fi

$\log (\mathrm{A})=3.2759-0.12758$ Sampling Seq

RSquare

Summary of Fit

RSquare Adj

Root Mean Square Error

Mean of Response
Observations (or Sum Wgts)

0.99922

0.999123

0.011445

2.574187
10

Parameter Estimates

Term Estimate Std Error t Ratio Prob $>|t|$

$\begin{array}{llllll}\text { Intercept } & 3.2758966 & 0.007818 & 419.01 & <.000\end{array}$

$\begin{array}{lrrrr}\text { Sampling Seq. } & -0.127584 & 0.00126 & -101.3 & <.0001\end{array}$
Ammonia Data(Study $=5$ Test Sequence $=6$ )

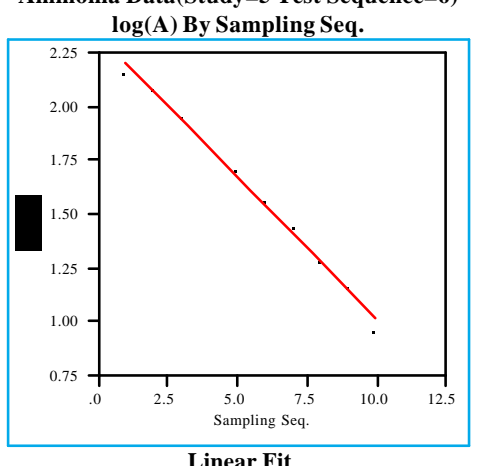

Linear Fit

$\log (\mathrm{A})=2.33228-0.13184$ Sampling Seq

$\begin{array}{ll}\text { RSquare } & \text { Summary of Fit } \\ \text { RSquare Adj } & 0.993864 \\ \text { Root Mean Square Error } & 0.993097 \\ \text { Mean of Response } & 0.033266 \\ \text { Orean } & 1.607177\end{array}$

Moot Mean of Response

Observations (or Sum Wgts)

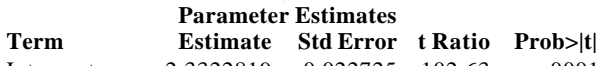

$\begin{array}{lrrrr} & \text { Estimate } & \text { Std Error } & \text { t Ratio } & \text { Prob }>|\mathbf{t}| \\ \text { Intercept } & 2.3322819 & 0.022725 & 102.63 & <.0001\end{array}$

$\begin{array}{llll}\text { Sampling Seq. } & -0.131837 & 0.003662 & -36.00\end{array}$

Ammonia Data(Study=5 Test Sequence=7)

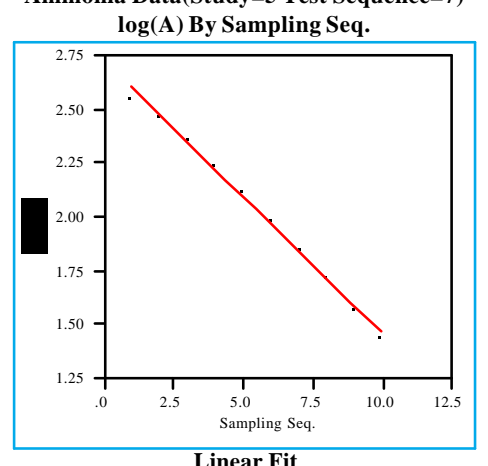

$\log (A)=2.72406-0.1261$ Sampling Seq

RSquare Summary of Fit

$\begin{array}{ll}\text { RSquare } & 0.997379 \\ \text { RSquare Adj } & 0.997051\end{array}$

Root Mean Square Error $\quad 0.020761$

$\begin{array}{lr}\text { Mean of Response } & 2.030486 \\ \text { Observations (or Sum Wgts) } & 10\end{array}$

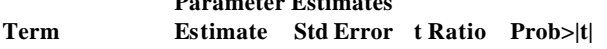

$\begin{array}{llllll}\text { Intercept } & 2.7240573 & 0.014182 & 192.07 & <.0001\end{array}$

$\begin{array}{lllll}\text { Sampling Seq. } & -0.126104 & 0.002286 & -55.17 & <.0001\end{array}$
Ammonia Data(Study=5 Test Sequence=8)

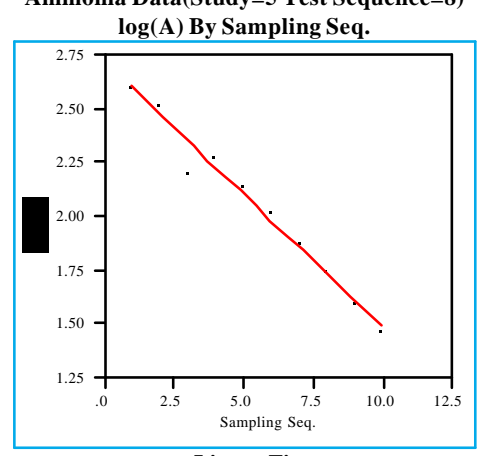

Linear Fit

Linear Fit
$\log (A)=2.71614-0.12262$ Sampling Seq

Summary of Fit $\quad 0.976805$

RSquare Adj $\quad 0.973905$

Root Mean Square Error $\quad 0.06068$

Observations (or Sum Wgts)

$\begin{array}{lrrrr} & \text { Parameter Estimates } & & \\ \text { Term } & \text { Estimate } & \text { Std Error } & \text { t Ratio } & \text { Prob }>|t| \\ \text { Intercept } & 2.7161392 & 0.041453 & 65.52 & <.0001\end{array}$ $\begin{array}{lllll}\text { Sampling Seq. } & -0.122623 & 0.006681 & -18.35 & <.0001\end{array}$

Ammonia Data(Study=5 Test Sequence=9)

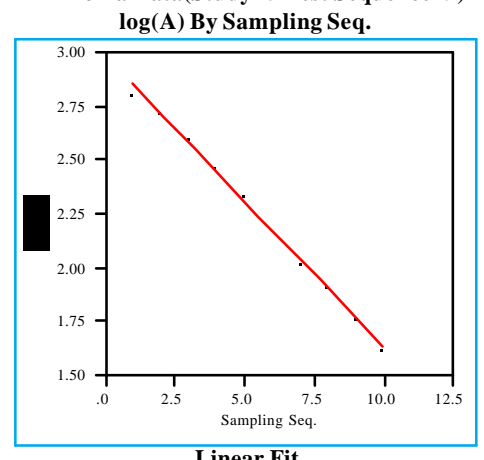

$\log (A)=2.98652-0.13547$ Sampling Se

Square Summary of Fit

0.996732 \begin{tabular}{ll} 
RSquare Adj & 0.996323 \\
Root Mean Square Error & 0.024912 \\
\hline
\end{tabular} Mean of Respons Observations (or Sum Wgts)

2.241408
10

Parameter Estimates

Term Estimate Std Error t Ratio Prob $>|\mathbf{t}|$ $\begin{array}{llllll}\text { Intercept } & 2.9865172 & 0.017018 & 175.49 & <.000\end{array}$ $\begin{array}{lllll}\text { Sampling Seq. } & -0.135474 & 0.002743 & -49.39 & <.0001\end{array}$
Ammonia Data(Study $=5$ Test Sequence $=10$

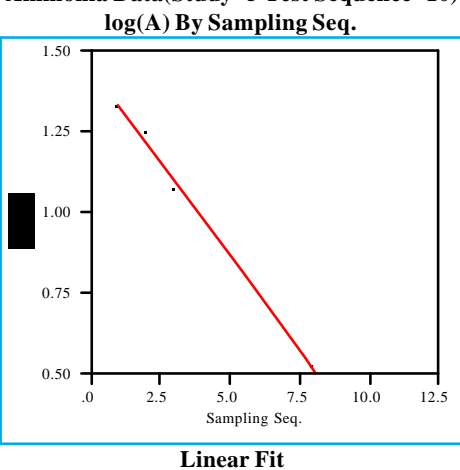

Linear Fit
$\log (\mathrm{A})=1.44613-0.11638$ Sampling Seq.

$\begin{array}{lr}\text { RSquare Summary of Fit } & \\ \text { RSquare Adj } & 0.99586 \\ \text { Root Mean Square Error } & 0.99379 \\ \text { Mean of Response } & 0.028573 \\ \text { Or } & 1.038791\end{array}$

Mean of Response

Observations (or Sum Wgts)

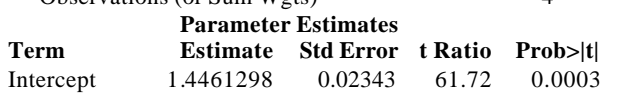

$\begin{array}{llllll} & 1.4461298 & 0.02343 & 61.72 & 0.0003\end{array}$

$\begin{array}{lllll}\text { Sampling Seq. } & -0.116382 & 0.005306 & -21.93 & 0.0021\end{array}$

Ammonia Data(Study $=5$ Test Sequence $=11$ )

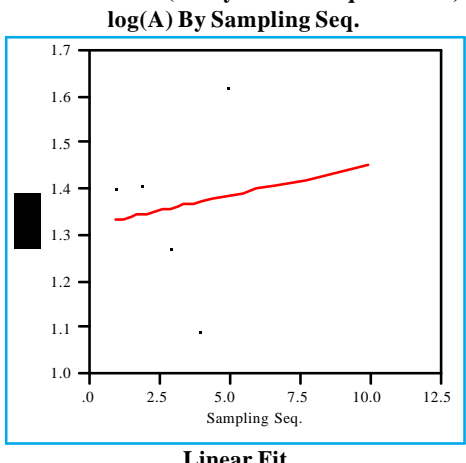

$\log (\mathrm{A})=1.31674+0.01328$ Sampling Seq Summary of Fit

RSquare

RSquare Adj

Root Mean Square Error

Mean of Response

0.011498
-0.318

Observations (or Sum Wgts)

0.224811
1.356583

Parameter Estimates

$\begin{array}{lrrrr}\text { Term } & \text { Estimate } & \text { Std Error } & \text { t Ratio } & \text { Prob }>|\mathbf{t}|\end{array}$ $\begin{array}{lrrrr} & 1.3167432 & 0.235784 & 5.58 & 0.0113 \\ \text { Sampling Seq. } & 0.01328 & 0.071091 & 0.19 & 0.8637\end{array}$ 
Exhibit B37: Common Logarithm of Peak Area Versus Sample Sequence for Ammonia Studies: Study 5; Tests 12-16; Study 6; Tests 1-3

Ammonia Data(Study=5 Test Sequence=12)

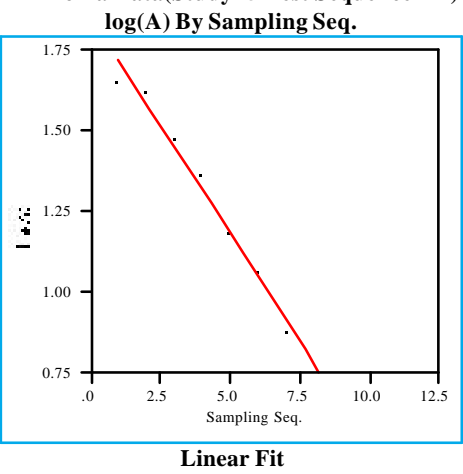

Linear Fit
$\log (\mathrm{A})=1.84697-0.13257$ Sampling Seq

\begin{tabular}{ll}
\multicolumn{1}{c}{ Summary of Fit } \\
RSquare & 0.979579 \\
RSquare Adj & 0.975495 \\
Root Mean Square Error & 0.045294 \\
Mean of Response & 1.316711 \\
Obse
\end{tabular}

Mean of Response

Observations (or Sum Wgts)

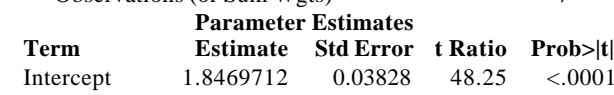

$\begin{array}{llll}0.03828 & 48.25 & <.000\end{array}$

$\begin{array}{lllll}\text { Sampling Seq. } & -0.132565 & 0.00856 & -15.49 & <.0001\end{array}$

Ammonia Data(Study $=5$ Test Sequence=13)

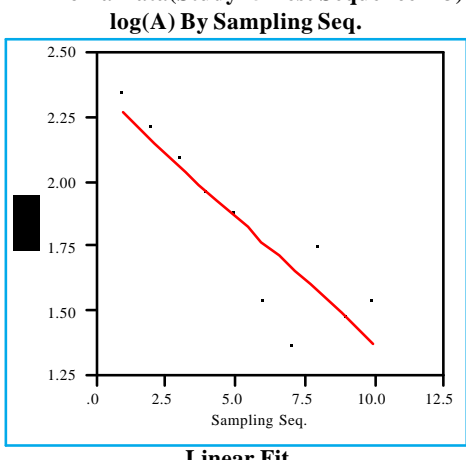

Linear Fit

$\log (A)=2.35853-0.09858$ Sampling Seq.

RSquare

Summary of Fit

$\begin{array}{lr}\text { RSquare Adj } & 0.785614 \\ \text { Root Mean Square Error } & 0.758816 \\ \text { Mean of Response } & 0.165371 \\ \text { Observations (or Sum Wgts) } & 1.816349 \\ & 10\end{array}$

Observations (or Sum Wgts)

Parameter Estimates

Term Estimate Std Error t Ratio Prob $>|\mathbf{t}|$ $\begin{array}{llllll}\text { Intercept } & 2.3585328 & 0.11297 & 20.88 & <.0001\end{array}$ $\begin{array}{lllll}\text { Sampling Seq. } & -0.098579 & 0.018207 & -5.41 & 0.0006\end{array}$
Ammonia Data $($ Study $=5$ Test Sequence=14)

$\log (\mathrm{A})$ By Sampling Seq.

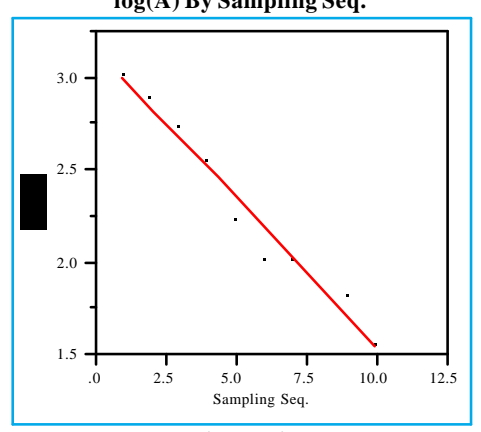

Linear Fit
$\log (\mathrm{A})=3.16109-0.16202$ Sampling Seq.

$\begin{array}{ll}\text { RSquare } & \text { Summary of Fit } \\ \text { RSquare Adj } & 0.970096 \\ \text { Root Mean Square Error } & 0.966358 \\ \text { Mean of Response } & 0.091352 \\ \text { O } & 2.269957\end{array}$

Root Mean Square Er

Observations (or Sum Wgts)
Parameter Estimates

0.966358

2.269957

Term $\begin{array}{llll}\text { Estimate } & \text { Std Error } & \text { t Ratio } & \text { Prob }>|t|\end{array}$

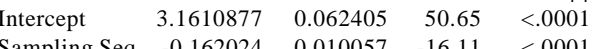

Ammonia Data(Study $=5$ Test Sequence $=15$ )

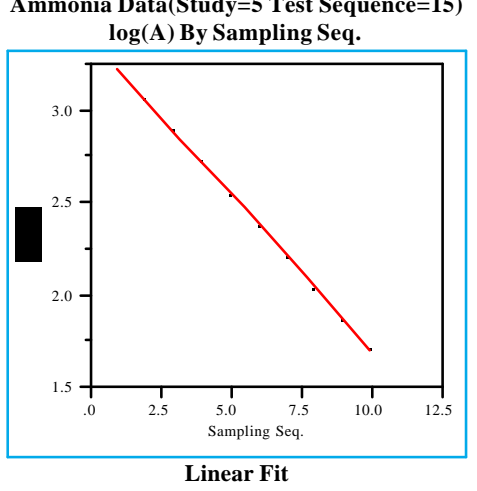

$\log (A)=3.38636-0.1685$ Sampling Seq.

RSquare Summary of Fit

$\begin{array}{ll}\text { RSquare } & 0.999723 \\ \text { RSquare Adj } & 0.999688\end{array}$

Root Mean Square Error $\quad 0.009013$

Mean of Response

2.459622
10

(or Sum Wgts)

Term Estimate Std Error t Ratio Prob $>|t|$

$\begin{array}{llllll}\text { Intercept } & 3.3863641 & 0.006157 & 549.99 & <.0001\end{array}$

$\begin{array}{lllll}\text { Sampling Seq. } & -0.168498 & 0.000992 & -169.8 & <.0001\end{array}$
Ammonia Data(Study=5 Test Sequence=16)

$\log ($ A) By Sampling Seq

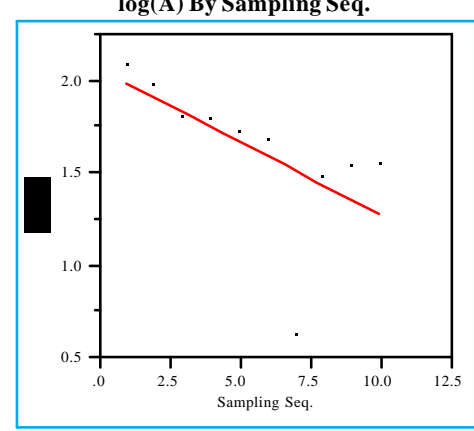

Linear Fit

$\log (\mathrm{A})=2.06136-0.07911$ Sampling Seq

$\begin{array}{lr}\text { RSquare } & \text { Summary of Fit } \\ \text { RSquare Adj } & 0.356063 \\ \text { Root Mean Square Error } & 0.275571 \\ \text { Mean of Response } & 0.34163 \\ \text { Oren } & 1.626268\end{array}$

Mean of Response

Observations (or Sum Wgts) \begin{tabular}{lrrrr}
\multicolumn{7}{c}{ Term } & Parameter Estimates & & \\
Estimate & Std Error & $\mathbf{t}$ Ratio & \multicolumn{1}{c}{ Prob $>|\mathbf{t}|$} \\
Intercept & 2.0613573 & 0.233378 & 8.83 & $<.0001$
\end{tabular}

Seq. $-0.079107 \quad 0.233378 \quad 8.83 \quad<$

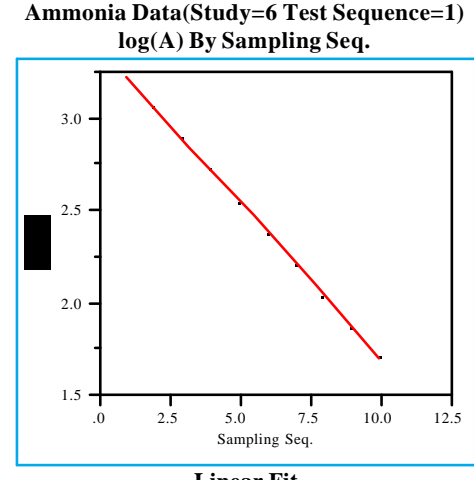

$\log (\mathrm{A})=3.3864-0.16849$ Sampling Seq

RSquare Summary of Fit

\section{RSquare Adj}

Root Mean Square Erro

Mean of Response

0.999729

0.008916

$\begin{array}{llll}\text { Term } & \text { Parameter Estimates } & & \\ \text { Estimate Std Error } & \text { t Ratio } & \text { Prob }>|t|\end{array}$ $\begin{array}{llllll}\text { Intercept } & 3.3863984 & 0.006091 & 555.97 & <.0001\end{array}$ $\begin{array}{lllll}\text { Sampling Seq. } & -0.16849 & 0.000982 & -171.6 & <.0001\end{array}$
Ammonia Data(Study $=6$ Test Sequence $=2$ )

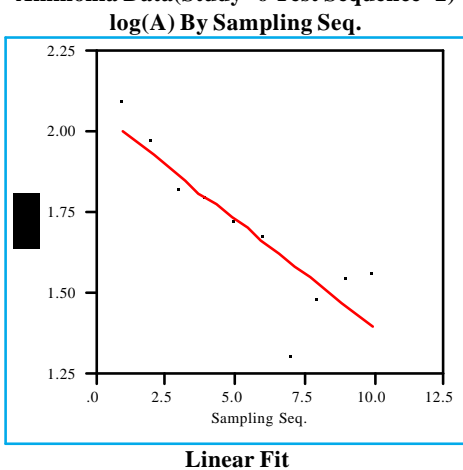

Linear Fit
$\log (\mathrm{A})=2.06136-0.06677$ Sampling Seq.

$\begin{array}{ll}\text { RSquare } & \text { Summary of Fit } \\ \text { RSquare Adj } & 0.732103 \\ \text { Root Mean Square Error } & 0.698616 \\ \text { Mean of Response } & 0.129711 \\ \text { Obse } & 1.694108\end{array}$

Mean of Response

0.698616

Observations (or Sum Wgts)

$\begin{array}{lrrrr}\text { Term } & \text { Parameter Estimates } & & \\ \text { Estimate } & \text { Std Error } & \text { t Ratio } & \text { Prob }>|\mathbf{t}| \\ \text { Intercept } & 2.0613573 & 0.08861 & 23.26 & <0001\end{array}$

$\begin{array}{rrrrr}2.0613573 & 0.08861 & 23.26 & <.0001\end{array}$

Ammonia Data(Study $=6$ Test Sequence $=3$ )

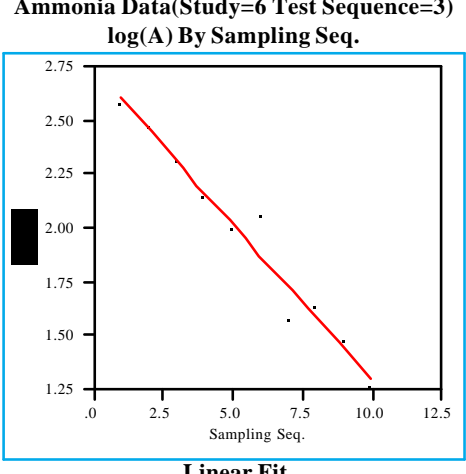

$\log (\mathrm{A})=2.7434-0.14479$ Sampling Seq

RSquare

Summary of Fit

$\begin{array}{lr} & 0.964694 \\ \text { RSquare Adj } & 0.96028\end{array}$

Root Mean Square Error $\quad 0.088948$

$\begin{array}{lr}\text { Mean of Response } & 1.94708 \\ \text { Observations (or Sum Wgts) } & 10\end{array}$

Parameter Estimates

Term Estimate Std Error t Ratio Prob $>|\mathbf{t}|$

$\begin{array}{lrrrr}\text { Intercept } & 2.7433981 & 0.060763 & 45.15 & <.0001 \\ \text { Sampling Seq. } & -0.144785 & 0.009793 & -14.78 & <.0001\end{array}$ 
Exhibit B38: Common Logarithm of Peak Area Versus Sample Sequence for Ammonia Studies: Study 6; Tests 4-5; Study 7; Tests 1-5

Ammonia Data(Study=6 Test Sequence=4)

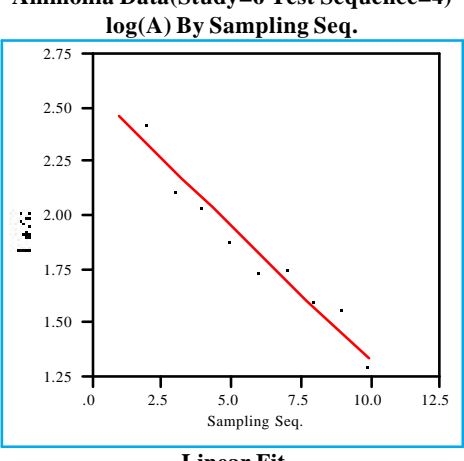

$\log (\mathrm{A})=2.58044-0.1258$ sit

$\log (A)=2.58044-0.12588$ Sampling Seq.

$\begin{array}{ll}\text { RSquare } & 0.960364 \\ \text { RSquare Adj } & 0.955409 \\ \text { Root Mean Square Error } & 0.082126 \\ \text { Mean of Response } & 1.888077\end{array}$

Moot Mean of Respone Ere

Observations (or Sum Wgts) $\begin{array}{lrrrr} & \text { Parameter Estimates } & & \\ \text { Term } & \text { Estimate } & \text { Std Error } & \text { t Ratio } & \text { Prob }>|\mathbf{t}| \\ \text { Intercept } & 2.5804381 & 0.056103 & 46.00 & <.0001 \\ \text { Sa } & 0.125884 & 0.009042 & 13.92 & <.0001\end{array}$

Ammonia Data(Study=6 Test Sequence $=5$ )

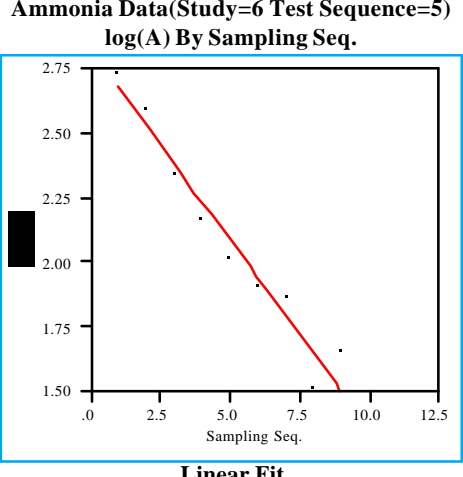

inear Fit

$\log (A)=2.82092-0.14623$ Sampling Seq.

RSquare

Summary of Fit

\begin{tabular}{lr} 
RSquare Adj & 0.952216 \\
Root Mean Square Error & 0.945389 \\
Mean of Response & 0.095906 \\
Observations (or Sum Wgts) & 2.089752 \\
\hline
\end{tabular}

Observations (or Sum Wgts)

Parameter Estimates

Term Estimate Std Error t Ratio Prob $>|\mathbf{t}|$

$\begin{array}{llllll}\text { Intercept } & 2.8209158 & 0.069674 & 40.49 & <.0001\end{array}$

$\begin{array}{lrrrr}\text { Sampling Seq. } & -0.146233 & 0.012381 & -11.81 & <.0001\end{array}$
Ammonia Data(Study=7 Test Sequence=1)

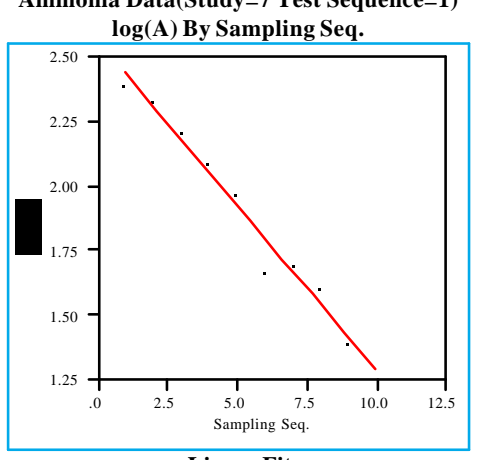

$\operatorname{lin}(\mathrm{A})=2.5585-0.12724$

$\log (\mathrm{A})=2.5585-0.12724$ Sampling Seq.

$\begin{array}{ll}\text { RSquare } & 0.969892 \\ \text { RSquare Adj } & 0.965591 \\ \text { Root Mean Square Error } & 0.065633 \\ \text { Mean of Response } & 1.922315\end{array}$

Moon of Response

Observations (or Sum Wgts)

$\begin{array}{lrrrr} & \text { Parameter Estimates } & & \\ \text { Term } & \text { Estimate } & \text { Std Error } & \text { t Ratio } & \text { Prob }>|t| \\ \text { Intercept } & 2.558504 & 0.047681 & 53.66 & <.0001 \\ \text { Sampling } & -0.127238 & 0.008473 & -15.02 & <.0001\end{array}$

Ammonia Data(Study=7 Test Sequence=2) $\log ($ A) By Sampling Seq.

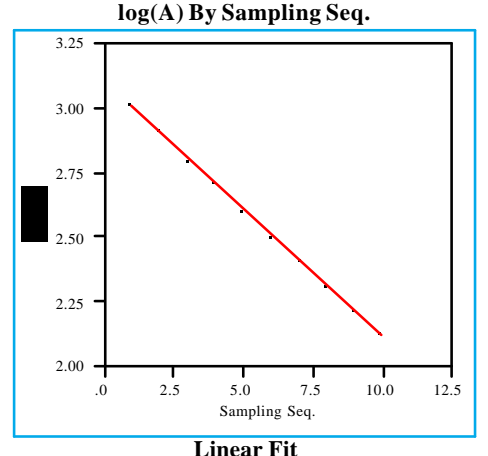

$\log (\mathrm{A})=3.10306-0.0985$ Sampling Se

RSquare Summary of Fit

$\begin{array}{ll}\text { RSquare } & 0.999114 \\ \text { RSquare Adj } & 0.9990021\end{array}$ Root Mean Square Error $\quad 0.999003$ $\begin{array}{lr}\text { Mean of Response } & 2.561325 \\ \text { Observations (or Sum Wgts) } & 10\end{array}$$$
\begin{array}{r}
561325 \\
10
\end{array}
$$

Term Estimate Std Error t Ratio Prob $>|t|$

$\begin{array}{lrrrrr}\text { Intercept } & 3.1030596 & 0.006436 & 482.17 & <.0001 \\ \text { Sampling Seq. } & -0.098497 & 0.001037 & -94.97 & <.0001\end{array}$
Ammonia Data(Study=7 Test Sequence=3)

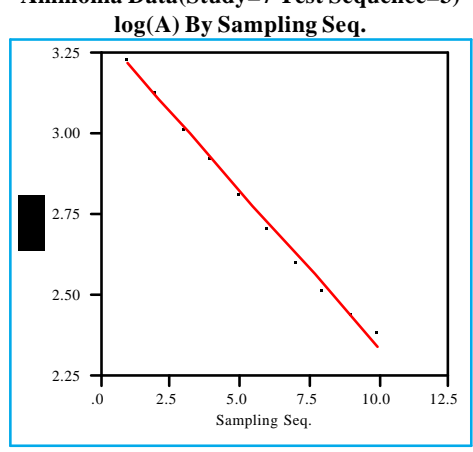

Linear Fit

$\log (\mathrm{A})=3.30663-0.09677$ Sampling Seq

\begin{tabular}{lr}
\multicolumn{2}{c}{ Summary of Fit } \\
RSquare & 0.994769 \\
RSquare Adj & 0.994115 \\
Root Mean Square Error & 0.022536 \\
Mean of Response & 2.774383 \\
Observations (or Sum Wgts) & 10
\end{tabular}

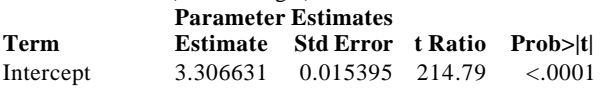

$\begin{array}{llllll}\text { tercept } & 3.306631 & 0.015395 & 214.79 & <.000\end{array}$

$\begin{array}{lllll}\text { Sampling Seq. } & -0.096772 & 0.002481 & -39.00 & <.0001\end{array}$

Ammonia Data(Study=7 Test Sequence=4)

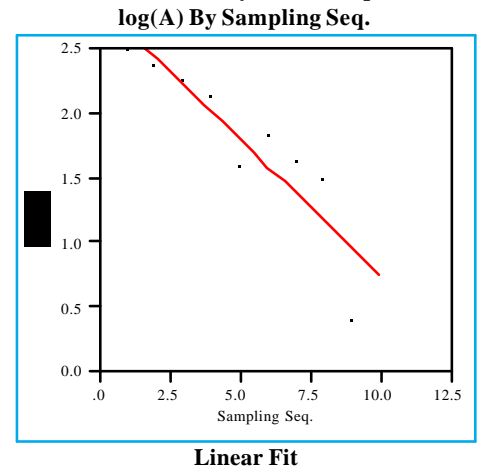

$\log (\mathrm{A})=2.84479-0.21043$ Sampling Seq

RSquare Summary of Fit

$\begin{array}{ll}\text { RSquare Adj } & 0.815104 \\ 0.78869\end{array}$

Root Mean Square Error $\quad 0.293417$

Mean of Response

Observations (or Sum Wgts)

1.79265

Parameter Estimates

$\begin{array}{lllll}\text { Term } & \text { Estimate } & \text { Std Error } & \text { t Ratio } & \text { Prob }>|t|\end{array}$ $\begin{array}{lrrrr} & 2.8447896 & 0.213162 & 13.35 & <.0001 \\ \text { Sampling Seq. } & -0.210427 & 0.03788 & -5.56 & 0.0009\end{array}$
Ammonia Data(Study=7 Test Sequence=5) $\log (\mathrm{A})$ By Sampling Seq.

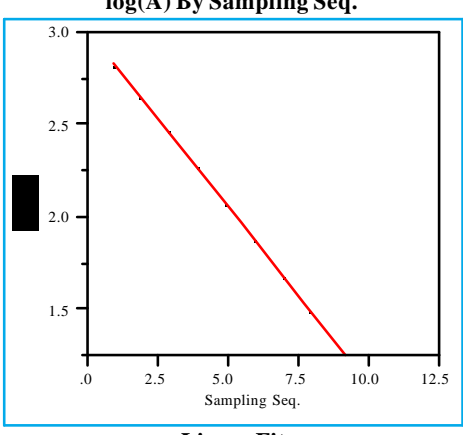

Linear Fit
$\log (\mathrm{A})=3.0165-0.19158$ Sampling Seq

RSquare
RSquare Ad

Summary of Fit

Root Mean Square Error

Mean of Response

0.999637

0.999577

2.15438

Observations (or Sum Wgts)

Parameter Estimates

Term Estimate Std Error t Ratio Prob $>|\mathbf{t}|$

$\begin{array}{lrrrr}\text { Intercept } & 3.0165036 & 0.007526 & 400.82 & <.0001 \\ \text { Sampling Seq. } & -0.191583 & 0.00149 & -128.6 & <.0001\end{array}$ 
Ammonia Data(Study=1 Test Sequence=1)

y By x

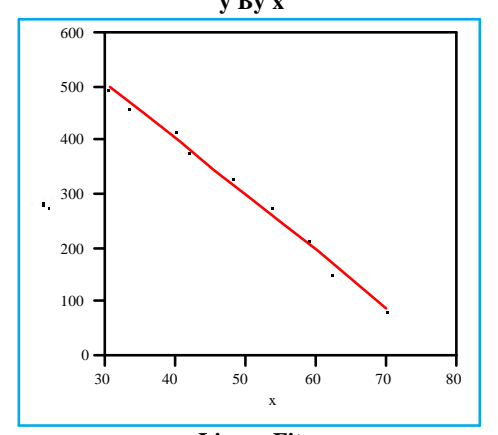

Linear Fit
$\mathrm{y}=820.974-10.438 \mathrm{x}$

RSquare

Summary of Fit

RSquare Adj

Root Mean Square Error

Mean of Response

0.991448

0.990226

3.9486

Observations (or Sum Wgts)

$\begin{array}{lrrrr} & \text { Parameter Estimates } & & \\ \text { Term } & \text { Estimate } & \text { Std Error } & \text { t Ratio } & \text { Prob }>|\mathbf{t}| \\ \text { Intercept } & 820.97412 & 18.56791 & 44.21 & <.0001 \\ \mathrm{x} & -10.43797 & 0.366405 & -28.49 & <.0001\end{array}$

Ammonia Data(Study=1 Test Sequence=2)

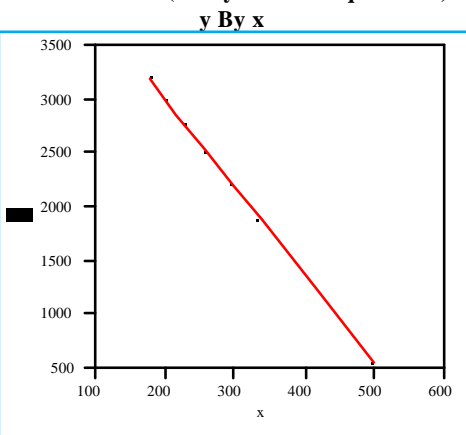

Linear Fit

$$
\begin{gathered}
\text { Linear Fit } \\
\mathrm{y}=4677.63-8.28219 \mathrm{x}
\end{gathered}
$$

RSquare

Summary of Fit

RSquare Adj

Error

0.99987

Root Mean Square

0.99985

Observations (or Sum Wgts)

11.07152

Observations (or Sum Wgts)

$\begin{array}{lrrrr}\text { Term } & \text { Estimate } & \text { Std Error } & \text { t Ratio } & \text { Prob }>|\mathbf{t}| \\ \text { Intercept } & 4677.6322 & 11.84454 & 394.92 & <.000)\end{array}$

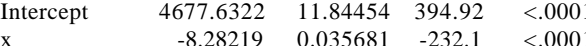

Exhibit B39: $y$ versus x for the Ammonia Studies: Study 1; Tests 1-8

Ammonia Data(Study=1 Test Sequence=3)

y By $\mathbf{x}$

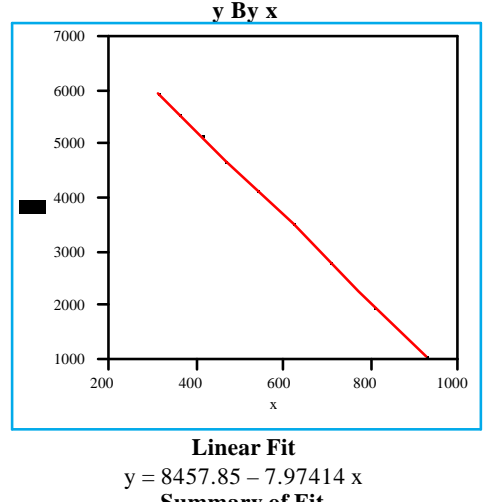

RSquare

$\begin{array}{lr}\text { RSquare } & 0.999892 \\ \text { RSquare Adj } & 0.999876 \\ \end{array}$

Root Mean Square Erro

Observations (or Sum Wgts)

3836.844

$\begin{array}{llll}\text { Term } & \text { Parameter Estimates } & & \\ \text { Estimate } & \text { Std Error t Ratio Prob }>|t|\end{array}$

$\begin{array}{lrrrr}\text { Term } & \text { Estimate } & \text { Std Error } & \text { t Ratio } & \text { Prob }>\mid \text { t } \mid \\ \text { Intercept } & 8457.8519 & 19.19619 & 440.60 & <.0001\end{array}$

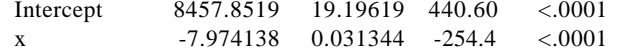

Ammonia Data(Study=1 Test Sequence=4)

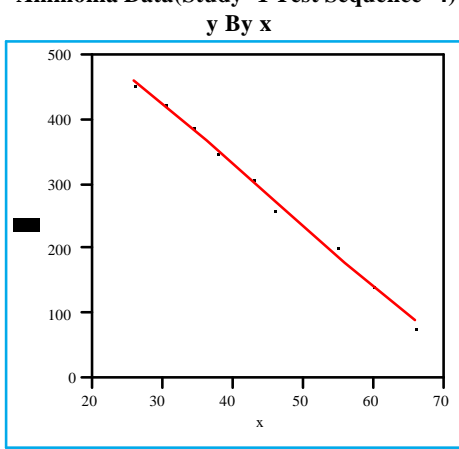

Linear Fit

$\mathrm{y}=702.605-9.32955 \mathrm{x}$

Summary of Fit

$\begin{array}{lr}\text { RSquare } & 0.99512 \\ \text { RSquare Adj } & 0.994422 \\ \text { Root Mean Square Error } & 9.58162 \\ \text { Mean of Response } & 286.7906\end{array}$

Observations (or Sum Wgts)

$\begin{array}{lrrrr}\text { Term } & \text { Estimate } & \text { Std Error } & \text { t Ratio Prob }>|t|\end{array}$

$\begin{array}{lrrrrr} & & \text { E2 } & \\ \text { Intercept } & 700464 & 11.46033 & 61.31 & <.0001 \\ \mathrm{x} & -9.329554 & 0.246946 & -37.78 & <.0001\end{array}$
Ammonia Data(Study $=1$ Test Sequence=5)

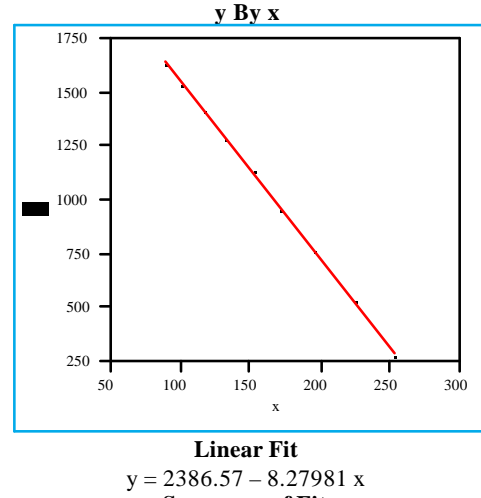

RSquare

RSquare Adj

Root Mean Square Er

Observations (or Sum Wgts)

0.999679

0.999634

Parameter Estimates

Term Estimate Std Error t Ratio Prob $>|t|$ $\begin{array}{lrrrrr}\text { Intercept } & 2386.5746 & 9.519921 & 250.69 & <.000\end{array}$

Ammonia Data(Study=1 Test Sequence=6)

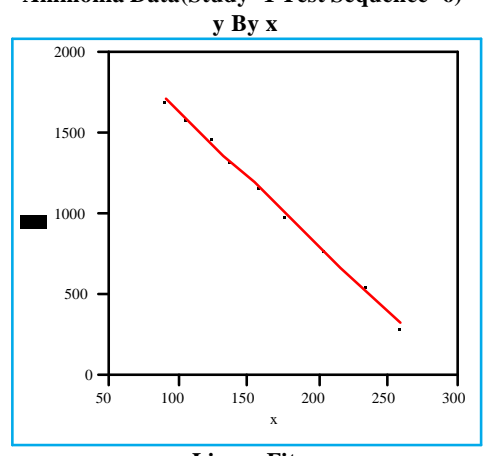

Linear F

$x=2468.28-8.3207 x$

Summary of Fit

RSquare

Root Mean Square Error

Mean of Response

Observations (or Sum Wgts)

0.998326

0.998087

21.00377

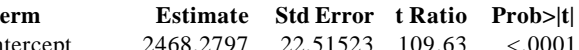

$\begin{array}{lllll} & & & \\ \mathrm{x} & 2468.2797 & 22.51523 & 109.63 & <.0001 \\ & -8.320699 & 0.128764 & -64.62 & <.0001\end{array}$
Ammonia Data(Study=1 Test Sequence=7)

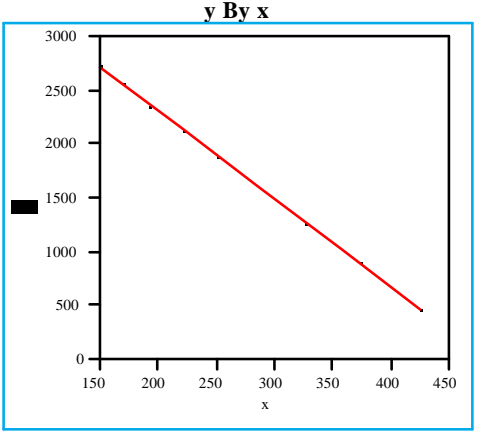

Linear Fi

$y=3968.39-8.24171 x$

RSquare

RSquare Adj

Mean Square Error

Mean of Response

(or Sum Wgts)

0.999829 0.999804 1757.294

Term $\begin{aligned} & \text { Parameter Estimates } \\ & \text { Estimate }\end{aligned}$ Std Error t Ratio Prob $>|t|$ $\begin{array}{llllll}\text { Intercept } & 3968.3916 & 11.52435 & 344.35 & <.000\end{array}$

(

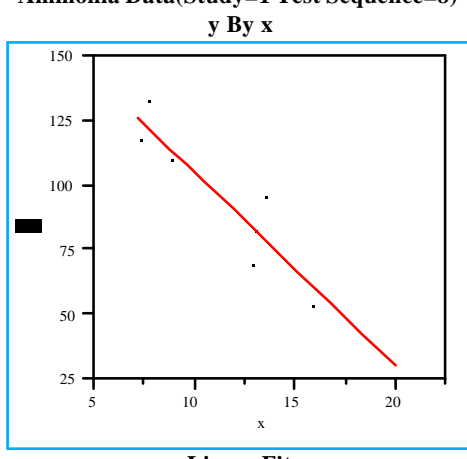

Linear Fit
$y=180.044-7.47962 x$

Summary of Fit

$\begin{array}{ll}\text { RSquare } & 0.921936 \\ \text { RSquare } & 0.910784\end{array}$

RSquare Ad $\quad 0.910784$

Mean of Response $\quad 10.20616$

Observations (or Sum Wgts)

$$
9
$$

$\begin{array}{lrrrr}\text { Term } & \text { Estimate } & \text { Std Error } & \text { t Ratio } & \text { Prob }>|\mathbf{t}| \\ \text { Intercept } & 180.04361 & 10.40049 & 17.31 & <.0001\end{array}$

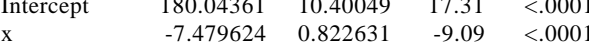


Exhibit B40: $y$ versus $x$ for the Ammonia Studies: Study 1; Tests 9-16

Ammonia Data(Study=1 Test Sequence=9) y By x

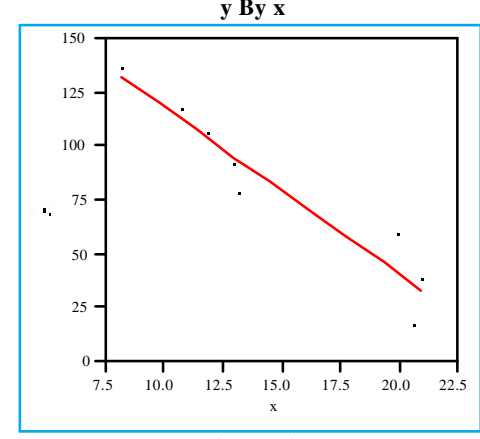

$$
\begin{aligned}
& \text { Linear Fit } \\
& \mathrm{y}=195.108-7.72844 \mathrm{x}
\end{aligned}
$$

Summary of Fit

$\begin{array}{lr} & \\ \text { RSquare } & \text { Summary of Fit } \\ \text { RSquare Adj } & 0.931209 \\ \text { Root Mean Square Error } & 11382 \\ \text { Mean of Response } & 11.5194 \\ \text { Oren } & 85.76456\end{array}$

Mean of Response

Observations (or Sum Wgts)

\begin{tabular}{lrrrr} 
& \multicolumn{2}{c}{ Parameter Estimates } & & \\
Term & Estimate & Std Error & t Ratio & Prob $>|\mathbf{t}|$ \\
Intercept & 195.10828 & 11.87092 & 16.44 & $<.0001$ \\
$\mathrm{x}$ & -7.728443 & 0.793934 & -9.73 & $<.0001$
\end{tabular}

Ammonia Data(Study=1 Test Sequence=10)

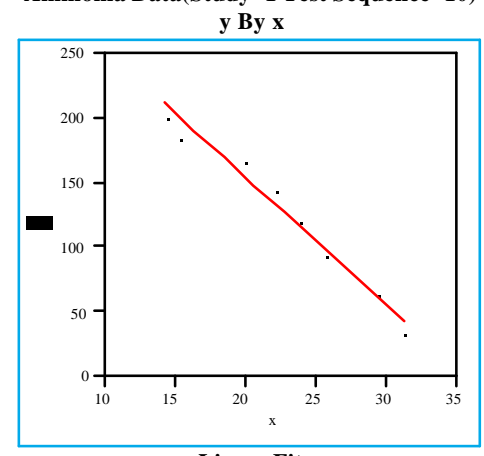

Linear Fit

$y=349.56-9.82141 x$
$\quad$ Summary of Fit

RSquare

RSquare Adj

Root Mean Square Error

Mean of Response

0.978783

Observations (or Sum Wgts)

0.975752
9.864684
133.7193

133.7193

Term Estimate Std Error t Ratio Prob $>|\mathbf{t}|$

$\begin{array}{lllrrr} & 349.5601 & 12.45311 & 28.07 & <.0001 \\ \mathrm{x} & -9.82141 & 0.546543 & -17.97 & <.0001\end{array}$
Ammonia Data(Study=1 Test Sequence=11) y By x

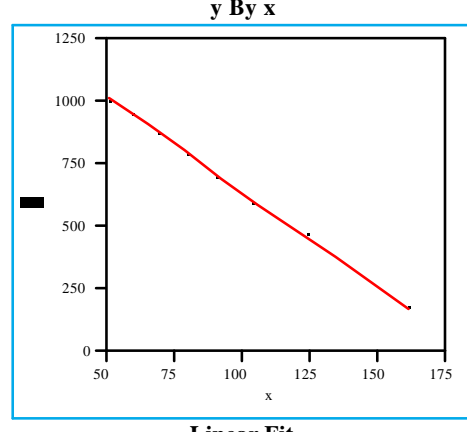

$y=1400.87-7.65422$

Summary of Fit

RSquare

Root Mean Square Error

Mean of Response

0.998608

0.998409
11.33795

649.1409

Observations (or Sum Wgts)

9

$\begin{array}{lrrrr}\text { Term } & \text { Parameter Estimates } & & \\ \text { Estimate } & \text { Std Error } & \text { t Ratio } & \text { Prob }>|\mathbf{t}|\end{array}$

$\begin{array}{lrrrr}\text { Intercept } & 1400.8711 & 11.26242 & 124.38 & <.0001 \\ \mathrm{x} & -7.654219 & 0.108026 & -70.86 & <.0001\end{array}$

Ammonia Data(Study=1 Test Sequence=12)

y By x

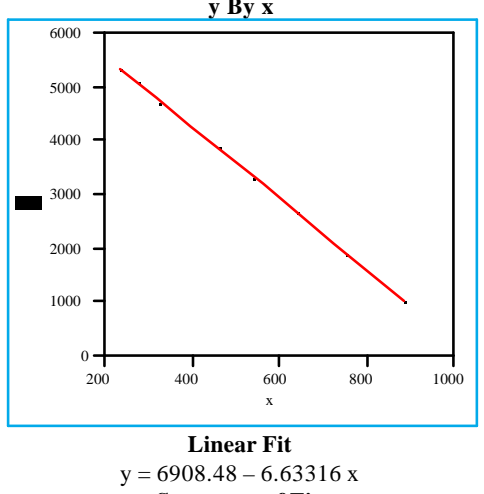

Summary of Fit

Root Mean Square Error

Mean of Response

0.999959

\begin{tabular}{lrrrr}
\multicolumn{5}{c}{ Parameter Estimates } \\
Term & Estimate & Std Error & t Ratio & Prob $>|t|$ \\
Intercept & 6908.4796 & 8.763954 & 788.28 & $<.0001$
\end{tabular}

$\begin{array}{lrrrrr}\text { Intercept } & 6908.4796 & 8.763954 & 788.28 & <.0001 \\ \mathrm{x} & -6.633157 & 0.015981 & -415.1 & <.0001\end{array}$
Ammonia Data(Study=1 Test Sequence=13) $\mathbf{y}$ By $\mathbf{x}$

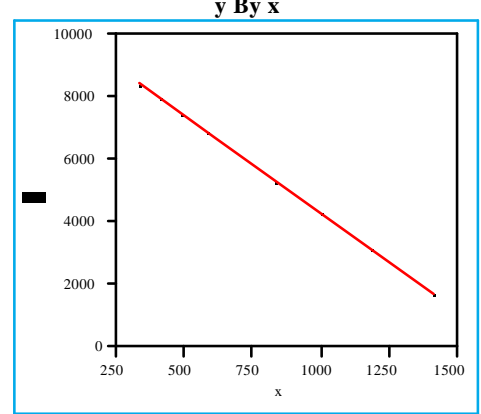

Linear Fi

$y=10456.8-6.18857$

\begin{tabular}{lr}
\multicolumn{1}{c}{ Summary of Fit } \\
RSquare & 0.999942 \\
RSquare Adj & 0.999933 \\
Root Mean Square Error & 18.70056 \\
Mean of Response & 5616.27 \\
Observations (or Sum Wgts) & 9
\end{tabular}

Term Parameter Estimates

$\begin{array}{lrrrr}\text { Term } & \text { Estimate } & \text { Std Error } & \text { t Ratio } & \text { Prob }>|t| \\ \text { Intercept } & 10456.825 & 15.30939 & 683.03 & <.0001\end{array}$

$\begin{array}{llllll}\mathrm{x} & -6.188569 & 0.017877 & -346.2 & <.0001\end{array}$

Ammonia Data(Study $=1$ Test Sequence $=14$ ) y By x

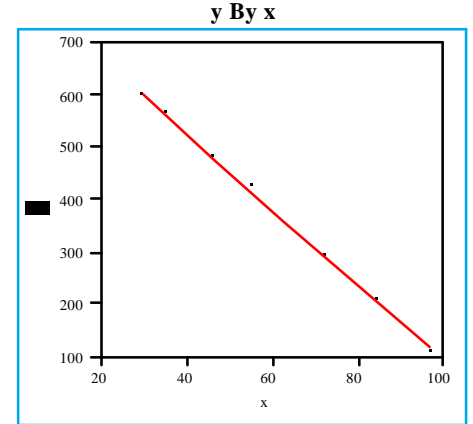

Linear Fit

$y=813.257-7.17568$

RSquare

Summary of Fit

RSquare Adj

Root Mean Square Erro

Mean of Response

Observations (or Sum Wgts)

0.998013

0.99772

7.99062

Parameter Estimates

$\begin{array}{lrrrr} & \text { Estimate } & \text { Std Error } & \text { t Ratio } & \text { Prob }>|\mathbf{t}| \\ \text { Intercept } & 813.25658 & 7.478799 & 108.74 & <.0001\end{array}$

$\begin{array}{llrrrr} & \mathrm{x} & 813.25658 & 7.478799 & 108.74 & <.0001 \\ \mathrm{I} & -7.175683 & 0.121018 & -59.29 & <.0001\end{array}$
Ammonia Data(Study=1 Test Sequence=15 y By $\mathbf{x}$

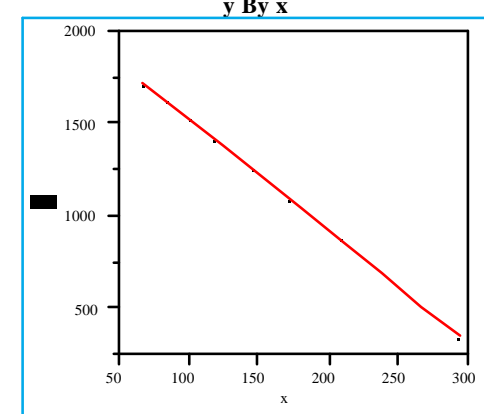

\section{Linear Fit}

$y=2137.69-6.11188$

$\begin{array}{ll}\text { RSquare } & 0.999648 \\ \text { RSquare } & 0.999597\end{array}$

$\begin{array}{ll}\text { RSquare Adj } & 0.999597 \\ \text { Root Mean Square Error } & 9.455191\end{array}$

\begin{tabular}{l}
0.455191 \\
\hline
\end{tabular}

Observations (or Sum Wgts)

Term $\quad \begin{array}{lll}\text { Parameter Estimates } & & \\ \text { Estimate } & \text { Std Error } & \text { t Ratio Prob }>|t|\end{array}$

$\begin{array}{lrrrr}\text { Term } & \text { Estimate } & \text { Std Error } & \text { t Ratio } & \text { Prob }>|t| \\ \text { Intercept } & 2137.6867 & 7.655672 & 279.23 & <.0001\end{array}$

$x \quad-6.111876 \quad 0.043361-141<000$

Ammonia Data(Study=1 Test Sequence=16)

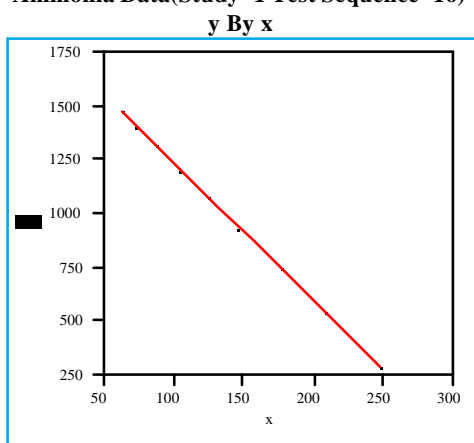

Linear Fi

$y=1869.53-6.34671 x$

Summary of Fit

RSquare Adj

Root Mean Square Error

Mean of Response

Observations (or Sum Wgts)

0.999719

0.999679

7.249283

Term Parameter Estimates

Intercept Estimate Std Error t Ratio Prob $>|\mathbf{t}|$

$\begin{array}{lrrrrr} & 1869.526 & 6.078798 & 307.55 & <.0001 \\ \mathrm{x} & -6.346708 & 0.040205 & -157.9 & <.0001\end{array}$ 
Exhibit B41: y versus x for the Ammonia Studies: Study 1; Tests 17-24

Ammonia Data(Study=1 Test Sequence=17)

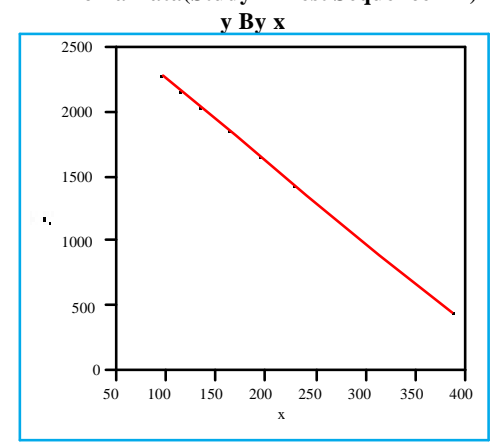

Linear Fit

$y=2888.87-6.31698$

$\begin{array}{ll}\text { RSquare } & 0.999964 \\ \text { RSquare Adj } & 0.999959 \\ \text { Root Mean Square Error } & 4.033632 \\ \text { Mean of Response } & 1529.292\end{array}$

Observations (or Sum Wgts)

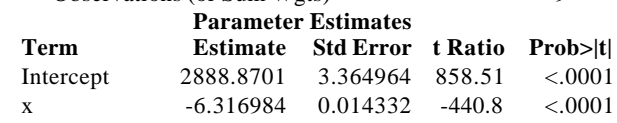

$\mathrm{x}=0.316984 \quad 0.014332-440.8<0001$

Ammonia Data(Study=1 Test Sequence=18) y By x

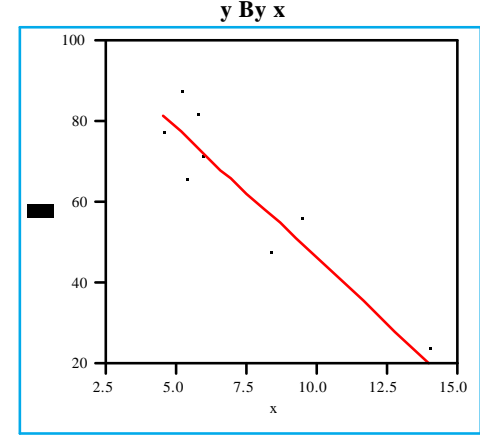

\section{Linear Fit}

$y=110.456-6.4377 x$

RSquare

Summary of Fit

RSquare Adj

Root Mean Square Error

Mean of Response

0.863186

0.843641

8.478532
60.85

Observations (or Sum Wgts)

9

Parameter Estimates

$\begin{array}{lrrrr}\text { Term } & \text { Estimate } & \text { Std Error } & \text { t Ratio } & \text { Prob>|t| } \\ \text { Intercept } & 110.45603 & 7.981578 & 13.84 & <.0001\end{array}$

Intercept

$\begin{array}{llll}-6.437697 & 0.968713 & -6.65 & 0.0003\end{array}$
Ammonia Data(Study=1 Test Sequence=19) y By x

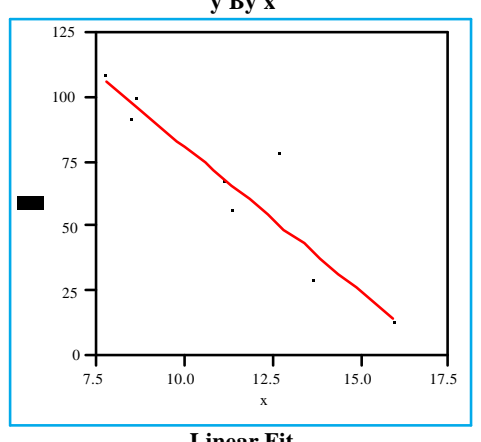
Linear Fit
$\mathrm{y}=193.073-11.1887$ $=193.073-11.1887 \times$
Summary of Fit

RSquare

RSquare Adj

Root Mean Square Erro

Mean of Response

(Parn Wgts)

$\begin{array}{lrrrr}\text { Term } & \text { Estimate } & \text { Std Error } & \text { t Ratio } & \text { Prob }>|\mathbf{t}| \\ \text { Intercept } & 193.07341 & 18.91583 & 10.21 & <.0001\end{array}$

$\begin{array}{lrrrr} & 13.07346 & 18.91583 & 10.21 & <.0001 \\ & -11.18866 & 1.61059 & -6.95 & 0.0002\end{array}$

Ammonia Data(Study=1 Test Sequence=20 y By $x$

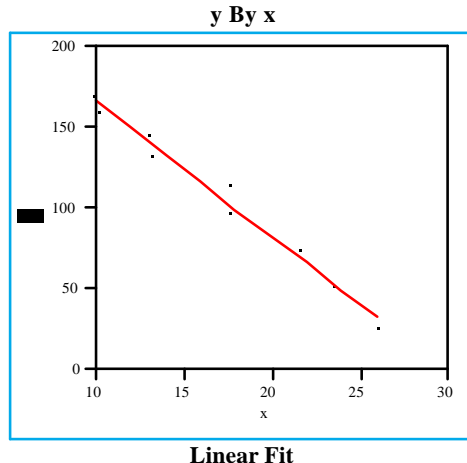

$=251.121-8.44087 x$

Summary of Fit

RSquare

RSquare Adj

Mean of Response

Observations (or Sum Wgts)

0.980959

0.978239
7.28068

107.2204

Term $\quad \begin{array}{lll}\text { Parameter Estimates } \\ \text { Estimate }\end{array}$ Std Error t Ratio Prob $>|t|$

$\begin{array}{lrrrrr}\text { Intercept } & 251.12132 & 7.956791 & 31.56 & <.0001 \\ \mathrm{X} & -8.440869 & 0.444486 & -18.99 & <.0001\end{array}$
Ammonia Data(Study=1 Test Sequence=21)

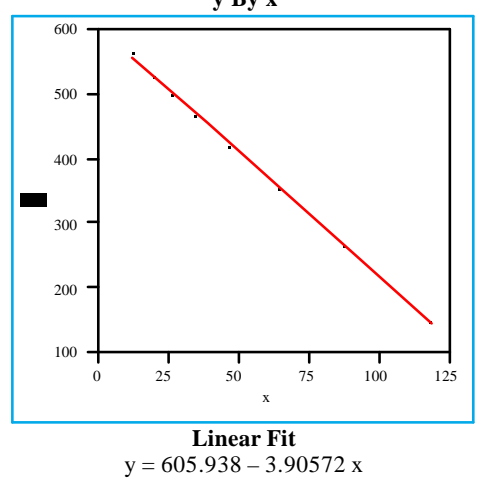

$=605.938-3.90572 \times$

$\begin{array}{ll}\text { RSquare } & \text { Summary of Fit } \\ \text { RSquare Adj } & 0.999084 \\ \text { Root Mean Square Error } & 0.998953 \\ \text { Mean of Response } & 4.570079 \\ \text { Ore } & 419.4402\end{array}$

Servations (or Sum Wgts) \begin{tabular}{lrrrr} 
& \multicolumn{1}{c}{ Parameter Estimates } & & \\
Term & Estimate & Std Error & t Ratio & Prob $>|\mathbf{t}|$ \\
Intercept & 605.93837 & 2.622068 & 231.09 & $<.0001$ \\
& -3.90572 & 0.044694 & -87.39 & $<.0001$
\end{tabular}

Ammonia Data(Study=1 Test Sequence $=22$ )

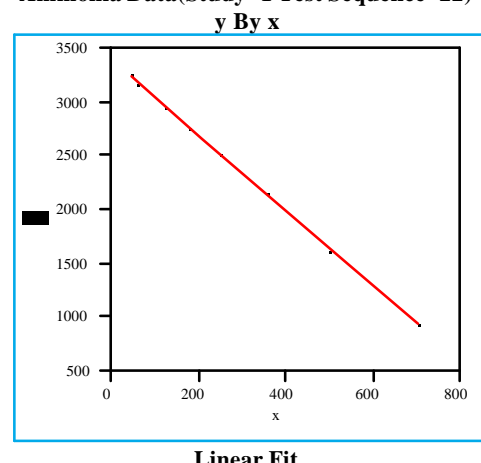

Linear Fit

$y=3408.42-3.51937 x$

RSquare

RSquare Adj

Mean of Response

0.999941 Observations (or

2479.74

Parameter Estimates

Term Estimate Std Error t Ratio Prob $>|t|$

$\begin{array}{lllll} & & & \\ \text { Intercept } & 3408.4217 & 3.442173 & 990.19 & <.0001 \\ \mathrm{x} & -3.519371 & 0.010178 & -345.8 & <.0001\end{array}$
Ammonia Data(Study=1 Test Sequence=23)

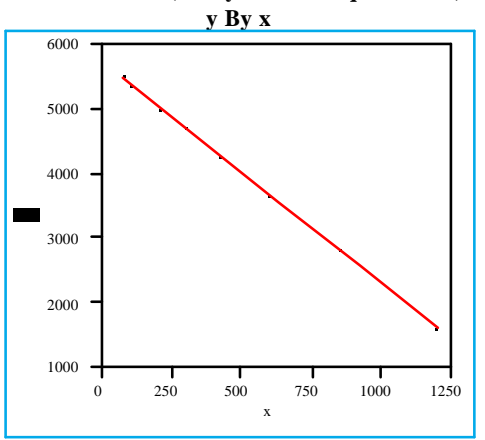

\section{Linear Fit}

$y=5748.93-3.45295 x$

RSquare $\quad 0.999966$

$\begin{array}{ll}\text { RSquare Adj } & 0.999961 \\ \text { Root Mean Square Error } & 8.259446\end{array}$

$\begin{array}{r}0.259446 \\ \hline\end{array}$

Observations (or Sum Wgts)

Term $\quad \begin{array}{lll}\text { Parameter Estimates } \\ \text { Estimate Std Error t Ratio Prob }>|t|\end{array}$

$\begin{array}{lrrrr}\text { Term } & \text { Estimate } & \text { Std Error } & \text { t Ratio } & \text { Prob }>\mid \text { t } \mid \\ \text { Intercept } & 5748.934 & 4.347931 & 1322.2 & <.0001 \\ \mathrm{x} & -3.452947 & 0.007637 & -452.1 & <.0001\end{array}$

Ammonia Data(Study=1 Test Sequence=24)

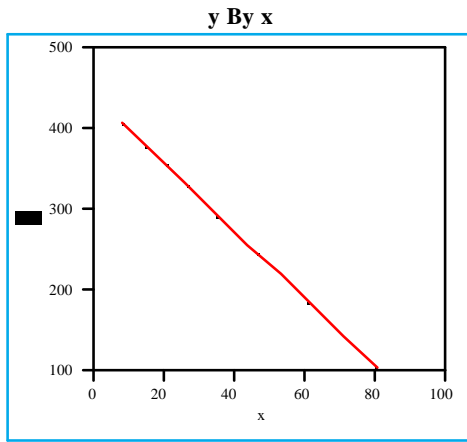

$y=441.399-4.20402 x$

RSquare

0.999731

0.999693

1.802911
296.5317

Observations (or Sum Wgts)

Parameter Estimates

Term Estimate Std Error t Ratio Prob $>\mid \mathbf{t}$

$\begin{array}{lrrrrr}\text { Intercept } & 441.39887 & 1.080616 & 408.47 & <.0001\end{array}$

$\begin{array}{lllll}\text { x } & -4.204018 & 0.026062 & -161.3 & <.0001\end{array}$ 
Exhibit B42: y versus $x$ for the Ammonia Studies: Study 1; Tests 25-32

Ammonia Data(Study=1 Test Sequence=25)

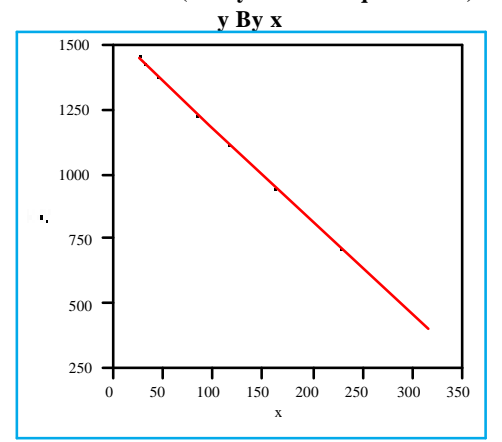

Linear Fit

$y=1548.9-3.64293 x$

$\begin{array}{ll}\text { RSquare } & 0.999697 \\ \text { RSquare Adj } & 0.999654 \\ \text { Root Mean Square Error } & 6.704102 \\ \text { Mean of Response } & 1108.109\end{array}$

Observations (or Sum Wgts)

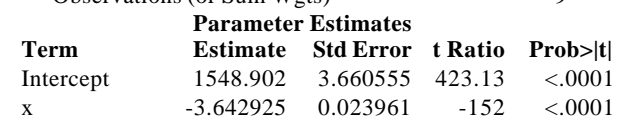

Ammonia Data(Study=1 Test Sequence=26)

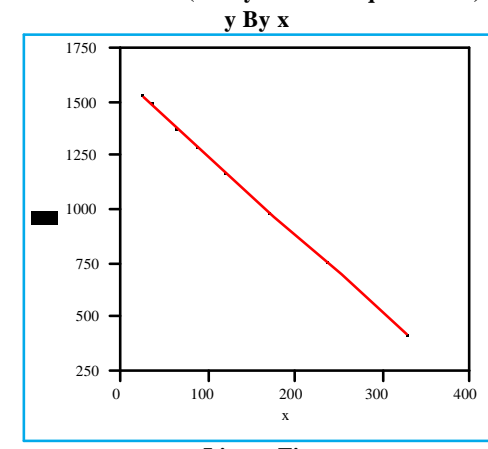

Linear Fit

$y=1618.41-3.62899 x$

RSquare

RSquare Adj

Root Mean Square Error

Mean of Response
Observations (or Su

0.999574

0.999513

8.27301

1161.313
9

Parameter Estimates

$\begin{array}{lrrrr} & \text { Parameter Estimates } & & \\ \text { Term } & \text { Estimate } & \text { Std Error } & \text { t Ratio } & \text { Prob }>|\mathbf{t}| \\ \text { Intercept } & 1618.4148 & 4.50935 & 358.90 & <.0001\end{array}$

Intercept

$\begin{array}{llll}-3.628985 & 0.028326 & -128.1 & <.0001\end{array}$
Ammonia Data(Study=1 Test Sequence=27)

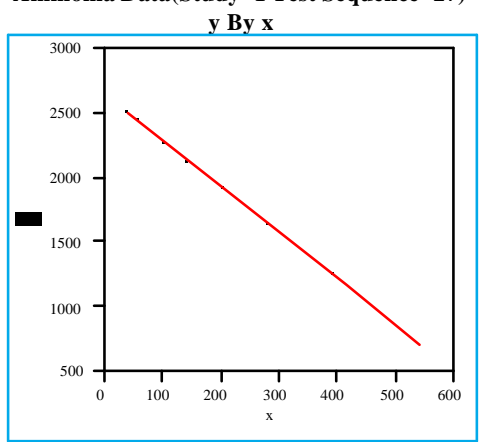

\section{Linear Fit}

$y=2646.19-3.55503$

RSquare Summary of Fit $\quad 0.999905$

$\begin{array}{ll}\text { RSquare Adj } & 0.999891 \\ \text { Root Mean Square Error } & 6.394006\end{array}$

Reon of Response $\quad 6.394006$

Observations (or Sum Wgts)

$\begin{array}{lrrrr} & \text { Parameter Estimates } & & \\ \text { Term } & \text { Estimate } & \text { Std Error } & \text { t Ratio } & \text { Prob }>|t|\end{array}$

$\begin{array}{lrrrr}\text { Intercept } & 2646.1915 & 3.429727 & 771.55 & <.0001\end{array}$

Ammonia Data(Study=1 Test Sequence=28)

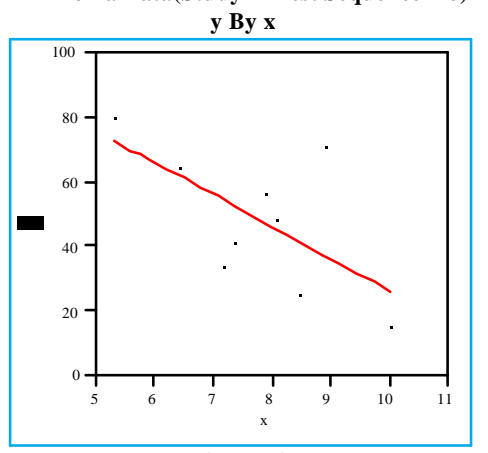

$y=125.331-9.92048$

RSquare

$\begin{array}{ll} & 0.403505 \\ \text { RSquare Adj } & 0.318291\end{array}$

Root Mean Square Error $\quad 17.82429$

Mean of Response

48.15611
9

Parameter Estimates
Pabservations (or Sum

$\begin{array}{llrrr}\text { Term } & \text { Estimate } & \text { Std Error } & \text { t Ratio } & \text { Prob }>\mid \text { t } \mid \\ \text { Intercept } & 125.3308 & 35.95964 & 3.49 & 0.0102\end{array}$

$\begin{array}{lrrrr}\mathrm{x} & -9.920476 & 4.558927 & -2.18 & 0.0660\end{array}$
Ammonia Data(Study=1 Test Sequence=29)

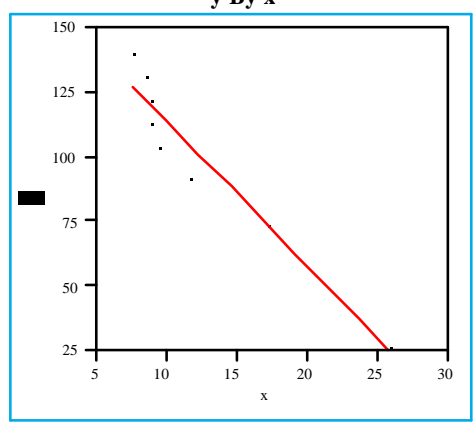

$$
\begin{aligned}
& \text { Linear Fit } \\
& \mathrm{y}=168.567-5.53553 \mathrm{x}
\end{aligned}
$$

Summary of Fit

\section{RSquare}

RSquare Adj

Root Mean Square Error

Oban or Response

(or Sum Wgts)

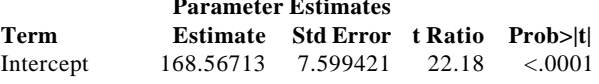
$\begin{array}{llllll} & 168.56713 & 7.599421 & 22.18 & <.0001\end{array}$

Ammonia Data(Study=1 Test Sequence=30)

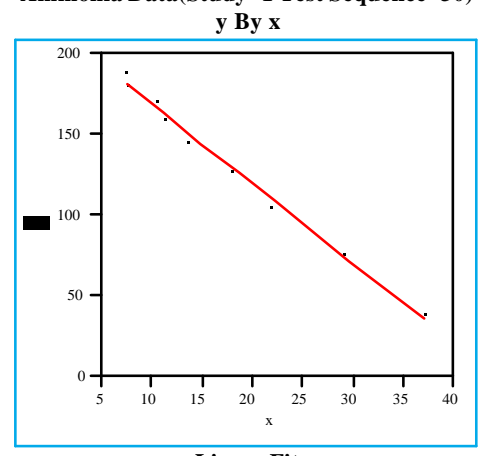

Linear Fit

$y=218.359-4.93163 x$

RSquare

RSquare Adj

Mean of Response

0.991626 Observations (or

Parameter Estimates

$\begin{array}{lrrrr} & \text { Estimate } & \text { Std Error } & \text { t Ratio } & \text { Prob }>|\mathbf{t}| \\ \text { Intercept } & 218.3592 & 3.438975 & 63.50 & <.0001\end{array}$

$\begin{array}{lllrr}\mathrm{x} & -4.93163 & 0.171294 & -28.79 & <.0001\end{array}$
Ammonia Data(Study=1 Test Sequence=31) y By $\mathbf{x}$

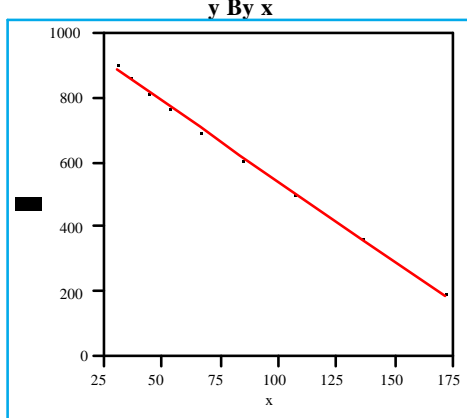

\section{Linear Fit}

$y=1036.86-4.96241 x$

RSquare $\quad 0.998779$

$\begin{array}{ll}\text { RSquare Adj } & 0.998604 \\ \text { Root Mean Square Error } & 8.974901\end{array}$

$\begin{array}{r}0.974901 \\ \hline\end{array}$

Observations (or Sum Wgts)

$\begin{array}{lrrrrr}\text { Term } & \text { Parameter Estimates } & & \\ \text { Intercept } & 1036.8551 & \text { Std Error } & \text { t Ratio } & \text { Prob }>|\mathbf{t}| \\ \text { Intimate } & 6.156496 & 168.42 & <.0001\end{array}$

$\begin{array}{lrrrr} & & & \\ \mathrm{I} & -4.96241 & 0.065586 & -75.66 & <.0001\end{array}$

Ammonia Data(Study=1 Test Sequence=32)

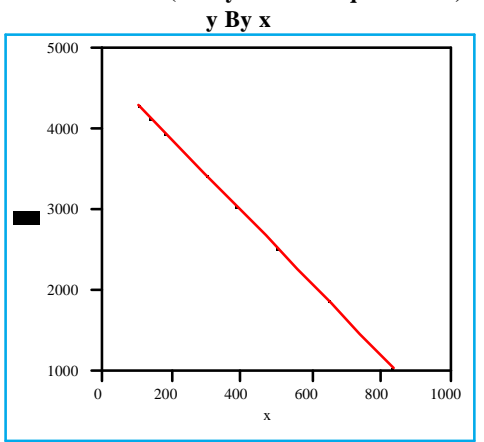

$y=4762.93-4.46917 x$

$\begin{array}{ll}\text { RSquare } & 0.999966 \\ \text { RSquare Adj } & 0.999962\end{array}$

Root Mean Square Error $\quad 6.883967$

$\begin{array}{lr}\text { Mean of Response } & 3090.504 \\ \text { Observations (or Sum Wgts) } & 9\end{array}$

Observations (orameter Estimates

Term Estimate Std Error t Ratio Prob $>|t|$

$\begin{array}{lrrrr}\text { Intercept } & 4762.9283 & 4.324144 & 1101.5 & <.0001\end{array}$

$\begin{array}{lllll}\mathrm{x} & -4.469175 & 0.009794 & -456.3 & <.0001\end{array}$ 
Ammonia Data(Study=1 Test Sequence=33) y By x

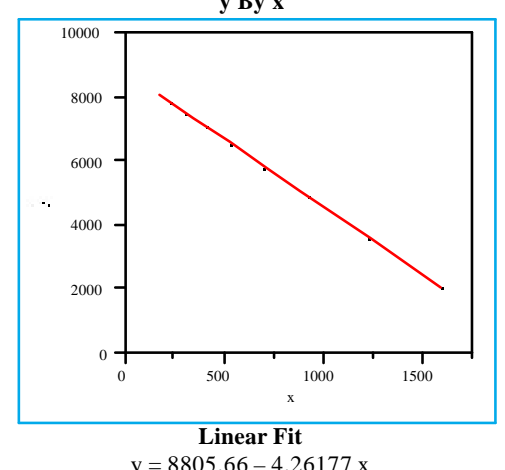

$=8805.66-4.26177$

RSquare

Summary of Fit

RSquare Adj

Root Mean Square Erro

0.999965

Mean of Response

0.99996

(or Sum Wgts)

5859.886

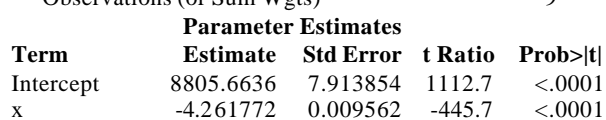

Ammonia Data(Study=1 Test Sequence=34) y By $\mathbf{x}$

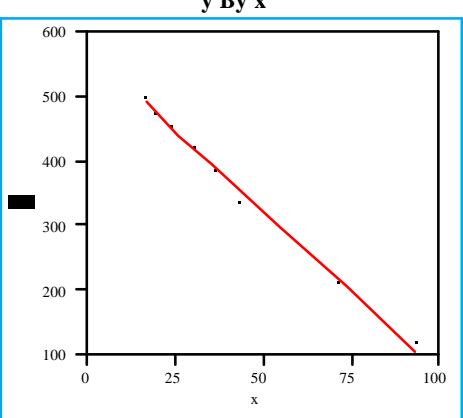

Linear Fit

$=572.029-4.98034$

RSquare

RSquare Adj

Root Mean Square Erro

Mean of Response

0.99574

0.995131

352.8277

Parameter Estimates

$\begin{array}{lrrrr}\text { Term } & \text { Estimate } & \text { Std Error } & \text { t Ratio } & \text { Prob }>|t|\end{array}$

$\begin{array}{lrrrrr}\text { Intercept } & 572.02942 & 6.186299 & 92.47 & <.0001\end{array}$

Exhibit B43: y versus x for the Ammonia Studies: Study 1; Tests 33-40

Ammonia Data(Study=1 Test Sequence=35)

y By $\mathbf{x}$

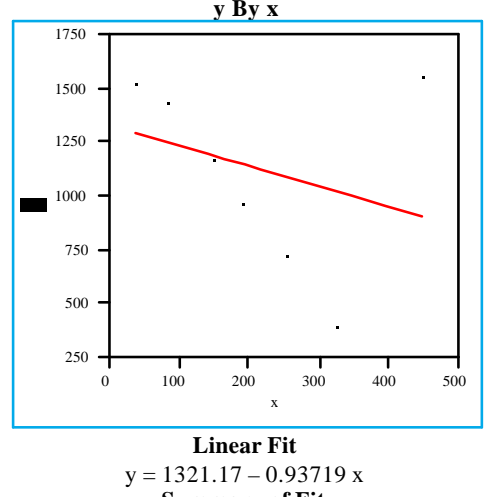

$=1321.17-0.93719$

RSquare

RSquare Adj

Root Mean Square Erro

Mean of Response

0.09366

$-0.0574$

Servations (or Sum Wgts)

1131.095

$\begin{array}{llll}\text { Term } & \text { Parameter Estimates } & & \\ \text { Estimate } & \text { Std Error t Ratio } & \text { Prob }>|t|\end{array}$

$\begin{array}{lrrrr}\text { Term } & \text { Estimate } & \text { Std Error } & \text { t Ratio } & \text { Prob }>|t| \\ \text { Intercept } & 1321.1729 & 284.8868 & 4.64 & 0.0035\end{array}$

$\begin{array}{lllll}\mathrm{N} & -1321.1729 & 284.8868 & 4.64 & 0.0035 \\ & -0.937186 & 1.190198 & -0.79 & 0.4610\end{array}$

Ammonia Data(Study=1 Test Sequence=36)

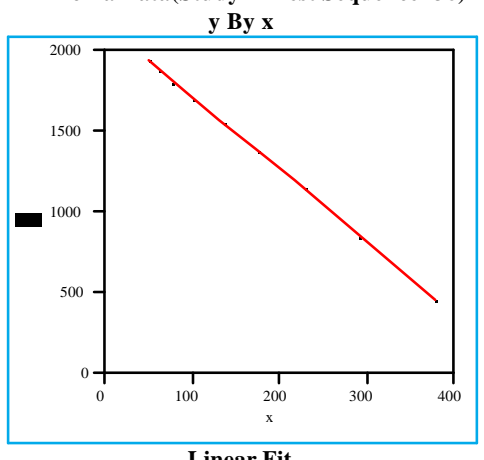

Linear Fit

$y=2160.76-4.47606$

$\begin{array}{ll}\text { RSquare } & 0.999833 \\ \text { RSquare Adj } & 0.999809\end{array}$

Summary of Fit

Root Mean Square Error $\quad 6.988227$

Mean of Response

6.988227
1397.514
9

Observations (or Sum Wgts)

Parameter Estimates

Term Estimate Std Error t Ratio Prob $>|t|$

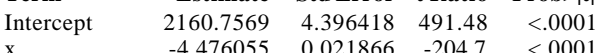

Ammonia Data(Study=1 Test Sequence=37)

$\mathbf{y}$ By $\mathbf{x}$

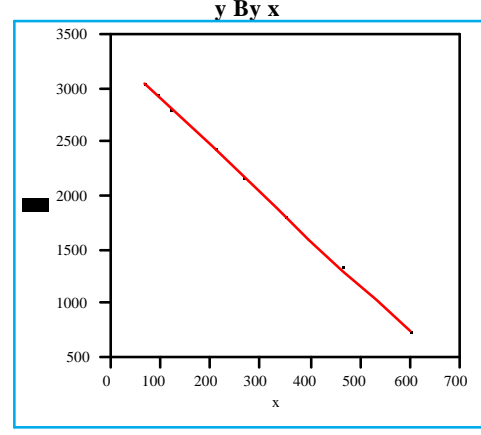

Linear Fit

$y=3350.2-4.36324 x$

RSquare

0.999929

RSquare Adj $\quad 0.999919$

Meor Mean Sque Eror

2199.18

Observations (or Sum Wgts) \begin{tabular}{lrrrr}
\multicolumn{5}{c}{ Parameter Estimates } \\
Term & Estimate & Std Error & t Ratio & Prob $>|\mathbf{t}|$ \\
Intercept & 3350.1973 & 4.343514 & 771.31 & $<.0001$ \\
$\mathrm{x}$ & -4.363237 & 0.013855 & -314.9 & $<.0001$
\end{tabular}

Ammonia Data(Study=1 Test Sequence=38) y By x

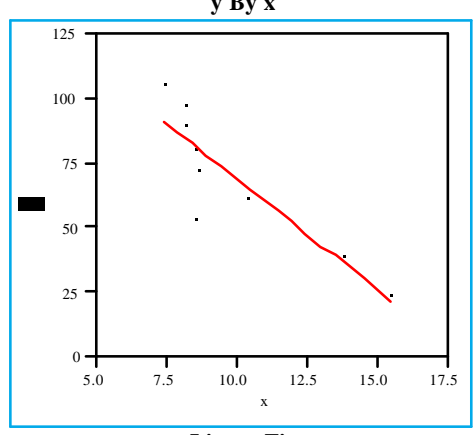

Linear Fit

$\mathrm{y}=154.305-8.57298 \mathrm{x}$

Summary of Fit

RSquare

RSquare Adj

Root Mean Square Error

Observations (or Sum Wgts)

0.787446

0.75708

68.9598

Parameter Estimates

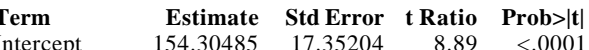

$\begin{array}{rrrr}154.30485 & 17.35204 & 8.89 & <.000 \\ -8.572979 & 1.683476 & -5.09 & 0.0014\end{array}$
Ammonia Data(Study=1 Test Sequence=39) $\mathbf{y}$ By $\mathbf{x}$

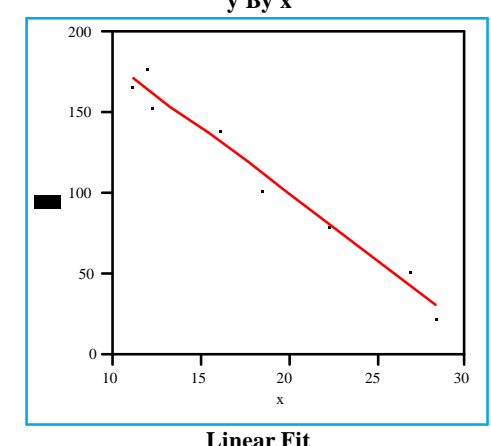

Linear Fit

$y=263.43-8.2043$

RSquare

RSquare Adj

Root Mean Square Error

Mean of Response

Observations (or Sum Wgts)

Term Estimate Std Error t Ratio Prob $>|\mathbf{t}|$

$\begin{array}{lllll}\text { Intercept } & 263.42999 & 9.236463 & 28.52 & <.0001 \\ \mathrm{x} & -8.204305 & 0.475491 & -17.25 & <.000\end{array}$

Ammonia Data(Study=1 Test Sequence $=40$ ) y By x

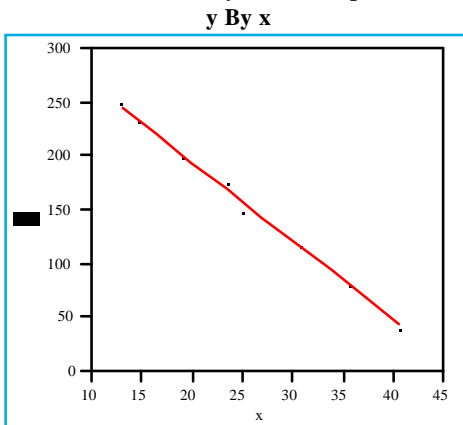

\section{Linear Fit}

$y=340.171-7.32688 x$

Summary of Fit

RSquare

RSquare Adj

Error

0.996567

Root Mean Square Error

Mean of Response

0.996077
4.443788

Observations (or Sum Wgts)

160.4823

Parameter Estimates

Term Estimate Std Error t Ratio Prob $>|t|$

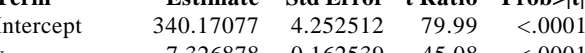

$\begin{array}{lllll}\mathrm{x} & -7.326878 & 0.162539 & -45.08<.000 & \end{array}$ 
Ammonia Data(Study=1 Test Sequence $=41$ )

By $x$

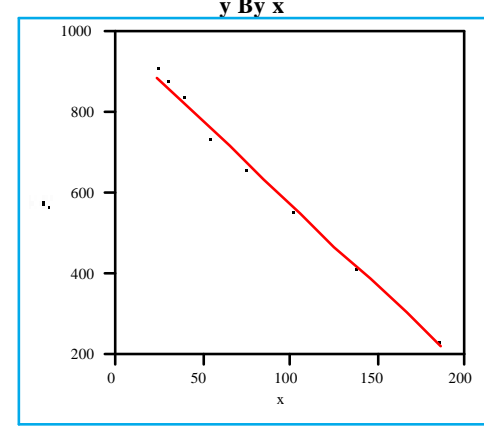

Linear Fit

$y=990.132-4.14381 x$

RSquare

Summary of Fit

RSquare Adj

Rquare Error

Mean of Response

0.994251
0.99343
18.53629

665.3419

Observations (or Sum Wgts)

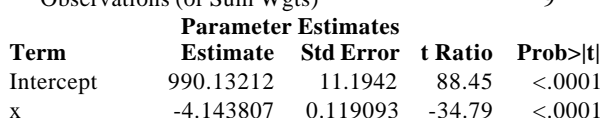

Ammonia Data $($ Study $=1$ Test Sequence $=42$ )

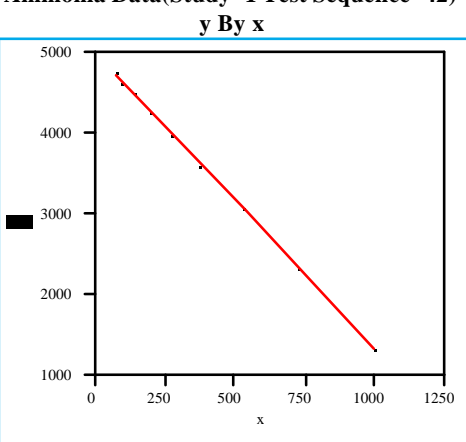

Linear Fit

$y=5003.46-3.66816 x$

RSquare

Summary of Fit

RSquare Adj

Root Mean Square Error

0.999953

Observations (or Sum Wgts)

0.999947

8.47018

Parameter Estimates

$\begin{array}{lrrrr}\text { Term Estimate } & \text { Std Error } & \text { t Ratio } & \text { Prob }>|t|\end{array}$

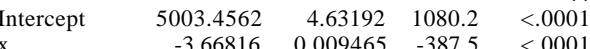

Exhibit B44: y versus x for the Ammonia Studies: Study 1; Tests 41-48

Ammonia Data(Study $=1$ Test Sequence $=43$ )

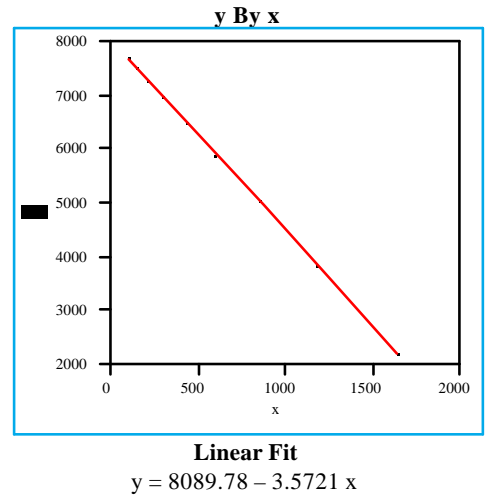

RSquare $=8089.78-3.5721 \times$

RSquare Adj $\quad 0.999987$

Mean of Response

0.999985

5863.245

ervations (or Sum Wgts)

Term Parameter Estimates $\quad$ Estimate Std Error t Ratio Prob $>\mid 4$

$\begin{array}{lrrrrr} & \text { Intercept } & 8089.7789 & 3.839369 & 2107.1 & <.000 \\ & -3.572104 & 0.004833 & -739 & <.000\end{array}$

Ammonia Data(Study=1 Test Sequence $=44$ ) y By x

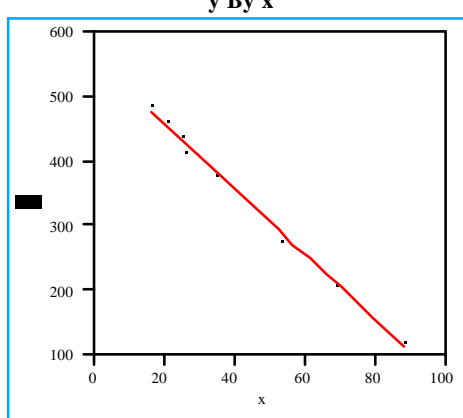

Linear Fit

$y=562.518-5.1137 x$

Summary of Fit

RSquare $\quad 0.993736$

RSquare Adj 0.992841

Root Mean Square Er

Observations (or Sum Wgts)

10.46862

Parameter Estimates

Term Estimate Std Error t Ratio Prob $>\mid t$

$\begin{array}{lrrrr} & & & \\ \text { Intercept } & 562.51803 & 7.408275 & 75.93 & <.0001 \\ & -5.113698 & 0.153451 & -33.32 & <.0001\end{array}$
Ammonia Data(Study $=1$ Test Sequence $=45$ )

y By x

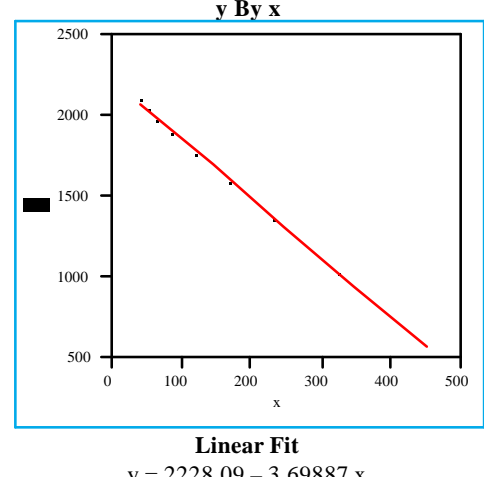

$=2228.09-3.69887 x$

RSquare

Square Adj $\quad 0.999074$

Square Error

0.99894

Mean of Response

servations (or Sum Wgts)

Term Parameter Estimates $\begin{array}{lrrrrr} & & \text { Partimate } & \text { Std Error } & & \\ \text { Intercept } & 2228.09 & 9.320405 & 239.06 & <.0001 \\ & -3.698866 & 0.042564 & -86.9 & <.001\end{array}$ $\begin{array}{lllll}-3.698866 & 0.042564 & -86.90 & <.0001\end{array}$

Ammonia Data(Study $=1$ Test Sequence $=46$ )

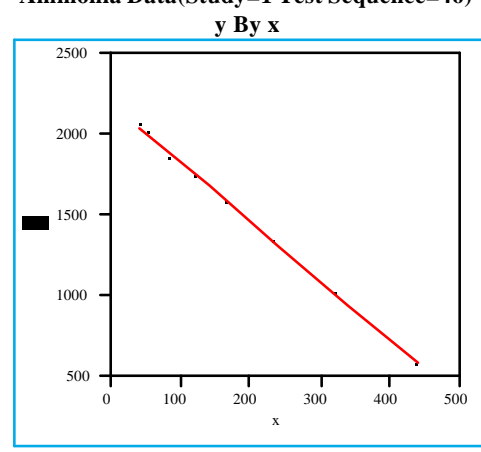

Linear Fit

$y=2197.08-3.70177 x$

RSquare

RSquare Adj

Mean of Response

Sum Wgts)

0.998844

$\begin{array}{lrrrrr} & & & \\ \mathrm{x} & 2197.0843 & 9.541192 & 230.27 & <.0001 \\ & -3.701775 & 0.04453 & -83.13 & <.0001\end{array}$
Ammonia Data(Study=1 Test Sequence $=47$ )

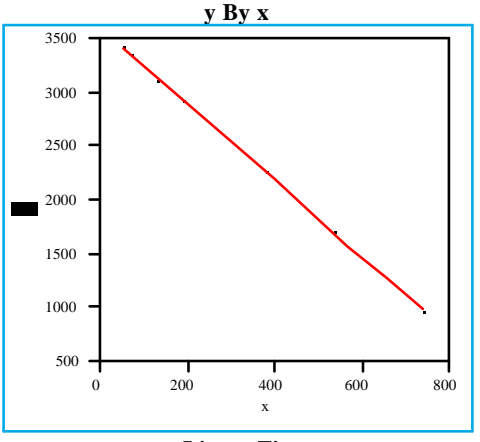

Linear Fit

$y=3607.47-3.55772 x$

RSquare

RSquare Adj
Root Mean Square Error

Mean of Response

Observations (or Sum Wgts)

0.999846 0.999824 2611.972

Parameter Estimates

Term Estimate Std Error t Ratio Prob $>|\mathbf{t}|$ $\begin{array}{llllll} & \text { Intercept } & 3607.4701 & 5.967486 & 604.52 & <.000 \\ \mathrm{x} & -3.557716 & 0.016712 & -212.9 & <.000\end{array}$

Ammonia Data(Study=1 Test Sequence=48) y By x

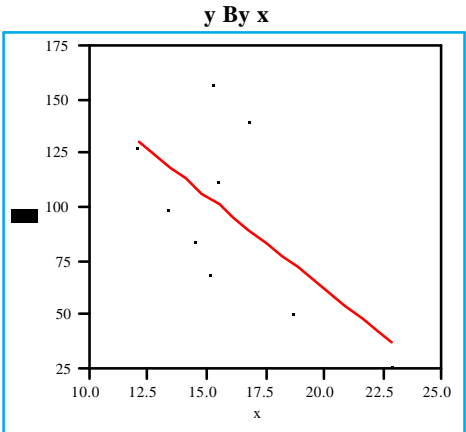

Linear Fit

$$
\begin{aligned}
& \text { Linear Fit } \\
& y=234.174-8.60321 x
\end{aligned}
$$

RSquare

Summary of Fit

RSquare Adj

Root Mean Square Error

Observations (or Sum Wgts)

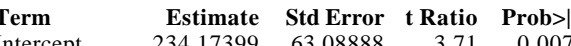

$\begin{array}{lrrrr} & 234.17399 & 63.08888 & 3.71 & 0.0075 \\ \text { Intercept } & -8.603206 & 3.849742 & -2.23 & 0.0605\end{array}$ 
Exhibit B45: y versus x for the Ammonia Studies: Study 1; Tests 49-50; Study 2: Tests 1-6

Ammonia Data(Study=1 Test Sequence=49) y By $x$

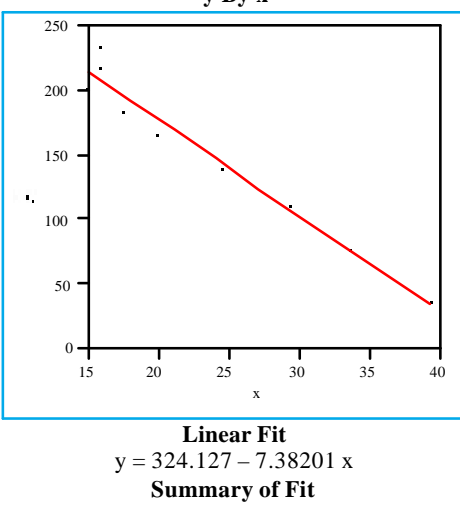

RSquare

RSquare Adj

Root Mean Square Error

Mean of Response

0.966369

0.961565

150.7721

Observations (or Sum Wgts)

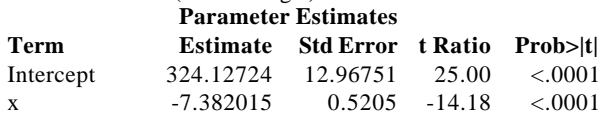

Ammonia Data(Study=1 Test Sequence $=50$ ) y By x

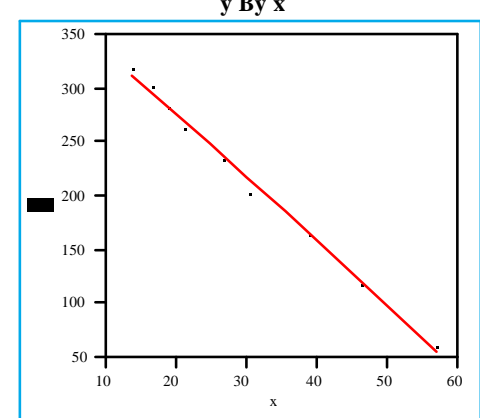

Linear Fit

$=395.344-5.94843$

Summary of Fit

RSquare

RSquare Adj

Root Mean Square Er

Observations (or Sum Wgts)

Parameter Estimates

$\begin{array}{lrrrr}\text { Term } & \text { Estimate } & \text { Std Error } & \text { t Ratio } & \text { Prob }>|\mathbf{t}|\end{array}$

$\begin{array}{lrrrrr}\text { Intercept } & 395.34374 & 5.135719 & 76.98 & <.0001 \\ \mathrm{x} & -5.948428 & 0.154034 & -38.62 & <.0001\end{array}$

0.995328

0.994661
6.412513

215.0086
Ammonia Data(Study=2 Test Sequence=1

y By $\mathbf{x}$

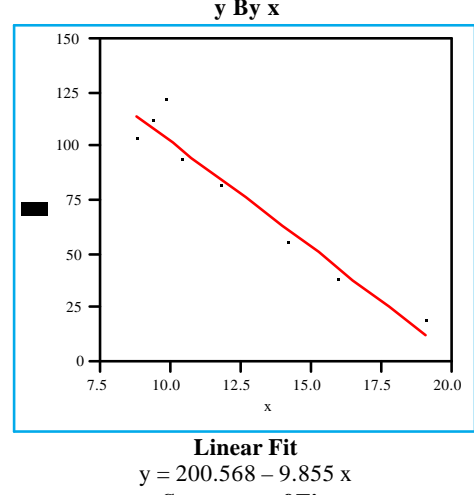

RSquare

RSquare Adj $\quad 0.933698$

Root Mean Square Error $\quad 9.575207$

Mean of Response

9.575207
77.29022

\begin{tabular}{lrrrr} 
Term & \multicolumn{2}{c}{ Parameter Estimates } & & \\
Estimate & Std Error & t Ratio & Prob $>|\mathbf{t}|$ \\
Intercept & 200.56754 & 12.82003 & 15.64 & $<.0001$
\end{tabular}

$\begin{array}{lrrrr}\text { Intercept } & 200.56754 & 12.82003 & 15.64 & <.0001 \\ \mathrm{X} & -9.855002 & 0.992585 & -9.93 & <.0001\end{array}$

Ammonia Data(Study=2 Test Sequence=2)

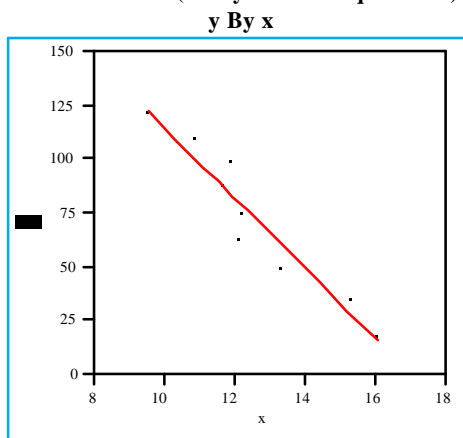

Linear Fit

$y=278.335-16.3473$

Summary of Fit

RSquare

0.919007

RSquare Adj

Square Error

0.907437
10.67179

72.87344

Observations (or Sum Wgts)

Term Estimate Std Error t Ratio Prob $>\mid \mathbf{t}$

$\begin{array}{lrrrr} & & \\ \text { Intercept } & 278.33483 & 23.32674 & 11.93 & <.0001 \\ \mathrm{x} & -16.34726 & 1.834253 & -8.91 & <.0001\end{array}$
Observations (or Sum Wgts)

Ammonia Data(Study=2 Test Sequence $=3$ )

y By $\mathbf{x}$

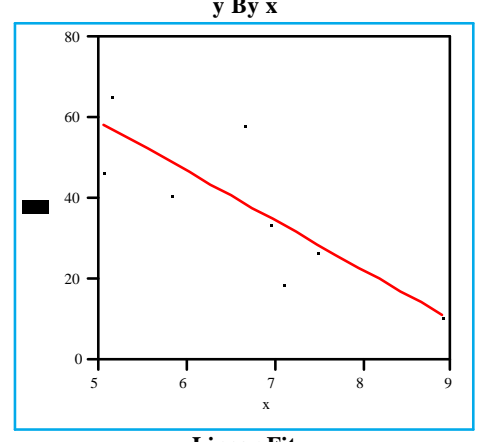

Linear Fit
$\mathrm{y}=119.812-12.2048$ Summary of Fit

RSquare

RSquare Adj

Root Mean Square Error

Mean of Response

Observations (or Sum Wgts)

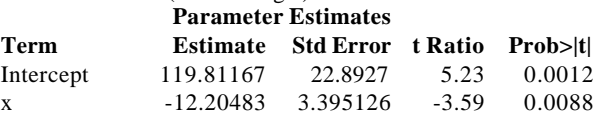

Ammonia Data(Study=2 Test Sequence=4) y By $x$

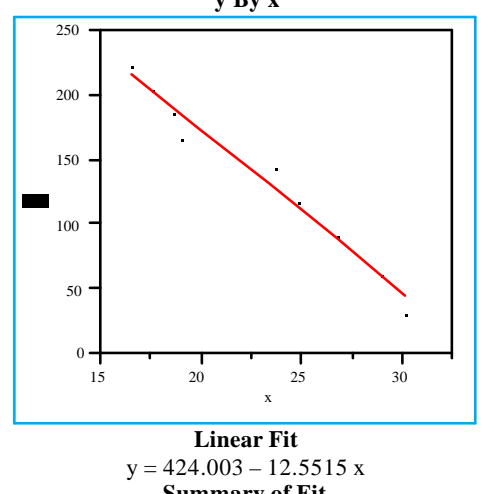

(

RSquare

RSquare Adj $\quad 0.969202$

Root Mean Square Error

Observations (or Sum Wots)

Observations (or Sum Wgts)
Term

ntercept

arameter Estimates

Estimate Std Error t Ratio Prob $>\mid t$ $\begin{array}{rrrr}24.0032 & 18.59408 & 22.80 & <.000\end{array}$
Ammonia Data(Study=2 Test Sequence=5) y By $\mathbf{x}$

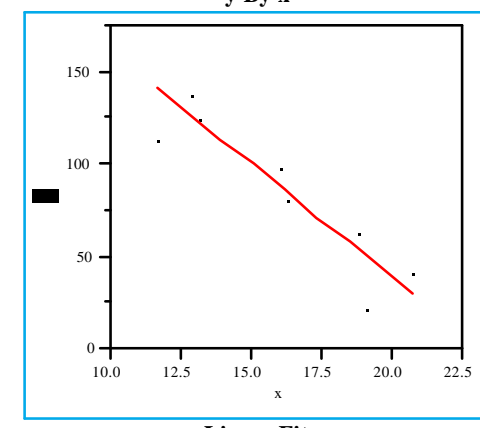

$y=284.237-12.2645 x$ Summary of Fit

RSquare

RSquare Adj

Root Mean Square Error

Mean of Response

Observations (or Sum Wgts)

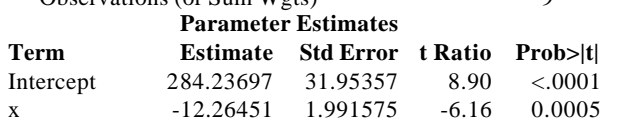

Ammonia Data(Study=2 Test Sequence $=6$ )

y By x

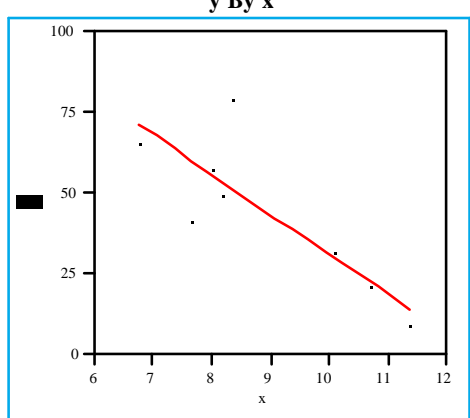

Linear Fit

$y=154.063-12.3061 x$

RSquare

RSquare Adj

Error

0.735473

oot Mean Square E

Observations (or Sum Wots)

0.697684

Parameter Estimates

$\begin{array}{lrrrr}\text { Term } & \text { Estimate } & \text { Std Error } & \text { t Ratio } & \text { Prob }>|\mathbf{t}|\end{array}$

$\begin{array}{lllll}\text { Intercept } & 154.06321 & 24.68092 & 6.24 & 0.0004 \\ \mathrm{x} & -12.30606 & 2.789466 & -4.41 & 0.003\end{array}$ 


\section{Exhibit B46: y versus x for the Ammonia Studies: Study 2: Tests 7-13; Study 3; Test 1}

Ammonia Data(Study=2 Test Sequence=7)

y By x

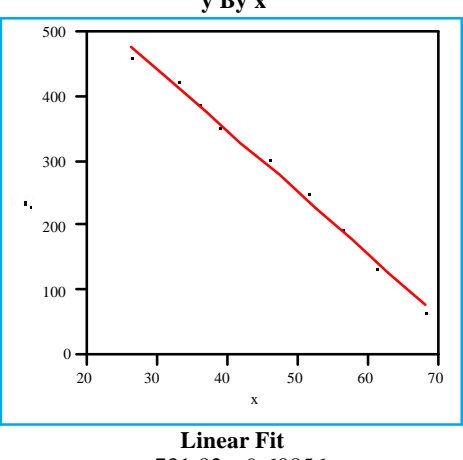

$y=731.82-9.60856 x$

RSquare

RSquare Adj

Root Mean Square Error

Mean of Response

Observations (or Sum Wgts)

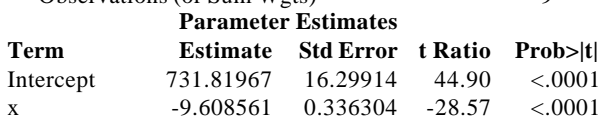

Ammonia Data(Study=2 Test Sequence=8) y By x

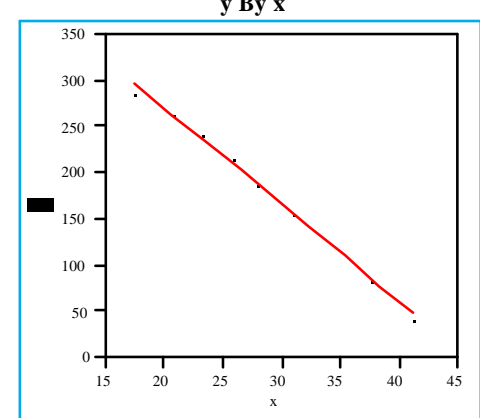

\section{Linear Fit}

$y=477.18-10.403 x$

RSquare

Summary of Fit

RSquare Adj

Root Mean Square Error

Mean of Response

0.994012

Observations (or Sum Wgs)

0.99315

Parameter Estimates

$\begin{array}{lrlrl}\text { Term } & \text { Estimate } & \text { Std Error } & \text { t Ratio } & \text { Prob }>|t|\end{array}$

$\begin{array}{llllll}\text { Intercept } & 477.18033 & 9.169576 & 52.04 & <.0001\end{array}$

$\begin{array}{llll}-10.40299 & 0.305178 & -34.09 & <.000\end{array}$
Ammonia Data(Study=2 Test Sequence=9)

y By $\mathbf{x}$

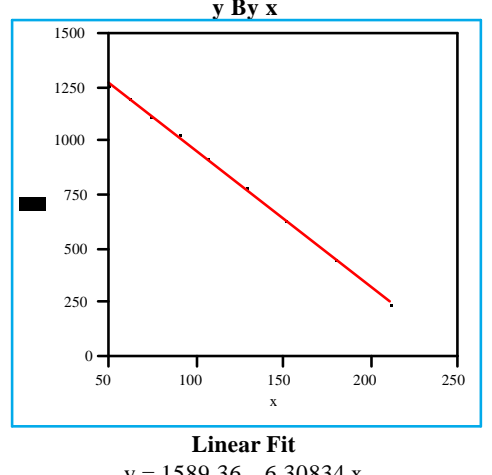

$y=1589.36-6.30834$

RSquare

ummary of Fit

$\begin{array}{lr}\text { RSquare } & 0.99895 \\ \text { RSquare Adj } & 0.9988\end{array}$

an Square Error

Mean of Response

Observations (or Sum Wgts)

12.06681

Parameter Estimates

Estimate Std Error t Ratio Prob $>\mid t$

$\begin{array}{lrrrrr}\text { Intercept } & 1589.3637 & 10.01015 & 158.78 & <.0001\end{array}$

Ammonia Data(Study=2 Test Sequence=10) y By x

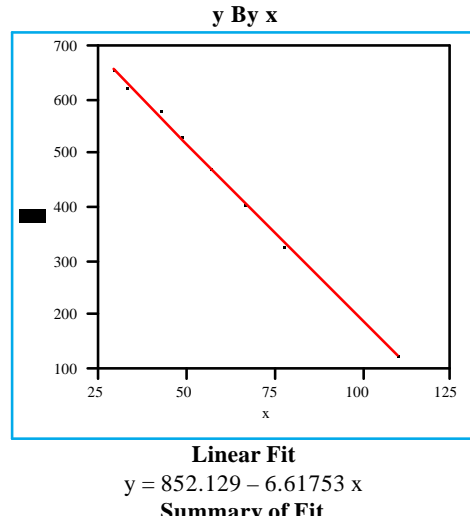

RSquare

Summary o

RSquare Adj

Root Mean Square Erro

0.998593
0.998392

7.33227

(um Wots)

Parameter Estimates

$\begin{array}{lrrrr}\text { Term } & \text { Estimate } & \text { Std Error } & \text { t Ratio } & \text { Prob }>|\mathbf{t}| \\ \text { Intercept } & 852.12924 & 6.382365 & 133.51 & <.000\end{array}$

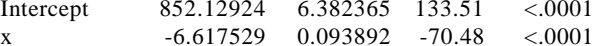

Ammonia Data(Study=2 Test Sequence=11)

$\mathbf{y}$ By $\mathbf{x}$

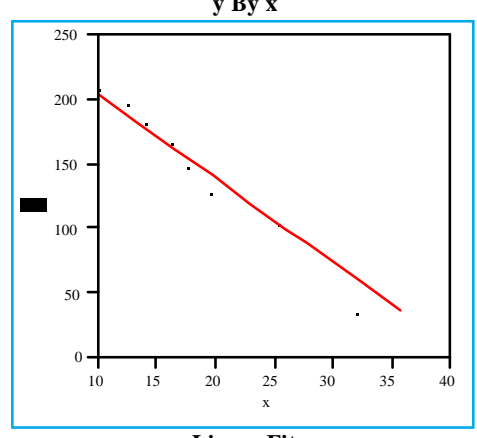

$y=268.539-6.47385$ Summary of Fit

RSquare

RSquare Adj

Meot Mean Square Err

Mean of Response

Observations (or Sum Wgts)

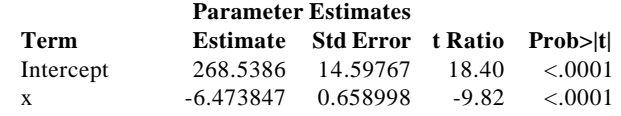

Ammonia Data(Study $=2$ Test Sequence=12) y By x

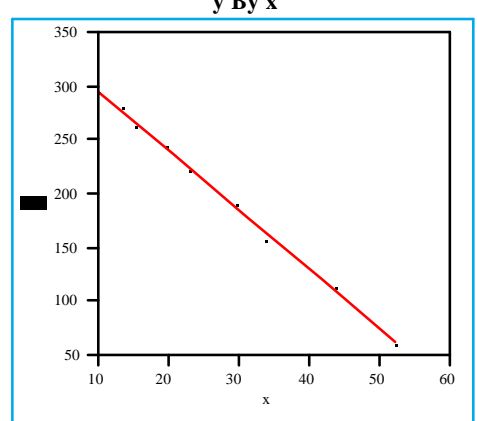

\section{Linear Fit}

$y=350.511-5.52918 x$

Summary of Fit

RSquare

RSquare Adj

Root Mean Square Erro

Observations (or Sum Wgts)

0.99774

0.997417

Parameter Estimates

Term Estimate Std Error t Ratio Prob>|t

$\begin{array}{lllll}\text { Intercept } & 350.51062 & 3.003601 & 116.70 & <.0001\end{array}$

$\begin{array}{llll}-5.529182 & 0.099462 & -55.59<0001\end{array}$
Ammonia Data(Study=2 Test Sequence=13) $\mathbf{y}$ By $\mathbf{x}$

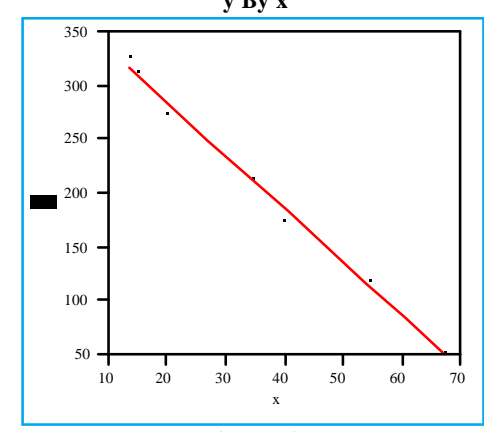

$\mathrm{y}=382.879-4.92812 \mathrm{x}$ Summary of Fit

RSquare

RSquare Adj

Root Mean Square Error

Mean of Response

Observations (or Sum Wgts)

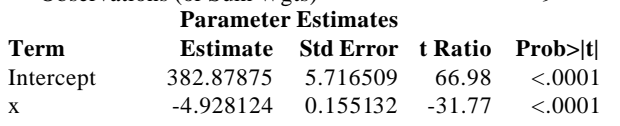

Ammonia Data(Study=3 Test Sequence=1)

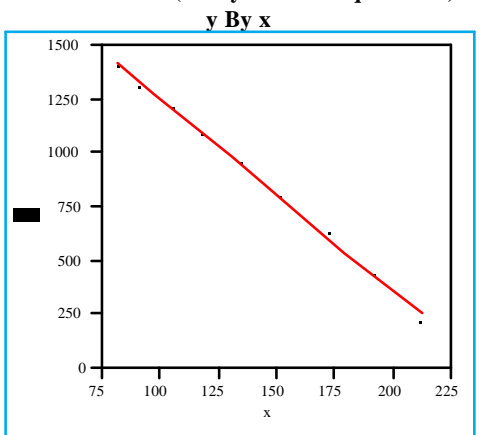

Linear Fit

$y=2137.17-8.88279 x$

Summary of Fit

RSquare

RSquare Adj

Error

Root Mean Square Er

Observations (or Sum Wgts)

0.998327

$\begin{array}{lrrrr}\text { Term } & \text { Estimate } & \text { Std Error t Ratio Prob }>|t|\end{array}$

$\begin{array}{lrrrrr} & 2137.1666 & 18.87375 & 113.23 & <.0001 \\ \text { Intercept } & -8.882787 & 0.128551 & -69.10 & <.0001\end{array}$ 
Exhibit B47: $y$ versus x for the Ammonia Studies: Study 3; Tests 2-9

Ammonia Data(Study $=3$ Test Sequence $=2$ ) y By x

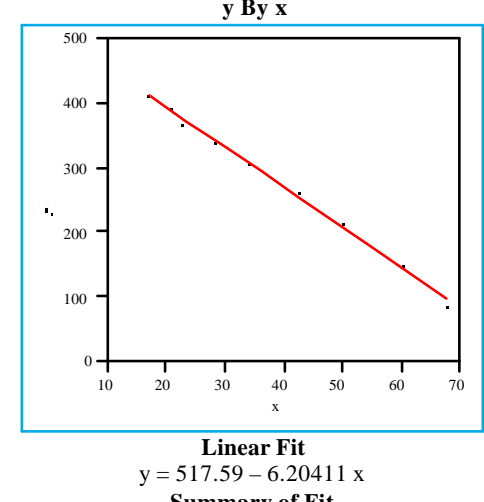

RSquare

0.995446

Root Mean Square Error

Mean of Response

0.994795

Observations (or Sum Wgts)

$$
\begin{array}{lcc} 
& \text { Parameter Estimates } \\
\text { Term } & \multicolumn{2}{c}{\text { Estimate Std Error }} \\
& \text { t Ratio Prob }>|\mathbf{t}| \\
\text { Intercept } & 517.58975 \quad 6.671958 \\
& 77.58 \quad<.0001 \\
\mathrm{x} & \multicolumn{2}{c}{-6.2041120 .158607}
\end{array}
$$

$(-39.123$ - 20001 Ammonia

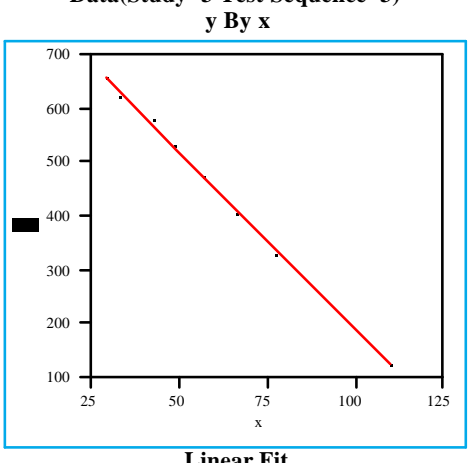

$852.129-6.61753$

Summary of Fit

RSquare

RSquare Adj

are Error

Mean of Response

0.998593

7.33227

436.5903

Parameter Estimates

Term Estimate Std Error t Ratio Prob $>\mid \mathbf{t}$

$\begin{array}{lrrrrr} & & & \\ \text { Intercept } & 852.12924 & 6.382365 & 133.51 & <.0001 \\ & -6.617529 & 0.093892 & -70.48 & <.0001\end{array}$
Ammonia Data(Study $=3$ Test Sequence $=4$ ) y By x

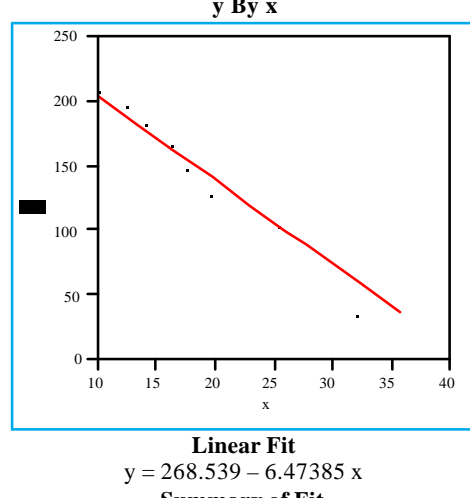

Summary of Fit

RSquare

RSquare Adj

Error

Mean of Response

Observations (or Sum Wgts)

$\begin{array}{lrrrr} & \text { Parameter Estimates } & & \\ \text { Term } & \text { Estimate } & \text { Std Error } & \text { t Ratio } & \text { Prob }>|\mathbf{t}| \\ \text { Intercept } & 268.5386 & 14.59767 & 18.40 & <.0001 \\ \text { x } & -6.473847 & 0.658998 & -9.82 & <.0001\end{array}$

Ammonia Data(Study $=3$ Test Sequence $=5$ )

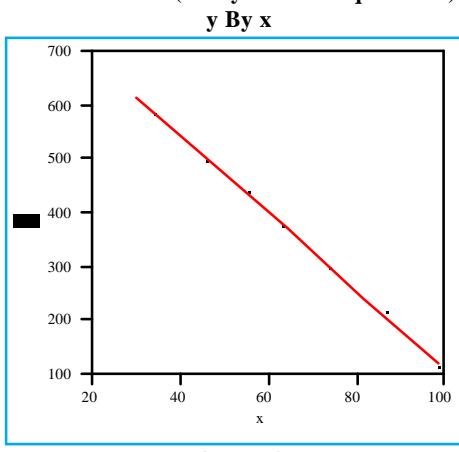

Linear Fit

$y=\underset{33.675-7.20821}{\text { Summary of Fit }}$

RSquare

RSquare Adj
Root Mean Square Erro

Mean of Response

Observations (or Sum Wgts)

0.999084

0.998953

5.575401

406.2331
9

Term Estimate Std Error t Ratio Prob $>|t|$

$\begin{array}{llllll}\text { Intercept } & 833.6749 & 5.234195 & 159.27 & <.0001\end{array}$ $\begin{array}{llll}-7.208206 & 0.082516 & -87.36 & <.0001\end{array}$
Ammonia Data(Study $=3$ Test Sequence $=6$ )

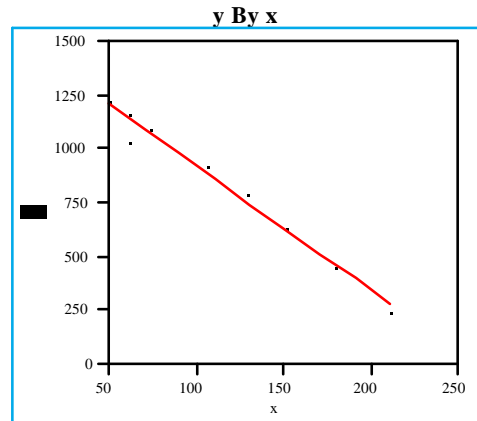

Linear Fit

$y=1502.75-5.80659$

Summary of Fit

$\begin{array}{lr}\text { RSquare } & 0.979068 \\ \text { RSquare Adj } & 0.976078 \\ \text { Root Mean Square Error } & 52.3152 \\ \text { Mean of Response } & 832.1426\end{array}$

Observations (or Sum Wgts)

$\begin{array}{lrrrr} & \text { Parameter Estimates } & & \\ \text { Term } & \text { Estimate } & \text { Std Error } & \text { t Ratio } & \text { Prob }>|\mathbf{t}| \\ \text { Intercept } & 1502.7513 & 40.959 & 36.69 & <.0001 \\ \mathrm{X} & -5.806594 & 0.320903 & -18.09 & <.0001\end{array}$

Ammonia Data(Study=3 Test Sequence=7)

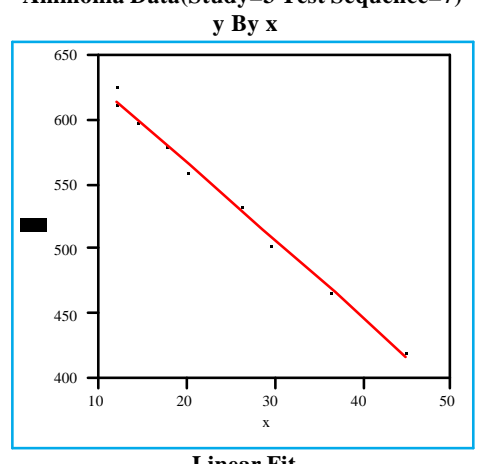

Linear Fit

$y=687.223-6.00148 x$

RSquare

RSquare Adj

Root Mean Square Erro

Mean of Response

Observations (or Sum Wgts)

0.993884

0.99301

5.811617

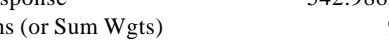

$\begin{array}{lrrrr}\text { Term } & \text { Estimate } & \text { Std Error } & \text { t Ratio } & \text { Prob }>|\mathbf{t}| \\ \text { Intercept } & 687.22253 & 4.694724 & 146.38 & <.0001\end{array}$ $\begin{array}{llll}-6.001483 & 0.177936 & -33.73 & <.0001\end{array}$
Ammonia Data $($ Study $=3$ Test Sequence $=8$ ) y By

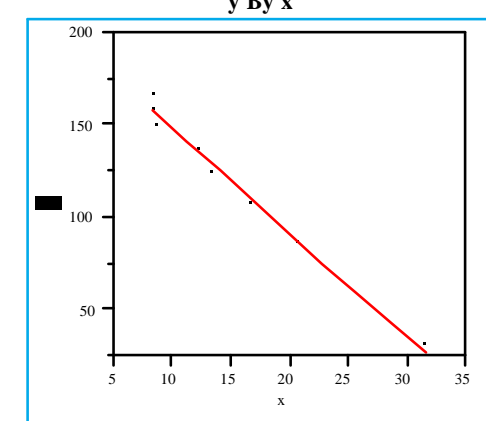

\section{Linear Fit}

$204.987-5.6646$

$\begin{array}{ll}\text { RSquare } & 0.985086 \\ \text { RSquare Adj } & 0.982955\end{array}$

0.982955
6.00608

Rer

Observations (or Sum Wgts)

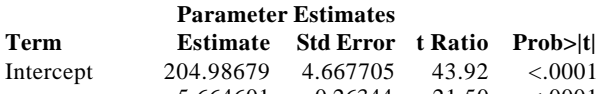

$\begin{array}{lrrrr}\text { Intercept } \quad 204.98679 & 4.667705 & 43.92 & <.0001\end{array}$

Ammonia Data(Study=3 Test Sequence=9) $\mathbf{y}$ By $\mathbf{x}$

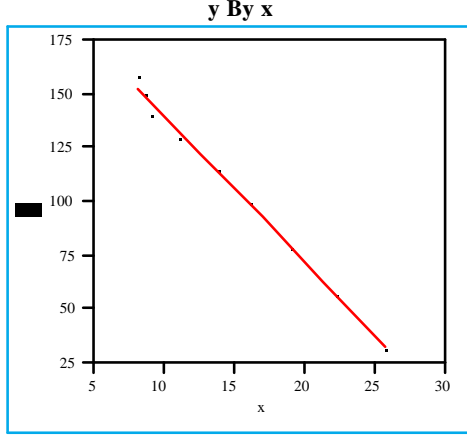

$\mathrm{y}=208.442-6.85374 \mathrm{x}$

Summary of Fit

Rquare

Root Mean Square Error

0.994488

0.993701

$\begin{array}{ll} & \\ \text { Observations (or Sum Wgts) } & 105.4991\end{array}$

$\begin{array}{lrrrr}\text { Estimate } & \text { Std Error } & \text { t Ratio } & \text { Prob }>|\mathbf{t}|\end{array}$ $\begin{array}{rrrrr} & & \\ \mathrm{X} & -6.44221 & 3.116364 & 66.89 & <.0001 \\ & -6.853735 & 0.192853 & -35.54 & <.0001\end{array}$ 
Exhibit B48: y versus $x$ for the Ammonia Studies: Study 4; Tests 1-8
Ammonia Data(Study=4 Test Sequence=1)

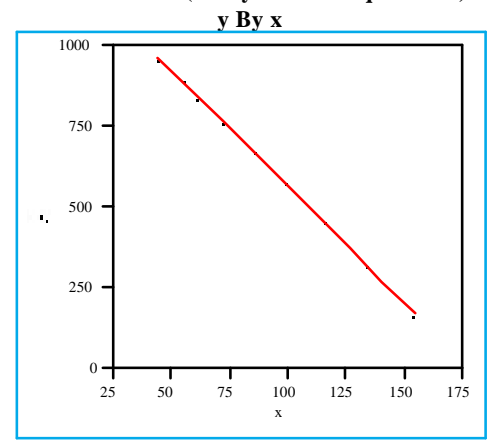

Linear Fit

$y=1280.43-7.17056 x$

RSquare

RSquare Adj

Response

Observations (or Sum Wgts)

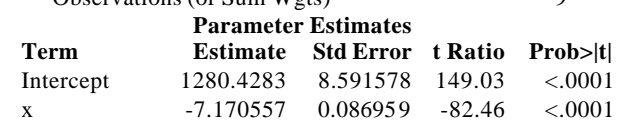

Ammonia Data(Study=4 Test Sequence $=2$ )

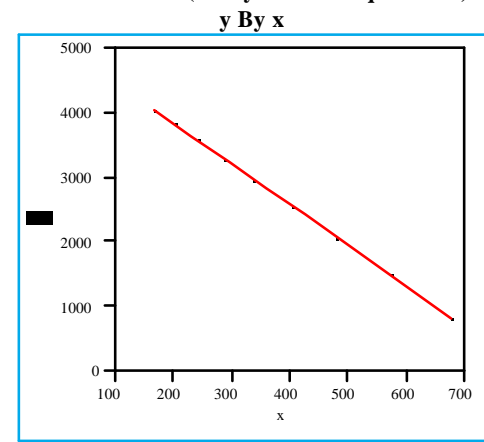

$y=5113.16-6.366 x$

RSquare

Summary of Fit

RSquare Adj

Root Mean Square Error

Mean of Response
Observations (or Sum Wgts)

0.999698

0.99965

20.53463

2701.726

Parameter Estimates

Term Estimate Std Error t Ratio Prob $>|\mathbf{t}|$

$\begin{array}{lrrrrr}\text { Intercept } & 5113.1618 & 17.25811 & 296.28 & <.0001 \\ \mathrm{X} & -6.365996 & 0.041823 & -152.2 & <.0001\end{array}$
Root Mean Square Error
Ammonia Data(Study=4 Test Sequence $=3$ )

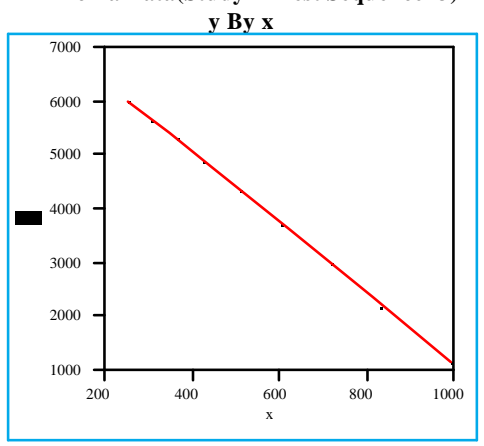

Linear Fit

$=7699.73-6.57471$

$\begin{array}{ll}\text { RSquare } & 0.999684 \\ \text { RSquare Adj } & 0.999639 \\ \text { Root Mean Square Error } & 31.23727 \\ \text { Mean of Response } & 3997.324\end{array}$

Observations (or Sum Wgts)

$\begin{array}{lrlll} & \text { Parameter Estimates } & & \\ \text { Term } & \text { Estimate } & \text { Std Error } & \text { t Ratio } & \text { Prob }>|\mathbf{t}|\end{array}$

$\begin{array}{llllll}\text { Intercept } & 7699.7323 & 26.97795 & 285.41 & <.0001\end{array}$

Ammonia Data(Study=4 Test Sequence=4)

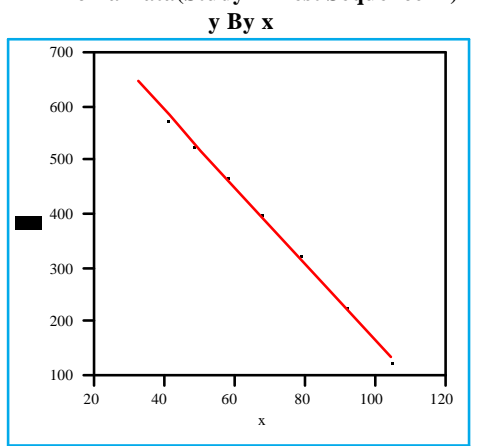

$y=878.13-7.12812 x$

RSquare

$\begin{array}{ll} & 0.998507 \\ \text { RSquare Adj } & 0.998294\end{array}$

Root Mean Square Error $\quad 7.501888$

Mean of Response

7.501888
432.0991
9

Parameter Estimates
Parstestions (or Sum

Term Estimate Std Error t Ratio Prob $>|\mathbf{t}|$

$\begin{array}{llllll}\text { Intercept } & 878.12957 & 6.981083 & 125.79 & <.0001 \\ \mathrm{x} & -7.128124 & 0.104163 & -68.43 & <.0001\end{array}$
Ammonia Data(Study=4 Test Sequence=5)

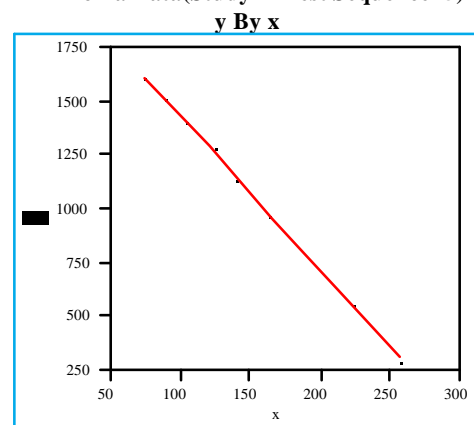

Linear Fit

$$
\begin{gathered}
\text { Linear Fit } \\
\mathrm{y}=2152.14-7.17319 \mathrm{x}
\end{gathered}
$$$$
\text { Summary of Fit }
$$

$\begin{array}{ll}\text { RSquare } & 0.999512 \\ \text { RSquare Adj } & 0.999443 \\ \text { Root Mean Square Error } & 10.53705 \\ \text { Mean of Response } & 1051.365\end{array}$

Mean of Response

Observations (or Sum Wgts) \begin{tabular}{lrrrr}
\multicolumn{5}{c}{ Parameter Estimates } \\
Term & Estimate & Std Error & t Ratio & Prob $>|\mathbf{t}|$ \\
Intercept & 2152.1356 & 9.837281 & 218.77 & $<.0001$ \\
& -7.173187 & 0.059879 & -119.8 & $<.0001$
\end{tabular}

Ammonia Data(Study=4 Test Sequence=6)

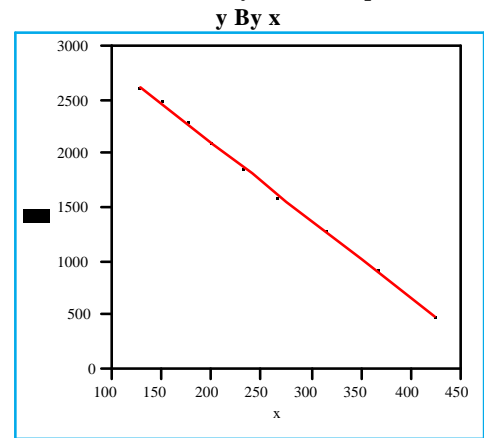

$$
\begin{gathered}
\text { Linear Fit } \\
y=3556.27-7.23884 x
\end{gathered}
$$$$
\text { Summary of Fit }
$$

RSquare

0.999427

RSquare Adj

Root Mean Square Error

Mean of Response

18.71909

Observations (or

$$
1727.633
$$

Parameter Estimates

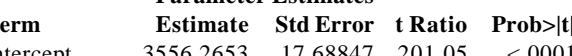

$\begin{array}{rrrr}3556.2653 & 17.68847 & 201.05 & <.0001 \\ -7.238838 & 0.06552 & -110.5 & <.0001\end{array}$
Ammonia Data(Study=4 Test Sequence=7) y By

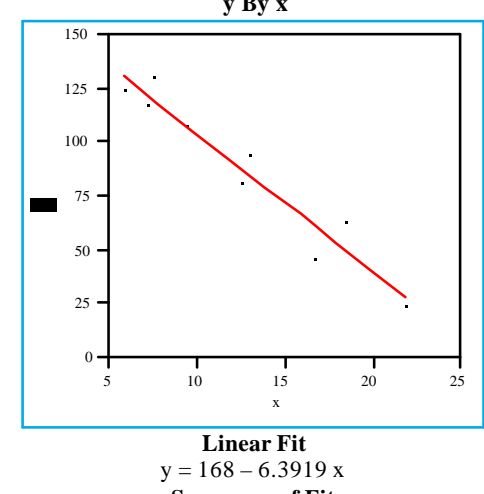

RSquare

RSquare Adj

Summary of Fit

Square Error

Mean of Response
Observations (or Sum Wgts)

Observations (or Sum Wgts)

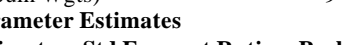

$\begin{array}{lrrrr}\text { Term } & \text { Estimate } & \text { Std Error } & \text { t Ratio } & \text { Prob }>|\mathbf{t}| \\ \text { Intercept } & 167.99969 & 8.742991 & 19.22 & <.0001 \\ & -6.391897 & 0.638 & -10.02 & <.0001\end{array}$

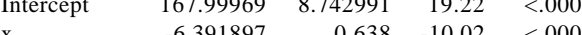

Ammonia Data(Study=4 Test Sequence $=8$ ) y By x

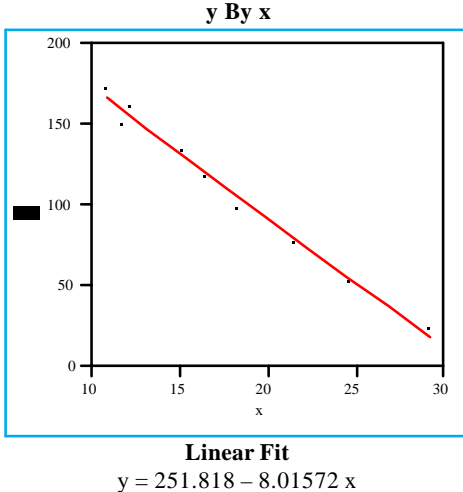

RSquare

Root Mean Square Error

0.985931

Mean of Response

6.425881

Observations (or Sum Wgts)

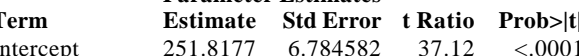

$\begin{array}{lrrrrr} & 251.8177 & 6.784582 & 37.12 & <.0001 \\ \mathrm{X} & -8.015724 & 0.361909 & -22.15 & <.0001\end{array}$ 
Exhibit B49: y versus x for the Ammonia Studies: Study 4; Tests 9-13; Study 5; Tests 1-3

Ammonia Data(Study=4 Test Sequence=9)

y By x

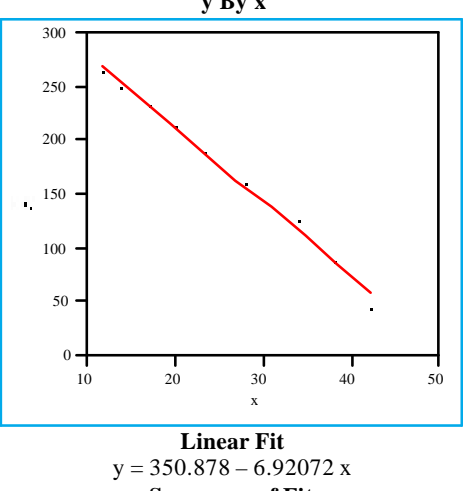

Summary of Fit

RSquare

RSquare Adj

Root Mean Square Erro

Mean of Response

0.991706

0.99052

7.361764
172.8987

Observations (or Sum Wgts)

172.8987
9

Intercet $\begin{array}{lllll}\text { Intercept } \quad 350.87811 & 6.623328 & 52.98 & <.000\end{array}$ $\begin{array}{llll}-6.920722 & 0.239219 & -28.93 & <.000\end{array}$

Ammonia Data(Study $=4$ Test Sequence $=10$ )

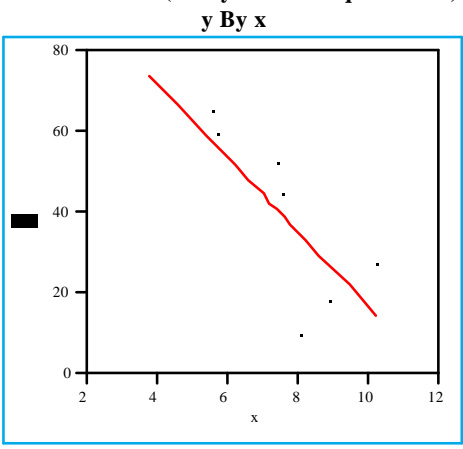

Linear Fit

$\mathrm{y}=108.985-9.21351$

Summary of Fit

RSquare

RSquare Adj

Root Mean Square Erro

Observations (or Sum Wots)

0.693194

0.649364

42.46267

Observations (or Sum Wgts)
Parameter Estimates

$\begin{array}{lrrrrr} & \text { Parameter Estimates } & & \\ \text { Term } & \text { Estimate } & \text { Std Error } & \text { t Ratio } & \text { Prob>|t| } \\ \text { Intercept } & 108.98521 & 17.25202 & 6.32 & 0.0004\end{array}$ $\begin{array}{llll}-9.213506 & 2.316759 & -3.98 & 0.0053\end{array}$
Ammonia Data(Study=4 Test Sequence=11)

y By x

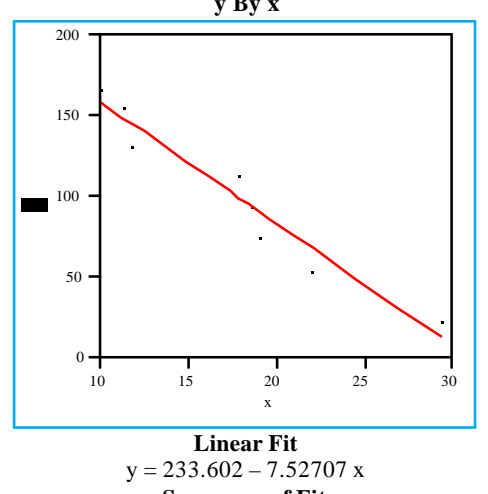

RSquare

RSquare Adj

Root Mean Square Erro

Mean of Response

0.941938

Observations (or Sum Wgts)

Term Estimate Std Error t Ratio Prob>|4

\begin{tabular}{lrrrr} 
Intercept & 233.60194 & 12.74798 & 18.32 & $<.000$ \\
\cline { 2 - 4 } & -7.527071 & 0.70634 & -10.66 & $<.000$
\end{tabular}

$\begin{array}{rrrr}233.60194 & 12.74798 & 18.32 & <.0001\end{array}$

Ammonia Data(Study=4 Test Sequence=12)

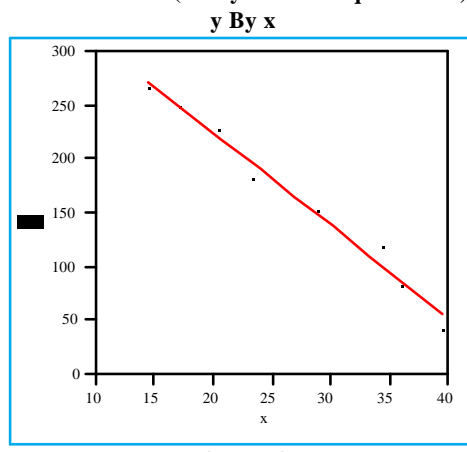

Linear Fit

$y=397.181-8.63346 x$

Summary of Fit

RSquare

RSquare Adj

Observations (or Sum Wots)

0.981284

0.97861
11.24608

ervations (or Sum Wgts)

$\begin{array}{lrrrr} & \text { Estimate } & \text { Std Error } & \text { t Ratio } & \text { Prob }>|t|\end{array}$

$\begin{array}{lrrrrr} & & \text { Est.18105 } & 12.52197 & 31.72 & <.0001 \\ \mathrm{x} & -8.633464 & 0.450661 & -19.16 & <.0001\end{array}$
Ammonia Data(Study=4 Test Sequence=13)

y By $\mathbf{x}$

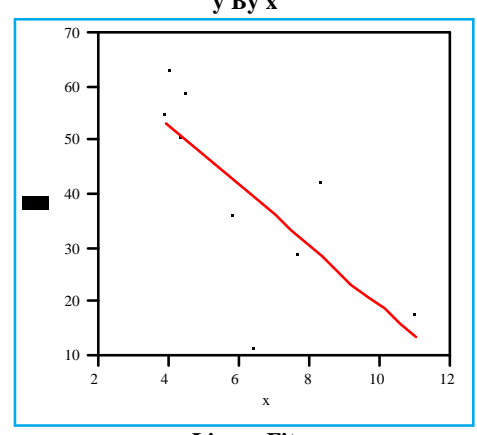

Linear Fit
$y=75.7758-5.66432 x$

\begin{tabular}{lr}
\multicolumn{2}{c}{ Summary of Fit } \\
RSquare & 0.539655 \\
RSquare Adj & 0.473892 \\
Root Mean Square Error & 13.38498 \\
Mean of Response & 40.263 \\
Observations (or Sum Wgts) & 9
\end{tabular}

Mean of Response

Parameter Estimates

$\begin{array}{llll} & \text { Parameter Estimates } & & \\ \text { Term } & \text { Estimate Std Error t Ratio Prob }>|\mathbf{t}|\end{array}$ $\begin{array}{lrrrr}\text { Intercept } & 75.775774 & 13.17549 & 5.75 & 0.0007 \\ \mathrm{x} & -5.664321 & 1.977343 & -2.86 & 0.0242\end{array}$

Ammonia Data(Study=5 Test Sequence=1)

y By x

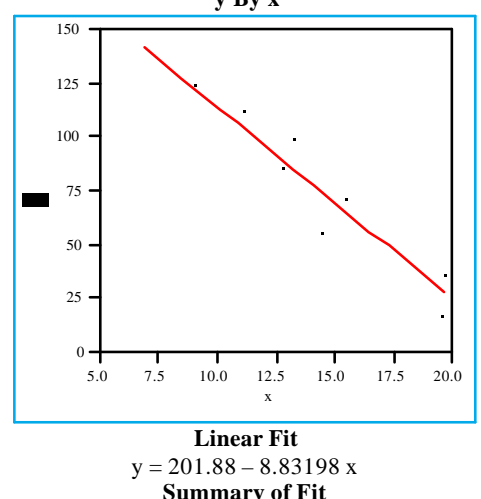

RSquare

Root Mean Square Error

Mean of Respons

0.924804

0.914061
11.67759

81.20511

Parameter Estimates

$\begin{array}{rrrr}\text { Estimate } & \text { Std Error } & \text { t Ratio } & \text { Prob }>|t|\end{array}$

$\begin{array}{rrrr}201.88042 & 13.57598 & 14.87 & <.000 \\ -8.831983 & 0.951881 & -9.28 & <.0001\end{array}$
Ammonia Data(Study=5 Test Sequence=2) $\mathbf{y}$ By $\mathbf{x}$

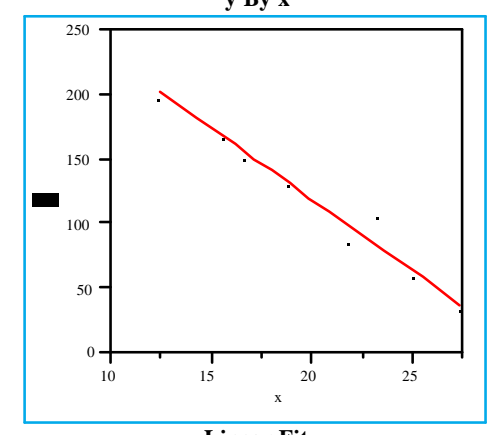

$y=339.261-11.0333 x$ Summary of Fit

RSquare

RSquare Adj

Root Mean Square Error

Mean of Response

Observations (or Sum Wgts)

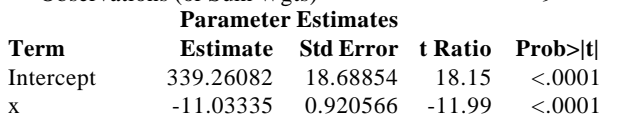

Ammonia Data(Study=5 Test Sequence=3)

y By x

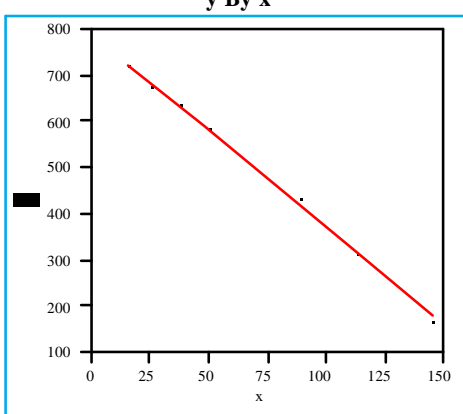

\section{Linear Fit}

$y=790.422-4.18498 x$

RSquare

Error

0.998176

Root Mean Square Error

0.997916
8.654599

Mean of Response

526.2176

Observations (or Sum Wgts)

Term Parameter Estimates

$\begin{array}{llll}\text { Term Estimate } & \text { Std Error } & \mathbf{t} \text { Ratio } & \text { Prob }>|t|\end{array}$

$\begin{array}{llllll} & 790.42201 & 5.15206 & 153.42 & <.0001\end{array}$ 
Exhibit B50: y versus x for the Ammonia Studies: Study 5; Tests 4-11

Ammonia Data(Study $=5$ Test Sequence $=4$ )

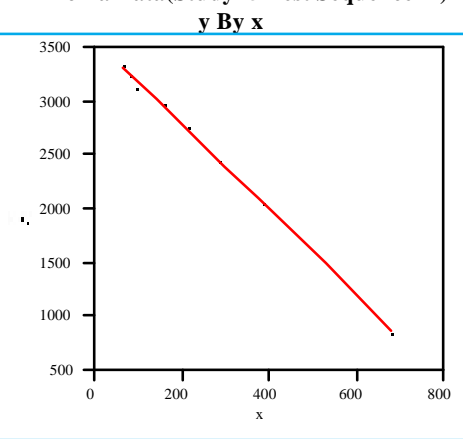

Linear Fit

$y=3588.12-3.97826 x$

RSquare

RSquare Adj

Root Mean Square Error

Mean of Response

0.999055

27.82184

2463.761

Observations (or Sum Wgts)

$$
\begin{array}{lrrrr} 
& \multicolumn{1}{c}{\text { Parameter Estimates }} & & \\
\text { Term } & \text { Estimate } & \text { Std Error } & \text { t Ratio } & \text { Prob }>|\mathbf{t}| \\
\text { Intercept } & 3588.1248 & 16.02856 & 223.86 & <.0001 \\
\mathrm{x} & -3.978263 & 0.046256 & -86.01 & <.0001
\end{array}
$$

Ammonia Data(Study=5 Test Sequence=5)

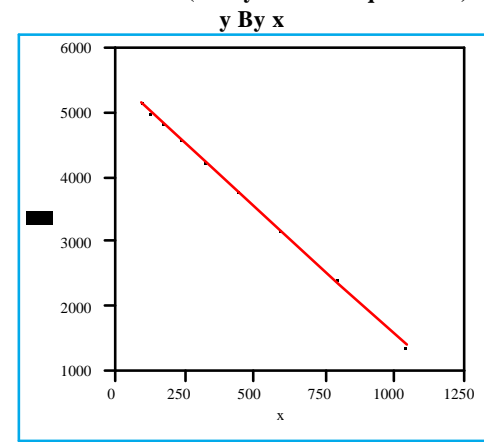

$$
y=5515.01-3.94391 x
$$

RSquare

Summary of Fit

RSquare Adj

Root Mean Square Error

Mean of Response
Observations (or Sum Wgts)

0.999643

0.999592

26.03199

3818.778

Parameter Estimates

Term Estimate Std Error t Ratio Prob $>\mid \mathbf{t}$

$\begin{array}{lrrrrr} & & & \\ \text { Intercept } & 5515.0136 & 14.89842 & 370.17 & <.0001 \\ \mathrm{x} & -3.943905 & 0.028158 & -140.1 & <.0001\end{array}$
Ammonia Data(Study $=5$ Test Sequence $=6$ )

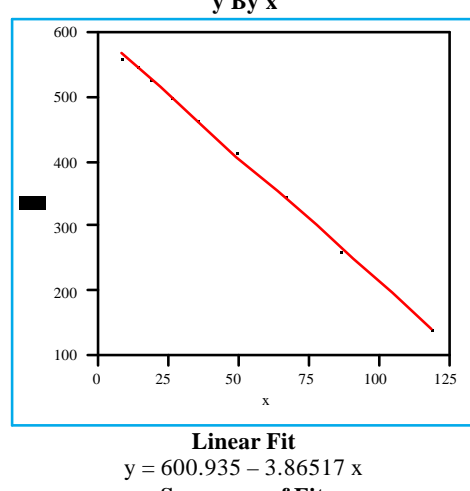

$=600.935-3.86517$

RSquare ummary of $\mathrm{Fit}$

RSquare Adj

Root Mean Square Error

Mean of Response
Observations (or Sum Wgts)

(Pats

Term $\quad 0.93544 \quad 2.43784$ Ratio Prob $>|\mathbf{t}|$

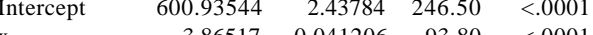

Ammonia Data(Study $=5$ Test Sequence $=7$ )

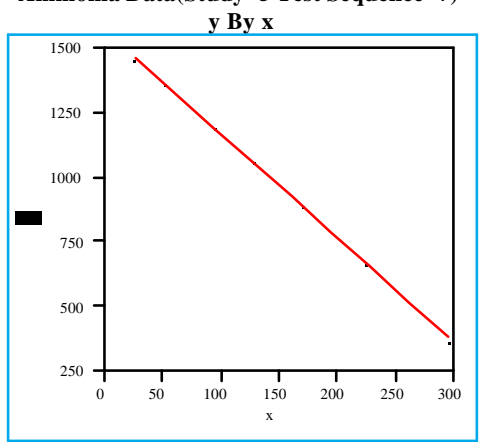

Linear Fit

$$
y=1566.43-4.01547
$$

$\begin{array}{ll}\text { RSquare } & 0.999164 \\ \text { RSquare Adj } & 0.999044\end{array}$

Root Mean Square Error $\quad 11.46612$

Mean of Response

11.46612
1071.039

Observations (or Sum Wgts)

9

eter Estimates

$\begin{array}{lrrrr}\text { Term } & \text { Estimate } & \text { Std Error } & \text { t Ratio } & \text { Prob }>|\mathbf{t}| \\ \text { Intercept } & 1566.4323 & 6.630169 & 236.26 & <.0001\end{array}$

$\begin{array}{llllll}\mathrm{x} & -4.015467 & 0.043914 & -91.44 & <.0001\end{array}$
Ammonia Data(Study=5 Test Sequence=8)

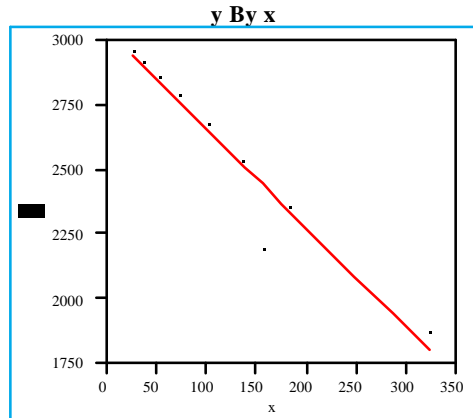

$$
\begin{gathered}
\text { Linear Fit } \\
\mathrm{y}=3041.84-3.8325 \mathrm{x}
\end{gathered}
$$

Summary of Fit

$\begin{array}{lr}\text { RSquare } & 0.93872 \\ \text { RSquare Adj } & 0.929965 \\ \text { Root Mean Square Error } & 97.53118 \\ \text { Mean of Response } & 2567.75\end{array}$

Observations (or Sum Wgts)

$\begin{array}{lrrrr}\text { Term } & \text { Parameter Estimates } & & \\ \text { Estimate } & \text { Std Error } & \text { t Ratio } & \text { Prob }>|t|\end{array}$ $\begin{array}{lrrrr}\text { Intercept } & 3041.8356 & 56.1513 & 54.17 & <.0001\end{array}$

Ammonia Data(Study=5 Test Sequence=9)

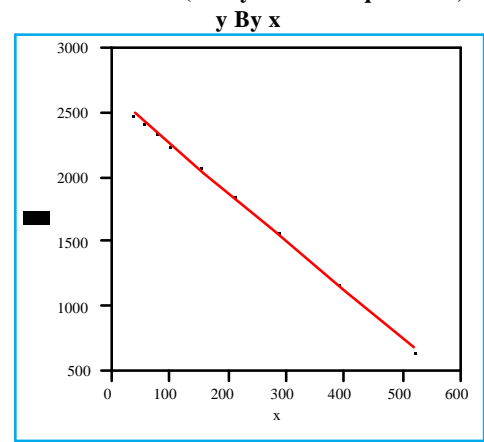

$$
\begin{aligned}
& \text { Linear Fit } \\
& y=2631.71-3.75649 x \\
& \text { Summary of Fit }
\end{aligned}
$$

RSquare

Root Mean Square Error

Mean of Response
Observations (or Sum Wgts)

0.998668

22.84247

Parameter Estimates

Term Estimate Std Error t Ratio Prob $>|t|$

Intercept

$\begin{array}{rrrr}2631.7141 & 12.62413 & 208.47 & <.000\end{array}$

$\begin{array}{rrrr}26.756489 & 0.048506 & -77.44 & <.0001 \\ -3.0001\end{array}$
Ammonia Data(Study=5 Test Sequence $=10$ ) y By x

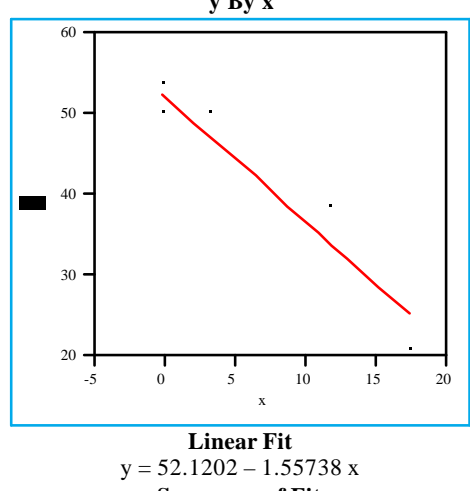

Summary of Fit

RSquare

Root Mean Square Error

0.925705

0.915091

Mean of Response

46.48544

Observations (or Sum Wgts)

Parameter Estimates

Term Estimate Std Error t Ratio Prob $>|t|$

$\begin{array}{llllll} & \text { Es.120214 } & 1.186381 & 43.93 & <.0001\end{array}$

Ammonia Data(Study $=5$ Test Sequence $=11$ )

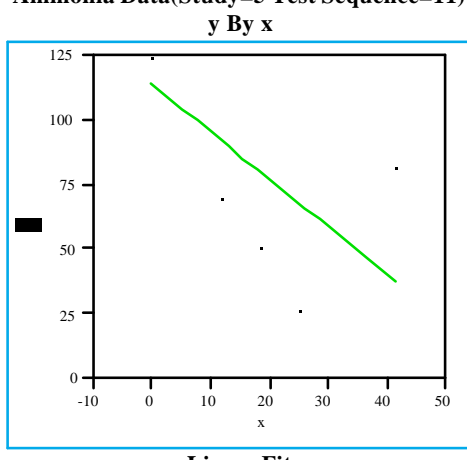

Linear Fit

$y=113.48-1.83634$

Square

Root Mean Square Error

ummary of Fit

0.525979

$\begin{array}{ll} & 28.13323 \\ \text { Observations (or Sum Wgts) } & 93.46644\end{array}$

Parameter Estimates

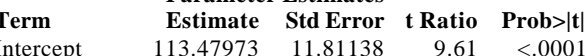

$\begin{array}{lrrrr} & 113.47973 & 11.81138 & 9.61 & <.0001 \\ \mathrm{X} & -1.836344 & 0.6589 & -2.79 & 0.0270\end{array}$ 
Exhibit B51: y versus x for the Ammonia Studies: Study 5; Tests 12-16; Study 6; Tests 1-3

Ammonia Data(Study=5 Test Sequence=12)

y By x

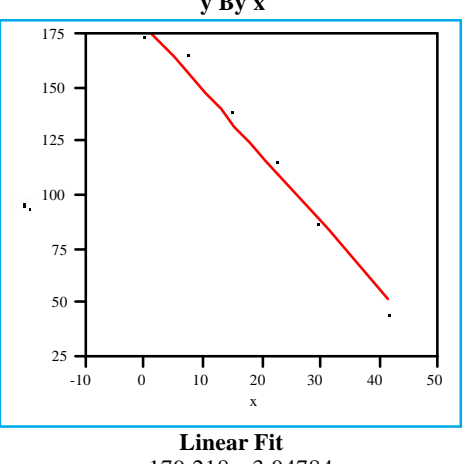

$=179.218-3.04784$

RSquare

RSquare Adj

Root Mean Square Error

Mean of Response

Observations (or Sum Wgts)

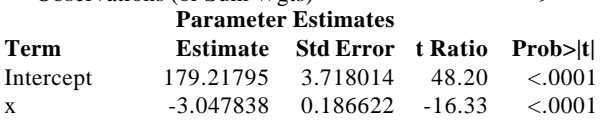

Ammonia Data(Study=5 Test Sequence=13) $\mathbf{y}$ By x

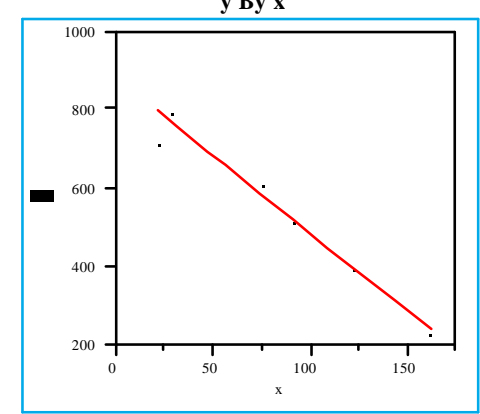

$y=\begin{gathered}886.539-3.99127 \\ \text { Summary of Fit }\end{gathered}$

RSquare

RSquare Adj

Root Mean Square Error

Mean of Respons

Observations (or Sum Wgts)

0.924383

0.91358

58.58884

Parameter Estimates

$\begin{array}{lrrrr}\text { Term } & \text { Estimate } & \text { Std Error } & \text { t Ratio } & \text { Prob }>|t|\end{array}$

$\begin{array}{lrrrrr}\text { Intercept } \quad 886.53902 & 36.06992 & 24.58 & <.0001\end{array}$

$\begin{array}{lllll}\mathrm{x} & -3.991271 & 0.431467 & -9.25 & <.0001\end{array}$
Ammonia Data(Study=5 Test Sequence=14)

y By x

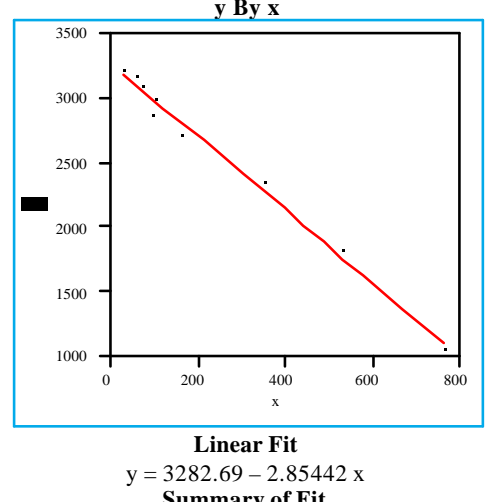

RSquare

RSquare Adj

Root Mean Square Erro

Mean of Response

0.990432

(or Sum Wgts)

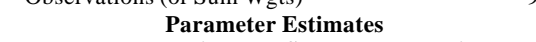

Term Estimate Std Error t Ratio Prob $>\mid t$ $\begin{array}{lrrrrr}\text { Intercept } & 3282.6944 & 36.4483 & 90.06 & <.0001\end{array}$

Ammonia Data(Study=5 Test Sequence=15)

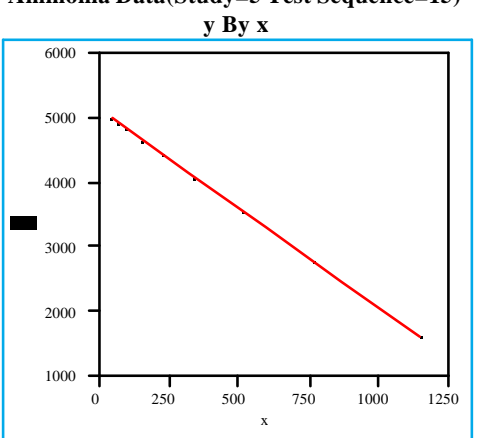

Linear Fit

$\mathrm{y}=5119.71-3.05525 \mathrm{x}$

Summary of Fit

RSquare

RSquare Adj

0.999954

Root Mean Square Er

0.999948

Observations (or Sum

8.266591
3957.867
9

Parameter Estimates

Term Estimate Std Error t Ratio Prob $>|t|$

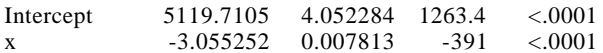

Ammonia Data(Study=5 Test Sequence=16)

y By $\mathbf{x}$

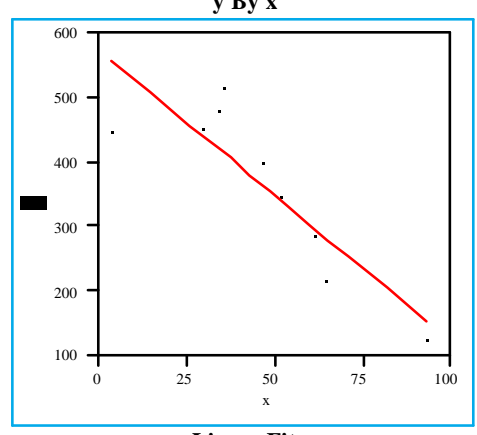

Linear Fit
$y=572.399-4.47456$

Summary of Fit

RSquare

Root Mean Square Erro

Mean of Response

Observations (or Sum Wgts)

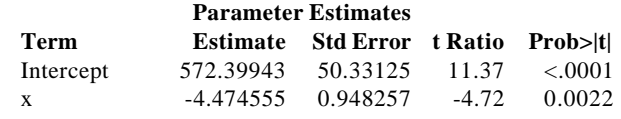

Ammonia Data(Study=6 Test Sequence=1)

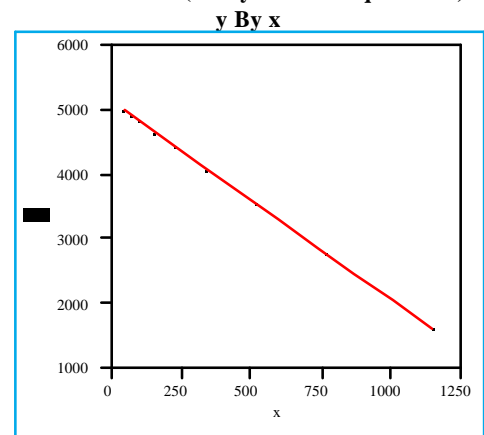

Linear Fit

$y=5120.12-3.05601$

Summary of Fit

0.760817 0.726648 579

$\mathbf{o b}>|\mathbf{t}|$
0.0022

\begin{tabular}{lr}
\multicolumn{2}{c}{$\quad \begin{array}{r}\text { Summary of Fit } \\
\text { RSquare }\end{array}$} \\
RSquare Adj & 0.999958 \\
Root Mean Square Error & 0.999952 \\
Mean of Response & 7.952921 \\
Observations (or Sum Wgts) & 3957.875 \\
\hline
\end{tabular}

Observations (or Sum Wgts)

$\begin{array}{lrrrr}\text { Term } & \text { Estimate } & \text { Std Error } & \text { t Ratio } & \text { Prob }>|t|\end{array}$ $\begin{array}{lrrrrr} & 5120.1213 & 3.89901 & 1313.2 & <.0001 \\ \mathrm{x} & -3.056009 & 0.007518 & -406.5 & <.0001\end{array}$
Ammonia Data(Study $=6$ Test Sequence $=2$ ) y By $\mathbf{x}$

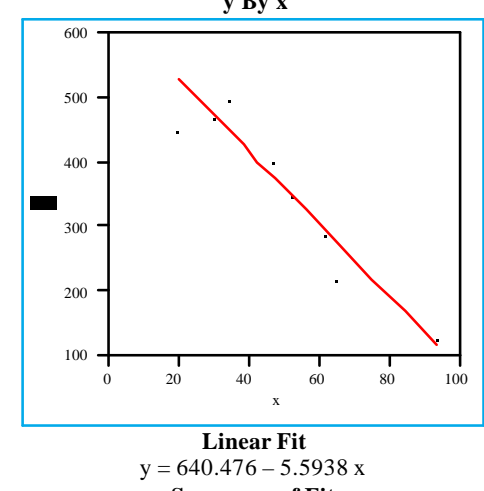

RSquare

RSquare Adj

Root Mean Square Error

Mean of Response

0.853523 0.832597

Observations (or Sum Wgts)

365.8481

Parameter Estimates

Term Estimate Std Error t Ratio Prob $>|\mathbf{t}|$ $\begin{array}{llllll}\text { Intercept } & 640.47572 & 46.83798 & 13.67 & <.000\end{array}$

Ammonia Data(Study $=6$ Test Sequence $=3$ )

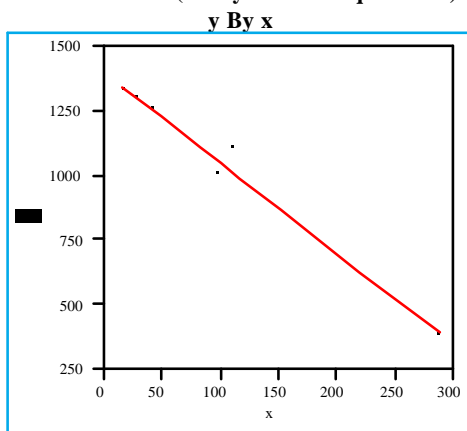

Linear Fit
$y=1399.65-3.51423 x$

Summary of Fit

RSquare

RSquare Adj

re Error

0.980045

Root Mean Square Error

Observations (or Sum Wgts)

0.977194

Parameter Estimates

$\begin{array}{lrrrr}\text { Term } & \text { Estimate } & \text { Std Error } & \text { t Ratio } & \text { Prob }>|t| \\ \text { Intercept } & 1399.6478 & 26.10861 & 53.61 & <.0001\end{array}$

$\begin{array}{llllll}\mathrm{x} & -3.514233 & 0.189533 & -18.54 & <.0001\end{array}$ 
Exhibit B52: y versus x for the Ammonia Studies: Study 6; Tests 4-5; Study 7; Tests 1-5

Ammonia Data(Study=6 Test Sequence=4) y By $x$

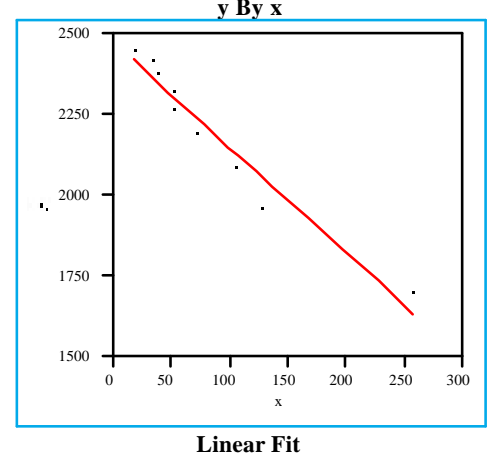
$\mathrm{y}=2475.2-3.25934 \mathrm{x}$

RSquare

RSquare Adj

Root Mean Square Error

Mean of Response

Observations (or Sum Wgts) \begin{tabular}{lrrrr}
\multicolumn{5}{c}{ Parameter Estimates } \\
Term & Estimate & Std Error & t Ratio & Prob $>|\mathbf{t}|$ \\
Intercept & 2475.1965 & 31.51122 & 78.55 & $<.0001$ \\
x & -3.25934 & 0.28511 & -11.43 & $<.0001$
\end{tabular}

Ammonia Data(Study=6 Test Sequence $=5$ )

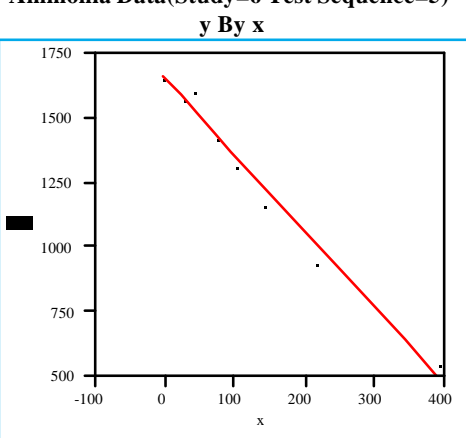

Linear Fit

$y=1653.54-2.95224$

RSquare

Summary of Fit

RSquare Adj

Root Mean Square Error

0.980189

of Response

0.977358

54.60119

Observations (or Sum Wgts)

Parameter Estimates

Term Estimate Std Error t Ratio Prob $>|t|$

$\begin{array}{lrrrr}\text { Intercept } & 1653.5419 & 26.61576 & 62.13 & <.0001 \\ \mathrm{x} & -2.952241 & 0.158637 & -18.61 & <.0001\end{array}$
Ammonia Data(Study=7 Test Sequence=1)

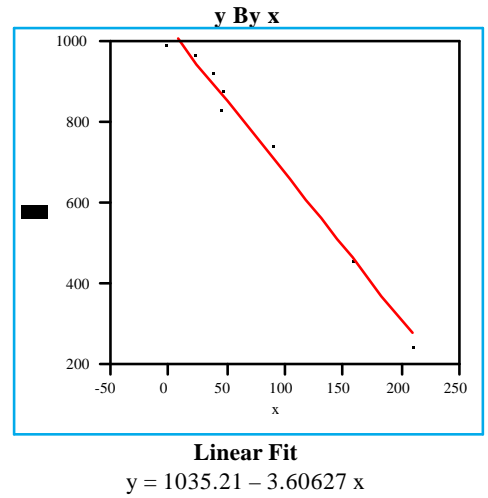

$=1035.21-3.60627$

RSquare

0.984779

RSquare Adj

Root Mean Square Erro

Mean of Response

0.982605

Observations (or Sum Wgts)

736.9561

Parameter Estimates

Term Estimate Std Error t Ratio Prob $>\mid$

$\begin{array}{lllll}\text { Intercept } & 1035.2064 & 17.90493 & 57.82 & <.0001\end{array}$

Ammonia Data(Study=7 Test Sequence $=2$ )

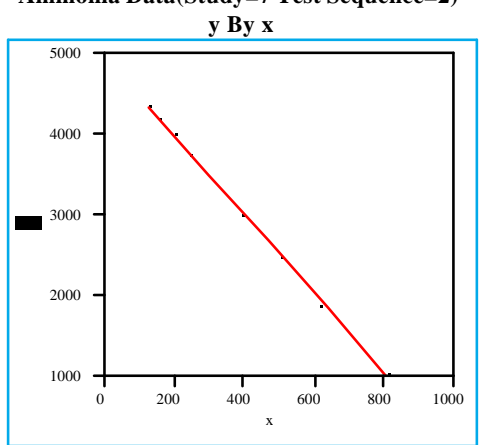

Linear Fit

$y=4971.59-4.87737$

$\begin{array}{ll}\text { RSquare } & 0.998939 \\ \text { RSquare Adj } & 0.998787\end{array}$

Root Mean Square Error $\quad 39.17847$

Mean of Response

39.17847

Observations (or Sum Wgts)

Parameter Estimates

Term Estimate Std Error t Ratio Prob $>|\mathbf{t}|$ $\begin{array}{lllll}\text { Intercept } & 4971.5873 & 26.41387 & 188.22 & <.0001 \\ \mathrm{x} & -4.877375 & 0.060088 & -81.17 & <.0001\end{array}$
Ammonia Data(Study=7 Test Sequence $=3$ )

y By x

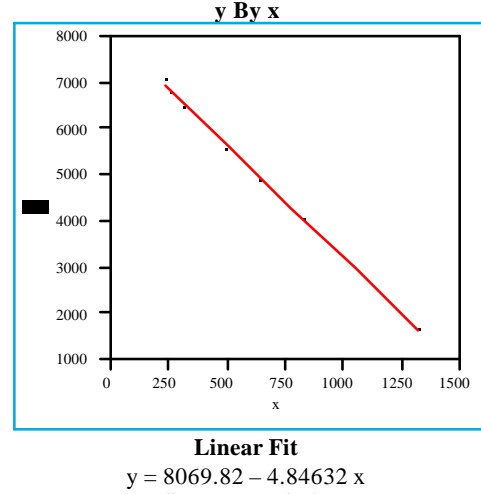

RSquare

Square Adj $\quad 0.99788$

0.997582
09.48618

Observations (or Sum Wgts) $\begin{array}{lrrrr} & \text { Parameter Estimates } & & \\ \text { Term } & \text { Estimate } & \text { Std Error } & \text { t Ratio } & \text { Prob }>|\mathbf{t}| \\ \text { Intercept } & 8069.8219 & 60.30176 & 133.82 & <.0001 \\ \mathrm{x} & -4.846323 & 0.084342 & -57.46 & <.0001\end{array}$

Ammonia Data(Study=7 Test Sequence=4)

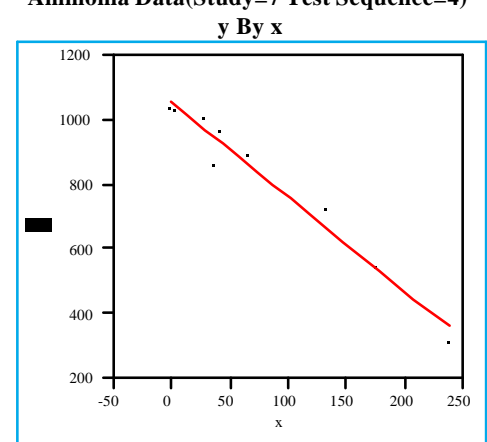

Linear Fi

$$
y=1054.37-2.94125 x
$$

RSquare

Root Mean Square Error $\quad 48.45794$

Mean of Response $\quad 817.3637$

Observations (or Sum Wgts)

Term Estimate Std Error t Ratio Prob $>|\mathbf{t}|$

$\begin{array}{lrrrrr} & \text { Intercept } & 1054.369 & 23.16468 & 45.52 & <.0001 \\ \mathrm{x} & -2.941247 & 0.206057 & -14.27 & <.0001\end{array}$
Ammonia Data(Study=7 Test Sequence=5)

y By $\mathbf{x}$

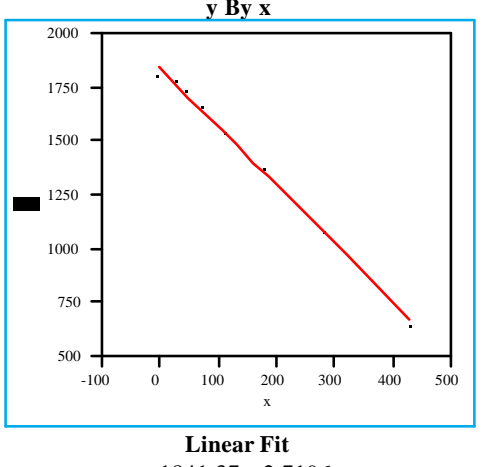

$=1841.37-2.7196 x$

ummary of Fit

RSquare

RSquare Adj
Root Mean Square Error

Mean of Response

0.996914

0.996474

1489.673

Observations (or Sum Wgts)

Parameter Estimates

Estimate Std Error t Ratio Prob $>|t|$ $\begin{array}{lrrrr}\text { Intercept } & 1841.3739 & 10.82723 & 170.07 & <.0001 \\ \mathrm{x} & -2.719597 & 0.057187 & -47.56 & <.0001\end{array}$ 
Exhibit B53: $\log (\mathrm{A})$ versus Sampling Sequence for the Special Salt Studies: Tests 1-8

Special Salt Solutions(Test Sequence=1)

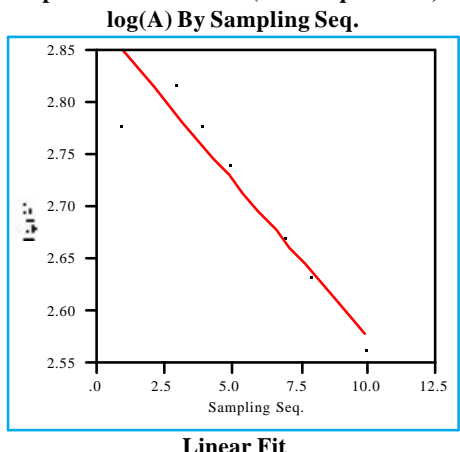

$\log (\mathrm{A})=2.87707-0.03017$ Sampling Seq.

RSquare Summary of Fit

RSquare Adj

Root Mean Square Erro

Mean of Response

0.906959

Observations (or Sum Wgts)
Parameter Estimates

Intercet $\quad 2.857$ Std Error t Ratio Prob>|t| $\begin{array}{lrrrrr} & 2.8770736 & 0.021197 & 135.73 & <.0001 \\ \text { Sampling Seq. } & -0.030167 & 0.003416 & -8.83 & <.0001\end{array}$

Special Salt Solutions(Test Sequence $=2$ )

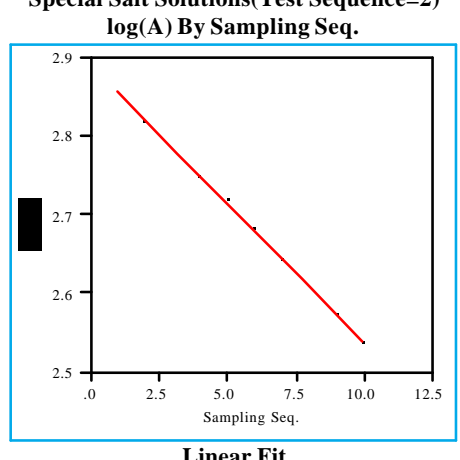

$\log (\mathrm{A})=2.89038-0.03519$ Sampling Seq. Summary of Fit

RSquare

RSquare Adj

Root Mean Square Error

0.999745

Mean of Response

0.999713

Observations (or Sum Wgts)

0.001806
2.696832

Parameter Estimates

10

Term Estimate Std Error t Ratio Prob $>|\mathbf{t}|$ $\begin{array}{lrrrrr}\text { Intercept } & 2.8903779 & 0.001234 & 2342.4 & <.0001\end{array}$ $\begin{array}{lllll}\text { Sampling Seq. } & -0.03519 & 0.000199 & -177 & <.0001\end{array}$
Special Salt Solutions(Test Sequence $=3$ )

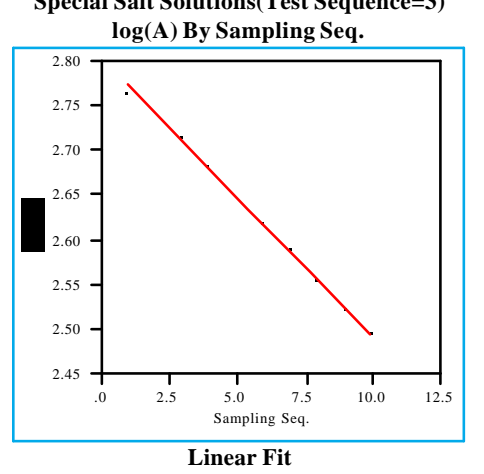

$\log (\mathrm{A})=2.80383-0.03107$ Sampling Seq Summary of Fi

RS

0.998263 RSquare Adj $\quad 0.998046$ Root Mean Square Error $\quad 0.004161$ Mean of Response 2.63297

Observations (or Sum Wgts) 10 \begin{tabular}{lrrrr}
\multicolumn{1}{c}{ Parameter Estimates } & & \\
Term & Estimate & Std Error & t Ratio & Prob $>|\mathbf{t}|$ \\
Intercept & 2.8038292 & 0.002843 & 986.37 & $<.0001$ \\
Sampling Seq. & -0.031065 & 0.000458 & -67.81 & $<.0001$
\end{tabular}

Special Salt Solutions(Test Sequence $=4$ )

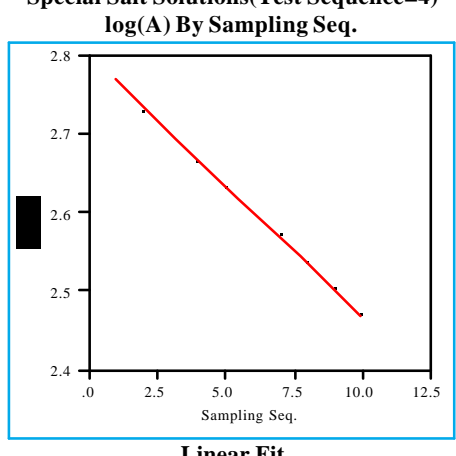

Linear Fit
$\log (\mathrm{A})=2.80301-0.03349$ Sampling Seq ummary of Fit

RSquare

RSquare Adj

Root Mean Square Erro

Mean of Response

0.996603

0.006279
2.618829

Observations (or Sum Wgts)

Term $\begin{array}{lll}\text { Parameter Estimates } & & \\ \text { Estimate Std Error t Ratio Prob }>|\mathrm{t}|\end{array}$

$\begin{array}{llllll}\text { Intercept } & 2.8030096 & 0.004289 & 653.49 & <.0001\end{array}$ $\begin{array}{lllll}\text { Sampling Seq. } & -0.033487 & 0.000691 & -48.44 & <.0001\end{array}$
Special Salt Solutions(Test Sequence=5)

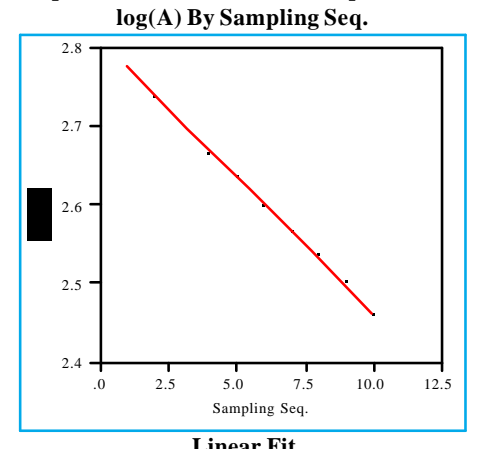

Linear Fit
$\log (\mathrm{A})=2.81028-0.03472$ Sampling Seq Summary of Fit

\section{RSquare}

RSquare Adj
Root Mean Square Error

0.997008

Mean of Response

0.99663

Observations (or Sum Wgts)

2.619305
10

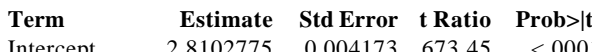
$\begin{array}{llllll} & 2.8102775 & 0.004173 & 673.45 & <.000\end{array}$ $\begin{array}{lllll}\text { Sampling Seq. } & -0.034722 & 0.000673 & -51.63 & <.0001\end{array}$

Special Salt Solutions $($ Test Sequence $=6$ )

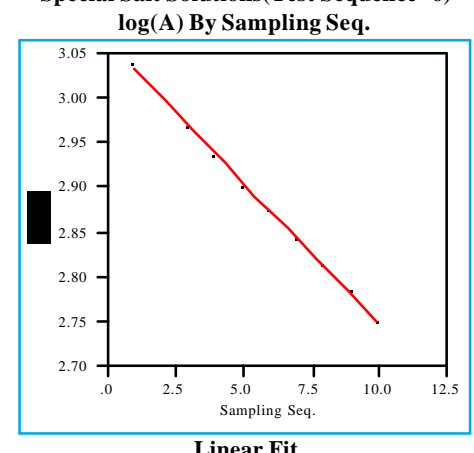

$\log (\mathrm{A})=3.06236-0.03145$ Sampling Seq Summary of Fit

RSquare

RSquare Adj

Root Mean Square Erro

Mean of Response

0.999091

0.998977 2.889375

Parameter Estimates

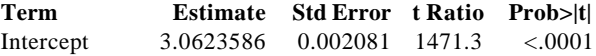
$\begin{array}{lllll}\text { Sampling Seq. } & -0.031452 & 0.000335 & -93.76 & <.0001\end{array}$
Special Salt Solutions(Test Sequence=7)

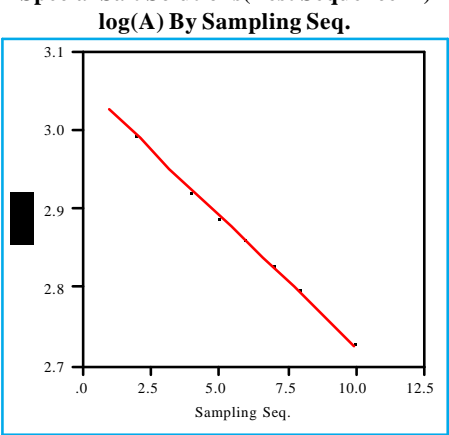

$\log (\mathrm{A})=3.0616-0.0337$ Sampling Seq

RSquare Summary of Fit

Root Mean Square Error

Mean of Response

0.993288

0.992449

0.008897

2.876223

Observations (or Sum Wgts)

10

Term Estimate Std Error t Ratio Prob $>|\mathbf{t}|$

$\begin{array}{lrrrrr}\text { Intercept } & 3.0615977 & 0.006078 & 503.73 & <.0001\end{array}$ $\begin{array}{lrrrr}\text { Sampling Seq. } & -0.033705 & 0.00098 & -34.41 & <.0001\end{array}$

Special Salt Solutions $($ Test Sequence $=8$ )

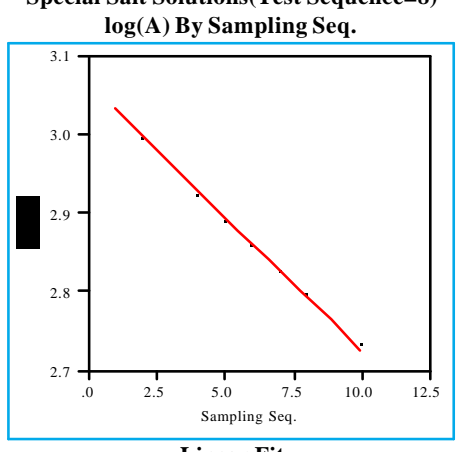

$\log (\mathrm{A})=3.06615-0.03412$ Sampling Seq

RSquare

Summary of Fit

0.990763

RSquare Adj

Root Mean Square Error

0.989608

Mean of Response

2.878474

Observations (or Sum Wgts)

Term Estimate Std Error $\quad$ t Ratio Prob $>\mid \mathbf{t}$ $\begin{array}{lrrrr}\text { Intercept } & 3.0661489 & 0.007228 & 424.22 & <.0001\end{array}$ $\begin{array}{lllll}\text { Sampling Seq. } & -0.034123 & 0.001165 & -29.29 & <.0001\end{array}$ 
Exhibit B54: $\log (\mathrm{A})$ versus Sampling Sequence for the Special Salt Studies: Tests 9-16

Special Salt Solutions (Test Sequence $=9$ ) $\log ($ A) By Sampling Seq.

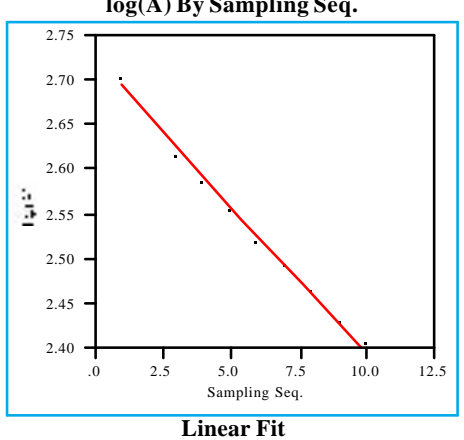

$\log (\mathrm{A})=2.72453-0.03293$ Sampling Seq Summary of Fit

RSquare

RSquare Adj

Root Mean Square Error

Mean of Response

\subsection{2} 0.99278 0.00849

Observations (or Sum Wgts) 2.543442

Parameter Estimates Estimate Std Error t Ratio Prob $>\mid \mathbf{t}$ $\begin{array}{lllllll}\text { Intercept } & 2.724534 & 0.005805 & 469.31 & <.000\end{array}$ $\begin{array}{lllll}\text { Sampling Seq. } & -0.032926 & 0.000936 & -35.19 & <.0001\end{array}$

\section{Special Salt Solutions(Test Sequence $=10$ )} $\log (\mathrm{A})$ By Sampling Seq.

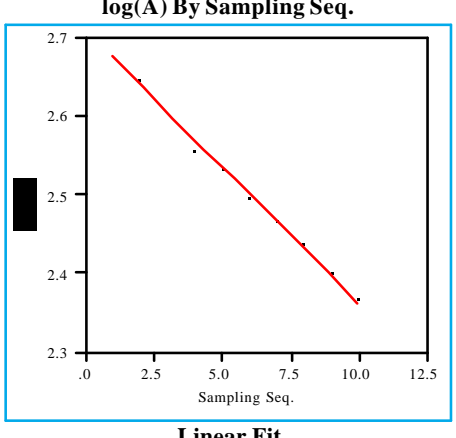

$\log (\mathrm{A})=2.71025-0.03472$ Sampling Seq

$\begin{array}{ll}\text { RSquare } & 0.987903 \\ \text { RSquare Adj } & 0.986391 \\ \text { Root Mean Square Error } & 0.012337 \\ \text { Mean of Response } & 2.519297\end{array}$

Mean of Response 2.519297

Observations (or Sum Wgts)$$
10
$$

$\begin{array}{lrrrr}\text { Term } & \text { Estimate } & \text { Std Error } & \text { t Ratio } & \text { Prob }>|t|\end{array}$ Sampling Seq. $-0.034719 \quad 0.001358 \quad-25.56<0001$
Special Salt Solutions(Test Sequence=11) $\log ($ A) By Sampling Seq.

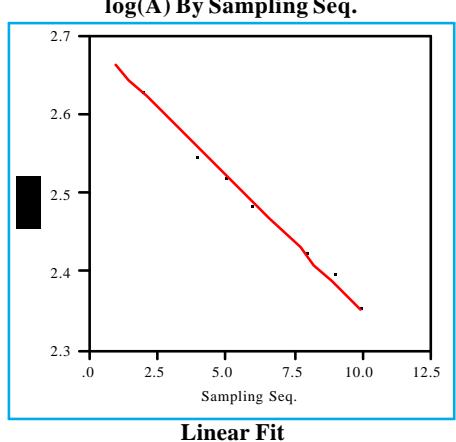

$\log (\mathrm{A})=2.69772-0.03469$ Sampling Seq Summary of Fi

$\begin{array}{lr}\text { RSquare } & 0.985364 \\ \text { RSquare Adj } & 0.983534 \\ \text { Root Mean Square Error } & 0.013577 \\ \text { Mean of Response } & 2.506921 \\ \text { Observations (or Sum Wgts) } & 10\end{array}$

Observations (or Sum Wgts) 10

$\begin{array}{lrrrr}\text { Term } & \text { Parameter Estimates } & & \\ & \text { Estimate } & \text { Std Error } & \text { t Ratio } & \text { Prob }>|t|\end{array}$ $\begin{array}{lrrrr} & 2.6977195 & 0.009275 & 290.86 & <.0001 \\ \text { Sampling Seq. } & -0.034691 & 0.001495 & -23.21 & <.0001\end{array}$

Special Salt Solutions(Test Sequence=12)

$\log (\mathrm{A})$ By Sampling Seq.

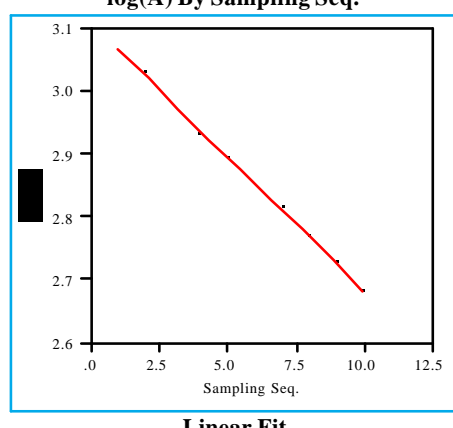

$\log (\mathrm{A})=3.11009-0.04265$

$\begin{array}{ll}\text { RSquare } & 0.997596 \\ \text { RSquare Adj } & 0.997295 \\ \text { Root Mean Square Error } & 0.006724 \\ \text { Mean of Response } & 2.875514\end{array}$

Mean of Response

2.875514

Observations (or Sum Wgts)

Term Estimate Std Error t Ratio Prob $>|t|$

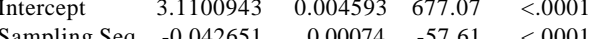

Special Salt Solutions(Test Sequence $=13$ ) $\log ($ A) By Sampling Seq.

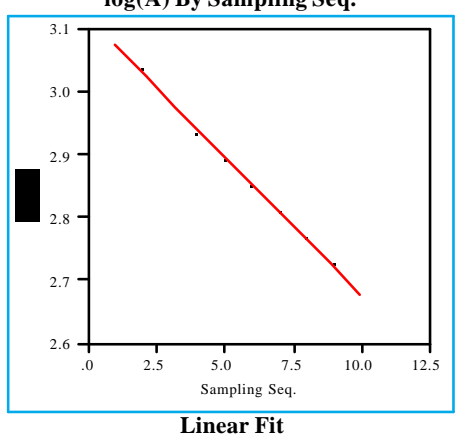

$\log (\mathrm{A})=3.11793-0.04423$ Sampling Seq Summary of Fit

\section{RSquare}

RSquare Adj

Error

Mean of Response

Observations (or Sum Wgts)

0.996458 0.996015 0.008469

Term Estimate Std Error t Ratio Prob $>\mid t$ $\begin{array}{llllll}\text { Intercept } & 3.1179268 & 0.005785 & 538.93 & <.0001\end{array}$ Sampling Seq. $\begin{array}{rllll}-0.044233 & 0.000932 & -47.44 & <.0001\end{array}$

Special Salt Solutions(Test Sequence=14)

$\log (\mathrm{A})$ By Sampling Seq.

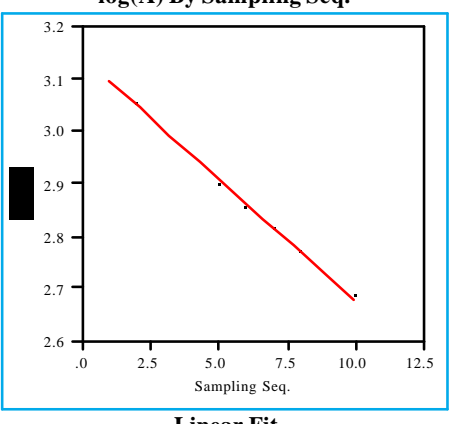

$\log (\mathrm{A})=3.14115-0.04645$

5 Sampling Seq.

$\begin{array}{lr}\text { RSquare } & 0.991681 \\ \text { RSquare Adj } & 0.990641 \\ \text { Root Mean Square Error } & 0.013662 \\ \text { Mean of Response } & 2.885666 \\ \text { Observations (or Sum Wgts) } & 10\end{array}$

Observations (or Sum Wgts)

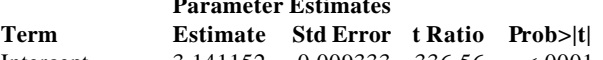

$\begin{array}{lrrrrr} & \text { Estimate } & \text { Std Error } & \text { t Ratio } & \text { Prob }>|t| \\ \text { Intercept } & 3.141152 & 0.009333 & 336.56 & <.0001\end{array}$ $\begin{array}{lrrrr}\text { Sampling Seq. } & -0.046452 & 0.001504 & -30.88 & <.0001\end{array}$
Special Salt Solutions(Test Sequence=15) $\log (\mathrm{A})$ By Sampling Seq.

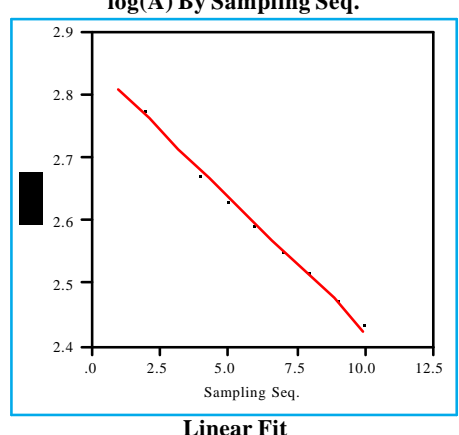

$\log (\mathrm{A})=2.8525-0.04264$ Sampling Se Summary of Fit

RSquare

RSquare Adj

Root Mean Square Error

Mean of Response

0.994559
0.993879

0.993879

Observations (or Sum Wgts)

.617961

Term Estimate Std Error t Ratio Prob $>\mid \mathbf{t}$ $\begin{array}{llllll}\text { Intercept } & 2.8524953 & 0.006919 & 412.27 & <.0001\end{array}$ $\begin{array}{lllll}\text { Sampling Seq. } & -0.042643 & 0.001115 & -38.24 & <.0001\end{array}$

Special Salt Solutions(Test Sequence=16)

$\log (\mathrm{A})$ By Sampling Seq.

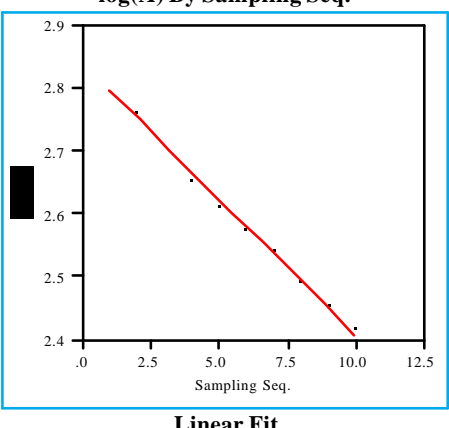

$\log (\mathrm{A})=2.84045-0.04329$ Sampling Seq.

\begin{tabular}{ll}
\multicolumn{1}{c}{ Summary of Fit } & \\
RSquare & 0.993649 \\
RSquare Adj & 0.992855 \\
Root Mean Square Error & 0.011113 \\
Mean of Response & 2.602363
\end{tabular}

Mean of Response

0.011113

Observations (or Sum Wgts)

Term Parameter Estimates

$\begin{array}{lllll} & \text { Estimate } & \text { Std Error } & \text { t Ratio } & \text { Prob }>|t|\end{array}$

Sampling Seq $-0.043288 \quad 0.001224 \quad-35.38<<0001$ 
Exhibit B55: $\log (\mathrm{A})$ versus Sampling Sequence for the Special Salt Studies: Tests 17-24

Special Salt Solutions(Test Sequence=17) $\log (A)$ By Sampling Seq.

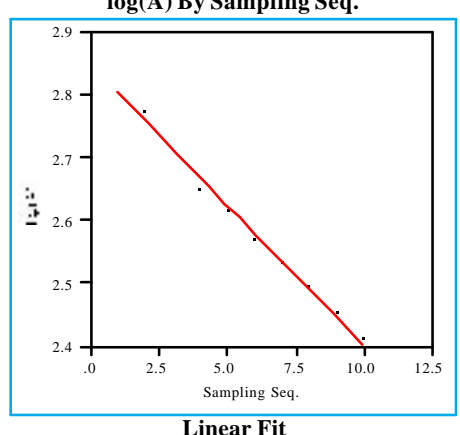

$\log (\mathrm{A})=2.84981-0.04487$ Sampling Se Summary of Fit

RSquare RSquare Adj

Root Mean Square Error

Mean of Response

0.988236

0.986766

Observations (or Sum Wgts)

10

$\begin{array}{lrrrr}\text { Term } & \text { Estimate } & \text { Std Error } & \mathbf{t} \text { Ratio } & \text { Prob }>|\mathbf{t}| \\ \text { Intercept } & 2.8498087 & 0.010739 & 265.37 & <.0001\end{array}$ Sampling Seq. $-0.044869 \quad 0.001731 \quad-25.92 \quad<.0001$

Special Salt Solutions(Test Sequence=18) $\log (\mathrm{A})$ By Sampling Seq.

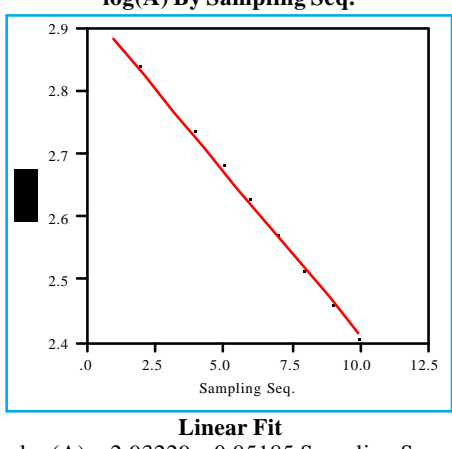

$\log (\mathrm{A})=2.93229-0.05185$ Sampling Seq

\begin{tabular}{ll}
\multicolumn{1}{c}{ Summary of Fit } & \\
RSquare & 0.988787 \\
RSquare Adj & 0.987386 \\
Root Mean Square Error & 0.017732 \\
Mean of Response & 2.647097
\end{tabular}

Meot Mean Square

0.017732

Observations (or Sum Wgts)

10

Term Estimate Std Error t Ratio Prob>|t| $\begin{array}{lllll} & 0.051853 & 0.001952 & -26.56\end{array}$

Special Salt Solutions(Test Sequence=19) $\log (\mathrm{A})$ By Sampling Seq.

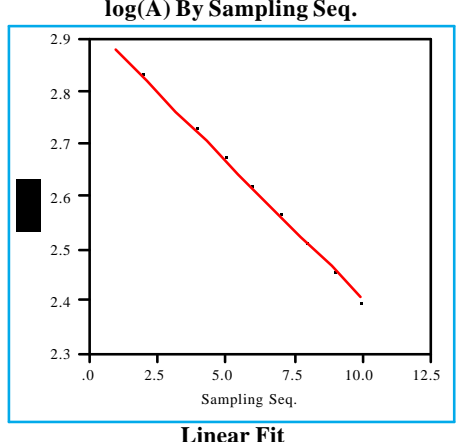

$\log (A)=2.93206-0.05264$ Sampling Seq Summary of Fit

$\begin{array}{lr}\text { RSquare } & 0.996819 \\ \text { RSquare Adj } & 0.996421 \\ \text { Root Mean Square Error } & 0.009549 \\ \text { Mean of Response } & 2.642559 \\ \text { Observations (or Sum Wots) } & 10\end{array}$
Observations (or S

0.009549

10

Term Estimate Std Error t Ratio Prob $>|\mathrm{t}|$ $\begin{array}{lrrrrr}\text { Intercept } & 2.9320585 & 0.006523 & 449.48 & <.0001 \\ \text { Sampling } & -0.052636 & 0.001051 & -50.07 & <.0001\end{array}$

Sampling Seq. -0.052636 (Test Sequence $=20$

Special Salt Solutions(Test Sequence $=$
$\log ($ A) By Sampling Seq.

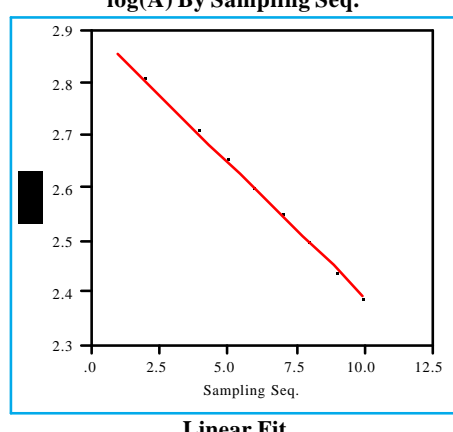

$\log (\mathrm{A})=2.90391-0.05111$

$$
\text { S91- } 0.05111 \text { Sampling Seq. }
$$

$\begin{array}{ll}\text { RSquare } & 0.996626 \\ \text { RSquare Adj } & 0.996204 \\ \text { Root Mean Square Error } & 0.009549 \\ \text { Mean of Response } & 2.622814\end{array}$

Rean of Response

Observations (or Sum Wgts)

Parameter Estimates

Term Estimate Std Error t Ratio Prob $>|t|$

$\begin{array}{llllll}\text { Intercept } & 2.9039107 & 0.006523 & 445.16 & <.0001\end{array}$

$\begin{array}{lllll}\text { Sampling Seq. } & -0.051109 & 0.001051 & -48.61 & <.0001\end{array}$
Special Salt Solutions $($ Test Sequence $=21$ )

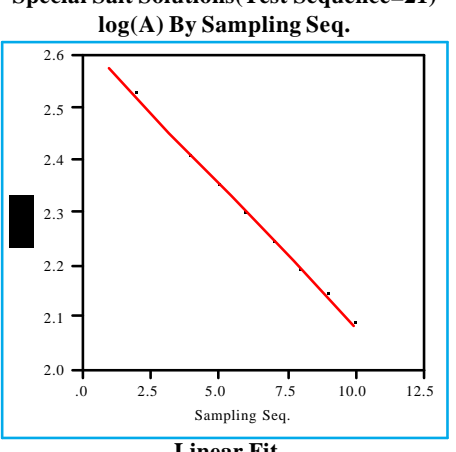

$\log (A)=2.62668-0.05418$ Sampling Seq Summary of Fit

$\begin{array}{lr}\text { RSquare } & 0.999169 \\ \text { RSquare Adj } & 0.999066 \\ \text { Root Mean Square Error } & 0.005016 \\ \text { Mean of Response } & 2.328688 \\ \text { Observations (or Sum Wgts) } & 10\end{array}$

.005016

Parameter Estimates

Term Estimate Std Error t Ratio Prob>|t| $\begin{array}{llllll}\text { Intercept } & 2.6266834 & 0.003427 & 766.52 & <.0001\end{array}$ $\begin{array}{lllll}\text { Sampling Seq. } & -0.054181 & 0.000552 & -98.10 & <.0001\end{array}$

Special Salt Solutions(Test Sequence $=22)$

$\log (\mathrm{A})$ By Sampling Seq.

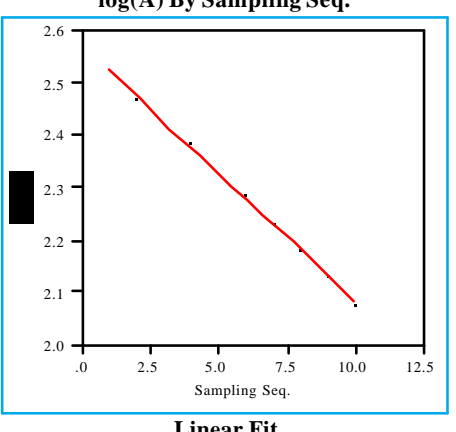

$\log (\mathrm{A})=2.571-0.04876$ Linear Fit

4876 Sampling Seq.

\begin{tabular}{lrrrr} 
RSquare & & 0.998125 \\
RSquare Adj & 0.997891 \\
Root Mean Square Error & 0.006787 \\
Mean of Response & 2.302823 \\
Observations (or Sum Wgts) & \multicolumn{2}{c}{10} \\
\multicolumn{5}{r}{ Parameter Estimates } \\
Term & Estimate & Std Error & t Ratio & Prob $>\mid$ t| \\
lntercept & 2.5709995 & 0.004636 & 554.55 & $<.0001$ \\
Sampling Seq. & -0.048759 & 0.000747 & -65.26 & $<.0001$
\end{tabular}

Special Salt Solutions(Test Sequence $=23$ )

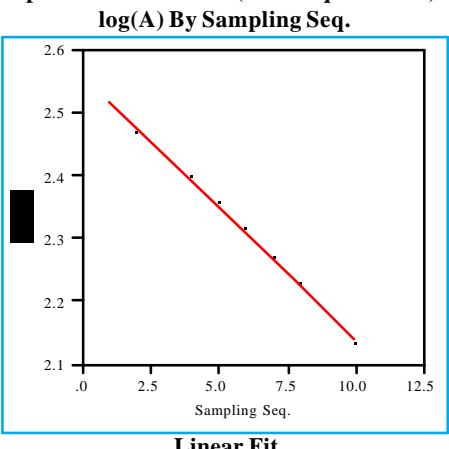

$\log (\mathrm{A})=2.55987-0.04179$ Sampling Seq Summary of Fit

$\begin{array}{lr}\text { RSquare } & 0.99773 \\ \text { RSquare Adj } & 0.997446 \\ \text { Root Mean Square Error } & 0.006402 \\ \text { Mean of Response } & 2.330027 \\ \text { Observations (or Sum Wgts) } & 10\end{array}$
Mean of Response

$\begin{array}{lllll} & \text { Estimate } & \text { Std Error } & \text { t Ratio Prob }>|\mathbf{t}|\end{array}$ $\begin{array}{lrrrrr} & 2.5598716 & 0.004373 & 585.34 & <.0001 \\ \text { Sampling Seq. } & -0.04179 & 0.000705 & -59.29 & <.0001\end{array}$

Special Salt Solutions(Test Sequence=24)

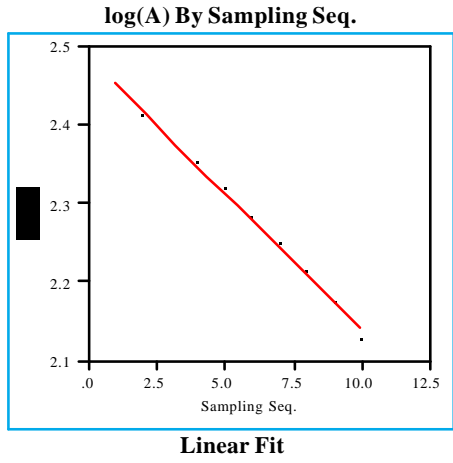

Linear Fit
$\log (\mathrm{A})=2.48712-0.03468$ Sampling Seq.

\begin{tabular}{lr}
\multicolumn{2}{c}{ Summary of Fit } \\
RSquare & 0.997102 \\
RSquare Adj & 0.99674 \\
Root Mean Square Error & 0.006004 \\
Mean of Response & 2.296378 \\
Observations (or Sum Wgts) & 10
\end{tabular}

Observations (or Sum Wgts) 96378

Term $\quad \begin{array}{llll}\text { Estimate } & \text { Std Error } & \text { t Ratio } & \text { Prob }>|t|\end{array}$ $\begin{array}{llllll} & \text { Eampling Seq } & 2.4871162 & 0.004101 & 606.40 & <.000\end{array}$ 
Exhibit B56: $\log (\mathrm{A})$ versus Sampling Sequence for the Special Salt Studies: Tests 25-32

Special Salt Solutions(Test Sequence=25) $\log (\mathrm{A})$ By Sampling Seq.

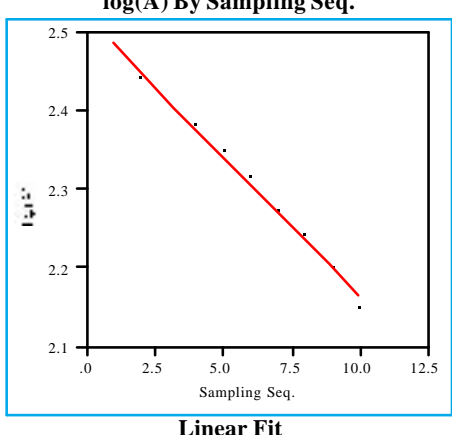

$\log (\mathrm{A})=2.52062-0.03559$ Sampling Seq.

\begin{tabular}{lr}
\multicolumn{2}{c}{ Summary of Fit } \\
RSquare & 0.996311 \\
RSquare Adj & 0.99585 \\
Root Mean Square Error & 0.006954 \\
Mean of Response & 2.32488 \\
Obs
\end{tabular}

Meot Mean of Response

Observations (or Sum Wgts)

10

Term Estimate Std Error t Ratio Prob>|t|

Sampling Seq $\quad \begin{array}{lllll}-0.035589 & 0.000766 & -46.48 & <.0001\end{array}$

Special Salt Solutions(Test Sequence=26)

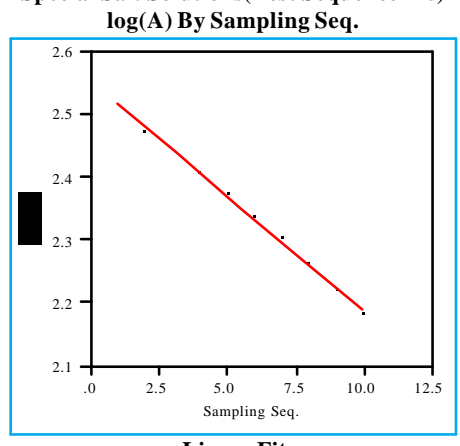

$\log (\mathrm{A})=2.55314-0.03655$ Sampling Seq

Summary of Fit

RSquare

RSquare Adj $\quad 0.997402$

0.997077
0.005989

Mean of Response

Observations (or Sum Wgts)

10

\begin{tabular}{lrrrrr} 
& \multicolumn{2}{c}{ Parameter Estimates } & & \\
Term & Estimate & Std Error & t Ratio & Prob $>|\mathbf{t}|$ \\
Intercept & 2.5531351 & 0.004092 & 623.99 & $<.0001$
\end{tabular} $\begin{array}{lrrrrr}\text { Intercept } & 2.5531351 & 0.004092 & 623.99 & <.0001 \\ \text { Sampling Seq. } & -0.036546 & 0.000659 & -55.42 & <.0001\end{array}$
Special Salt Solutions(Test Sequence=27) $\log (A)$ By Sampling Seq.

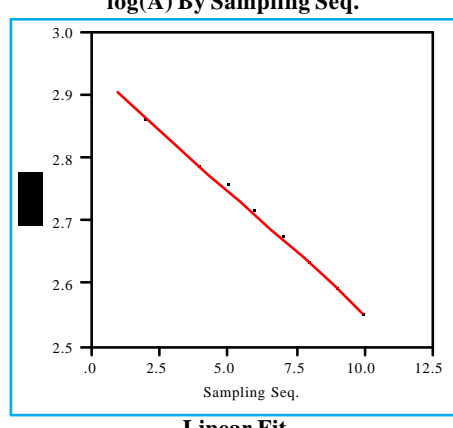

Linear Fit

$\log (\mathrm{A})=2.94212-0.03869$ Sampling Seq

\begin{tabular}{lr}
\multicolumn{1}{c}{ Summary of Fit } \\
RSquare & 0.996089 \\
RSquare Adj & 0.9956 \\
Root Mean Square Error & 0.007785 \\
Mean of Response & 2.72934
\end{tabular}

Mean of Response

Observations (or Sum Wgts)

Term Estimate Std Error t Ratio Prob $>|\mathbf{t}|$

$\begin{array}{lrrrr} & \text { Estimate } & \text { Std Error } & \text { t Ratio } & \text { Prob }>|t| \\ \text { Intercept } & 2.94212 & 0.005318 & 553.22 & <.0001\end{array}$

$\begin{array}{lllll}\text { Sampling Seq. } & -0.038687 & 0.000857 & -45.14 & <.0001\end{array}$

Special Salt Solutions $($ Test Sequence $=28$ )

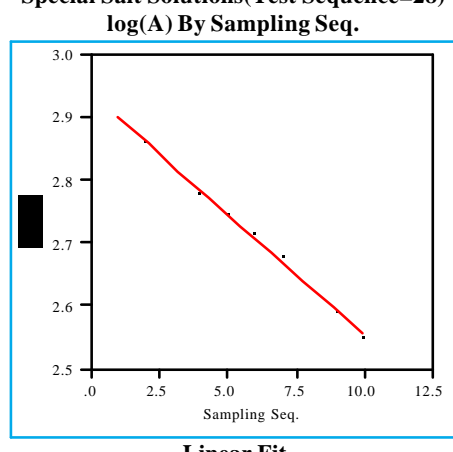

$\log (\mathrm{A})=2.93815-0.03825$ Sampling Seq$$
\text { Summary of Fit }
$$
$\begin{array}{lrrrr} & \text { Parameter Estimates } & & \\ \text { Term } & \text { Estimate } & \text { Std Error } & \text { t Ratio } & \text { Prob }>|\mathbf{t}| \\ \text { Intercept } & 2.9565841 & 0.006193 & 477.37 & <.0001 \\ \text { S } & -0.03924 & 0.000998 & -39.31 & <.0001\end{array}$

RSquare

Root Mean Square Erro

0.99599
0.995488

0.007794

Observations (or Sum Wgts)

$$
2.727795
$$

Parameter Estimates

Estimate Std Error t Ratio Prob $>|\mathbf{t}|$

$\begin{array}{lrrrrr}\text { Intercept } & 2.9381467 & 0.005324 & 551.87 & <.0001 \\ \text { Sampling Seq } & -0.038246 & 0.000858 & -44.57 & <.0001\end{array}$ pecial Salt Solutions $($ Test Sequence $=29$ ) $\log (\mathrm{A})$ By Sampling Seq.

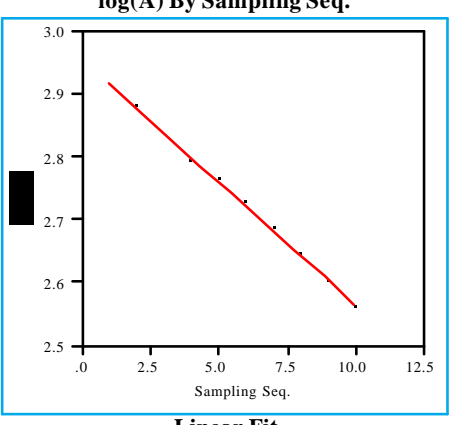

$\log (\mathrm{A})=2.95658-0.03924$ Sampling Seq.

$$
\text { Summary of Fit }
$$

RSquare

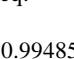

Root Mean Square Error

0.994206

of Response

2.740766

Observations (or Sum Wgts)

Special Salt Solutions $($ Test Sequence $=30$ )

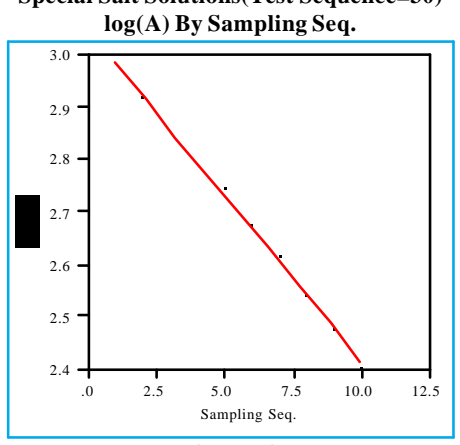

$\log (\mathrm{A})=3.04726-0.06321$ S

Special Salt Solutions(Test Sequence=31) $\log (\mathrm{A})$ By Sampling Seq.

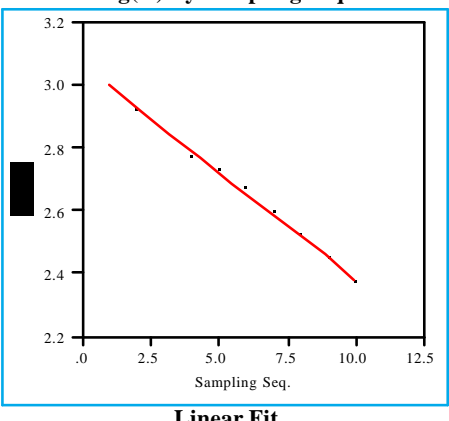

$\log (\mathrm{A})=3.06298-0.06793$ Sampling Seq

\begin{tabular}{ll}
\multicolumn{1}{c}{ Summary of Fit } \\
RSquare & 0.993883 \\
RSquare Adj & 0.993118 \\
Root Mean Square Error & 0.017115 \\
Mean of Response & 2.689344 \\
Ore &
\end{tabular}

Mean of Response

0.017115

Observations (or Sum Wgts)

$\begin{array}{lrrrr} & \text { Estimate } & \text { Std Error } & \text { t Ratio } & \text { Prob }>|\mathbf{t}|\end{array}$

Sampling Seq $-0.067934 \quad 0.001884 \quad-36.05<000$

Special Salt Solutions(Test Sequence $=32$ )

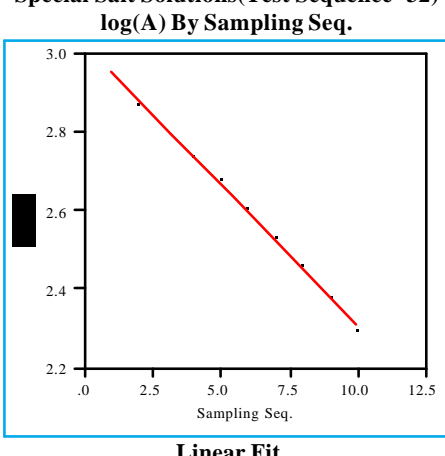

$\log (\mathrm{A})=3.0247-0.07149$ Sampling Seq

\section{RSquare}

Summary of Fit

$\begin{aligned} \log (\mathrm{A}) & =3.0247-0.07149 \mathrm{Sam} \\ & \text { Summary of Fit }\end{aligned}$

0.99718
0.996828

Root Mean Square Erro

0.010793

Mean of Response

2.699622

Parameter Estimates

Root Mean Square Error

0.997359

Mean of Response

0.011812

Observations (or Sum Wgts)

2.631528

Term Estimate Std Error t Ratio Prob $>|t|$ $\begin{array}{llllll} & 3.0472559 & 0.007373 & 413.30 & <.000\end{array}$

Term Parameter Estimates

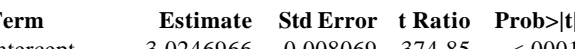

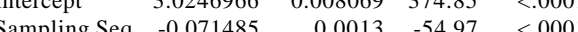


Exhibit B57: $\log (\mathrm{A})$ versus Sampling Sequence for the Special Salt Studies: Tests 33-40

Special Salt Solutions(Test Sequence=33)

$\log (\mathrm{A})$ By Sampling Seq.

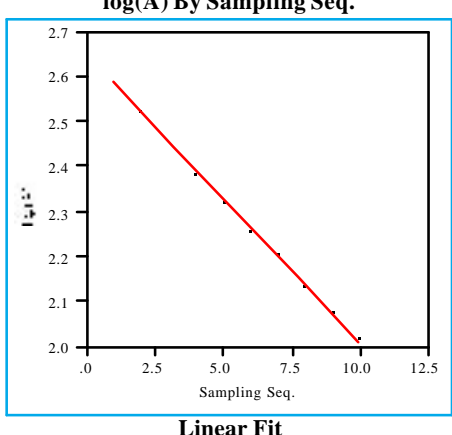

$\log (A)=2.65197-0.06445$ Sampling Seq.

\begin{tabular}{ll}
\multicolumn{2}{c}{ Summary of Fit } \\
RSquare & 0.998363 \\
RSquare Adj & 0.998158 \\
Root Mean Square Error & 0.008381 \\
Mean of Response & 2.297518 \\
Ore & 10
\end{tabular}

0.998158

Observations (or Sum Wgts)

Term $\quad \begin{array}{llll}\text { Estimate } & \text { Std Error } & \text { t Ratio } & \text { Prob }>|t|\end{array}$

Special Salt Solutions(Test Sequence=34)

$\log (\mathrm{A})$ By Sampling Seq.

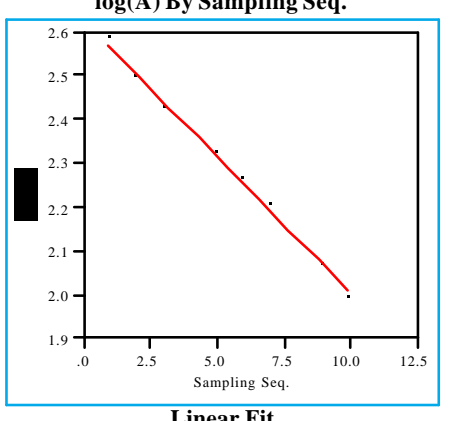

$\log (\mathrm{A})=2.63084-0.06212$ Sampling Se$$
\text { Summary of Fit }
$$

$$
\text { RSquare }
$$

RSquare Adj

are Error

0.992665
0.991748

Root Mean Square Error

0.991748

Mean of Response

2.289192

Observations (or Sum Wgts)$$
10
$$

Term Estimate Std Error t Ratio Prob $>|\mathbf{t}|$ $\begin{array}{lrrrrr}\text { Intercept } & 2.630842 & 0.011714 & 224.59 & <.0001 \\ \text { Sampling Seq } & -0.062118 & 0.001888 & -32.90 & <.0001\end{array}$

Special Salt Solutions(Test Sequence=35)

$\log (\mathbf{A})$ By Sampling Seq.

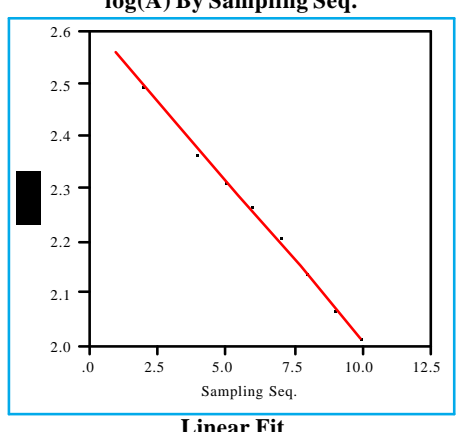

$\log (\mathrm{A})=2.62089-0.06083$

$\begin{array}{lr}\text { RSquare } & 0.99769 \\ \text { RSquare Adj } & 0.997402 \\ \text { Root Mean Square Error } & 0.009398 \\ \text { Mean of Response } & 2.286343\end{array}$

Mean of Respons

0.997402

286343

Parameter Estimates
Prentions (or Sum Wgts)

Term Estimate Std Error t Ratio Prob $>|t|$

$\begin{array}{llllll} & 2.6208872 & 0.00642 & 408.22 & <.0001\end{array}$

Special Salt Solutions(Test Sequence $=36$ )

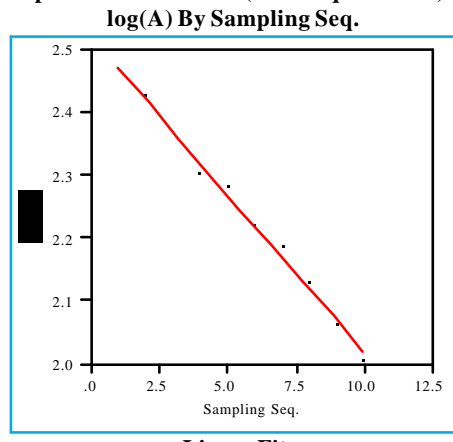

Linear Fit
$\log (\mathrm{A})=2.51999-0.05027$ Sampling Seq

$$
\text { Summary of Fit }
$$

$\log (\mathrm{A})=2.52958-0.04888$ Sampling Seq.

$$
\text { Summary of Fit }
$$

RSquare

RSquare Adj

Root Mean Square Er

Observations (or Sum Wgts)

2.26074

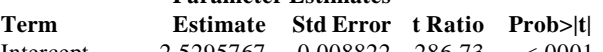
$\begin{array}{lrrrrr} & 2.5295767 & 0.008822 & 286.73 & <.0001 \\ & & 0.048879 & 0.001422 & -34.38 & <.0001\end{array}$

Special Salt Solutions(Test Sequence=38)

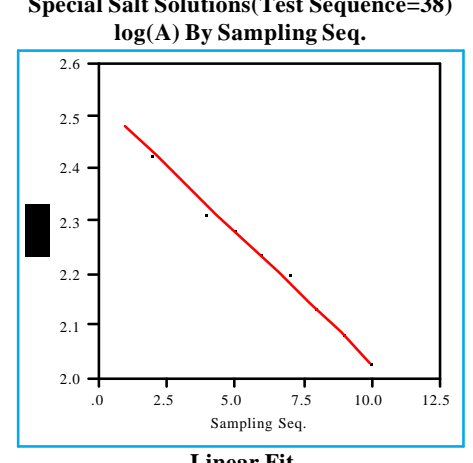

Linear Fit
$\log (\mathrm{A})=2.53148-0.05018$ Sampling Seq

$\begin{array}{ll}\text { RSquare } & 0.992759 \\ \text { RSquare Adj } & 0.991854 \\ \text { Root Mean Squar Error } & 0.013786\end{array}$

Root Mean Square Error $\quad 0.013786$

Mean of Response

2.243523

Observations (or Sum Wgts)

Term Estimate Std Error t Ratio Prob $>|\mathbf{t}|$

$\begin{array}{llllll}\text { Intercept } & 2.5199873 & 0.009418 & 267.59 & <.0001\end{array}$

$\begin{array}{lllll}\text { Sampling Seq. } & -0.050266 & 0.001518 & -33.12 & <.0001\end{array}$ RSquare Summary of Fit RSquare Adj $\quad 0.991372$ Root Mean Square Error $\quad 0.014165$ Mean of Response
Observations (or Sum Wgts)

$\begin{array}{lrrrrr} & \text { Parameter Estimates } & & \\ \text { Term } & \text { Estimate } & \text { Std Error } & \text { t Ratio } & \text { Prob }>|\mathbf{t}| \\ \text { Intercept } & 2.5314849 & 0.009677 & 261.61 & <.0001\end{array}$ $\begin{array}{lrrrr}\text { Intercept } & 2.5314849 & 0.009677 & 261.61 & <.0001 \\ \text { Sampling Seq. } & -0.050176 & 0.00156 & -32.17 & <.0001\end{array}$ pecial Salt Solutions $($ Test Sequence $=37)$ $\log (\mathrm{A})$ By Sampling Seq.

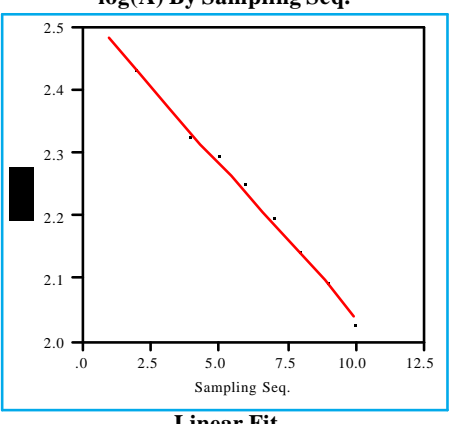

Special Salt Solutions(Test Sequence $=39$ )

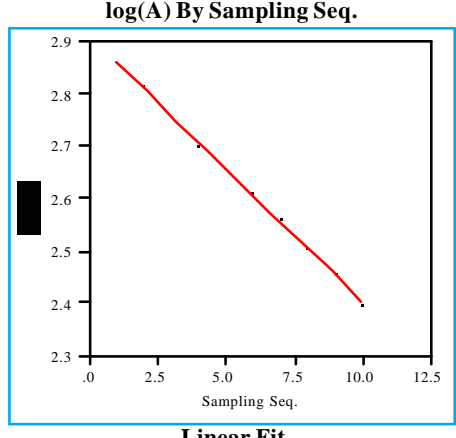

$\log (\mathrm{A})=2.91157-0.05096$ Sampling Seq.

\begin{tabular}{ll}
\multicolumn{1}{c}{ Summary of Fit } \\
RSquare & 0.997539 \\
RSquare Adj & 0.997231 \\
Root Mean Square Error & 0.008129 \\
Mean of Response & 2.631274 \\
Ore &
\end{tabular}

Mean of Response

0.097231

Observations (or Sum Wgts)

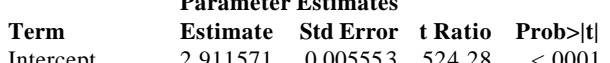

$\begin{array}{llllll}\text { Intercept } & 2.911571 & 0.005553 & 524.28 & <.000 \\ \text { Sampling Seq. } & -0.050963 & 0.000895 & 56.94 & <.0001\end{array}$

Special Salt Solutions(Test Sequence $=40$ )

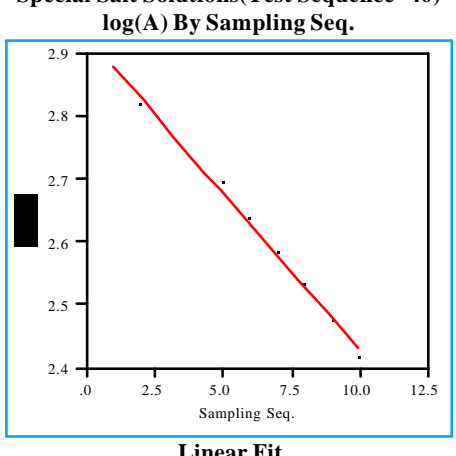

$\log (A)=2.92803-0.04985$ Sampling Seq. $\log (A)=2.92803-0.04985$ Sampling Seq.
Summary of Fit RSquare

RSquare Adj

Root Mean Square Erro
Mean of Response

0.996607

0.00934

Observations (or Sum Wgts)

2.653859

Term Parameter Estimates

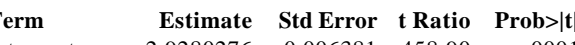
Sampling Seq. $-0.049849 \quad 0.001028$ 
Exhibit B58: $\log (\mathrm{A})$ versus Sampling Sequence for the Special Salt Studies: Tests 41-48

Special Salt Solutions(Test Sequence $=41$ )

$\log (A)$ By Sampling Seq.

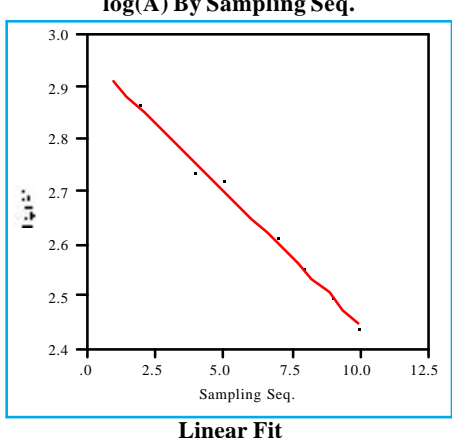

$\log (\mathrm{A})=2.95945-0.05127$ Sampling Seq

Summary of Fit

RSquare Adj

Root Mean Square Error

0.995274

Mean of Response

0.994683

2.677442

Observations (or Sum Wgts)

10

Term Estimate Std Error t Ratio Prob>|t|

$\begin{array}{lllll} & -0.05 & <001\end{array}$

Special Salt Solutions(Test Sequence $=42$ )

$\log (\mathbf{A})$ By Sampling Seq.

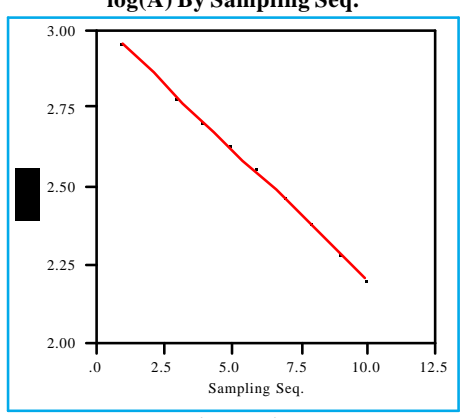

Linear Fit
$\log (\mathrm{A})=3.03505-0.083$ Sampling Seq.

RSquare

Summary of Fit

Root Mean Square Error

0.998427

Mean of Response

0.99823

Observations (or Sum Wgts)

2.578527$$
10
$$

Term

Parameter Estimate

$\begin{array}{llll}2 & \text { Partio } & \text { Prob }>|t|\end{array}$

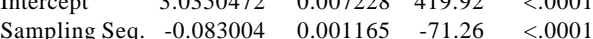

Special Salt Solutions $($ Test Sequence $=43$ )

$\log (\mathrm{A})$ By Sampling Seq.

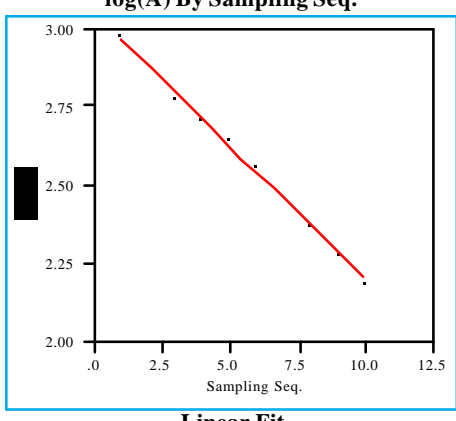

$\log (\mathrm{A})=3.05099-0$.

$5099-0.08495 \mathrm{~S}$

$\begin{array}{ll}\text { RSquare } & 0.996513 \\ \text { RSquare Adj } & 0.996077 \\ \text { Root Mean Square Error } & 0.016138 \\ \text { Mean of Response } & 2.583741\end{array}$

Parameter Estimates

Term Estimate Std Error t Ratio Prob $>|\mathbf{t}|$ $\begin{array}{lrrrrr} & 3.0509851 & 0.011024 & 276.76 & <.0001 \\ \text { Sampling Seq. } & -0.084954 & 0.001777 & -47.82 & <.0001\end{array}$

Special Salt Solutions(Test Sequence=44) $\log (\mathrm{A})$ By Sampling Seq.

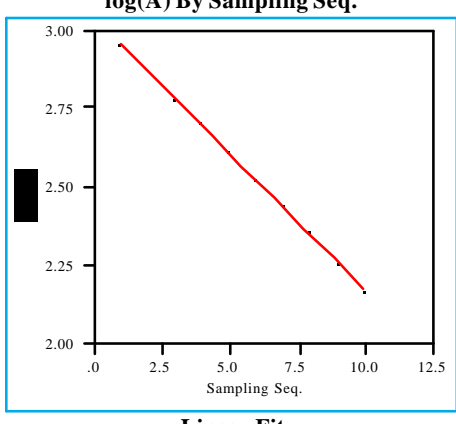

Linear Fit
$\log (\mathrm{A})=3.04218-0.08708$ Sampling Seq.

\begin{tabular}{ll}
\multicolumn{2}{c}{ Summary of Fit } \\
RSquare & 0.999421 \\
RSquare Adj & 0.999349 \\
Root Mean Square Error & 0.006729 \\
Mean of Response & 2.563257
\end{tabular}

Root Mean Square

0.9996729

Observations (or Sum Wgts)

Parameter Estimates

$\begin{array}{lrrrr}\text { Term } & \text { Estimate } & \text { Std Error } & \text { t Ratio } & \text { Prob }>|\mathbf{t}|\end{array}$

$\begin{array}{lrrrr}\text { Intercept } & 3.0421826 & 0.004597 & 661.83 & <.0001 \\ \text { Sampling Seq. } & -0.087077 & 0.000741 & -117.5 & <.0001\end{array}$
Special Salt Solutions $($ Test Sequence $=45$ )

$\log (\mathrm{A})$ By Sampling Seq.

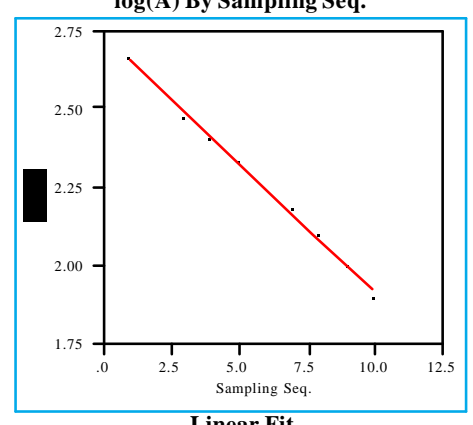

$\log (\mathrm{A})=2.73141-0.08127$ Sampling Seq$$
\text { Summary of Fit }
$$

RSquare

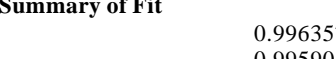

RSquare Adj

0.996357

0.01578

Mean of Respons

2.284451

Observations (or Sum Wgts) 10

$\begin{array}{lrrrr}\text { Term } & \text { Estimate } & \text { Std Error } & \text { t Ratio } & \text { Prob }>|t|\end{array}$ $\begin{array}{lrrrrr} & & & \\ \text { Sampling Seq. } & -0.081265 & 0.001737 & -46.77 & <.0001\end{array}$

Special Salt Solutions(Test Sequence $=46$ )

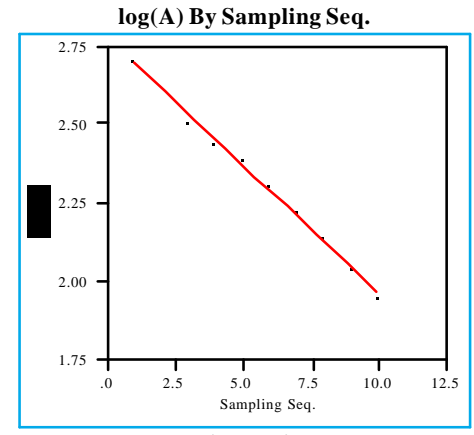

Linear Fit

$\log (A)=2.77329-0.08107$ Sampling Seq

\begin{tabular}{lrrrr}
\multicolumn{2}{c}{ Summary of Fit } & \\
RSquare & & 0.996091 \\
RSquare Adj & & 0.995603 \\
Root Mean Square Error & 0.016308 \\
Mean of Response & 2.327414 \\
Observations (or Sum Wgts) & \multicolumn{2}{c}{10} \\
\multicolumn{5}{c}{ Parameter Estimates } \\
Term & Estimate & Std Error & t Ratio & Prob>|t| \\
ntercept & 2.773288 & 0.01114 & 248.94 & $<.0001$ \\
lampling Seq. & -0.081068 & 0.001795 & -45.15 & $<.0001$
\end{tabular}

$\begin{array}{lllll}0.0114 & 248.94 & <.0001\end{array}$
Special Salt Solutions(Test Sequence=47)

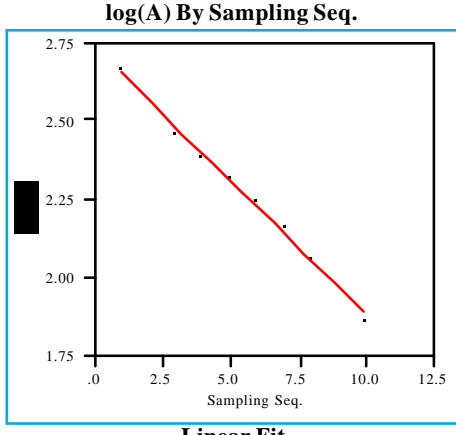

$\log (\mathrm{A})=2.73559-0.08516$ Sampling Seq.
Summary of Fit

RSquare

$\begin{array}{lr}\text { RSquare } & 0.994567 \\ \text { Root Mean Square Error } & 0.993888 \\ \text { Mean of Response } & 0.020211 \\ & 2.26724\end{array}$

Mean of Response

2.2672

Observations (or Sum Wgts)

$\begin{array}{lrrrr}\text { Term } & \text { Estimate } & \text { Std Error } & \text { t Ratio } & \text { Prob }>|t|\end{array}$

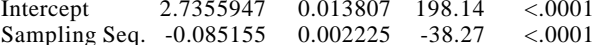

Special Salt Solutions(Test Sequence=48)

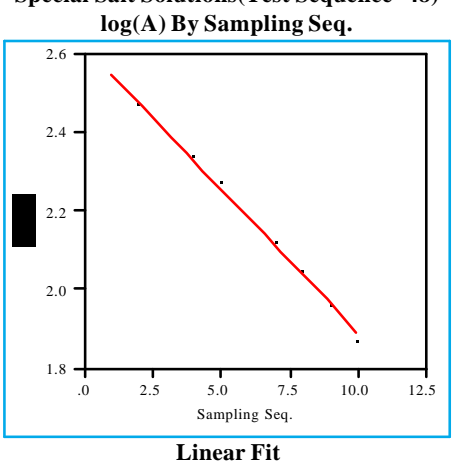

Linear Fit
$\log (\mathrm{A})=2.62227-0.07302$ Sampling Seq.

$$
\begin{array}{r}
\log (A)= \\
\text { Summary of Fit }
\end{array}
$$

RSquare

Root Mean Square Error

0.996372

Observations (or Sum Wgts)

0.995918

Parameter Estimates

10

Term
Intercep

$\begin{array}{lrrrr} & \text { Partatio } & \text { Prob }>|t|\end{array}$

Sampling Seq $-0.073018 \quad 0.001558 \quad-46.87 \quad<.0001$ 
Exhibit B59: $\log (\mathrm{A})$ versus Sampling Sequence for the Special Salt Studies: Tests 49-54

Special Salt Solutions(Test Sequence=49) $\log ($ A) By Sampling Seq.

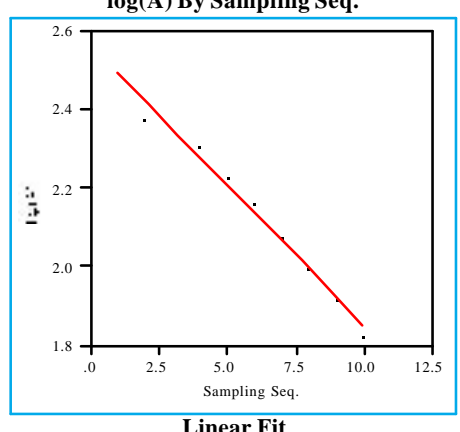

$\log (A)=2.56189-0.07087$ Sampling Seq. Summary of Fit

RSquare RSquare Adj

Root Mean Square Error

Mean of Response

0.986866

0.985224

Observations (or Sum Wgts)

0.026255

10

Term Estimate Std Error t Ratio Prob $>\mid \mathbf{t}$ $\begin{array}{llllll}\text { Intercept } & 2.5618901 & 0.017935 & 142.84 & <.0001\end{array}$ $\begin{array}{lllll}\text { Sampling Seq. } & -0.070868 & 0.002891 & -24.52 & <.0001\end{array}$

Special Salt Solutions(Test Sequence $=50$ ) $\log (\mathrm{A})$ By Sampling Seq.

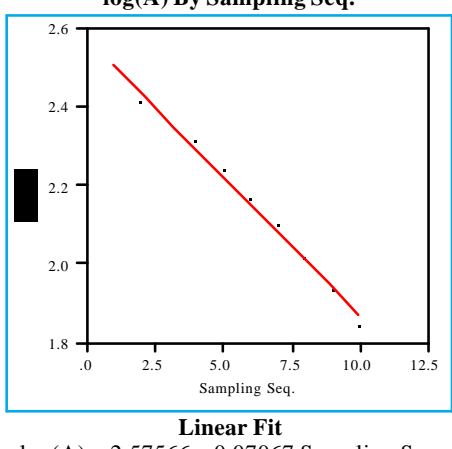

$\log (\mathrm{A})=2.57566-0.07067$ Sampling Seq.

\begin{tabular}{ll}
\multicolumn{2}{c}{ Summary of Fit } \\
RSquare & 0.994033 \\
RSquare Adj & 0.993287 \\
Root Mean Square Error & 0.017583 \\
Mean of Response & 2.186987
\end{tabular}

$\begin{array}{ll}\text { Root Mean Square Error } & 0.017583 \\ \text { Mean of Response } & 2.186987\end{array}$

Observations (or Sum Wgts)

10

Term Estimate Std Error t Ratio Prob $>|\mathbf{t}|$ \begin{tabular}{llllll}
2.5756646 & 0.012011 & 214.43 & $<.000$ \\
\hline
\end{tabular} $\begin{array}{lllll}\text { Sampling Seq. } & -0.070669 & 0.001936 & -36.51 & <.0001\end{array}$
Special Salt Solutions(Test Sequence=51)

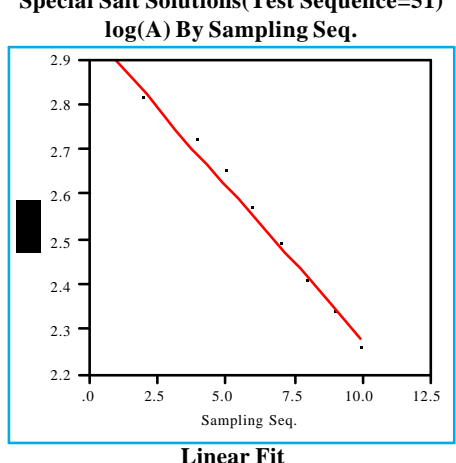

$\log (\mathrm{A})=2.96662-0.06873$ Sampling Seq Summary of Fit

$\begin{array}{lr}\text { RSquare } & 0.98635 \\ \text { RSquare Adj } & 0.984643 \\ \text { Root Mean Square Error } & 0.025966 \\ \text { Mean of Response } & 2.588588\end{array}$
Mean of Response

Term Estimate Std Error t Ratio Prob $>|\mathrm{t}|$ $\begin{array}{llllll}\text { Intercept } & 2.9666235 & 0.017738 & 167.24 & <.0001\end{array}$ $\begin{array}{lllll}\text { Sampling Seq. } & -0.068734 & 0.002859 & -24.04 & <.0001\end{array}$

Special Salt Solutions(Test Sequence $=52$ )

$\log ($ A) By Sampling

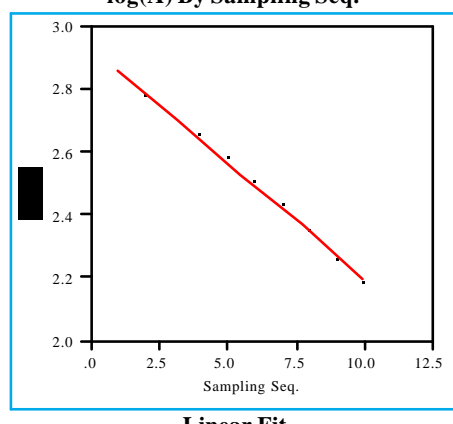

$\log (\mathrm{A})=2.93514-0.07354$

Sum 0.07354 Sampling Seq

$\begin{array}{lr}\text { RSquare } & 0.99617 \\ \text { RSquare Adj } & 0.995691 \\ \text { Root Mean Square Error } & 0.014644 \\ \text { Mean of Response } & 2.530668\end{array}$

Mean of Response

2.530668

Observations (or Sum Wgts)

10

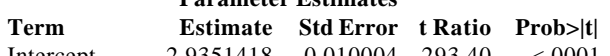

$\begin{array}{llllll}2.9351418 & 0.010004 & 293.40 & <.0001\end{array}$
Special Salt Solutions $($ Test Sequence $=53$ ) $\log (\mathrm{A})$ By Sampling Seq.

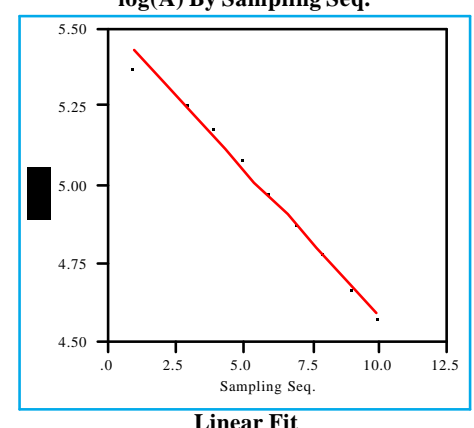

$\log (\mathrm{A})=5.51939-0.09304$ Sampling Seq Summary of Fit

\section{RSquare}

RSquare Adj

0.992069

Root Mean Square Error

Mean of Response

0.02671

Observations (or Sum Wgts)

5.00769

Parameter Estimates

Term Estimate Std Error t Ratio Prob $>|t|$ $\begin{array}{llllll}\text { Intercept } & 5.5193854 & 0.018249 & 302.45 & <.0001\end{array}$ $\begin{array}{lllll}\text { Sampling Seq. } & -0.093035 & 0.002941 & -31.63 & <.0001\end{array}$

Special Salt Solutions(Test Sequence $=54$ )

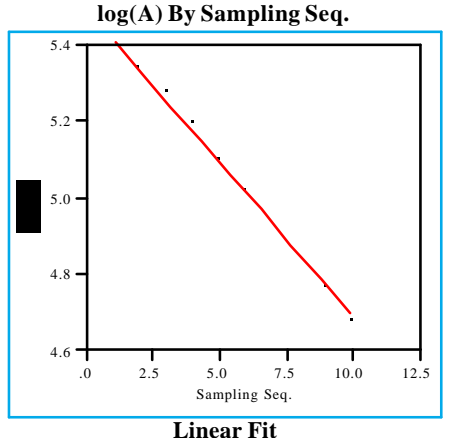

$\log (\mathrm{A})=5.49186-0.0793$ Sampling Seq

RSquare Summary of Fit

\subsection{5}

RSquare Adj 0.991736

Root Mean Square Error $\quad 0.02190$

Mean of Response

5.055733
10

Observations (or Sum Wgts)

Estimate Std Error t Ratio Prob $>|\mathbf{t}|$

$\begin{array}{lrrrr}\text { Intercept } & 5.4918622 & 0.014964 & 367.01 & <.0001 \\ \text { Sampling Seq. } & -0.079296 & 0.002412 & -32.88 & <.0001\end{array}$
Special Salt Solutions(Test Sequence $=55$ ) $\log (A)$ By Sampling Seq.

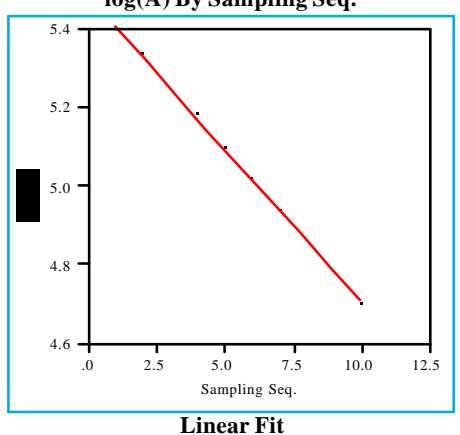

$\log (\mathrm{A})=5.48265-0.07769$ Sampling Seq

RSquare

RSquare Adj

Summary of Fit

Root Mean Square Error

Mean of Response

0.997247
0.996902

0.996902

5.055378

Observations (or Sum Wgts)

Term Estimate Std Error t Ratio Prob $>\mid \mathbf{t}$ $\begin{array}{llllll}\text { Intercept } & 5.4826495 & 0.008955 & 612.25 & <.0001\end{array}$ $\begin{array}{lllll}\text { Sampling Seq. } & -0.077686 & 0.001443 & -53.83 & <.0001\end{array}$

Special Salt Solutions(Test Sequence=56)

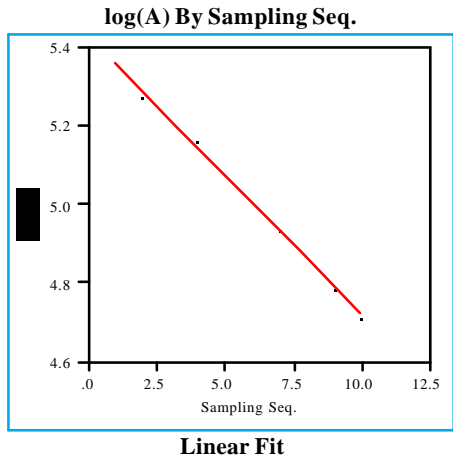

$\log (\mathrm{A})=5.4303-0.07113$ Sampling Seq.

\begin{tabular}{ll}
\multicolumn{1}{c}{ Summary of Fit } & \\
RSquare & 0.997078 \\
RSquare Adj & 0.996713 \\
Root Mean Square Error & 0.012364 \\
Mean of Response & 5.039112
\end{tabular}

Mean of Response

0.996713

$\begin{array}{lllll}\text { Term } & \text { Parameter Estimates } & & \\ \text { Estimate } & \text { Std Error } & \text { t Ratio } & \text { Prob }>|t|\end{array}$ $\begin{array}{lrrrrr} & \text { E.4303029 } & 0.008446 & 642.91 & <.000\end{array}$ 
Exhibit B60: $\log (\mathrm{A})$ versus Sampling Sequence for the Special Salt Studies: Tests 55-61

Special Salt Solutions(Test Sequence $=57$ )

$\log (\mathbf{A})$ By Sampling Seq.

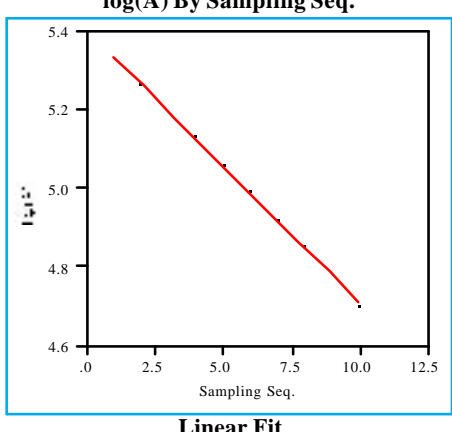

$\log (\mathrm{A})=5.40545-0.06974$ Sampling Seq

$$
\begin{array}{lr}
\multicolumn{1}{c}{\text { Summary of Fit }} \\
\text { RSquare } & 0.999277 \\
\text { RSquare Adj } & 0.999186 \\
\text { Root Mean Square Error } & 0.006026 \\
\text { Mean of Response } & 5.021877 \\
\text { Observations (or Sum Wgts) } & 10
\end{array}
$$

Observations (or Sum Wgts)

Term Estimate Std Error t Ratio Prob $>|\mathbf{t}|$ $\begin{array}{rrrr}5.4054469 & 0.004116 & 1313.1 & <.0001\end{array}$ Sampling Seq $\quad-0.06974 \quad 0.000663 \quad-105.1<000$

Special Salt Solutions(Test Sequence=58)

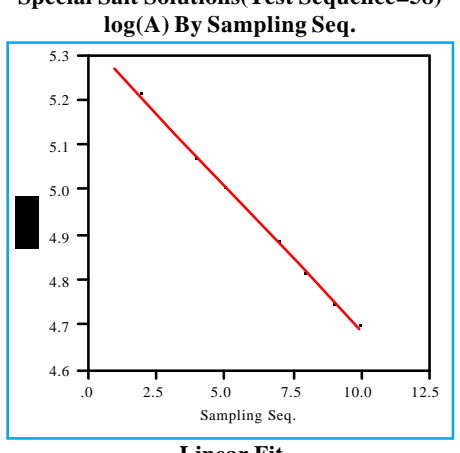

$\begin{aligned} \text { Linear Fit } & \\ \log (\mathrm{A})= & 5.3349-0.06457 \text { Sampling Seq }\end{aligned}$

Summary of Fit

0.998065

RSquare Adj

Root Mean Square Error

0.997823

Mean of Response

Observations (or Sum Wgts)

-10
10

Term Estimate Std Error t Ratio Prob $>|\mathbf{t}|$

$\begin{array}{llllll}\text { Intercept } & 5.3349043 & 0.006237 & 855.36 & <.000\end{array}$

$\begin{array}{lllll}\text { Sampling Seq. } & -0.064572 & 0.001005 & -64.24 & <.000\end{array}$
Special Salt Solutions(Test Sequence $=59$ )

$\log (A)$ By Sampling Seq.

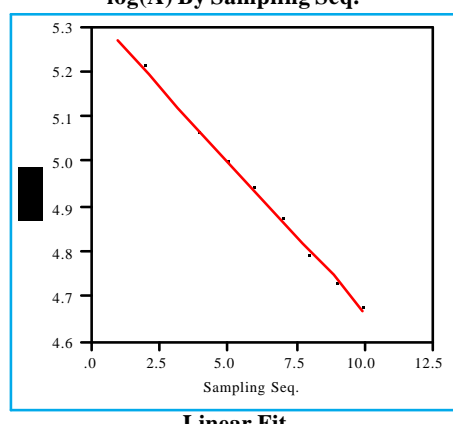

Linear Fit

$\log (\mathrm{A})=5.33543-0.06667$ Sampling Seq

$\begin{array}{lr}\text { RSquare } \quad \text { Summary of Fit } & \\ \text { RSquare Adj } & 0.99886 \\ \text { Root Mean Square Error } & 0.998718 \\ \text { Mean of Response } & 0.007233 \\ \text { Orens } & 4.968719\end{array}$

Mean of Response

4.968719

Observations (or Sum Wgts)

Term Estimate Std Error t Ratio Prob $>|\mathbf{t}|$

$\begin{array}{llllll}\text { Intercept } & 5.3354287 & 0.004941 & 1079.8 & <.0001\end{array}$

$\begin{array}{lllll}\text { Sampling Seq. } & -0.066675 & 0.000796 & -83.73 & <.0001\end{array}$

Special Salt Solutions (Test Sequence $=60$ )

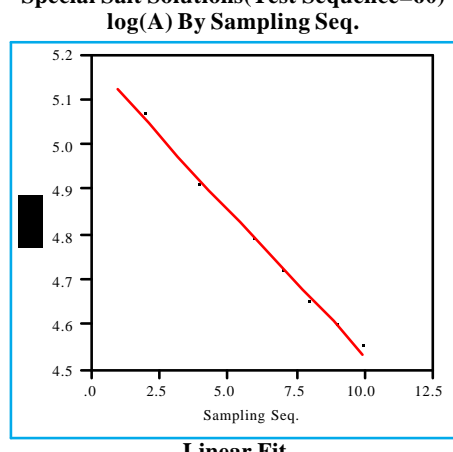

$\log (A)=5.18432-0.06512$ Sampling Seq

\begin{tabular}{lrrrr}
\multicolumn{3}{c}{ Summary of Fit } & \\
RSquare & & 0.996351 \\
RSquare Adj & & 0.995895 \\
Root Mean Square Error & 0.012655 \\
Mean of Response & 4.826186 \\
Observations (or Sum Wgts) & & 10 \\
\multicolumn{4}{c}{ Parameter Estimates } \\
m & Estimate & Std Error & $\mathbf{t}$ Ratio & Prob $>|\mathbf{t}|$ \\
rcept & 5.1843205 & 0.008645 & 599.71 & $<.0001$ \\
npling Seq. & -0.065115 & 0.001393 & -46.74 & $<.0001$
\end{tabular}

Special Salt Solutions $($ Test Sequence $=61)$

$\log (\mathrm{A})$ By Sampling Seq.

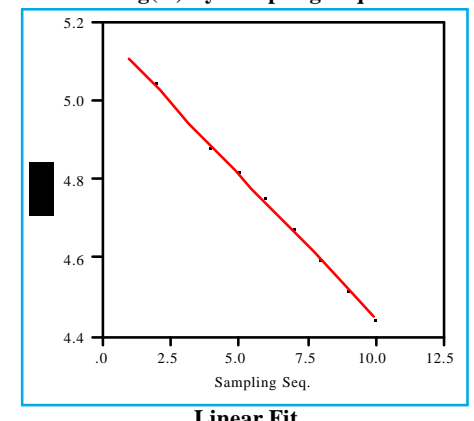

$\log (\mathrm{A})=5.17916-0.07307$ Sampling Seq

\begin{tabular}{lr} 
RSquare $\quad$ Summary of Fit & \\
RSquare Adj & 0.998269 \\
Root Mean Square Error & 0.998053 \\
Mean of Response & 0.009771 \\
Observations (or Sum Wgts) & 4.777249 \\
\hline$\quad 10$
\end{tabular}

Observations (or Sum Wgts)

Term Estimate Std Error t Ratio Prob $>\mid \mathbf{t}$

$\begin{array}{llllll}\text { Intercept } & 5.1791594 & 0.006675 & 775.95 & <.0001\end{array}$

$\begin{array}{lllll}\text { Sampling Seq. } & -0.073075 & 0.001076 & -67.93 & <.0001\end{array}$ 
Special Salt Solutions(Test Sequence=1) y By x

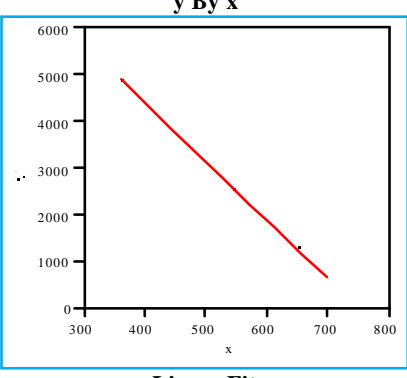

$y=9433.18-12.5263 x$

RSquare

Summary of Fit

RSquare Adj

Root Mean Square Error

Observations (or Sum Wgts)

\subsection{8}

0.999392

36.22062

2951.076

Term Estimate Std Error t Ratio Prob $>|t|$ $\begin{array}{llllll}\text { Intercept } & 9433.1754 & 57.78845 & 163.24 & <.000 \\ & & -12.52625 & 0.109208 & -114.7 & <.000\end{array}$ $\begin{array}{llll}-12.52625 & 0.109208 & -114.7 & <.0001\end{array}$

Special Salt Solutions(Test Sequence $=2$ )

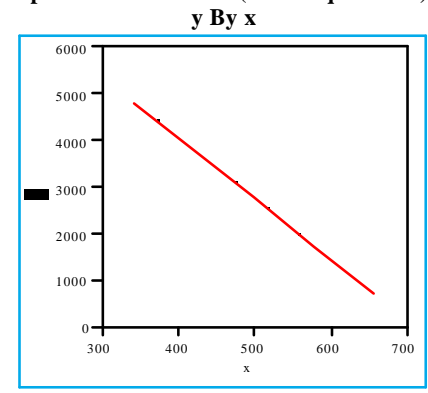
Linear Fit $y=9219.39-12.8765$

RSquare

RSquare Adj
Root Mean Square Error

Summary of

Mean of Response

0.999647

27.86794

Observations (or Sum Wgts)

Term Parameter Estimates

$\begin{array}{lrrrr} & \text { Estimate } & \text { Std Error } & \text { t Ratio } & \text { Prob }>|t|\end{array}$

$\begin{array}{lrrrrr} & & 9219.3893 & 45.63123 & 202.04 & <.0001\end{array}$

\section{Exhibit B61: y versus $x$ for the Special Salt Studies: Tests 1-8}

Special Salt Solutions(Test Sequence $=3$ )

y By x

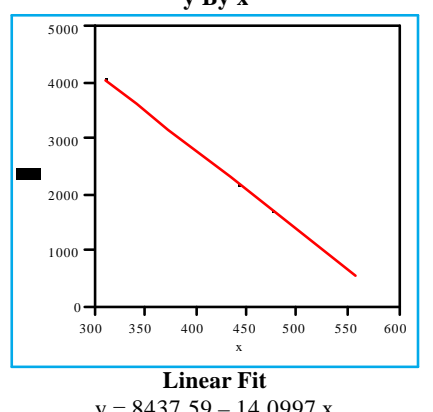

$y=8437.59-14.0997 x$

RSquare

RSquare Ad $j$

Root Mean Square Erro

Mean of Response

Observations (or Sum Wgts)

Term Estimate Std Error t Ratio Prob $>|\mathrm{t}|$ $\begin{array}{lrrrrr}\text { Intercept } & 8437.5946 & 62.29152 & 135.45 & <.0001 \\ \mathrm{X} & -14.0997 & 0.144811 & -97.37 & <.0001\end{array}$

Special Salt Solutions $($ Test Sequence $=4$ )

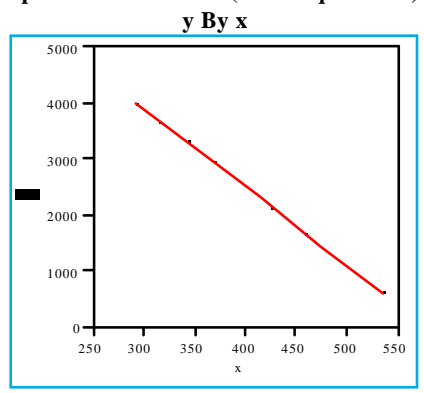

Linear Fit

$$
y=8102.54-13.9693
$$

Summary of Fit

RSquare

Error

Root Mean Square Error

Observations (or Sum Wgts)

Parameter Estimates

Estinate Std Error tRatio Prob>14

$\begin{array}{lrrrr}\text { Term } & \text { Estimate } & \text { Std Error } & \text { t Ratio } & \text { Prob }>|t| \\ \text { Intercept } & 8102.5378 & 33.40369 & 242.56 & <.0001\end{array}$ $\begin{array}{rrrr}8102.5378 & 33.40369 & 242.56 & <.0001 \\ -13.96935 & 0.080813 & -172.9 & <.0001\end{array}$

0.999766

0.999732
Special Salt Solutions(Test Sequence=5) y By x

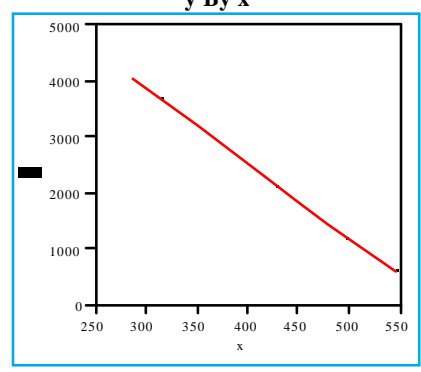

$$
\text { Linear Fit }
$$$$
y=7887.76-13.3721
$$

RSquare Summary of $F$

Rquare Adj 0.999058

Root Mean Square Erro

Observations (or Sum Wgts)

0.99892

37.82081
2448.782

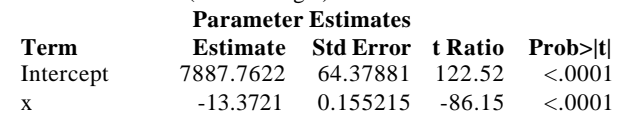

Special Salt Solutions(Test Sequence $=6$ )

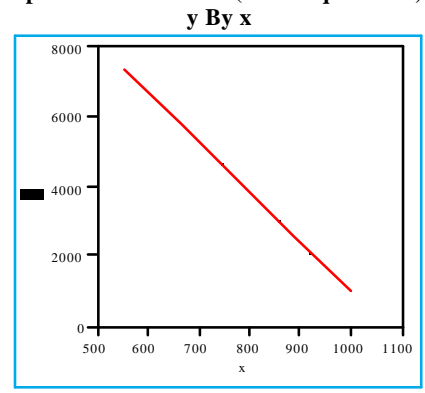

\section{Linear Fi}

$\mathrm{y}=15435.5-14.4218 \mathrm{x}$

RSquare

RSquare Adj

Error

Mean of Response

Observations (or Sum Wots)

Term Estimate Std Error t Ratio Prob>

$\begin{array}{lrrrr}\text { Intercept } & 15435.539 & 105.4776 & 146.34 & <.0001\end{array}$

$\begin{array}{lllll} & & & \\ \text { Intercept } & 15435.539 & 105.4776 & 146.34 & <.0001 \\ \mathrm{x} & -14.42181 & 0.136597 & -105.6 & <.0001\end{array}$
Special Salt Solutions(Test Sequence=7)

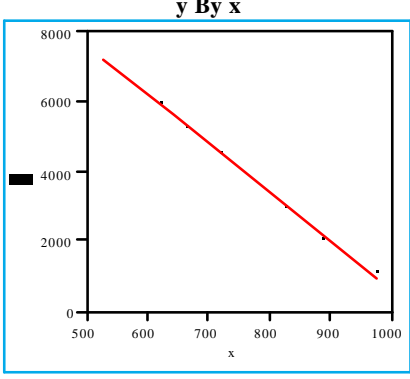

Linear Fi

$y=14607-13.9127$

RSquare

RSquare Adj

Root Mean Square Er

Mean of Response

Observations (or Sum Wgts)

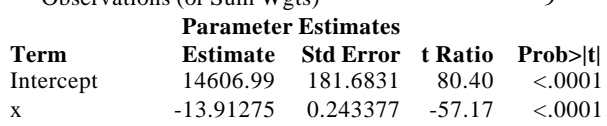

Special Salt Solutions $($ Test Sequence $=8$ )

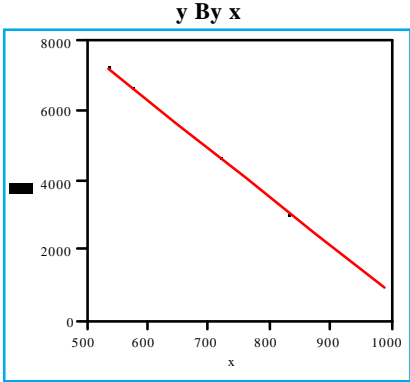

\section{Linear Fit}

$y=14609.4-13.8142 x$

Summary of Fit

RSquare

Error

0.997122

Root Mean Square E

0.996711

Observations (or Sum Wots)

4441.143

Term Estimate Std Error t Ratio Prob $>|t|$

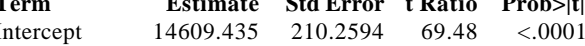

$\begin{array}{lllll}\mathrm{x} & -13.81422 & 0.280499 & -49.25 & <.0001\end{array}$ 


\section{Exhibit B62: y versus $x$ for the Special Salt Studies: Tests 9-16}

Special Salt Solutions $($ Test Sequence $=9$ )

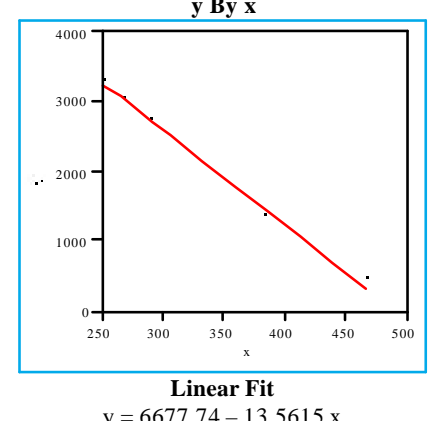

$y=6677.74-13.5615 x$

RSquare

Rquare Adj

Root Mean Square Error

Mean of Response

Observations (or Sum Wgts)

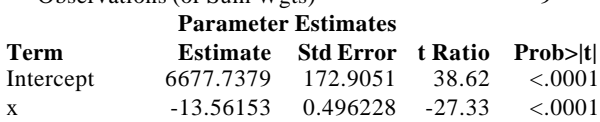

Special Salt Solutions(Test Sequence $=10)$

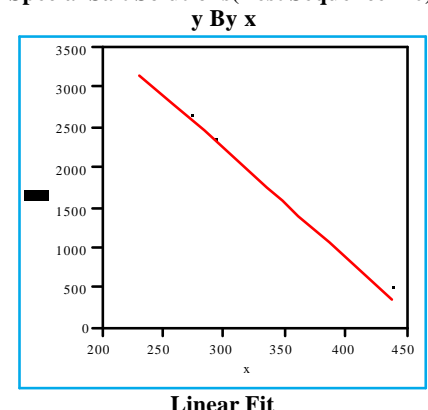

$y=6286.08-13.4761 x$

Summary of Fit

RSquare

RSquare Adj

Root Mean Square Error

Mean of Response

0.991932

Observations (or Sum Wgts)

Term Stimate Std Error t Ratio Prob $>|t|$

$\begin{array}{lrrrrr} & 6286.079 & 150.6708 & 41.72 & <.0001 \\ \mathrm{x} & -13.47609 & 0.459361 & -29.34 & <.0001\end{array}$
Special Salt Solutions(Test Sequence=11)

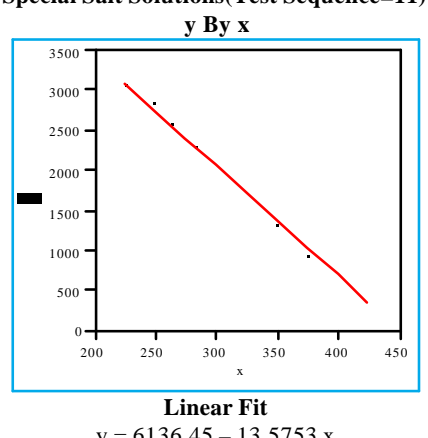

$y=6136.45-13.5753 x$

$\begin{array}{ll}\text { RSquare } \quad \text { Summary of Fit } & \\ \text { RSquare Adj } & 0.992214 \\ \text { Root Mean Square Error } & 0.991102 \\ \text { Mean of Response } & 83.14941 \\ \text { Oren } & 1894.877\end{array}$

Me

Observations (or Sum Wgts)

Parameter Estimates

Term Estimate Std Error t Ratio Prob $>\mid \mathbf{t}$

$\begin{array}{lrrrr}\text { Intercept } & 6136.4483 & 144.6914 & 42.41 & <.0001 \\ \mathrm{x} & -13.57534 & 0.454516 & -29.87 & <.0001\end{array}$

Special Salt Solutions(Test Sequence=12)

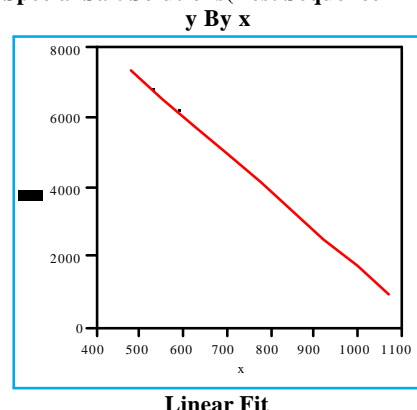

$12459.5-10.6715 x$

Summary of Fit

RSquare

RSquare Adj

Mean of Response

Observations (or Sum Wgts)

0.995847

0.995254
144.3445

4604.137

Parameter Estimates

Term Estimate Std Error t Ratio Prob $>\mid \mathbf{t}$

$\begin{array}{lrrrrr}\text { Intercept } & 12459.54 & 197.6851 & 63.03 & <.0001 \\ \mathrm{x} & -10.67148 & 0.260477 & -40.97 & <.0001\end{array}$
Special Salt Solutions $($ Test Sequence $=13$
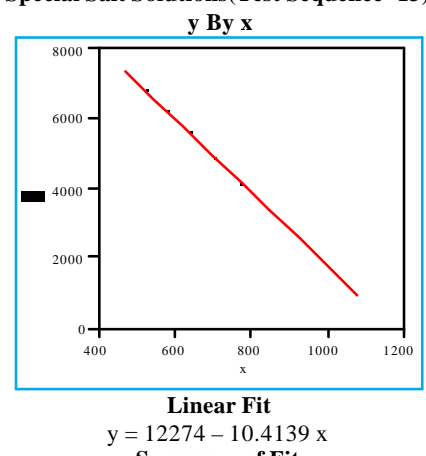

Summary of Fit

RSquare Adj

0.995972

Root Mean Square Erro

0.995397

Mean of Response

141.6886

Observations (or Sum Wgts)

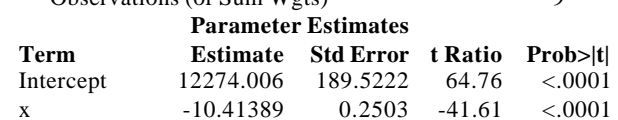

$\begin{array}{llll}-10.41389 & 0.2503 & -41.61 & <.0001\end{array}$

Special Salt Solutions(Test Sequence=14)

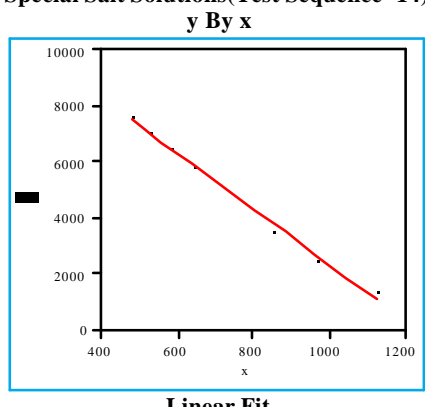

$y=12299.1-9.96608 x$

RSquare

RSquare A

Root Mean Square Error

Mean of Response

0.992633

Observations (or Sum Wgts)$$
182.9616
$$

Parameter Estimates

$\begin{array}{lrrrr} & \text { Estimate } & \text { Std Error } & \text { t Ratio } & \text { Prob }>|\mathbf{t}| \\ \text { Intercept } & 12299.079 & 235.5315 & 52.22 & <.000\end{array}$

$\begin{array}{lllrr}\mathrm{x} & -9.966083 & 0.303399 & -32.85 & <.0001\end{array}$
Special Salt Solutions(Test Sequence=15)

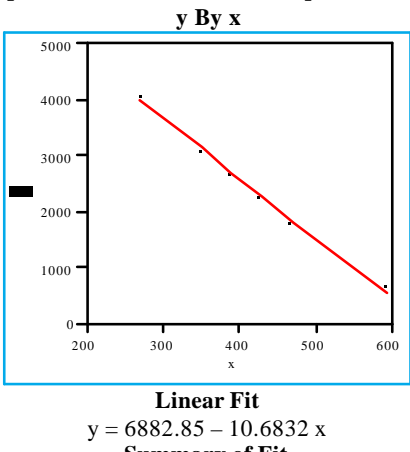

$\begin{array}{lr}\text { RSquare } \quad \text { Summary of Fit } & 0.994707 \\ \text { RSquare Adj } & 0.993951 \\ \text { Root Mean Square Error } & 89.48244 \\ \text { Mean of Response } & 2549.302 \\ \text { Observations (or Sum Wgts) } & 9\end{array}$

$$
\begin{aligned}
& \begin{array}{lrrrr} 
& \text { Parameter Estimates } & & \\
\text { Term } & \text { Estimate } & \text { Std Error } & \text { t Ratio } & \text { Prob }>\mid \text { t } \mid \\
\text { Intercept } & 6882.8498 & 123.1472 & 55.89 & <.0001
\end{array} \\
& \begin{array}{lrrrr} 
& 6882.8498 & 123.1472 & 55.89 & <.000
\end{array}
\end{aligned}
$$

Special Salt Solutions(Test Sequence=16)

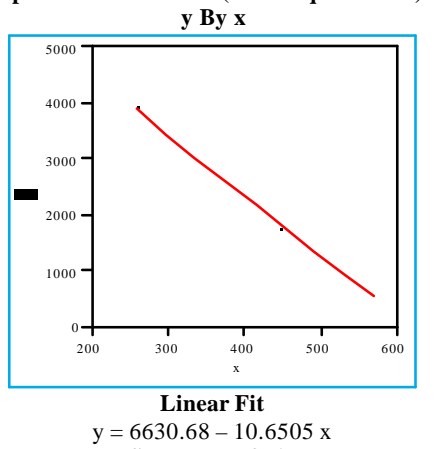

$=6630.68-10.6505$

RSquare

Root Mean Square Error

0.994612

Mean of Response

87.08709

Observations (or Sum Wgts)

2468.323
9

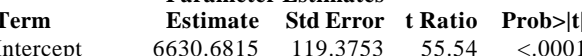

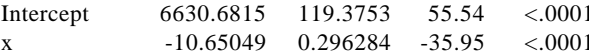


Exhibit B63: y versus $x$ for the Special Salt Studies: Tests 17-24

Special Salt Solutions(Test Sequence=17) y By x

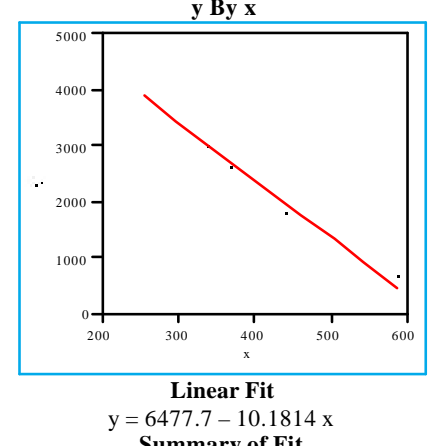

RSquare

Summ

RSquare Adj

Root Mean Square Erro

Mbservations (or Sum Wots)

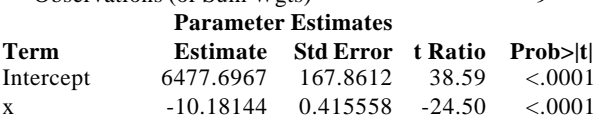

Special Salt Solutions(Test Sequence $=18)$

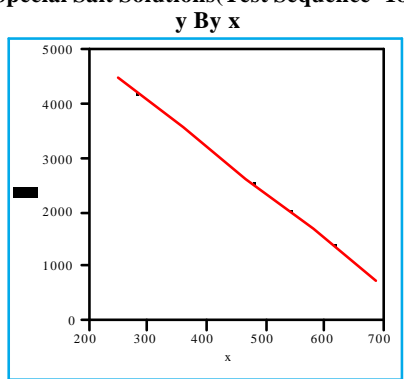

Linear Fit
$y=6602.15-8.44505 x$ Summary of Fit

RSquare

RSquare Adj

Root Mean Square Error

Mean of Response

0.999661

Observations (or Sum Wgts)

0.999612

25.33323

$\begin{array}{llll} & \text { Parameter Estimates } & \\ \text { Term } & \text { Estimate Std Error t Ratio Prob }>\mid 4\end{array}$

$\begin{array}{lrrrr}\text { Intercept } & 6602.1506 & 27.52021 & 239.90 & <.000 \\ & -8.45055 & 0.058816 & -143.6 & <.0001\end{array}$

$\begin{array}{rrrrr}6602.1506 & 27.52021 & 239.90 & <.0001 \\ -8.445055 & 0.058816 & -143.6 & <.000\end{array}$
Special Salt Solutions(Test Sequence=19)

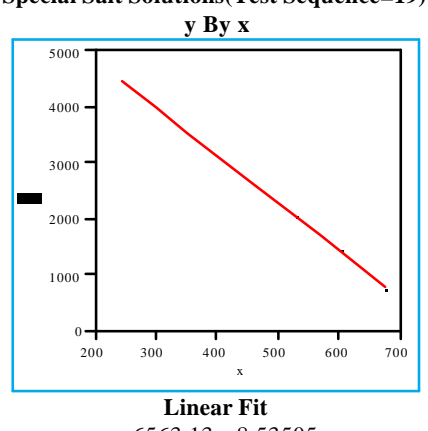

$y=6563.13-8.53505 x$

\begin{tabular}{ll}
\multicolumn{2}{c}{ Summary of Fit } \\
RSquare & 0.999806 \\
RSquare Adj & 0.999778 \\
Root Mean Square Error & 18.79823 \\
Mean of Response & 2828.436
\end{tabular}

Observations (or Sum Wgts)

\begin{tabular}{llrrrr} 
& \multicolumn{2}{l}{ Parameter Estimates } & & \\
Term & Estimate & Std Error & t Ratio & Prob $>|\mathbf{t}|$ \\
Intercept & 6563.127 & 20.63985 & 317.98 & $<.0001$
\end{tabular}

$\begin{array}{lllll}\text { lintercept } & 6563.127 & 20.63985 & 317.98 & <.0001\end{array}$

Special Salt Solutions $($ Test Sequence $=20)$

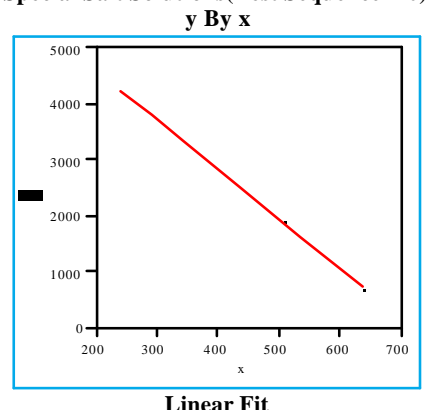

Linear Fit

$y=6355.96-8.80547 x$

RSquare

RSquare Adj

Mean of Response

Observations (or Sum Wgts)

0.99933

0.999234
33.31207

2680.336
9

Parameter Estimates

Term Estimate Std Error t Ratio Prob $>\mid t$

$\begin{array}{lrrrrr}\text { Intercept } & 6355.9617 & 37.65521 & 168.79 & <.0001 \\ \mathrm{x} & -8.80547 & 0.086197 & -102.2 & <.0001\end{array}$
Special Salt Solutions $($ Test Sequence $=21$ )

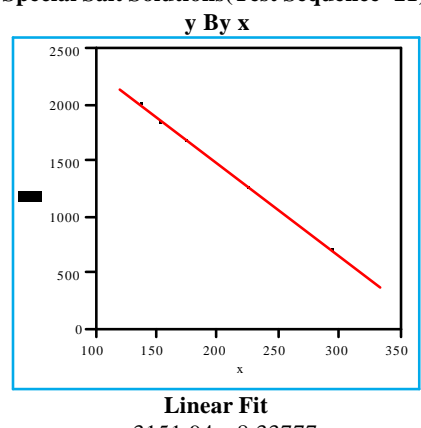

$$
\begin{gathered}
\text { Linear Fit } \\
y=3151.94-8.33777 x
\end{gathered}
$$

RSquare Summary of Fit $\quad 0.99941$

$\begin{array}{ll}0.999327 \\ \text { RSquare Adj } & 15.76361\end{array}$

Root Mean Square Erro

Observations (or Sum Wgts)

5.7636

\begin{tabular}{lrrrr}
\multicolumn{7}{c}{ Parameter Estimates } & & \\
Term & Estimate & Std Error & t Ratio & Prob $>|\mathbf{t}|$ \\
Intercept & 3151.9351 & 17.0048 & 185.36 & $<.0001$ \\
x & -8.337769 & 0.076497 & -109 & $<.0001$
\end{tabular}

Special Salt Solutions(Test Sequence $=22$

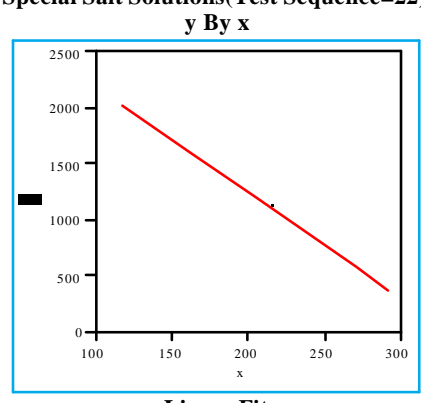

Linear Fit

$y=3131.74-9.38091 x$

RSquare

RSquare Adj

Mean of Response

Observations (or Sum Wgts)

0.998275 0.998028
25.42612

Term

Intercept

Estimate Std Error t Ratio Prob $>\mid \mathbf{t}$

$\begin{array}{rrrr}3131.7407 & 30.49584 & 102.69 & <.0001 \\ -9.380906 & 0.147409 & -63.64 & <.0001\end{array}$
Special Salt Solutions $($ Test Sequence $=23$ )

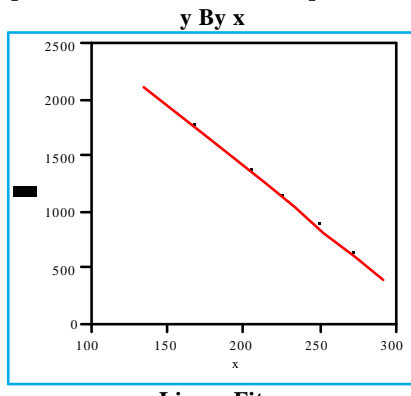

\section{Linear Fit}

$=3603.46-10.9314$

\begin{tabular}{llrrr}
\multicolumn{4}{c}{ Summary of Fit } & \\
RSquare & & 0.997568 \\
RSquare Adj & 0.997221 \\
Root Mean Square Error & 31.84127 \\
Mean of Response & 1300.776 \\
Observations (or Sum Wgts) & & 9 \\
\multicolumn{5}{c}{ Parameter Estimates } \\
erm & Estimate & Std Error & t Ratio & Prob $>|\mathbf{t}|$ \\
tercept & 3603.4558 & 44.26431 & 81.41 & $<.0001$ \\
& -10.93137 & 0.204003 & -53.58 & $<.0001$
\end{tabular}

$\begin{array}{lllll}3603.4558 & 44.26431 & 81.41 & <.0001\end{array}$

Special Salt Solutions $($ Test Sequence $=24)$

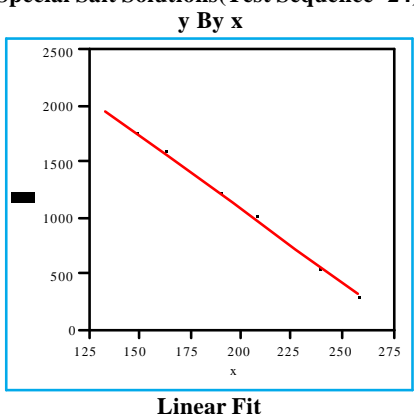

$y=3712.28-13.1221 x$

Summary of Fit

RSquare

Root Mean Square Error

Mean of Response

0.997078
0.99666

Observations (or Sum Wgts)

$\begin{array}{llll}\text { Term } & \text { Estimate } & \text { Std Error t Ratio Prob }>|\mathbf{t}|\end{array}$

$\begin{array}{lrrrr} & 3712.2753 & 53.27673 & 69.68 & <.0001 \\ \mathrm{X} & -13.12211 & 0.2685 & -48.87 & <.0001\end{array}$ 
Exhibit B64: y versus $x$ for the Special Salt Studies: Tests 24-32

Special Salt Solutions(Test Sequence $=25$ ) y By

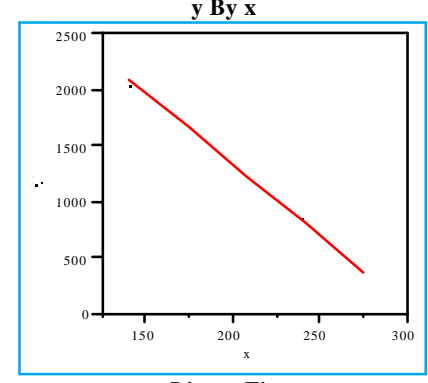

$$
\begin{aligned}
& \text { Linear Fit } \\
& y=3903.2-12.7955 x
\end{aligned}
$$

RSquare

Summ

Root Mean Square Error

Mean of Response

Observations (or Sum Wgts)

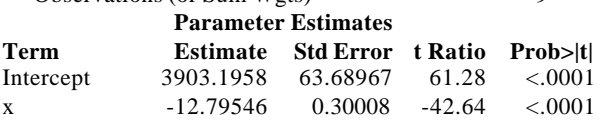

$x-12.79546 \quad 0.30008 \quad 42.64<.0001$

Special Salt Solutions(Test Sequence $=26)$

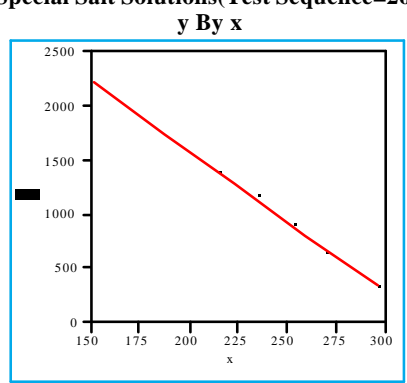

$$
\begin{gathered}
\text { Linear Fit } \\
y=4144.64-12.7631 x
\end{gathered}
$$

$=4144.64-12.7631$
Summary of Fit

RSquare

RSquare Adj

Root Mean Square Error

Mean of Response

0.997789

Observations (or Sum Wgts)

0.997473

1334.545

Term $\quad$ Parameter Estimates

Intercept Estimate Std Error t Ratio Prob $>\mid \mathbf{t}$

$\begin{array}{lrrrr}\text { Intercept } & 4144.6437 & 51.0931 & 81.12 & <.0001 \\ \mathrm{x} & -12.76313 & 0.227098 & -56.20 & <.0001\end{array}$
Special Salt Solutions(Test Sequence=27)

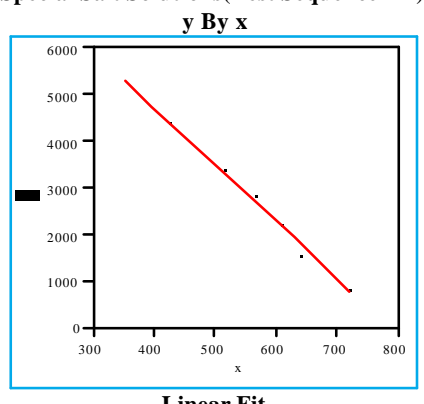

$$
\begin{gathered}
\text { Linear Fit } \\
y= \\
9534.96-12.0352
\end{gathered}
$$

RSquare Summary of Fit $\quad 0.994805$

RSquare Adj $\quad 0.994063$

Root Mean Square Erro

Observations (or Sum Wgts)

0.994063
115.4715

115.4715
3215.779

Observations (or Sum Wgts)
Parameter Estimate

Term Estimate Std Error $\quad$ t Ratio Prob $>\mid \mathbf{t}$

$\begin{array}{rrrr}9534.959 & 176.8344 & 53.92 & <.0001\end{array}$

Special Salt Solutions $($ Test Sequence $=28$ )

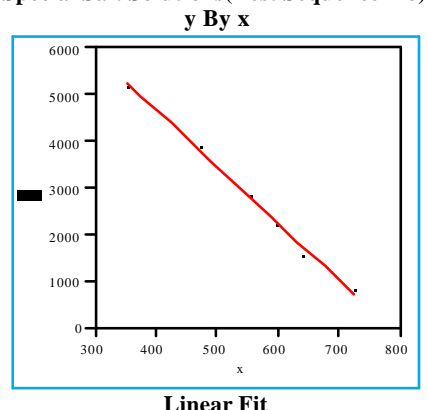

$9545.7-12.1407$

Summary of Fit

RSquare

RSquare Adj

Mean of Response

Observations (or Sum Wgts)

0.995623

0.994998

105.2499

Parameter Estimates

Term Estimate Std Error t Ratio Prob $>\mid t$

$\begin{array}{lrrrrr}\text { Intercept } & 9545.7023 & 162.864 & 58.61 & <.0001 \\ \mathrm{x} & -12.14066 & 0.304238 & -39.91 & <.0001\end{array}$
Special Salt Solutions $($ Test Sequence $=29$ )

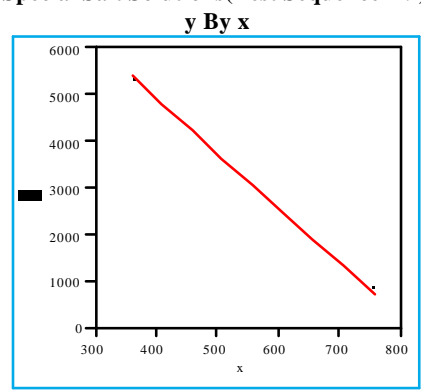

\section{Linear Fit}

$=9672.83-11.8083$

RSquare Summary of Fit $\quad 0.993016$

RSquare Adj 0.992018

Root Mean Square Erro

Observations (or Sum Wgts)

Observations (or Sum Wgts)
Parameter Estimates

\begin{tabular}{lrrrr}
\multicolumn{1}{c}{ Parameter Estimates } & & \\
Term & $\begin{array}{r}\text { Estimate } \\
\text { Std Error }\end{array}$ & t Ratio & Prob $>|\mathbf{t}|$ \\
Intercept & 9672.8349 & 206.6617 & 46.81 & $<.0001$ \\
x & -11.80829 & 0.374303 & -31.55 & $<.0001$
\end{tabular}

Special Salt Solutions $($ Test Sequence $=30$

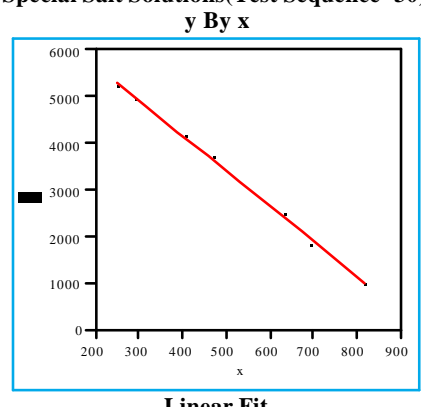

$y=7179.73-7.52037 x$

Summary of Fit

RSquare

Root Mean Square Error

Mean of Response

Observations (or Sum Wgts)

0.996189

0.995644

$\begin{array}{lrrrrr}\text { Term } & \text { Estimate } & \text { Std Error } & \text { t Ratio } & \text { Prob }>|t| \\ \text { Intercept } & 7179.7291 & 93.6107 & 76.70 & <.0001\end{array}$

$\begin{array}{lrrrr}\mathrm{x} & -7.520371 & 0.175811 & -42.78 & <.0001\end{array}$
Special Salt Solutions(Test Sequence $=31$ )

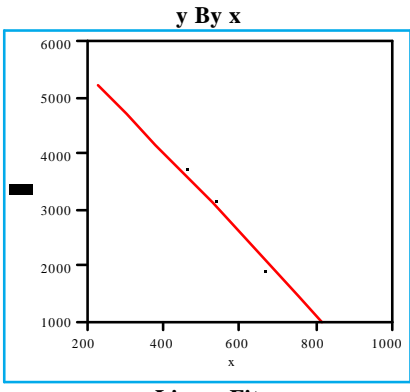

Linear Fit

$y=6930.92-7.17495$

$\begin{array}{lr}\text { RSquare } & 0.993085 \\ \text { RSquare Adj } & 0.992097 \\ \text { Root Mean Square Error } & 125.584 \\ \text { Mean of Response } & 3452.061\end{array}$

Parameter Estimates
Observations (or Sum Wgts

$\begin{array}{lrrrr}\text { Term } & \text { Estimate } & \text { Std Error } & \text { t Ratio } & \text { Prob }>|t|\end{array}$

$\begin{array}{llllll}\mathrm{x} & 630.9247 & 117.439 & 59.02 & <.0001\end{array}$

Special Salt Solutions(Test Sequence $=32$ )

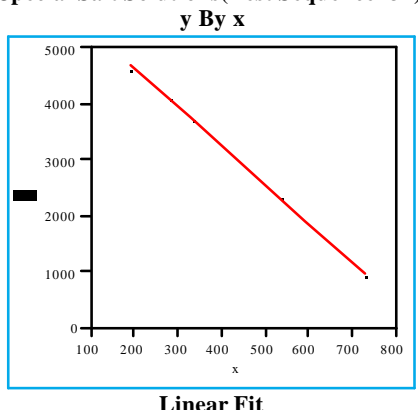

$6012.73-6.86496 x$

Summary of Fit

RSquare

RSquare Adj

Root Mean Square Error

0.997696

0.997367

Observations (or Sum Wgts) $\quad 3072.966$

Term $\quad \begin{array}{llll}\text { Parameter Estimates } & & \\ \text { Estimate } & \text { Std Error } & \mathbf{t} \text { Ratio } & \text { Prob }>|\mathbf{t}|\end{array}$

$\begin{array}{llllr}\text { Intercept } & 6012.7262 & 57.54527 & 104.49 & <.0001\end{array}$

$\begin{array}{llllll}\mathrm{x} & -6.864962 & 0.124683 & -55.06 & <.0001\end{array}$ 
Exhibit B65: y versus $x$ for the Special Salt Studies: Tests 33-40

Special Salt Solutions(Test Sequence $=33$ ) y By

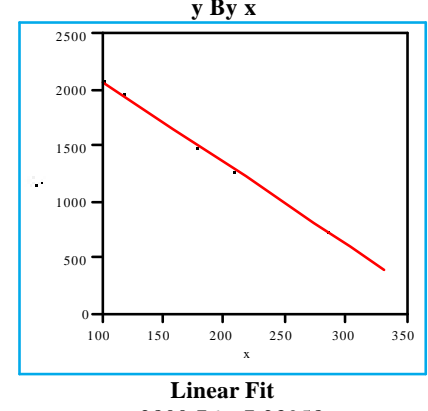

$y=2800.76-7.23958$

RSquare

RSquare Adj

Summary of Fit

Root Mean Square Error

Mean of Response

Observations (or Sum Wgts)

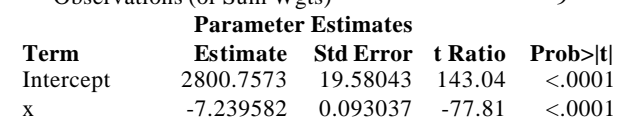

Special Salt Solutions(Test Sequence $=34)$

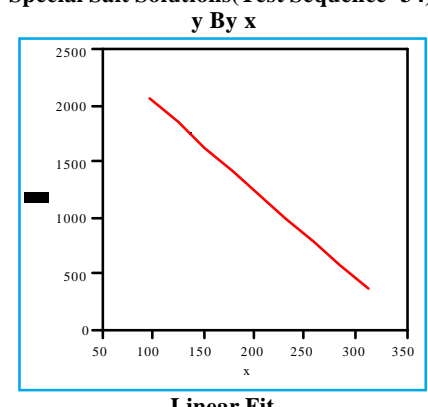

$2830.67-7.83279$

Summary of Fit

RSquare

RSquare Adj

Root Mean Square Error

Mean of Response

Observations (or Sum Wgts)
Parameter Estimat

\subsection{6}

0.993046

46.3977

Term Estimate Std Error t Ratio Prob $>|t|$

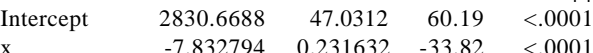

Special Salt Solutions(Test Sequence=35)

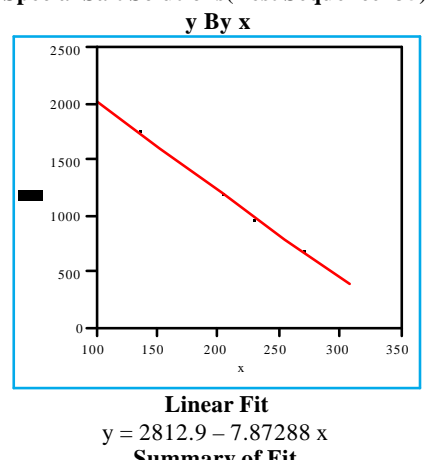
$\begin{aligned} & y= 2812.9-7.8728 \\ & \text { Summary of Fit }\end{aligned}$

RSquare

Root Mean Square Error

Mean of Response

Observations (or Sum Wgts)

Parameter Estimates

$\begin{array}{lrrrr} & \text { Estimate } & \text { Std Error } & \text { t Ratio } & \text { Prob }>|\mathbf{t}| \\ \text { Intercept } & 2812.9039 & 21.46851 & 131.02 & <.0001\end{array}$

$\begin{array}{lllll}\mathrm{x} & -7.872882 & 0.106192 & -74.14 & <.0001\end{array}$

Special Salt Solutions $($ Test Sequence $=36$ )

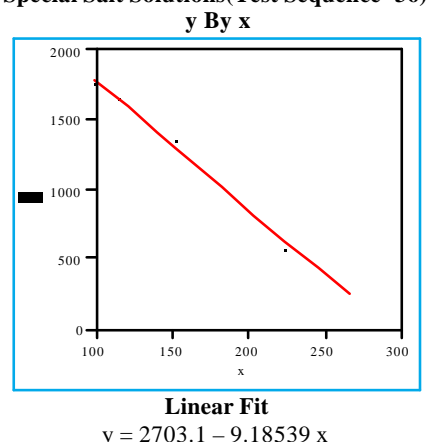

Summary of Fit

RSquare

RSquare Adj

Mean of Response

Observations (or Sum Wgts)

0.989784

0.988324

53.71888
1116.281

Parameter Estimates

Term Estimate Std Error t Ratio Prob $>\mid t$

$\begin{array}{lrrrr}\text { Intercept } & 2703.1025 & 63.51028 & 42.56 & <.0001 \\ \mathrm{x} & -9.185393 & 0.352718 & -26.04 & <.0001\end{array}$
Special Salt Solutions $($ Test Sequence $=37$ )

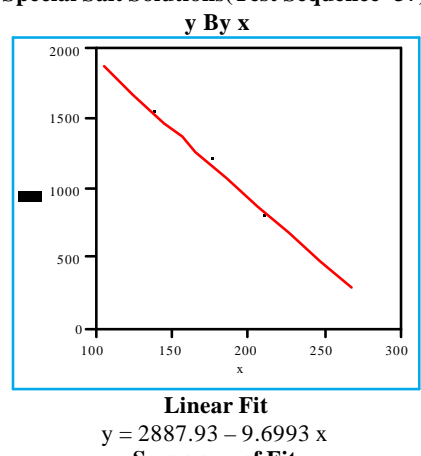

RSquare $\quad$ Summary of Fit $\quad 0.991687$

RSquare Adj

Root Mean Square Error $\quad 50.16136$

Observations (or Sum Wgts)

\begin{tabular}{lrrrr} 
& \multicolumn{2}{c}{ Parameter Estimates } & & \\
Term & Estimate & Std Error & t Ratio & Prob $>|t|$ \\
Intercept & 2887.9324 & 62.2933 & 46.36 & $<.0001$ \\
$x$ & -9.699297 & 0.33564 & -28.90 & $<.0001$
\end{tabular}

Special Salt Solutions(Test Sequence $=38$ )

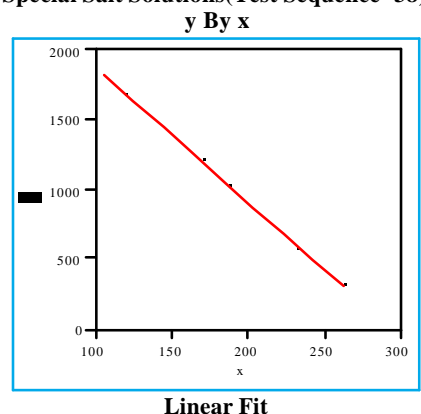

$=2836.91-9.58428$

Summary of Fit

RSquare

Root Mean Square Error

Mean of Response

0.995918

Observations (or Sum Wgts)

4.4825

151.64

Parameter Estimates

$\begin{array}{lrrrr} & \text { Estimate } & \text { Std Error } & \text { t Ratio } & \text { Prob }>|\mathbf{t}| \\ \text { Intercept } & 2836.9119 & 42.37102 & 66.95 & <.0001\end{array}$

$\begin{array}{lllrr}\mathrm{x} & -9.584278 & 0.231932 & -41.32 & <.0001\end{array}$
Special Salt Solutions(Test Sequence=39)

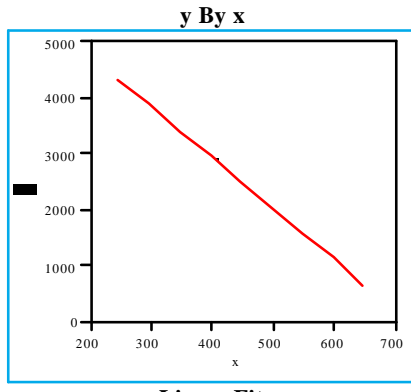

$$
\begin{gathered}
\text { Linear Fit } \\
\mathrm{y}=6555.97-9.04331 \mathrm{x} \\
\text { Summary of Fit }
\end{gathered}
$$

RSquare Summary of Fit

0.995865

RSquare Adj $\quad 0.995274$

Root Mean Square Error

Mean of Response

83.51961
2737.144

Observations (or Sum Wgts)

$$
\begin{array}{lrrrr}
\multicolumn{7}{c}{\text { Parameter Estimates }} & & \\
\text { Term } & \text { Estimate } & \text { Std Error } & \text { t Ratio } & \text { Prob }>|t| \\
\text { Intercept } & 6555.9692 & 97.08474 & 67.53 & <.0001 \\
\text { x } & -9.043306 & 0.22025 & -41.06 & <.0001
\end{array}
$$

Special Salt Solutions (Test Sequence $=40$ )

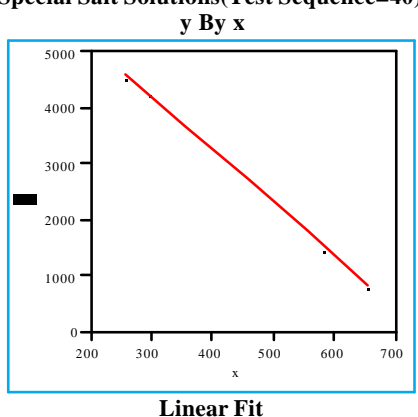

$y=7041.4-9.39792$

Summary of Fit

Square

Root Mean Square Error

Mean of Response

0.995315

Observations (or Sum Wgts)

$\begin{array}{lllll} & \text { Estimate } & \text { Std Error } & \text { t Ratio } & \text { Prob }>|t|\end{array}$

$\begin{array}{lrrrrr} & 7041.397 & 112.8801 & 62.38 & <.0001 \\ \mathrm{x} & -9.397917 & 0.243707 & -38.56 & <.0001\end{array}$ 


\section{Exhibit B66: y versus $x$ for the Special Salt Studies: Tests 41-48}

Special Salt Solutions(Test Sequence $=41$ ) y By x

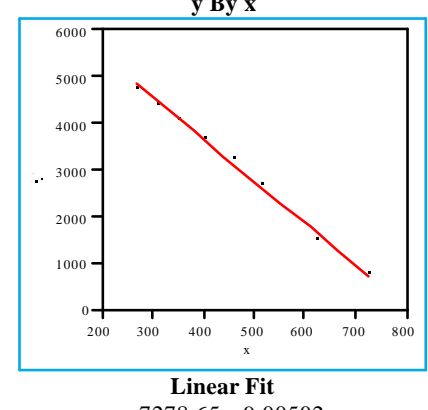

$$
\begin{gathered}
\text { Linear Fit } \\
y=7278.65-9.00502 x \\
\text { Summary of Fit }
\end{gathered}
$$

RSquare

Summary of Fit

Root Mean Square Erro

Mean of Response

Observations (or Sum Wgts)

$$
\begin{array}{lrrrr} 
& \text { Parameter Estimates } & & \\
\text { Term } & \text { Estimate } & \text { Std Error } & \text { t Ratio } & \text { Prob }>|\mathbf{t}| \\
\text { Intercept } & 7278.6547 & 140.7612 & 51.71 & <.0001 \\
\mathrm{x} & -9.005016 & 0.286927 & -31.38 & <.0001
\end{array}
$$

Special Salt Solutions(Test Sequence $=\mathbf{4 2}$ )

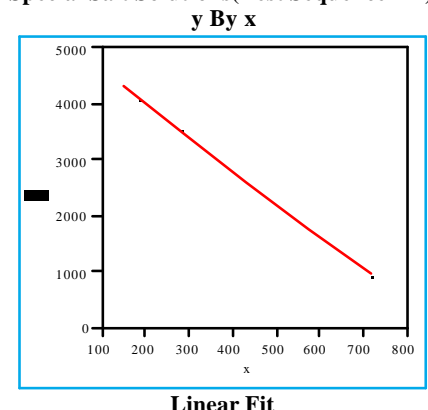

$y=5198.37-5.92149 x$

Summary of Fit

RSquare

RSquare Adj

Root Mean Square Error

Mean of Response

0.998745

Observations (or Sum Wgts)

0.998566

43.19212

Parameter Estimates

Term Estimate Std Error t Ratio Prob $>|\mathbf{t}|$

$\begin{array}{lrrrrr}\text { Intercept } & 5198.3657 & 33.89636 & 153.36 & <.0001 \\ \mathrm{x} & -5.921487 & 0.079322 & -74.65 & <.0001\end{array}$
Special Salt Solutions(Test Sequence $=43$ )

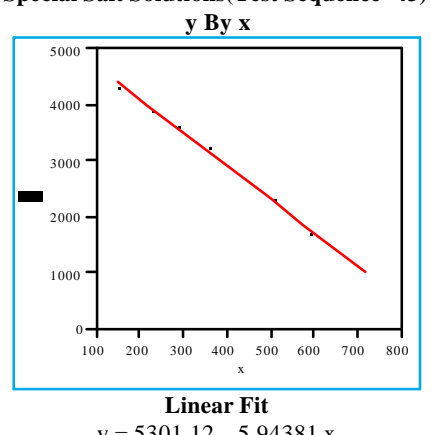

$y=5301.12-5.94381$

\begin{tabular}{lr} 
RSquare $\quad$ Summary of Fit & \\
RSquare Adj & 0.996514 \\
Root Mean Square Error & 0.996016 \\
Mean of Response & 72.86301 \\
Observations (or Sum Wgts) & 2982.79 \\
\hline Pars & 9
\end{tabular}

Parameter Estimates

$\begin{array}{lrrrr}\text { Term } & \text { Estimate } & \text { Std Error } & \text { t Ratio } & \text { Prob }>|t|\end{array}$

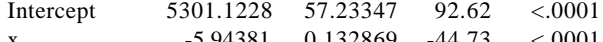

Special Salt Solutions $($ Test Sequence $=44$ )

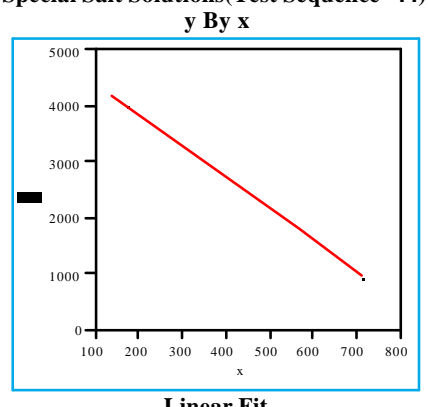

$y=4993.66-5.61006$

RSquare Root Mean Square Error $\quad 37.06673$ Mean of Response $\quad 2879.136$

Observations (or Sum Wgts)

Term Estimate Std Error t Ratio Prob $>|\mathbf{t}|$

$\begin{array}{lrrrr}\text { Intercept } & 4993.6591 & 27.8077 & 179.58 & <.0001\end{array}$

$\begin{array}{lllll}\mathrm{x} & -5.610064 & 0.066094 & -84.88 & <.0001\end{array}$
Special Salt Solutions $($ Test Sequence $=45$

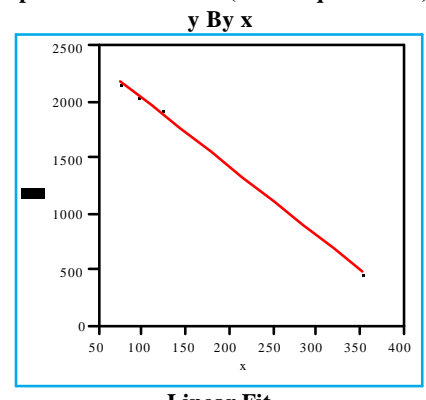

$$
\begin{array}{r}
\text { Linear Fit } \\
y=2659.61-6.13272
\end{array}
$$

$=2659.61-6.13272 \times$

\begin{tabular}{lr} 
RSquare & Summary of Fit \\
RSquare Adj & 0.997839 \\
Root Mean Square Error & 0.99753 \\
Mean of Response & 28.64144 \\
Oro & 1461.482 \\
\hline
\end{tabular}

$$
\text { Mean }
$$

Observations (or Sum Wgts)

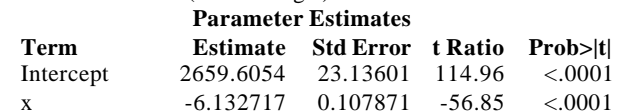

$\begin{array}{lllllll}-6.132717 & 0.107871 & -56.85 & <.0001\end{array}$

Special Salt Solutions(Test Sequence $=46$ )

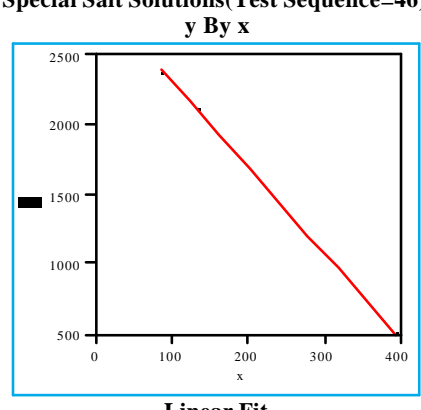

$=2931.46-6.11709 x$

Summary of Fit

RSquare

RSquare Adj

Mean of Response

Observations (or Sum Wgts)

0.996322
0.995796

Term

Intercept

Parameter Estimates

Std Error t Ratio Prob>|t|

$\begin{array}{rrrr}2931.4596 & 33.19141 & 88.32 & <.0001 \\ -6.117085 & 0.140482 & -43.54 & <.0001\end{array}$
Special Salt Solutions(Test Sequence $=47$ )

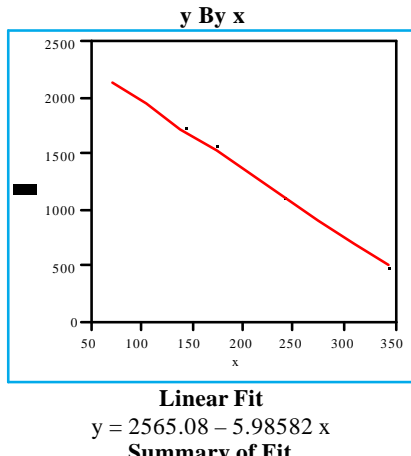

\begin{tabular}{ll}
\multicolumn{1}{c}{ Summary of Fit } & \\
RSquare & 0.996288 \\
RSquare Adj & 0.995757 \\
Root Mean Square Error & 36.25674 \\
Mean of Response & 1439.967
\end{tabular}

Observations (or Sum Wgts)

Parameter Estimates

Term Estimate Std Error t Ratio Prob $>|\mathbf{t}|$

$\begin{array}{lll}x-5.985816 & 0.138102 & -43.34\end{array}$

Special Salt Solutions(Test Sequence $=48$ )

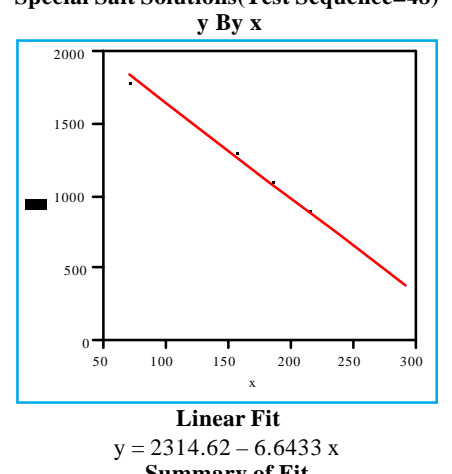

RSquare $\quad 0.997164$

Roure Ad

0.14761
1197.822

Observations (or Sum Wgts)

Parameter Estimates

Term Estimate Std Error t Ratio Prob $>\mid$ |

$\begin{array}{rrrrr} & 2314.6217 & 24.3896 & 94.90 & <.0001\end{array}$

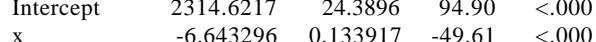


Exhibit B67: y versus $x$ for the Special Salt Studies: Tests 49-56

Special Salt Solutions(Test Sequence $=49$ )

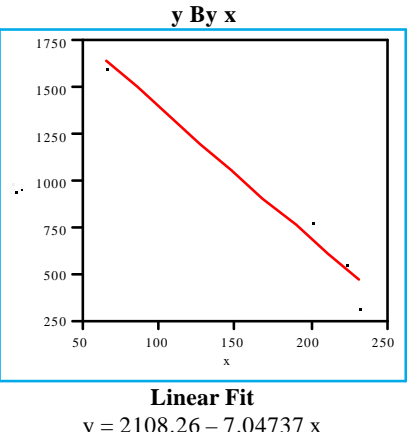

$y=2108.26-7.04737 x$

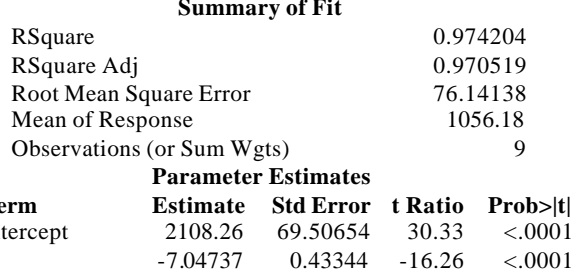

$\begin{array}{lrrrr} & 2108.26 & 69.50654 & 30.33 & <.000 \\ \text { Intercept } & -7.04737 & 0.43344 & -16.26 & <.000\end{array}$

Special Salt Solutions(Test Sequence $=50)$

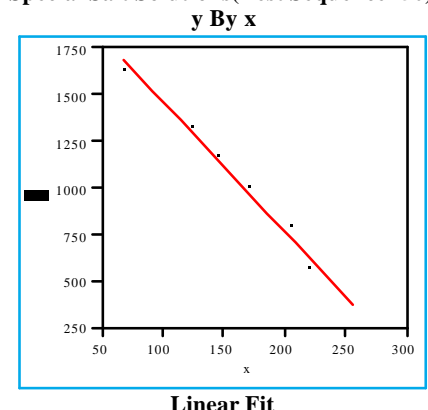

$y=2173.44-6.99345 x$

Summary of Fit

RSquare

RSquare Adj

Root Mean Square Error

Mean of Response

0.99172
0.990537

Observations (or Sum Wgts)

Parameter Estimates

Term Estimate Std Error t Ratio Prob>|t|

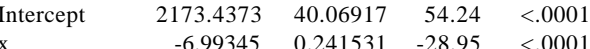

Special Salt Solutions (Test Sequence $=51$ )

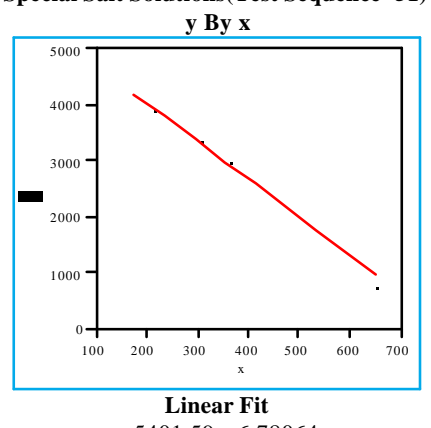

$y=5401.59-6.78064 x$

\begin{tabular}{ll}
\multicolumn{1}{c}{ Summary of Fit } & \\
RSquare & 0.989489 \\
RSquare Adj & 0.987988 \\
Root Mean Square Error & 128.3034 \\
Mean of Response & 2712.288
\end{tabular}

Observations (or Sum Wgts)

Parameter Estimates

$\begin{array}{lrrrr}\text { Term } & \text { Estimate } & \text { Std Error } & \text { t Ratio } & \text { Prob }>|t|\end{array}$

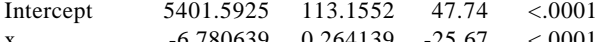

Special Salt Solutions(Test Sequence $=52$ )

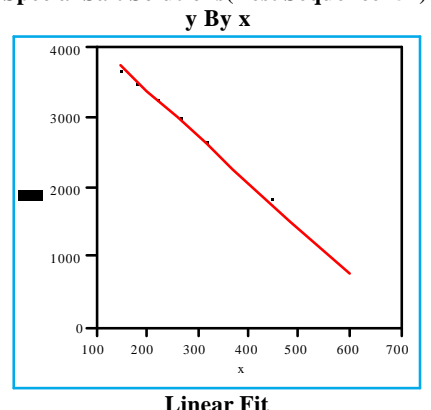

Linear Fit $63772 x$

Summary of Fit

RSquare

RSquare Adj

Mean of Response

0.997247

2450.015

Parameter Estimates

Term Estimate Std Error t Ratio Prob $>|t|$

$\begin{array}{lrrrrr}\text { Intercept } & 4704.5875 & 48.62651 & 96.75 & <.0001 \\ \mathrm{x} & -6.537718 & 0.129823 & -50.36 & <.0001\end{array}$
Special Salt Solutions $($ Test Sequence $=53$

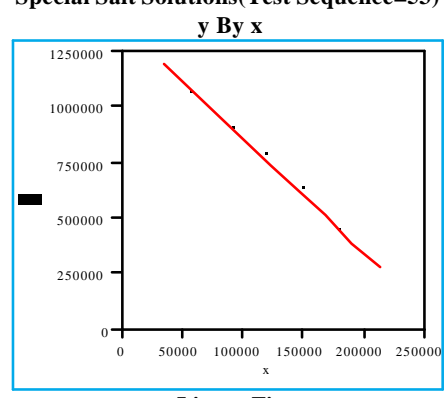

Linear Fit

$$
y=1382946-5.17008 x
$$

Summary of Fit

$$
\begin{array}{lr}
\text { RSquare } & 0.996353 \\
\text { RSquare Adj } & 0.995832 \\
\text { Root Mean Square Error } & 20927.9 \\
\text { Mean of Response } & 820644.5
\end{array}
$$

Observations (or Sum Wgts)
Parameter Estimates

$\begin{array}{lrrrr} & \text { Parameter Estimates } & & \\ \text { Term } & \text { Estimate } & \text { Std Error } & \text { t Ratio } & \text { Prob }>|\mathbf{t}| \\ \text { Intercept } & 1382945.8 & 14628.04 & 94.54 & <.0001 \\ \mathrm{x} & -5.170077 & 0.118218 & -43.73 & <.0001\end{array}$

Special Salt Solutions $($ Test Sequence $=54)$

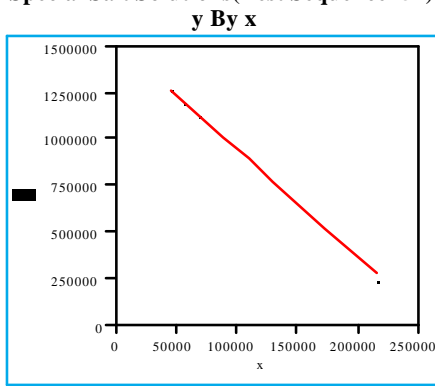

$$
\begin{gathered}
\text { Linear Fit } \\
y=1538184-5.84027 x \\
\text { Summary of Fit }
\end{gathered}
$$

RSquare Summary of Fit

Root Mean Square Error

Mean of Response

0.997131

Observations (or

0.99672

19951.09

(orsum Wgts)

Term Parameter Estimates

Estimate Std Error t Ratio Prob $>\mid \mathbf{t}$

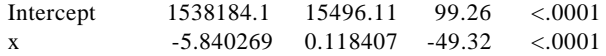

Special Salt Solutions(Test Sequence $=55$ )

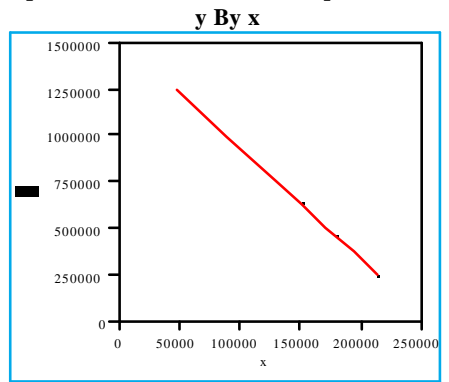

\section{Linear Fit}

$y=1542315-5.99378$

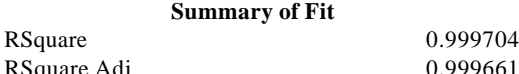

RSquare Adj $\quad 0.999661$

$\begin{array}{lr} & 6308.84 \\ \text { Root Mean Square Eror } & 842057.7\end{array}$

Observations (or Sum Wgts)

\begin{tabular}{lrrrr}
\multicolumn{7}{c}{ Parameter Estimates } & & \\
Term & Estimate & Std Error & t Ratio & Prob $>|t|$ \\
Intercept & 1542314.9 & 5018.966 & 307.30 & $<.0001$
\end{tabular}

$\begin{array}{llllll}\mathrm{x} & -5.993777 & 0.039006 & -153.7 & <.0001\end{array}$

Special Salt Solutions(Test Sequence $=56$ )

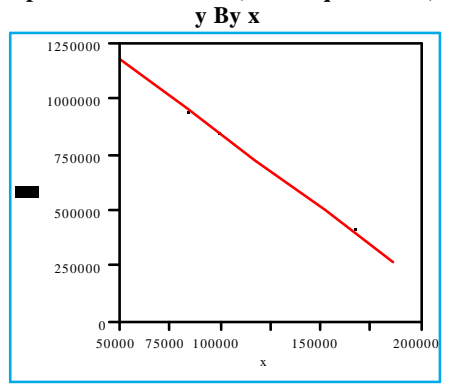

$$
\begin{gathered}
\text { Linear Fit } \\
y=1521878-6.69628 x \\
\text { Summary of Fit }
\end{gathered}
$$

RSquare

RSquare Adj

Root Mean Square Error

Mean of Response

Observations (or Sum Wgts)

0.995165

0.994475

780917.6

Term Estimate Std Error t Ratio Prob $>|\mathbf{t}|$

$\begin{array}{lllll}\text { Intercept } & 1521878 & 21114.82 & 72.08 & <.0001\end{array}$

$\begin{array}{lrrrr}\mathrm{x} & -6.696276 & 0.176407 & -37.96 & <.0001\end{array}$ 


\title{
Exhibit B68: y versus $x$ for the Special Salt Studies: Tests 57-61
}

Special Salt Solutions(Test Sequence $=57$ )

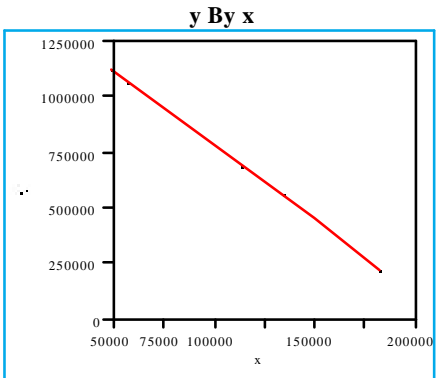

Linear Fit

$y=1461317-6.74504 x$

$\begin{array}{ll}\text { RSquare } & 0.999663 \\ \text { RSquare Adj } & 0.999615 \\ \text { Root Mean Square Error } & 6077.751 \\ \text { Mean of Response } & 746129.9\end{array}$

Mean of Response

Observations (or Sum Wgts)

$$
\begin{array}{lrrrr} 
& \text { Parameter Estimates } & & \\
\text { Term } & \text { Estimate } & \text { Std Error } & \text { t Ratio } & \text { Prob }>|t| \\
\text { Intercept } & 1461316.5 & 5362.666 & 272.50 & <.0001 \\
\mathrm{x} & -6.745036 & 0.046828 & -144 & <.0001
\end{array}
$$

Special Salt Solutions(Test Sequence $=58$ )

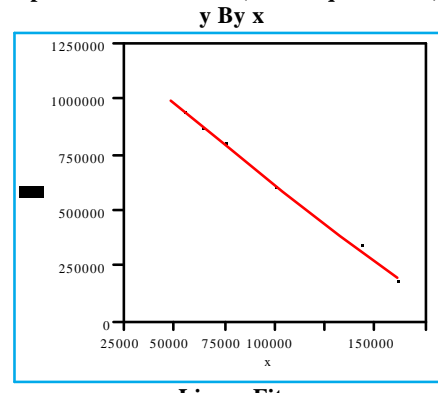

$$
\begin{gathered}
\text { Linear Fit } \\
=1333743-7.04204 x \\
\text { Summary of Fit }
\end{gathered}
$$

RSquare Summary of Fit

RSquare Adj

Root Mean Square Erro

Mean of Response

Observations (or Sum Wgts)

0.998053

0.997775

13097.74

658838.8

Parameter Estimates

Term Estimate Std Error t Ratio Prob $>\mid \mathbf{t}$

$\begin{array}{llllll}\text { Intercept } & 1333743 & 12083.37 & 110.38 & <.000\end{array}$

$\begin{array}{lllll}\mathrm{x} & -7.042043 & 0.117562 & -59.90 & <.0001\end{array}$
Special Salt Solutions $($ Test Sequence $=59$ )

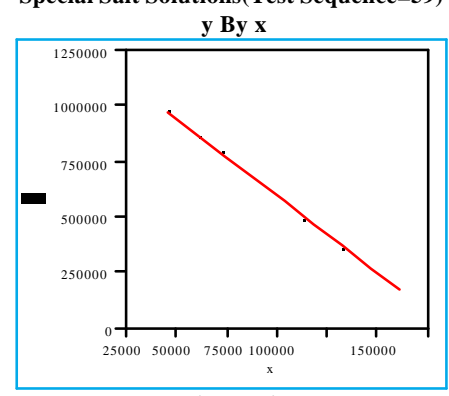

Linear Fit

$y=1298344-6.95273 x$

$\begin{array}{lll} & \text { Summary of Fit } & \\ \text { RSquare } & & 0.997811\end{array}$

RSquare Adj $\quad 0.997498$

Root Mean Square Error $\quad 13485.78$

Observations (or Sum Wgts)

$\begin{array}{llll} & \text { Parameter Estimates } & & \\ \text { Term } & \text { Estimate Std Error } & \text { t Ratio Prob }>|\mathbf{t}|\end{array}$

$\begin{array}{lrrrrr} & \text { Estimate } & \text { Std Error } & \text { t Ratio } & \text { Prob }>\mid \\ \text { Intercept } & 1298344.4 & 12304.27 & 105.52 & <.0001\end{array}$

$\begin{array}{lllll}\mathrm{x} & -6.952727 & 0.123096 & -56.48 & <.0001\end{array}$

Special Salt Solutions(Test Sequence $=60$ )

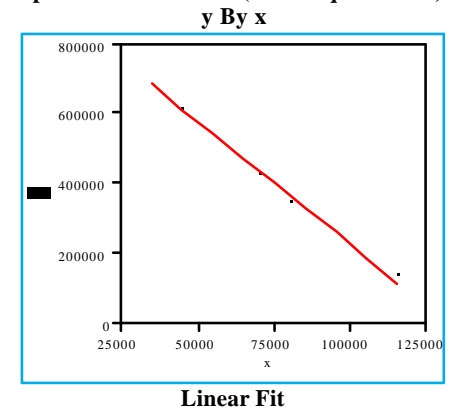

$y=\begin{aligned} & 934651-7.01618 \\ & \text { Summary of Fit }\end{aligned}$

RSquare

RSquare Adj $\quad 0.995411$

Root Mean Square Error $\quad 13893.53$

$\begin{array}{lr}\text { Mean of Response } & 467134.2 \\ \text { Observations (or Sum Wgts) } & 9\end{array}$

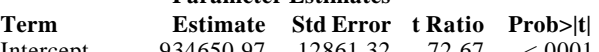

$\begin{array}{lrrrrr} & & \text { Estimate } & & \\ \text { Intercept } & 934650.97 & 12861.32 & 72.67 & <.0001 \\ \mathrm{x} & -7.016178 & 0.180067 & -38.96 & <.0001\end{array}$
Special Salt Solutions $($ Test Sequence $=61$

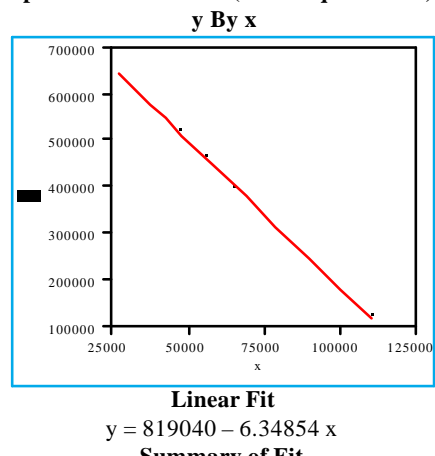

RSquare Summary of Fit $\quad 0.9976$

Root Mean Square Error $\quad 9271.835$

Mean of Response

Term Estimate $\begin{array}{llll}\text { Std Error } & \text { t Ratio } & \text { Prob }>|t|\end{array}$

$\begin{array}{llllll}\mathrm{x} & -6.348541 & 0.117696 & -53.94 & <.0001\end{array}$

\begin{abstract}
$-7.016178<0.18006-38.96<<001$
\end{abstract}




\section{DISTRIBUTION}

C. I. Aponte, 703-H

M. J. Barnes, 773-A

C. J. Berry, 704-8T*

C. S. Boley, 703-H

T. E. Britt, 703-H*

W. C. Clark, 704-56H

T. B. Edwards, 773-42A*

S. D. Fink, 773-A

F. F. Fondeur, 773-A

D. T. Hobbs, 773-A*

R. A. Jacobs, 704-3N

M. D. Johnson, 703-H

M. T. Keefer, 706-8C

B. L. Lewis, 703-H

T. J. Lex, 703-H

S. W. McCollum, 773-A

M. S. Miller, 704-56H

M. J. Montini, 703-H

J. P. Morin, 703-H

C. A. Nash, 773-42A

L. M. Papouchado, 773-A

S. F. Piccolo, 703-H

M. R. Poirier, 773-42A*

V. D. Stewart, 704-8T

L. A. Strong, 703-H

P. C. Suggs, 704-196N

R. F. Swingle, 773-A

W. L. Tamosaitis, 773-A

G. A. Taylor, $704-196 \mathrm{~N}$

T. C. Temple, 703-H

W. B. Van Pelt, 773-42A*

D. D. Walker, 773-A

W. R. Wilmarth, 773-42A

J. E. Young, 773-A*

LWP Files c/o C. Canada, 773-A*

STI, 703-43A*

* These recipients receive the main report and both appendices. Other recipients receive only the main report and Appendix A. 